\title{
Lehr- und Verhaltensstile von Sportlehrkräften: \\ Evasives Bewältigungsverhalten im Sportunterricht
}

\author{
Dissertation \\ zur Erlangung des sozialwissenschaftlichen Doktorgrades \\ der Sozialwissenschaftlichen Fakultät \\ der Georg-August-Universität Göttingen
}

vorgelegt von

Christian Belz

aus Fulda

Göttingen, 2012 
Erstgutachterin: Prof. Dr. Katja Schmitt

Zweitgutachter: Prof. Dr. Marcus Hasselhorn

Tag der mündlichen Prüfung: 08.03.2013 


\section{Inhaltsverzeichnis}

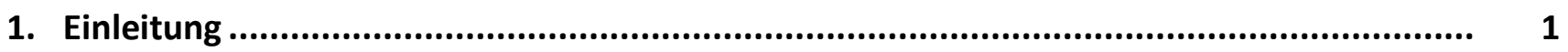

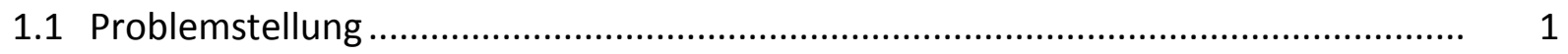

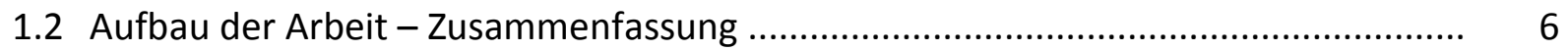

\section{Theoretischer Teil}

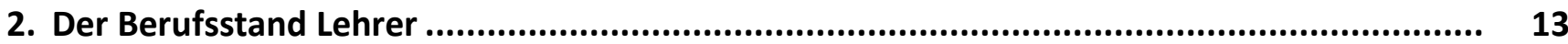

2.1 Motive zur Berufswahl - Rekrutierungscharakteristik im Lehrerberuf.......................... 13

2.2 Die Besonderheiten des Lehrberufs, Aufgaben, Antinomien und Rollenerwartungen 25

2.2.1 Die Institutionalisierung und Entwicklung des Lehrens und Lernens ....................... 29

2.2.2 Aufgabenspektrum von Lehrkräften .................................................................... 41

2.2.3 Antinomien, Rollenerwartungen und Rollenkonflikte im Lehrberuf........................... 48

2.2.4 Der Lehrerberuf in der Gesellschaft........................................................................ 52

2.3 Die besondere Bedeutung und Anforderungsstruktur des Sportunterrichts und des Sportlehrerberufs......................................................................................... 55

2.3.1 Die Entwicklung des Sportunterrichts in Deutschland .............................................. 56

2.3.2 Der Auftrag des Sportunterrichts ......................................................................... 60

2.3.3 Aufgabenspektrum und Arbeitsalltag von Sportlehrkräften..................................... 69

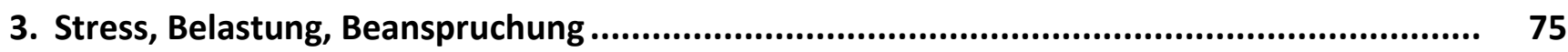

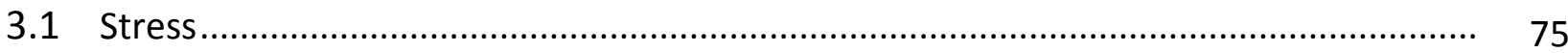

3.1.1 Reaktionsbezogene Stresskonzeption ............................................................... 76

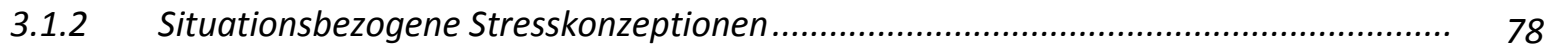

3.1.3 Stress als relationaler Begriff -Transaktionales Stresskonzept .................................. 79

3.2 Belastungs- und Beanspruchungsmodelle............................................................. 86

3.2.1 Das Belastungs-Beanspruchungsmodell von Rudow .............................................. 87

3.2.2 Das Modell des Lehrerstresses von Rudow ........................................................... 92

3.3 Belastungen des Lehrers im Allgemeinen und des Sportlehrers im Speziellen ............. 95

3.3.1 Arbeitsaufgaben und schulorganisatorische Bedingungen......................................... 97

3.3.2 Arbeitshygienische Bedingungen ..................................................................... 106

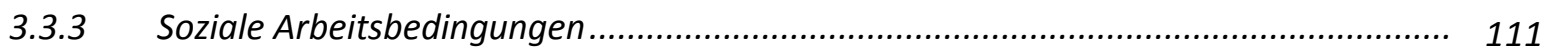

3.3.4 Gesellschaftlich-kulturelle Bedingungen ................................................................ 117 
4.1 Erste Überlegungen zu Bewältigungsprozessen - Beginn der Forschungsaktivitäten.... 120

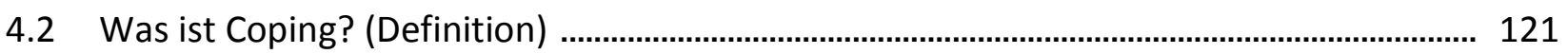

4.3 (Dispositionsähnlicher) Charakter von Coping!? ...................................................... 122

4.4 Formale Eigenschaften von Situationen ................................................................ 123

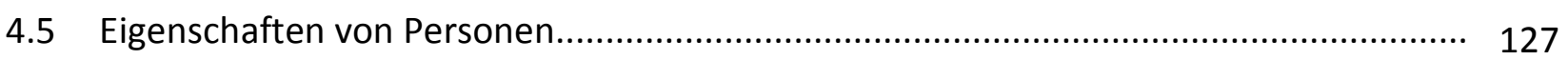

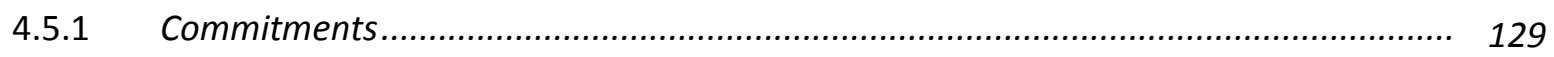

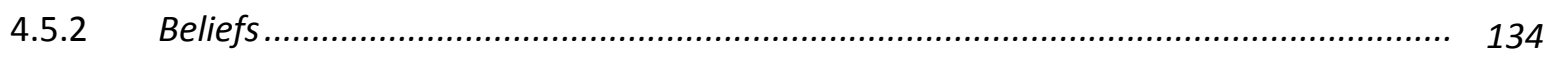

4.5.2.1 Selbstwirksamkeitserwartung (self-efficacy) ............................................................ 135

4.5.2.2 Kontrollüberzeugungen (locus of control) ................................................................ 140

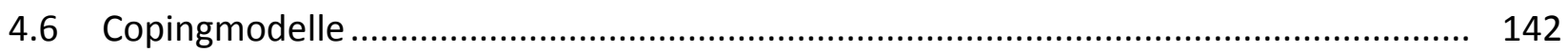

4.7 Kategorisierung von Bewältigungsformen und -arten........................................... 144

4.8 Messinstrumente zur Erfassung von Coping - Differenzierung von Bewältigungspro-

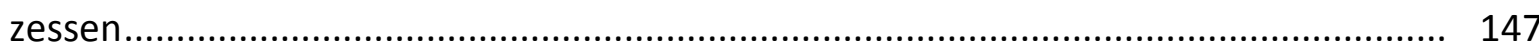

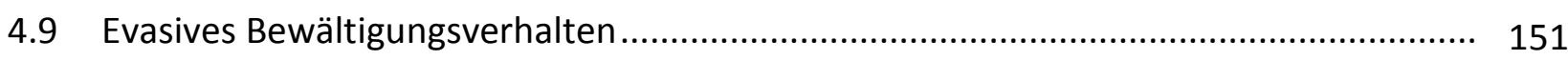

4.9.1 Einflussfaktoren auf das Unterrichten ............................................................... 156

4.9.2 Konflikte und ihre Bedeutung........................................................................ 160

4.9.3 Einbettung von evasivem Verhalten in Stress- bzw. Verhaltensmodelle ..................... 162

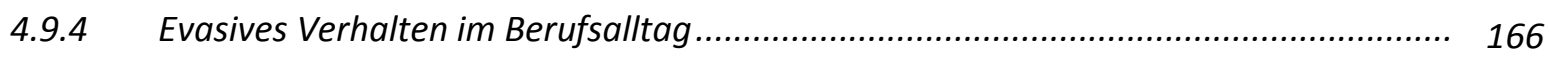

4.9.4.1 Evasives Verhalten im Bezug auf den Umgang mit Schülerinnen und Schülern................. 168

4.9.4.2 Evasives Verhalten im Bezug auf Inhalte .................................................................... 171

4.9.4.3 Evasives Verhalten im Bezug auf äußere Rahmenbedingungen...................................... 173

4.9.5 Beanspruchungsreaktionen und Beanspruchungsfolgen resultierend aus evasivem

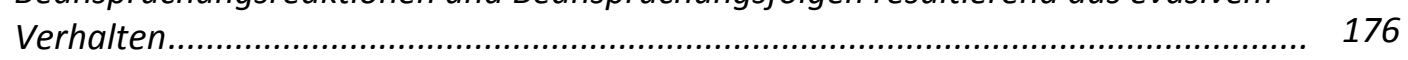

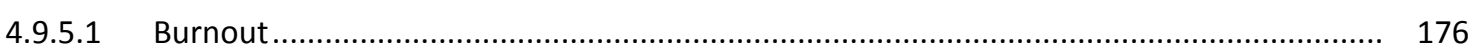

4.9.5.2 Innere Kündigung............................................................................................... 183

\section{Methodischer Teil}

5. Ableitung der Fragestellungen und Hypothesenbildung

5.1 Untersuchungskomplex 1: Persönliche und berufsbiographische Merkmale - „Wer nutzt evasive Strategien im Unterricht?".....

5.2 Untersuchungskomplex 2: Unterrichtliche Charakteristik: „In welchen Aspekten unterscheidet sich der Unterricht von eher evasiv und eher invasiv Unterrichtenden?".....

5.3 Untersuchungskomplex 3: Verhalten und Erleben im bzw. von Sportunterricht „Welche Merkmale sind in Bezug auf das Verhalten und Erleben im bzw. von Sportunterricht kennzeichnend für Personen mit ausgeprägtem evasiven Potenzial?". 
6.1 Konstruktion des Erhebungsinstruments evasiver Sportunterricht (EESU) .................. 202

6.1.1 Zielsetzung und theoretische Grundlagen............................................................ 202

6.1.2 Skalenkonstruktion und -modifikation ............................................................ 203

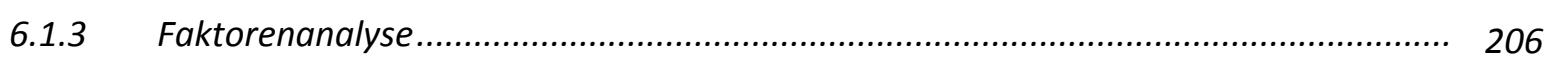

6.1.3.1 Voraussetzungen der Faktorenanalyse und Umgang mit fehlenden Werten ................... 207

6.1.3.2 Faktorenextraktion ....................................................................................... 209

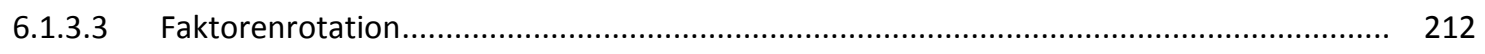

6.2 Interpretation der beiden Faktoren............................................................. 216

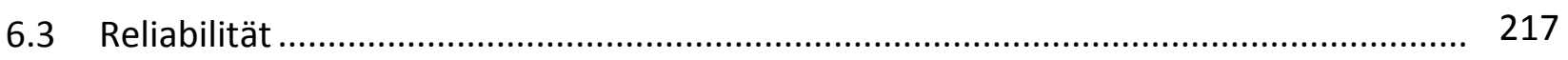

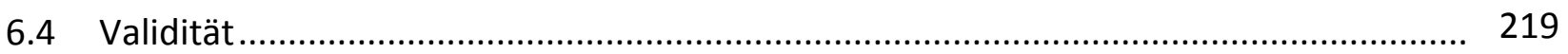

6.5 Durchführung der Hauptuntersuchung .................................................................. 221

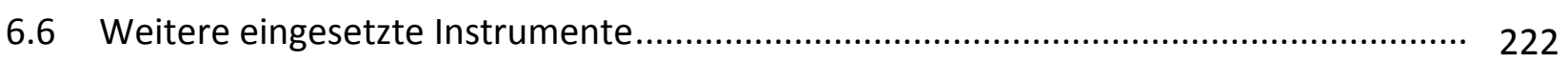

6.6.1 Arbeitsbezogene Verhaltens- und Erlebensmuster (AVEM) ..................................... 223

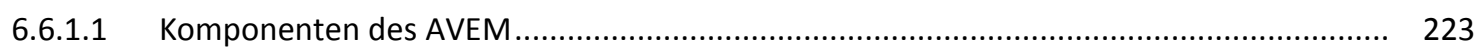

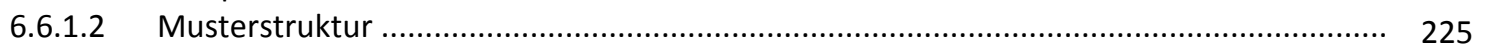

6.6.2 Fragebogen zu Kontroll- und Kompetenzüberzeugungen (FKK) ............................... 228

6.6.3 Stressverarbeitungsfragebogen (SVF 120).......................................................... 231

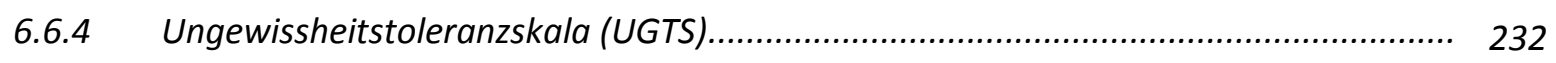

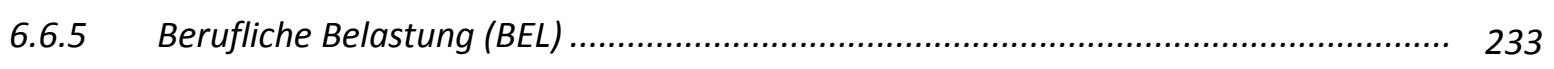

6.6.6 Achievement-Motive-Scale (AMS-kurz)............................................................. 233

6.6.7 Oldenburger Burn-Out-Inventar (OLBI) .............................................................. 234

6.6.8 Skala zu Lehrerselbstwirksamkeitserwartung (WIRKLEHR) ...................................... 235

6.6.9 Persönliche, berufsbiographische und didaktisch-pädagogische Daten, Einstellungen und Überzeugungen der Lehrkräfte .....

\section{Empirischer Teil}

7. Darstellung der Ergebnisse

7.1 Stichprobe.

7.2 Deskriptive Darstellung sportunterrichtsspezifischer Aspekte .................................. 242

7.2.1 Kriterien der Inhaltswahl.............................................................................. 242

7.2.2 Durchführungshäufigkeit verschiedener Inhalte.................................................... 243

7.2.3 Bedeutsamkeit von Vermittlungsaspekten ........................................................... 244

7.2.4 Lehrweise und Organisationsform ......................................................................... 245

7.2.5 Technisch-taktisches und konditionelles Aspirationsniveau ...................................... 246

7.2.6 Sportartspezifische Praxis- und Theoriekompetenz der Lehrkräfte ............................. 247

7.2.7 Aktive Teilnahme am Sportunterricht und Spaß am Unterrichten.............................. 248 
7.2.8 Belastungsfaktoren im Sportunterricht 250

7.2.9 Zeitaufwand für Vor- und Nachbereitung ............................................................ 251

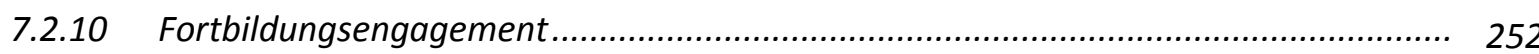

7.2.11 Individuelles Fühlen und Erleben im bzw. von Sportunterricht ................................. 252

7.3 Untersuchungsschwerpunkt 1: Persönliche und berufsbiographische Merkmale und evasives Unterrichten: „Wer nutzt evasive Strategien im Unterricht"?

7.3.1 Alter und Berufserfahrung 255

7.3.2 Geschlechtsvergleich 255

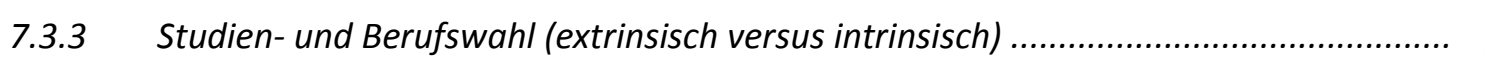

7.3.4 Praktische und theoretische Vermittlungskompetenz 260

7.3.5 Individuelle Sportaffinität (Fitnesszustand, Sportaktivität in der Freizeit, Vereinstrainerschaft, aktuell oder in der Vergangenheit betriebener Wettkampfsport, Stellenwert von Sport im Privatleben).

7.3.6 Kombination von Fachrichtungen bzw. Haupt- und Nebenfächern.

7.3.7 Gesamtstundenverpflichtung, Sportstundenzahl und prozentualer Anteil des Sportunterrichts an der Gesamtlehrverpflichtung ...

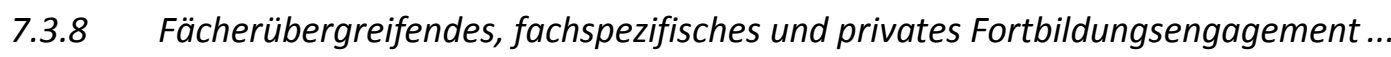

7.3.9 Zusammenfassende Interpretation der Ergebnisse

7.4 Untersuchungsschwerpunkt 2: Unterrichtliche Charakteristik: „In welchen Aspekten unterscheidet sich der Unterricht von eher evasiv und eher invasiv Unterrichtenden?"

7.4.1 Kriterien der Inhaltswahl

7.4.2 Durchführungshäufigkeit von Sportarten 271

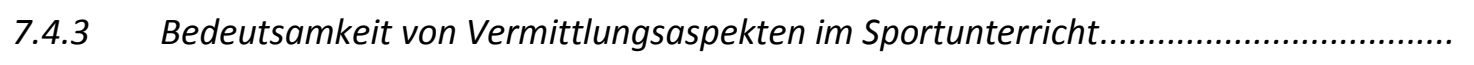
272

7.4.4 Aktive Teilnahme am Sportunterricht. 272

7.4.5 Arbeitsaufwand für Vor- und Nachbereitung. 273

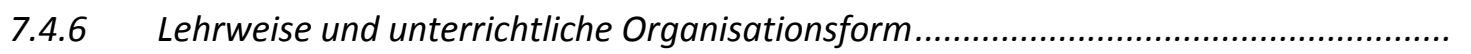
274

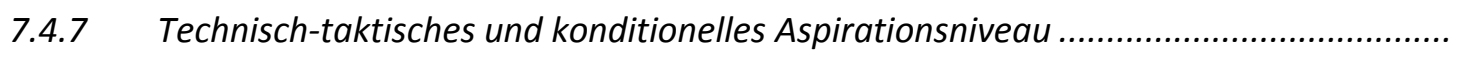
275

7.4.8 Subjektive Einschätzung des Stellenwerts des Sportunterrichts im Fachvergleich ........ 276

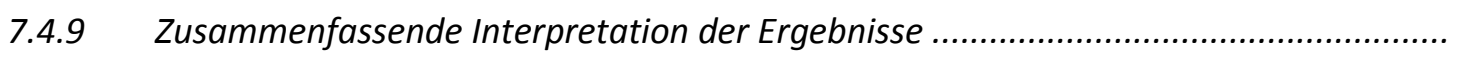
277 277

7.5 Untersuchungsschwerpunkt 3: Verhalten und Erleben im bzw. von Sportunterricht: "Welche Merkmale sind in Bezug auf das Verhalten und Erleben im bzw. von Sportunterricht kennzeichnend für Personen mit ausgeprägtem evasiven Potenzial?".....

7.5.1 Erleben von beruflicher Belastung ....................................................................... 281

7.5.2 Arbeitsbezogene Verhaltens- und Erlebensmuster ................................................. 283

7.5.3 Berufsbedingte Erschöpfung, arbeitsbezogenes (Dis-) Engagement .......................... 286

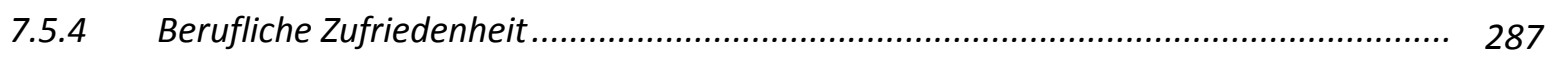

7.5.4.1 Arbeitszufriedenheit und Arbeitsüberforderung ......................................................... 288

7.5.4.2 Zufriedenheit mit der Stundenverteilung ................................................................. 288

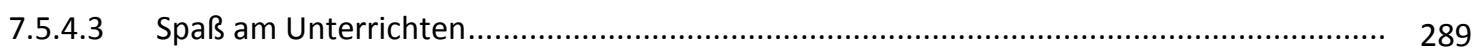

7.5.4.4 Wahrgenommene Atmosphäre im Sport- und Gesamtkollegium ..................................... 289

7.5.4.5 Gefühle mit der bisherigen Karriere, Gefühle für die Zukunft......................................... 290 
7.5.4.6 Erneute Berufswahl

7.5.5 Persönlichkeitseigenschaften: Lehrerselbstwirksamkeitserwartung, Kompetenzund Kontrollüberzeugungen, Leistungsmotivation, Ungewissheitstoleranz

7.5.5.1 Lehrerselbstwirksamkeitserwartung

7.5.5.2 Kompetenz- und Kontrollüberzeugungen

7.5.5.3 Leistungsmotivation

7.5.5.4 Ungewissheitstoleranz.

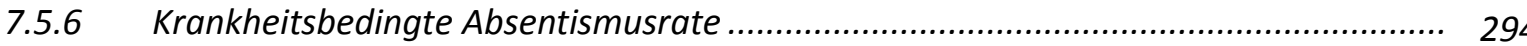

7.5.7 Zusammenfassende Interpretation der Ergebnisse ............................................... 295

8. Diskussion

8.1 Zusammenfassende Diskussion der Ergebnisse

8.2 Ausblick, Desiderata, Fazit

\section{Anhang}

I. 1. Fragebogenentwurf (Pilotierung 1)

II. 2. Fragebogenentwurf (Pilotierung 2)

III. Informationsflyer

IV. Fragebogen Erhebungsinstrument evasiver Sportunterricht - EESU (Endversion)

V. Lebenslauf 
Im Folgenden wird zugunsten der Lesbarkeit des Textes für Lehrerinnen und Lehrer sowie Schülerinnen und Schüler lediglich die männliche Form, verstanden als grammatikalische Neutralität, verwendet. Grundsätzlich sind beide Geschlechter angesprochen, sollte der Bezug nur für eines der beiden Geschlechter gelten, wird dies durch die spezifische Nutzung der entsprechenden Form deutlich gemacht. 


\section{Einleitung}

\subsection{Problemstellung}

Der Beruf Lehrer ist, wie keine andere gewerbsmäßig ausgeführte Tätigkeit, im Zentrum der Gesellschaft verankert. Durch die Schülerrolle hat nahezu jeder in der modernen Gesellschaft Kontakt mit Personen, die dafür bezahlt werden, Bildungs- und Erziehungsprozesse zu initiieren bzw. zu unterstützen. Nicht verwunderlich ist es, dass dementsprechend auch viele Meinungen und Ansichten, seien sie nun positiv oder negativ, zu diesem Berufsfeld vorliegen. Ebenso vielfältig stellen sich die Forschungsaktivitäten rund um den Lehrerberuf dar. Während ein Forschungszweig sich mit der Qualität vom Lehren und Lernen und damit einhergehend auch mit der Ausbildung der Lehrer beschäftigt, stellt die Belastungsproblematik von Lehrern einen zweiten umfangreichen Bereich dar. Abgeleitet von der Belastungsforschung etablierte sich ein Forschungsfeld, bei dem man sich mit der Gesundheit von Lehrkräften und den flankierenden beruflichen Anforderungen befasst. Im Rahmen dieser Arbeit soll schwerpunktmäßig das Bedingungsgefüge zwischen dem Berufsalltag mit den dort vorliegenden objektiven Belastungen, der letztendlichen subjektiven Manifestation, der konkreten Realisation des Unterrichts sowie den Auswirkungen auf die Gesundheit der Lehrer untersucht werden. Im Zuge der zunehmenden Arbeitsbelastung und den ansteigenden Zahlen von Burnout-Fällen in der Berufsgruppe der Lehrer soll eine spezielle Form der Bewältigung aufgearbeitet und sowohl gesundheitsrelevante als auch unterrichtsbezogene Schlussfolgerungen gezogen werden. Der unter dem Begriff Bewältigung zusammengefasste Prozess stellt sich als ein hoch komplexes Gefüge dar, das sich aus den Eigenschaften der Situation und den Persönlichkeitsmerkmalen der Lehrkraft konstituiert. Einen weiteren Beleg für die besonders hohe Relevanz des Umgangs mit Belastungen lieferte zuletzt eine Studie des Instituts für Demoskopie Allensbach im Jahr 2012, in der sich herausstellte, dass jeder zweite Lehrer von einer Verschärfung der Belastungssituation in den letzten Jahren berichtet. 44\% der Lehrer gaben sogar an, dass es Zeitpunkte in ihrem Berufsleben gab, in denen sie die durch die Arbeit in der Schule resultierende Belastung als unerträglich einstuften (Süßlin, 2012).

Im Speziellen soll in dieser Arbeit eine Bewältigungsstrategie in den Fokus genommen werden, die von Kolb und Wolters (2000) bezogen auf den Sportunterricht unter dem Ausdruck evasives Unterrichten beschrieben wurde. Bei dieser Bewältigungsstrategie wird statt einer offensiven Problembewältigung eine Vermeidungshaltung eingenommen, im Rahmen derer Konflikte ignoriert, positiv umgedeutet, verleugnet oder gar von Fluchttendenzen begleitet werden. Diese Verhaltensweise, die keineswegs nur in 
der Schule bzw. im Sportunterricht zu finden ist, kann in verschiedensten Situationen zur Anwendung kommen. Das evasive Handeln wird dabei teils von motivationalen Aspekten beeinflusst und wird entweder bewusst eingesetzt oder aber kommt unbewusst zum Einsatz.

Als einfaches Beispiel aus dem Alltag, wenn auch durch einen plakativen Geschlechtsstereotypus geprägt, kann man sich die Situation vorstellen, in der eine Ehefrau sich daran stört, dass ihr Mann seine Socken nach dem Tragen nicht in den dafür vorgesehenen Wäschekorb wirft. In den meisten Fällen wird die Ehefrau ihrem Mann das Fehlverhalten vor Augen führen und ihn bitten, in Zukunft die getragenen Socken in den dafür vorgesehen Behälter zu werfen. Beim erneuten Auftreten dieser Situation werden jedoch bei der Ehefrau verschiedene Denkoperationen in Gang gesetzt:

„War meine Aussage letztes Mal nicht deutlich genug?“, „Wieso macht er mir das Leben so schwer?“, „Was soll ich denn sonst machen, außer es ihm einfach zu sagen?“, „Auf diese Diskussion habe ich jetzt gar keine Lust - ich habe wirklich wichtigere Dinge zu tun, bei denen ich meine Kräfte sinnvoller einsetzen kann!“, „Versuche ich noch mal mein Glück und spreche es an - möglicherweise reagiert er dann genervt oder ist schlecht gelaunt - oder aber werfe ich die Socken selbst in den Wäschekorb, dann habe ich weniger Ärger und die Sache ist erledigt?".

Entscheidet sich die Ehefrau dafür, den Konflikt zu umgehen, indem sie selbst die getragenen Socken in den Wäschekorb wirft und damit nicht den Weg einer offensiven Problembewältigung einschlägt, ist der aktuelle Störungszustand schnell beendet und sie kann zur Tagesordnung zurückkehren. Auf lange Sicht wird sich vermutlich aber herausstellen, dass ihr Umgang mit dem Problem, bezogen auf die Zielsetzung, dass der Mann zukünftig seine getragenen Socken selbst in den Wäschekorb wirft, wenig erfolgreich war. Auch in Zukunft wird das Fehlverhalten auftreten und Spannungen und Ärger auslösen. Fasst die Frau sich ein Herz und spricht die Situation nach einiger Zeit erneut an, wäre eine Reaktion des Ehemanns im Sinne von: „Das habe ich doch immer so gemacht!" nicht verwunderlich.

In der dargestellten fiktionalen Situation, die wie bereits zuvor beschrieben auf einem veralteten Rollenbild von Mann und Frau und deren Beziehung zueinander basiert, wird deutlich, was im Rahmen dieser Arbeit mit dem evasiven Unterrichten und dessen Wirkungen gemeint ist: Durch evasives Verhalten ist es möglich, unangenehme Situationen zu beenden, sie zu umgehen oder ihnen zu entfliehen, wobei es sich jedoch lediglich um eine Scheinlösung handelt, bei der nicht das Problem gelöst, sondern nur dessen Symptome bzw. aktuelle Manifestation beseitigt wird. Während in der zuvor be- 
schriebenen Ehesituation die Folgen eines solchen Bewältigungsverhaltens je nach Charaktereigenschaften der Beteiligten von völliger Folgenlosigkeit bis hin zu einem ausgewachsenen Ehekrach reichen können und der Kreis der Betroffenen sehr begrenzt ist, zieht dieses Bewältigungsverhalten im schulischen Alltag weitreichendere Folgen nach sich. Auf der einen Seite ist es wichtig, in einem Beruf, der sich durch einige Besonderheiten wie mangelnde Gratifikation und zunehmende Entgrenzung des Arbeitsauftrages kennzeichnet, effektiv mit Alltagsproblemen umzugehen, um die eigene Gesundheit zu erhalten. Andererseits sollen Lehrer gemäß ihres Bildungs- und Erziehungsauftrags bei den Schülern nachhaltige Verhaltensänderungen bewirken, was vor allem aufgrund zunehmend fehlender häuslicher Sozialisation und der in diesem Zusammenhang zunehmenden erzieherischen Verantwortung der Lehrer zu sehen ist.

Die Gründe dafür, dass dennoch evasiv gehandelt wird, sind sowohl für das Sockenbeispiel als auch für den Alltag in der Schule vielfältig. Während das Fehlen von Handlungsalternativen eher in den Bereich der Hilflosigkeit eingeordnet werden kann, was meist auch mit fehlendem Vertrauen in die eigenen Fähigkeiten einhergeht, entspricht ein evasives Verhalten aus hedonistischen Beweggründen eher dem von Schaarschmidt \& Fischer (1996) als "Schonungstyp“ bezeichneten Verhaltensprofil (siehe Kap. 6.6.1). Solange das Verhalten nach außen nicht auffällt, wird der Weg des geringsten Widerstands gewählt, da die Motivation, mit dem eigenen Verhalten etwas bewirken zu wollen, nicht ausgeprägt ist. Bei dieser Musterstruktur, bei der bewusst (nicht) gehandelt wird, weist nicht das Können, sondern eher das Wollen handlungsleitende Funktion auf. Neben den psychologischen Aspekten, bezogen auf die Persönlichkeit der Lehrkraft, ist das ausweichende Verhalten auch durch den besonderen Charakter des Lehrerberufs bedingt. Anders als z.B. bei Handwerkern, die produktorientiert arbeiten, tritt schnell das Gefühl ein, nie fertig zu werden. Dadurch kann sich eine resignative Grundhaltung ausbilden, die bis zur schmerzlichen Erkenntnis führen kann, dass es sich um ein aussichtsloses Bemühen handelt. Auch in der Studie vom Institut für Demoskopie Allensbach gaben 56\% der Befragten an, dass es Klassen gibt, die man nicht in den Griff kriegen kann, was das Potenzial der Lehrer zu resignieren bzw. zu kapitulieren unterstreicht (Süßlin, 2012). Neben der Gestaltung der schulischen Praxis trotz widersprüchlichen Anforderungen und Erwartungen, fehlendem Respekt und widrigen Arbeitsbedingungen wird der Berufsgruppe zusätzlich die Last auferlegt, für die Rettung der Gesellschaft und den Erhalt der Werte verantwortlich zu sein. Meist befindet sich jedoch nicht die Ursache von Problemen im Einflussbereich von Lehrern, sondern nur deren Manifestation im Unterricht, wodurch es zu Resignationsprozessen kommen kann. Auch durch Verhaltensmodelle (Allmer, 1982; Heckhausen \& Rhein- 
berg, 1980) wird deutlich, dass bei Verhaltensweisen wie das evasive Unterrichten unterschiedliche Motive in Betracht gezogen werden müssen.

Grundsätzlich gilt es nun noch zu klären, wieso gerade der Sportunterricht als Fach ausgewählt wurde, um das evasive Verhalten von Lehrkräften genauer zu erforschen. Der Sportunterricht nimmt im schulischen Fächerkanon aufgrund seiner besonderen Rolle als Bewegungsfach eine Sonderrolle ein. Er stellt auch bezogen auf die Anerkennung und Wertschätzung der beruflichen Leistung den Extrempol dar, bei dem Geringschätzung und fehlender Respekt durch eine zunehmende Erweiterung bzw. Ausbeulung des Arbeitsauftrages und Verschlechterung der materiellen Arbeitsbedingungen flankiert wird, was zu einem ausgeprägten Ungerechtigkeitsempfinden der Sportlehrer führt. In einer Studie von Schaarschmidt (2005c) stellte sich heraus, dass bezogen auf das arbeitsbezogene Verhalten und Erleben bei Sportlehrern eine bedenkliche Situation vorzuliegen scheint: $47 \%$ der Sportlehrerinnen werden in das gesundheitsgefährdende Burnoutmuster, 40\% der Sportlehrer in das durch geringes Engagement geprägte Schonungsmuster eingruppiert. Die zweifellos einzigartigen erzieherischen Möglichkeiten des Sport- bzw. Bewegungsunterrichts wurden bereits zum Ende des 18. Jahrhunderts von den Philanthropen um GuthsMuths herausgestellt, die die wissenschaftliche Ausbildung stets in Verbindung mit der Entwicklung der Persönlichkeit sahen. Im Rahmen eines ganzheitlichen Erziehungsverständnisses wurden die Ansichten von Pestalozzi, der in seiner Elementargymnastik einen Beitrag zur Menschenbildung sah, praktisch in einer vernünftig-natürlichen Erziehung umgesetzt (Krüger, 2005). Nach einer kurzen Phase, in der der Sportunterricht durch außerschulische Aspekte legitimiert wurde, nehmen in jüngster Vergangenheit zunehmend erzieherische Aspekte u.a. aufgrund gesellschaftlicher Veränderungsprozesse (siehe Kap. 2.3.2) eine zentrale Stellung ein (Größing, 2007). Auch die besondere Belastungssituation im Sportunterricht legt nahe, dass gerade in diesem Unterrichtsfach Bestrebungen, belastungsreduzierende Strategien einzusetzen, zu erwarten sind. Der Arbeitsalltag von Sportlehrenden ist gekennzeichnet durch chronische Stressoren wie z.B. dem stetig hohen Lärmpegel oder das dauerhaft bestehenden Unfallrisiko (siehe Kap. 2.3.3). Hinzu kommt eine große Anzahl kleiner Hinder- und Ärgernisse, die im Sportunterricht als Bewegungsfach besonders häufig auftreten und als Stressorensequenzen oft Mehrfachanforderungen in einem häufig wechselnden Ordnungsrahmen darstellen und so multiplikative Wirkung haben. Durch die vielen Parallelschauplätze des Unterrichts ist der Unterrichtsalltag durch ein stetiges Orientieren in einer fast nicht zu kontrollierenden bzw. schlecht überschaubaren Unterrichtssituation geprägt. Diese Eigenschaften des Sportunterrichts, die Multidimensionalität und Simultanität der Aufgabenstruktur 
führen dazu, dass sich fast naturgegeben ein Bedürfnis nach Handlungssicherheit entwickelt. Rauin (2010) formuliert, dass Lehrer in solchen Situation teils keine Lösungen, sondern nur einen Ausweg suchen würden. Einen weiteren Faktor, der in seiner extremen Ausprägung spezifisch für den Sportunterricht ist, stellt die Ambiguität bzw. Ungewissheit dar: Aufgrund der Tatsache, dass der Sportunterricht meist das einzige Bewegungsfach ist, übernimmt er für die zum Sitzen und Lernen gezwungenen Schüler eine Ventilfunktion, die ihren Bewegungsdrang ausleben möchten. Hierbei kommt es oft dazu, dass die Schüler über das Ziel hinausschießen. Gleichzeitig werden jedoch auch hohe Anforderungen an die Kooperationsfähigkeit der Schüler gestellt, wodurch eine Vielzahl an kleinen und größeren Konflikte resultiert, die bei einem produktiven Umgang eine wichtige Rolle im Erziehungsprozess einnehmen. Keine Seltenheit ist es, dass in Konfliktsituationen eine unmittelbare Reaktion von der Lehrkraft erfordert wird, wodurch schnell eine Drucksituation entsteht, die teils affekthaltige Reaktionen auslöst. Während sich in anderen Fächern die Möglichkeit bietet, Probleme unter den Teppich zu kehren, finden sich Sportlehrer oft in der Zwangslage, handeln zu müssen. Ebenso wie bei dem Phänomen der Unfallflucht können in solchen Bedrohungssituationen statt rationalen Entscheidungen Verhaltensweisen wie Flucht oder Erstarren zu Tage treten, die kurzfristig entlastend wirken, sich langfristig jedoch als kontraproduktiv erweisen (siehe Kap. 4.9).

Im Sportunterricht entstehen naturgemäß viele Handlungs- und Konfliktsituationen, in denen man handeln kann, aber nicht immer muss. Neben den Konflikten, deren Lösung man in die Hände der Schüler legen sollte, um deren Selbstständigkeit und Konfliktfähigkeit zu fördern, gibt es auch Konflikte, die von der Lehrkraft im Sinne einer Ablauforientierung nicht stetig aktiv angenommen werden müssen bzw. sollten. Würde man auf alle Konflikte konsequent eingehen, würde der Handlungsfluss permanent unterbrochen und so die Bewegungs- und Erfahrungsmöglichkeiten des Unterrichts bei Weitem nicht ausgeschöpft. Die in Kapitel 2.2.3 aufgeführten Antinomien des Lehrerhandelns stellen Spannungsverhältnisse zwischen zwei gegensätzlichen Polen dar, die für sich betrachtet jedoch Berechtigung aufweisen und anzustreben sind. Während sich die von Helsper (1996) formulierten Antinomien auf Aspekte des Verhältnisses der Lehrkraft zu Schülern, Inhalten, der Ausrichtung des Unterrichts und der Schulorganisation beziehen, scheint also auch bezogen auf die Bewältigung eine Antinomie vorzuliegen. Während durch eine ausgeprägte evasive Tendenz die Übernahme von erzieherischer Verantwortung weitestgehend abgelehnt wird und so der Erziehungs- und Bildungsauftrag des Sportunterrichts gefährdet ist, kann ein stetig konsequent offensives Konfliktverhalten dazu führen, dass der Sportunterricht seinen bewegungsintensiven 
und freudvollen Charakter gänzlich verliert. Des Weiteren ist aufgrund der zuvor beschriebenen Anforderungsstruktur des Sportunterrichts bei solch einer Konfliktstrategie davon auszugehen, dass eine ernsthafte Gefahr für die psychische aber auch physische Gesundheit der Lehrkraft besteht.

Im Rahmen dieser Arbeit soll durch eine Fragebogenerhebung herausgefunden werden, inwieweit evasive Verhaltenstendenzen im Sportunterricht verbreitet sind, auf welche Facetten unterrichtlichen Handelns sie sich beziehen und durch welche biographischen Faktoren und Persönlichkeitseigenschaften diese bedingt sind. Desweiteren soll geprüft werden, inwieweit Zusammenhänge zwischen dem beschriebenen Bewältigungsverhalten und dem arbeitsbezogenen Verhalten und Erleben bzw. der Gesundheit bestehen. Außerdem soll geprüft werden, ob die Ausprägung des evasiven Verhaltenspotenzials Auswirkungen auf die Gestaltung des Unterrichts hat. Aufgrund des vorgestellten Ansatzes besteht die Möglichkeit anhand der Ergebnisse herauszustellen, in welchen Bereichen z.B. Fortbildungen wünschenswert sind und welche Änderungen in der Ausbildung von Sportlehrkräften notwendig sind. Auf diese Art und Weise kann ein weiterer Schritt im Sinne der Entwicklung der Qualität von Schule und Unterricht angestrebt werden, die neben pädagogischem und didaktischem Geschick sowie infrastrukturellen Gegebenheiten vor allem auf einer psychophysisch stabilen, motivierten und gesunden Lehrperson gründet.

\subsection{Aufbau der Arbeit - Zusammenfassung}

Bevor im methodischen Teil ein Überblick über den Ablauf des Forschungsprojekts sowie die in diesem Rahmen eingesetzten Methoden und Instrumente gegeben wird, soll in einem ausführlichen theoretischen Teil die Grundlage für die Interpretation der im empirischen Teil dargestellten Ergebnisse geschaffen werden. Während das zweite Kapitel ganz von dem Berufsstand Lehrer geprägt ist, werden im dritten Kapitel die Aspekte Stress, Belastung und Beanspruchung bearbeitet, bevor in Kapitel 4 der Umgang mit Belastungen bzw. Konflikten und dessen Folgen thematisiert wird.

Der Einstieg in diese Arbeit erfolgt in Kapitel 2.1 anhand der Darstellung der Besonderheiten der Prozesse rund um die Berufsentscheidung Lehrer. Es wird aufgezeigt, dass oft naive Vorstellungen darüber vorliegen, was einen erwartet, wenn man sich für den Lehrerberuf entscheidet. Im realen Berufsleben kommt es daher zu Enttäuschungen und Unzufriedenheit. Durch die karrierebezogene Einbahnstraße von Studium und Referendariat kommt es so zu einem gezwungenen Festhalten der „Falschen“ an dem eingeschlagenen Berufsweg. Ebenso wird dargestellt, welch unterschiedliche Motive bzw. Motivationen dafür verantwortlich sind, dass die individuelle Entscheidung Lehrer 
zu werden getroffen wird. Während Eduard Spranger (1963) auch davon spricht, dass die Liebe zum Kind durch Erschöpfung und Resignation schwinden kann, stellen pragmatische bzw. hedonistische Gründe für die Berufswahl negative Anfangsvoraussetzungen dar. Nach Bauer (2008) verspielt man so sein eigenes Lebensglück für Jahrzehnte und auch die Erziehungs- und Bildungsziele sind in Gefahr, denn nur wer von einer Sache begeistert ist, kann den Funken zu seinen Schülern überspringen lassen. Daher werden in Kapitel 2.1 statistische Daten vorgestellt, die zeigen, dass auch die wirtschaftliche Situation, der soziale Status des Elternhauses sowie die dort vorgelebte Karriere die Berufswahl beeinflussen. Während in anderen Branchen ein verfehlter Beruf meist nur individuelle Auswirkungen hat, multipliziert sich das Negative bei Lehrern auf das Vielfache und prägt damit zukünftige Generationen. Aufgrund dieser Tatsache ist es wichtig, dass sich nur geeignete Personen für den Beruf entscheiden, was durch Forderungen nach einer frühzeitigen Prüfung der beruflichen Eignung begleitet wird.

Im sich anschließenden Kapitel 2.2 werden die Besonderheiten des Lehrerberufs, wie z.B. die rechtlichen Besonderheiten, die der Beamtenstatus mit sich bringt, vorgestellt. Die Redensart "des Kaisers Kleider sind eng - halten aber warm" fasst diesen Aspekt relativ gut und knapp zusammen. Zudem wird das berufliche Aufgabenspektrum vorgestellt, das von der Kultusministerkonferenz im Jahr 2004 in die vier Kompetenzbereiche Unterrichten, Erziehen, Beurteilen und Innovieren differenziert wurde und zu vielgestaltigen Rollenkonflikten führen kann. Außerdem soll die geschichtliche Entwicklung des Lehrens und Lernens ausgehend von dessen Institutionalisierung dargestellt werden, wodurch die Bedeutung des Lehrerberufs für die Gesellschaft deutlich wird. Danach wird aufgezeigt welche Meinungen und Ansichten in der Gesellschaft über Lehrer bzw. deren Beruf vorliegen bzw. vorlagen, was sich unter anderem auch in Musik und Literatur widerspiegelt. All diese Aspekte stellen für die Belastungsthematik u.a. für das Verständnis des in Kapitel 4.9 angesprochenen Missverhältnisses von Aufwand und Ertrag eine bedeutsame Grundlage dar.

Kapitel 2.3 ist ganz dem Sportunterricht und dessen Eigen- bzw. Besonderheiten gewidmet. Auch hier wird als Grundlage zunächst die historische Entwicklung der Leibeserziehung bzw. des Sportunterrichts in Deutschland erarbeitet, bevor darauf aufbauend der aktuelle äußerst vielschichtige Auftrag des Faches in komprimierter Form angeführt wird. Während in der Vergangenheit eine Vielzahl unterschiedlicher teils gegenläufiger Strömungen bzw. Ansichten zur Ausrichtung des Sportunterrichts vorlagen, besteht etwa seit Beginn des 21. Jahrhunderts Einigkeit darüber, dass eine Konzeption, die in Anlehnung an Herbart als „Erziehender Sportunterricht" bezeichnet wird, einen 
sinnvollen Konsens darstellt (Stibbe, 2000). Neben der „Entwicklungsförderung durch Bewegung, Spiel und Sport" stellt die „Erschließung der Bewegungs-, Spiel- und Sportkultur" den zweiten Aspekt des "Doppelauftrags" des Sportunterrichts dar (Laging, 2004, S. 546 f.). Aufbauend auf Kapitel 2.2 folgt eine kurze Aufarbeitung des Arbeitsalltags und des Aufgabenspektrums von Sportlehrkräften die zeigt, wie bedeutsam die Persönlichkeit des Lehrenden aufgrund des im Unterricht stattfindenden Lernens am Modell ist und wie weit die Außenwahrnehmung des Faches und die unterrichtliche Realität voneinander entfernt sind.

Im dritten Kapitel erfolgt eine intensive Auseinandersetzung mit den Begriffen Stress, Belastung und Beanspruchung. Hierzu werden in Kapitel 3.1 mit dem reaktionsbezogenen, dem situationsbezogenen und dem interaktionistischen Stresskonzept drei verschiedene Ansätze vorgestellt, bevor der letztgenannte Ansatz in Belastungs- und Beanspruchungsmodellen bzw. Modellen zum Lehrerstress auf die Unterrichtstätigkeit angewandt wird und anhand von Beispielen aus dem Sportunterricht auf die Praxis Verdeutlichung findet. Während man früher den „Gestressten“ als Opfer der Umstände sah, weiß man heute, dass subjektive Einschätzungsprozesse und Bewältigungskompetenzen eine große Rolle spielen. Der Vorgang der Widerspiegelung einer Situation wird als entscheidende Instanz zwischen den für alle Lehrkräfte ähnlichen objektiven Belastungen und der individuell verspürten subjektiven Belastung identifiziert. Gleichzeitig wird die Frage nach den Faktoren aufgeworfen, die für die interindividuellen Unterschiede der Widerspiegelung von Situationen verantwortlich sind. Neben Belastungen außerhalb des Berufs bzw. dem Maß an sozialer Unterstützung und sozialem Rückhalt sind in diesem Zusammenhang vor allem Persönlichkeitseigenschaften ausschlaggebend, die in Kapitel 4 unter dem Schlagwort Bewältigung berücksichtigt werden. Zuvor wird jedoch in Kapitel 3.3 die Belastungssituation in der Schule, speziell bezogen auf den Sportunterricht ausführlich dargestellt. Während der Blick von außen auf den Sportunterricht eher ein geringes Belastungspotenzial erwarten lässt, schätzt hingegen etwa ein Drittel der Sportlehrkräfte die Belastung als hoch bis sehr hoch ein, was durch arbeitswissenschaftliche Untersuchungen bestätigt wird (Schönwälder, Berndt, Ströver \& Tiesler, 2003). Während einige Faktoren wie der nach oben nicht begrenzte Auftrag identisch mit allen anderen Fächern ist, existieren verschiedene Belastungsfaktoren die exklusiv im Sportunterricht vorliegen. Neben dem stetig vorliegenden Verletzungsrisiko und den Anforderungen, die ein Bewegungsfach stellen, sind dies vor allen Dingen die physischen Belastungen und die schlechten Arbeitsbedingungen. Neben fehlenden Pausenzeiten und einem die Gesundheit gefährdenden Lautstärkepegel werden in einigen Studien vor allem die sozialen Arbeitsbedingungen, die 
durch den Umgang mit Schülern, Eltern, Kollegen und der Schulleitung geprägt sind, als Hauptbelastungsquelle beschrieben (z.B. Ulich, 1996; Kraemis-Aebischer, 1996; Heim \& Klimek, 1999; van Dick, 2006, König, 2008).

Ausgehend von der Klärung des Begriffs Bewältigung bzw. Coping steht in Kapitel 4 die Bewältigung von Belastungen, Stressoren und Konflikten im Mittelpunkt. Die in Kapitel 3 herausgestellten antezedenten Bedingungen der Widerspiegelung und damit auch jeglichen Bewältigungshandelns, die Situationscharakteristika sowie die Persönlichkeitseigenschaften werden differenziert dargestellt. Während bei den formalen Situationscharakteristika immer wieder Bezüge zum Lehrer- und Sportlehrerberuf hergestellt werden, sind die Erläuterungen zu den die Bewältigung beeinflussenden Eigenschaften der Persönlichkeit überwiegend theoretisch geprägt. Neben der Leistungsmotivation und der Lehrerselbstwirksamkeitserwartung werden in diesem Rahmen zudem die Kompetenz- und Kontrollüberzeugungen herangezogen. In den beiden folgenden Unterkapiteln werden die verschiedenen Ansätze, Bewältigungsvorgänge phasen- bzw. prozesshaft zu beschreiben bzw. zu kategorisieren vorgestellt. In Vorbereitung auf das in Kapitel 4.9 beschriebene evasive Bewältigungsverhalten werden in Kapitel 4.8 unterschiedliche Erhebungsinstrumente beschrieben und auf ihre Eignung bezüglich der Messung des ausweichenden Agierens im Sportunterricht überprüft. In dem zentralen Unterkapitel zum evasiven Unterrichten wird zunächst die Verhaltensweise genau erläutert, die Bedeutung eines sinnvollen Umgangs mit Konflikten herausgestellt und die unterrichtliche Realität durch die Identifizierung der Einflussfaktoren genauer bestimmt. Durch die Einbettung des evasiven Verhaltens in die bereits in Kapitel 3.1 und 3.2 beschriebenen Stress- und Verhaltensmodelle können die Gründe und die unterschiedlichen Bedingungen des evasiven Verhaltens in Unterrichtssituationen nachvollzogen werden. Anhand eines Auszugs aus dem Film „Die Feuerzangenbowle“ erfolgt anschließend eine beispielhafte Aufarbeitung von ausweichendem Verhalten im schulischen Berufsalltag, das bezogen auf die Einflussfaktoren des Unterrichtens, d.h. den Umgang mit Schülerinnen und Schülern, Inhalten sowie äußeren Rahmenbedingungen konkretisiert wird. Die Beanspruchungsreaktionen und Beanspruchungsfolgen sind Thema von Kapitel 4.9.5, in dem neben Burnout mit der Inneren Kündigung eine weitere verwandte Beanspruchungsfolge in zusammengefasster Form vorgestellt wird.

Zu Beginn des methodischen Teils werden, basierend auf dem voranstehenden Theorieteil, eine Reihe von Fragestellungen abgeleitet, die zu insgesamt drei Untersuchungskomplexen zusammengefasst wurden. Der erste Untersuchungskomplex summiert schwerpunktmäßig Fragestellungen rund um die Persönlichkeit und die berufsbiographischen Merkmalen der Lehrkräfte, was in der Frage: „Wer nutzt evasive Stra- 
tegien?" deutlich wird. Im zweiten Untersuchungskomplex wird in insgesamt acht Teilfragen die unterrichtliche Realität in Augenschein genommen, was im Ergebnisteil eine Antwort auf die Frage "In welchen Aspekten unterscheidet sich der Unterricht von eher evasiv und eher invasiv Unterrichtenden?" ermöglichen soll. Das Verhalten und Erleben des Unterrichts bzw. des Arbeitsalltags wird im dritten und letzten Untersuchungskomplex im Bezug auf die Ausprägung des evasiven Verhaltens untersucht. Während die ersten beiden Untersuchungskomplexe eher Rückschlüsse bezüglich zukünftiger Personalwahl bzw. der Gestaltung von Aus- und Weiterbildung ermöglichen sollen, wird im Rahmen dieses Komplexes geprüft, ob die Ausprägung des evasiven Verhaltens Gesundheitsrelevanz aufweist. Des Weiteren wurden bei jedem Untersuchungskomplex zu den Teilfragen jeweils Hypothesen formuliert, deren Verifizierung bzw. Falsifizierung im Ergebnisteil (siehe Kapitel 7) erfolgt. In Kapitel 6, der methodischen Umsetzung, wird zunächst der Ablauf des Forschungsprojekts beschrieben und die Notwendigkeit der Neukonstruktion eines Erhebungsinstruments begründet. Neben der Selektion von Items und der Faktorenanalyse wird zudem die Prüfung der Gütekriterien dokumentiert. Wie sich zeigte, konnte ein ökonomisch einsetzbares, aber dennoch differenzierendes Instrument, bezogen auf die Ausprägung des evasiven Verhaltenspotenzials, geschaffen werden. In dem Unterkapitel 6.6 werden mit dem Fragebogen von Schaarschmidt zur Erfassung der "Arbeitsbezogenen Verhaltens- und Erlebensmuster" (AVEM), dem „Fragebogen zu Kompetenz- und Kontrollüberzeugungen“ (FKK) von Krampen, dem "Stressverarbeitungsfragebogen“ (SVF120) von Janke, Erdmann und Kallus, der „Ungewissheitstoleranzskala“ (UGTS) von Dalbert, dem Instrument zur Erfassung der beruflichen Belastung (BEL) von Enzmann und Kleiber, der „Achievement-Motive-Scale“ (AMS-kurz) von Engeser, dem „Oldenburger-BurnoutInventar" (OLBI) von Demerouti und der "Skala zur Lehrerselbstwirksamkeitserwartung" (WIRKLEHR) von Schmitz und Schwarzer die eingesetzten Instrumente beschrieben.

Der empirische Teil dieser Arbeit ist, nachdem die Eigenschaften der Stichprobe (siehe Kap. 7.1) und die deskriptiven Daten sportunterrichtsspezifischer Aspekte (siehe Kap. 7.2) dargestellt wurden, an der in Kapitel 5 vorgestellten Struktur orientiert.

Nachdem die einzelnen Teilfragen der drei Untersuchungskomplexe bezüglich der vorab formulierten Hypothesen bearbeitet wurden, erfolgt jeweils eine interpretative Zusammenfassung. Untersuchungsmethodisch wurde eine klassische Vorgehensweise realisiert: Neben der Berechnung von Pearsons Korrelationskoeffizienten $(r)$ zwischen den verschiedenen Angaben der Sportlehrkräfte und dem evasiven Verhaltenspotenzi- 
al wurden zudem t-Tests sowie Mann-Whitney-U-Tests ${ }^{1}$ gerechnet, um die Bedeutsamkeit von Mittelwertdifferenzen bezüglich der Signifikanz einzuschätzen. Um statistische Zusammenhänge zwischen verschiedenen Gruppen differenziert darzustellen, wurden einfaktorielle Varianzanalysen (ANOVA) durchgeführt und hier ebenso wie bei den $t$-Tests die Effektstärke Cohens $d$ mit dem Programm G-Power berechnet. Des Weiteren wurde die Ausprägung des evasiven Verhaltens an einigen Stellen aufgrund der größeren Anschaulichkeit in Quartile eingeteilt. Auf diese Art und Weise ist eine leichtere Interpretation und grafische Darstellung der Abhängigkeiten bzw. Zusammenhänge möglich.

Die Ergebnisse der im Rahmen des ersten Untersuchungskomplexes bearbeiteten Teilfragen (siehe Kap. 7.3) ermöglichen es, ein genaueres Bild des evasiven Lehrertyps zu formulieren. Zentrale Bedeutung in diesem Zusammenhang nimmt die Grundeinstellung bzw. Überzeugung ein, mit der man seinen Beruf auswählt bzw. ausübt. Rein pragmatische Gründe für die Berufswahl sowie eine fehlende Affinität zu Bewegung, Spiel und Sport scheinen im Sinne eines angemessenen Bewältigungsverhaltens negative Anfangsvoraussetzungen darzustellen. Des Weiteren zeigen sich einige Zusammenhänge zwischen der Ausprägung des evasiven Verhaltenspotenzials und persönlichen bzw. berufsbiographischen Merkmalen wie z.B. dem Alter oder dem Fortbildungsengagement.

Anhand der Auswertung des zweiten Untersuchungskomplexes (siehe Kap. 7.4) zeigte sich, dass fast alle untersuchten Aspekte, die die Ausgestaltung des Unterrichts betreffen, Zusammenhänge zur Ausprägung des evasiven Verhaltenspotenzials aufweisen. Dies gilt sowohl für im Vorfeld getroffene Entscheidungen, die präventiv die Konfliktwahrscheinlichkeit senken wie z.B. die Inhaltswahl, als auch für die konkrete Umsetzung des Unterrichts, z.B. das Anspruchsniveau betreffend. Die Einstellung der evasiv unterrichtenden Lehrkräfte ist, was die Ausrichtung und Organisation des Unterrichts betrifft, eher traditionell bzw. konservativ und verkennt dabei teils Kernaspekte, die die Eckpfeiler moderner Legitimationsansätze des Sportunterrichts darstellen. Auffällig ist hingegen, dass die Einsatzbereitschaft bei evasiv Unterrichtenden scheinbar nicht in geringerem Maß ausgeprägt ist, wofür der nahezu identische Zeitaufwand für die Vorund Nachbereitung des Unterrichts ein Indikator ist.

In Kapitel 7.5 sind die Ergebnisse des dritten Untersuchungskomplexes zusammengefasst, der sich schwerpunktmäßig um das Fühlen und Erleben der Sportlehrkräfte im Alltag dreht. Wie sich zeigte, kann das Profil einer evasiv unterrichtenden Lehrkraft weder auf die AVEM-Musterstruktur von Schaarschmidt noch auf andere bereits exis-

\footnotetext{
${ }^{1}$ Der Mann-Whitney-U-Test wurde eingesetzt, falls die Voraussetzungen für die Durchführung eines tTests nicht erfüllt sind.
} 
tierende Kategorisierungsmöglichkeiten problemlos übertragen werden. Es stellte sich heraus, dass evasives Verhalten eine unangemessene Möglichkeit des Umgangs mit Belastungen und Konflikten des Alltags darstellt. Eine Strategie, die belastungsmindernd wirken soll, führt letztendlich zu einer Verschärfung der Belastungssituation, die das Selbst bedrohende Züge aufweisen kann. Während bei dem von Schaarschmidt beschriebenen Schonungsmuster eine relativ hohe Lebenszufriedenheit und Gelassenheit vorherrscht bei gleichzeitig ausgeprägtem Laissez-faire-Verhalten, sind bei evasiv unterrichtenden Personen diese protektiv wirkenden Faktoren gegenteilig ausgeprägt. Ein auf Schonung der Ressourcen angelegtes Verhalten führt zu einem ausgeprägten Belastungsempfinden, begleitet mit beruflicher Unzufriedenheit, und weist letztendlich deutliche Parallelen zu dem in Kapitel 4.9.5.1 beschriebenen Burnout-Syndrom auf.

In Kapitel 8 werden Schlussfolgerungen gezogen und weitere Fragestellungen abgeleitet, die Anregungen und Ideen enthalten, das evasive Verhalten in seinen verschiedenen Facetten genauer darzustellen und das umgebende Bedingungs- und Begründungsgefüge konkreter zu untersuchen. 


\section{Der Berufsstand Lehrer}

In diesem Kapitel werden einleitend grundsätzliche Aspekte der Berufswahl in zusammenfassender Form dargestellt. Daran anknüpfend werden die Motive, den Lehrerberuf zu ergreifen und die Rekrutierungscharakteristik des Berufes vorgestellt und Entscheidungsmuster nachvollzogen. Neben Besonderheiten bei der Berufsentscheidung im Lehramt, werden Ergebnisse statistischer Erhebungen und die Auswirkungen von Fehlentscheidungen skizziert (siehe Kap. 2.1). Im Anschluss werden grundlegende Eigenschaften des Lehrerberufs vorgestellt (siehe Kap. 2.2) sowie basierend auf der geschichtlichen Entwicklung des Berufsstandes, den Aufgaben, den Rollenerwartungen und den daraus resultierenden Spannungen und Schwierigkeiten das allgemeine Berufsbild Lehrer skizziert. Darauf aufbauend werden in Kapitel 2.3 die Besonderheiten des Sportlehrerberufs dargestellt. Neben den kennzeichnenden Merkmalen, die dem Auftrag, Schüler in dem Fach Sport zu unterrichten inne wohnen, wird die besondere Anforderungsstruktur, mit der sich Lehrkräfte in diesem Fach konfrontiert sehen, erläutert.

\subsection{Motive zur Berufswahl - Rekrutierungscharakteristik im Lehrerberuf}

Eduard Spranger (1963) schreibt in seinem Werk "Der geborene Erzieher", dass ein Drittel bis die Hälfte von dem, was die Persönlichkeit einer Lehrkraft ausmacht, ausbildungsunabhängig ist und durch die eigene Sozialisation in Kindheit und Jugend bedingt ist. Pädagogische Liebe lasse sich nicht lernen, wohl aber ist es möglich, dass die Liebe zum Kind durch Erschöpfung und Resignation, aber auch durch Routine schwinden kann. Auch Bauer (2008, S. 77) betont die Bedeutsamkeit der hinter einer Berufsentscheidung stehenden Motive:

Wer bereits bei Studienbeginn vor allem auf der Suche nach einer sicheren Stelle ist, der sollte nicht Lehrer werden! Auch wer sich nur wegen einer Begabung oder Neigung zu diesem oder jenem Fach für diesen Beruf entscheidet, verspielt sein Lebensglück, denn er wird, nachdem er jahrelang unzählige Kinder und Jugendliche frustriert hat, wahrscheinlich wegen vorzeitiger Krankheit in den Ruhestand gehen müssen.

Mit dem Abschluss der schulischen Ausbildung rücken für junge Erwachsene zentrale Fragen ins Bewusstsein, nämlich die, mit welcher Tätigkeit man lang-, mittel- oder kurzfristig beabsichtigt, den Lebensunterhalt zu bestreiten. Kinder- und Jugendträume 
werden dann nur noch in den seltensten Fällen tatsächlich als reale Zielvorstellungen verfolgt. Die letztendliche Entscheidung für einen bestimmten Studiengang bzw. für einen Beruf ist abhängig von Motiven, die in Abgrenzung zur Motivation, konstanter, d.h. situations- und zeitüberdauernd sind (Brühwiler \& Spychiger, 1997). Nach Ginzberg et al. (1951) ist entwicklungsbedingt langfristig eine Genese der Studien- und Berufswahlmotive festzustellen, die in verschiedene Stufen eingeteilt wird. Nachdem der erste Entwicklungsabschnitt, die fantasy stage, oft noch stark von naiven und romantischen Motiven geprägt ist, schließt sich die tentative stage der Teenagerzeit an, in der Jugendliche bereits über ihre Interessen, Fähigkeiten und Werte nachdenken. In der Adoleszenz manifestieren sich konkreter werdende Berufswünsche in der realistic stage. Am Ende der Schulzeit heißt es dann oft für die Jugendlichen Abschied zu nehmen von fantasievoll ausgemalten, an subjektiven Bedürfnissen und Interessen orientierten Berufswünschen und Tagträumen, dem Nacheifern von Idolen und Vorbildern. Es erfolgt eine objektivere Abschätzung von dem, was die gesellschaftliche Umwelt zu bieten hat. Der Politik und der Gesellschaft bleiben daher Probleme mit einer Schwemme an Tierärztinnen, Sänger- und Tänzerinnen, Lokomotivführern, Agenten, Fußballprofis, Artistinnen, Feuerwehrmännern, Polizisten sowie Tierpflegern und Zoodirektoren erspart. Wie bei den meisten anderen Entwicklungsmerkmalen wird diese Phase, in der ernsthafte Berufsvorstellungen ausgebildet werden, von Mädchen meist früher erreicht als von Jungen (Kniveton, 2004).

Neben den entwicklungsbedingt realistischer werdenden Vorstellungen bezogen auf die Ausübung von Berufen, sind in der letzten Zeit zudem wachsende Zukunftssorgen im Denken von Kindern und Jugendlichen zu finden. Sicherheits- und Wohlstandsstreben sind dabei, den Traum von einem in den Tag lebenden Abenteurer abzulösen. Auch die Landesbausparkassen (LBS) verwerteten dieses veränderte Zukunftsdenken in einem Werbespot, in dem ein kleines Mädchen ihrem alternativen Vater in einem „Jute-statt-Plastik-Wohnwagen-Camp" eröffnet, dass sie, wenn sie groß ist, auch Spießer werden möchte. Matzig (2005) spricht in diesem Zusammenhang von einer Renaissance alter Rollenbilder und zunehmend mehr Neocons, eine auf Sicherheit setzende neokonservative Jugendkultur, die sich entgegen der üblichen Klischees der Jugendkultur positioniert. Statt Rebellion und Unangepasstheit inszenieren sich Kinder und Jugendliche biedermeierlich, streben nach klassischen Statussymbolen wie einem Eigenheim, einem Auto und einem schönen Porzellanservice. Weiterhin stellt die Ausrichtung nach konservativen Werten auch eine Reaktion der Jugendlichen auf die sogenannten Best Agers dar, jenem Anteil der Erwachsenengeneration, der sich verhaltensmäßig an der Phase des Jugendalters orientiert. Dass der Lehrerberuf, der in wei- 
ten Teilen Deutschlands mit dem zukunftssicheren Beamtenstatus eng verknüpft ist, in diesem Zusammenhang eine attraktive Alternative darstellt, ist naheliegend. Gerade in Zeiten zunehmender wirtschaftlicher Schwankungen, in denen existenzbedrohende Entwicklungen scheinbar augenblicklich und ohne Vorwarnungen auf bestimmte Berufsgruppen hereinbrechen, scheint ein gesteigertes Sicherheitsdenken geradezu notwendig zu sein. In der Studie des Instituts für Demoskopie Allensbach wurde genau diese Tendenz bestätigt: Während die Sicherheit des Arbeitsplatzes im Lehrerberuf, der in den meisten Bundesländern noch den Beamtenstatus mit sich bringt, für $40 \%$ der Junglehrer, die weniger als fünf Jahre in dem Beruf sind, als Motiv für die Berufswahl Bedeutung hat, waren es bei den Lehrern mit mehr als 20 Jahren Berufserfahrungen nur 22\% (Süßlin, 2012).

Wann und wie letztendlich die Entscheidung für einen Ausbildungs- oder Studiengang getroffen wird, ist oft sehr unterschiedlich und läuft auf verschiedenen Ebenen ab. Schwänke (1988) warnt vor allzu festen Typisierungen bzw. starren Mustern, die für die Studien- und Berufswahl verantwortlich gemacht werden, da bereits die Vorstellungen der angestrebten Berufstätigkeit aufgrund von biographisch bedingten und psychischen Bedürfnissen, sozial vermittelten Einstellungen sowie rationalen Entscheidungen äußerst vielschichtig sind und einen hohen Komplexitätsgrad aufweisen. Oft handelt es sich bei der Studien- und Berufswahl um einen langfristigen Prozess, in dem kein eindeutiger Zeitpunkt bestimmt werden kann, an dem die Entscheidung getroffen wird. Neben bewussten Motiven spielen zum Großteil unbewusste Prozesse eine entscheidende Rolle, die meist einen diffusen Charakter aufweisen und sich unserer Wahrnehmung entziehen bzw. nicht kategorisiert oder einer bestimmten Richtung zugeordnet werden können (Brühwiler \& Spychiger, 1997).

Des Weiteren stellte sich in verschiedenen Untersuchungen heraus, dass Studien- bzw. Ausbildungs- und Berufswahlmotive keine kongruenten Motivkategorien darstellen. Während unter dem Studien- bzw. Ausbildungsmotiv lediglich die subjektive Begründung zur Wahl des Studiums bzw. der Ausbildung verstanden wird, beziehen sich die Berufswahlmotive darauf, am Ende der Ausbildung auch tatsächlich einen bestimmten Beruf zu ergreifen und diesen den Rest des Lebens auch ausüben zu wollen (Brühwiler \& Spychiger, 1997). Bisher ist es nicht gelungen, eine befriedigende Theorie des Berufswahlprozesses zu generieren und so kann nur von Indizien, nicht aber von klar nachvollziehbaren Entscheidungsschemata für oder gegen einen bestimmten Beruf gesprochen werden. Neben dem eigenen Ich sind es oft auch äußere Umstände, die das Für und Wider im individuellen Denken beeinflussen. Auch die Einflüsse von außen auf die Entscheidung sind nicht zu unterschätzen, da Berufswünsche von Kindern und 
Jugendlichen fast immer Reflexionen dessen sind, was sie in der Welt der Erwachsenen erleben und bewundern. Studien zur beruflichen Sozialisation haben ergeben, dass es gerade Erfahrungen aus der Kindeszeit sind, die entscheidenden Einfluss auf die Berufswahl haben. Ein als bedeutsam erlebtes Berufsfeld führt dazu, dass der Wunsch besteht, selbst Teil dieses bedeutsamen Systems werden zu wollen (Evans, 1993). Auch Personen des näheren Umfelds wie Freunde, Verwandte und vor allem die Eltern beeinflussen durch Beratung bzw. das Vorleben von Berufskarrieren die Entscheidung des potenziellen Absolventen. Kniveton (2004) untersuchte die Qualität des Einflusses verschiedener Bezugspersonen auf die Berufswahl. Sowohl bei Jungen als auch bei Mädchen stellen die Eltern die einflussreichsten Personen im Entscheidungsprozess dar, wobei noch hinzuzufügen ist, dass eine klare Dominanz des Einflusses des gleichgeschlechtlichen Elternteils vorliegt. Deutlich weniger Einfluss auf die Berufswahl üben Lehrer, Freunde und Geschwister aus. Doch auch durch gesellschaftliche und wirtschaftliche Veränderungen ändert sich zunehmend der Berufswahlprozess. Durch die verstärkte Trennung der Lebensbereiche Arbeiten und Wohnen ist bei dem Erwerb von Wissen und dem Sammeln von Erfahrungen über bzw. mit verschiedenen Berufen ein Wandel festzustellen. Während unmittelbare primäre Erfahrungen vom Handwerksbetrieb um die Ecke fast verschwunden sind, prägen hauptsächlich medial aufbereitete Darstellungen das Bild, das Kinder und Jugendliche von Berufen haben (Kaiser, 2006). Für den Lehrerberuf gilt dieser Wandel nur bedingt, da unmittelbare Erfahrungen mit dem für jeden in einem bestimmten Lebensabschnitt bedeutsamen Systems unumgänglich sind. Die mediale Aufbereitung stellt lediglich eine zusätzliche Einflussgröße dar, die teils zu einem gewandelten Blick auf den Beruf führt (siehe Kap. 2.2.4).

Im folgenden Abschnitt wird der Frage „Warum denn Lehrer werden?" genauer nachgegangen. Neben den zahlreichen Faktoren die gegen die Berufsentscheidung Lehramt sprechen, wie Mängel in der Ausbildung, hohe Berufsanforderungen, fehlende Aufstiegschancen oder geringe gesellschaftliche Anerkennung, entschließen sich dennoch viele junge, meist von ihrer Entscheidung überzeugte Menschen dazu, eine Berufskarriere als Lehrer einzuschlagen. Mehrere Studien haben sich das Ziel gesetzt, die Faktoren und Motive aufzudecken, die dafür verantwortlich sind, warum ein Lehramtsstudium aufgenommen wird bzw. der Lehrerberuf als berufliches Ziel angestrebt wird. Stephens (1969, zitiert nach Evans, 1993) geht mit seiner Ansicht, das Lehren als evolutionären Trieb des Menschen zu bezeichnen, sogar noch einen Schritt weiter: Er stellt eine enge Verbindung zwischen dem Aufstieg der menschlichen Rasse und der Entwicklung in Form einer Professionalisierung des Berufsstandes her. 
Von großer Bedeutung bei der Berufswahl sind realistische Einschätzungen des zukünftigen Arbeitsumfelds und den Anforderungen, mit denen man im Laufe eines Berufslebens konfrontiert wird. Wie sich in Studien herausstellte, sind die Motive und Erwartungen, die mit dem Beruf des Lehrers von Schülern und Studenten verbunden werden, oft naiver Natur (Kiel et al., 2007). Diese teils romantischen und zum Großteil positiv geprägten Vorstellungen und Erwartungen führen beispielsweise dazu, dass $40 \%$ der sich im Berufsleben befindenden Lehrer, die Situation in der Schule als schlechter als zuvor erwartet einstufen (Ulich, 2004). Diese negative Bewertung bezieht sich vor allem auf das doch geringere $M a ß$ an Freiheit in der Berufsausübung und die weitaus höheren Belastungen. Die Fehleinschätzungen, d.h. die Diskrepanz zwischen Erwartung und Realität, münden nicht selten in Enttäuschungen und Frustrationen, was gerade für den Lehrerberuf von hoher Relevanz ist, denn mit der Studienentscheidung Lehramt legt man sich bereits weitgehend auf ein sehr enges Berufsfeld fest. Weitere akademische Berufe sind durch die Studienentscheidung meist versperrt und Fehlentscheidungen sowie Überraschungen im realen Berufsleben sind nur durch großen finanziellen und zeitlichen Einsatz zu korrigieren. Da sich ein realistischer Blick auf den Beruf und vor allem bezogen auf die Passung der eigenen Fähigkeiten, Kompetenzen und Persönlichkeitsmerkmale mit den Berufsmerkmalen und Anforderungen meist erst nach einer beträchtlichen Studienzeit, im Rahmen von Praktika oder gar erst im Referendariat ausbildet, halten viele an dem Berufsziel Lehrer fest, auch wenn Zweifel und Ängste auftauchen, eine falsche Entscheidung getroffen zu haben. Fehlerwartungen, die häufig in Enttäuschungen münden, begünstigen Prozesse des Ausbrennens (siehe Kap. 4.9.5.1) und sollten durch Reflexion der eigenen Motive und Erwartungen an die zukünftige Arbeitssituation vor der Aufnahme bzw. während des Studiums, als auch im Rahmen des Referendariats verhindert werden.

Wer wird also Lehrer und wer sollte Lehrer sein? Am Zentrum für Lehrerbildung der Universität Kassel beschäftigt man sich im Rahmen der Erarbeitung und Umsetzung des "Trainingsmoduls Psychosozialer Basiskompetenzen im Lehrerberuf" unter anderem damit, was man schon in der ersten Phase der Lehrerausbildung tun kann, um Studierende frühzeitig zu beraten, die nicht über die für den Lehrerberuf grundlegenden sozialen und personalen Kompetenzen verfügen (Zentrum für Lehrerbildung, 2008). In einem ähnlichen Projekt beschäftigten sich bereits seit 1994 Schaarschmidt und Herlt damit, die individuelle Eignung für den Beruf zu überprüfen. Beide kombinieren in ihrem Instrument „Fit für den Lehrerberuf" sowohl Selbsterkundungs- als auch Fremdeinschätzungsverfahren und richten sich vornehmlich an Personen, die mit dem Gedanken spielen, ein Lehramtsstudium aufzunehmen. Aber auch diejenigen, die be- 
reits ein Studium aufgenommen haben, können das Instrument nutzen, um eigene Schwächen zu diagnostizieren und daran zu arbeiten, bzw. frühzeitig eine berufliche Neuorientierung in Gang zu setzen (Schaarschmidt \& Herlt, 2007). Ebenso zielt auch das internetbasierte Verfahren „Career Counselling for Teachers (CCT)“ durch Informationstexte und Selbsterkundungsverfahren in adaptierter Version in mehreren Ländern darauf ab, zielgruppenorientierte Beratung für Studieninteressierte, Studierende, Berufseinsteiger und erfahrene Lehrkräfte zu bieten (Häcker \& Zopfi, 2006; Sieland \& Nieskens, 2001).

Grundlage für die zuvor dargestellten Anstrengungen sind die oft beobachteten und berichteten Reaktionen von Berufseinsteigern und Junglehrern, die im Zusammenhang mit dem sogenannten Praxisschock einhergehen. Aufgrund der Unvereinbarkeit von Erwartung und Wirklichkeit werden die Maßstäbe des eigenen Handelns oft weit gesenkt, das praktizierte Handeln im Laufe des Berufslebens und der sogenannte heimliche Lehrplan legitimiert. Auf eine kritische Reflexion des eigenen Handelns wird weitgehend verzichtet (Meier, 1983).

Bei der Berufswahlentscheidung wird grundsätzlich zwischen extrinsischen, intrinsischen und tätigkeitsorientierten Motiven unterschieden. Unter extrinsischen Gründen den Beruf Lehrer bzw. das Lehramtsstudium zu wählen summieren sich Eigenschaften des Lehrerberufs, die von Außenstehenden oft als Grundvorzüge des Berufs gesehen werden. Neben langen Ferienzeiten, einem guten Gehalt und einem sicheren, durch den Beamtenstatus geschützten Arbeitsplatz ist es auch die geringe vorgeschriebene verbindliche wöchentliche Arbeitsverpflichtung, die als vorteilhaft im Vergleich zu anderen Berufen angesehen wird. Bei der Studienwahl verbindet man mit dem Lehramtsstudium ein geringes Anspruchsniveau und einen meist abwechslungsreichen Verlauf des Studiums. Teilweise nutzen Frauen die Lehramtsausbildung auch dazu, um der Verantwortung für die Erziehung der eigenen Kinder gerecht zu werden. Struck (1994) fasst diese Motive unter dem Begriff Berufshygiene zusammen. Diese extrinsische Motivkategorie wird verstärkt entscheidungsrelevant für Personen, deren eigentlicher Berufswunsch aufgrund von nicht erfüllten Zugangsvoraussetzungen versperrt bleibt oder aber auch für die Unentschlossenen. Viele, die sich noch für keinen Beruf entschieden haben "studieren erst mal Lehramt". Baumert (2009) stellt jedoch heraus, dass Berufswahlmotive wie die Freiheit im Zeitmanagement oder die Ferienzeiten lediglich ein Sekundärmotiv darstellen sollten und in Form eines Zusatzbonus neben der eigentlichen Primärmotivation aufzufassen sind.

In der intrinsisch-altruistischen Kategorie sind vor allem Motive subsummiert, die die Arbeit mit Kindern und Jugendlichen, die Förderung derer Entwicklung sowie den 
durch die Bildung zu erfüllenden gesellschaftlichen Auftrag betonen. Struck (1994) bezeichnet die auf diese Art und Weise Motivierten als die besten Lehrer, die an den Schulen aber nur etwa zehn bis 20 Prozent ausmachen. Neben dem reinen Unterricht sind diese Lehrer auch bereit, sich mit den Problemen und Bedürfnissen der Schüler auseinanderzusetzen und entwickeln ihre Kenntnisse und Kompetenzen stetig weiter. Des Weiteren können auch gesellschaftspolitische und fachliche Interessen neben der Arbeit mit Kindern und Jugendlichen zu einer intrinsisch bedingten Berufswahlmotivation führen. Lehrer, die aufgrund gesellschaftsbezogener und politischer Beweggründen den Beruf gewählt haben, sind oft außergewöhnlich engagiert, jedoch am Schicksal von einzelnen Kindern oft weit weniger interessiert als die intrinsisch-altruistisch Motivierten. Sie interpretieren ihren Erziehungs- und Bildungsauftrag globaler und weitreichender. Auch individuelles Fachinteresse kann zu einer intrinsisch bedingten Berufswahl führen. Struck (1994) stellte jedoch fest, dass Lehrer, die hauptsächlich aus Fachinteresse den Lehrerberuf gewählt haben, zwar durchaus oft gute Didaktiker sind, auf Zuwendungen zu Schülern und zu deren Problemen jedoch weitestgehend verzichten. Tätigkeitsorientierte Motive schließlich sind für den Lehrerberuf immanent durch die Selbstständigkeit, den hohen Anteil an frei einteilbarer Arbeitszeit und der guten Vereinbarkeit von Beruf und Familie. In Abgrenzung zu den extrinsischen Motiven ist diese Motivkategorie stark durch den individuellen Charakter und die Ausgestaltung der Berufstätigkeit bestimmt, die jedoch weiterhin im Mittelpunkt steht, wohingegen extrinsische Motive, wie zuvor beschrieben, eine deutliche Verschiebung zu den Begleitumständen beinhalten.

Neben diesen kategorisierbaren Motiven kann auch ein biographisch-kompensierender Ansatz der Studien- und Berufswahl zugrunde liegen. Lehrer zu werden bedeutet auch immer eine Begegnung mit der eigenen Vergangenheit (Struck, 2007). Negative Erfahrungen aus der eigenen Kindheit bzw. der Schulzeit werden durch das Studium von Pädagogik und Psychologie aufgearbeitet und es wird oft die Zielsetzung verfolgt, es besser zu machen als die Lehrer, die die eigene Schulzeit geprägt haben (Struck, 1994). Grundsätzlich darf bezüglich der Faktoren bzw. Motive, die für die Studien- und Berufsentscheidung verantwortlich sind, kein unkritischer Umgang erfolgen, denn sie können durchaus negative Anfangsvoraussetzungen darstellen. So sind neben hedonistischen bzw. extrinsischen Motiven auch solche, die allzu sehr von Idealismus geprägt sind, nicht als völlig unbedenklich einzustufen, denn hier besteht die besondere Gefahr, dass sich im Berufsleben schnell Frustration einstellt. Neben der Gefahr, den eigenen Ansprüchen nicht gerecht werden zu können, stehen hier oft Aufwand und Ertrag der Arbeit in keinem Verhältnis zueinander. 
Wie sich in Studien zu den Berufswahlmotiven von Lehramtsstudenten bzw. -absolventen (Dann \& Lechner, 2001; Kramis-Aebischer, 1996; Oesterreich, 1987; Schwänke, 1988; Urban, 1985; Ulich, 2004) herausstellte, sind es vor allem pädagogische und zwischenmenschliche Motive wie z.B. Freude an der Arbeit mit Kindern und Jugendlichen, menschliche Begegnung oder Interesse an Erziehung, die für eine Entscheidung Pro-Lehramt verantwortlich gemacht werden. Diese Kategorie enthält neben der positiven Bewertung der Arbeit mit Kindern und Jugendlichen auch gesellschaftliche Aspekte. Als zweitwichtigste Motivkategorie sind tätigkeitsorientierte Begründungsmuster zu nennen, die vor allem auf den Besonderheiten des Lehrberufs wie freie Zeiteinteilung, Selbstständigkeit und einem abwechslungsreichen Berufsalltag basieren. Bei Frauen, die sich für den Beruf entscheiden, spielt vor allem auch die gute Vereinbarkeit von Beruf und Familie eine nicht zu unterschätzende Rolle.

Entgegen der landläufigen Meinung spielen extrinsische Motive wie der sichere Beamtenstatus, die langen Ferienzeiten und das gute und vor allem sichere Gehalt in den zuvor genannten Studien nur eine nachgeordnete Rolle. Auffällig ist hingegen in der geschlechtsspezifischen Betrachtung, dass Studenten doppelt so oft extrinsische Gründe anführen als Studentinnen, die offensichtlich in stärkerem Maße die Arbeit mit Kindern und Jugendlichen sowie die Vereinbarkeit mit dem Familienleben schätzen. Weiterhin ist ein Anstieg des Fachinteresses als Studien- und Berufswahlmotiv von der Grundschule über die Haupt- und Realschule bis hin zum Gymnasium festzustellen, was mit dem ausgebildeten intellektuellen Leistungspotenzial der Lehrkräfte korrespondiert (Gold \& Rauin, 2007).

Auch in internationalen Studien werden ähnliche Motivkategorien identifiziert, die für die Berufswahl als ausschlaggebend angesehen werden. Stiegelbauer (1992) befragte im Jahr 1992 insgesamt 203 Studenten der Faculty of Education der University of Toronto nach den Gründen für die Entscheidung, Lehrer zu werden. Neben dem Anspruch, die Gesellschaft zu verbessern, stellte sich der Wunsch, eigenes Wissen mit Schülern zu teilen und das Lehrer-Schüler-Verhältnis für eine beidseitige Entwicklung zu nutzen, als bedeutsamste Begründungen heraus. In einer anderen Studie gaben die meisten Studenten der Northwestern Oklahoma State University an, dass sie „a positive difference in the lives of the children" bewirken möchten und aus dieser Zielsetzung ihre Motivation für die Berufswahl Lehramt entstammt (Bastick, 2000, S. 343).

Weiner (1993) verglich die Berufswahlmotivation von Harvard-Studenten mit der von Studenten aus ländlichen Regionen und stellte zudem die Gründe für die Berufswahl Lehramt von reichen Industriestaaten und Entwicklungsländern gegenüber. Es stellte sich heraus, dass bei geringerem sozialen Status der Eltern extrinsische Motive deutlich 
häufiger eine Rolle im Entscheidungsprozess spielen. In Weiners Vergleich stellte sich jedoch ebenfalls heraus, dass sowohl bei den Harvard-Studenten als auch bei den aus ländlichen Regionen stammenden Studenten überwiegend idealistische Begründungen für die Berufswahl vorliegen. Diese idealistischen Begründungen sind jedoch bei der zweitgenannten Gruppe deutlich fokussierter auf die Arbeit mit den Kindern und weniger global bzw. gesellschaftlich ausgerichtet als bei den Harvard-Studenten. In der weiteren Auswertung der Daten zeigte sich, dass bei den Harvard-Studenten Unabhängigkeit und Freiheit, also tätigkeitsorientierte Motive als deutlich bedeutsamere und entscheidungsrelevantere Berufsmerkmale eingestuft wurden, als bei der Vergleichsgruppe. Die Sicherheit des Arbeitsplatzes und das Gehalt bezeichnen lediglich 15,1\% der Studenten in ländlichen Gebieten als eher unwichtig, während der Anteil bei den Harvard-Studenten mit $49 \%$ deutlich höher ist.

Noch deutlicher wird der offensichtliche Zusammenhang von sozio-ökonomischem Hintergrund und den Beweggründen für eine Lehramtskarriere in der Studie von Yong (1995) zur Berufswahlmotivation von Lehramtsstudenten in Brunei Darussalam. 46\% gaben an, den Lehrerberuf aufgrund von extrinsischen Aspekten gewählt zu haben, während 31,6\% intrinsische und 22,4\% altruistische Gründe angaben. Unter den extrinsischen Begründungen wurde die Antwort „ich hatte keine andere Wahl" am häufigsten angegeben. Der Einfluss von bedeutsamen Personen im privaten Umfeld, das Gehalt, die Sicherheit des Arbeitsplatzes und die Arbeits- bzw. Ferienzeit waren die nächstgenannten Beweggründe. Schlussfolgernd resümiert Yong, dass viele Studenten in Brunei Darussalam den Lehrberuf als letzten Ausweg sehen und ein wahres Bedürfnis zu unterrichten selten vorzufinden sei.

Watt und Richardson (2007) belegten die Relevanz der motivationalen Faktoren, die dazu führen, eine Karriere im Lehrerberuf anzustreben. Sie verglichen in einer Längsschnittstudie die Eintrittsmotivation von Lehramtsanwärtern zu Beginn ihrer Ausbildung mit verschiedenen Faktoren zu einem Zeitpunkt nach der Ausbildung, die auf eine professionelle und engagierte Berufsauffassung hindeuten. Intrinsische und altruistische Motivation bei der Berufswahl korrelierten positiv mit dem gemessenen beruflichen Engagement sowie dem Fort- und Weiterbildungsbestreben. Zwischen extrinsischen Motiven, den Beruf zu ergreifen und den unterstützenden positiven Eigenschaften und Überzeugungen, bestehen keine bzw. negative Zusammenhänge. In dieselbe Richtung deuten die Ergebnisse von Rauin (2007), der Zusammenhänge zwischen Eintritts-Typisierungen von Studenten anhand der Motivation und dem Engagement sowie ihren erworbenen Kompetenzen zum Ende des Studiums nachweisen konnte. Im weiteren Verlauf manifestiert sich fehlendes Engagement und Kompetenz in einem 
verstärkten Belastungserleben. Dieses führt, entgegen den Annahmen, verstärkt bei denjenigen, die nie gebrannt haben, in seiner Endform zu Burnout. Jedoch wirkt sich mangelnde Motivation und Engagement nicht nur auf die Lehrperson selbst negativ aus, auch die Qualität des Unterrichts und damit die Bildung und Erziehung der Kinder und Jugendlichen wird gefährdet. Der Enthusiasmus in dem Fach zu unterrichten und eine allgemeine Lust auf Schule sind die entscheidenden Faktoren für die Unterrichtsqualität und die Lernentwicklung von Schülern (Baumert, 2009). Hiermit wird die Aussage von Bauer (2008) bestätigt: Fachinteresse und extrinsische Motivation reichen nicht aus, um langfristig glücklich, zufrieden und vor allem gesund im Beruf zu agieren und gleichzeitig dem Anspruch gerecht zu werden, Kinder und Jugendliche in ihrer Entwicklung optimal zu fördern.

Nachdem sich in dem vorherigen Abschnitt alles um die Studien- und Berufswahlmotive gedreht hat, soll im Folgenden die Rekrutierungscharakteristik etwas genauer herausgearbeitet werden. Während der Lehrerberuf in der Vergangenheit für Töchter aus höheren Schichten als standesgemäßer Überbrückungsberuf zum Hausfrauendasein galt, waren es unter den Männern oft diejenigen, die entweder aus ungünstigen sozioökonomischen Verhältnissen stammten oder für andere Studienfächer keine ausreichende Begabung aufwiesen. Auf der Basis der Allgemeinen Bevölkerungsumfrage der Sozialwissenschaften (ALLBUS) der Jahre 1980 bis 2002 eruierte Kühne (2006) das soziale Rekrutierungsfeld der Gegenwart bzw. jüngeren Vergangenheit von Lehrkräften. Im Vergleich zu anderen akademischen Berufsgruppen weist der Lehrerberuf eine deutlich niedrigere soziale Herkunft auf und galt bzw. gilt als klassischer Aufsteigerberuf. Juristen, Architekten und Mediziner rekrutieren sich zu ca. 50\% aus der oberen Dienstklasse, während lediglich jeder fünfte Lehrer diesem Milieu entstammt. Eine Besonderheit, die man außer bei Lehrern auch oft bei Ärzten beobachtet, sind regelrechte Dynastien, in denen sich die Berufswahl über mehrere Generationen stetig fortpflanzt und erkennbare Neigungen und Talente scheinbar eine weniger ausschlaggebende Rolle spielen. Bezogen auf die Berufsvererbungsprozesse liegt bei Lehrern die Selbstrekrutierungsrate ${ }^{1}$ an höheren Schulen mit $18 \%$ deutlich höher als bei den meisten anderen Berufen. Differenziert man nicht nach den verschiedenen Lehrämtern, d.h. legt man keinen Wert auf eine Übereinstimmung der Schulform des Elternteils und der Nachkommen, liegt die Quote sogar bei 24\%. Lediglich die Mediziner vererben häufiger den Beruf an ihre Nachkommen weiter. Jedes vierte Kind eines Arztes schlägt ebenfalls diesen vorgelebten Berufsweg ein. Besonderheiten sind noch in der Ge-

\footnotetext{
${ }^{1}$ Die Selbstrekrutierungsrate besagt, dass die Kinder eines Lehrers einer höheren Schule ebenfalls eine Lehramtskarriere an einer höheren Schule aufnehmen.
} 
schlechtsspezifik herauszuheben, denn während beim Lehrerberuf vor allem die Töchter den Vätern in den Beruf folgen, sind bei Ärzten vornehmlich die Söhne diejenigen, die in die Fußstapfen ihrer Väter treten (Kühne, 2006).

Neben der Charakteristik eines klassischen Aufsteigerberufs und der überdurchschnittlichen Selbstrekrutierung ist zudem auffallend, dass sich die leistungsstärksten Absolventen der Gymnasien sehr selten für ein Lehramtsstudium entschließen und eher anderen Studiengängen nachgehen. Wößmann (2009, zitiert nach Baumert, 2009, S. 72) sieht in den schlechteren Abiturnoten von Lehramtsstudenten im Vergleich mit anderen Studiengängen einen verlässlichen Hinweis auf eine geringere Leistungsbereitschaft im Beruf, die auch durch das besonders stark ausgeprägte soziale Interesse nicht mehr kompensierbar sei. Die Entscheidung der leistungsstärksten Absolventen sieht Wößmann in dem Charakter des Lehrerberufs begründet: „Alles an der Struktur der Personalpolitik spricht dagegen, dass sich leistungsfähige und -bereite Menschen für den Lehramtsberuf entscheiden. Dass es viele aufgrund einer an Kindern und Jugendlichen ausgerichteten Motivation dennoch tun, ist ihnen umso höher anzurechnen“. Baumert (2009) konnte jedoch in der COACTIV-R-Studie zeigen, dass zwischen der durchschnittlichen Abiturnote und der Unterrichtsqualität sowie der Leistungsentwicklung von Schülern keinerlei Zusammenhänge bestehen. Baumert sieht weiterhin in der Rekrutierung keinen dringlichen Handlungsbedarf, sondern vielmehr in der Ausbildung von Lehrkräften. Eine weitere Problematik im Zusammenhang mit der Berufswahl Lehrer ergibt sich aus der verbreiteten gesellschaftlichen Einschätzung von Studium und Berufsleben. Sowohl das Lehramtsstudium als auch der Beruf wird oft als Weg des geringsten Widerstandes gesehen. „Lehrer werden ist nicht schwer“, „dann kann mein Leben so bleiben, wie es jetzt ist“" da keine große Umstellung zum bisher bekannten Schulleben erforderlich ist ${ }^{2}$, (Kiel et al., 2007, S. 12). Tatsächlich stellt das Lehramtsstudium oft eine Negativauswahl dar, wenn man sich ein reines Fachstudium nicht zutraut oder aufgrund von Zulassungsbeschränkungen in anderen Studiengängen vorerst oder auch final im Lehramt bzw. dessen Studium parkt (Struck, 1994). Für den Sportlehrerberuf bzw. dessen Studium konnten Weiß und Kiel (2010) in einer Studentenbefragung empirisch nachweisen, dass angehende Sportlehrer von geringeren Ansprüchen und Anforderungen im Studium ausgehen als in anderen Fächern. Das Lehramtsstudium wird jedoch nicht nur als Sekundärwahl herangezogen, sondern stellt auch eine Ad-hoc-Entscheidung dar. Birk et al. (1978) fanden heraus, dass mehr als die Hälfte derjenigen Studenten, die kurz vor dem Abitur noch nicht wussten, welche Studienrichtung sie wählen sollten, ein Lehramtsstudium aufnahmen (Birk et al., 1978, zitiert

\footnotetext{
${ }^{2}$ Aussage einer 14jährigen Schülerin
} 
nach Schwänke, 1988). Dieser Anteil an Spätentschlossenen die sich dafür entscheiden, den Ausbildungsweg eines Lehrers zu wählen, hat sich aufgrund der sich deutlich verschlechterten Einstellungsvoraussetzungen gegenüber den späten 70er Jahren etwas abgesenkt. Der Anteil dieser Notlösungs-Lehramtsstudenten beträgt jedoch immer noch $25 \%$ (Kiel et al., 2007).

Neben dem bereits zuvor angesprochenen geringeren Niveau der Hochschulzugangsberechtigung der Lehramtsanwärter bzw. Lehrer ergab eine repräsentative Studie mit kurz vor dem Abschluss stehende Gymnasiasten des Kantons Bern, dass die Lehramtskandidaten in Bezug auf Motivation und Engagement eine Haltung aufweisen, die entgegen den Bestrebungen der Neuorientierung des Lehrerberufs und der Ausbildung steht. Der Frage nachgehend "die Ausbildung der Lehrpersonen wandelt sich - aber wandeln sich auch die Studierenden?" wurden die Berufswahlmotive von Maturanden mit dem Ziel Lehramt mit anderen Maturanden verglichen. In den Ergebnissen zeigte sich, dass angehende Lehramtskandidaten signifikant weniger an einer persönlichen Weiterentwicklung im Beruf sowie wissenschaftlichem Arbeiten interessiert sind, als Maturanden, die sich für andere Studiengänge bzw. Berufe entscheiden. Maturanden, die sich für den Lehrerberuf interessieren, bewerten damit gerade die zentralen Aspekte der auf Professionalisierung des Berufs abzielenden reformierten Ausbildung als wenig bedeutsam. Sie stellen damit gleichzeitig ihre eher durch Pragmatismus charakterisierte Einstellung einer erfolgreichen Umsetzung der Ansätze zur Neuorientierung infrage. Differenzieren muss man diese Feststellungen jedoch noch in Bezug auf die bereits in diesem Kapitel beschriebene sozio-ökonomische Herkunft der Lehramtsanwärter. Während der Pragmatismus bei Maturanden aus bildungsferneren Schichten deutlicher ausgeprägt ist, ist bei Maturanden aus höheren Bildungsschichten ein höheres Interesse an wissenschaftlichem Arbeiten während der späteren beruflichen Tätigkeit festzustellen (Denzler, Fiechter \& Wolter, 2005).

In diesem Kapitel wurde deutlich, dass die Motive, die für die Studien- bzw. Berufswahl verantwortlich sind, bereits einen relativ aussagekräftigen Indikator für die Art und Weise der Interpretation und Ausgestaltung des Berufes darstellen. Im weiteren Verlauf dieser Arbeit wird sich zeigen, dass die Berufswahlmotivation eine bedeutsame Einflussgröße auf den weiteren Karriereverlauf hat. Gemäß des Zitats des französischen Schriftstellers Honoré de Balzac „ein verfehlter Beruf verfolgt uns durch das ganze Leben“ nimmt diese Karriereentscheidung eine Schlüsselposition im Leben ein und sollte genau abgewägt werden (zitiert nach Brinkmann \& Stapf, 2005, S. 114). Durch die Langfristigkeit der Auswirkungen sollte eine solch bedeutsame Entscheidung aufgrund einer soliden Begründungsbasis getroffen werden und nicht aus einer spontanen 
Laune heraus bzw. aufgrund mangelnder Alternativmöglichkeiten. Hierfür ist es notwendig, dass Jugendliche über realistische Einschätzungen über die Berufe verfügen, die ihren Interessen, Neigungen und Kompetenzen entsprechen.

\subsection{Die Besonderheiten des Lehrberufs, Aufgaben, Antino- mien und Rollenerwartungen}

Nachdem sich im voranstehenden Unterkapitel alles um die Wahl des Berufes drehte, soll in diesem Kapitel der Beruf selbst im Zentrum der Betrachtung stehen. Für eine sinnvolle Auseinandersetzung mit den Berufsanforderungen, Aufgaben und den damit verbundenen Belastungen, ist es zunächst wichtig zu wissen, was den Lehrerberuf überhaupt auszeichnet, wie er definitorisch umrissen ist, welche Aufgabenbereiche und Verpflichtungen er umfasst und wie man ihn in der Gesellschaft wahrnimmt und wertschätzt.

Sucht man im Internet nach dem Begriff "Lehrer" liefert die derzeitig populärste Suchmaschine "Google“ über 44 Millionen Suchtreffer. Unter dem ersten Treffer verbirgt sich eine Beschreibung der freien Enzyklopädie „Wikipedia“", die mit einer Definition startet, in der Lehrer als Personengruppe beschrieben werden, „deren Aufgabe es ist, andere dabei zu unterstützen, sich Bildung bzw. Ausbildung anzueignen und ihre Persönlichkeit weiterzuentwickeln, damit sie in der Gesellschaft erfolgreich agieren können“. Vom Europäischen Rat werden Lehrer als „die wichtigsten Akteure jeder auf die Förderung der Entwicklung der Gesellschaft und Wirtschaft gerichteten Strategie “ verstanden und sollen jungen Menschen dazu verhelfen, sich in einer sich stetig wandelnden Welt zurechtzufinden, indem sie Einfluss auf die Persönlichkeitsentwicklung junger Menschen nehmen (Eurydice, 2002, S. 3). Während diese beiden Definitionen sehr allgemein und global beschreiben, welche Aufgaben Lehrer haben und sehr viel Spielraum für Interpretation lassen, wird in den Standards für die Lehrerbildung: Bildungswissenschaften (2004) genauer beschrieben, was die Aufgaben von Lehrern sind. Lehrer werden als Fachleute für das Lehren und Lernen bezeichnet, deren Kernaufgabe darin besteht, anhand wissenschaftlicher Erkenntnisse gezielt Lehr- und Lernprozesse zu planen, zu initiieren, zu organisieren und zu reflektieren. Individuelle Bewertung und systemische Evaluation komplettieren das Anforderungsspektrum, das durch die Lehrkräfte erfüllt werden soll (Kultusministerkonferenz, 2005.

Im Schuljahr 2006/2007 unterrichteten in Deutschland etwa 792.000 Lehrerinnen und Lehrer. Statistisch auffällig war und ist der überproportionale Anteil von 65\% Frauen unter den Lehrkräften, woran sich auch in Zukunft nichts ändern dürfte, denn auch

\footnotetext{
${ }^{3}$ Zugriff am 16.10.2009.
} 
unter den 208.800 Studierenden in den Lehramtsstudiengängen waren mit 68\% deutlich mehr Frauen als Männer eingeschrieben (Statistisches Bundesamt, 2007b). Grundsätzlich handelt es sich um einen akademischen Beruf, den man nach einer zweiphasigen Ausbildung ausübt, die sich aus einem weitgehenden theoretisch gestalteten Teil an einer Hochschule sowie einem Praxisanteil im Referendariat zusammensetzt. In dem Kultur-, Gesellschafts- und Sozialberuf wird neben der Enkulturation und Bildung Heranwachsender Einfluss auf die ökonomische, politische und soziale Entwicklung genommen, wodurch die Interessen verschiedener gesellschaftlicher Gruppen vertreten werden. Letztendlicher Zweck von Organisation und Durchführung des Unterrichts ist jedoch das Lernen der Schüler (Ulich, 1996).

Auch in den jeweiligen Schulgesetzen der Länder ist definiert, welche Vorstellungen der jeweilige Dienstherr von seinen Bediensteten, den Lehrkräften, hat. Im hessischen Schulgesetz wird im siebten Teil (Hessischer Landtag, 2005, § 86) spezifiziert, was das Gesetz unter Lehrerinnen und Lehrern versteht.

(1) Lehrerin oder Lehrer im Sinne dieses Gesetzes ist, wer an einer Schule selbstständig Unterricht erteilt. Lehrerinnen und Lehrer an öffentlichen Schulen sind in der Regel Bedienstete des Landes. Sie sind in der Regel in das Beamtenverhältnis zu berufen.

In dem Auszug aus dem Hessischen Schulgesetz wird bereits eine Dependenz vom Dienstherrn angedeutet. Im niedersächsischen Schulgesetz wird diese Abhängigkeit mit den zugehörigen Verpflichtungen, denen die relative Freiheit der Berufsausübung unterliegen, deutlicher formuliert. Dies zeigt der Auszug aus dem dritten Teil (Niedersächsischer Landtag, 1998, §50).

(1) Die Lehrkräfte erziehen und unterrichten in eigener pädagogischer Verantwortung. Sie sind an Rechts- und Verwaltungsvorschriften, Entscheidungen der Schulleiterin oder des Schulleiters oder der kollegialen Schulleitung, Beschlüsse des Schulvorstands, Beschlüsse der Konferenzen und deren Ausschüsse nach $\S 39$ Abs. 1 und 2 sowie an Anordnungen der Schulaufsicht gebunden.

(2) Die Lehrkräfte an den öffentlichen Schulen stehen in einem unmittelbaren Dienstverhältnis zum Land.

Aus den Gesetzesauszügen der beiden Bundesländer wird deutlich, dass Lehrpersonen sich durch die Kulturhoheit in einem Dienstverhältnis des jeweiligen Landes befinden, das in den meisten alten Bundesländern im Regelfall als Beamtenstatus umgesetzt ist. 
In den neuen Bundesländern werden Lehrer oft als Angestellte beschäftigt. Der Beamtenstatus ist mit einigen Vorzügen versehen, sorgt jedoch auch dafür, dass man sich in einem Abhängigkeitsverhältnis zum Dienstherrn, im Fall des Lehrers, des Landes, befindet. Neben der Weisungsabhängigkeit und den damit verbundenen Vorschriften sind Lehrer zudem dazu verpflichtet, einen Diensteid zu leisten, der zur Verfassungstreue verpflichtet. Doch der Lehrerberuf kennzeichnet sich auch durch Widersprüchlichkeiten. Auf der einen Seite sollen Lehrpersonen Kinder und Jugendlichen zu einer kritischen, demokratischen und unabhängigen Einstellung und Meinung verhelfen, müssen dies aber aus einer Position heraus bewältigen, die durch politische Treuepflicht und Streikverbot zu politischer Abstinenz und Standpunktlosigkeit verpflichtet (Ulich, 1996). Im Hinblick auf das Beamtentum und den damit verbundenen Privilegien und Einschränkungen beschreibt die metaphorische Betrachtung "des Kaisers Rock ist eng, hält aber warm" die Situation, in der sich viele Beamte wiederfinden, relativ anschaulich. Im siebten Teil des Hessischen Schulgesetzes wird in $\S 86$ (Hessischer Landtag, 2005) deutlich in welcher Art und Weise Freiheiten der Lehrkräfte eingeschränkt werden:

(3) Zur Gewährleistung der Grundsätze des $\S 3$ Abs. 1 haben Lehrkräfte in Schule und Unterricht politische, religiöse und weltanschauliche Neutralität zu wahren; $\S 8$ bleibt unberührt. Insbesondere dürfen sie Kleidungsstücke, Symbole oder andere Merkmale nicht tragen oder verwenden, die objektiv geeignet sind, das Vertrauen in die Neutralität ihrer Amtsführung zu beeinträchtigen oder den politischen, religiösen oder weltanschaulichen Frieden in der Schule zu gefährden. Bei der Entscheidung über das Vorliegen der Voraussetzungen nach Satz 1 und 2 ist der christlich und humanistisch geprägten abendländischen Tradition des Landes Hessen angemessen Rechnung zu tragen. Für Lehrkräfte im Vorbereitungsdienst kann die zuständige Behörde auf Antrag abweichend von Satz 2 im Einzelfall die Verwendung von Kleidungsstücken, Symbolen oder anderen Merkmalen zulassen, soweit nicht zwingende öffentliche Interessen entgegenstehen.

Auch im niedersächsischen Schulgesetz (Niedersächsischer Landtag, 1998, § 51) existieren sogenannte dienstliche Sonderregelungen, die weitere Verpflichtungen und Vorschriften enthalten:

(1) Die Lehrkräfte erteilen Unterricht grundsätzlich in solchen Fächern und Schulformen, für die sie die Lehrbefähigung erworben haben, die Lehrkräfte mit der Lehrbefähigung für Schulformen der allgemein bildenden Schulen 
auch in Gesamtschulen. Darüber hinaus haben die Lehrkräfte Unterricht in anderen Fächern und Schulformen zu erteilen, wenn es ihnen nach Vorbildung oder bisheriger Tätigkeit zugemutet werden kann und für den geordneten Betrieb der Schule erforderlich ist. Vor der Entscheidung sind sie zu hören. Sie sind verpflichtet, Aufgaben im Rahmen der Eigenverwaltung der Schule und andere schulische Aufgaben außerhalb des Unterrichts zu übernehmen.

(2) Die Lehrkräfte sind verpflichtet, sich zur Erhaltung der Unterrichtsbefähigung in der unterrichtsfreien Zeit fortzubilden.

(3) Das äußere Erscheinungsbild von Lehrkräften in der Schule darf, auch wenn es von einer Lehrkraft aus religiösen oder weltanschaulichen Gründen gewählt wird, keine Zweifel an der Eignung der Lehrkraft begründen, den Bildungsauftrag der Schule (§ 2) überzeugend erfüllen zu können. Dies gilt nicht für Lehrkräfte an Schulen in freier Trägerschaft.

Diese Einschränkungen führen dazu, dass man lediglich von einer pädagogischen Restfreiheit spricht, im Rahmen derer Lehrkräfte ihren Unterricht frei gestalten können. Trotz der Weisungsabhängigkeit lassen jedoch in der konkreten Unterrichtssituation Lehrpläne, Curricula und Bildungsstandards dem Lehrer eigene Ausgestaltungsmöglichkeiten, sodass sich der Lehrerberuf in einer Schwebelage zwischen staatlicher Reglementierung und pädagogischer Freiheit befindet und in gewissem Maße auch die Umsetzung eigener Interessen zulässt (Rothland \& Terhart, 2007). Die Arbeit in der Klasse wird vom Lehrer allein ausgeführt und ist nicht direkt reglementierbar, die pädagogischen Freiheiten sind jedoch durch Rechtsvorschriften eingeschränkt. Diese Restfreiheit, die durch staatliche Vorschriften, Verfügungen, Beschlüsse des Kollegiums und Weisungen der Schulleitung bleibt, reduziert sich durch die Forcierung der Profilbildung von Schule, die in jüngerer Vergangenheit die Schulorganisation bestimmt. Als Konsequenz auf den Geburtenrückgang und den darauf einsetzenden Wettbewerb um Schüler sind Schulen dazu aufgefordert, sich von anderen Schulen durch spezielle Schwerpunktsetzungen abzugrenzen und möglichst die eigene Attraktivität durch diese Profilbildung zu steigern (Ulich, 1996). Ein weiterer Faktor, der Druck auf die Lehrkräfte verstärkt und die Freiheit einschränkt, stellt die Output-Orientierung der Post-PISASchock-Periode dar, die sich durch externe Evaluation und zentrale Leistungsvergleiche kennzeichnet. Trotz des zunehmenden Drucks auf die Lehrer von außen ist der Beruf in der Praxis im weitesten Sinne vom Leistungsprinzip entbunden und Unterschiede im Gehalt kommen lediglich aufgrund der abgeleisteten Dienstjahre und der Vorbildung zustande. Da innerberufliche Karrierechancen so gut wie nicht bestehen, geht das En- 
gagement von Lehrern oft auf berufsferne Bereiche über, wodurch das verstärkte soziale und politische Engagement von Lehrern erklärt werden kann. Man spricht bei dem Lehrerberuf oft von einem „Beruf ohne Karriere“, was auch motivational zu Problemen führen kann und im weiteren Verlauf der Arbeit erneut an verschiedenen Stellen aufgegriffen werden wird (Rothland \& Terhart, 2007, S. 16).

\subsubsection{Die Institutionalisierung und Entwicklung des Lehrens und Lernens}

Um genauer das Aufgabenspektrum, den Berufsstand und die damit verbundenen Anforderungen von bzw. an Lehrkräfte darzulegen, ist es wichtig, eine begründungstheoretische Basis, sowohl im Bezug auf das Lehren und Lernen generell, als auch bezogen auf die geschichtliche Entwicklung des Schulwesens zu schaffen. Der Pädagoge Friedrich Paulsen stellte bereits 1885 fest, dass die Beschäftigung mit der Vergangenheit dazu beitragen kann, zukunftsbezogen Folgerungen zu ziehen bzw. gegenwärtig existente Situationen und Entwicklungen leichter verstehbar machen.

Im Folgenden soll ausgehend von der Institutionalisierung des Lehrens und Lernens über die geschichtliche Entwicklung die aktuelle Situation der Lehr-Lern-Kultur dargestellt werden, bevor darauf aufbauend im nächsten Unterkapitel das Aufgabenspektrum von Lehrern zusammengefasst wird.

Ausgangs- und Ansatzpunkt für die Entwicklung der Bildungseinrichtung Schule und den darin unterrichtenden Lehrkräften stellt die Grundanforderung des Lebens an die Menschen das Lernen dar, das meist unbewusst und beiläufig erfolgt. Nach Hasselhorn und Gold (2006, S. 35) ist Lernen „ein Prozess, bei dem es zu überdauernden Änderungen im Verhaltenspotenzial als Folge von Erfahrungen kommt“. Grundlage von Lernprozessen ist das Erkennen von Zusammenhängen zwischen Ereignissen, d.h. von dem grundlegenden Reiz, dem an den Tag gelegten Verhalten und den resultierenden Konsequenzen. In den behavioristischen Ansätzen der ersten Hälfte des 20. Jahrhunderts bezog sich der Lernprozess lediglich auf das beobachtbare Verhalten, das im Rahmen von Banduras sozial-kognitive Lerntheorie durch kognitive-mentale Prozesse und Strukturen erweitert wurde. Auch Behavioristen kamen nicht umher diese bedeutende Erweiterung bereits bestehender Lerntheorien anzuerkennen, wollten sie Lernen und Verhalten ganzheitlich und umfassend erforschen. Im Kognitivismus werden innere, bewusste Vorgänge des Lernprozesses in den Vordergrund gerückt, während in konstruktivistischen Ansätzen Wissen durch subjektive Interpretation und Konstruktion entsteht. Lernen wird hier als selbstgesteuerter, aktiver Prozess begriffen. Ohne zu lernen ist der Mensch nicht in der Lage, sich von der Abhängigkeit von seinen Fürsorgepersonen zu befreien, denn nur wer in der Lage ist, sich bestimmte Dinge anzueig- 
nen, wird sich in seiner Umwelt zurechtfinden und am gesellschaftlichen Leben erfolgreich partizipieren können. In seiner basalen Form hat Lernen mit Spaß oder Freude wenig zu tun, sondern sichert grundständig zunächst das Leben und Überleben. Es stellt eine Antwort auf Not, Elend, Hunger und Unsicherheit dar, die als Antrieb, als Ausgangsmotivation zu verstehen sind. Durch diesen Zusammenhang wird auch verständlich, wie Kinder in Entwicklungsländern unter unzumutbaren Bedingungen hochmotiviert zu Werke gehen, da die Beziehung von Lernen und Leben bzw. Überleben noch existiert, während in hochtechnisierten Sozialstaaten wie Deutschland das Lernen mit zunehmendem Luxus in eine Krise gerät (Steiner, 2001).

Seit Menschengedenken sind Behütung, Pflege und Unterweisung des Nachwuchses notwendige Voraussetzungen, um die individuelle und die kollektive gesellschaftliche Entwicklung voranzutreiben. Dementsprechend, ungeachtet ihrer pädagogischen oder methodischen Fähigkeiten, waren und sind Lehrer unverzichtbar für die Gemeinschaft, deren Fortbestand und Weiterentwicklung (Arnhardt, Hofmann \& Reinert, 2000). Bevor die Institutionalisierung des Wissens durch die Einrichtung von Schulen erfolgte, waren es Personen, die Dinge besser konnten als andere, die sich spezialisierten und Fertigkeiten entwickelten bzw. Wissen aufbauten. Problematisch und immer wieder für das Stagnieren der Entwicklung verantwortlich war jedoch, dass der Erhalt von Fertigkeiten und Wissen, durch den Tod des Individuums gefährdet war. Der erste Entwicklungsschritt wurde durch die Weitergabe von Wissen an Jüngere durch die Erfindung der Schrift bewältigt. Durch die Möglichkeit Wissen zu konservieren und weiterzugeben, war keine direkte Interaktion mit dem Wissenden notwendig, der längst verstorben oder an einem ganz anderen Ort leben konnte. Voraussetzung für die Verbreitung von Wissen war und ist jedoch das Beherrschen der Techniken des Lesens und Schreibens.

Um das dem Menschen angeborene Potenzial auszuschöpfen und weiterzuentwickeln, wurden Systeme geschaffen, um Fertigkeiten, Kenntnisse, Normen und Überzeugungen adäquat und zielgerichtet zu vermitteln (Hasselhorn \& Gold, 2006). Die kulturelle Erfindung, durch die Wissen und Fertigkeiten aufbewahrt bzw. weitergegeben werden können, ist in der Grundform die häusliche Erziehung, deren Weiterentwicklung der Unterricht in einer Schule darstellt, der durch seine Distanz zum Alltagsleben charakterisiert ist (Konrad, 2007). Lehrkräfte sind weder die Ältesten und Weisesten, noch diejenigen, die mit Alltagsproblemen am besten umgehen können, sondern es sind Spezialisten für die Aufbewahrung, Gestaltung, Strukturierung und Vermittlung von Wissen im Unterricht (Giesecke, 2001). Lehrern fiel in allen Epochen der menschlichen Entwicklung die Aufgabe zu, Wissen, Können, Einsichten und Fertigkeiten an die nächste 
Generation weiterzureichen. Die ersten Schulen, die auch in neueren wissenschaftlichen Darstellungen als solche bezeichnet werden, waren seit dem 6. Jahrhundert Internate, die an Klöster bzw. Bischofsschulen angeschlossen waren und der Ausbildung des Klerikernachwuchses dienten. Neben der Rekrutierung von Klerikern wurden von Anfang an, entgegen Befürchtungen einer drohenden Verweltlichung, auch Kinder und Jugendliche aufgenommen, die nicht den Kirchendienst anstrebten. In der weiteren Entwicklung unterrichteten Dom- und Klosterschulen neben den Internatsschülern immer mehr Schüler des Ortes bzw. der Stadt. Nicht zuletzt waren es adelige Eltern, die bereit waren, für die Ausbildung ihrer Söhne zu zahlen und damit dafür sorgten, dass neben den klosterinternen scholae interiores zunehmend scholae exteriores eingerichtet wurden, die weltlichen Zwecken dienten. Im Laufe der weiteren Entwicklung der Gesellschaft und den einsetzenden Stadtgründungen wurde seit dem 13. Jahrhundert damit begonnen, Schulen unter städtischem Patronat einzurichten, was nicht über den engen Zusammenhang von Klerikalität und Bildung im Mittelalter hinwegtäuschen soll. Die Expansion des Wissens und die einsetzende Spezialisierung führten dazu, dass sich im 13. Jahrhundert aus verschiedenen Schulen die ersten europäischen Universitäten bildeten, auf die der kirchliche Einfluss von Beginn an deutlich geringer war (Konrad, 2007).

Im Spätmittelalter registrierte man neben den sich zunehmend öffnenden kirchlichen Schulen sogenannte Schreibschulen. An diesen Institutionen, die auch als deutsche Schulen bezeichnet wurden, boten Schreibmeister ihre Dienste in Form von Schreibunterricht und Buchhaltung in koedukativem Unterricht, also auch für Mädchen, an. Latein wurde durch die jeweilige Landessprache abgelöst und kirchliche Bildung verlor zunehmend an Bedeutung im Vergleich zu weltlichem Wissen, da den Anforderungen des Handels und des produzierenden Gewerbes entsprochen werden musste. Städtische Magistrate reagierten auf diese Bedürfnisse mit der Gründung eigener Schulen (Konrad, 2007). Die Schulmeister an den Volksschulen traten als eigener Berufsstand auf, standen jedoch in völliger Abhängigkeit ihrer Dienstherren, der Eltern und auch der Schüler. Sie wurden minimal besoldet und auch ihr Ansehen war sehr gering, was sich auch in der Einordnung der Berufe in einer Sparte mit Nachtwächter und Mäusefänger zeigt (Adrion \& Schneider, 1979). Neben den institutionalisierten Formen hatten sich zusätzlich Familien- und Privatunterricht durch Kleriker und Wanderlehrer etabliert. Diese Formen des Unterrichtens übten einen nicht zu vernachlässigenden Einfluss auf das Bildungswesen dieser Zeit aus (Zymek, 2004). Bis zum letzten Drittel des 18. Jahrhunderts, als eine unübersehbare Bildungsbewegung ganz Europa erfasste und das sogenannte Jahrhundert der Erziehung begann, ist keine einheitlich stringente 
Bildungs- bzw. Schulentwicklung festzustellen (Arnhardt, Hofmann \& Reinert, 2000). Weder die Schule noch die Lehrer waren in dieser Zeit großen Veränderungen ausgesetzt (Adrion \& Schneider, 1979).

Durch die Aufklärung rückten Kinder und Jugendliche verstärkt in den Fokus der Aufmerksamkeit, was in zahlreichen europäischen Ländern zu Schulreformen und damit verbunden zu Veränderungen der sozialen Geltung und Ausbildung von Lehrern führte. Als Beispiel hierfür sei die theresianisch-josephinische Schulreform angeführt, im Zuge derer die "Allgemeine Schulordnung für Normal-, Haupt- und Trivialschulen" aus dem Jahr 1774 erlassen wurde, in der Unterricht zur Beseitigung der Finsternis der Unwissenheit in standesgemäßer Ausgestaltung vorgesehen war (Arnhardt, Hofmann \& Reinert, 2000). Es entstand bald überall ein mehr oder weniger ausdifferenziertes Schulangebot und man strebte das Ziel an, durch Elementarschulen die ganze Bevölkerung zu Landeskindern zu machen. Hiermit war die Schaffung eines einheitlichen nationalen Schulsystems verbunden, was sich als schwieriges und vor allem langwieriges Unterfangen erweisen sollte. Selbst die flächendeckende Einführung der Elementarschule und demzufolge eine umfassende Schulpflicht konnten erst in den letzten Jahrzehnten des 19. Jahrhunderts realisiert werden (Zymek, 2004). Aufklärung, Kapitalismus und nicht zuletzt die Industrialisierung führten im 18. und 19. Jahrhundert zu einem geistigen, ökonomischen und politischen Wandel, der vor allem durch das aufstrebende Bürgertum bedingt wurde. Qualifizierte Arbeitskräfte wurden in höherem Maße benötigt und Lehrpläne mussten modifiziert werden, um die Ansprüche der deutschen Industrie an ihre Mitarbeiter zu erfüllen (Herrlitz et al., 2005). Kirchlicher Dogmatismus wurde durch eine Erziehung des eigenen Verstandes abgelöst. Pädagogisches Experimentieren und Publizieren sowie das Vorantreiben der Gedanken der Aufklärung in Erziehung und Schule prägten diese Epoche (Konrad, 2007). In dieser Zeit kam es auch zum Zusammenbruch des preußischen Reiches 1806/07 und damit einhergehend zur grundlegenden Erneuerung des gesellschaftlichen Lebens. Die neuhumanistische Bildungsreform spielte eine zentrale Rolle in der preußischen Reformbewegung. Wilhelm von Humboldt war für die Bildungsreform zuständig und forderte in seinen bildungstheoretischen Prinzipien Beteiligungschancen aller an der Bildung, was von Fichte als Anspruch aller auf gleiche Bildungsrechte bezeichnet wurde. Die Realisierung der Reformen blieb jedoch zum Großteil aus und stellte sich als hartnäckige Aufgabe auch für kommende Generationen heraus. Selbst heute ist dies immer noch Thema in den Bildungsdiskussionen (Herrlitz et al., 2005, S. 31). Nach der Befreiung von der napoleonischen Besatzung strebten die Lehrkräfte an, ihr Wirken nicht nur auf die Schulstube zu beschränken, sondern volkserzieherisch im weitesten Sinne tätig zu werden (Adrion \& 
Schneider, 1979). In der Schulentwicklung ist diese Phase gekennzeichnet durch die Absonderung des höheren Schulwesens vom niederen, auch bezogen auf die Lehrerstände. Während ein Landschullehrer meist alleine eine Dorfschule führte und hierfür in einem Lehrerseminar oder vom örtlichen Pfarrer ausgebildet wurde, genossen die gymnasialen Stadtschullehrer meist ein universitäres Studium und verdienten ungefähr das Dreifache (Schwänke, 1988). Während sich der Beruf des Gymnasiallehrers eher der Laufbahn von Beamten des höheren Dienstes anglich, wurden die Volksschullehrer als Proletarier der Gelehrtenklasse angesehen. Die Ausrichtung der Lehrer orientierte sich vor allem an den höheren Schulen an neuhumanistischen Idealen. Im Selbstverständnis sehen sich die Lehrkörper eher als Philologen denn als Pädagogen. Die meisten Gymnasien, unter denen die altsprachlichen Formen dominierten, waren auf soziale Exklusivität angelegt, sie durften nach den revolutionären Gleichheitsversprechen aber keine reine Standesschule sein. Je nach Bedarfslage waren sie für begabte Aufsteiger teilweise zugänglich (Herrlitz et al., 2005).

Neben dem Typus Lehrer der eher als Fachwissenschaftler und nicht als Pädagoge, Psychologe oder Erzieher auftrat, etablierte sich an der Schwelle vom 18. zum 19. Jahrhundert mit dem Philanthropismus eine zweite Strömung. Im Geiste des Philanthropismus beschäftigte man sich mit dem Einklang von angemessener wissenschaftlichen Ausbildung und Persönlichkeitsentwicklung durch Erziehungs- und Unterrichtskunst. Der Philanthropismus sollte sich in der Folgezeit als zukunftsbestimmende Ausrichtung der Lehrerschaft erweisen, die als Wortführer des liberalen Fortschritts bezeichnet werden können. Neben diesen auf Verbesserung und Weiterentwicklung der Gesellschaft abzielenden Lehrern, wuchsen andere in die Rolle des weltfremden Paukers hinein, der Vorbild für verschiedenste übersteigerte Zerrbilder in Literatur, Musik und Film wurde (Arnhardt, Hofmann \& Reinert, 2000).

Doch die Entwicklung und der breite Ausbau des Schulwesens hatte nicht nur Befürworter. Von dem Ziel, allen Kindern eine grundständige Schulbildung zu ermöglichen, waren Gutsherren nicht begeistert, die die Landbevölkerung in ihren Diensten ausnutzten. Sie befanden daher, dass Bauernkinder nichts lernen müssten, um sie als billige Arbeitskräfte nicht zu verlieren (Konrad, 2007). Zusätzlich wurden Großgrundbesitzer bei der Finanzierung der ländlichen Schulen wirtschaftlich belastet, da der Grundbesitz als Bemessungsgrundlage herangezogen wurde, obwohl sie selbst diese Dorfschulen für ihren eigenen Nachwuchs nicht in Anspruch nahmen (Herrlitz et al., 2005). Aufgrund der weitgehenden Erfolglosigkeit der Bildungsreformen im Zuge der restaurativen Phase, wurden die zuvor unter amtlichem Wohlwollen existierenden Lehrerverbände in den 40er Jahren des 19. Jahrhunderts zunehmend radikaler und beschäftig- 
ten sich mit bildungspolitischen und pädagogischen Fragen. Der Widerstand konservativer Kräfte sowie von Vertretern höherer Schulen und Universitäten, die um ihren privilegierten Status fürchteten, führte dazu, dass 1854 in den Stieh/schen Regulativen berufliche Vereinigungen von Lehrern verboten wurde und das Ausbildungsniveau radikal gesenkt wurde, um letztendlich Lehrer und Erzieher als dienstwillige Untertanen zu haben (Arnhardt, Hofmann \& Reinert, 2000). Des Weiteren wurde in der Volksschule die Klassenstärke heraufgesetzt und die Schulbesuchsdauer reduziert (Konrad, 2007). Gegen diese staatlich verordnete Unbildung regte sich bald breiter und heftiger Widerstand, was zur Abschaffung der Regulative im Jahr 1872 führte (Arnhardt et al., 2000). Die Entfaltungsmöglichkeiten des Einzelnen in der vorindustriellen Zeit, die durch Geburtsstände stark prädisponiert waren, wurden mit dem Zugang breiterer Bevölkerungsschichten zu schulischer Bildung zunehmend ausgeweitet (Herrlitz et al., 2005).

Als Anpassung an die einsetzende Industrialisierung wurde in der Folgezeit der Ausbau der Volksschule vorangetrieben. Die Bedeutung der Schule und des Lehrers für die Entwicklung von Wirtschaft und Kultur wurde zunehmend anerkannt. Aufgrund des Mangels an Nachwuchs in sämtlichen akademischen Berufen in den deutschen Fürstentümern, wurde eine soziale Besserstellung der Lehrer erreicht, um für diesen Beruf gute Köpfe zu gewinnen. Schulorganisatorisch entwickelte sich das Bildungssystem zu einer vertikalen Form, die die gesellschaftliche Schichtung nicht nur abbildete, sondern weiter verstärkte. Die Schere zwischen Arm und Reich öffnete sich weiter (Konrad, 2007). Dem realen Bildungsanspruch, der sich zu einem Modernisierungszwang für den Bildungssektor entwickelte, folgten sogenannte Realgymnasien und Oberrealschulen, die nicht altsprachlich organisiert waren und damit mehr Lebensnähe und Praxisrelevanz aufwiesen. Die Gleichstellung mit den humanistischen Gymnasien konnte jedoch erst mit einem Erlass von Wilhelm II. im Jahre 1900 erreicht werden, durch den das Monopol der Gymnasien beim Zugang in die akademischen Berufe aufgebrochen wurde und das Schulsystem so an die veränderten Bedingungen der kapitalistischen Gesellschaft angepasst wurde. Ein weiterer Schritt hin zur Gleichberechtigung bedeuteten die Bestimmungen vom August 1908, in denen auch Frauen der Zugang zum Abitur und einem sich anschließenden Studium ermöglicht wurde (Herrlitz et al., 2005).

Durch die Reformen in der Zeit des Übergangs vom 19. zum 20. Jahrhundert wurde der entscheidende Schritt hin zu einem modernen nationalen Schulsystem bewerkstelligt und die damals bestehenden Teilbereiche systematisch zueinander in Beziehung gesetzt, auch wenn in der Zeit des wilhelminischen Deutschen Reichs die Jugend in den Schulen den Ungeist des Nationalismus und Militarismus gelehrt bekam (Zymek, 2004). 
Das gesellschaftliche Ansehen, die Besoldung und damit die soziale Stellung der Lehrer besserten sich mit dem Beginn des 20. Jahrhunderts entscheidend. Die Beziehung zu Schülern war in dieser Zeit stark durch einen autoritären Umgang geprägt und Züchtigung als Erziehungsmittel war eine weit verbreitete Methode (Adrion \& Schneider, 1979).

In struktureller Hinsicht boten die Jahre nach dem ersten Weltkrieg keine großen Neuerungen. Durch die einsetzende Reformpädagogik bzw. deren Wiederaufleben und den Aktivitäten verschiedener Lehrervereine wurden jedoch Fortbildungsprogramme ins Leben gerufen, Zeitschriften gegründet und verstärkt pädagogisch geforscht. Nach und nach richtete sich der Blick der Lehrer auf die Entfaltung der Individualität des Einzelnen. In einer national und sozial geprägten Gemeinschaft basierte die Reflexion der Pädagogik auf dem philosophischen Zeitgeist von Wilhelm von Humboldt, Friedrich Nietzsche, Max Weber und Søren Aaby Kierkegaard. Die pädagogisch-didaktische Öffnung schlägt sich schließlich in den Ansätzen zu Bildung und Erziehung von Maria Montessori, Berthold Otto oder Peter Petersen nieder und stellt ein Gegenbild zur verknöcherten und weltfremden Bildung zu diesem Zeitpunkt dar. Einschränkend muss trotz all der Neuerungen jedoch erwähnt werden, dass meist nur die neuen d.h. jungen frisch ausgebildeten Lehrkräfte in der Lage waren, von Tradition und Erfahrung unbeeinflusst neue und fortschrittliche Aspekte des Unterrichtens zu berücksichtigen. Lehrer und Schüler fanden in dieser Zeit zunehmend zusammen (Arnhardt, Hofmann \& Reinert, 2000). Es gelang in ersten Ansätzen, die Lehrerbildung in das wissenschaftliche Hochschulwesen einzugliedern und so wurden z.B. in Hamburg ab 1926 auch Volksschullehrer an der Universität ausgebildet (Schwänke, 1988). Das humanistische Gymnasium begann nach 1918 deutlich zu schrumpfen, die soziale Öffnung der höheren Bildung wurde zunehmend forciert. Wie in allen Lebensbereichen zeichnete sich auch das Schulwesen in dieser Zeit durch eine massive Vielfalt aus, was in der amtlichen Hochschulstatistik nach 1924/25 deutlich wird. Hier wurden 25 verschiedene Vorbildungsarten unterschieden, die den Zugang zur Hochschule ermöglichten. Waren im Jahr 1890 noch 85\% der Studenten an preußischen Universitäten Absolventen eines humanistischen Gymnasiums, reduzierte sich diese Zahl bis ins Jahr 1930 auf 40\%.

Die Schulen waren jedoch neben der Ausbildung von Fachwissen für den beruflichen Wettbewerb nicht in der Lage, eine weitreichendere Orientierung zu liefern. So verwundert es nicht, dass es gerade die Studenten waren, die sich bereits 1930 für nationalsozialistisches Gedankengut begeisterten (Herrlitz et al., 2005). Zusätzlich negativ auf die Schule und Lehrer wirkte sich die Weltwirtschaftskrise aus, die auf den Börsencrash des schwarzen Freitags im Oktober 1929 folgte, denn der volkswirtschaftliche 
Einbruch machte auch vor den Schulen nicht halt. Staatliche Mittel wurden eingespart, die Besoldung der Lehrer gekürzt und Lehrerstellen abgebaut. Die faschistische Indoktrination führte letztendlich dazu, dass die Reformbewegung in Deutschland ihr jähes Ende fand und das Schulsystem eine andere Ausrichtung bekam (Arnhardt, Hofmann \&Reinert, 2000).

Im Deutschen Reich zwischen 1933 und 1945 änderte sich am äußeren Gewand der Schule relativ wenig und auch Lehrpläne wurden kaum verändert. Was sich jedoch änderte, war der grundsätzliche Auftrag der Schule, die sich von einer Stätte der Menschenbildung zu einem Werkzeug politischer Macht entwickelte und damit einen fundamentalen Gegensatz zur christlich-humanistischen Tradition darstellte (Adrion \& Schneider, 1979). Aus dem Bildungswesen entwickelte sich eine Schule der Diktatur. Das, was auf revolutionäre Weise nicht gelang, versprach der Führerstaat mit diktatorischen Mitteln zu erreichen (Herrlitz et al., 2005). Wie die Mehrzahl der Deutschen verhielt sich auch der Großteil der Lehrer gegenüber dem nach Macht strebenden Faschismus wehrlos und hörig (Arnhardt, Hofmann \& Reinert, 2000). Der Philologenverband stand schon immer den Rechtsparteien nahe und auch in der Volksschullehrerschaft löste der Abbau von Staatsleistungen im Bildungs- und Versorgungsbereich einen Loyalitätskonflikt aus, der zu einem Identifikationsbruch mit dem Verfassungsstaat führte (Herrlitz et al., 2005). Viele Lehrkräfte verhielten sich apolitisch und konservativ, andere traten dem Nationalsozialismus mutig entgegen, riskierten damit jedoch ihre soziale, oft sogar physische Existenz. Die Mitglieder von Lehrervereinen wurden automatisch in den nationalsozialistischen Lehrerbund überführt. Durch diese Gleichschaltung sollte aus der gesellschaftlich zentralen Position des Lehrers, Humanismus und Liberalismus zugunsten des völkischen Gedankengutes ausgemerzt werden. Statt Bildungspluralismus standen Vereinheitlichung und Homogenisierung des Schulwesens auf der Charta der Nationalsozialisten. Die Entwicklung rassischer Elemente und das Heranzüchten kerngesunder Körper waren die Primärzielsetzung, geistige Ausbildung hatte nur nachgeordnete Bedeutung in dieser Zeit. Diese Schwerpunktsetzung machte sich im regelmäßigen Unterrichtsausfall zugunsten von HJ-Aktivitäten bemerkbar (Konrad, 2007). Die Ausbildung von Lehrern wurde im Fahrtwind der generellen Aversion gegenüber intellektueller Bildung beschränkt und streng reglementiert, um ein Optimum an nationalsozialistischer Beeinflussung zu erzielen (Arnhardt, Hofmann \& Reinert, 2000). Die Ansätze, die Lehrerausbildung an den Universitäten zu institutionalisieren, wurden wieder rückgängig gemacht und die Lehrerbildung wurde in seminarähnlichen Lehrerbildungsanstalten durchgeführt, die militärisch geprägt und lagermäßig organisiert waren (Schwänke, 1988). Für Lehramtsstu- 
denten war eine Mitgliedschaft in der $\mathrm{HJ}$ vorgeschrieben und während der Ausbildung war Uniform zu tragen. Ähnlich wie in der preußischen Regulativpolitik führten auch die völkischen Schulreformen zur Gesinnungsbildung und einem rapiden Absinken des gesamten Bildungsniveaus an Grund-, Volks- und Mittelschulen. (Arnhardt, Hofmann \& Reinert, 2000).

Nach dem Zusammenbruch des nationalsozialistischen Staates dominierte im Osten wie im Westen das Ideal, basierend auf humanistischer Tradition, die Heranwachsenden in Toleranz, Solidarität und Friedensliebe zu schulen. Hierfür wurde sogar mit der Gemeinschaftskunde ein eigenes Schulfach implementiert. Von Seiten der Behörden wurde in dieser Zeit kaum reglementiert und so hatten Lehrende nahezu uneingeschränkte Freiheiten und knüpften an reformpädagogische Ansätze an, die zur Menschlichkeit in entwicklungsgemäßer Form vom Kinde aus erziehen wollten (Adrion \& Schneider, 1979). Ab den 50er Jahren des 20. Jahrhunderts drifteten die Schulsysteme In Ost- und Westdeutschland zunehmend in unterschiedliche Richtungen (Baumert, Cortina \& Leschinky, 2005). In der sowjetischen Besatzungszone war die aus dem Boden gestampfte Schulverwaltung damit beschäftigt, den eklatanten Lehrermangel zu beheben, um zumindest eine notdürftige Beschulung in der Einheitsschule zu gewährleisten (Arnhardt, Hofmann \& Reinert, 2000). Ausgehend von antifaschistischdemokratischer Erneuerung, einem an der Weltlichkeit orientierten Schulwesen, machte sich jedoch schnell sowohl im Staat als auch in der Schule zunehmend eine sich selbst pervertierende Machtpolitik breit, die durch Politisierung, Ideologisierung und Sowjetisierung gekennzeichnet war. Auf weitgehende Autonomie in der Lehrerarbeit folgte also politische Bekenntnisbereitschaft (Arnhardt, Hofmann \& Reinert, 2000). Die deutsche Tradition der Dichotomie von volkstümlicher und höherer Bildung wurde aufgehoben und zusätzliche alternative Wege zur Hochschulreife installiert und kontinuierlich stabilisiert (Herrlitz et al., 2005). Der Unterricht war bald überall uniform in seiner Gestalt, da neben der zentralen Steuerung und Organisation ein detailliertes Lehrwerk für vergleichbare Verhältnisse sorgte. Die achtjährige Einheitsschule wurde 1959 durch zwei weitere Schuljahre in Allgemeine Polytechnische Oberschule (POS) umbenannt bzw. aufgestockt. Kinder und Jugendliche sollten bereits während der Schulzeit mit Betrieben und der Arbeitswelt bekannt gemacht werden, was in vielen Fällen jedoch lediglich ein Nutzen billiger Arbeitskräfte darstellte. An die POS schloss sich die Erweiterte Oberschule (EOS) an, die in zwei Jahren zum Abitur führte. Ergänzend zu diesen beiden Formen existierten für die Bereiche Sport, Mathematik, Sprache, Technik und Kunst zusätzliche Eliteschulen, die wahre Kaderschmieden waren. Für die Talentförderung im Bereich Sport stellten die sogenannten Eliteschulen der ehema- 
ligen DDR, bezogen auf die Organisation der sportlichen Entwicklung, Vorbilder für die Installation von Förderzentren und Internaten im westlichen Deutschland in der Zeit nach der Grenzöffnung dar. Ein weiteres Beispiel für die Politisierung und Ideologisierung ist die Verschmelzung von schulischer und außerschulischer Erziehung. Neben Unterstützungsangeboten für schwache Schüler waren FDJ-Gruppen zusätzlich für die Verankerung der sozialistischen Ideologie der Schüler verantwortlich und auch Lehrkräfte waren sowohl in ihrer Ausbildung als auch später im Beruf dazu verpflichtet, politisch-pädagogische Dienste zu leisten. Nach der Wiedervereinigung passte man sich zwar weitgehend dem westlichen System an, bewahrte aber dennoch seinen eigenen Charakter, was sich beispielsweise in der teilintegrierten Struktur im Sekundarschulbereich erkennen lässt (Konrad, 2007). Neben all den negativen Tendenzen in der sowjetischen Besatzungszone müssen jedoch der starke Ausbau der Vorschulerziehung, die niedrige Sitzenbleiberquote sowie geringe Zahlen von Schülern ohne Schulabschluss gewürdigt werden (Herrlitz et al., 2005).

In den westlichen Besatzungszonen strebten die Alliierten die Implementierung eines einheitlich-horizontal strukturierten Schulsystems an. Primäres Ziel war es, der Basisinstitution Schule wieder zu einem ordentlichen Betrieb zu verhelfen. Beträchtliche infrastrukturelle Probleme verhinderten eine grundlegende Strukturreform. Viele Schulen waren nach dem Krieg zerstört oder wurden von den Besatzungsmächten in Anspruch genommen. Doch nicht nur an unterrichtsgemäßen Räumlichkeiten mangelte es, auch Bücher, Lehrmittel und Heizungen waren nicht vorhanden bzw. nicht funktionsfähig. Hinzu kam, dass ein extremer Lehrermangel vorherrschte, der durch die Entnazifizierung und Kriegsgefangenschaft bzw. das Fallen im Krieg von Lehrern bedingt wurde. Während in manchen gesellschaftlichen Bereichen Nationalsozialisten wegen ihrer Fachkenntnis im Beruf belassen wurden, bedachten die Alliierten die Entnazifizierung des Lehrerstandes aufgrund der Bedeutsamkeit der Schule für die demokratische Zukunft Deutschlands mit besonders großer Gründlichkeit und Sorgfalt (Gutte, 1994). Die neukonstituierte Ständige Kultusministerkonferenz der Länder entschied sich für die Wahrung der Dreigliedrigkeit (Arnhardt, Hofmann \& Reinert, 2000). Neben den infrastrukturellen Hindernissen, waren es auch der einsetzende Kalte Krieg und die abschreckende Wirkung der Implementierung der Einheitsschule in der sowjetischen Besatzungszone, die den Widerstand der Alliierten gegen die Dreigliedrigkeit hemmten. Im weiteren Verlauf kam es dazu, dass der beginnende wirtschaftliche Aufschwung zu einer stiefmütterlichen Behandlung des Bildungssystems führte. Die Bildungspolitik wurde von konservativen Kräften bestimmt und das komplette Bildungssystem verhielt sich reformresistent. Die Menge an qualifiziertem Arbeitspersonal, die 
in der Zeit des Wirtschaftswunders verstärkt benötigt wurde, konnte von den Schulen nicht bedarfsdeckend ausgebildet werden (Konrad, 2007). Bildungspolitisch sowie bezogen auf die Organisation von Schule, kann man diese Phase als Stagnation bezeichnen (Herrlitz et al., 2005). Als Wachrüttler dieser Epoche für daraufhin einsetzende Reformbemühungen kann man den Sputnikschock sowie die Aufsätze über „Die deutsche Bildungskatastrophe" von Georg Picht aus dem Jahr 1964 bezeichnen. Auch die 1968er Bewegung wendete sich gegen die autoritären Strukturen und die Bevormundung, die von der Institution Schule ausgingen. Im Laufe der Zeit erkannte man, dass man mit tradiertem Bildungsgut den Problemen und Ansprüchen der Gegenwart nicht mehr gerecht werden konnte und die Basiskompetenzen, die für eine spätere Arbeitstätigkeit und das gemeinschaftliche gesellschaftliche Leben notwendig sind, nicht vermittelt wurden (Arnhardt, Hofmann \& Reinert, 2000).

Im Jahr 1965 wurde der Deutsche Bildungsrat gegründet, der Reformvorschläge erarbeitete, die hin zu einer wissenschaftsorientierten Schule führen sollten. In diesem Zusammenhang änderte sich sowohl die Ausbildung als auch die Rolle der Lehrer. Neben der Neudefinition der Aufgabenbereiche von Lehrkräften, war das Stichwort Chancengleichheit von zentraler Bedeutung in den Bemühungen des Bildungsrats. Mehr Kinder sollten zu höheren Bildungsabschlüssen geführt werden, um die brachliegenden Begabungsreserven auszuschöpfen. Wie sich im Verlauf zeigte, konnten solch weitreichende Reformen wie dies der Bildungsrat vorsah nicht zügig und planmäßig realisiert werden. Die zunächst unumstrittene vollständige Umsetzung des Bildungsgesamtplans scheiterte unter anderem an der Weigerung der Finanzminister, Steuererhöhungen bzw. Umschichtungen des Etats zur Finanzierung der Reformen vorzunehmen. Als Verstärker in diesem Zusammenhang wirkte die Ölkrise und der damit verbundene Einbruch des Wirtschaftswachstums (Herrlitz et al., 2005).

Durch die Reform der gymnasialen Oberstufe im Jahr 1972 versuchte man, den Neigungen der Schüler besser zu entsprechen und zunehmend Lebensnähe herzustellen. Der Unterricht wurde auf den Entwicklungsstand der Schüler abgestimmt und die Benachteiligung der Mädchen war weitestgehend verschwunden (Konrad, 2007). Ein Diskussionspunkt war der Werteverlust, dem das Bildungssystem mit einem Wiederaufleben des Erziehenden Unterrichts im Herbartschen Sinne entgegentrat (Arnhardt, Hofmann \& Reinert, 2000). Generell war diese Zeit gekennzeichnet durch Bildungsexpansion und wachsende Anforderungen an die Qualifikationen des Einzelnen in der postindustriellen Wissens- und Dienstleistungsgesellschaft der 1970er und 1980er Jahre, wodurch die Schule von einem eher randständigen Bestandteil des Lebens mehr ins Zentrum rückt. Ab den 80er Jahren des 20. Jahrhunderts, als man sich das Scheitern 
vieler der vom Bildungsrat angedachten Reformen eingestehen musste, setzte man verstärkt auf die sogenannte Schulreform von unten. Der Einzelschule wurde zunehmend mehr Autonomie gewährt, um eine Öffnung und Weiterentwicklung zuzulassen. Die Veränderungen waren diesmal jedoch nicht von der Schulverwaltung angeordnet, sondern stellten eher einen basisnahen Veränderungsprozess dar, dem die Schulverwaltung beratend und unterstützend zur Seite stand (Konrad, 2007).

Die Wiedervereinigung brachte vielschichtige Aufgaben und Anforderungen mit sich, denen entgegengetreten werden musste. Im Einigungsvertrag vom 31.08.1990 wurde die Entscheidung der DDR-Seite, die eine Anpassung an das westliche Schulsystem vorsah, bestätigt. Strukturen und Lehrinhalte wurden zum Großteil aus den alten Bundesländern übernommen, landespolitisch wurden jedoch auch bestimmte Besonderheiten des Schulaufbaus und Curriculums ermöglicht, sodass keine Rede von einem einheitlichen Auf- und Ausbau der Schulen in den neuen Bundesländern sein kann. Die Zusammenlegung von Haupt- und Realschulen in einigen neuen Ländern fußte neben bildungstheoretischen Begründungen auch auf Prognosen und Berechnungen bezüglich der demographischen Entwicklung, die einen starken Geburtenrückgang sowie Migrationsbewegungen in den Westen registrierten bzw. voraussagten (Herrlitz et al., 2005).

Modelle und Reformen die sowohl vor als auch nach der Wiedervereinigung angedacht wurden, scheiterten an personeller und materieller Knappheit. Aufgrund der veränderten häuslichen Verhältnisse rückt die Schule als Institution der Erziehung mehr in die Verantwortung, dem sie mit dem Ausbau von Ganztagsbetreuung nachzukommen versuchte. Die Reform von unten, die Synthese unverwechselbarer und attraktiver Schulprofile, wurde durch sinkende Geburtenraten und dem daraus resultierenden Kampf um die Schüler angekurbelt und durch die Politik zusätzlich unterstützt. Die seit dem Beginn des 21. Jahrhunderts einsetzenden Diskussionen und Verabschiedungen um bzw. von Bildungsstandards und zentralen Prüfungen sind als Reaktionen auf eine ausgeprägte Heterogenität der Schulen zurückzuführen. Die Anschlussfähigkeit der Bildungsgänge wurde und wird zunehmend infrage gestellt. Die erste sogenannte PISAStudie und der darauf in Deutschland einsetzende „PISA-Schock“ verstärkten die Rufe nach Reformen, da neben dem generellen schlechten Abschneiden der Schüler zusätzlich die Streubreite der Leistungen extrem und der Zusammenhang von sozialem Hintergrund und der Zugangswahrscheinlichkeit zu höherer Bildung sowie erzielter Leistung stark ausgebildet war (Konrad, 2007). Der selbstgenügsamen Schulpolitik der 80er und 90er Jahre des 20. Jahrhunderts wurde unvermittelt und unverblümt aufgezeigt, wie rückständig und reformbedürftig sie war. Analog zu den 1960er Jahren zeigte sich 
dieser Modernitätsrückstand zuerst im internationalen Vergleich (Herrlitz et al., 2005). Zwischen den einzelnen Bundesländern zeichneten sich zudem erhebliche Leistungsunterschiede von bis zu einem Schuljahr Differenz ab, dem zukünftig durch Bildungsstandards, Kerncurricula und externer Evaluation begegnet werden soll. Viele der Probleme werden auf das dreigliedrige System zurückgeführt, da sich in Studien im Grundschulbereich zeigte, dass es hier offensichtlich besser gelingt, mit Differenzen umzugehen und international gesehen bessere Leistungen erzielt werden. Deutsche Schüler können zum Ende ihrer Grundschulzeit besser lesen als neuseeländische Schüler, die jedoch fünf Jahre später um ein ganzes Schuljahr voraus sind. Nach den jahrzehntelangen Grabenkämpfen von Politikern und Bildungswissenschaftlern scheint es dennoch unrealistisch zu sein, ein integriertes Schulsystem in absehbarer Zeit flächendeckend implementieren zu können (Konrad, 2007). Durch die Einrichtung von Ganztagsschulen, die Einführung von Bildungsstandards sowie einer Neuausrichtung der Lehrerbildung soll eine Neujustierung des Bildungssystems erfolgen (Herrlitz et al., 2005).

Wie sich im Laufe dieses Kapitels gezeigt hat, nimmt der Lehrerberuf eine zentrale Stellung im gesellschaftlichen und politischen Leben in Deutschland ein und unterliegt zyklischen Schwankungen in der erziehungs- und sozialwissenschaftliche Zuwendung sowie der Reformintensität. Genauso wie der Wechsel von Überfüllungs- und Mangelsituationen gehört auch die Vielfalt der Lehrerschaft zu den Eigenarten des Berufs. Neben warmherzigen Elternfiguren und bildungspolitischen Reformern sind genauso praxislustige Quereinsteiger, enttäuschte Idealisten, scharfsinnige Spezialisten, phantasieleere Scharfmacher, lebenssatte Ironiker und ahnungslose Gutwillige zu finden (von Thadden, 1994). Während das Schulsystem mehreren Wandlungsprozessen unterzogen war, wandelten sich auch das Verständnis des Berufes, das Selbstverständnis der Lehrer sowie die Lehrerrolle in der Gesellschaft mehrfach. Unabhängig von Wandlungsprozessen und Reformen sind Lehrer jedoch stets als einflussreiches Bindeglied zwischen den Generationen anzusehen, die neben der Weitergabe von Wissen zunehmend erzieherische Aufgaben übernehmen müssen, wodurch ihre gesellschaftliche Bedeutung, aber auch die Verantwortung stetig zunimmt.

\subsubsection{Aufgabenspektrum von Lehrkräften}

In diesem Kapitel sollen die Aufgaben, die an eine Lehrkraft gestellt werden näher betrachtet werden. Fragen nach dem genauen Aufgabenbereich von Lehrkräften und die Abgrenzung gegenüber anderen im Erziehungs- und Bildungsbereich tätigen Personen sind so alt wie der Beruf des Lehrers selbst. Wie in Kapitel 2.2.1 deutlich wurde, differierte das Aufgabenspektrum in der Vergangenheit aufgrund der unterschiedlichen pädagogischen Traditionen und den Absichten des jeweiligen Dienstherrn stark. Wäh- 
rend vor der Institutionalisierung, d.h. in der Zeit des Lehrens und Lernens in Form von Kloster- und Klerikerschulen Lehrer als geistliche Führer auftraten, kann man sie in der Folgezeit als eine Art Handwerker beschreiben, deren Einfluss und Ansehen als eher gering zu bezeichnen ist. An den elitären humanistisch und altsprachlich geprägten Gymnasien verstanden sich Lehrer als Anwender und Umsetzer von Wissenschaft. In der Folgezeit, die immer wieder geprägt war von Lehrern, die sich als pädagogische Künstler oder Entwicklungshelfer der Schüler sahen, wie z.B. in der Reformpädagogik oder dem Philanthropismus, wurden Lehrer auch als politische Agenten eingesetzt. Sowohl in der Weimarer Republik und im Deutschen Reich zwischen 1933 und 1945, als auch in der sowjetischen Besatzungszone zwischen 1945 und 1989 wurde die schulische Erziehung politisch verzweckt. Neben diesen bereits genannten Lehrerbildern können Lehrer auch im Rollenverständnis als verbeamtete Staatsdiener, personale Erzieher oder als Kulturvermittler verstanden werden (Terhart, 2001). Die Vielschichtigkeit und die Bandbreite der Rollendefinitionen der Vergangenheit zeigen, dass die Frage nach dem Auftrag bzw. dem Mandat von Lehrern eine entscheidende Funktion in der Organisation von Bildung und Erziehung hat. Eng verknüpft mit diesem Fragenkomplex ist die Professionalisierungsdebatte, die ihren Anfang zum Ende der 1960er Jahre nahm. Die Gegner der Professionalisierung des Lehrerberufs sehen in der Verwissenschaftlichung eine Gefahr, während Befürworter darin eine Chance sehen, die Lehrertätigkeit zu modernisieren.

Um das Mandat der Lehrer genauer zu formulieren, skizzierte der deutsche Bildungsrat im Jahr 1970 die Arbeit von Lehrern mit den Bereichen Lehren, Erziehen, Beurteilen, Beraten und Innovieren. In der Folgezeit wurden unzählige Änderungen vorgenommen und Reformen umgesetzt wie z.B. die der gymnasialen Oberstufe im Jahr 1972, bevor die Kultusministerkonferenz in ihrem Beschluss vom 5. Oktober 2000 zu den Aufgaben der Lehrer einen neuen, jedoch scheinbar deckungsgleichen Katalog vorlegte, der auch in Schulgesetzen und den Standards für die Lehrerbildung so auftaucht (Gudjons, 2007).

Im siebten Abschnitt des Hessischen Schulgesetzes ${ }^{4}$ (Hessischer Landtag, 2005) wird das Aufgabenspektrum von Lehrern, im Wesentlichen auf der Basis der vom Deutschen Bildungsrat formulierten Bereiche, geregelt:

(3) Die Lehrerinnen und Lehrer erziehen, unterrichten, beraten und betreuen in eigener Verantwortung im Rahmen der Grundsätze und Ziele der $\S \S 1$ bis 3 sowie der sonstigen Rechts- und Verwaltungsvorschriften und der Konfe-

\footnotetext{
${ }^{4}$ Hessisches Schulgesetz $\S 86$, Punkt 2.
} 
renzbeschlüsse. Die für die Unterrichts- und Erziehungsarbeit der Lehrerin oder des Lehrers erforderliche pädagogische Freiheit darf durch Rechts- und Verwaltungsvorschriften und Konferenzbeschlüsse nicht unnötig oder unzumutbar eingeengt werden. Lehrerinnen und Lehrer sind verpflichtet, sich regelmäßig fortzubilden und einen Nachweis über die Erfüllung dieser Verpflichtung zu führen.

In der „Vereinbarung der Standards für die Lehrerbildung: Bildungswissenschaft" formuliert die Kultusministerkonferenz (KMK) in ihrem Beschluss vom 16.12.2004 vier, jetzt als Kompetenzbereiche formulierte Aufgaben des Lehrerberufs. Diese vier Bereiche entsprechen den bereits vom Bildungsrat formulierten Aufgabenbereichen, erfahren aber eine aktualisierte Ausgestaltung und Gewichtung (Kultusministerkonferenz, 2005).

Im Kompetenzbereich Unterrichten geht es sowohl um die fachlich und sachlich korrekte Planung und Durchführung von Unterricht als auch um die Unterstützung der Schüler beim Lernen durch die Gestaltung von motivierenden Lernumgebungen bzw. Lernsituationen, in denen die Fähigkeiten zum selbstbestimmten Lernen und Arbeiten gefördert werden. Grundsätzlich sind Lehrer also Fachleute für das Lehren und Lernen (Gudjons, 2007). Im Planungsprozess müssen neben den fachspezifischen Inhalten ebenso wissenschaftliche Erkenntnisse in Bezug auf die Vermittlung berücksichtigt werden, die u.a. eine angemessene Beachtung der Lernausgangslage der Schüler inkludieren. Wissen muss so strukturiert dargeboten werden, dass es von den Schülern verstanden werden kann und vor allem auch verstanden werden will, wodurch der motivationale Aspekt gleichbedeutende Relevanz zugesprochen bekommt (Giesecke, 2001). Bauer (2008) vergleicht die Motivationsproblematik mit dem häuslichen Kochen, bei dem die Kinder vom Geruch in die Küche gelockt werden. Ebenso sollte auch die Schule möglichst die Schüler zum eigenständigen Lernen und Forschen aktivieren. Mit der Planung, Organisation und Durchführung des Unterrichts endet jedoch dieser Aufgabenbereich noch nicht, denn nur die Auswertung und Evaluation von Lehr-LernProzessen ermöglicht erfolgreiches Lehren und Lernen in der Zukunft. Übergreifende Zielsetzung ist nicht nur das unmittelbare Lernen, sondern vor allem die Kompetenzen der Schüler insoweit zu entwickeln, dass sie durch spezifische Kenntnisse und Methoden zu einem selbstständigen und lebenslangen Lernen in der Lage sind (Kultusministerkonferenz, 2000). Während der Kernaufgabe des Unterrichtens ist der Lehrer stets mit einem hohen Anteil an Ambiguität konfrontiert, denn ob ein Schüler lernt oder nicht, ist ein stetiger Ungewissheitsfaktor und der Glaube an die Berufung meist lebensnotwendig, um die Dauerüberraschung kompensieren zu können (Scheunpflug, 
2005). Lernen ist ein idiosynkratrischer Prozess des Schülers, der ihm nicht durch die Lehrkraft abgenommen werden kann und sich einstellende Unterrichtserfolge sind meist nicht eindeutig auf das Lehrerhandeln zurückzuführen. (Baumert \& Kunter, 2006). Während in anderen Berufen die Erfolgsgenese klar auf das eigene Handeln, anhand von Werkstücken, Vertragsabschlüssen oder Verkaufszahlen zurückzuführen ist, ist das Erreichen anspruchsvoller Bildungsziele aufgrund der fehlenden eindeutigen Kausalbeziehung kaum ermittelbar. Ziel des Lehrers kann nur sein, möglichst optimale Lernbedingungen zu schaffen. Anders als die diffusen und meist nur langfristig zu erzielenden Erfolge, sind Misserfolge meist unmittelbar und sehr deutlich wahrnehmbar, sodass man oft eine unausgeglichene Gratifikationsbilanz vorfindet. Lehrer sind nie fertig und sehen am Ende des Tages, dass sie stets nur einen kleinen Teil der Herausforderungen schaffen können (Struck, 2007). Das Unterrichten stellt den Kernbereich der Arbeit des Lehrers dar, trotz des immer mehr diffuser werdenden Berufsbildes.

Um jedoch überhaupt zum Unterrichten zu kommen, müssen hierfür oft erst die Voraussetzungen geschaffen werden, wodurch der zweite Kompetenzbereich, das Erziehen, zunehmend an Bedeutung gewinnt (Terhart, 2006). Zwar gibt es im Herbartschen Sinne keinen Unterricht, der nicht erzieht, denn bereits die soziale Gestalt des Unterrichts, die Regeltreue, die Pünktlichkeit und die geordnete Unterrichtsführung erziehen (Baumert \& Kunter, 2006). In jüngster Vergangenheit fand jedoch eine Akzentveränderung in Richtung Erziehung statt, die sich neben, zum Teil sogar vor den Unterricht schiebt (Tenorth, 2006). Der Spagat zwischen Sozialpädagogik und der Vermittlung von Fachwissen erfordert eine neue Lehrerrolle, die eines Kümmerers, der nicht vorhandene Bildungsvoraussetzungen als elementare Basis schafft, wobei jedoch der beobachtete Mangel nur zu einem kleinen Teil in der Reichweite des Einflussbereichs der Schule liegt (Giesecke, 2001). Während das Gymnasium in den letzten vier Jahrzehnten noch empört die Übernahme sozialer und erzieherischer Aufgaben ablehnte und diese Verantwortung eindeutig im familären Bereich sah, werden heute in starkem Maß, auch von Gymnasien erzieherisch-sozialisatorische Hilfestellungen erwartet (Terhart, 2001). Damit Lehrer ihrer Erziehungsaufgabe gerecht werden und die Entwicklung ihrer Schüler positiv beeinflussen können, stellt das Wissen über soziale und kulturelle Lebensbedingungen von Schülern die Grundlage dar. Neben der Lösung von Konflikten und Schwierigkeiten in Schule und Unterricht geht es vor allem um selbstbestimmtes Urteilen und um die Vermittlung von generellen Werten und Normen, die über den Rahmen der Schule hinausgehen, also um eine absichtsvolle Einflussnahme auf die Entwicklung der Persönlichkeit. Grundvoraussetzung für gelungene Erziehungsprozesse, wie die Einübung von Regelbewusstsein und die Bereitschaft und Fähigkeit zum 
sozialen Miteinander, ist das vorbildhafte Verhalten von Lehrern und Eltern. Neben diesen beiden Kontaktpersonen ist auch ein Zusammenarbeiten mit weiteren pädagogischen oder psychologischen Fachkräften außerhalb der Schule notwendig (Kultusministerkonferenz, 2000). Das schlichte Schaffen von Unterrichtsvoraussetzungen durch die Vermittlung von basalen Umgangsformen wird ergänzt durch Drogenprophylaxe, den Umgang mit Verhaltensproblemen sowie die Kompensation familiärer Defizite (Gudjons, 2007). Diese notwendigen Erziehungsmaßnahmen beschränken sich nicht auf Grund-, Haupt- und Realschulen, sondern betreffen vor allem auch das Gymnasium. „Das Gymnasium als auf das Studium vorbereitende, rein kognitiv-fachbezogen orientierte Wissenschafts- oder Studienschule ist heute als Regelfall nicht mehr existent und auch nicht existenzfähig" (Terhart, 2001, S. 121). Während in der Vergangenheit Schüler, die kognitiv oder sozial nicht den Ansprüchen genügten, aussortiert wurden, prägt heute die Förderung und Differenzierung den Arbeitsalltag auch von Gymnasiallehrkräften (Bosse, 2004a).

Der dritte Anforderungsbereich stellt der Kompetenzbereich Beurteilen dar, der die Aufgabe beinhaltet, Lernvoraussetzungen und Lernprozesse von Schülern zu diagnostizieren und auf dieser Grundlage gezielt zu fördern bzw. zu beraten. Daneben wird zudem der Anspruch auf eine Erfassung schulischer Leistungen von Schülern basierend auf transparenten Beurteilungsmaßstäben erhoben. Letztendlich besteht die Aufgabe aber auch darin, Berechtigungen für weitere Ausbildungswege zu vergeben und Schülern dabei zu helfen, sich selbst realistisch einzuschätzen sowie beratende Funktion im Falle von Lernschwierigkeiten bzw. Schullaufbahnentscheidungen zu übernehmen (Kultusministerkonferenz, 2000; 2005; Rothland \& Terhart, 2007). Eine Verschärfung in der Praxis stellt jedoch der Anspruch dar, Kinder und Jugendliche individuell zu fördern, ihren Leistungsfortschritt zu berücksichtigen und auf der anderen Seite, Kinder und ihre Leistung durch Standard- und Outputorientierung an einem zentral vorgegebenen Maßstab zu messen (Gudjons, 2007).

Der Kompetenzbereich Innovieren umfasst die ständige Weiterentwicklung von Kompetenzen und das Auffassen des Berufs als ständige Lernaufgabe. Lehrer haben dafür Sorge zu tragen, dass sie ihre eigenen fachdidaktischen, fachwissenschaftlichen und allgemeindidaktischen Kompetenzen stets ausbauen und anhand neuer wissenschaftlicher Erkenntnisse aktualisieren, aktiv an Fortbildungsmaßnahmen teilnehmen und vor allem auch ihrem Charakter entsprechende und vertretbare Bewältigungsstrategien entwickeln. Im Bereich der Schulentwicklung tragen Lehrer dazu bei, die eigene Schule zu einem lernförderlichen Raum zu formen, ein angenehmes Schulklima zu schaffen und eine gemeinsame Identität zu entfalten, die sich in einem Schulprofil bzw. Schul- 
programm widerspiegelt. Hierzu ist Engagement in schulinternen und schulübergreifenden Gremien, Teamarbeit, Zusammenarbeit mit außerschulischen Einrichtungen sowie die Mitwirkung an externen und internen Evaluationen notwendig (Kultusministerkonferenz, 2000; 2004; Rothland \& Terhart 2007).

Die Aufgabenstruktur im Lehrerberuf zeigt einen hohen Komplexitätsgrad, der vor allem im Unterrichten bei der zeitlich parallelen Bewältigung verschiedener Teilaufgaben deutlich wird (Ulich, 1996). Im Zentrum der Lehrerarbeit befindet sich zweifellos der Unterricht, die anderen dargestellten Bereiche beziehen sich alle auf diesen Kern. Für Lehrkräfte an Gymnasien macht der Sachanspruch im Unterricht einen größeren Anteil aus als in anderen Schulformen, in denen dafür in größerem Maße Erziehungsansprüche verfolgt werden (Terhart, 2001). Schaarschmidt (2005a) kritisiert, dass das Aufgabenspektrum, das Lehrer abdecken sollen, zunehmend ausgeweitet wird, während die Bedingungen der Berufsausführung sich weiter verschlechtern. Unzureichende räumliche und materielle Ausstattungen, Schülerfehlverhalten sowie nachlassende Unterstützung durch die Eltern problematisieren den Beruf zunehmend (siehe Kap. 3.3). Um den Anforderungen, die in den beschriebenen Aufgabenbereichen enthalten sind zu genügen, sind verschiedene individuelle Kompetenzen auf Seiten der Lehrer notwendig. Ulich (2004) sieht grundsätzlich Selbstkompetenz, Beziehungskompetenz und Fach- bzw. Sachkompetenz als Grundvoraussetzungen an, die die veränderte Situation in Schule und Unterricht unabdingbar machen. Um auf neue Anforderungen und Aufgaben reagieren zu können sowie den Wandel aktiv mitgestalten zu können, sind sowohl Reform- als auch auf Analyse zielende Reflexionskompetenz, bezogen auf jegliches berufliche Handeln notwendig. Die neuen Anforderungen bringen jedoch nicht nur Veränderungen der Lehrerrolle mit sich, sondern bergen auch die Gefahr der Entgrenzung des Arbeitsauftrages, denn Professionalisierung zeichnet sich per definitum durch einen gewissen Grad an Partikularität aus, die durch die Ausbeulung der Aufgaben gefährdet scheint (Gudjons, 2007).

Bauer (2008) thematisiert in seinem Buch „Lob der Schule“ auf einer anderen Ebene die Voraussetzungen von erfolgreichem Lernen. Er sieht die Ausstrahlung und Vorbildfunktion von Lehrern als Grundlage jeglichen Unterrichts- und Lernerfolgs. Denn nur wer selbst von einer Sache begeistert ist, kann für die zu erreichenden Ziele begeistern und den Funken auf die Schülerin bzw. den Schüler überspringen lassen. Mit der Vorbildfunktion verbunden ist es, sich als Mensch mit Eigenschaften erkennbar zu geben und vital und lebensbejahend aufzutreten, für seinen Lebensstil und Werte einzutreten und gleichzeitig menschlich zu bleiben. Diese Einstellung, die man auch als Enthusiasmus bezeichnet, zeichnet sich auch äußerlich durch ausgeprägte Gestik, wechselnde 
Intonation, Humor, häufiger Standortwechsel in der Klasse, lebendige Beispiele sowie eine lebendige und überzeugende Kommunikation mit den Schülern aus. Hinzuzufügen ist jedoch, dass es bei Enthusiasmus nicht um ein Maximum, sondern um ein Optimum geht (Helmke, 2003). All dies stellt die Grundlage für ein Lernen am Modell dar wie dies bereits Bandura (1979) aufdeckte und Mitte der 1990er Jahre als neurobiologisches System unter dem Schlagwort Spiegelneurone bestätigt wurde. Beobachtetes Verhalten anderer Menschen wird durch die Beobachtenden simuliert, im eigenen Gehirn nachgespielt und Emotionen, Empfindungen und Denkweisen unbewusst nachgeahmt. Dieses Mit-Tun stellt die Grundlage für das Modelllernen dar. Zusammengesetzt ergeben diese Denk-, Emotions- und Stimmungsfragmente das Bild bzw. den Eindruck, den man von einer Person hat (Bauer, 2008).

Um die an sie gestellten Aufgaben und Anforderungen möglichst gut zu erfüllen bzw. zu bewältigen, durchlaufen Lehramtsanwärter in Deutschland eine zweiphasige, in staatlicher Verantwortung liegende Ausbildung, die sich aus dem universitären Studium und dem Vorbereitungsdienst zusammensetzt. Ausgehend von einer theoretischen Basis, gestaltet sich der Verlauf der Ausbildung zunehmend praxisorientiert, bis schließlich im Vorbereitungsdienst die reflexive theoriegeleitete Praxis im Mittelpunkt steht. Wie bereits in Kapitel 2.1 aufgeführt, kamen Lehramtsstudenten jedoch bisher sehr spät in ihrem Studienverlauf mit der tatsächlichen Praxis des Lehrerberufs in Berührung: Stellt man nach dem umfangreichen Vorstudium zum Beispiel in den Praktika fest, dass man ungeeignet für den Beruf ist, ist ein Wechsel des Studiengangs nur mit großen Schwierigkeiten verbunden. Bauer (2008) hält ein Frühwarnsystem für Studentinnen und Studenten des Lehramts für unbedingt notwendig und vergleicht die Ausbildung von zukünftigen Lehrern mit der Ausbildung eines Raubtierdompteurs:

Der Auftritt eines Lehrers/einer Lehrerin in der Klasse gleicht in gewisser Weise dem eines Löwen- oder Tigerbändigers in der Manege. Um in diesem Bild zu bleiben: Bildet man Dompteure so aus, wie wir derzeit in Deutschland Lehrer ausbilden, hätten sie keine Chance, lange zu überleben. Der Grund: Man würde die Ausbildung darauf beschränken, dem angehenden Dompteur mit allerlei Informationen über die Zoologie der Raubtiere zu versorgen, zum Beispiel, welche Stadien der Entwicklung sie durchlaufen, während sie aufwachsen, wann und warum sie welches Futter fressen, dass sie scharfe Zähne und dicke Felle haben. Man würde ihm erzählen, wie hoch sie springen können und - natürlich - welche Kunststücke sie beherrschen sollen. Dann brächte man ihm noch das ein oder andere über den Zirkusbetrieb und die dort geltenden Vorschriften bei - und fertig wäre der frischgebackene Dompteur! Ein paar Dinge allerdings hät- 
te er nicht gelernt: wie man in der Manege mit Raubkatzen umgeht, wie man ihnen begegnet, mit ihnen arbeitet, auf welche Zeichen man zu achten hat, welche Zeichen man ihnen geben muss und wie man brenzlige Situationen meistert (Bauer, 2008, S. 79 f.).

In diesem kritischen Vergleich macht Bauer deutlich, dass Lehramtsstudenten kein bzw. kaum praktisches anwendbares Wissen erlangen, um auf die dynamischen Vorgänge in Klassen und Störungen im Unterricht angemessen reagieren zu können. Kritik wird oft auch aufgrund der fehlenden Verzahnung der beiden Ausbildungsphasen geäußert, die bei wechselseitigem Bezug ein deutlich effektiveres Lernen ermöglichen würden (Terhart, 2001). Erste neuere Reformen sehen Praktika vor, die dem Studium vorgeschaltet sind. Ergänzend und oft übersehen ist die Fort- und Weiterbildung ausgebildeter Lehrkräfte als dritte Phase der Lehrerbildung im Sinne eines lebenslangen Lernens (Kultusministerkonferenz, 2004). Trotz aller Bemühungen, Lehramtsstudentinnen und Lehramtsstudenten als auch im Beruf befindliche Lehrkräfte zu einer professionellen Kompetenz zu führen, die sowohl die kognitive als auch die soziale, personale, intuitive und kreative Dimension umfasst, ist die tatsächliche Bildung einer Lehrkraft von der Eigentätigkeit des Individuums abhängig und nur schwer von außen steuerbar (Terhart, 2001).

\subsubsection{Antinomien, Rollenerwartungen und Rollenkonflikte im Lehrberuf}

Wie zuvor dargestellt werden Lehrer vielfältig gefordert und unterschiedliche Interessengruppen haben differente Erwartungen, sodass Lehrer diese Erwartungen in einem Für- und Wider-Prozess abwägen müssen, um ihre eigenen Prioritäten in der Ausrichtung des Handelns zu definieren. Zu jedem Aspekt des schulischen Handelns haben Schüler, Eltern, Kollegen, Vorgesetzte sowie die gesellschaftliche Öffentlichkeit unterschiedliche Erwartungsspektren, die den Lehrer oftmals zwangsläufig in Rollenkonflikte bringen. Der Lehrer wird so situationsabhängig immer wieder neu mit der schwierigen Aufgabe konfrontiert, sich für eine bestimmte Rolle und der damit verbundenen Handlungsweise zu entscheiden, auch wenn er damit bei anderen Interaktionspartnern auf Ablehnung, Unverständnis und Konfrontation stößt (Rothland \& Terhart, 2007).

Auf einer höher gelagerten abstrakteren Ebene spricht Helsper (1996; 2000) im Bezug auf das Handeln im Unterricht von Antinomien des Lehrerhandelns. Antinomien stellen Spannungsverhältnisse zwischen zwei gegensätzlichen, jedoch jeweils für sich betrachtete, berechtigte Pole dar. Beide Pole sind zwar positiv und demzufolge auch anzustreben, zusammen sind sie jedoch nicht erreichbar und müssen daher situationsspezifisch unterschiedlich gewichtet werden. 
Unter Nähe-Distanz-Antinomie ist das bipolare Verhältnis zu den Schülern gemeint. Auf der einen Seite meint dies die Bestrebung, in einen direkten affektiven Kontakt mit den Schülern zu treten, um möglichst gut auf jedes Individuum eingehen zu können, Lernschwierigkeiten zu identifizieren und zu überwinden und zur Ausbildung der Persönlichkeit und psychosozialer Integrität beizutragen. Der Lehrer versucht, die Person mit ihrem Bemühen und ihren Motiven wahrzunehmen. Auf der andern Seite ist es jedoch anzustreben, Ziele zu formulieren, Kritik zu üben und zu den eigenen Forderungen und Ansprüchen zu stehen sowie eine gewisse professionelle Distanz und ein Mindestmaß an Neutralität zu den Schülern zu wahren, um eine gerechte Gleichbehandlung optimal umzusetzen (Helsper, 1996). Dieser Balanceakt zwischen verstehender Zuwendung und Führung gelingt dann am besten, wenn die Schüler als Person wahrgenommen werden, gleichzeitig der Lehrer aber selbst als Person mit bestimmten Eigenschaften auftritt und dadurch sowohl Spontanität als auch Authentizität im alltäglichen Unterricht verkörpert. Durch diese professionelle Distanz wird die Antinomie entschärft, da Fürsorge im pädagogischen Handeln weder Intimität noch Therapie bedeutet, sondern professionelle Förderung auf der Grundlage des zuvor beschriebenen Verhaltens erfolgt, das auch klare Grenzziehungen erfordert (Baumert \& Kunter, 2006). Den meisten Lehrkräften gelingt diese Ausgewogenheit intuitiv, einige verschanzen sich jedoch auch hinter einer starren Führungsrolle. Sie tun dies aus Angst, die Kontrolle zu verlieren. Doch auch diejenigen Lehrkräfte, die die Führung außer Acht lassen und mit reiner Menschlichkeit und Nächstenliebe ihren Unterricht über die Beziehungsebene definieren, drohen zu scheitern, denn Schüler wollen und brauchen sowohl Nähe als auch Distanz (Bauer, 2008).

Die Antinomie von Person und Sache beschreibt die polare Beziehung zwischen der Vermittlung von abstrakten Inhalten auf universalistischer Art und Weise auf der einen und dem individuellen Schülerbezug auf der anderen Seite. Während die Vermittlung eines fachlichen Wissensgegenstands unzweifelhaft den Auftrag des Lehrers darstellt, soll gleichzeitig die Lebenswelt der Schüler als Bezugspunkt genutzt werden, um nach Klafki den Bildungsgehalt sowohl in Bezug auf das Elementar als auch das Fundamentale und das Exemplarische zu erschließen. Die Aufgabe des Lehrers ist es also, durch Reduktion und Rekonstruktion den Gegenstand partikular in lebensweltlich alltagsnaher Ausformung zu erschließen und lebensgeschichtlich, kulturell und milieuspezifisch einzubetten. Hier besteht stets die Gefahr, das eine auf Kosten des anderen zu vernachlässigen und die Vermittlung dadurch einseitig zu gestalten (Helsper, 2000). Gerade nach PISA stellt die Forderung nach Problemorientierung und Ganzheitlichkeit im Lernprozess eine von der breiten Mehrheit geforderte Veränderung der Lernkultur dar, 
die sich in der Antinomie von Person und Sache verorten lässt. Während reine Wissensvermittlung in der didaktisch-pädagogischen Diskussion an Stellenwert eingebüßt hat, wird die Förderung der Lernkompetenz, in der der untrennbare Zusammenhang von Sach-, Selbst-, Methoden- und Sozialkompetenz aufgeht, die Zukunft des Lernens und Lehrens prägen (Vollstädt, 2004).

Ein weiteres Spannungsverhältnis des Lehrerberufs zeigt sich in der Antinomie von Einheitlichkeit und Differenz. Die Gleichbehandlung aller Schüler in allen Belangen ist eine der am eindeutigsten formulierten Anforderung an Lehrpersonen, die jedoch sobald sich ein Lehrer intensiver um einen Schüler mit Lernproblemen kümmert verletzt wird. In solchen Situationen wird dem Rest der Klasse nicht die gleiche Aufmerksamkeit und Zuwendung zu Teil wie dem einzelnen Schüler. Die Spannung besteht also darin, dem Anspruch gerecht zu werden, universalistischen und gleichzeitig individuell differenzierend im Unterricht zu agieren. In der Vergangenheit versuchte man diesem Spannungsverhältnis dadurch entgegenzutreten, dass alle Schüler zur gleichen Zeit dasselbe lernen sollen (Rothland \& Terhart, 2007). Aufgrund der Zunahme an Heterogenität, die durch lebenslagespezifische, familiäre und milieuspezifische Bedingungen und dem daraus teils folgenden Ressourcenmangel in Bezug auf Bildung und Erziehung zustande kommt, würde eine strikte Gleichbehandlung jedoch eine weitere Benachteiligung bedeuten (Helsper, 2000). Statt in einer verkappten Form eine universalistische Gleichbehandlung anzustreben, sollten Lehrer in schülerorientierten Formen des Unterrichts mehr als Berater tätig werden, um jedem Schüler die ihm gemäße Hilfe und Beratung, aber auch Freiheit und Selbstständigkeit zu gewähren. In schülerorientierten Unterrichtsorganisationen ist ein genaues Aufrechnen von individueller Förderzeit obsolet. Auch in der Oberstufe, bei der man in der Vergangenheit von nahezu leistungs- und interessenshomogenen Gruppen ausgegangen ist, kommt man um Differenzierungsmaßnahmen nicht mehr herum. Heterogenität kann auch hier nicht mehr ignoriert werden, sondern muss in produktiver Art und Weise thematisiert und genutzt werden (Bosse, 2004a).

In weiten Teilen der Forschung besteht die Ansicht, dass die Interaktion zwischen Schülern und Lehrern und die individuellen Lernprozesse durch prinzipielle Offenheit gekennzeichnet sein sollten. Dem zuwider ist jedoch die Vorstrukturierung des Handlungsspielraums durch einen organisatorischen Rahmen, der sich durch einen wöchentlichen starren Unterrichtsrhythmus mit unbeweglichen Zeittakten und Fachstrukturen kennzeichnet. Unterricht wirkt damit in abstrakter Art und Weise stark formalisierend und die emergente Offenheit des professionellen Handelns ist bedroht. Andererseits bietet jedoch die organisatorische Institutionalisierung die Gewährleistung 
allgemeingültiger Standards. Dieses Spannungsverhältnis wird von Helsper (2000) als Antinomie von Organisation und Interaktion bezeichnet. In Schulentwicklungsprojekten, in der Literatur oft in Praxisbeispielen der Laborschule Bielefeld oder der HeleneLange-Schule in Wiesbaden dargestellt, versucht man durch das Aufnehmen eines eigenen Arbeitsrhythmus weitestgehend ohne äußeren Zeit-, Leistungs- und Zensurendruck den natürlichen Lernbedürfnissen von Kindern und Jugendlichen nachzukommen. Auf diese Art und Weise versucht man individuelles Lernen zu ermöglichen (Thurn \& Tillmann, 1997). Auch Struck (2007) sieht beispielsweise in der Bündelung der Fächer zu Lernbereichen den notwendigen Schritt, um ein zeitgemäßes und zukunftstragendes Lernen zu ermöglichen.

In der Antinomie von Autonomie und Heteronomie wird eine weitere widersprüchliche Charaktereigenschaft der Schule deutlich. Auf der einen Seite sollen Schüler zu selbstständigem und selbstbestimmtem Handeln erzogen werden, auf der anderen Seite ist die Schülerrolle per Definition durch Abhängigkeit und Unselbstständigkeit, erzeugt durch Vorgaben und Weisungen der Lehrer und der Institution, gekennzeichnet. So stellt sowohl die ausgeprägte Heteronomie als auch das genau Gegenteil, eine Überspitzung der Autonomie, eine Gefahr dar, wenn Schüler durch die Zuschreibung von Eigenverantwortlichkeit überfordert werden und es dadurch zu wiederkehrendem Scheitern kommt. „Genau dies markiert jene konstitutive Spannung, die immer wieder als pädagogisches Grunddilemma seit Kant formuliert wird: Autonomie auf der Grundlage noch bestehender Heteronomie im Rahmen sozialer Zwänge zu generieren“ (Helsper, 2000, S. 151).

Die vorgestellten Antinomien des Lehrerhandelns führen dazu, dass der Berufsalltag stetig durch ein Handeln und Entscheiden in Unsicherheit geprägt ist und notwendigerweise Entscheidungen getroffen werden müssen, die den teils gegensätzlichen Geltungsansprüchen nicht gleichzeitig entsprechen können. Einerseits sorgen diese Spannungsverhältnisse dafür, dass durch stetiges Hinterfragen von Vorgehensweisen und Methoden ein reflexives Berufsverständnis unterstützt wird. Andererseits kann diese emergente Offenheit auch dazu verleiten, das eigene Verhalten als Schutzmechanismus unreflektiert einseitig zur Routine werden zu lassen, um die Unsicherheit als zentrales Belastungsmoment für sich selbst zu reduzieren (Helsper, 2000). Tenorth (2004) sieht sogar im Gelingen von Lehren und Lernen, trotz der Unsicherheit und den vorliegenden widersprüchlichen Erwartungen und Anforderungen, die eigentlich hervorzuhebende Leistung, die den Kern der Professionalität von Lehrkräften ausmacht. 


\subsubsection{Der Lehrerberuf in der Gesellschaft}

Bis heute hat sich das gesellschaftliche Bild des Lehrers zwar gegenüber den Stereotypen der Vergangenheit gewandelt, die negative Grundattribution ist aber weiterhin geblieben. Das Bild des sadistischen Tyrannen wurde durch den „modernen“ Lehrer ersetzt, der nicht ausreichend qualifiziert ist, bei maximaler Arbeitsminimierung übermäßig entlohnt wird, oft krank ist und sich frühpensionieren lässt. In Deutschland hat nahezu jeder direkten und ausgiebigen Kontakt zu Lehrern, kann also vergleichen und hat Effekte des Wirkens am eigenen Leib erlebt. In unserer Gesellschaft spielt sich ein großer Teil der Entwicklungsphasen Kindheit und Jugend im Einflussbereich von Lehrern ab und somit dürfte das Produkt aus Beobachterzahl, nämlich alle Personen, die je eine Schule besucht haben, und Beobachtungsdauer, gemeint ist die Zahl der erlebten Unterrichtsstunden, von kaum einem anderen Berufsstand erreicht werden. Diese unmittelbar gemachten Erfahrungen sind unerreicht in unserer modernen Gesellschaft und demzufolge existierten und existieren zu kaum einer anderen Berufsgruppe so ausgebildete Meinungsstrukturen wie zum Beruf des Lehrers. Keine andere Berufsausübung vollzieht sich so unter aller Augen (Rheinberg \& Bromme, 2001).

Aus der scheinbaren Transparenz der Berufsausübung und den direkten Erfahrungen resultieren oft Be- und Verurteilungen des Berufes, wie sich auch in der Literatur und anderen Medien wie Fernsehen und Musik zeigte. Kritisch anzumerken ist jedoch, dass dieses Bild, genau wie das der Eltern, stets eine Außensicht darstellt und nur ein kleiner Teil des tatsächlichen Berufes in Urteile und Bewertungen einbezogen wird. Geht es nach Lotte Kühn, der Autorin des Lehrerhasserbuchs, ist „jeder Mensch, der jemals eine Schule besucht hat, ein Experten auf dem Gebiet Lehrerbeurteilung " (Kühn, 2005, S. 12). Ein fehlendes Berufsgeheimnis, z.B. in Form einer Fachsprache wie in der Medizin, verhindert die Exklusivität der Arbeit. Es existiert keine spezifische Arbeitsweise oder Technologie, die als Besonderheit gilt. Beim Lehrerberuf spricht man gegenüber Juristen und Ärzten von einem gewissen Aroma des gesellschaftlich nicht ganz Vollkommenen, obwohl der Beruf die Grundsäule für den Fortbestand der Gesellschaft darstellt (Bastian \& Combe, 2003; Ulich, 1996). Der ehemalige Bundespräsident Horst Köhler machte diese zentrale Rolle in der Gesellschaft in seiner Berliner Rede vom 21.09.2006 deutlich, in der er Lehrer als "Helden des Alltags" bezeichnete (Volkery, 2006).

"Nichts besonderes", lautet dennoch der gesellschaftliche Tenor auf die Frage, was ein Lehrer tut. Ein Lehrer stellt nicht wirklich etwas her, was anderen unmittelbar von Nutzen sein könnte. Seine visiblen Tätigkeiten sind u.a. etwas an der Tafel zu zeigen, Fragen zu stellen, Schülern zu antworten, Klausuren zu korrigieren und Arbeitsblätter aus- 
zuteilen. Dies alles scheint nicht besonders anspruchsvoll zu sein, zumal er Schülern etwas vermittelt, was er selbst schon weiß. Mit solchen wechselseitigen Situationen wird jeder in seinem Leben konfrontiert, sei es im Sportverein, in der Familie oder unter Freunden und sie stellen selten ein Problem dar. Erziehen und Unterrichten scheint eine Fähigkeit zu sein, die jeder irgendwie hat und naturgemäß kann (Giesecke, 2001). Auch in literarischer Hinsicht kommen Lehrer in den seltensten Fällen gut weg. In der meist energisch vorgetragenen Schulkritik werden Lehrer entweder als tyrannischer Sadist oder als pedantischer Pauker dargestellt. Hermann Hesse bezeichnet in seinem Werk „Unterm Rad“ Lehrer als Unmenschen, als Angstmacher, die für sich beanspruchen den einzigen richtigen Weg zu kennen (Hesse, 1972). Mit demselben Stereotyp wird auch der begeisterte, menschliche und zugängliche Mr. Keating in N.H. Kleinbaums "Dead Poets Society" konfrontiert, der sich als Anreger des freien Denkens sieht, der auf die Schüler eingeht, sie motiviert und dennoch deren Respekt genießt (Lange \& Melches, 1999). Die ablehnende Haltung gegenüber dem Typus Lehrer manifestiert sich auch in der Musik. In dem Lied "Another brick in the wall" der britischen Rockband Pink Floyd wird diese kritische Einstellung mehr als deutlich. In ihrem Lied, inspiriert durch eigene Erfahrungen eines Bandmitglieds, wird eine ablehnende Haltung gegenüber dem englischen Bildungssystem eingenommen „we don't need no education", das den Individualismus unterdrücke, "we don't need no thought control" und geprägt ist von einer sarkastischen, zynischen und kalten Lehrerschaft, was in dem Textauszug „no dark sarcasm in the classroom“ deutlich wird (Grunder, 1999b).

Neben den Darstellungen in der klassischen Literatur, wie bei Hesse und Kleinbaum, spiegeln auch die Medien der jüngeren Vergangenheit das negative Bild wider, wie die Analyse verschiedener Nachrichtenmagazine von Blömeke aus dem Jahr 2005 deutlich macht. Von insgesamt 42 Artikeln, die sich schwerpunktmäßig mit Lehrern beschäftigten, waren 32 negativ geprägt und spiegelten althergebrachte Klischees wider. Eine genauere Betrachtung macht jedoch deutlich, dass das gesellschaftliche Urteil bzw. die Wertschätzung gespalten ist. Zum einen in Anforderungen des Berufs, die von $74 \%$ der Befragten einer demoskopischen Umfrage des Instituts Allensbach als schwer eingestuft werden, und zum anderen die ausübenden Personen, mit denen negative Attributionen verbunden werden (Rothland \& Terhart, 2007). Diese negative Einstellung gegenüber Lehrpersonen wurde ebenso in der Studie des Marktforschungsinstituts Forsa bestätigt, in der Eltern gefragt wurden, ob sie die Lehrer ihrer Kinder bzw. ihres Kindes feuern würden, wenn sie könnten. 39\% der Väter und 28\% der Mütter beantworteten diese Frage mit ja. Bei der Begründung für die Entlassung gaben $54 \%$ an, dass sie die Lehrkraft für unfähig halten (Struck, 2007). Zwischen den verschiedenen Lehr- 
ämtern sind jedoch weitere Differenzierungen bezogen auf das gesellschaftliche Urteil notwendig. Während die gesellschaftliche Meinung von Grundschullehrkräften überwiegend positiv geprägt ist, bestehen vor allem gegenüber Lehrern, die an Gymnasien unterrichten, Vorurteile und negative Meinungen. Während Lehrkräfte des Primarbereichs auf dem vierten Platz der Berufsprestigeskala nach Arzt, Pfarrer/Geistlicher und Hochschulprofessor rangieren, liegen Studienräte auf dem zwölften Platz von insgesamt 17 Berufen, die den Befragten zur Einschätzung vorlagen (Institut für Demoskopie Allensbach, 2008).

In der letzten Zeit, vor allem nachdem Studien zur Belastungs- und Beanspruchungssituation (Bauer, 2004; Schaarschmidt \& Fischer, 2001; Schaarschmidt 2005a; 2008; Schönwälder et al., 2003) ins gesellschaftliche Bewusstsein rücken, wird die Geringschätzung durch Mitleid und Bedauern abgelöst. Aus dem "faulen Sack“ wird das "arme (kranke) Schwein“, was die derzeitige Problematik des Lehrermangels weiter verschärft. Potenzielle Lehramtsstudenten werden abgeschreckt, sich bereits im Beruf befindliche Lehrer fühlen sich aufgrund der gesellschaftlichen Einschätzung der Berufsgruppe gekränkt und belastet (siehe Kap. 3 und Demmer, 2003, S. 22). Obwohl schon allein darin eine professionelle Leistung der Lehrer besteht, trotz all der Unsicherheiten, dem fehlendem Respekt, den widrigen Arbeitsbedingungen, den widersprüchlichen Anforderungen und Erwartungen sowie einem mannigfaltigen Aufgabenspektrums die schulische Praxis zu gestalten, wird der Berufsgruppe zusätzlich die Last auferlegt, für die Rettung der Gesellschaft und den Erhalt der Werte verantwortlich zu sein (Demmer, 2003; Rothland \& Terhart, 2007). Struck (2007, S. 10) vergleicht die Situation der Lehrer mit einem „Schiffsbrüchigen, der in tosender See auf einem Floß sitzt und mit Hilfe einer leeren Apfelsinenkiste um inn herum schwimmenden Delfinen beizubringen versucht, wie ein Computer funktioniert." Während Kritiker davor warnen, dass die Schule sich als Reparaturbetrieb der Gesellschaft verzwecken lässt, wird sich an der Vereinseitigung der einstmalig vorhandenen Arbeitsteilung von Schule und Familie bei Bildung und Erziehung in nächster Zukunft nichts ändern. Die Schule wird auch weiterhin der einzige Ort sein, an dem alle Kinder und Jugendliche erreicht und erzieherische Bemühungen wirksam werden können. Um diese neuen und erweiterten Aufgaben, die ergänzend zum zuvor genannten Aufgabenspektrum hinzukommen, annehmen zu können und ihnen gerecht zu werden, ist es jedoch notwendig, dass sich die Schule, vor allem aber die Unterstützung derselben durch Politik und Gesellschaft wandelt.

Wie in diesem Kapitel deutlich wurde, befindet sich der Lehrerberuf seit der Institutionalisierung des Lehren und Lernens in einer zentralen und bedeutsamen Position in 
der Gesellschaft und wird diese notwendigerweise auch in Zukunft behalten. Trotz eines umfangreichen und anspruchsvollen Spektrums an Aufgaben und Erwartungen erfahren Lehrkräfte weder durch gesellschaftliche Anerkennung und Wertschätzung noch durch die angemessen Gestaltung ihres Arbeitsplatzes und Arbeitsalltags die Unterstützung, die für ein erfolgreiches und nachhaltiges Lehren und Lernen unabdingbar sind. In Kapitel 2.3 wird nun nachfolgend die besondere Aufgaben- und Anforderungsstruktur von Sportlehrkräften beschrieben. In Kapitel 3 soll basierend auf einer theoretischen Grundlegung die Belastungs-Beanspruchungsproblematik aufgearbeitet werden. In Kapitel 4 werden letztendlich die verschiedenen Möglichkeiten des Umgangs mit Belastungen und Beanspruchungen im Sinne von Bewältigungsreaktionen sowie Reaktionen und Folgen dargestellt.

\subsection{Die besondere Bedeutung und Anforderungsstruktur des Sportunterrichts und des Sportlehrerberufs}

Der Lehrer hat die Aufgabe, eine Wandergruppe mit Spitzensportlern und Behinderten bei Nebel durch unwegsames Gelände zu führen, und zwar so, dass alle bei bester Laune und möglichst gleichzeitig an drei verschiedenen Zielorten ankommen (Gudjons, 1993, S. 81).

Für wohl kein anderes Fach scheint das obenstehende Zitat treffender zu sein als für den Sportunterricht, der im Folgenden mitsamt seiner sozialgeschichtlichen Entwicklung und dem spezifischen Auftrag sowie dem damit verbundenen Aufgabenspektrum für die Lehrkräfte vorgestellt wird.

In allen aktuellen Lehrplänen, Rahmenrichtlinien und Kerncurricula findet man den Schulsport unter verschiedenen, zumindest partiell als synonym anzusehenden Bezeichnungen im Fächerkanon wieder. Bereits an den verschiedenen Bezeichnungen für das Fach kann man Unterschiede im Verständnis bezüglich der Ziele und Inhalte sowohl in der geschichtlichen Genese als auch in der aktuellen fachdidaktischen Kultur erkennen. Während in dem Begriff Leibeserziehung deutlich und unverkennbar die Erziehung des Leibes bzw. durch körperliche Aktivität akzentuiert ist, offeriert der Begriff Sportunterricht eine Fokussierung auf den zu unterrichtenden Sport. Die in jüngerer Vergangenheit verstärkt auftretende Bezeichnung Bewegung, Spiel und Sport zeigt, dass sich der Schulsport immer weiter öffnet, was durch die Ausdifferenzierung der Sportkultur bedingt wird (siehe Kap. 2.3.2). Neben einzelnen normativen Setzungen ist es in letzter Zeit also vor allem der Auftrag des Faches selbst, der die fachdidaktischen Diskussionen bestimmt. 


\subsubsection{Die Entwicklung des Sportunterrichts in Deutschland}

Als Startschuss der Entwicklung des Schulsports in Deutschland sind die theoretischen und praktischen Ansätze der Philanthropen wie Salzmann, GutsMuths und Basedow im ausgehenden 18. Jahrhundert zu sehen. In seinem Werk „Gymnastik für die Jugend“ aus dem Jahr 1793 nimmt zwar eine systematisch-generische Stoffsammlung den größten Anteil ein, zusätzlich legitimiert Johann Christoph Friedrich GutsMuths seine Gymnastik jedoch auch theoretisch und es sind erste, teils bis heute gültige, unterrichtsorganisatorisch-methodische Hinweise und Leitsätze enthalten (Größing, 2007). Zum ersten Mal wurden körperliche Ertüchtigung und Spiele im Rahmen der Ausbildung genutzt, um ein ganzheitliches Erziehungsprogramm im Sinne von Pestalozzis Menschenbildung umzusetzen. Pestalozzi sah seine Elementargymnastik als einen Teil der Ganzheit der menschlichen Bildung an und beschäftigte sich mit dem Unterricht der Leibesübungen. Neben den rousseauistischen Zielsetzungen Naturnähe, einfaches Leben, Lernen aus Erfahrung und körperliche Abhärtung strebten die Philanthropen im Rahmen der vernünftig-natürlichen Erziehung an, die Kinder und Jugendlichen zu gesunden, tüchtigen, praktischen, fleißigen und aufgeklärten Bürgern heranzuziehen, die berufstüchtig und mit einem guten Erwerbssinn ausgestattet sein sollen, um sich in der Welt zurechtfinden zu können. Mit ihrem auf das Individuum gerichteten, an funktionalen Nützlichkeitsaspekten orientierten Erziehungsverständnis und den damit verbundenen Zielsetzungen sprachen die Philanthropen vor allem bürgerliche und adelige Eltern an (Grupe \& Krüger, 1997). Im Rahmen dieser Erziehungsvorstellung führte GutsMuths ein Bewertungssystem ein, meldete Erfolge an die Eltern und prämierte Bestleistungen öffentlich an der Meritentafel (Funke-Wienecke, 2007).

In der Folgezeit, die in starkem Maße politisch geprägt war, wurden turnerische Übungen, die bereits von den Philanthropen um GutsMuths verwendet wurden, durch verschiedene neue Turngeräte ergänzt. Durch die Belegung mit dem turnerischen Geist wollte Friedrich Ludwig Jahn die körperliche Ertüchtigung zur Erziehung des gesamten Volkes nutzen. Jahn verstand sein deutsches Turnen vor allem als Kritik an den herrschenden Verhältnissen in Politik, Gesellschaft und Kultur. Aufgrund von Verbindungen zur politischen Opposition und umstürzlerischen studentischen Burschenschaften wurde jedoch das Turnen im Jahre 1820 durch die sogenannte Turnsperre verboten und erst im Jahre $1842 \mathrm{im}$ Gewand des Spießschen Turnens als Schulturnen rehabilitiert. Im preußischen Schulturnerlass aus dem Jahr 1844 wurden letztlich „Leibesübungen als ein notwendiger und unentbehrlicher Bestandteil der männlichen Erziehung förmlich anerkannt und in den Kreis der Volkserziehungsmittel aufgenommen " (Krüger, 2005, S. 81). Auch in den anderen Ländern des Deutschen Bundes werden in der 
Folgezeit die Voraussetzungen für die Einführung des Turnens im schulischen Rahmen geschaffen. Zwar begann man ausgehend von Berlin damit, offiziell Turnlehrer auszubilden, zu einem einheitlichen Berufsbild führten diese von den Ländern nach unterschiedlichsten Prinzipien ins Leben gerufene Ausbildungsgänge jedoch nicht. In Preußen waren es meist Vereinsturnlehrer oder Volksschullehrer, die eine schmale Turnausbildung absolvierten und danach den Unterricht an den höheren Schulen übernahmen. Ein geringes Ansehen in Philologenkreisen war die Folge, das sich in abgeschwächter Form bis heute fortgepflanzt hat. Seit dem Schulturnerlass und dem damit verbundenen Lehrermangel wurde die Ausbildung forciert. Trotz der Anstrengungen ist jedoch auch Ende des 19. Jahrhunderts die Erteilung von Turnunterricht ohne staatliche Ausbildung keine Seltenheit (Voltmann-Hummes, 2008).

Wie oben bereits angedeutet, machte Adolf Spieß das Turnen mit seinen Frei- und Ordnungsübungen schul- und unterrichtsfähig. Die Übungen, die sich nach dem Grundprinzip der Elementarisierung und damit der Zergliederung von Bewegungen orientierte, ermöglichten es, möglichst viele Schüler effektiv und gleichzeitig zu unterrichten. In der Realität strahlte das Schulturnen dieser Zeit jedoch eher eine starre Kasernenhofatmosphäre aus, anstelle „Lebensfrische, Leibesschönheit und veredelte Körperlichkeit“, wie Spieß es vorsah zu verkörpern (Krüger, 2005, S. 117). Die Kritik an dem systematischen und erstarrten Turnen ließ nicht lange auf sich warten. Die Spielbewegung und die Reformpädagogik stellten die nächsten, die Entwicklung des Schulsports beeinflussenden Strömungen dar. Die durch den Spielerlass im Jahr 1882 offiziell unterstützte Spielbewegung griff den Sports aus England, mit Sport- und Laufspielen, Ballschlagen und ähnlichen freien Formen auf, bei denen Willenskraft, Selbstständigkeit und Geistesgegenwart in einem deutlich höheren Maße gefordert und gefördert wurden, als dies beim Turnen der Fall war (Grupe \& Krüger, 1997). Diese staatliche Unterstützung war auch als Reaktion auf die, durch die einsetzende Industrialisierung verstärkte Verschlechterung der Gesundheit vieler Menschen und dem rapiden Absinken der Wehrtauglichkeit junger Männer zu sehen (Krüger, 2005).

$\mathrm{Ab}$ dem 20. Jahrhundert war in den meisten Ländern des Reiches nun auch für Mädchen das Fach Turnen als verbindlicher Unterrichtsinhalt vorgesehen, was nicht zuletzt durch das Argument des Turnlehrers Wilhelm Klumpp „dass nur gesunde und kräftige Mütter auch gesunde und kräftige Kinder gebären können, und dass das leibliche Wohl und Wehe unserer ganzen Zukunft von dieser Bedingung abhängt" initiiert wurde (Krüger, 2005, S. 178).

In der Zeit nach dem ersten Weltkrieg fand mit der Reformpädagogik eine weitere einflussreiche Strömung Niederschlag in der Fachkultur, in der man sich genau wie in allen 
anderen Gesellschaftsbereichen gegen das Alte und für die Erneuerung aussprach. Das alte System von Schule und Erziehung sollte abgelöst werden durch eine Erziehung vom Kinde aus, die Abkehr nimmt von der formalisierten Lernschule und die die Entwicklung und Erziehung des Kindes in den Mittelpunkt stellt. Ein weiterer Vorsatz war die Professionalisierung der Ausbildung von Turnlehrern, was bereits von Spieß gefordert, jedoch stets stiefmütterlich behandelt wurde. Bekannte Vertreter des reformpädagogischen Schulturnens, das auch als Natürliches Turnen bezeichnet wurde, waren Karl Gaulhofer, Margarete Streicher und Erich Harte (Grupe \& Krüger, 1997). Als Gegenbewegung zum unnatürlichen, formalisierten und starren Schulturnen wird hier auf kindgemäße Art und Weise das Sammeln von Erfahrungen und das selbstständige Lösen von gestellten Bewegungsaufgaben ermöglicht (Krüger, 2005).

Nachdem der Schulsport durch die Nationalsozialisten im Dritten Reich durch die Prinzipien Volksgemeinschaft, Wehrhaftigkeit, Rassenbewusstsein, Auslese der Stärksten und Führertum zu politischen Zwecken missbraucht wurde, galt es, im Nachkriegsdeutschland das Fach zu reorganisieren und neu zu legitimieren. Nach dem Zweiten Weltkrieg kam es zu einem, durch verschiedene Gründe bedingten eklatanten Sportlehrermangel, dem wiederum eine Phase der Einstellung von nicht- bzw. schlecht ausgebildeten Lehrkräften folgte (Voltmann-Hummes, 2008). Inhaltlich kam es direkt nach Kriegsende zunächst zu einer Renaissance der Reformpädagogik, die durch die Machtübernahme der Nationalsozialisten 1933 abrupt aus der Schule verschwand, bevor in den „Empfehlungen zur Förderung der Leibeserziehung in den Schulen“ klassischhumanistische Bildungsideale in den Mittelpunkt traten (Größing, 2007). In seiner Ausrichtung stand nun nicht der Sport als gesellschaftliches Phänomen im Mittelpunkt, sondern vielmehr die Erziehung des Leibes und des ganzen Menschen. In dieser bildungstheoretischen Phase setzte sich in den westlichen Besatzungszonen eine Eigenwelttheorie des Sports durch, die Autonomie gegenüber Politik und Wirtschaft vorsah und bis in 1970er Jahre die dominierende Idee der Positionierung der Leibeserziehung darstellte. Man verstand das Fach in dieser Zeit nicht als ein Unterrichtsfach wie die anderen, sondern viel mehr als ein Prinzip der Erziehung mit überfachlichem Auftrag, dessen Erfüllung jedoch dadurch infrage gestellt wurde, dass jede dritte Lehrkraft die im Schuljahr 1972/73 Sport unterrichtete, keine Lehrbefähigung für das Fach besaß (Grupe \& Krüger, 1997; Voltmann-Hummes, 2008).

Im Zusammenhang mit den grundlegenden Bildungsreformen der 1960er und 1970er Jahre, die der Schule zu mehr Lebensnähe verhelfen sollten, wurde 1972 das „Aktionsprogramm für den Schulsport" verabschiedet. In dieser nun einsetzenden curriculumsund lerntheoretischen Phase sollte statt abstrakter Bildung konkretes Lernen fürs Le- 
ben - im Falle des Schulsports das, was Schüler für den Sport in ihrer Freizeit benötigen - im Mittelpunkt stehen. Breiten-, Freizeit und auch der Leistungssport starteten, ausgelöst durch den auf die Olympischen Spiele 1972 folgenden Sportboom, eine Erfolgskarriere, die auch an der Leibeserziehung nicht folgenlos vorüberging (Balz \& Kuhlmann, 2004). Neben der Veränderung des Inhaltsspektrums hin zu einer Öffnung gegenüber den vielfältigen Erscheinungsformen des Sports, wandelten sich simultan auch die fachspezifischen Zielsetzungen (Grupe \& Krüger, 1997). Diese einsetzende Versportlichung der Leibeserziehung und das Zurücktreten pädagogischer Argumente führten auch dazu, dass das Fach verbreitet als Sportunterricht bezeichnet wurde. Die Erziehung durch Leibesübungen bzw. Sport musste nach und nach einer Erziehung zum Sport weichen. Das Fach legitimierte sich damit stärker durch außerschulische als durch innerschulische Argumente (Größing, 2007).

In der Folgezeit mehrte sich heftige Kritik an der einseitigen sportiven Ausrichtung des Sportunterrichts und im "Zweiten Aktionsprogramm für den Schulsport" rückt die Handlungsfähigkeit des Schülers, und damit auch verstärkt wieder die Erziehung in das Zielspektrum des Schulsports. Diese Position stellt einen pragmatischen Kompromiss zwischen traditionellem Sportartenlernen und alternativer Bewegungserziehung dar (Grupe \& Krüger, 1997). Die Diskussion bezüglich der Ausrichtung des Sportunterrichts ist in der Folgezeit jedoch keineswegs geradlinig und übersichtlich verlaufen. Nach und nach kristallisierten sich mit der konservativen, der pragmatischen, der alternativen und der fundamentalen Position vier konkurrierende fachdidaktische Konzeptionen heraus. Nachdem ausgehend vom Bundesland Nordrhein-Westfalen der Erziehende Sportunterricht seit dem Übergang zum 21. Jahrhundert mit seinem charakteristischen Doppelauftrag das in Lehrplänen und Literatur dominierende Konzept darstellte, bestimmen derzeit vor allem Kompetenzen und Standards die Curricula- und Fachdiskussionen (siehe Kap. 2.3.2).

Wie gezeigt werden konnte, erwies sich das Berufsbild der Sportlehrkräfte in den verschiedenen Phasen der Entwicklung des Sportunterrichts stets als inhomogen, was auch heute noch teilweise der Fall ist. Die Akademisierungsbestrebungen des Sportlehrerberufs wurden und werden durch die administrative Steuerung mitsamt Entlassungen oder Einstellung von Fachfremden oder Nichtausgebildeten stets behindert. Auch heute noch besteht Heterogenität in der Sportlehrerschaft, was die Berechtigung zur Erteilung des Sportunterrichts betrifft. Während an Gymnasien mit den DiplomSportlehrkräften eine Gruppe zu finden ist, die keine allgemeinpädagogische Ausbildung durchlaufen hat, wird an Grundschulen auch heute noch ein erheblicher Stundenanteil durch fachfremd Unterrichtende abgedeckt. All diese Uneinheitlichkeiten 
führen dazu, dass eine selbstbewusste Berufsidentität als nur ansatzweise gegeben scheint und das Fach in der Schule meist nur randständige Wahrnehmung und Anerkennung findet (Voltmann-Hummes, 2008).

\subsubsection{Der Auftrag des Sportunterrichts}

Während man in den 1970er Jahren im Sportunterricht hauptsächlich das Ziel verfolgte, durch die Vermittlung von Fähigkeiten, Fertigkeiten und Kenntnissen die Schüler auf den außerschulischen Sport vorzubereiten, hat sich der Auftrag des Sportunterrichts bis zum heutigen Tag entscheidend gewandelt und ausgeweitet (Scherer, 2008). Der Sportunterricht stellte damals eine Doppelwelt zur außerschulischen Sportrealität dar, die möglichst unverfälscht in den Unterricht transportiert wurde. Im Sport allein wurde genug erzieherisches und pädagogisches Potenzial gesehen, sodass dieser auch möglichst abbildhaft in der Schule umgesetzt werden sollte (Balz \& KuhImann, 2004).

Seit den frühen 80er Jahren des letzten Jahrhunderts wurde jedoch zunehmend Kritik am Sportunterricht geübt und sogar Söll (2000), der wohl bekannteste Verfechter und Mitbegründer des Sportartenkonzepts sieht im Sport allein und dessen Tradition keine ausreichende Legitimationsgrundlage. Heutzutage reicht also Sport als alleiniger Legitimationsaspekt nicht mehr aus, da vielfältige Möglichkeiten und Chancen vergeben werden, die das Fach zusätzlich in sich birgt. Der außerschulische Sport stellt aber dennoch eine wichtige Bezugsebene dar und liefert dem Schulsport Ziele, Inhalte, Beispiele und Anregungen (Scherer, 2008). Tiefgreifende Veränderungen des außerschulischen Sports führten jedoch dazu, dass der Sportunterricht vor ernsthafte Orientierungsprobleme gestellt wurde und wird. Zwar zählt Sport auch heute noch zu den Kulturgütern, die in der Schule gepflegt werden sollten, existiert in der Gesellschaft aber nicht mehr in solch klarer Form wie dies vor allem in den 1970er Jahren der Fall war (Grupe \& Krüger, 2000). Durch die Ausweitung und Differenzierung der Sport- und Bewegungsformen ist es allein aufgrund der Menge und Vielgestaltigkeit unmöglich, den außerschulischen Sport ungefiltert in die Schule in Form eines einheitlichen Inhaltskatalogs zu transportieren. Neben der Neuerfindung von Sportarten werden auch bereits existente Formen wiederentdeckt, modifiziert und weiterentwickelt oder mit anderen Sportarten kombiniert. Aufgrund der Erschließung neuer Sozial-, Raum- und Zeitdimensionen ist Sport nicht mehr festgelegt und es wird flexibel variiert, wer, wie, mit wem, wann und wo Sport treibt. Das traditionelle Sportverständnis befindet sich in einem Auflösungsprozess und erweiterte Deutungsspielräume erleichtern den Zugang zu Bewegung, Spiel und Sport und eröffnen mehr Möglichkeiten der Befriedigung individueller Bedürfnisse. Wettkampf als alleinige Motivation wurde abgelöst durch eine 
zunehmende Orientierung an Spaß, Geselligkeit, Erlebnis und Fitness, was sich oft auch in den zugehörigen Szenen und Kulturen zeigt (Bräutigam, 2003).

Neben der Berücksichtigung der offensichtlich vernachlässigten Potenziale, die dem Sportunterricht innewohnen, muss das Fach also zusätzlich durch einen flexiblen und offenen Gegenstandsbereich für künftige Entwicklungen zugänglich bleiben. Ein weiterer Aspekt, der vor allem im methodischen Bereich Berücksichtigung finden muss, sind die Veränderungen im Aufwachsen von Kindern und Jugendlichen. Unter dem Begriff Veränderter Kindheit werden Wandlungen in der bewegungsbezogenen Sozialisation, als auch Einschränkungen der sozialen Interaktionskompetenz zusammengefasst (dvs, DSLV, DOSB \& FSW, 2008). In diesem Zusammenhang spricht man z.B. von einer Verinselung der Lebensräume von Kindern. Während früher Wegstrecken zwischen dem Zuhause und der Schule, dem Training im Sportverein oder dem Unterricht in der Musikschule einen zusätzlichen Erfahrungsbereich darstellten, werden diese Wege heute meist aufgrund infrastruktureller Gegebenheiten mit dem Pkw der Eltern zurückgelegt. Dieser lebensweltliche Wandel durch zunehmende Bebauung und Verkehrsdichte sowie die expansive Mediennutzung stellt eine weitreichende Veränderung der Bewegungskultur dar und muss Berücksichtigung in der Ausgestaltung des Unterrichts finden (Laging \& Klupsch-Sahlmann, 2001). Pühse (1994; 1999) stellt zusätzlich eine Einbuße von Aussagekraft und Verbindlichkeit traditioneller Werte durch die Erosion traditioneller Sinninstanzen fest. Die Familie verliert als Ort der sozialen Geborgenheit und Vermittlungsinstanz sozialer Kompetenz an Bedeutung und vor allem bezogen auf den Sport nehmen Individualisierungsprozesse ungekannte Ausmaße an. Pühse bezeichnet diesen Prozess metaphorisch als soziale Abschottung durch einen Kokon (Pühse, 1994; 1999). Während altruistische, soziale, kollektive und ethische Werthaltungen bei den Jugendlichen im Vergleich zu früher in geringerem Maße ausgeprägt sind, ist ein Anstieg hedonistischer Interessen festzustellen, die auf Spaß und Genuss ausgerichtet sind. Im Zuge dieser Entwicklungen werden Forderungen laut, dass vor allem dem sozialen Lernen an Schulen einen größeren Stellenwert beigemessen werden muss. Der Sportunterricht muss also sowohl die aktuellen Voraussetzungen von Kindern und Jugendlichen berücksichtigen, als auch sich wandelnde Chancen und Probleme der Sacherschließung einkalkulieren und neuere Vorstellungen von den Vorgaben und Aufgaben der Schule annehmen (Balz \& Kuhlmann, 2004).

Den Auftrag des Sportunterrichts zu beschreiben stellt heutzutage ein äußerst vielschichtiges Vorhaben dar, da eine sehr breite Palette an Kompetenzen im fachlichen aber vor allem im überfachlichen Bereich den eigentlichen Ertrag des Faches bestimmt. Der Sportunterricht in der Schule ist von besonderer Bedeutung, denn er bietet sowohl 
im körperlichen als auch im sozialen Bereich unmittelbare Handlungs- und Erfahrungsmöglichkeiten. Zudem ist der Sportunterricht der einzige Sport für alle bei dem Kinder und Jugendliche ein qualifiziertes Bewegungsangebot erhalten. Durch den schulischen Sportunterricht werden alle Kinder, unabhängig davon wie körperlich leistungsfähig, wie alt sie sind, welches Geschlecht sie haben oder welchen sozialen Status sie aufweisen, erreicht (Söll, 2000; dvs et al., 2008). Gerade in der heutigen Zeit in der, wie zuvor bereits beschrieben, Kinder und Jugendliche in veränderten Lebensverhältnissen aufwachsen, häufig unter Bewegungsmangel und den daraus resultierenden körperlichen und gesundheitlichen Folgen leiden sowie Probleme im sozialen Bereich aufweisen, kann das Fach Sport kompensatorisch wirken. Bewegung, Spiel und Sport im Unterricht soll einerseits dazu dienen die Gesundheit und die Fitness zu verbessern, gleichzeitig aber auch das Selbstvertrauen stärken und soziale Kontakte ermöglichen (dvs, DSLV, DOSB \& FSW, 2008).

Der breit gefächerte Auftrag wird in der folgenden Definition von Laging aus dem Jahr 2004 deutlich. Laging reduziert das Fach nicht auf die Vermittlung von sportartspezifischen Techniken und Taktiken, sondern sieht den „Erziehenden Sportunterricht" im Rahmen des Bildungs- und Erziehungsauftrag der Schule in einem größeren Zusammenhang:

Der Sportunterricht ist für die Schulpflichtzeiten der verschiedenen Abschlüsse [...] ein verbindliches Unterrichtsfach der Schule. [...] Der Sportunterricht ist in den pädagogischen Auftrag der Schule eingebunden, der durch die Lehrpläne und Rahmenrichtlinien seine jeweilige Konkretisierung erhält. Entsprechend ist für den Sportunterricht eine enge Verbindung von Bildung, Erziehung und Lernen herzustellen. Die Inhalte des Sportunterrichts sind somit nicht die Sportarten bzw. deren einzelne motorische Fertigkeiten an sich, sondern die pädagogisch ausgelegten Formen der bewegungsbezogenen Auseinandersetzung mit Lernaufgaben zur Lösung sich stellender Bewegungsprobleme im sportlichen wie nichtsportlichen Sinnhorizont. Insofern ist der Sportunterricht eine Veranstaltung organisierten Lernens im Kontext von Bildung und Erziehung in der Schule unter Leitung und in Verantwortung einer akademisch ausgebildeten Sportlehrerin bzw. eines Sportlehrers (Laging, 2004b, S. 546f.).

In der Definition von Laging wird deutlich, dass im Sportunterricht mit „Entwicklungsförderung durch Bewegung, Spiel und Sport“ - „Erschließung der Bewegungs-, Spielund Sportkultur" ein Doppelauftrag verfolgt wird (Kurz, 2000, S. 14). In diesem Doppelauftrag gehen die Ziele der beiden Hauptlinien der fachdidaktischen Ansätze der 
letzten Jahrzehnte auf, die Prohl (2006) als pragmatisch-qualifikatorische Strömung und kritisch-emanzipatorische Strömung bezeichnet. Lange Zeit bestimmten heftige Diskussionen die Ausrichtung des Sportunterrichts. Vertreter der pragmatischqualifikatorischen Strömung fokussieren vor allem auf das Kulturgut Sport, das es in der Schule zu vermitteln gilt, ohne dass Sportarten durch pädagogische Funktionalisierung bis zur Unkenntlichkeit verändert werden (Prohl, 2006). Den Gegenpol stellen die Vertreter der kritisch-emanzipatorischen Strömung dar, die hauptsächlich die pädagogisch-erzieherischen Aspekte von Bewegung, Spiel und Sport nutzen möchten. In den neueren Lehrplänen und Kerncurricula schlugen sich letztendlich mit dem Erziehenden Sportunterricht beide Positionen nieder. Dieser Doppelauftrag sieht sowohl eine Erziehung zu, als auch durch Bewegung, Spiel und Sport vor (Scherer, 2008). Diese Orientierung stellt, in Zeiten, die geprägt sind durch gestiegenen Orientierungsbedarf das Wiederaufleben des bildungstheoretischen Denkens im herbartschen Sinne dar. Ausgehend von der Lehrplanrevision in Nordrhein-Westfalen hat der Erziehende Sportunterricht auch viele andere Bundesländer erfasst und wurde im Anschluss ministeriell verordnet, da Einigkeit darin besteht, dass der Sportunterricht dabei helfen muss, sowohl die Bewegungskultur zu erschließen, als auch die Persönlichkeit zu entwickeln (Prohl, 2008). Bewegung, Spiel und Sport ist in diesem Konzept sowohl der Gegenstand der materialen Bildung als auch Medium einer formalen Bildung (dvs, DSLV, DOSB \& FSW, 2008).

Als Vermittler zwischen gesellschaftlichen und individuellen Ansprüchen nimmt der Sportunterricht gegenwärtig eine bildungstheoretische Position ein, die stärker pädagogisch akzentuiert ist als in früheren Konzepten (Stibbe, 2000). Dabei wird letztendlich die enge Anbindung an den Sport ein Stück weit gelöst und durch die Orientierung an breiter gefassten Bewegungsfeldern sowohl Veränderungs- als auch Ausdifferenzierungstendenzen des Sports in der Gesellschaft Rechnung getragen. Bewegungsfelder stellen eine Ausweitung des Inhaltskanons dar, bei dem, was umstritten ist, auch das bewegungskulturelle Umfeld des Sports zum Thema des Unterrichts werden kann und soll. Während man früher von Leichtathletik als Unterrichtsinhalt sprach, fasst man heute mit "Laufen, Springen, Werfen" einen grundlegenden und umfassenderen Bereich zusammen, in dem aufgezeigt wird, dass Laufen, Springen und Werfen in weit mehr sportlichen Bewegungsformen Bedeutung hat, als in den leichtathletischen Grunddisziplinen (Kurz, 2008). Gleichzeitig ist das Fach durch einen weiteren Gegenstandsbereich ohne inhaltliche Verbindlichkeiten offener und flexibler für zukünftige Entwicklungen (Stibbe, 2000). Würde man sich neuen Formen von Bewegung, Spiel und Sport und den veränderten Lebensbedingungen der Kinder und Jugendlichen ver- 
schließen und in der Schule eine Gegenwelt aufbauen, die bewusst einen Kontrast zu der Art des Sporttreibens, das außerhalb der Schule stattfindet, herstellt, würde man Kinder und Jugendliche auf eine Sportwelt vorbereiten, die in der Form längst nicht mehr existiert (Stibbe, 2000; Balz \& Kuhlmann, 2004). In Bewegungsfeldern sollen Erfahrungen ermöglicht und grundlegende Einstiegsqualifikationen erworben werden, die den Zugang zu sportlichen Aktivitäten erschließen. Diese sportartübergreifende Elementarisierung ersetzt nicht die Sportarten, sondern stellt eine Ergänzung zu den traditionellen Sportarten dar, die Teil der Bewegungsfelder sind (Stibbe, 2000).

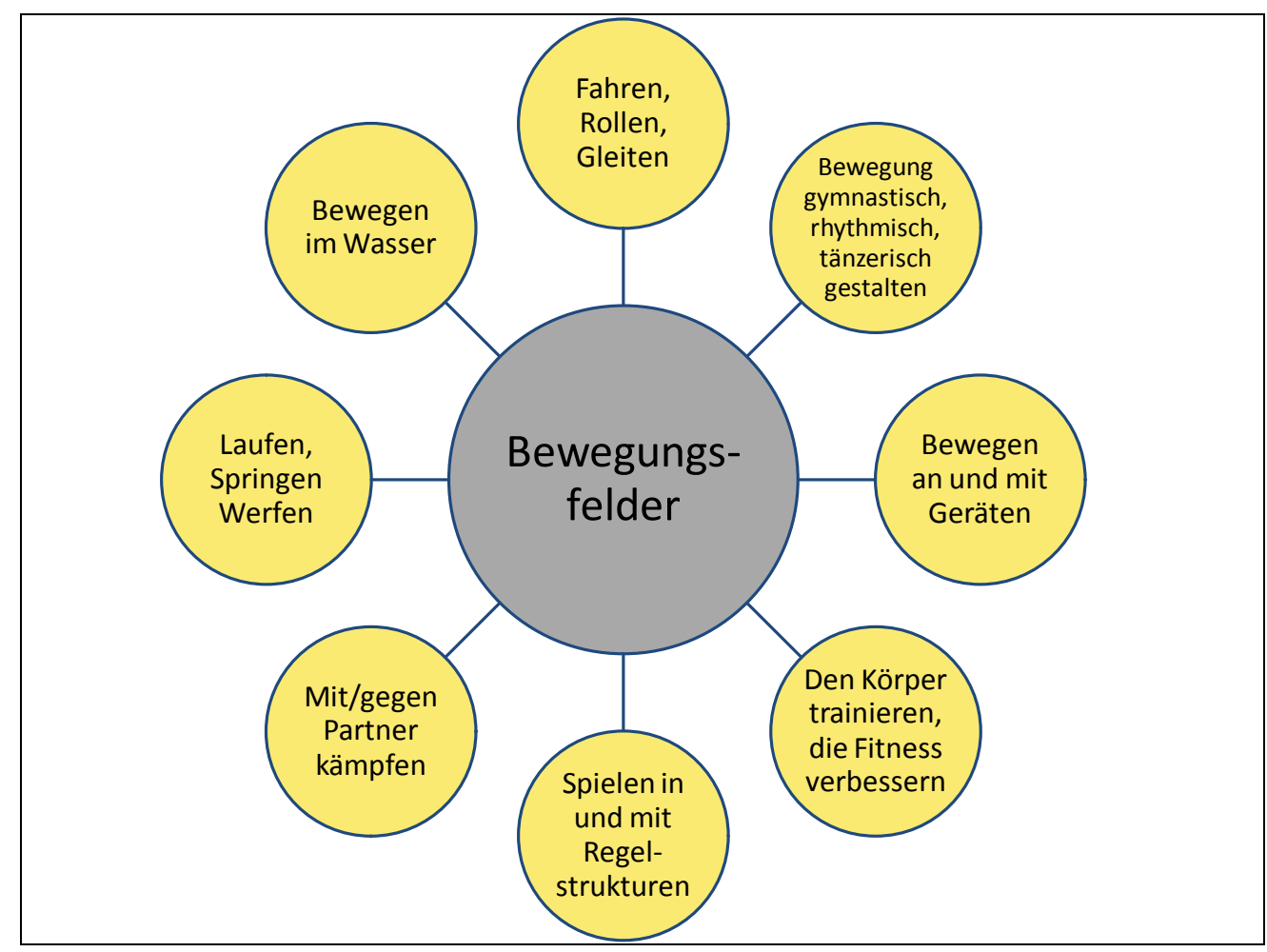

Abb. 1: Bewegungsfelder im Sportunterricht (angelehnt an Hessisches Kultusministerium, 2010)

Schülern, die aufgrund unterschiedlichster Voraussetzungen dem sportlichen Treiben mit einer gewissen Distanz gegenüberstehen, z.B. wenn sie aus einer sport- oder bewegungsfernen Familie kommen, können durch Bewegungsfelder einen leichteren und motivierenden Einstieg in die sportliche Bewegungswelt finden, als dies in den reglementierten traditionellen Sportarten der Fall ist (dvs, DSLV, DOSB \& FSW, 2008).

Neben der inhaltlichen Neuordnung in Form von Bewegungsfeldern stellen die pädagogischen Perspektiven den zweiten Kernaspekt moderner Vorstellungen von Sportunterricht dar. Orientiert an empirischen Untersuchungen zu Sportmotiven und Einstellungen zu sportlicher Aktivität und den daraus hervorgehenden Dimensionen, stellen pädagogische Perspektiven bestimmte Standpunkte dar, von denen ein Objekt aus betrachtet werden kann. Die von Kenyon (1968) beschriebenen Dimensionen wurden 
bis heute immer wieder bestätigt und stellen die grundlegende Systematik und Begründung der Akzentuierungen in Form der pädagogischen Perspektiven dar. „Jede Perspektive erschließt zunächst von einem besonderen Standpunkt aus, inwiefern sportliche Aktivität pädagogisch wertvoll sein kann. Anders ausgedrückt: Jede Perspektive bietet eine besondere Auslegung, inwiefern Sportunterricht die Entwicklung Heranwachsender fördern kann“ (Kurz, 2000, S. 25). Pädagogische Perspektiven beschreiben, was im Sportunterricht gelernt werden soll und kann und bringen erzieherische Wertvorstellungen auf den Punkt (Bräutigam, 2003). Durch die pädagogischen Perspektiven sollen beide Seiten des Doppelauftrages erfüllt werden: einerseits die Einlösung des allgemeinen Erziehungs- und Bildungsauftrages der Schule und andererseits die Erschließung der Bewegungs-, Spiel- und Sportkultur durch das Anknüpfen an den individuellen Beweggründen der Kinder und Jugendlichen (Stibbe, 2000).

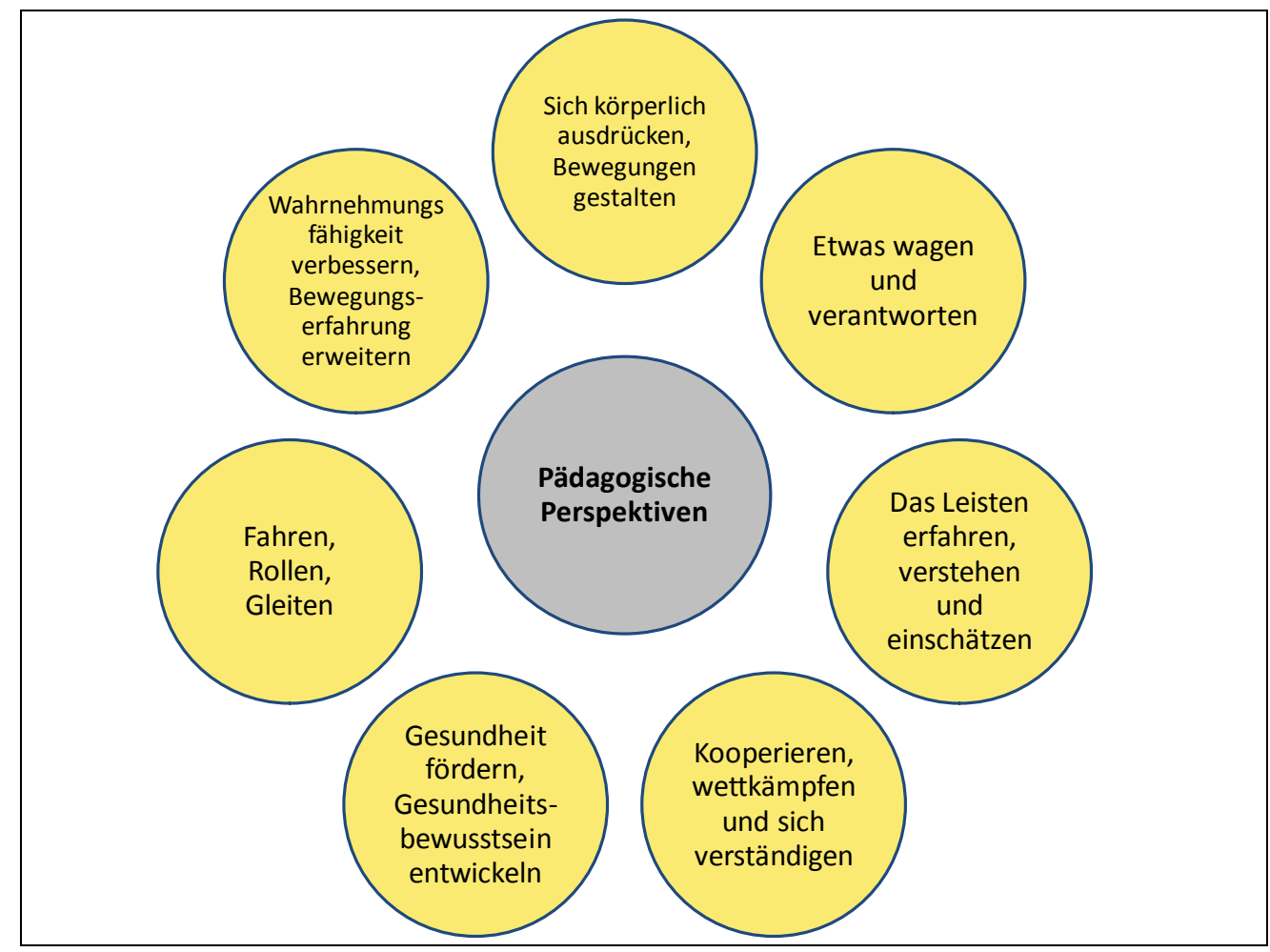

Abb. 2: Die pädagogischen Perspektiven des Sportunterrichts (mod. nach Hessisches Kultusministerium, 2010, S. 5)

Durch die Akzentuierung der Bewegungsfelder anhand der pädagogischen Perspektiven wird das pädagogisch Bedeutsame erschlossen, d.h. die eigentlichen Inhalte des Sportunterrichts entstehen erst durch didaktische und pädagogische Deutung (Kurz, 2008; Scherer, 2008). Die Verknüpfung von pädagogischen Perspektiven, Bewegungsfeldern sowie fachlichen Kenntnissen und Methoden zum selbstständigen Arbeiten im Rahmen eines Unterrichtsvorhabens bezeichnet man als Thema. Neben dem „Was" 
d.h. den Inhalten, besteht immer auch ein enger Bezug zu dem "Wozu“, den Zielen des Unterrichts. Ergänzend muss hinzugefügt werden, dass manche Inhalte sich besser eignen, um bestimmte Ziele zu erreichen als andere, sodass eine gründliche theoretische Vorbereitung von Unterricht unerlässlich ist (Balz \& Kuhlmann, 2004). „Zusammengenommen bieten die Perspektiven eine gedankliche Ordnung für pädagogisch bedeutsame Möglichkeiten, sportliche Aktivitäten mit Sinn zu belegen“ (Kurz, 2000, S. 27). Kern des pädagogischen Konzepts ist die Mehrperspektivität, in der es im bildungstheoretischen Sinne um eine Perspektivenerweiterung geht. Kinder und Jugendliche sollen ihren Horizont erweitern und lernen, mit anderen Augen zu sehen. Im Rahmen eines mehrperspektivisch ausgelegten Sportunterrichts lernen Kinder und Jugendliche, dass Bewegung, Spiel und Sport durch das Einnehmen unterschiedlicher Blickwinkel vielschichtige, teils kontrastierende Sinnzuschreibungen ermöglichen, wodurch sich die sportlichen Aktivitäten selbst verändern (Stibbe, 2000). Durch Kontrastierung verschiedener Perspektiven können zudem unterschiedlichste Deutungs- und Umsetzungsmöglichkeiten erschlossen werden (Bräutigam, 2003).

Inwieweit der Doppelauftrag zu einem höheren Ertrag im Vergleich zu einer einseitigen Sportorientierung im Sinne einer Vermittlung von Sportarten führt, zeigt ein von Kurz (2008) vorgestelltes Beispiel zur Ausdauerschulung im Sportunterricht: Eine Steigerung der Ausdauerleistung kann nur dann gelingen, wenn Kinder auch außerhalb des Sportunterrichts trainieren. Mit zwei oder drei Sportstunden pro Woche allein, ist kein bemerkbarer Einfluss auf den Gesundheitszustand der Kinder und Jugendlichen zu erreichen. Lediglich durch eine drastische Stundenerhöhung wäre ein merklicher und direkter Effekt auf das körperliche Leistungsvermögen bzw. die Gesundheit zu erzielen, was bei der derzeitigen Situation im Bildungssystem jedoch unrealistisch erscheint (Rehfuss, 1995). Die Entwicklungsförderung hingegen wirkt auch in kleinen Mengen. Dazu gehört z.B. zu wissen, wie man seine Laufausdauer verbessern kann, warum sie sich verbessert und welche Effekte in welcher Zeit zu erwarten sind. Weiterhin kann vermittelt werden, welche Alternativen es zum Laufen gibt und warum bei manchen Kindern schneller Fortschritte festzustellen sind als bei anderen. Ein weiteres zentrales Lernziel im Sinne einer Kompetenzorientierung im Rahmen einer solchen Einheit ist es zu erkennen, welche Bedeutung die Ausdauer für die Gesundheit hat und wie man durch Ausdauerbelastungen das Gewicht reduzieren kann. Kurz betont, dass unterrichtliche und erzieherische Ziele nicht losgelöst voneinander bedacht und umgesetzt werden sollen, sondern integrativen Charakter haben, denn „es kommt nicht nur darauf an, dass die Schüler das Laufen lernen, sondern auch darauf, was sie am Laufen lernen“ (Kurz 2008, S. 217). 
Zielsetzung des Sportunterrichts ist es also, körperliche, sozial-emotionale und kognitive Bildungsprozesse im Umfeld von Bewegung, Spiel und Sport zu initiieren und gesunderhaltende körperliche Freizeitaktivitäten in das Leben der Schüler langfristig zu integrieren. Die Bedeutsamkeit des Sportunterrichts ergibt sich ergänzend dadurch, dass die Kinder, die besonderen Förderungsbedarf sowohl im körperlichen als auch im sozialen Bereich aufweisen, meist von außerschulischen Angeboten nicht erreicht werden. Gerade diesen Kindern und Jugendlichen kann im Sportunterricht als Hauptberührungspunkt mit sportlicher Bewegung aufgezeigt werden, dass sportliches Bewegen auf mehreren Ebenen eine gewinnbringende Betätigung sein kann. Kinder und Jugendliche, die aufgrund ihrer außerschulischen sportlichen Sozialisation als sportaffin zu bezeichnen sind, realisieren ihr Können und freudvolles Erleben vor allem im Kontext von traditionellen Sportarten, sodass auch diese Berücksichtigung in der Ausgestaltung der Bewegungsfelder finden sollen und müssen (dvs, DSLV, DOSB \& FSW, 2008). Der Sportunterricht sollte es außerdem ermöglichen, dass Kinder und Jugendliche Kompetenzen, die sie im außerschulischen Sport erworben haben, in den schulischen Sport einbringen können. Wenn die Vielfalt ein wesentliches Merkmal des außerschulischen Sports ist, dann sollte es die Aufgabe des Schulsports sein, diese Vielfalt der Sinnorientierungen, Praxis- und Inszenierungsformen erlebbar zu machen. Die Schule sollte den Jugendlichen helfen, sich in den Bedingungen der alltäglichen Realität zurechtzufinden, da diese oft unstrukturiert ist, was vor allem durch die selbstständige Lösung von Aufgaben und Problemen erprobt werden kann (Wopp, 1999).

Insgesamt wird aktuell also eine mittlere Position eingenommen, in der sich der außerschulische Sport und der Sportunterricht wechselseitig aufeinander beziehen. Im Sportunterricht wird an die Sportrealität angeknüpft, jedoch nicht um diese zu reproduzieren wie dies in den 1970er Jahren der Fall war, sondern um sie als Ausgangs- und Bezugspunkt zu nutzen. Im Unterricht wird die gesellschaftliche Sportwirklichkeit überschritten und weitergeführt und durch die pädagogischen Perspektiven alternative Formen und Deutungsmuster aufgezeigt, die Sport und Bewegung als veränderbares Konstrukt interpretieren und als den eigenen Bedürfnissen und Interessen anpassbar beschreibt. Es soll also einerseits auf das außerschulische Sporttreiben vorbereitet werden, gleichzeitig aber auch Erfahrungen ermöglicht werden, die sonst nicht möglich wären (Bräutigam, 2003).

Dass ein Kind geschwitzt, gespielt oder sich bewegt hat oder zumindest beschäftigt war, stellt keinesfalls eine Haltung dar, die man hinnehmen kann im Angesicht der Möglichkeiten, die der Sportunterricht für die Förderung der Entwicklung der Schüler bietet (dvs, DSLV, DOSB \& FSW, 2008). Im Sportunterricht besteht wie in keinem ande- 
ren Fach die Möglichkeit, soziales Lernen in Verbindung mit praktischer Erfahrung und Reflexion unmittelbar zu ermöglichen. In engem Zusammenhang damit ist die Erweiterung und Intensivierung des Erfahrungshorizontes zu sehen, bei der an Vorerfahrungen angesetzt wird (Stibbe, 2000). Durch den Sportunterricht werden Lernprozesse initiiert die einen Beitrag zur Persönlichkeitsentwicklung leisten wie Fairness, Teamgeist, Anstrengungs- und Leistungsbereitschaft, Empathie- und Kooperationsfähigkeit, Durchhaltevermögen und Integration von Schwächeren, aber vor allem auch das Gewinnenund Verlieren-Können (dvs, DSLV, DOSB \& FSW, 2008). Der Sportunterricht eignet sich zwar wegen seiner Struktur und seinen Inhalten besonders dazu, sozial-kooperatives Verhalten zu initiieren, trotzdem muss aber klar sein, dass die Schule und mit ihr der Sportunterricht kein Allheilmittel und keine Reparaturwerkstatt für gescheiterte Sozialisation darstellt. Neben der parallel zur Schule ablaufenden gesellschaftlichen Sozialisation bestimmen Erfahrungen und Sozialisierungseinflüsse der ersten Lebensjahre das Verhalten maßgeblich und relativieren so die Einflüsse des Sportunterrichts (Pühse, 1994).

Seit dem Jahr 2003 wendet man sich im Sportunterricht genau wie in der Schule insgesamt (siehe Kap. 2.2.1) zunehmend einer Output-Orientierung zu. In Kerncurricula werden verbindliche Standards formuliert, in denen spezifische Erwartungen an den Unterricht formuliert sind. Anhand der Ergebnisse von Lehr- und Lernprozessen soll die Qualität von Schule und Unterricht gemessen werden und Rückschlüsse auf erfolgreiche schulische Programme gezogen werden. Bevor eine fachdidaktische Diskussion über Chancen und Gefahren der Standards einsetzte, wurden bereits in vielen Bundesländern verbindliche Anforderungen unterschiedlicher Qualität formuliert (Aschebrock \& Stibbe, 2008).

Im niedersächsischen Kerncurriculum Sport beispielsweise sind neben den inhaltsbezogenen Kompetenzbereichen, die fachbezogen sind, ebenso prozessbezogene Kompetenzbereiche berücksichtigt, bei denen es um Fähigkeiten und Fertigkeiten geht, die die Anwendung von Wissen ermöglichen. Neben „Erkenntnisse gewinnen“, „Beziehungen entwickeln“, "Lernen lernen“ gehört auch der Aspekt „Bewegungen evaluieren“ zu den prozessbezogenen Kompetenzbereichen. Die Umsetzung soll in Form selbsttätiger Auseinandersetzung mit Bewegungsproblemen und Aufgaben erfolgen (Niedersächsisches Kultusministerium, 2007). Die Überprüfung der prozessbezogenen Kompetenzen erweist sich jedoch als schwierig und die Maßgaben für die inhaltsbezogenen Bereiche scheinen, wenn man die Standards verschiedener Bundesländer vergleicht, zufällig festgelegt. Sportunterrichtsspezifisch kommt hinzu, dass die Leistungen im motorischen Bereich zum Großteil durch die im privaten Bereich stattfindende Sport- und 
Bewegungssozialisation bedingt sind und tatsächlich auf den Sportunterricht zurückzuführende Leistungen äußerst schwer mess- und prüfbar sind (Aschebrock \& Stibbe, 2008). In der Regel sind die bisher existenten Standards überwiegend als Bewegungsstandards formuliert und kognitive, sozial-affektive und emotional-motivationale Dimensionen bleiben weitgehend unberücksichtigt (Balz, 2008). Sportliche Leistung als Indikator für die Qualität des Unterrichts anzusehen greift also zu kurz und verkennt die Bedeutung des sozialen Lernens, dem zentralen Legitimationsargument des Sportunterrichts. Im Rahmen von Bewegungsstandards und der damit verbundenen pädagogisch-didaktischen Verknappung steht der Sportunterricht vor einem ernsthaften Legitimationsproblem, da ein Bezug zum Bildungsauftrag des Faches nicht oder nur kaum erkennbar ist. Balz (2008) fordert daher, dass bei der Implementierung von Standards im Sportunterricht über die Bewegungskompetenzen hinaus erzieherische Ansprüche inkludiert werden müssen, um weiterhin die ganzheitliche Entwicklungsförderung von Kindern und Jugendlichen anzustreben. Balz sieht in der Werkstattarbeit in Nordrhein-Westfalen, bei der den pädagogischen Perspektiven entsprechende Qualitätsstandards zugeordnet werden, einen sinnvollen und weiter zu verfolgenden Ansatz. Im Rahmen eines erziehenden Sportunterrichts, der sowohl Sacherschließung als auch Entwicklungsförderung berücksichtigt, werden die Qualitätsstandards inhaltlich durch Aufgabenbeispiele und Qualitätsindikatoren bzw. -kriterien konkretisiert.

\subsubsection{Aufgabenspektrum und Arbeitsalltag von Sportlehrkräften}

Sportlehrer im weiteren Sinne ist die Sammelbezeichnung für alle Lehrkräfte, die Sportunterricht erteilen. Nach dem Grundsatz der Gewerbefreiheit ist es jedermann gestattet, auch ohne Qualifikation und entsprechende Prüfungen, eine Sportlehrertätigkeit auszuüben. Ausgenommen ist die Unterrichtserteilung, die landesrechtlich in einem Lehrplan eingebaut worden ist, bzw. die durch Sportverbände oder andere Verbände geregelt ist. Im engeren Sinne werden solche Personen als Sportlehrer bezeichnet, die besondere Qualifikationen erworben und diese durch Prüfungen nachgewiesen haben (Laging, 2004a, S. 518f).

Neben Sportlehrern für den außerschulischen Bereich gibt es mit Sportlehrkräften mit staatlichem Abschluss für den schulischen Bereich, eine zweite Gruppe, die eine zentrale Rolle in dieser Arbeit einnimmt.

Die Aufgaben von Sportlehrern unterscheiden sich auf den ersten Blick nicht von den Aufgaben von Lehrkräften anderer Fächer. Auch Sportlehrer sollen lehren, erziehen, beraten, beurteilen und innovieren (siehe Kap. 2.2.2). Wie in den anderen Fachrich- 
tungen liegt auch im Sportunterricht die Hauptaufgabe im Lehren bzw. dem Unterrichten. Trotz oft nicht zufriedenstellender Wirkung geht es darum, Lernprozesse vorzubereiten, zu initiieren, zu unterstützen und zu korrigieren. Gleichzeitig müssen sich Sportunterrichtende, wie jede andere Lehrkraft auch, als Teil des Systems Schule auffassen und sich dementsprechend an deren Entwicklung beteiligen. Gerade im Sportunterricht ist eine stetige Reformbereitschaft aufgrund der bereits beschriebenen weitreichenden gesellschaftlichen Veränderungen notwendig (Zoglowek, 2008). Gerade in der aktuellen Entwicklung, die geprägt ist von einer verstärkten Hinwendung zur Organisation der Schule als Ganztagsangebot, kommt den Sportlehrkräften eine besondere Rolle zu. Im Rahmen der Profilbildung von Schulen nimmt der Sportunterricht bzw. der Schulsport eine zentrale Stellung ein, indem ein wichtiger Beitrag dazu geleistet wird, die Schule zu einem Lebensraum umzugestalten und mit außerschulischen Angeboten zu vernetzen. Neben dem Sportunterricht, der den Kern von Bewegung, Spiel und Sport in der Schule bildet, sind Sportlehrer bei der Umsetzung des Konzepts der Bewegten Schule, den Elementen des Bewegten Lernens, in sportbetonten AGAngeboten oder dem Pausensport gefordert (dvs, DSLV, DOSB \& FSW, 2008). Im Kollegium ist der Sportlehrer normalerweise derjenige, der als besonders beliebt bei den Schülern gilt und ein gutes Verhältnis zu diesen hat. Als Begründung hierfür wird oft das größere Interesse der Schüler an dem Fach gesehen und die Abwechslung, die das Fach vom Unterricht im Klassenraum verschafft (Lange, 1981). Das Bild des braungebrannten Sportlehrers, der sein Hobby zum Beruf gemacht hat, existiert immer noch und die Arbeit, die Sportlehrer im Unterricht zu verrichten haben, wird meist nicht als besonders anspruchsvoll eingeschätzt. Kinder und Jugendliche seien automatisch motiviert und der Unterricht verläuft somit eigentlich von alleine (Zoglowek, 2008). Tatsächlich jedoch ist das Verhältnis zu den Schülern nicht naturgegeben, sondern muss erst erarbeitet werden, da es sich beim Sportunterricht ebenso wie bei jedem anderen Schulfach um eine Veranstaltung mit Zwangscharakter handelt, in der Lehrer als Kontrolleure auftreten müssen. Die Zensurengebung, die Einhaltung von Vorschriften und Erlassen sowie sonstigen Dienstverpflichtungen widersprechen oft den Wünschen der Schüler, sodass es übertrieben wäre, den Sportunterricht als ein Wunschkonzert zu betrachten (Lange, 1981). Da unterrichtliches Handeln ebenso vom Organisationswissen abhängig ist, ist neben der didaktischen Kompetenz zusätzlich die Schulkompetenz von Bedeutung, um den Unterricht im gegebenen Rahmen überhaupt durchführen zu können (Zoglowek, 2008). In der praktischen Umsetzung stellen die derzeitigen Kerncurricula einen Orientierungsrahmen dar, der schulintern in Form eines schuleigenen Curriculums präzisiert werden soll. Dieses schulinterne Curriculum stellt die zentrale 
Vermittlungsinstanz zwischen den Vorgaben aus dem Kerncurriculum und der individuellen Unterrichtsplanung dar (Aschebrock \& Stibbe, 2008). Erfahrungen haben jedoch gezeigt, dass häufig Unklarheiten bestehen, auf welchem Weg man zu den festgelegten Zielen gelangen soll bzw. kann und Sportlehrkräfte fühlen sich teils alleine gelassen und überfordert (Kurz, 2008).

Die bereits angesprochene Ausgleichs- und Ventilfunktion des Sportunterrichts, die häufig als Vorteil des Sportunterrichts angegeben wird, entwickelt sich in der Unterrichtsrealität oft zu einer Besonderheit des Sportunterrichts mit hohem Konfliktpotenzial. Der Sportunterricht wird von Schülern oft dazu genutzt, Frustrationen und Unterdrückung, bedingt durch den Schulbetrieb, auszuleben und einfach mal Dampf abzulassen (Lange, 1981). So haben Disziplinprobleme und Konflikte weniger ihren Ursprung in der Planung bzw. Durchführung des Sportunterrichts, sondern vielmehr z.B. in Verständnisschwierigkeiten in Mathematik oder in einer schlechten Bewertung in einem anderen Fach. Auch weitere komplexe gesellschaftliche Bedingungen, die nicht im Handumdrehen aus der Welt zu schaffen sind, können dafür verantwortlich sein, dass Verhaltensweisen an den Tag gelegt werden, die nicht toleriert werden können. So liegt es in der Natur des Unterrichtsfaches Sport, dass hier Aggressivität deutlicher zum Ausdruck kommen kann, als dies in anderen Fächern der Fall ist, in denen sich Konflikte mehr oder weniger elegant unter den Teppich kehren lassen. Der Sportunterricht nimmt eine kompensatorische Rolle ein und stellt ein notwendiges Ventil im Unterrichtsalltag für Schüler dar (Storf, 1981). Wenn auch teilweise widrige Bedingungen vorliegen, sei es aufgrund räumlich-materieller Gegebenheiten, dem Verhalten der Schüler oder der fehlenden Wertschätzung Dritter für die Durchführung des Sportunterrichts, sollte es dennoch stets das Ziel sein, den Bedingungen entgegenzuwirken und zum Wohl der Kinder und Jugendlichen das enorme Potenzial des Sportunterrichts zu nutzen. Zielsetzung der Sportlehrkräfte ist es einerseits, das Selbstbewusstsein jedes Einzelnen zu stärken, gleichzeitig aber auch respektvolles Verhalten gegenüber den Mitschülern zu fördern, das auf den außersportlichen Bereich übertragen werden soll (dvs, DSLV, DOSB \& FSW et al, 2008).

Wenn man sich den Alltag des Sportunterrichts ansieht, scheinen Schüler hauptsächliches Interesse an Inhalten zu zeigen und auch bei den Lehrern bestimmte lange Zeit das „Was“ des Unterrichtens die Überlegungen und Planungen (Bräutigam, 2003). Neben der reinen Vermittlung von Inhalten, ist es auch die Aufgabe des Sportlehrers, bei den Schülern die Übernahme von Selbstverantwortung für die eigene Entwicklung und das Lernen anzuregen, um durch diese Art der Unterrichtsgestaltung die Persönlichkeitsentwicklung der Schüler zu unterstützen. Diese, eher als prospektiv bezeichnete 
Erziehungsaufgabe, soll die Schüler zu mehr Selbstständigkeit, zur Übernahme von Verantwortung in einem eigenverantwortlich organisierten und gestalteten Leben erziehen. Die Zielsetzung, die Schüler zu eigenständig denkenden und unabhängigen Persönlichkeiten zu formen und auf der anderen Seite die Gemeinschaft und die Zusammenarbeit zu fördern, stellt eine berufstypische Grundspannung dar. Die Schüler sollen einerseits ihre Kindheit ausleben, aber gleichzeitig auch auf die Erwachsenenwelt vorbereitet werden. Zu weiteren Spannungen führt der Anspruch, dass auf der einen Seite der Lehrplan möglichst komplett umgesetzt werden soll, gleichzeitig aber auch Schülerwünschen in gewissem Maße entsprochen werden soll (Zoglowek, 2008). Durch Situationen, die Schüler mit Bewegungsaufgaben konfrontieren und eigenständig erarbeitete Lösungswege erfordern, versucht man einen sinnvollen Umgang mit dem eigenen Körper anzuregen, Bewegungsbedeutungen sollen aufgespürt und soziales Handeln initiiert werden. Zudem sollten Möglichkeiten des Reflektierens von gemachten Bewegungs- und Sozialerfahrungen in den Unterricht eingebaut werden (Köppe, 1999). Der Person bzw. der Persönlichkeit des Lehrers kommt im Sportunterricht eine besondere Bedeutung zu. Gegenüber den Schülern befindet der Sportlehrer sich in einer Vorbildfunktion aufgrund der er sein Handeln und Wirken kontrollieren muss, da Schüler nicht nur vom sondern vor allem auch am Lehrer lernen (Bräutigam, 2003). Auf der Suche nach Orientierungspunkten verschmilzt der Sportlehrer in der Wahrnehmung der Schüler mit den Inhalten und den Methoden zu einem Gesamtbild. Nach Ansicht der Schüler sollte ein Sportlehrer sowohl sportlich-trainiert als auch kameradschaftlich und gerecht sowie schwungvoll, freundlich und geduldig sein. Weiterhin wünschen sich Schüler, dass ihr Lehrer sie für den Sport begeistern kann, auf ihre Wünsche eingeht und Verständnis auch für Schwächere oder Ängstliche zeigt (Zoglowek, 2008). Im Sportunterricht findet wie in keinem anderen Fach Modelllernen am Vorbild Lehrer statt (Heine, 1981). Sportlehrer müssen in ihrem Unterricht Spaß an der Bewegung vermitteln, dabei die Qualitäten eines Entertainers aufweisen und selbst aktiv und mit Spaß bei der Sache sein. Bandura (1979) wies nach, dass vor allem bei Kindern das Verhalten stark durch das Modelllernen beeinflusst wird, indem Handlungen, Einstellungen oder Verhaltensweisen von Modellen imitiert werden. Ob eine Person als Modell angenommen wird, hängt davon ab, ob sie geachtet wird, dem Beobachter ähnlich ist, fachliche Kompetenz oder soziales Prestige besitzt. Lehrer, die im Unterricht nicht oder nur selten zeigen, dass sie mit ihrem Körper umzugehen wissen und meist passiv sind, werden von Schülern nicht anerkannt. Bei dem als Wahrnehmungslernen bezeichneten Vorgang handelt es sich nicht um ein bewusstes Nachmachen, sondern um einen unbewussten Prozess, der umso ausgeprägter ist, desto positiver der sozial- 
emotionale Bezug zur Modellperson ist, sodass beliebte und als emotional warm erlebte Sportlehrer besonders zur Übernahme von Verhaltenstendenzen, Normen und Einstellungen anregen (Zimmer \& Volkamer, 1981). Vieles ist von der Kommunikationsund Interaktionsfähigkeit des Lehrers abhängig, anhand der sich entscheidet, ob sich Kinder auf den Unterricht einlassen oder nicht. Als Berater ist der Lehrer manchmal die einzige Ansprechperson, den die Schüler zu Rate ziehen, wenn es darum geht, die Planung des eigenen Lebens anzugehen (Zoglowek, 2008). Unerlässlich in diesem Zusammenhang ist es, den Schülern glaubwürdig zu vermitteln, dass man sich auf der gleichen Seite befindet und gemeinsam versucht, bestimmte Zielsetzungen zu erreichen (Lange, 1981). Eine ausgeprägte Selbstkompetenz stellt dabei den Schlüssel für das authentische Denken und Handeln sowie die Wahrung eines individuellen Stils gemäß der eigenen Persönlichkeit dar (Zoglowek, 2008). So ist es nicht verwunderlich, dass in Befragungen von Schülern zwar mit dem Tyrann, dem Frühpensionär, dem lockeren Typ, dem Handwerker, dem harten Hund und dem Enthusiast verschiedene Lehrertypen voneinander unterschieden werden können, eine eindeutige Aussage, welcher Typ ausschließlich als positiv bzw. ausschließlich negativ empfunden wird, nicht getroffen werden kann. Die Aussage so schlecht ist er auch wieder nicht zeigt, dass es nicht ausschließlich auf die Art und Weise des Unterrichtens ankommt, sondern immer auch Authentizität eine Rolle spielt. Deutlicher fällt hingegen das Urteil der Schüler bezüglich der Sündenfälle der Sportlehrkräfte auf. Als kapitale Fehler sehen Schüler es an, wenn langweiliger Unterricht durchgeführt wird, indem man immer dasselbe auf die dieselbe Art und Weise vermittelt und wenig Abwechslung geboten wird oder Sportlehrkräfte sich einfach über die Schülerwünsche und Anregungen hinwegsetzen. Ebenso bemängeln Schüler ungenügende Fachkompetenz und eine schlechte Organisation des Unterrichts. Wenig Engagement und ein unangemessener durch häufiges Meckern gekennzeichneter Umgang mit Schülern wird ebenfalls als störend wahrgenommen (Bräutigam, 2004). Neben der Selbstkompetenz und der Sachkompetenz, die sich oft in der Auswahl und Aufbereitung von Unterrichtsinhalten niederschlägt, ist die didaktische Kompetenz vor allem dafür verantwortlich, ob sich die Kinder und Jugendlichen auf einen Unterrichtsgegenstand einlassen oder nicht. Die zwischenmenschliche Komponente ist demnach genauso wichtig wie die fachliche und ebnet oft erst Möglichkeiten für emotionale, kommunikative und soziale Entwicklungschancen.

Da der Ablauf des Sportunterrichts mehr als in anderen Fächern durch Unplanbarkeit geprägt ist, muss sich die Lehrkraft stetig auf sich rasch ändernde Bedingungen einstellen und Einflüsse von außen auf den Unterricht verarbeiten. Während für manche Lehrkräfte die paradoxen und konfliktbehafteten Situationen, die eine unmittelbare 
Reaktion erfordern, den vitalen Kern des Berufs darstellen, wirkt diese Eigenart auf andere Sportlehrer belastend (Zoglowek, 2008). Auf einer theoretischen Aufbereitung der Themenbereiche Stress, Belastung und Beanspruchung aufbauend, wird in Kapitel 3 auf die Belastungssituation von Lehrkräften im Allgemeinen und der Sportlehrkräfte im Speziellen eingegangen. 


\section{Stress, Belastung, Beanspruchung}

In diesem Kapitel erfolgt eine Auseinandersetzung mit den Begriffen Stress (siehe Kap. 3.1), Belastung und Beanspruchung (siehe Kap. 3.2), bevor im vierten Kapitel Bewältigungs- bzw. Copingstrategien dargestellt werden. In Anknüpfung an das Kapitel 2 erfolgt eine Analyse der speziellen Belastungssituation von Lehrkräften. Besonderes Augenmerk wird hierbei auf die dem Sportunterricht genuin innewohnenden Belastungen gelegt (siehe Kap. 3.3). Neben den Belastungen durch die Arbeitsaufgaben und den schulorganisatorischen und arbeitshygienischen Bedingungen, werden zudem Belastungen aufgrund sozialer und gesellschaftlich-kultureller Einflüsse thematisiert, deren Genese und Entwicklung anhand von Kapitel 2 ableitbar sind.

\subsection{Stress}

Der Begriff Stress wird oft als Resultat von Belastung und Beanspruchung gesehen, aber auch als deren Synonym verwendet. Wenn man im Alltag von Stress spricht, wird dies meist mit einem unangenehmen Erlebniszustand verbunden. Stress vermag aber auch, die Leistungsfähigkeit zu erhöhen. Wir sprechen von Arbeits-, Freizeit-, Familien-, Verkehrs-, Behörden- oder Prüfungsstress und es gibt kaum einen Lebensbereich, der nicht mit dem Begriff Stress in Zusammenhang gebracht werden kann.

Im Verständnis und der Nutzung der Begrifflichkeit besteht keineswegs Eindeutigkeit, denn einerseits bezeichnet man mit „ich bin gestresst" einen aktuellen Zustand, der auf den Reaktionen des Selbst basiert, andererseits wird durch die Bezeichnung "ich habe Stress" die umgebende Umwelt beschrieben. Das heißt, Stress wird sowohl genutzt für die Reaktion des Individuums als auch für den auslösenden Stimulus (Reicherts, 1988). In extremen Situationen, die von der Umwelt an Personen gestellt werden, wie z.B. Krieg, Naturkatastrophen oder Folter, entsteht für nahezu jeden Stress. Extremereignisse sind zwar nicht völlig ausgeschlossen, bestimmen aber nicht den Alltag. Neben der Charakteristik des Stressereignisses selbst, spielt auch die Wahrnehmung desselbigen eine bedeutende Rolle und bedingt bei Individuen eine hohe Variationsbreite an Reaktionen, sodass eine innere und eine äußere Komponente des Stresses unterschieden werden kann. Bezüglich der Intensität eines als stressig erlebten Ereignisses kann man zumindest eine Regelmäßigkeit ableiten: Je mehr sich die Bedingungen von den genannten Extremerlebnissen entfernen, desto mehr zeigen sich Unterschiede im Erleben und Reagieren auf diese Umweltanforderungen (Lazarus \& Folkman, 1984). In Situationen, in denen man sich "gestresst fühlt" oder "Stress hat" wird die individuelle Homöostase gestört, was sich meist in einem angespannten Verhältnis von Person und Umwelt manifestiert und als Überforderung oder Überlastung 
wahrgenommen wird. Genau hier setzen verschiedene Anti-Stress-Programme an, die möglichst schnell den Ausgangszustand wiederherstellen helfen, bzw. die Störungen präventiv verhindern. Andauernder Stress kann zu langfristigen Schädigungen und Beeinträchtigungen der psychischen und physischen Gesundheit führen kann (siehe Kap. 4). Bevor die Belastung und Beanspruchungsreaktion bzw. -folge im Bezug auf den Lehrerberuf definiert wird, soll zunächst der Begriff Stress genauer analysiert werden.

Ähnlich den Verwirrungen im alltäglichen Sprachgebrauch, stellt sich auch die Situation in der Wissenschaft dar. Nachdem der Begriff Stress im Jahre 1917 durch Cannon als eine mehr oder weniger reflexhafte Reaktion in überraschenden, neuartigen oder gefährlichen Situationen in die psychologische Fachliteratur eingebracht wurde und seit den 40er Jahren des vergangenen Jahrhunderts auch die Begriffe Belastung und Beanspruchung als Fachbegriffe der Psychologie übernommen wurden, entstanden unzählige Definitionen und Modellvorstellungen. Einige Definitionen deuten in ähnliche Richtungen, andere widersprechen sich vollends. Einige Wissenschaftler bezeichneten den Stressbegriff aufgrund der Unschärfe des Gegenstandsbereiches sogar als unnütz und obsolet (Kramis-Aebischer, 1996). Lazarus und Folkman (1984) sehen in Stress jedoch eine übergreifende und unverzichtbare Konzeption zum Verständnis der Konfrontation mit, und der sich anschließenden Bewältigung von Problemen. Der Anspruch, eine möglichst universalistische Begriffsbestimmung zu liefern, stellt sich in vielen Definitionsversuchen als problematisch dar, denn eine enorme Fülle an Aspekten wird mit dem Stressbegriff in Verbindung gebracht. Für die vorliegende Arbeit soll eine modellhafte Darstellung der Belastungs-Beanspruchungs-Reaktion erfolgen (siehe Kap. 3.2). Bezogen auf den von Cannon eingeführten und von Selye popularisierten Stressbegriff, bestehen heutzutage im Verständnis des Begriffes große Diskrepanzen. Die Bezeichnung Stress hat sich zu einem Modebegriff entwickelt, denn wo vorher von Angst, Konflikten, Frustration, Verstörtheit oder Anomien die Rede war, wird nun Stress als begriffliches Sammelbecken genutzt, was zu einer Vieldeutigkeit des Begriffes führt. Aufgrund der unzähligen Beschreibungen, Erläuterungen und Ansichten bezüglich des Phänomens Stress, erfolgt die Darstellung systematisiert in reaktionsbezogene, situationsbezogene und interaktionistische Konzepte, in denen sich vor allem unterschiedliche theoretische Grundlegungen in Bezug auf das Person-Umwelt-Verhältnis niederschlagen (Herzog, 2007).

\subsubsection{Reaktionsbezogene Stresskonzeption}

"Stress ist der Zustand, der sich als spezifisches Syndrom manifestiert, das aus allen unspezifischen induzierten Veränderungen innerhalb eines biologischen Systems besteht" (Selye, 1976; zitiert nach Laux, 1983, S. 457). Stress ist also ein Syndrom physio- 
logischer Veränderung, als Reaktion auf eine Störung der Homöostase, die unabhängig von der Art und der Qualität des jeweiligen Stressors auftritt. Stress ist eine charakteristische Erscheinungsform, die auf unterschiedliche Ursachen zurückgeführt werden kann und in gewissem Maß mit jeder menschlichen Aktivität verbunden ist. Lediglich der Todeszustand ist eine Situation, in der kein Stress auftritt. Zeitlich verläuft die Stressreaktion in drei aufeinander folgende Phasen, die als Alarmreaktion, Widerstandsphase und Erschöpfungsphase bezeichnet werden. Während in der Alarmreaktion verschiedene physiologische Veränderungen, vor allem im vegetativen Bereich, nach einer initialen Schockphase zu einem Ankurbeln von Verteidigungsmechanismen in der Gegenschockphase führen, ist die Widerstandsphase durch eine erhöhte Widerstandsfähigkeit gegenüber dem jeweiligen Stressor gekennzeichnet. Ergänzend muss hinzugefügt werden, dass die erhöhte Widerstandsfähigkeit gegenüber dem spezifischen Stressor durch eine Reduktion der Widerstandsfähigkeit gegenüber anderen Stressoren erworben wird. In der finalen Erschöpfungsphase ist der Organismus nicht mehr in der Lage, die zur Adaption notwendigen Mechanismen aufrechtzuerhalten und verfällt in einen Zustand irreversibler Alarmreaktionen (Laux, 1983). Besonderer Bedeutung wird in diesem Konzept dem spezifischen Reaktionsmuster, d.h. der interindividuellen Variabilität in der Reaktion zugeschrieben (Herzog, 2007). In dieser endokrinologischen Konzeption von Stress können neben physiologischen auch psychologische Belastungen relevante Reize darstellen.

Von der Annahme ausgehend, dass Stress die unspezifische Reaktion auf jegliche Art von Anforderung sei, können auch freudige Ereignisse wie z.B. ein Lottogewinn oder ein Heiratsantrag psychophysiologische Veränderungen bewirken, die man als Stress bezeichnet (Jerusalem, 1990). Unterschieden wird daher in diesem Konzept zwischen Eustress, dem nützlichen Stress, und Distress, dem Stress, dessen Auswirkungen Selye langfristig als schädlich ansieht. Grundlage des endokrinologischen Konzepts stellen Ergebnisse von Tierversuchen dar, die auf den Humanbereich übertragen wurden (Laux, 1983). Nach Selye besitzen Menschen ein unterschiedliches Maß an Anpassungsenergie, die bei jeder biologischen Aktivität etwas verbraucht wird. Verbrauchte Energie kann wieder aufgebaut werden, bei rücksichtslosem Umgang jedoch kann es zu gesundheitlichen und psychischen Problemen kommen (Barth, 1997). An den reaktionsorientierten Konzeptionen wird jedoch kritisiert, dass eigentlich jede menschliche Handlung und Regung zu einem Verlassen der Homöostase führt und die Definition eines Steady-State-Zustands, dessen Verlassen Stress bedeuten würde kaum möglich ist (Lazarus \& Folkman, 1984). Weiterhin wird an Selyes Stresskonzeption die NichtBerücksichtigung moderierender psychischer Bewertungsvorgänge kritisiert (Jerusa- 
lem, 1990). Selye selbst betonte im Jahr 1980, dass nicht der von außen einwirkende Reiz, sondern emotionale Erregung als zentraler Mediator in der Stressreaktion auftritt und für die Unspezifität des Stress-Syndroms verantwortlich ist (Lazarus \& Folkman, 1984; Laux, 1983). In neueren reaktionsbezogenen Stresskonzeptionen stellt die Interaktion der Person mit der Umwelt, d.h. kognitive Bewertungsvorgänge, ein zusätzliches Element dar, wodurch Unterschiede zwischen Personen im Stresserleben erklärt werden können (Jerusalem, 1990).

\subsubsection{Situationsbezogene Stresskonzeptionen}

Während in der reaktionsbezogenen Stresskonzeption die Person im Zentrum der Diagnose von Stress steht, werden in situations- bzw. reizbezogenen Konzepten den Bedingungen der Umgebung mit ihren unterschiedlichen Intensitäten und Qualitäten verstärkte Aufmerksamkeit geschenkt (Jerusalem, 1990). Auch die Unterscheidung zwischen einer dauerhaften oder einer akuten Anforderung ist relevant in situationsbezogenen Konzepten (Lazarus \& Folkman, 1984). Die Bedingungen, die potenzielle Stresserzeuger, bezeichnet man als Stressoren, die in vier Kategorien einteilbar sind: Neben akuten zeitlich begrenzten Stressoren und Stressorensequenzen unterscheiden die beiden Autoren zusätzlich zwischen chronischen Stressoren, die mit Unterbrechungen auftreten und solchen die ununterbrochen, d.h. tatsächlich chronischen Verlauf zeigen (Herzog, 2007, Lazarus \& Folkman, 1984). Stressrelevante Situationen können durch Anwesenheit, als auch durch Abwesenheit von Reizen entstehen, wie z.B. durch Monotonie, Isolation oder Reizdeprivation (Reicherts, 1988).

Unter dem Oberbegriff des Situationsbezugs, wird je nach der erforderlichen Readaptionszeit und dem Ausmaß der negativen Valenz, zwischen kritischen Lebensereignissen (Life-Events) und den alltäglichen Ärgernissen und Sorgen (Daily-Hassles) unterschieden. Die arbeitswissenschaftliche Stresskonzeption stellt den dritten Ansatz der situationsbezogenen Stresskonzepte dar (Kramis-Aebischer, 1996).

In der Life-Event-Forschung beschäftigt man sich mit kritischen Lebensereignissen, die man auch retrospektiv als Einschnitt bzw. Übergang im Lebenslauf identifizieren kann und die zu grundlegenden Veränderungen bzw. Umorientierungen von Menschen führen können (Herzog, 2007). Heirat, Tod des Ehegatten oder Schwierigkeiten mit Vorgesetzten sind Beispiele für solch einschneidenden Erlebnisse, die Abwehrstrategien in Gang setzen und teils auch in Beziehung zu körperlichen Erkrankungen und psychischen Störungen stehen (Laux, 1983, Cooper \& Dewe, 2007). In der Anfangszeit sah man das Individuum als passives Opfer, während in neueren Ansätzen kritische Ereignisse als Produkt der Lebensführung angesehen und vermehrt subjektive Einschät- 
zungsprozesse und Bewältigungskompetenzen mit einbezogen werden (Jerusalem, 1990).

In Daily-Hassles-Forschungsansätzen beschäftigt man sich mit den vielen alltäglichen kleinen, teils banalen Ärgernissen, Widrigkeiten und Irritationen des psychischen und physischen Wohlbefindens. Neben den negativen Alltäglichkeiten werden mit den uplifts auch die kleinen Freuden einbezogen, die kompensatorische Funktion aufweisen können. In diesem Ansatz wird weniger den großen Lebensereignissen als vielmehr den alltäglichen Problemen und Missgeschicken eine Bedeutung beigemessen (Lazarus \& Folkman, 1984). In diesem Ansatz fasst man daily hassles als subjektive Konstrukte auf, die aufgrund ihrer Bedeutung für die Person in irgendeiner Form Präsenz in der individuellen Erinnerung aufweisen (Cooper \& Dewe, 2007).

In arbeitswissenschaftlichen Konzeptionen untersucht man das Auftreten und die Wirkung von objektiven Faktoren wie z.B. Lärm und Zeitdruck, die als spezifisch für das berufliche Profil gelten. In der Erforschung von organisationsspezifischem Stress werden arbeitsspezifische Stressoren in Verbindung mit Persönlichkeitsmerkmalen in ihrer Manifestation als Arbeitszufriedenheit oder physiologischer Erregung gemessen. Durch Belastungsanalysen und den daraus abgeleiteten Konsequenzen wird versucht, arbeitsbedingte Erkrankungen beispielsweise durch die Gestaltung des Arbeitsplatzes zu verhindern (Jerusalem, 1990).

In den situationsbezogenen Konzeptionen wurde, genau wie zuvor bei den reaktionsbezogenen Ansätzen, in jüngerer Vergangenheit die Bedeutsamkeit der Bewertungsprozesse des Individuums erkannt und mit einbezogen. Stress ist auf der Grundlage der Reizcharakteristik nicht umfassend erklärbar und kann nur durch den Wechselwirkungsprozess zwischen Person und Umwelt nachvollzogen werden (Kramis-Aebischer, 1996).

\subsubsection{Stress als relationaler Begriff - Transaktionales Stresskonzept}

Wie bereits in den beiden vorangehenden Abschnitten deutlich wurde, versteht man unter Stress in neueren Konzeptionen ein Konstrukt, das sich durch die Wechselwirkungen von Person und Situation konstituiert. In einem Abgleich werden die Situationsanforderungen mit den personenspezifischen Handlungsmöglichkeiten konfrontiert und dann als Stress wahrgenommen, wenn die Anforderungen gleich stark oder stärker gegenüber der eigenen Kompetenz sind. In diesem Ansatz ist für die Entstehung von Stress von großer Bedeutung, wie die eigene Kompetenz eingeschätzt wird (Kramis-Aebischer, 1996). Da die zeitliche Dynamik jedoch ebenfalls einen konstituierenden Faktor darstellt, wurde diese im „Transaktionalen Stresskonzept" von Lazarus zu- 
sätzlich einbezogen, was die kognitive Wende in der Stressforschung einleitete (BöhmKasper, 2004).

Das transaktionale Stresskonzept geht im Wesentlichen auf Lazarus und seine Mitarbeiter zurück. Stress wird in dieser Konzeption nicht als reine Input-Output-Reaktion angesehen, sondern stellt einen wechselseitigen Prozess dar, bei dem eine sich verändernde Situation mit einem denkenden, fühlenden und handelnden Individuum in Beziehung steht (Lazarus \& Folkman, 1984; Herzog, 2007). Der Begriff Transaktion wird der Verwendung von Interaktion vorgezogen, da nicht nur ein einseitiges kausales Wechselgefüge besteht, sondern sowohl die Situation auf die Person einwirkt, als auch die Person bestimmenden Einfluss auf die Situation ausübt. Entscheidend im Konzept von Lazarus sind Bewertungsprozesse und Einschätzungen sowie die verfügbaren Bewältigungsmöglichkeiten. Menschen, ebenso wie Tiere, bewerten ständig Situationen bezüglich des Einflusses auf ihr eigenes Wohlergehen (Lazarus \& Folkman, 1987). Diese Besonderheit der Stresskonzeption spiegelt sich auch in der von Lazarus und Folkman (1984, S. 19) formulierten Definition von Stress wider: „Psychological stress is a particular relationship between the person and the environment that is appraised by the person as taxing or exceeding his or her resources and endangering his or her wellbeing". Das in der Definition angesprochene Person-Umwelt-Verhältnis wird moderiert durch kognitive Bewertungsvorgänge und Coping, also den Bewältigungsprozessen, die von der Person initiiert werden, um das Wohlbefinden aufrechtzuerhalten bzw. wiederherzustellen. Stress entsteht dann, wenn das Ereignis „die adaptiven Mittel einer Person übersteigt“ (van Dick \& Stegmann, 2007, S. 37). D.h., wenn eine Diskrepanz zwischen der perzipierten subjektiven Stressexposition und den wahrgenommenen Response-Kapazität besteht (Reicherts, 1988). Neben den Eckpfeilern Bewertung und Bewältigung bezieht Lazarus zudem Gefühle und Emotionen als Folge- und Begleiterscheinungen der subjektiven Bewertungsvorgänge mit ein und spricht von einer kognitiv-relationalen Theorie der Emotion und Bewältigung. (Jerusalem, 1990).

Lazarus differenziert innerhalb des evaluativen Prozesses in eine primäre und eine sekundäre Bewertung, wobei sich diese Einteilung in mehrfacher Hinsicht als unglücklich erwiesen hat. Die Bezeichnung primär und sekundär im Bewertungsprozess soll weder auf eine festgelegte Reihenfolge hindeuten, noch soll hierdurch eine größere Bedeutsamkeit des einen gegenüber dem anderen ausgedrückt werden. Ein weiterer Schwachpunkt ist die Inhaltslosigkeit der Begriffe bezüglich der Charakteristik des jeweiligen Bewertungsprozesses (Lazarus \& Folkman, 1984). Ein umgekehrter oder simultaner Ablauf ist ebenso möglich wie ein, der wörtlichen Bezeichnung ${ }^{1}$ entspre-

${ }^{1}$ primär = zuerst, sekundär $=$ an zweiter Stelle/danach 
chender, Verlauf. Im Folgenden soll aufgezeigt werden, wie durch Bewertungsprozesse dieselben Stimuli von Personen in unterschiedlicher Art und Weise empfunden werden und somit auch die Bewältigung individuell erfolgt (siehe Kap. 4). Ob eine Situation nun als stressig empfunden wird, wird durch das Verhältnis der situativen Stressrelevanz und den zur Verfügung stehenden individuellen Bewältigungsmöglichkeiten im Sinne einer Person-Umwelt-Transaktion bestimmt, die in der Theorie von Lazarus zentrale Bedeutung hat (Herzog, 2007).

\section{Primäre Bewertung (primary appraisals)}

Im ersten Schritt wird zunächst die grundsätzliche Bedeutsamkeit der Situation eingeschätzt. Mögliche Ergebnisse dieses Prozesses sind, dass die Situation entweder als irrelevant, angenehm-positiv oder als stressrelevant bewertet wird (Herzog, 2007). Auf irrelevante Situationen wird normalerweise mit Gleichgültigkeit reagiert. Bei als angenehm-positiv bewerteten Situationen wird das Wohlbefinden gewahrt oder gar gesteigert, die eigenen Kompetenzen werden als den Situationsanforderungen eindeutig überlegen angesehen (Jerusalem, 1990). Assoziierte Emotionen sind Freude, Liebe, Fröhlichkeit und Heiterkeit. Jedoch gibt es auch das Phänomen, dass momentane Freude letztendlich mit antizipiertem späterem Unheil ausgeglichen wird, sodass selbst diese Situationen als bedrohlich wahrgenommen werden. Sowohl bei Irrelevanz als auch bei angenehm-positiven Situationen ist in der Regel keine Bewältigungsmaßnahme notwendig. Wird eine Situation als stressrelevant bewertet, wird die Situation in einem kognitiven Prozess entweder als Herausforderung (challenge), Bedrohung (threat), Verlust (loss) oder als Schädigung (harm) eingeschätzt (Lazarus \& Folkman, 1984). Stressrelevanz bedeutet, dass wichtige Aspekte wie Gesundheit, beruflicher Erfolg oder soziale Anerkennung durch die Situation potenziell beeinflusst werden, und das Kräfteverhältnis zwischen Situation und Person unklar oder gar ungünstig erscheint.

Bei der Einschätzung als Herausforderung (challenge) werden Anstrengungen aufgenommen, um einen potenziellen Gewinn aus dem Ereignis zu erzielen, möglicherweise kann durch die Bewältigung sogar etwas hinzugelernt werden. Die Anforderungen, die durch die Situation gestellt werden scheinen angemessen, d.h., sie bewegen sich in einem Bereich, der in etwa den eigenen Kompetenzen entspricht. In der Bewertung als Herausforderung ist jedoch trotz der positiven Erlebnisqualität stets auch eine gewisse Wahrscheinlichkeit einer Bedrohung bzw. des Scheiterns enthalten (Lazarus \& Folkman, 1987). Eifer, Erregung, Interesse, Heiterkeit und Zuversicht dominieren das subjektive Erleben und es liegt die subjektive Erwartung vor, wenn man sich genügend anstrengt, die Situation durch den Einsatz der eigenen Fähigkeiten erfolgreich meistern 
zu können. Den positiven Bewertungskognitionen wurden in der Weiterentwicklung des Ursprungsmodells durch die Einführung der Bewertung als benefit zusätzliche Bedeutung beigemessen (Jerusalem, 1990).

Die Bewertung als Bedrohung (threat) ist mit der zuvor dargestellten Herausforderung verwandt und tritt oft simultan zu dieser auf. Bedrohungsgefühle beziehen sich ebenfalls auf zukünftige Begebenheiten, stellen jedoch weniger Anforderungen, als vielmehr Überforderungen für das Individuum dar (Lazarus \& Folkman, 1984). Wenn das gestörte Person-Umwelt-Verhältnis als kontrollierbar eingestuft wird, ist die Wahrscheinlichkeit erhöht, dass der Bewertungsprozess im Verspüren einer Herausforderung mündet. Erwartet man ein Scheitern oder einen drohenden Misserfolg, stellen Angst, Besorgnis und Verdruss, die mit der Bewertung als Bedrohung verbundenen Emotionen dar (Jerusalem, 1990). Auch die Vereitelung einer Bedürfnisbefriedigung kann eine Bewertung als Bedrohung auslösen (Laux, 1983). Antizipativ erwartet man eine Schädigung oder einen Verlust, dem man durch präventive Bewältigungsversuche entgegenwirken kann, um die Konfrontation zu verhindern oder den Verlauf abzuschwächen. Die Anforderungen der bedrohlichen Situation scheinen deutlich über den eigenen Möglichkeiten zu liegen, sodass Furcht vor einem Misserfolg mit geringer Hoffnung auf Erfolg einhergeht (Jerusalem, 1990).

Während die Einstufung als Herausforderung oder Bedrohung sich antizipativ auf zukünftige Situationen beziehen, sind Verlust (loss) und Schaden (harm) auf die Gegenwart bzw. die Vergangenheit bezogen (Lazarus \& Folkman, 1984). Die negativen Beeinträchtigungen sind in diesem Fall nicht mehr durch Bewältigungsmaßnahmen abzuwenden, lediglich Linderung bzw. Wiederherstellung des Wohlbefindens lassen sich durch angemessene Bewältigung erzielen. Schaden stellt eine Sammelbezeichnung sowohl für physische als auch für psychische Schädigungen wie Beeinträchtigungen des Selbstwertgefühls oder Verhinderung von Bedürfnisbefriedigung dar, während Verlust sowohl materielle als auch emotionale Aspekte beinhaltet. Assoziierte Emotionen sind Trauer, Schmerz oder Resignation (Jerusalem, 1990). Aus Schaden und Verlust können auch Bedrohungsgefühle für zukünftige Ereignisse resultieren, wenn durch die schlechte gegenwärtige Situation auch negative Auswirkungen für die Zukunft befürchtet werden. So kann z.B. der Verlust eines Angehörigen gegenwärtig zu der Bewertung Schaden und Verlust führen und gleichzeitig als Bedrohung interpretiert werden, wenn man davon ausgeht, zukünftige Situationen im Leben ohne den jeweiligen Menschen nicht meistern zu können (Laux, 1983).

Die im Laufe der primären Bewertung ablaufenden Einschätzungsprozesse in Herausforderung, Bedrohung, Schaden und Verlust sind jedoch nicht als Alles-oder-Nichts- 
Prozesse zu sehen, sondern sie sind gekennzeichnet durch Mehrdeutigkeit und Ambiguität, wobei durchaus auch Mischformen auftreten können (Herzog, 2007). Weiterhin ist es möglich, die verschiedenen Kategorien der primären Bewertungsvorgänge z.B. in somatische oder selbstwertbezogene Prozesse etc. zu untergliedern (Laux, 1983). Es besteht aufgrund der unterschiedlichen Qualitäten der Bewertungen eine Ähnlichkeit zu den von Selye eingeführten Begriffen Eustress und Distress, da es Situationen gibt, die sowohl als positiv-konstruktiv oder als negativ-destruktiv eingeschätzt werden können (Jerusalem, 1990).

\section{Sekundäre Bewertung (secondary appraisals)}

Nachdem eine stressrelevante Situation als Herausforderung, Schaden, Verlust oder Bedrohung eingeschätzt wurde, geht das Transaktionale Stressmodell von Lazarus und Folkman (siehe Abb. 3) davon aus, dass in einem sekundären Bewertungsvorgang ein Abgleich mit den zur Verfügung stehenden Bewältigungsmöglichkeiten basierend auf den eigenen Fähigkeiten und Ressourcen vorgenommen wird.

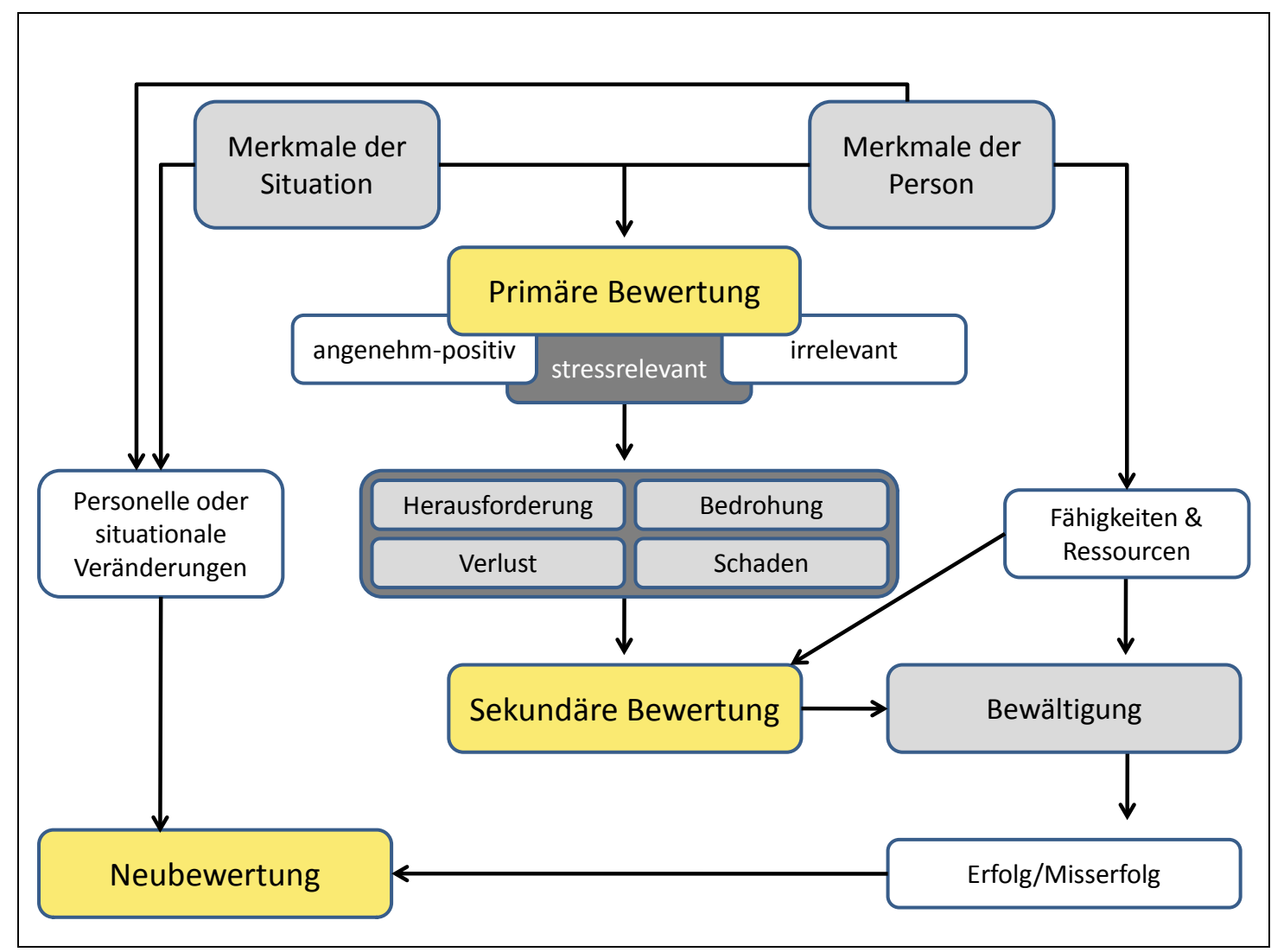

Abb. 3: Vereinfachte grafische Darstellung des transaktionalen Stressmodells nach Lazarus \& Folkman, 1984.

Es erfolgt kein simples Auskundschaften der zur Verfügung stehenden Handlungsalternativen, sondern es wird anhand der eigenen Ressourcen abgewogen, inwieweit ver- 
schiedene Bewältigungsmöglichkeiten erfolgsversprechend angewendet werden können. In dem sekundären Bewertungsprozess werden „die verfügbaren Bewältigungsmöglichkeiten, deren verschiedene Erfolgswahrscheinlichkeiten und die Wahrscheinlichkeit, dass man selbst bestimmte Strategien wirksam einsetzen kann", anhand körperlicher, materieller, sozialer und psychologischer Ressourcen und Fähigkeiten kalkuliert (Jerusalem, 1990, S. 11). Neben der Breite des Handlungsrepertoires, das durch Kenntnisse und bereits gemachten Erfahrungen bestimmt wird, ist die Motivation ein weiterer wichtiger Moderator dieses Prozesses und prädiktiv an der Wahl einer Bewältigungsstrategie beteiligt (Herzog, 2007). Abgesehen von den zur Verfügung stehenden Möglichkeiten und der Motivation sind zudem verschiedene Persönlichkeitseigenschaften sowie interne und externe Ressourcen an den bewältigungsbezogenen Einschätzungsprozessen beteiligt. Die zuvor im Zitat beschriebenen Erwartungen entsprechen den von Bandura unterschiedenen Konstrukten der Kompetenz- bzw. Wirksamkeitserwartung (efficacy expectation) sowie der Konsequenz- bzw. Ergebniserwartung (outcome expectancy), die die Erfolgswahrscheinlichkeit von Bewältigungsmöglichkeiten beschreiben (Bandura 1977). Unter Wirksamkeitserwartung oder Selbstwirksamkeit versteht man die Überzeugung einer Person, Handlungen, die erfolgsversprechend sind, auch plangemäß ausführen zu können (Jerusalem, 1990). Beispielhaft ist das Wissen über den angemessenen und erfolgreichen Umgang mit Disziplinkonflikten (Konsequenzerwartung) im Unterricht die notwendige Grundlage für eine effektive und sinnvolle Bewältigung. Ohne das Zutrauen in die eigene Lehr- und Sozialkompetenz (Kompetenzerwartung) wird jedoch die Lehrkraft entweder eine Strategie mit subjektiv höherer Erfolgswahrscheinlichkeit wählen oder eine Handlung wird komplett vermieden. Es reicht also nicht aus zu wissen, mit welcher Handlung man zu dem gewünschten Ergebnis gelangt (Ergebniserwartung), sondern man muss es sich auch selbst zutrauen, den Weg erfolgreich und effektiv meistern zu können (Kompetenzerwartung). Weitere einflussausübende Konstrukte stellen Selbstbewusstsein, Selbstachtung, Überzeugungen und Kontrollüberzeugungen dar (Lazarus \& Folkman, 1987). Von sich überzeugte Menschen, die sich selbst eine hohe Kompetenz zuschreiben, werden zudem Situationen eher als herausfordernd auffassen als durch Unsicherheit geprägte Personen, die sich selbst weniger zutrauen und schneller dazu neigen, Situationen als bedrohlich einzuschätzen (Jerusalem, 1990).

In der vereinfachten grafischen Darstellung des transaktionalen Stressmodells von Lazarus und Folkman (siehe Abb. 3) sind zugunsten der Übersichtlichkeit interdependente Beziehungen zwischen den Einschätzungsprozessen, der Bewältigung sowie den Fähigkeiten und Ressourcen nicht dargestellt. Ebenfalls sei darauf hingewiesen, dass 
die im Modell scheinbar vorliegenden klar strukturierten zeitlichen Abfolgen von primärer und sekundärer Bewertung sowie dem Bewältigungshandeln konzeptionell eine andere Struktur aufweisen können. Zwischen der primären und sekundären Bewertung besteht ebenfalls eine variable interdependente Beziehung, denn neben dem aufeinander aufbauenden Verhältnis von primärer und sekundärer Bewertung kann ebenso das Wissen über eventuelle Hilflosigkeit oder eingeschränkte Handlungsfähigkeit (sekundäre Bewertung) die primäre Einschätzung beeinflussen. Desweiteren ist es möglich, dass Bewältigungsformen bereits vor dem eigentlichen Ereignis und der damit verbundenen primären Bewertung gespeichert werden und im konkreten Fall abrufbereit vorliegen. Bei dem Auftreten des potenziell bekannten Ereignisses wird dann eine Art Automatismus in Gang gesetzt, im Rahmen dessen eine Bewältigungshandlung unmittelbar initiiert werden kann (Laux, 1983).

Auch hinsichtlich Bewertung und Bewältigung ist das Konzept so ausgelegt, dass ein wechselseitiges Beziehungsgeflecht entsteht. Als beispielhafte Situation aus dem schulischen Alltag, die die Variabilität des Konzeptes hinsichtlich Bewertung und Bewältigung sowie deren Wechselwirkungen verdeutlicht, kann man die Inhaltswahl im Sportunterricht heranziehen. Das Wissen über die Frustration und die drohenden Disziplinkonflikte mit Schülern auf die Ankündigung des meist unbeliebten Unterrichtsinhalts Gerätturnen veranlasst Sportlehrer dazu, bereits vor dem primären Bewertungsvorgang und der Einschätzung als Bedrohung eine sekundäre Bewertung und Bewältigungsprozesse zu initiieren. Anhand eigener Ressourcen und Fähigkeiten wird a priori eine Strategie auf die erwartete Bedrohung erarbeitet bzw. vorbereitet, die auf die in der realen Situation erfolgende primäre Bewertung schnell einsetzbar ist. Im konkreten Fall wäre dies beispielsweise das in Aussicht stellen eines beliebten und freudvollen Abschlusspiels im Anschluss an den Unterrichtsinhalt Gerätturnen. In Kapitel 4 wird dieses Verhalten, die teilweise auch notwendige Routinisierung des Alltags mit dem Abzielen auf Entlastung und Handlungssicherheit, genauer beschrieben und Abgrenzungen zum evasiven Verhalten im Sportunterricht (siehe Kap. 4.9) aufgezeigt.

\section{Neubewertung (reappraisal)}

Konzeptionell unterscheidet sich dieser Vorgang nicht von den Prozessen der ersten und zweiten Bewertung, sondern stellt lediglich eine zeitlich spätere Wiederholung dar. Neubewertungen erfolgen als Reaktion auf Veränderungen der situativen und personalen Bedingungen und bewirken eine Änderung bzw. Bestätigung der ursprünglichen Bewertung (Herzog, 2007). Normalerweise ist das Eintreffen neuer Informationen und Hinweise über die Situation im Sinne einer veränderten Problemlage der Auslöser für eine neue Bewertung. Es kann auch sein, dass durch Nachdenken eine neue, 
vielleicht angemessenere oder effektivere Problemlösestrategie entdeckt wird und daher eine Neubewertung erfolgt. Grundsätzlich sind in irgendeiner Art und Weise Rückmeldungen und die damit verbundenen Emotionen Auslöser für Neubewertungen (Jerusalem, 1990). Informationen über Erfolg oder Misserfolg der aktuellen Bewältigung kann ebenfalls mit in die Rückmeldeschleife einfließen (van Dick \& Stegmann, 2007). In einer Sonderform, der defensiven Neubewertung (defensive reappraisal) wird versucht, der Bedrohung durch intrapsychische Manöver wie Verneinung oder Intellektualisierung zu begegnen. Es erfolgt eine positivere Neubewertung der Situation, nicht aufgrund neuer Informationen, die der Umwelt entstammen, sondern aufgrund von inneren Prozessen, die die Vergangenheit, gegenwärtige Schädigungen oder Verluste in einem besseren Licht erscheinen lassen und in der Situation einen neutralen oder sogar angenehm-positiven Charakter aufweist (Lazarus \& Folkman, 1984). So kann eine Lehrkraft, die aufgrund von Disziplinproblemen zunächst eine erfolglose konfrontative Strategie im Sinne von hartem und strengem Durchgreifen als Bewältigungsmaßnahme wählte, zu einer positiven Umdeutung/Neubewertung der Situation gelangen. Auf diese Art und Weise könnte respektloses Verhalten der Schüler durch die Lehrkraft als normales und entwicklungsgemäßes Verhalten interpretiert und so die Störungen im Nachhinein legitimiert werden. Durch die Neubewertung ist ein weiteres Eingreifen nicht notwendig und auch die negativen Emotionen des bereits Geschehenen erscheinen in einem neuen, angenehmeren Licht. Solche Rückmeldeschleifen, sind, ebenso wie die bereits angesprochenen Interdependenzen, in der vereinfachten grafischen Darstellung des transaktionalen Stresskonzepts von Lazarus und Folkman nicht enthalten.

\subsection{Belastungs- und Beanspruchungsmodelle}

Das in Kapitel 3.1 beschriebene transaktionale Stresskonzept von Lazarus stellt die Grundlage verschiedener Modelle dar, in denen handlungs- und stresstheoretische Perspektiven zusammengeführt werden. Belastungs- und Beanspruchungsmodelle dienen dazu, die Arbeit zum Beispiel von Lehrern zu verstehen, zu analysieren und zu verbessern. Prozessuale Abläufe werden in diesen Modellen ausgehend vom Arbeitsauftrag und den Arbeitsbedingungen über die konkrete Arbeitssituation bis hin zum Output in modellhafter Art und Weise dargestellt. Handlungen und die damit verbundenen Ziele werden strukturiert und zueinander in Beziehung gesetzt. Zu zentralen Begriffen der Analyse in diesem Problemfeld haben sich die Begriffe Belastung und Beanspruchung entwickelt, die in Kapitel 3.2.1 definiert und voneinander abgegrenzt werden (Herzog, 2007). Rudow führt in seinem Modell zum Lehrerstress (siehe Kap. 3.2.2), das basierend auf dem folgenden allgemeinen Belastungs- 
Beanspruchungsmodell von Rudow dargestellt werden soll, arbeitswissenschaftliche und stresspsychologische Konzepte zusammen (Rudow, 1995; Ulich, 1996).

\subsubsection{Das Belastungs-Beanspruchungsmodell von Rudow}

Das Rahmenmodell von Rudow soll dazu genutzt werden, ein Verständnis von Belastung und Beanspruchung und deren Beziehung untereinander zu erlangen. Rudow unterscheidet zwischen objektiven und subjektiven Belastungen, Beanspruchungsreaktionen und Beanspruchungsfolgen (Rudow, 1995).

Im alltäglichen Sprachgebrauch werden die Begriffe Belastung und Beanspruchung meist synonym verwendet für Situationen, die in irgendeiner Art und Weise beeinträchtigenden Einfluss auf Physis und Psyche ausüben. Erst seit den 1970er Jahren unterscheidet man zwischen diesen beiden Begriffen auch inhaltlich (Böhm-Kasper, 2004). Assoziationen mit Belastung sind Stress, Unwohlsein, Überforderung oder Langeweile (Krause, 2003). Der Begriff Belastung ist im direkten Vergleich etwas negativer belegt als Beanspruchung, denn wenn man davon spricht, dass jemand oder eine bestimmte Situation für einen selbst eine Belastung darstellt, meint dies, dass die persönliche Situation besser und angenehmer wäre ohne diesen Einfluss. Beanspruchung wird hingegen auch in Situationen verwendet, in denen, wie bereits der Wortstamm aussagt, ein gewisser Anspruch der Bewältigung im Sinne einer Herausforderung gehegt wird, um anderen oder sich selbst Kompetenz, Stärke, Ausdauer oder Willen zu beweisen.

Unter Belastungen werden ausschließlich Anforderungen und Einwirkungen von außen an bzw. auf das Individuum verstanden, die potenziell Beanspruchungen hervorrufen können. Diese Definition folgt eher arbeitswissenschaftlicher Tradition und bezieht den Begriff Belastung nicht auf die Reaktion der Person, sondern beinhaltet alle möglichen äußeren Einflussfaktoren, die von den Arbeitsbedingungen und dem Arbeitsauftrag ausgehen und personenunabhängig wirken (Krause, 2003). Gemäß dieser Definition sind Belastungen wertneutral (Herzog, 2007). Der Stellenwert und die Bedeutsamkeit psychischer Belastungen durch, während oder in sonstigem Zusammenhang mit der beruflichen Tätigkeit wird darin deutlich, dass selbst die Bundesanstalt für Arbeitsschutz und Arbeitsmedizin (2009) eine eigene DIN-Norm zur psychischen Belastung am Arbeitsplatz definiert hat. Laut DIN Norm EN ISO 1075 ist die psychische Belastung als „die Gesamtheit aller erfassbaren Einflüsse, die von außen auf den Menschen zukommen und psychisch auf ihn einwirken" definiert (Bundesanstalt für Arbeitsschutz und Arbeitsmedizin, 2009). Ulich (1985, S. 74) definiert Belastungen im Vergleich zu der arbeitswissenschaftlichen Begriffsbestimmung und der Formulierung in der DIN-Norm 
deutlich personenbezogener als „Beeinträchtigungen der individuellen Befindlichkeit und Stimmung, der Erlebnis- Verarbeitungs- und Handlungsmöglichkeiten einer Person in einer gegebenen Situation, die subjektiven Leidensdruck hervorrufen. Belastung ist also Zustand des Erleidens von Beeinträchtigungen und Mangelzuständen, das Erleben von negativen Veränderungen oder Einbußen an positiven Erlebnis- und Handlungsmöglichkeiten" und bezieht damit bereits zum Teil die Reaktionen des Individuums mit ein, was später unter dem Begriff der Beanspruchung vertieft wird. Konkret auf den Lehrerberuf bezogen können, nach dem didaktischen Modell von Scherler (siehe Kap. 4.9.1), der Umgang mit Schülerinnen und Schülern, mit Inhalten sowie mit äußeren Rahmenbedingungen potenzielle Belastungen darstellen. Charakteristisch für die Lehrerarbeit ist vorwiegend die psychische Belastung, d.h. dass die Aufnahme, Verarbeitung und Vermittlung von Informationen gegenüber muskulärer bzw. körperlicher Anstrengung deutlich akzentuiert ist (Rudow, 1995). Im Sportunterricht liegen neben den kognitiven und emotionalen Belastungsfaktoren auch verstärkt körperliche Belastungen vor, die in Kapitel 3.3 ausführlich erläutert werden.

Rudow (1995) unterscheidet grundsätzlich zwischen objektiver und subjektiver Belastung. Objektive Belastungen stellen solche Umgebungsvariablen dar, die unabhängig vom Individuum potenziell Beanspruchungen bedingen können. An sich haben objektive Belastungen weder einen positiven noch einen negativen Charakter, sondern stellen lediglich die Arbeitsaufgaben und die Bedingungen in wertindifferenter Weise dar, unter denen die berufliche Tätigkeit stattfindet. Dieser neutrale Status, der erst durch die Konfrontation mit den Eigenschaften der Person eine positive oder negative Prägung erhält, lässt sich anhand eines Zitats aus Shakespeares Hamlet verdeutlichen: "There is nothing either good or bad, but the thinking makes it so" (Shakespeare, 1603, zitiert nach Lazarus \& Folkman, 1984, S. 24). Objektive Belastungen sind personenunabhängige Faktoren wie z.B. die durch den Lehrplan festgelegten Bildungs- und Erziehungsziele, Konflikte zwischen und mit Schülern, große Klassen, ein hoher Lautstärkepegel in der Klasse oder das schlechte Image des Lehrerberufs in der Gesellschaft. Der Begriff Stressor bzw. potenzieller Stressor wird als Synonym zu Belastung bzw. objektiver Belastung angesehen (van Dick, 2006).

In einem dreistufigen Prozess, den man als Widerspiegelung bezeichnet, werden objektive Belastungen bei Relevanz zu individuellen subjektiven Belastungen, bei denen zwischen emotionalen und kognitiven Belastungen unterschieden wird. Vergleichbar mit der primären Bewertung im transaktionalen Stresskonzept von Lazarus ist dieser Vorgang notwendig, um die psychologische Relevanz der Belastung zu messen. Die Widerspiegelung setzt sich aus der Wahrnehmung objektiver Belastung, die von Person 
zu Person unterschiedlich erfolgen oder auch ausbleiben kann, dem Bewertungsvorgang sowie der kognitiven Verarbeitung zusammen (siehe Abb. 4). Es erfolgt ein Abgleich der aktuellen Situationsanforderungen mit Motiven, Einstellungen, der emotionalen und psychovegetativen Stabilität, Berufserfahrung, Bedürfnissen und pädagogischen, sozialen und fachlichen Qualifikationen, die man zusammenfassend als Leistungs- und Handlungsvoraussetzungen bezeichnen kann. Je nachdem wie weit Motive und Bedürfnissen und deren antizipierten Realisierungsmöglichkeiten in der vorliegenden Situation voneinander abweichen, desto stärker ist die emotionale Belastung, die sich durch angenehme bzw. unangenehme Gefühle bei der Aufgabenerfüllung manifestiert. Rudow (1995, S. 43) führt in diesem Zusammenhang kognitive Belastung und Selbstbelastung als zwei Sonderformen auf. Als kognitive Belastung bezeichnet er „die Inanspruchnahme kognitiver Leistungs- und Handlungsvoraussetzungen zur Bewältigung geistiger Anforderungen“ wohingegen die Selbstbelastung einen Belastungsbereich bezeichnet, dem man sich selbst aussetzt. Bei Lehrpersonen stellen mangelnde Vorbereitung der Unterrichtsstunden oder ein ineffektiver Gesprächsstil verfestigte, teils automatisierte Verhaltensweisen dar, die selbstbelastend wirken (Herzog, 2007). Grundsätzlich ist also die subjektive Belastung stets ein Person-Umwelt-Verhältnis, denn weder die persönlichen Voraussetzungen noch die Umweltbedingungen alleine konstituieren eine Belastung, sondern lediglich deren Differenzen. Proportional zur entstandenen Diskrepanz empfindet das Individuum eine subjektive Belastung, die im Gedächtnis als Erfahrung gespeichert wird (Rudow, 1995). Die subjektiv erlebte Belastung kann im konkreten Fall also sehr unterschiedliche Formen und Ausprägungen annehmen. Während eine Lehrkraft, einen hohen Lautstärkepegel in einer Gruppenarbeitsphase als Angriff gegen die eigene Autorität ansieht und neben der Belastung durch die Lautstärke zusätzlich die eigene pädagogische Kompetenz anzweifelt, kann eine zweite Lehrkraft die Situation völlig anders bewerten, indem sie die wahrgenommen Lautstärke als fruchtbare Unruhe auffasst, die das Interesse und Engagement der Schüler widerspiegelt. Beide Lehrkräfte sind mit identischen Arbeitsaufgaben und Bedingungen konfrontiert, individuelle Wahrnehmung und Interpretation führt jedoch zu einer stark unterschiedlich erlebten subjektiven Belastung. Der Prozess der Widerspiegelung objektiver Belastungen stellt also einen Vermittlungsprozess zwischen der Person und der Umwelt dar. Inwieweit subjektive Belastungen der Vergangenheit, die als Erfahrungen im Gedächtnis gespeichert sind, den zukünftigen Prozess der Widerspiegelung bedingen wird in Kapitel 4 näher ausgeführt. Subjektive Belastungen resultieren in Beanspruchungen, genauer gesagt in dem Interaktionsphänomen der Beanspruchungsreaktion. Die Beanspruchungsreaktion stellt ein psychophysisches Phänomen 
dar, das auf die Konfrontation der psychischen und physischen Handlungsvoraussetzungen und den Anforderungen durch die Situation folgt und mit erhöhter psychophysischer Aktivität einhergeht (Rudow, 1995). Beanspruchungsreaktionen können physischer, kognitiver, affektiver oder verhaltensmäßiger Natur sein. Kurzfristige Reaktionen auf Beanspruchungen im physiologischen Bereich erfolgen in Form von somatischen Veränderungen wie Puls- und Blutdrucksteigerung und der Aktivierung des Hormonsystems.

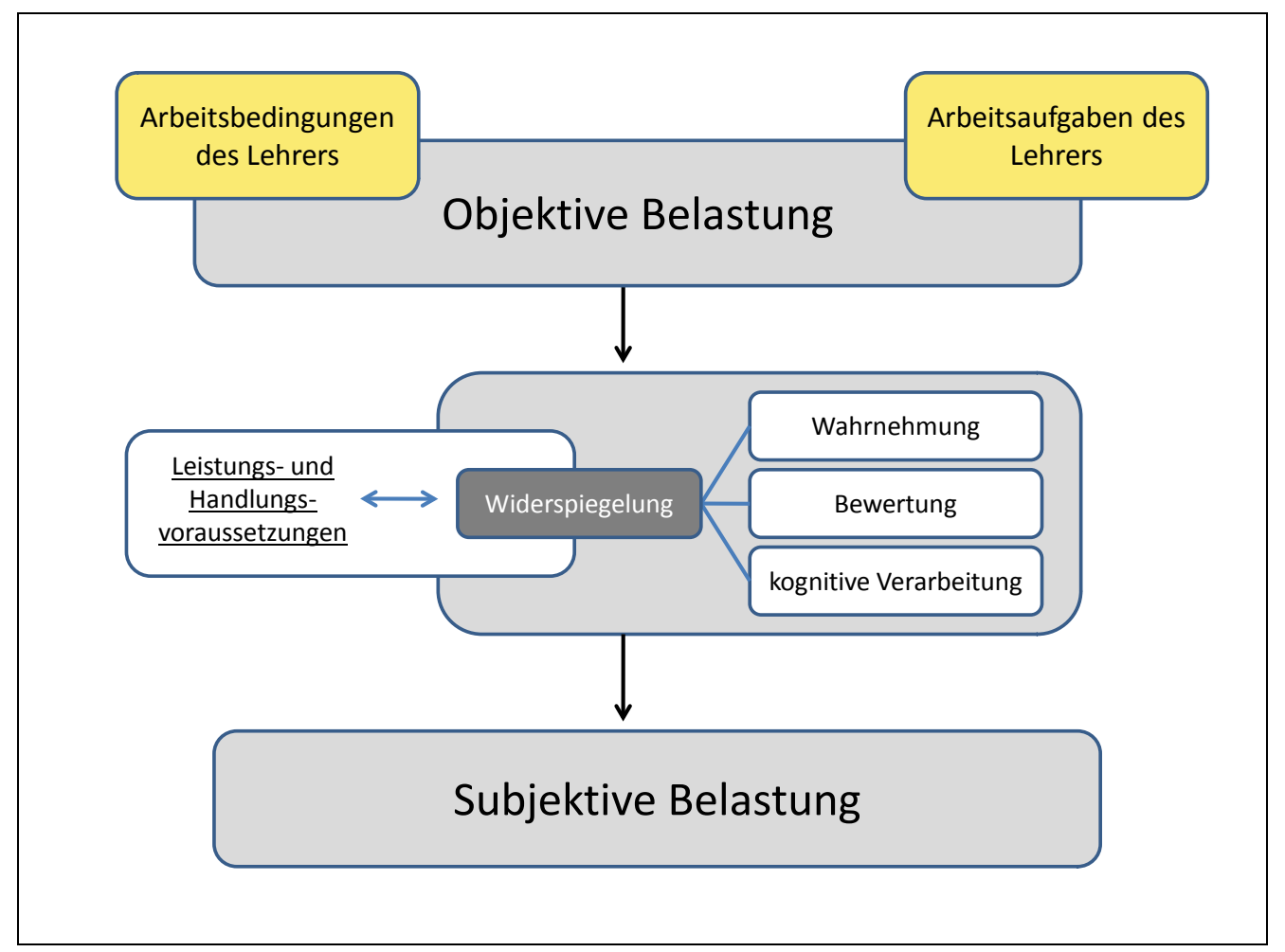

Abb. 4: Objektive und subjektive Belastung (mod. nach Rudow, 1995, S. 43)

Die Bundesanstalt für Arbeitsschutz und Arbeitsmedizin (2009) definiert die psychische Beanspruchung in Abgrenzung zur physischen Belastung als „die unmittelbare (nicht die langfristige) Auswirkung der psychischen Belastung im Individuum in Abhängigkeit von seinen jeweiligen überdauernden und augenblicklichen Voraussetzungen, einschließlich der individuellen Bewältigungsstrategien“. In dieser Definition wird deutlich, dass Beanspruchungsreaktionen kurzfristig auftreten, reversibel sind und sowohl negativ als auch positiv wirken können, indem sie regulativ auf die Handlungsvoraussetzungen einwirken. Einen positiven Einfluss kann z.B. ein gewisses geistiges Aktivitätsniveau, das leistungsfördernd wirkt, ausüben. Affektive Beanspruchungsreaktionen umfassen sowohl negative als auch positive Emotionen wie Gereiztheit, Ärger, Unsicherheit, Irritation, Sättigung, Freude, Ruhe, Flow-Erlebnisse oder aber auch Gelassenheit (Krause \& Dorsemagen, 2007). Negative Beanspruchungsreaktionen können ne- 
ben den eindeutig unangenehmen Eigenschaften aber auch positive Momente aufweisen, da sie als Warnsignale dienen können um Schlimmeres zu verhindern (Rudow, 1995). Neben der Differenzierung in positiv und negativ kann man bei den Beanspruchungsreaktionen auch zwischen kognitiven und verhaltensmäßigen Reaktionen unterscheiden. Unter kognitiven Beanspruchungsreaktionen versteht man Reaktionen wie das Empfinden von Monotonie, psychischer Ermüdung oder Sättigung, die durch mentale Prozesse, die sich während des Unterrichts im Kopf der Lehrer abspielen, ausgelöst werden. Zu den verhaltensmäßigen Beanspruchungsreaktionen rechnet man alle beobachtbare Verhaltensäußerungen, die aus der Ausübung der Tätigkeit resultieren wie z.B. das Verhalten in Pausen oder nach dem Unterricht (Krause \& Dorsemagen, 2007).

Werden negative Beanspruchungsreaktionen nicht in geeigneter Weise kompensiert, bzw. treten sie in dauernder Folge auf (siehe Abb. 5), kann es zu Chronifizierungen kommen, die als Beanspruchungsfolgen bezeichnet werden und die pädagogische Handlungskompetenz einschränken sowie die Zielbildung und die Verarbeitung von Rückmeldungen stören (Rudow, 1995).

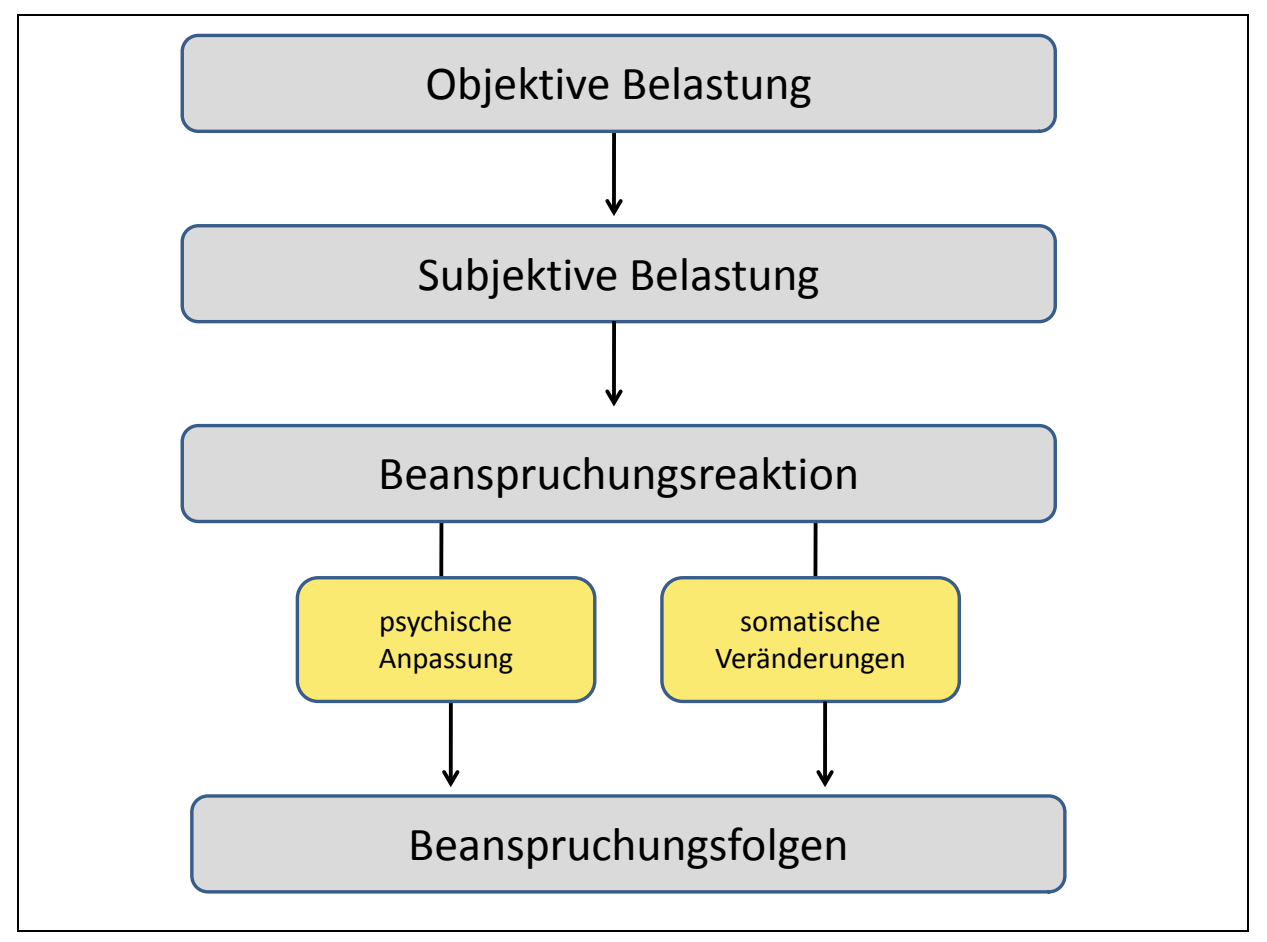

Abb. 5: Beanspruchung, Beanspruchungsreaktionen und Folgen (Rudow, 1995, S. 46)

Beanspruchungsfolgen sind im Gegensatz zu den Beanspruchungsreaktionen nur bedingt reversibel und nehmen oft einen chronischen Verlauf (Böhm-Kasper, 2004). Beanspruchungsfolgen können sowohl positive als auch negative Wirkung haben. Im positiven Fall eignen sich Lehrkräfte zusätzliche Handlungsmuster und Strategien an und 
profitieren im Sinne einer verbesserten Handlungskompetenz, einer Steigerung der psychischen Gesundheit oder einem Anstieg der Arbeitszufriedenheit (Krause, 2003; Ulich, 1996). Erworbene Handlungsstrategien und -muster werden im operativen Abbildsystem eingeordnet und können zur emotionalen und kognitiven Stabilität beitragen und damit die individuelle Weiterentwicklung der Persönlichkeit unterstützen. Emotionale Stabilität und der Status des operativen Abbildsystems stellen die entscheidenden Bedingungen für die Ausbildung pädagogischer Handlungskompetenz dar. Bei den negativen Beanspruchungsfolgen ist eine weitere Differenzierung in physiologische, affektive, kognitive und verhaltensmäßige Auswirkungen möglich. Im physischen Bereich kann sich eine berufsbedingte Dauerbelastung bis hin zu psychosomatischen Gesundheitsstörungen entwickeln, die zu weiterer Verstärkung der negativen Beanspruchungsreaktionen führen können. Länger andauernde emotionale Zustände wie Burnout (siehe Kap. 4.9.5.1), Phobien, Ängste, Übermüdung, chronischer Stress, Veränderung der Arbeitsunzufriedenheit und generelle Einschränkung der Leistungsfähigkeit werden unter dem Begriff der affektiven Folgeerscheinungen zusammengefasst (Rudow, 1995). Im kognitiven Bereich sind vor allem Frühpensionierungsabsichten, Drop-out-Intention, innere Kündigung, Resignation, erlernte Hilflosigkeit sowie eine Beeinflussung des Selbstwertgefühls und des Selbstkonzepts als Folgen zu nennen. Verhaltensmäßige Beanspruchungsfolgen, die als Folgen sich wiederholender oder nicht kompensierter Beanspruchungen genannt werden, sind z.B. Sucht-oder Risikoverhalten, Absentismus, Veränderungen im Gesundheitsverhalten oder die Reduzierung der Lehrverpflichtung (Krause \& Dorsemagen, 2007).

\subsubsection{Das Modell des Lehrerstresses von Rudow}

Seit den 70er Jahren des vergangenen Jahrhunderts setzte ein wahrer Forschungsboom ein und man beschäftigte sich verstärkt mit Lehrerstress, Berufszufriedenheit von Lehrern und Lehrerbelastung, aber auch die gesundheitlichen Aspekte blieben nicht unberücksichtigt (Rudow, 1995). Kyriacou und Sutcliffe erarbeiteten bereits 1978 ein Lehrerstress-Modell basierend auf dem allgemeinen Stresskonzept von Lazarus (1966), reduzierten die Umweltbedingungen jedoch auf potenzielle Stressoren und vernachlässigten die Bewältigungskompetenz sowie die Attributionsmuster. Berücksichtigung fanden jedoch Rückkopplungsschleifen. Schönwälder setzte 1988 ein Belastungsmodell in einem arbeitswissenschaftlichem Bezugsrahmen um und berücksichtigt sowohl Arbeitsbedingungen als auch den Arbeitsauftrag, die beide objektive Belastungsquellen darstellen und durch Interaktion mit den individuellen Leistungsvoraussetzungen subjektive Belastungen auslösen können. Das Modell des Lehrerstresses von Rudow integriert letztendlich verschiedene Ansätze auf der Grundlage des transaktio- 
nalen Stresskonzepts und führt sie weiter aus, sodass Einzelphänomene wie Stress oder Burnout, die oft als Globalkonzepte genutzt werden, anhand der Arbeitsaufgaben und Arbeitsbedingungen eingeordnet werden können (Böhm-Kasper, 2004).

Im Modell von Rudow (siehe Abb. 6) werden potenzielle Stressoren, die im Arbeitsalltag von Lehrkräften auftreten im Rahmen einer primären Bewertung bezüglich der Gefährdung tätigkeitsbestimmender Bedürfnisse eingeschätzt. Endet die primäre Bewertung mit dem Ergebnis der Stressrelevanz, spricht man von einem Stressor oder einer subjektiven Belastung, die aus einer Gegenüberstellung von Tätigkeitsmerkmalen und Persönlichkeitsmerkmalen resultiert. Beispielhaft können Disziplinprobleme mit Schülern im Unterricht als Gefährdung des Selbstkonzeptes interpretiert werden. Ob, wie, mit welcher Intensität und in welcher Art und Weise Motive bzw. die Befriedigung von Bedürfnissen oder die Gesundheit als gefährdet eingeschätzt werden, ist individuell verschieden. Während manche Lehrkräfte solche eine Situation als höchst belastend einstufen, schätzen andere Lehrkräfte die Situation nicht einmal als stressrelevant ein (Rudow, 1995).

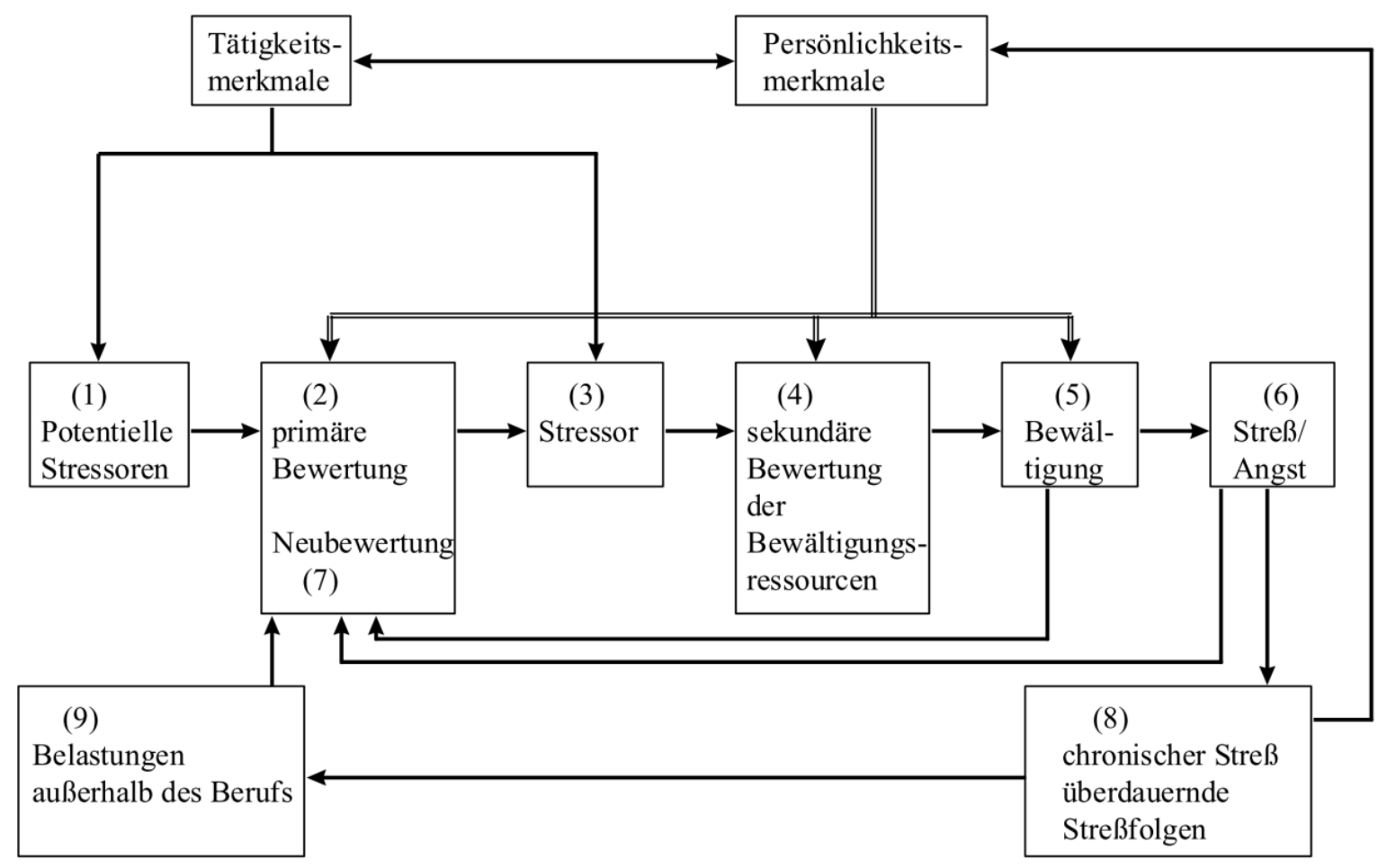

Abb. 6: Modell des Lehrerstresses von Rudow (mod. Fassung von van Dick, 2006, S. 37)

Auf der Grundlage der primären Bewertung werden die Stressoren anhand der zur Verfügung stehenden Ressourcen, bezogen auf eine bevorstehende Bewältigung in einem sekundären Bewertungsprozess eingeschätzt. Während des Bewertungsprozesses findet ein Abgleich statt, welche Bewältigungsmöglichkeiten in der jeweiligen Situ- 
ation zur Verfügung stehen (van Dick, 2006). Im Rahmen der Bewältigungsreaktion werden eine oder mehrere Strategien eingesetzt, um den Stressor zu entschärfen bzw. zu beseitigen. Wird in der Neubewertung der Situation festgestellt, dass die Strategie erfolgreich war, kommt es nicht zu weiteren Stressreaktionen und der Prozess ist beendet. Misslingt hingegen der Bewältigungsversuch, kann auf verschiedenen Regulationsebenen eine Stressreaktion auftreten. Es kommt zu einem weiteren Durchlaufen des Bewertungs-Bewältigungsprozesses, was im wiederholten Fall zu einer Chronifizierung von Stress führen kann. Stressfolgen manifestieren sich letztendlich in physischen, psychischen und/oder psychosomatischen Störungen oder gar Erkrankungen, die auch Einfluss auf das Privatleben aufweisen (Rudow, 1995). Aufgrund der angespannten Situation im Berufsleben können alltägliche Belastungen in der Familie bzw. in anderen außerberuflichen Interaktionssituationen verstärkt werden. Probleme im Privatleben können im Umkehrschluss eine größere Stressempfänglichkeit und Sensibilität im Berufsleben bedingen. Personen, die ein stabiles und unterstützendes privates Umfeld haben, gelten als deutlich stressresistenter und erfolgreicher im beruflichen Bewältigungshandeln. Das Modell von Rudow sieht zusätzlich eine weitere Rückkopplungsschleife vor: Chronischer Stress wirkt sich auf die Persönlichkeitsmerkmale und damit auch auf zukünftige Bewertungs- und Bewältigungsvorgänge aus, da diese hauptsächlich von den Eigenschaften der Persönlichkeit abhängig sind. Des Weiteren sind auch die Tätigkeitsmerkmale, die durch ihre Komplexität, Variabilität und Transparenz Einfluss auf die primäre Bewertung ausüben, von entscheidender Bedeutung für das Stresserleben. Zwischen den Tätigkeitsmerkmalen und den Persönlichkeitseigenschaften besteht eine wechselseitige Beziehung, da beispielsweise die Wahrnehmung der beruflichen Tätigkeit als angenehm und interessant, positiven Einfluss auf die Persönlichkeit hat. Umgekehrt können Persönlichkeitseigenschaften, die einen positiven und selbstbewussten Charakter aufweisen, dazu führen, dass die Merkmale der Tätigkeit eher positiv wahrgenommen werden (van Dick, 2006). In der Praxis bedeutet dies, dass z.B. eine Lehrkraft, die gerne ihren Beruf ausübt und mit dem notwendigen Selbstbewusstsein ausgestattet ist, berufstypische Tätigkeitsmerkmale wie Ereignisunsicherheit oder mangelnde Anerkennung in deutlich geringerem Maß negativ wahrnimmt als Lehrkräfte, die den Beruf aufgrund von extrinsischen Motivationen wie dem Gehalt oder den langen Ferienzeiten gewählt haben oder ein geringeres Selbstbewusstsein haben. 


\subsection{Belastungen des Lehrers im Allgemeinen und des Sport- lehrers im Speziellen}

Während der bisherige Verlauf des Kapitels 3 weitestgehend durch die inneren Komponenten des Stress- und Belastungsempfindens geprägt waren, folgt im Anschluss eine Skizze der äußeren Komponenten wie z.B. Anforderungen auf organisatorischer Ebene, Unterrichtsgestaltung, Initiierung von Erziehungsprozessen oder Elternarbeit. Zwar zeichnete sich der Lehrerberuf schon immer durch ein hohes Maß an psychischer Belastung aus (siehe Kap. 2.2), in letzter Zeit kommt es jedoch zu einer Zuspitzung der Belastungssituation aufgrund der Expansion des Auftrags, bei gleichzeitiger Verschlechterung der Bedingungen, unter denen der Unterricht bzw. die Lehrtätigkeit stattfindet (Schaarschmidt, 2005b). Für den Sportunterricht fanden Kastrup, Dornseifer und Kleindienst-Cachay (2008) heraus, dass sich nahezu ein Drittel der Sportlehrkräfte stark bzw. sehr stark belastet fühlen, wohingegen lediglich knapp ein Fünftel den individuellen Belastungsgrad als gering bzw. sehr gering einschätzten. Allen Untersuchungen und Abhandlungen, die sich mit dem Thema Lehrerbelastung beschäftigen, ist gemein, dass weniger die sporadisch großen Konflikte auf den Magen schlagen sondern die kleinen Reibereien, Probleme und Ärgernisse, mit denen Lehrer in ihrem Alltag stetig und wiederkehrend konfrontiert werden (Treutlein, Janalik \& Hanke, 1996).

Die offensichtlichste Besonderheit des Lehrerberufs, die sowohl be- als auch entlastend wirken kann, ist die strukturelle Trennung der Arbeit auf zwei Arbeitsplätze, die so extrem in keinem anderen Beruf ausgeprägt ist. Die Arbeit des Lehrers findet an zwei völlig verschiedenen Orten statt und das Selbstbild der Lehrkräfte ist dadurch stark antagonistisch geprägt. Den einen Pol des Arbeitsverhältnisses stellt die Arbeit in der Schule dar, zu der neben dem Unterrichten und den Konferenzen auch Elterngespräche gehören. Lehrkräfte stehen stets unter Druck, den Lehrstoff durchzukriegen und müssen sich gemäß Regelungen und Vorgaben verhalten (Altrichter, Gather Thurler \& Heinrich, 2005). Den zweiten Teil der Arbeit, meist durch Pendeln und den Pädagogenschlaf vom Unterrichtsgeschäft in der Schule abgetrennt, erledigen Lehrkräfte zu Hause. Neben den Korrekturen ist es vor allem die Vor- bzw. Nachbereitung des Unterrichts, die in den heimischen vier Wänden stattfindet und wo es oft zu einer Überlagerung von Berufs- und Privatexistenz kommt (Ulich, 1996). Welche Folgen und Auswirkungen im Sinne von Belastungen durch die Trennung des Arbeitsplatzes hervorgerufen werden können, wird im Laufe dieses Kapitels geklärt. Gleichzeitig zählt diese Besonderheit jedoch auch zu den Vorteilen und Besonderheiten des Berufes, denn durch die zeitliche und räumliche Flexibilität der Arbeit ist ein hohes Maß an selbstbestimmter Arbeitsorganisation möglich. 
In der Literatur existiert eine Vielfalt von Belastungsfaktoren im Lehrerberuf, da dieses Thema nach wie vor einen Schwerpunkt der empirischen Lehrerforschung darstellt (Rothland, 2007c). Vor allem im Kontext der Burnout-Forschung wurden die Belastungen, die der Lehrerberuf mit sich bringt, verstärkt ins Zentrum des Forschungsinteresses gerückt. Es existieren Einteilungen in Kategorien, die aufgrund unterschiedlichster Maßstäbe voneinander abgegrenzt werden und teils willkürliche unsystematische Aufzählungen darstellen. Eine schnelle und eindeutige Benennung und Beschreibung der Belastungen, denen Lehrer ausgesetzt sind, ist nicht ohne Weiteres in wenigen Sätzen abzuhandeln (Wendt, 2001). Während Kramis-Aebischer (1996) Belastungen anhand organisationspsychologischer Kriterien in Systemebene, Individuumsebene und Organisationsebene einteilt, klassifiziert Rudow (1995) die Belastungsfaktoren mit Arbeitsaufgaben und schulorganisatorische Bedingungen, arbeitshygienische Bedingungen, soziale Arbeitsbedingungen und gesellschaftlich-kulturelle Bedingungen in vier Kategorien (siehe Tab. 1).

Tab. 1: Belastungskategorien und -faktoren im Lehrerberuf (Rudow, 1995, S. 60)

\begin{tabular}{|c|c|c|c|}
\hline $\begin{array}{l}\text { Arbeitsaufgaben und } \\
\text { schulorganisatorische } \\
\text { Bedingungen }\end{array}$ & $\begin{array}{l}\text { Arbeitshygienische Be- } \\
\text { dingungen }\end{array}$ & $\begin{array}{l}\text { Soziale Arbeitsbedin- } \\
\text { gungen }\end{array}$ & $\begin{array}{l}\text { Gesellschaftlich- } \\
\text { kulturelle Bedingun- } \\
\text { gen }\end{array}$ \\
\hline Arbeitsaufgaben & Lärm & Schüler & Schulkultur/-klima \\
\hline Arbeitszeit/Pausenzeit & Mikroklima & Kollegen & $\begin{array}{l}\text { Gesellschaftliche } \\
\text { Erwartungen }\end{array}$ \\
\hline Unterrichtsfach & Luftbeschaffenheit & Schulleitung & Medien \\
\hline Lehrplan & Beleuchtung & Eltern/-beirat & $\begin{array}{l}\text { Berufsstatus, Berufs- } \\
\text { image/-anerkennung }\end{array}$ \\
\hline Klassenfrequenz & Klassenraum & Schulbehörden & Gehalt \\
\hline Klassenrekrutierung & Bildschirmarbeit & Betriebe & $\begin{array}{l}\text { Schulreformen/ } \\
\text {-innovationen }\end{array}$ \\
\hline Stundenplan & $\begin{array}{l}\text { Unterrichtsfachspezifische } \\
\text { Faktoren }\end{array}$ & $\begin{array}{l}\text { Sozialarbeiter/ } \\
\text {-pädagogen }\end{array}$ & Schulimage \\
\hline Raumplan/-wechsel & $\begin{array}{l}\text { Pausen- } \\
\text { /Entspannungsraum }\end{array}$ & Externe Fachkräfte & \\
\hline Schultyp/-größe & Schulgebäude & Schulsekretärin & \\
\hline Lehrerfunktionen & Schulausstattung & Hausmeister & \\
\hline Unterrichtsmethode & Sanitärräume & & \\
\hline Lehr/Lernmittel & Schulstandort(e) & & \\
\hline Prüfungen & Infektionsgefahr & & \\
\hline Weiterbildung & & & \\
\hline Physische Belastungen & & & \\
\hline Sprechbelastung & & & \\
\hline
\end{tabular}


Die folgenden Abschnitte in denen die berufsbezogenen Belastungen von Lehrern im Allgemeinen und von Sportlehrern im Speziellen vorgestellt werden, entsprechen diesem Gliederungsmuster. Es wird sich zeigen, dass trotz der Einteilung in verschiedene Kategorien die Belastungsfaktoren nicht isoliert voneinander betrachtet werden können, da es sich bei dem Unterrichten um eine Tätigkeit handelt, die durch einen hohen interaktiven Anteil gekennzeichnet ist. Findet der Unterricht mit einer großen Klasse beispielsweise in einer engen und schlecht ausgestatteten Sporthalle statt, führt dies durch verschiedene verstärkende Wechselwirkungen zu einer veränderten Belastungssituation bzw. dem Auftreten von Belastungen in anderen Bereichen ${ }^{2}$. Neben der Beschreibung der einzelnen Belastungskategorien werden zusätzlich Ergebnisse von Studien, die sich mit dem Thema Lehrerbelastung auseinandergesetzt haben mit einbezogen und an einigen Stellen Reaktionen und Folgen der Belastungen bzw. Beanspruchung angesprochen. In Tabelle 1 wurden einzelne potenzielle Belastungsfaktoren in das Kategorisierungsschema eingeordnet, die zum Teil in den folgenden Abschnitten genauer erläutert werden und in Bezug auf die besondere Situation im Rahmen des Sportunterrichts spezifiziert werden.

\subsubsection{Arbeitsaufgaben und schulorganisatorische Bedingungen}

An Lehrer werden, ausgehend von den Vorgaben der Kultusministerkonferenz (siehe Kap. 2.2.2), verschiedene Aufgaben gestellt, die sie im Rahmen ihrer Tätigkeit zu erfüllen haben und mit denen Anforderungen verbunden sind, die potenzielle Belastungen darstellen. Der Arbeitsauftrag an sich stellt in diesem Zusammenhang bereits den ersten belastenden Faktor dar, denn während z.B. Bankkaufleute oder Handwerker klar definierte Aufgaben zu erfüllen haben und gesetzte Ziele erreichen müssen, ist der Auftrag an Lehrer breit, offen und nach oben hin nicht begrenzt. In Interviews wird immer wieder deutlich, dass Lehrer nie zum Ende kommen und eigentlich immer etwas zu tun haben (Czerwenka, 1996; Ulich, 1996). Eine klare Grenzziehung, wann der Arbeitsauftrag erfüllt ist, ist im Lehrerberuf nicht möglich, denn es gibt immer ein mehr bzw. besser, was auch große interindividuelle Unterschiede in der wöchentlichen Arbeitszeit bedingen (Rothland \& Terhart, 2007). Neben der eigentlichen Kernaufgabe, dem Unterrichten, Beraten, Erziehen, Beurteilen und Innovieren, sind es die zusätzlich anfallenden, vom Unterricht abhängigen und unabhängigen Verwaltungsaufgaben, die vor allem aufgrund des großen Zeitaufwandes und der relativen Anspruchslosigkeit als sehr belastend wahrgenommen werden (Rudow, 1995). Auch Barth (1997, S. 24) betont, dass durch die Einspannung in die „bürokratische Maschinerie“ Lehrer zu Tätig-

\footnotetext{
${ }^{2}$ Z.B. Anstieg des Verletzungsrisikos oder entstehende Wartezeiten bedingt durch die beengende Halle und das daraus resultierendes Schülerfehlverhalten.
} 
keiten verpflichtet und in ihrer Arbeit behindert werden. „Der ganze Bürokram, den man machen muss, also Klassenlisten, Lehrpläne, Wochenpläne, vor allem so pingelig, wie man es machen muss, das nervt" (Ulich, 1996, S. 59). Die Verwaltungsarbeiten sind der einzige Faktor, den Lehrer als belastender wahrnehmen als Lehrerinnen. In den übrigen Kategorien bzw. bei den übrigen Belastungsfaktoren weisen Lehrerinnen stets ein höheres Belastungsempfinden auf als ihre männlichen Kollegen. In diesen Bereich fällt auch die Konfrontation mit Erlassen, Vorschriften und Richtlinien, die oft als Entmündigung bzw. Entwertung der eigenen Kompetenzen, durch die als inkompetent eingestufte Schulverwaltung aufgefasst wird. Unsicherheit und Angst entsteht dadurch, dass die Regelungen oft kompliziert und unüberschaubar sind, dennoch aber eingehalten werden müssen (Ulich, 1996). Für den Sportunterricht ergibt sich die gesonderte Situation, dass in Verbindung mit Schul- und Rechtsvorschriften stets auch der Sportunfall einen nicht zu vernachlässigenden Faktor darstellt. Zwar sind disziplinarische, arbeitsrechtliche und strafrechtliche Maßnahmen erst dann zu befürchten, wenn das Handeln der Sportlehrkraft als grob fahrlässig zu bezeichnen ist und ein schwerer Unfall die Konsequenz ist, dennoch stellen Unfälle im Sportunterricht und die damit verbundene Unsicherheit eine erhebliche Belastungsquelle dar. Unzureichende Vorbereitung in der Ausbildung und Tabuisierung des Unfallrisikos im Alltag führen dazu, dass sich Hilflosigkeit und Überforderungserleben einstellt und Sportlehrkräfte sogar Angst vor der Vermittlung verschiedener Inhalte haben und diese daher aus ihrem Unterricht ausklammern (Feuß, 1998; Hundeloh, 2005).

Stets ein brisantes und heikles Thema stellt die Arbeitszeit von Lehrern dar. Während in der öffentlichen Meinung Lehrer mit ihrem Beruf gesegnet sind, sich in einem privilegierten Arbeitsverhältnis befinden, das sich durch lange Ferienzeiten und einen täglichen Arbeitsumfang eines „Halbtagsjobbers" auszeichnet, ergeben Arbeitszeitanalysen meist ein völlig anderes Bild. Auf den Unterricht entfallen nur etwa 40\% der Gesamtarbeitszeit, sodass etwa $60 \%$ für Unterrichtsvor- und -nachbereitung, Konferenzen, Korrekturen von Klassenarbeiten oder Beratung von Schülern und Eltern investiert werden (Dorsemagen, Lacroix \& Krause, 2007). Unter methodischen Aspekten sind Zweifel an der Objektivität der Arbeitszeituntersuchungen zwangsläufig, da diese stets auf Lehrerbefragungen basieren. Trotz der Anfälligkeit solcher Untersuchungen für Verfälschungen aufgrund des Effekts der sozialen Erwünschtheit sollten sie nicht im Vorhinein als Übertreibungen aufgefasst werden (Ulich, 1996). Seit den 1950er Jahren wurde eine Vielzahl von Studien durchgeführt, die sich mit den durchschnittlichen Wochenarbeitszeiten beschäftigten und zu unterschiedlichen Ergebnissen führten. In allen Untersuchungen deuten die Ergebnisse jedoch in dieselbe Richtung. Während in eini- 
gen Studien (Häbler \& Kunz, 1985; Wulk, 1988; Hübner \& Werle, 1997; Barth, 1997; Mummert \& Partner, 1999) eine Durchschnittsarbeitszeit von unter 50 Wochenstunden ermittelt wurde, deuten sich in anderen Studien (Seegerer, Wulsten \& Ulich, 1975; Müller-Limmroth, 1980; v. Engelhardt, 1982) Wochenarbeitszeiten von über 50 Stunden an. Die gleichlautende Aussage aller Autoren ist jedoch, dass Lehrer im Vergleich zu vielen anderen Berufsgruppen eine überdurchschnittlich hohe Zeit für ihren Beruf investieren, wodurch die mangelnde Erfüllung der Gütekriterien in den Einzeluntersuchungen kompensiert und von einer empirischen Absicherung ausgegangen werden kann (Rudow, 1995). Häbler und Kunz (1985) fanden zudem heraus, dass Teilzeitlehrkräfte in Bezug auf den Faktor Zeit, relativ gesehen zu ihrer vorgeschriebenen Arbeitszeit, am stärksten belastet sind, was in der Studie von Landert (1999) anhand von selbstadministrativen Arbeitszeitprotokollen bestätigt werden konnte. Auch die Berücksichtigung der Ferienzeiten und die sich anschließende Hochrechnung auf ein reguläres Arbeitsjahr ergaben stets noch überdurchschnittliche Stundenzahlen im Vergleich zu den meisten anderen Arbeitnehmern (Hübner \& Werle, 1997).

Bei einer Analyse des Belastungsfaktors Arbeitszeit wird deutlich, dass weniger die Zeitinvestition für Berufliches selbst, sondern vielmehr die gesellschaftliche Meinung darüber als negativ und störend wahrgenommen wird. Während lediglich ein Drittel der Lehrkräfte eine direkte Belastung durch den zeitlichen Umfang der Arbeit empfindet, nehmen etwa zwei Drittel die gesellschaftliche Meinung, dass Lehrer zu viel Freizeit hätten, als Belastung wahr. Diese Tatsache führt zu einer Überschneidung zwischen der Kategorie gesellschaftlich-kulturelle Bedingungen und den Arbeitsaufgaben und schulorganisatorischen Bedingungen, in denen die Arbeitszeit eigentlich einzuordnen ist. Die Diskontinuität der Arbeitszeit im Schuljahresverlauf, d.h. die periodischen Schwankungen durch die Korrektur von Klassenarbeiten, Konferenzen, Fortbildungsveranstaltungen oder das Zeugnisschreiben wirkt zusätzlich als Belastung (Ulich, 1996). Ob die hohen Arbeitszeiten jedoch ausschließlich aufgrund der an die Lehrkräfte gestellten Anforderungen zustande kommen oder ob auch ineffektive Arbeitstechniken zum Teil dafür verantwortlich sind, ist ungeklärt. Wulk (1988) vermutet, dass der Freiraum, den Lehrer in der Ausführung ihrer Arbeit haben, dazu führen kann, dass Arbeiten vor sich hergeschoben werden und so die Gesamtbelastung, sowohl psychisch als auch zeitlich ansteigt. Bezogen auf die außerunterrichtliche Arbeitszeit handelt es sich beim Sportunterricht, bis auf im Leistungsfach Sport, um ein Fach mit eher geringerem außerunterrichtlichem Zeitaufwand. Wie anhand der weiteren Bedingungen und Anforderungen des Fachs deutlich werden wird, ist der Sportunterricht zwar durch seinen zeitlich entlastenden Nicht-Korrektur-Status gekennzeichnet, weist aber dennoch eine 
Reihe von Belastungsfaktoren auf. Im Hamburger Arbeitszeitmodell für Lehrer (AZM) wurde pauschal, aufgrund des zeitlichen Korrekturaufwandes ein Modell implementiert, in dem die einzelnen Unterrichtsfächer einen, die wöchentliche Lehrverpflichtung bestimmenden Zeitfaktor zugewiesen bekamen. Die Stunden im Fach Sport werden seitdem mit dem Faktor 1,25 gewertet, dem niedrigsten Wert aller Fächer, was dazu führte, dass die Sportlehrkräfte etwa ein Drittel mehr Stunden pro Woche unterrichten müssen (Zimmermann, 2006). In einer Interviewstudie von Kastrup \& Mylius (2012) konnte gezeigt werden, dass in Lehrerkollegien Diskussionen über Arbeitszeitmodelle aus Angst vor Konflikten grundsätzlich abgelehnt wird. Außerdem zeigte die Befragung, dass sich die Sportlehrer aufgrund der Andersartigkeit der Belastung, trotz der Entlastung durch die fehlenden Korrekturen, mindestens in gleichem Maße wie ihre Kollegen belastet sehen, wie ein Interviewauszug zeigt (Kastrup \& Mylius, 2012, S. 69).

Die Belastung, die die Sportkollegen haben durch Lärm, also durch dieses ganze Umfeld, das können sie einfach nicht aufrechnen in Punkten. Das geht einfach nicht. Denn psychische Belastung kann man ja nicht anfassen. Also ich kann da drei Stapel Korrekturen hinlegen und sagen: 'Oh, ist der Kollege aber belastet'. Aber ich kann nicht die Belastung sichtbar machen, die ein Sportlehrer hat. Die Belastung ist einfach eine andere, die verschiebt sich (Frau ZG - 40 Jahre, Gymnasium, Sport/Spanisch).

Die Untersuchung von Schönwälder et al. (2003) zeigte jedoch, dass allein der zeitliche Umfang verschiedener beruflicher Tätigkeiten nur sehr bedingt Aufschluss über die perzipierte physische und psychische Belastung liefern kann. Sie gehen vielmehr davon aus, dass die Qualität der belastenden Tätigkeiten eine von der absoluten Arbeitszeit unabhängig Komponente darstellt. Miethling und Sohnsmeyer (2009) zeigten auf, dass der Anteil der Lehrer, die dem Muster Burnout (siehe Kap. 4.9.5.1) zugeordnet werden, im Stadtstaat Hamburg im Vergleich zu den Bundesländern Baden-Württemberg und Schleswig Holstein deutlich überrepräsentiert ist, was unter anderem mit der höheren zeitlichen Belastung und der damit verbundenen Geringschätzung der eigenen Tätigkeit zusammenhängen könnte.

Ein weiterer potenzieller Belastungsfaktor, der in direkter Beziehung zur Arbeitszeit zu sehen ist und für die spezifische Belastungssituation von Sportlehrenden von besonderer Bedeutung ist, sind die Unterrichtspausen. Wie sich in einer Interviewstudie von Wulk (1988) herauskristallisierte, stellen die Pausen für Lehrkräfte aller Fachrichtungen keine wahren Erholungszeiten dar. Die Lehrkräfte gaben an, dass meist Tätigkeiten mit einem hohen Belastungsgrad, wie z.B. Verwaltungsarbeiten, Gespräche mit Eltern oder 
Schülern oder das Vorbereiten von Experimenten bzw. der Folgestunden Inhalt der Pausen sind. So werden Phasen, die zur Erholung und zum Belastungsausgleich gedacht sind zu einer eigenen Belastungsquelle. In der Potsdamer Lehrerstudie gaben zwei Drittel der Befragten an, „die Pausen selten oder nie zur Entspannung nutzen zu können“ (Ksienzyk \& Schaarschmidt, 2005, S. 77). Für Sportlehrkräfte resultiert aus der Aufteilung des Unterrichts auf Klassen- bzw. Fachräume und auf die Sporthalle bzw. den Sportplatz ein zusätzlicher enormer Zeitdruck. Sportlehrkräfte haben stets die Aufgabe, die Schüler an bzw. in der Halle als Erster zu empfangen, Aufbauten und Hilfsmittel vorzubereiten, gleichzeitig aber auch bei eventuell auftretenden Streitereien in den Umkleideräumen einzugreifen, sich selbst umzuziehen und den Unterricht pünktlich zu beginnen (Frommel, 2006). Besonders anschaulich schilderte Frommel (2006, S. 242 f.) den Alltag einer Sportlehrerin:

Es ist 11.13 Uhr, Englisch in Klasse 5d. Mit schlechtem Gewissen beende ich den Unterricht 2 Minuten vor dem Läuten. In 7 Minuten beginnt der Sportunterricht in Klasse $5 d$, aber vorher gibt es noch viel zu tun: Ich muss das Klassenzimmer abschließen, bevor ich den Lauf zur Sporthalle beginne. Das ginge auch sehr schnell, wären da nicht Max und Dennis, die eine enorme Gelassenheit beim Zusammenpacken ihrer Unterrichtsmaterialien erworben haben. Ich versuche ruhig zu bleiben, sie trotzdem zur Schnelligkeit zu bewegen, indem ich schon mal den Schlüssel ins Schloss stecke. Geschafft! Mal sehen ob ich heute meinen Schnelligkeitsrekord einstellen kann. Mit Schultasche und CD-Player bepackt eile ich aus dem 3. Stock die Treppe hinunter, die Gänge entlang - es ähnelt einem Slalomlauf, schließlich befindet sich eine beträchtliche Anzahl der 1200 Schüler mit mir in den Gängen. Jetzt noch schnell ins Lehrerzimmer, den CDPlayer an den dafür vorgesehenen Platz abstellen. Hoffentlich will niemand der Kollegen etwas von mir. Glücklicherweise spricht mich niemand an, traut sich wahrscheinlich keiner bei dem gehetzten Anblick, der sich ihnen bietet. Ich verlasse das Schulgebäude, sprinte - soweit das eben mit meinen eleganten Schuhen geht - über den Schulhof, hinein in die Lehrerumkleide. Meine Sportkleidung habe ich schon heute Morgen bereitgelegt. Die Kassette mit der Aufwärmmusik nicht vergessen und hinein in die Sporthalle. Es ist 11:23 Uhr, nicht schlecht, es ist mir gelungen, vor den letzten Schülern (ich bemerke ungern, dass es meistens Schülerinnen sind, die etwas länger brauchen) in der Halle zu sein. [...] Es bleiben mir 35 Minuten für Aufwärmen, Aufbau, Spannungsübungen, Hauptteil, Abbau und Ausklang. 
Selbst Zeit für Toilettengänge, waschen oder umkleiden bleibt meist keine Zeit, sodass der sich anschließenden Unterricht unter wenig angenehmen hygienischen Bedingungen stattfindet oder eben verspätet beginnt. Präventives Verhalten von Lehrern, um Schwitzen zu vermeiden, wird von Schülern in Studien als negativ und weniger motivierend beschrieben und stellt daher ebenfalls keine Alternative dar (Rohnstock, 2000; Frommel, 2006). Für Sportlehrkräfte sind zudem häufig Springstunden im Stundenplan vorgesehen sowie zusätzliche Zeiten für das Training bzw. die Wettkämpfe von Schulmannschaften, sodass sich die schulischen Verpflichtungen meist bis weit in den späten Nachmittag ohne nennenswerte Pausen ausdehnen (Rohnstock, 2000).

Curriculare Vorgaben können für Lehrkräfte nicht nur eine Hilfe oder ein Orientierungsrahmen sein, sondern je nach Ausgestaltung, auch eine Quelle der Verunsicherung oder Einengung darstellen. Differenzen zwischen den gesetzten Lehrzielen und den tatsächlichen Leistungen bzw. dem Verhalten von Schülern führen zu einer Unzufriedenheit, die zur Belastung werden kann (Trachsler, 1990). Aufgrund der Zunahme der Heterogenität im motorischen Bereich und im sozialen Verhalten der Schüler wirken curriculare Vorgaben vor allem im Sportunterricht belastend und sind damit stresswirksam (Heim \& Klimek, 1999; Frommel, 2006; Gröbe 2006). Als Reaktion auf die desolaten Ergebnisse der PISA-Studie wurden bereits früh verbindliche Anforderungen in Curricula festgelegt, bevor eine umfassende fachdidaktische Diskussion über Chancen und Probleme stattfand. Sportlehrerende sehen sich derzeit mit komplett neuen und teiweise widersprüchlichen Vorgaben der Bildungsverwaltungen konfrontiert, wodurch Unsicherheit und Verwirrung entsteht (Aschebrock \& Stibbe, 2008). Während in der Stichprobe von Heim und Klimek (1999) curriculare Vorgaben nur eine zweitrangige Rolle im Stresserleben spielen, erreicht diese Subskala in der Untersuchung von Miethling und Brand (2004) in Baden-Württemberg mit Abstand den höchsten Wert. Ob die Ergebnisse durch die Tatsache zustande kommen, dass Miethling und Brand ausschließlich Sportlehrer in der ersten Berufsphase befragten und diese die Vorgaben durch den Lehrplan aufgrund fehlender Erfahrungen als verunsichernd wahrnehmen, ist unklar. Ebenso könnte es jedoch auch sein, dass in der Studie aus dem Jahr 2004 bereits die Lehrplanrevision im Land Baden-Württemberg Einfluss auf die perzipierte Belastung genommen hat. Ein weiteres Spannungsverhältnis resultiert aus dem curricularen Auftrag des Sportunterrichts, der neben einer möglichst intensiven Verwendung der Zeit für die sportliche Bewegung auch den Erwerb von sozialen Kenntnissen und Kompetenzen vorsieht. Meist sind hierfür die Bewegungszeit reduzierende Reflexionsphasen notwendig, die aufgrund der großen Stoffmenge zu einer dauerhaften Hektik und zeitlichen Problemen führt (Neumann, 2003). 
Wie bereits in Kapitel 2.3.2 dargestellt, befindet sich die gesellschaftliche Bewegungskultur in einem stetigen Veränderungsprozess sowohl in der Breite als auch in der Tiefe, vor dem sich auch der Sportunterricht nicht verschließen darf. Die Sache, die dem Lernenden vermittelt werden soll, stellt sich für heutige Sportlehrkräfte als reichlich diffus dar (Kugelmann \& Klupsch-SahImann, 2000). Die Sportlehrkraft muss selbst entscheiden, welche neuen Techniken und Übungen, Trendsportarten und sonstige Entwicklungen unter den Bedingungen der Schule sinnvoll und realisierbar sind. In diesem Zusammenhang ist der Auf- und Ausbau von Qualifikationen und Kompetenzen im Rahmen von Weiter- und Fortbildungsmaßnahmen unabdingbar (Gröbe, 2006). Fortbildungen werden aber vor allem durch junge Lehrer auch als Belastung wahrgenommen, da sie häufig viel ihrer privaten Zeit dafür investieren müssen. Meist werden solche Veranstaltungen als notwendiges Übel akzeptiert. Langfristig tragen Weiterbildungsmaßnahmen jedoch zur Reduzierung von Beanspruchungen durch die Steigerung der individuellen Kompetenz der Lehrkräfte bei (Rudow, 1995). Regelmäßige Weiterbildungsmaßnahmen dienen dazu, Qualifikationen zu erhalten und zu erweitern, verhindern das Verbleiben in Alltagsroutinen und bilden das Bewusstsein aus, dass unterrichtliches Handeln regelmäßig überprüft werden muss. Obwohl fast alle Lehrkräfte in Befragungen Fort- und Weiterbildungsmaßnahmen als sinnvoll einschätzen, ist das tatsächliche Engagement hingegen äußerst gering (Balster, 1990).

Die Klassengröße steht immer wieder im Zentrum bildungspolitischer Diskussionen, genau wie in Untersuchungen zur Belastung und Beanspruchung von Lehrern. Neben zu großen Klassen werden auch zu kleine Klassen als unangenehm wahrgenommen (Rudow, 1995; Czerwenka, 1996). Eine zu große Klasse stellt einen Faktor dar, der neben direkten Verbindungen zu Leistungs- und Verhaltensproblemen von Schülern auch in direkter Beziehung zum Lärmpegel im Unterricht zu sehen ist. In der SPRINT-Studie ergab die Auswertung der Antworten der teilnehmenden Lehrer, dass die Größe der Lerngruppe die am bedeutendste Einflussgröße für die Unterrichtsqualität im Sportunterricht darstellt (Oesterreich \& Heim, 2006). Sowohl in der Längsschnittstudie zu den Prädiktoren des Belastungserlebens im Lehrerberuf von Abele und Candova (2007) als auch in der Studie des Instituts für Demoskopie Allensbach (Süßlin, 2012) wird diesem Faktor das größte Belastungspotenzial beigemessen. In der Untersuchung von van Dick (2006) werden „zu große Klassen“ ebenfalls als Faktor mit dem größten Belastungspotenzial eingeschätzt. Selbst die in der Studie zur Lehrergesundheit von Schaarschmidt als besonders positiv, widerstandsfähig und ausgeglichen beschriebenen Lehrer des Verhaltensmusters Gesundheit (siehe Kap. 6.3.2.1), beschreiben große Klassen als belastend (Schaarschmidt, 2005a). Durchschnittlich bestehen die Lerngruppen an deut- 
schen Gymnasien aus 24 Schülern. Während sehr kleine ${ }^{3}$ und eher kleine ${ }^{4}$ Klassen mit insgesamt 19\%, genau wie sehr große ${ }^{5}$ Klassen mit 7\% geringe Häufigkeiten aufweisen, sind es immerhin $36 \%$ der Lerngruppen, die als gro $\beta^{6}$ zu bezeichnen sind. Die restlichen $38 \%$ entfallen auf die von Lehrern als am angenehmsten wahrgenommene mittlere $^{7}$ Gruppengröße (Oesterreich, 2008). Im Sportunterricht beinhaltet die Größe der Lerngruppe durch die spezifischen Sicherheitsanforderungen und dem meist stark begrenzten Platzangebot an der oft schlecht ausgestatteten Sportstätte ein im Vergleich zu den anderen Fächern höheres Belastungspotenzial. Der Unterricht wird folglich meist durch technische und organisatorische Maßnahmen bestimmt, die eine Daueraufmerksamkeit der Lehrkraft erfordern und einer individuellen Förderung von einzelnen Kindern entgegenwirkt (Frommel, 2006). In einigen Studien (Malanowsky \& Wood, 1984; Mclntyre, 1983) finden sich sogar lineare Zusammenhänge zwischen der Klassengröße und der Ausprägung von Burnout.

Die Verfügbarkeit von Lehr- und Lernmittel stellt ebenso einen Faktor dar, der im negativen Fall belastende Auswirkungen haben kann. Gehmacher (1980) stellt fest, dass sowohl der Mangel an Lehrmitteln als auch die schlechte Qualität selbiger eine sich unmittelbar auf die Qualität des Unterrichts auswirkende Belastungsquelle darstellt. Meist bleibt den Sportlehrkräften nichts anderes übrig, als selbst Zeit für die Herstellung von Lehr- und Lernmittel zu investieren bzw. solche käuflich zu erwerben. Die in den Schulen für den Sportunterricht verfügbaren Lehr- und Lernmittel z.B. Schautafeln mit Illustrationen von Bewegungsabläufen oder Lehrvideos sind meist in schlechtem Zustand und selten aktuell. Allein das technisch-modisch veraltete äußere Erscheinungsbild löst oft bei den Schülern Unruhe und Gelächter aus. Engagierte Lehrer müssen die in Eigenarbeit selbst hergestellten Lehrmittel immer mit in die Sporthalle bringen. Eine Deponierung in der Halle ist meist nicht möglich, da oft keine sicheren Lagermöglichkeiten vorhanden sind oder die Materialien an verschiedenen Standpunkten benötigt werden.

Eine meist unterschätzte Belastungsdimension stellen physische Belastungen im Rahmen der Lehrtätigkeit dar. Selbst für den Klassenunterricht wurde durch Messungen des Kalorienverbrauchs und der Herzfrequenz ermittelt, dass es sich beim Unterrichten um eine mittelschwere Tätigkeit handelt, die sich vor allem durch Belastungen des Halteapparates auszeichnet (Rudow, 1995). Entgegen dem Vorurteil: „Er macht sein

\footnotetext{
${ }^{3}$ Unter 15 Schüler.

${ }^{4} 16$ bis 20 Schüler.

${ }^{5} 31$ bis 40 Schüler.

${ }^{6} 26$ bis 30 Schüler.

${ }^{7} 21$ bis 25 Schüler.
} 
Hobby zum Beruf und hält sich im Sportunterricht fit" werden Sportlehrkräfte im Gegenteil physisch belastet, teils sogar geschädigt (Gröbe, 2006, S. 305). Im Vergleich mit den Lehrkräften anderer Fachrichtungen werden sie mit hohen Anforderungen an die persönliche Fitness konfrontiert. Neben der Demonstration von Bewegungen sind es vor allem Hilfestellungen und die Handhabung von Großgeräten bei Auf- und Abbau, die kraftraubend wirken (Rohnstock, 2000). Zusammenhänge zwischen der mangelnden Ausstattung der Halle, z.B. aufgrund veralteter Geräteausstattung und physischen Belastungen bzw. Verletzungen werden häufig berichtet. Nicht verwunderlich in diesem Zusammenhang ist die Tatsache, dass sich Sportlehrer mit einem überproportionalen Unterrichtseinsatz im Fach Sport physisch stärker belastet fühlen als die Kollegen, die einen geringeren prozentualen Anteil des Sportunterrichts an der Gesamtlehrverpflichtung aufweisen (Weiß, 2004). Gröbe (2006) führt zudem an, dass der körperliche Einsatz der Sportlehrer meist ohne ausreichende Erwärmung erfolgt und so mit zunehmendem Alter immer häufiger zu gesundheitlichem Verschleiß des aktiven und passiven Bewegungsapparats führt. Zusätzlich sieht er in den körperlich-motorischen Defiziten der Schüler eine erhöhte Beanspruchung für die Hilfestellung gebende Lehrkraft. Allgemein kann man der Aussage zustimmen, dass mit zunehmendem Alter die körperliche Beanspruchung ansteigt. Aufgrund der starken persönlichen Verbundenheit mit der eigenen Physis „erzeugt ein Körper, der nicht mehr leisten kann, was ihm bisher abverlangt wurde, Angst und Verunsicherung" (Kugelmann \& KlupschSahlmann, 2000, 6 f). In Langzeit-Herzfrequenzmessungen ermittelten Schönwälder et al. (2003) auffällig hohe Werte des Arbeitspulses bei Sportlehrkräften. Rohnstock (2000) vergleicht die durch Unterrichtsbeanspruchung ausgelösten Kreislaufwerte und den gestiegenen Hormonspiegel sogar mit denen von Formel-1-Rennfahrern. Auch Heim und Klimek (1999) sehen in körperlichen Beanspruchungen eine nicht zu vernachlässigende Belastungsquelle für Sportlehrer.

Die Sprechbelastung stellt eine weitere physische Belastungsquelle dar, die in engem Zusammenhang mit dem Lärmpegel während des Unterrichts zu sehen ist, der unter den arbeitshygienischen Bedingungen in Kapitel 3.3.2 aufgearbeitet wird. Neben den Geräuschen durch die Schüler, die aufgrund der Ventilfunktion des Sportunterrichts oft aufgedreht sind und Probleme haben, sich zu regulieren, kommen meist noch Geräuschbelastungen durch weitere Klassen hinzu (Rohnstock, 2000). Im Rahmen von Mehrfachbelegungen der Sporthallen findet der Unterricht von mehreren Klassen meist parallel statt. Die Trennwände stellen lediglich einen Sichtschutz dar, sodass der mit der eigenen Stimme bei verbalen Lehrinstruktionen zu begegnende Geräuschpegel enorm ist. Langfristig kann dies zu stimmlicher Überbeanspruchung in Form von Ent- 
zündungen des Rachenraumes, permanenter Heiserkeit und Stimmbandirritationen führen (Frommel, 2006). Der Sprechpegel sollte im Interesse der Lehrkräfte nicht über 60 Dezibel liegen, was, wie sich in Kapitel 3.3.2 zeigen wird, für den Sportunterricht kaum realisierbar ist (Krause \& Dorsemagen, 2007).

\subsubsection{Arbeitshygienische Bedingungen}

Bei den arbeitshygienischen Bedingungen ist meist der in der Klasse vorherrschende Lärmpegel der am häufigsten erforschte und bearbeitete Aspekt. Die Lautstärke im Unterricht wird sowohl durch von außen eindringende Geräusche, vor allem aber durch die Schüler und den Lehrer selbst bestimmt. Besonders in diesem Zusammenhang ist, dass die subjektiv wahrgenommenen lärmbedingten Belastungen von Lehrkräften auch in wissenschaftlichen Messungen bestätigt wurden. Die Bedeutung des Lärmpegels im Unterricht wird in verschiedenen Studien (Leuschner, 1979; Wulk, 1988; Sust \& Lazarus, 1997; Schönwälder et al., 2003) durch stets niedrige Positionen in den Rangreihen der Belastungsfaktoren unterstrichen. Nach der Arbeitsstättenverordnung sollte der Hintergrund-Schallpegel bei geistigen Tätigkeiten den Grenzwert von 55 Dezibel nicht überschreiten, wenn es sich um Organisationsformen mit Sprechen und Zuhören handelt, beträgt der Grenzwert sogar 35 Dezibel. Aufgrund der notwendigen Pegeldifferenz zwischen dem Hintergrundschallpegel und der Sprache von ca. 15 Dezibel ergibt sich ein Sprechpegel von maximal 50 Dezibel (Heyse, 2008). Sust und Lazarus (1997) fanden heraus, dass jede fünfte Lehrkraft einen Sprechpegel von mindestens 75 Dezibel erreicht (Heyse, 2008). Der durchschnittliche Geräuschpegel liegt im Sportunterricht bei etwa 90 Dezibel, Spitzenwerte, die bei über 100 Dezibel liegen, entsprechen dem Geräuschpegel, den ein Düsenjet in 200 Meter Entfernung erzeugt (GEWSportkommission, 2005). Der Vergleich dieser Werte mit den Vorgaben der Arbeitsstättenverordnung macht deutlich, wie erheblich die Lärmbelastung und damit auch die Sprechbelastung im Sportunterricht sind. Besonders hoch ist die Lärmexposition im Schwimmunterricht (Miethling, 2001). An industriellen Arbeitsplätzen wäre aufgrund arbeitsrechtlicher Bestimmungen bei einem dem Sportunterricht entsprechenden Lärmpegel, längst ein Gehörschutz zwingend vorgeschrieben (Rohnstock, 2000). Die Tatsache, dass im Jahr 2004 zum ersten Mal Lärmschwerhörigkeit als Berufskrankheit anerkannt wurde, unterstützt die These, dass Sportlehrkräfte unter äußerst kritischen Bedingungen ihrer Arbeit nachgehen (Hundeloh, 2005). Ein Zitat aus Interviews, die Allmer (1982) mit Sportlehrern führte zeigt deutlich, inwieweit Lärm, Klassengröße, Heterogenität und Lernerfolge bei den Schülern miteinander in Beziehung stehen. Durch die Kombination mehrerer Faktoren erweisen sich selbst einfache Aufgabenstellungen als schwer bewältigbar: 
Wir haben so eine Dreifachturnhalle. Wenn du da z.B. in der mittleren Halle bist und auf der einen Seite Ball gespielt wird und auf der anderen Seite auch, das ist ein ohrenbetäubender Lärm. Manchmal konnte ich mich nur verständigen, indem ich da in die Klasse hinein gebrüllt habe. Hinterher bist du dann ganz schön geschafft. Auf jeden Fall herrscht immer so eine Unruhe in der Klasse, es sind 36 Schüler. Was ich normal in drei Monaten übe, das schaffe ich in der Klasse in sechs Monaten. Jedes Mal sage ich am Ende: Mensch bin ich geschafft! Das Schlimmste war, dass bei 35 Schülern die Organisation so schwierig war und so viel Aufmerksamkeit erforderte, dass man das kaum schaffen konnte als einzelner Lehrer. Weil das unheimlich anstrengend war und man so viel Aufmerksamkeit haben musste für jede Gruppe und nicht nur für die Gruppen, sondern auch für jeden einzelnen Schüler. Wenn aber ein so richtiges Gefälle da ist, eine Hälfte ganz schlecht und die andere sehr gut, dann ist das natürlich sehr, sehr schwierig, die in einen Pott zu bringen (Allmer, 1982, S. 67).

$\mathrm{Zu}$ den unterrichtsfachspezifischen Belastungen zählen die Mehrfachanforderungen und der häufig wechselnde Ordnungsrahmen. Der Unterricht findet viel stärker als in anderen Fächern an Parallelschauplätzen statt, sodass der Alltag von Sportlehrern durch stetiges Orientieren gekennzeichnet ist. Zudem müssen zahlreiche Situationen aufgrund der hohen Interaktionsdichte des Unterrichts in Bruchteilen von Sekunden eingeschätzt werden, um eine umgehende Reaktion initiieren zu können. Der resultierende permanente Handlungs- und Orientierungsdruck führt dazu, dass der Sportunterricht als besonders intensiv und belastend wahrgenommen wird (Treutlein, Janalik \& Hanke, 1996). Im Vergleich zum Unterricht in der Klasse, in denen Stillarbeitsphasen von Lehrern als Entlastung wahrgenommen werden, kennzeichnet sich der Sportunterricht durch eine Dauerkonzentrations-Anspannung auf mehreren Ebenen:

Sie fungieren als Schiedsrichter, was Ihre gesamte Konzentration in Anspruch nimmt. Aber so ganz nebenbei werden Sie dann von einem Schüler befragt, der Ihnen eine kleine Wunde hinhält, ob Sie ein Pflaster hätten; und so ganz nebenbei weisen Sie einige zuschauend und sich kabbelnde Schüler zurecht; und sie passen außerdem auf, dass niemand unerlaubt die Halle verlässt und beantworten noch nebenbei einem anderen Schüler die Frage, welche Mannschaft als nächstes spielt. Natürlich haben sie immer noch das Spielgeschehen haargenau zu beobachten und ja kein Foul zu übersehen (Rohnstock, 2000, S. 108). 
Belastungen im Sportunterricht werden in besonderem Maße durch die Multidimensionalität und Simultanität der Aufgabenstruktur bedingt, sodass davon ausgegangen werden kann, dass sowohl eine additive als auch eine multiplikative Verknüpfung von Einzelbelastungen erfolgt (Rudow, 1995). Weiterhin gibt es kein anderes Schulfach, das sich durch ein höheres Maß an Unvorhersehbarkeit der Ereignisse auszeichnet, denn "was, wann, wo, warum, wie und ob überhaupt" im Sportunterricht passiert ist meist im Vorfeld überhaupt nicht absehbar (Klusmann et al., 2006). Oft stellen die gleichzeitig zu bewältigenden Aufgaben isoliert betrachtet keine nennenswerte Schwierigkeit dar und übersteigen die Personenressourcen nicht. Durch die zeitliche Bündelung und Kombination verschiedenster Tätigkeiten kann es jedoch zu erheblichen Problemen aufgrund der Verdichtung von Anforderungen und der stetigen unterschwelligen Belastung kommen (Czerwenka, 1996). Ein weiteres Spezifikum des Sportunterrichts, das als belastend wahrgenommen wird, ist das unangemessene Aufwand-Effekt-Verhältnis, denn trotz aufwendiger Bemühungen bleibt oft der intendierte Effekt aus (Allmer, 1982). Gerade was physische Leistungsparameter angeht ist der Einfluss des Sportunterrichts aufgrund der geringen Wochenstundenzahl unbedeutend. Die Erreichung der langfristigen Ziele des Sportunterrichts, die Erziehung zu Bewegung, Spiel und Sport im weiteren Lebensverlauf, liegt meist nicht mehr im Erfahrungsbereich der Sportlehrkräfte, sodass diese, was den eigentlichen Ertrag ihrer Arbeit angeht, meist mit leeren Händen dastehen.

Eine weitere Besonderheit des Faches Sport ist, dass der Unterricht neben der Öffentlichkeit, die bereits durch die Klassensituation gegeben ist, zusätzlich vom Frühjahr bis zum Herbst oft außerhalb der Halle stattfindet, sodass deren Schutz nicht mehr gegeben ist. Der Sportunterricht wird von Außenstehenden oft als oberflächlich eingeschätzt und Einzelsituationen ohne Kenntnisse der Schüler- und Lehrerhandlungen bewertet. Auf Seiten der Lehrer entsteht ein stetiger Druck, die eigenen Kompetenzen gegenüber der Öffentlichkeit unter Beweis stellen zu müssen (Gröbe, 2006).

Belastungen durch die klimatischen und hygienischen Bedingungen sind im Sportunterricht stärker ausgeprägt als in anderen Fächern. Während der Unterricht im Winter bei niedrigen Temperaturen in einer meist zugigen Halle stattfindet, gleichen die Sporthallen im Sommer einem Brutkasten, der sich durch eine hohe Luftfeuchtigkeit aufgrund mangelnder Belüftung kennzeichnet. Weiterhin klagen Lehrer nicht nur über fehlende Zeit für den Toilettengang, selbiger kostet oftmals zusätzlich ein gehöriges Maß Überwindung, aufgrund des teils katastrophalen hygienischen und bautechnischen Zustands der Sanitäranlagen von Sporthallen. Wie bereits in Kapitel 3.3.1 dargestellt leiden Sportlehrkräfte unter einem enormen Zeitdruck, sodass auch die persönliche Hy- 
giene oft vernachlässigt werden muss. Nur $18 \%$ der Sportlehrenden geben an, dass die Zeit zwischen dem Sportunterricht und dem Klassenzimmerunterricht ausreicht, um sich zu waschen und umzuziehen, oft unterrichten sie zwangsläufig auch in den Klassenräumen in ihrer Sportkleidung (Frommel, 2006).

Ein weiterer für das Fach Sport spezifischer belastender Faktor stellt die stetige Verletzungsgefahr der Schüler und die damit verbundene Anspannung bei der Übungswahl und den Sicherungsvorkehrungen dar (siehe Kap. 3.3.1). Die Studie von Dallermassen (1986) zeigt, dass Verletzungen, Unfälle und Sicherheitsvorkehrungen vordere Plätze in den Problembereichen des Sportunterrichts einnehmen und häufig als schwierige Unterrichtssituationen wahrgenommen werden (Frommel, 2006).

Ich hatte ein Basketballspiel zu leiten und habe das gar nicht mitgekriegt und plötzlich hört man nach Sekunden einen Schrei und das nimmt mich dann doch jedes Mal mit. Da liegt erst einmal ein Schüler, schreit. Ob er jetzt vom Trampolin gefallen ist oder sonst wie oder auch nur vom Kasten gefallen ist, man hat doch ständig Angst: was ist es? (Allmer, 1982, S. 71).

Aufgrund der zunehmenden Verschlechterung der sensorischen und motorischen Eigenschaften von Schülern wird der Lehrer immer häufiger mit Situationen konfrontiert, die eine beständige Einschätzung von Gefahrenmomenten unabdingbar machen (Rohnstock, 2000). Unterstützt wird diese These durch die gestiegenen Unfallzahlen im Sportunterricht, bei gleichzeitig rückläufiger Anzahl an erteilten Sportstunden. Da in der Ausbildung das Thema Unfälle im Schulsport nur nebensächlich behandelt wird, sind Sportlehrkräfte meist nicht richtig vorbereitet und weisen ein grundsätzlich schüler- und zufallzentriertes Unfallverständnis auf, was zu dauerhaften Beanspruchungssituationen durch Hilflosigkeit und Überforderung führt. Die Sportlehrkräfte erleben sich selbst als machtlos im Entstehungsprozess von Unfällen, wodurch dieses Thema oft tabuisiert wird. Eine reflexive, unvoreingenommene und konstruktive Analyse von Unfällen stellt eine wichtige Voraussetzung für die Verbesserung der Unterrichtsqualität und der Sicherheit im Schulsport dar und sollte sowohl in Aus- als auch Weiterbildungsmaßnahmen eine zentrale Stellung einnehmen (Hundeloh, 2005). Neben dem Unterrichten, Erziehen und Beraten gibt es zudem eine Reihe weitere Aufgaben, die Sportlehrkräfte zu erfüllen haben. Hierzu gehört z.B. die Überprüfung, ob die Schüler angemessene Sportkleider und Schuhe tragen und ob Schmuck und Uhren abgelegt wurden, da dies unmittelbaren Einfluss auf die verantwortbare Teilnahme am Unterrichtsgeschehen hat (Frommel, 2006). Vor allem bei räumlich weitläufigen Organisationsformen ist eine genaue Planung und Instruktion der Schüler bezüglich der Verhaltensregeln sowie die Überprüfung deren Einhaltung notwendig. In einer konkreten 
Unfallsituation müssen Lehrkräfte die Ernsthaftigkeit der Verletzung einschätzen, notwendige Hilfs- bzw. Rettungsmaßnahmen einleiten und gleichzeitig weiterhin die Verantwortung für den Rest der Gruppe tragen (Voltmann-Hummes, 2008). Nicht nur in Unfallsituationen, sondern auch im normalen Alltagsbetrieb sind Sportlehrkräfte in höherem Maß als andere Lehrkräfte von Infektionen mit Krankheiten bedroht. Hilfestellungen oder Geräteaufbauten erfordern meist den direkten Körperkontakt mit Schülern, der, in Verbindung mit fehlenden oder schlecht ausgestatteten Waschgelegenheiten, die Weitergabe von Krankheiten, die sich über Tröpfchen- oder Schmierinfektion ausbreiten, begünstigt.

Eine weitere Quelle potenzieller Belastungen stellt der außerunterrichtliche Schulsport dar, der sich schnell als Arbeitsfeld ohne Grenzen herausstellt. Neben dem verbindlichen Sportunterricht gibt es an vielen Schulen ein Angebot an Sportaktivitäten, die neben individuellen Fördermaßnahmen, vor allem Interessens- und Neigungsgruppen umfassen. Schulsportliche Wettkämpfe, Spiel- und Sportfeste, Pausensport und einoder mehrtägige Veranstaltungen mit sportlichem Schwerpunkt sind Formen des außerunterrichtlichen Schulsports, der abhängig vom sportpädagogischen Profil der Schule und dem Willen der Schulgemeinde mehr oder weniger stark ausgeprägt ist. Während vielfach angenommen wird, dass die Organisation von Sportveranstaltungen auf Schulfesten und die Betreuung von Mannschaften bei schulsportlichen Wettkämpfen das private Hobby von Sportlehrkräften sei, nehmen diese, die teils in Schulgesetzen und Verordnungen vorgeschriebenen Aufgaben als einen Bereich wahr, in dem harte Arbeit geleistet werden muss, die durchaus belastendes Potenzial beinhaltet. Neben der Auswahl der Schüler, der Organisation der Anreise und dem meist großem zeitlichen Aufwand kommt der Druck durch den Lehrplan hinzu, der aufgrund des Unterrichtsausfalls des regulären Unterrichts entsteht (Stündl \& Zimmermann, 2006).

Der Zustand der Sportstätten und der dort vorhandenen Geräte und Sportmaterialien erfordern von Lehrkräften in Zeiten von Sparmaßnahmen der Kommunen und der Bildungsverwaltung Flexibilität und Kreativität (Frommel, 2006). Der geringe Schuletat geht häufig zu Lasten der materiellen Ausstattung der Sportstätten, was sowohl den Ersatz fehlender bzw. defekter Geräte und Materialien sowie die Anschaffung neuartiger Sportmaterialien betrifft. Dadurch, dass mehr Schüler unter schlechteren Bedingungen unterrichtet werden, kommt es zu einer höheren Auslastung der Sportstätten durch Hallenmehrfachbelegungen, die neben der bereits beschriebenen Lärmbelastung auch zu weiteren gravierenden Organisationsschwierigkeiten führen (Miethling, 2000). Sowohl nicht vorhandene als auch beschädigte Materialien führen dazu, dass man Lernumwege in Kauf nehmen oder andere Geräte und Materialien zweckent- 
fremden muss. Auch Kleingeräte, die sich nicht auf ihrem Platz befinden, können durch die notwendige Suche und die in dieser Zeit unbeaufsichtigte Klasse zu unnötigen Drucksituationen führen. Meist sind solche ungeordneten Materialräume die Folge des Zeitdrucks, unter dem Lehrer Auf- und Abbauten meist selbst bewerkstelligen müssen oder aber sie sind durch externe Nutzer wie z.B. Vereine oder sonstige Sportgruppen verursacht worden (Frommel, 2006). Oesterreich (2008) fand heraus, dass 10 bis $25 \%$ der Lehrer die Sportstättensituation als schlecht einschätzen und dadurch eine starke Einschränkung der Unterrichtsqualität wahrnehmen. Im Gegensatz zu den zuvor dargestellten Ergebnissen ergab die Befragung von Heim und Klimek (1999), dass organisatorische und räumliche Rahmenbedingungen eine zweitrangige Rolle in der Belastungswahrnehmung spielen.

Ein weiteres Problem für Sportlehrkräfte stellt die meist räumliche Trennung der Sporthalle bzw. des Sportplatzes und dem Schulgebäude dar. Die Lehrer müssen dafür Sorge tragen, dass die Schüler rechtzeitig und sicher im sich anschließenden Unterricht erscheinen. Hierdurch wird die oft schon sehr knapp bemessene Zeit des Unterrichts zusätzlich verkürzt. Durch die schlechte Ausstattung der Sportstätten kommt es auch dazu, dass teilweise Materialien von einer Sportstätte zur anderen transportiert werden müssen, wofür meist Pausen oder Freistunden verwendet werden (Frommel, 2006).

\subsubsection{Soziale Arbeitsbedingungen}

Unter den sozialen Arbeitsbedingungen sind die Beziehungen zwischen Lehrern und allen, in irgendeiner Form am Unterricht beteiligten bzw. den Unterricht beeinflussenden Personen, subsummiert. Hauptaugenmerk bei der Betrachtung dieser Belastungskategorie soll auf den Beziehungen von Lehrkräften zu den Schülern, zu den Kollegen, zu der Schulleitung, zu den Eltern und zu der Schulbehörde liegen. Weitere, wenn auch nur in geringerem Maße soziale Einflussgrößen, die belastend wirken können, stellen Hausmeister, Sekretärinnen, externe Fachkräfte, Sozialarbeiter und Pädagogen sowie Betriebe und Vereine dar, mit denen Lehrer zuweilen in Kontakt treten. Ein für den Lehrerberuf häufig verwendeter Begriff lautet Einzelkämpfertum, worin zum Ausdruck kommt, dass neben der Unterrichtssituation, in der Lehrkräfte alleine in einem geschlossenen Raum mit vielen Kindern arbeiten, auch im sonstigen Berufsleben fachliche und menschliche Unterstützung sowie Anerkennung oft nicht gegeben ist (KramisAebischer, 1995; Barth, 1997).

Hauptinteraktionspartner von Lehrern stellen die Schüler dar und der Sozialkontakt zu diesen wird oft als vorrangige Belastungsquelle angegeben (Ulich, 1996; Kraemis- 
Aebischer, 1996; Heim \& Klimek, 1999; van Dick, 2006, König, 2008). Probleme und Konflikte mit und zwischen Schülern treten vor allem dann auf, wenn diese in näheren Kontakt zueinander treten, kooperativ handeln sollen oder sich genau an vorgegebene Regeln und Vorschriften halten müssen. Der Sportunterricht stellt durch die direkte und indirekte Interaktion der Schüler untereinander in den Umkleidekabinen, bei Hilfestellungen oder im sportlichen Tun ein Schulfach dar, in dem deutlich engere und intensivere Kontaktmöglichkeiten als in anderen Fächern bestehen, was das Konfliktpotenzial erhöht. In Mannschaftsspielen und Partner- bzw. Gruppenübungen stellt der Sportunterricht einen hohen Anspruch an die Kooperation der Schüler, da oft der Erfolg vom Zusammenspiel aller abhängig ist. Weiterhin stellen Regeln und Vorschriften beim Spielen, Aufbau oder dem Transport von Geräten Nährboden für Konflikte dar (Rohnstock, 2000). Wolters (2008) sieht die Ursache vieler Konflikte in den schwierigen Lebenslagen, in denen Schüler aufwachsen und daher im System Schule Schwierigkeiten haben, sich auf Lernprozesse einzulassen. Gerade für Schüler, die es nicht gewöhnt sind, sich über längere Zeit in kognitiv geprägten Phasen zu konzentrieren, nimmt der Sportunterricht eine wichtige Ausgleichs- und Ventilfunktion ein, kann sich jedoch auch zu einem eigendynamischen, kaum noch steuerbaren Handlungsprozess entwickeln (Miethling, 1989).

In der Studie von Heim und Klimek (1999) werden den Probleme bei der Interaktion mit Schülern besonderes Gewicht zugesprochen und die Zusammenhänge zu BurnoutFaktoren wie Depersonalisierung oder psychosomatischen Beschwerden als durchaus signifikant und markant beschrieben. Aufgrund der Bedeutsamkeit des Einflussfaktors Schüler ist in ihrem Instrument zur Erfassung fachunterrichtlicher Stressoren mit den Subskalen "Mangelnde Disziplin der Schüler" sowie "Motivations- und Benotungsprobleme“ ein Großteil des Erhebungsinstruments hierdurch geprägt. Auch bei van Dick (2006) bewerteten die Lehrkräfte „mangelnde Motivation bzw. Konzentrationsfähigkeit der SchülerInnen“, "geringe Lernbereitschaft der SchülerInnen“ sowie „Disziplinprobleme“ im Vergleich zu den anderen Faktoren als überdurchschnittlich belastend. Neben Disziplin- und Motivationsschwierigkeiten, allgemeiner Unruhe und Desinteresse beklagen Lehrer meist auch die Einstellung der Schüler zum Lerngegenstand und der Schule insgesamt (König, 2008). Meist wird es als belastender erlebt, Schüler zu motivieren, die wenig Interesse zeigen, als mit Verhaltensauffälligkeiten konfrontiert zu werden (Kramis-Aebischer, 1996). Im Geschlechtervergleich fällt auf, dass Lehrerinnen sich deutlich mehr durch schülerspezifische Probleme belastet fühlen als Lehrer, was nach Schaarschmidt (2005a) daran liegen kann, dass Frauen eher mit emotionaler Labilisierung und Resignation auf Belastungen reagieren und sich Verhaltensprobleme 
zu Herzen nehmen, wohingegen Männer verstärkt abwehrende Strategien wie z.B. Zynismus oder Desengagement anwenden (Ulich, 1996). Lehrerinnen sehen in dem Fehlverhalten der Schüler immer auch einen gewissen Anteil an eigener Schuld und neigen zu Selbstzweifeln (Ulich, 1996). Gerade in der Pubertät sind störende Verhaltensweisen oft nicht durch den Lehrstil bedingt, sondern stellen entwicklungsbedingte Phänomene dar, die in der Bewertung von Unterrichtsstörung berücksichtigt werden müssen. Nimmt ein Lehrer das Desinteresse und die Abwehr von Seiten der Schüler hingegen persönlich, entsteht Stress (Barth, 1997). Wenn im Unterricht Disziplinprobleme auftreten, sind diese nicht ausschließlich isoliert für sich zu sehen, sondern können Ausdruck von fehlender Motivation, Vergesslichkeit, fehlendem Können oder fehlender Einsicht sein (Treutlein, Janalik \& Hanke, 1996).

Eine weitere Subkategorie der Belastung, bedingt durch den Umgang mit den Schülern, ist das stark heterogene allgemeine Fähigkeitsniveau der Schüler, welches durch extreme Leistungsunterschiede geprägt ist (siehe Kap. 3.3.1). Im Sportunterricht ist die Zunahme der Heterogenität nicht nur im körperlich-motorischen Bereich, sondern vor allem auch im sozialen Bereich festzustellen, was zu einem stetigen Anstieg der Belastungen führt (Dordel, 2000; Gröbe, 2006). Im Vergleich zu früher schätzen Lehrer die Schüler als deutlich unruhiger, unkonzentrierter und weniger ausdauernd ein, wenn es darum geht, länger an einer Sache festzuhalten. Schüler streben vermehrt nach Action und Abwechslung und sind für bestimmte Inhalte, wie z.B. Turnen kaum zu motivieren (Miethling, 2001). Gesellschaftliche Veränderungen werden in die Schule getragen und Lehrkräfte werden zunehmend im Unterricht mit verhaltensauffälligen Schülern konfrontiert (Krause \& Dorsemagen, 2007). Grundlegende Regeln des Umgangs mit Gleichaltrigen und Erwachsenen werden nicht eingehalten und Lehrende müssen einen großen Anteil ihrer Unterrichtszeit für die Schaffung grundlegender Unterrichtsvoraussetzungen investieren. In der SPRINT-Studie stellte sich heraus, bezogen auf den Bereich Disziplin und Zeitnutzung, dass Lehrer in allen Altersstufen lange warten müssen, bis Ruhe in den Klassen eingekehrt ist und mit zunehmendem Alter die Schüler häufig zu spät im Unterricht erscheinen (Gerlach et al., 2006). Im Unterricht sehen sich Sportlehrer oftmals Strukturen gegenüber, die von extremer Heterogenität im motorischen Bereich geprägt sind. Auf der einen Seite müssen Lehrkräfte versuchen, Schüler, die nicht einmal die grundlegenden alterstypischen konditionellen und koordinativen Voraussetzungen mitbringen, möglichst gut zu fördern und auf der anderen Seite muss auch den Schülern mit überdurchschnittlichem Leistungsniveau ein attraktives Bewegungsangebot offeriert werden (Gröbe, 2006). Durch umfangreiche Differenzierungs- 
maßnahmen muss der Sportlehrer dafür sorgen, möglichst passende Herausforderungen zu schaffen, um weder die Schüler zu unter- noch zu überfordern (Wolters, 2008).

80\% der Sportlehrer sehen insgesamt eine Verschlechterung der motorischen Eigenschaften der Schüler in den letzten Jahren im Vergleich zu früheren Schülergenerationen (Rohnstock, 2000; Miethling, 2001). Alles in allem wird durch die Lehrerangaben der SPRINT-Studie deutlich, dass Schüler die Unterrichtsqualität am stärksten beeinflussen. Die mangelnden motorischen Fähigkeiten werden in dieser Studie jedoch als deutlich beeinträchtigender eingeschätzt als die eher nachrangigen Faktoren Disziplinund Motivationsprobleme (Oesterreich \& Heim, 2006). Eine weitere Besonderheit des Sportunterrichts ist, dass Schüler mehr als in anderen Fächern Interessen, Wünsche und Vorstellungen, bezogen auf den Inhalt und den Ablauf des Sportunterrichts aufweisen und auf deren Umsetzung drängen. Oftmals konfligieren jedoch diese Schülervorstellungen mit denen der Lehrkraft bzw. mit den Lehrplanvorgaben und es resultieren Konflikte und Spannungen, die sich letztendlich auf alle Bereiche des Sportunterrichts auswirken (Wolters, 2008). Auch in der Interviewstudie mit Lehrern von Ulich (1996), nehmen Leistungs- und Verhaltensprobleme in der Rangreihe beruflicher Belastungen den ersten Platz ein, d.h. diese Probleme wurden von den Lehrern am häufigsten genannt. In den Interviews wurde deutlich, dass sowohl ein weit auseinanderliegendes Leistungsspektrum innerhalb der Klasse als auch Disziplin- und Motivationsprobleme bedeutsame Belastungsquellen darstellen.

Das Verhältnis zu den Schülern wird jedoch nicht ausschließlich als negativ wahrgenommen, da der Umgang mit jungen Menschen von einem Großteil der Lehrkräfte als ein bedeutsamer Vorteil des Berufs angesehen wird (Rudow, 1995). Auch in der Erhebung von Kramis-Aebischer (1996) stellte sich heraus, dass die Anstrengungen, die von Seiten der Lehrkraft zu erbringen sind, um eine gute Beziehung unter den Schülern zu fördern, nur von etwa jeder dritten Lehrkraft als belastend wahrgenommen wird. Das Verhalten, die Leistung oder auch die Anerkennung und Zuneigung, die Schüler im Unterricht gegenüber der Lehrkraft zeigen, sind primäre Quelle des beruflichen Erfolgserlebens von Lehrern (Ulich, 1996).

Der Umgang mit Eltern wird von Lehrern zunehmend als belastend wahrgenommen und ist meist von Spannungen geprägt. Neben fehlendem Interesse, Unverständnis und übergroßem Engagement nehmen Lehrkräfte auch Einmischungen in den Unterricht von Eltern als belastend wahr. Das Haupthindernis für einen engeren kooperativen Umgang mit Eltern sehen Lehrkräfte in der eigenen mangelnden Qualifikation. Besonders problematisch scheint das Verhältnis zu den Eltern im Grundschulbereich zu sein, was sich in einer Studie von Terhart (1993, zitiert nach Ulich, 1996) herausstellte, 
in der fast $40 \%$ der Lehrer angeben, durch den Kontakt zu Eltern belastet zu sein. An weiterführenden Schulen sind es weniger als 20\%, die den Umgang mit Eltern als belastend einschätzen. Ein ungetrübtes und durchweg positives Verhältnis zu Eltern stellt heutzutage die Ausnahme dar (Ulich, 1996). Während früher die Lehrenden sich auf die Unterstützung der Elternschaft verlassen konnten, berichten Lehrer in der letzten Zeit häufig von Eltern, die bedenkenlos Partei für ihre Kinder ergreifen und Erziehungsaufgaben an die Lehrkräfte delegieren (Miethling, 2001). Eltern hegen oft einen ausgeprägten Anspruch an die Ausbildung ihrer Kinder und fordern häufig genaue Notenbegründungen (Ulich, 1996). Sie tauchen in der Schule meist nur auf, wenn es Probleme gibt, eine wirkliche Zusammenarbeit mit den Eltern scheint es aber nicht zu geben, wodurch die Lehrer sich oft ziemlich allein gelassen fühlen (Barth, 1997). Die Ergebnisse der Studie von van Dick (2006) belegen, dass sich etwa die Hälfte der Lehrer durch den Umgang mit Eltern belastet fühlt. Besondere Geringschätzung bringen die Eltern offensichtlich dem Sportunterricht entgegen, was in dem großzügigen und bedenkenlosem Ausstellen von Entschuldigungen für die Befreiung vom Sportunterricht Ausdruck findet. Während die Anerkennung von Sportlehrern in der Schülerschaft meist deutlich ausgeprägt ist, sehen Eltern in ihnen Personen, die ihrem Hobby im Beruf nachgehen und keinen bedeutsamen Beitrag zur Allgemeinbildung ihres Kindes leisten (Voltmann-Hummes, 2008).

Mangelnde Unterstützung im Kollegium stellt einen weiteren Faktor dar, der als bedeutender Aspekt beruflicher Belastung angesehen wird. Das zum Teil von Konkurrenzdenken geprägte Verhältnis von Kollegen untereinander, erzeugt häufig Disharmonien, die überdauernd und auch im eigenen Unterricht als belastend wahrgenommen werden (Ulich, 1996). Etwa die Hälfte der Lehrer empfindet das Klima im Kollegium als belastend und fühlt sich alleine und verunsichert (Kramis-Aebischer, 1996). Dies zeigt auch die folgende Aussage eines Lehrers:

Belastend wirkt auf mich, wenn die Atmosphäre im Lehrerzimmer unter Kollegen nicht gut ist; wenn ich merke, dass ich für allgemeine Erziehungsaufgaben keine Stärkung habe im Kollegium, dass ich also alleine unterwegs bin (Ulich, 1996, S. 147).

Behrens-Tönnies \& Tönnies (1986) konnten zwischen der Qualität kollegialer Beziehungen und dem Belastungsempfinden von Lehrern Zusammenhänge ermitteln. Je mehr sich Lehrer von ihren Kollegen geachtet fühlen, desto geringer ist das Belastungserleben. Auch in der Potsdamer Lehrerstudie fand man heraus, dass an Schulen, die sich durch günstige Beanspruchungsverhältnisse auszeichnen, stets auch ein gutes 
soziales Klima herrscht (Schaarschmidt \& Kieschke, 2007). Auch die Geringschätzung bezüglich der Arbeit der Sportlehrenden und die daraus resultierende geringe Reputation im Kollegium stellt eine nicht zu unterschätzende Belastungsquelle dar. Meist haben die Kollegen aus den anderen Fächern lediglich eine vage Vorstellung darüber, was die Arbeit im Sportunterricht eigentlich ausmacht und orientieren sich an dem unvollständigen Bild, das durch die Medien, die Elternschaft und die Schulverwaltung gezeichnet wird (Thomann, 2006). Lehrerinnen fühlen sich generell durch die geringe Statusperzeption deutlich mehr belastet als ihre männlichen Kollegen und verspüren einen erhöhten Legitimationsdruck sowie geschlechtsspezifischen Diskriminierungen (Scheffel \& Palzkill, 1994; Firley-Lorenz, 1994). In einer Studie von van Dick (2006) machten etwa $10 \%$ der Lehrkräfte Angaben, die auf Mobbing im Kollegium bzw. durch die Schulleitung hindeuten. Trotz der Tatsache, dass das soziale Klima und die gegenseitige Unterstützung und Solidarität im Sportkollegium offensichtlich meist als angenehm bezeichnet wird bzw. gegeben scheint, definieren Sportlehrer sich meist über ihr Zweitfach aufgrund der dort entgegengebrachten höheren Wertschätzung (Miethling, 2001). Entgegen den zuvor dargestellten Ergebnissen aus der Interviewstudie von Miethling werden in quantitativen Studien (Heim \& Klimek, 1999; Miethling \& Brand, 2004) die Items der Subskala „Probleme in der Interaktion mit Kollegen“ hingegen als so belastend eingeschätzt, dass dieser Bereich nach der Subskala "Unangemessene curriculare Vorgaben" als am meisten belastend wahrgenommen wird.

Die Schulleitung hat einen beträchtlichen Einfluss auf das Kollegiums- und Schulklima und damit auch auf jede einzelne an der Schule beschäftigte Lehrkraft (Ulich, 1996). Das Verhältnis zu der Schulleitung wird von Sportlehrern meist als eher distanziert beschrieben. Die Organisationskompetenz der Sportlehrer wird für die Außendarstellung oft genutzt, die Anerkennung, dass Sportlehrer sich ebenso beansprucht fühlen wie Kollegen, die ein Korrekturfach unterrichten, bleibt jedoch aus (Miethling, 2001; Thomann, 2006). Van Dick (2006) fand heraus, dass die Schulleitung die am wirkungsvollste Unterstützungsquelle darstellt, zu der jedoch ein ambivalentes Verhältnis besteht, da etwa die Hälfte der Antwortenden keine bzw. mangelnde Unterstützung im praktischen und emotionalen Bereich beklagt. Ein Großteil der Lehrer nehmen die Beziehung zur Schulleitung als belastend wahr, da sie sich weder als gleichberechtigte Partner fühlen, noch bei wichtigen Entscheidungen mit einbezogen werden und sich zudem mangelhaft unterstützt fühlen. Diese Unzufriedenheit mit dem Führungsstil nimmt vordere Ränge in den als belastend wahrgenommenen Faktoren von Lehrern ein (Rudow, 1995; Jehle \& Schmitz, 2007). Weiteren Ausdruck findet die schulinterne Geringschätzung des Faches zudem darin, dass bei Krankheit bzw. Verhinderung des Sport- 
lehrers im Fächervergleich am wenigsten vertreten wird und die Sportstunde entweder ausfällt oder in Form von Klassenunterricht vertreten wird. Ärger und Probleme mit den Schulbehörden sehen etwa 30\% der Lehrer als belastend an (van Dick, 2006). Wulk (1988) kommt zu dem Schluss, dass ein ungünstiges Führungsklima für die hohe Belastungsbewertung von Vorschriften, Gesetzen und Erlassen verantwortlich ist. Lehrpersonen fühlen sich durch die strukturellen und organisatorischen Vorgaben vor allem aber auch durch die Schulaufsicht selbst in ihrer Freiheit eingeschränkt.

\subsubsection{Gesellschaftlich-kulturelle Bedingungen ${ }^{8}$}

Das Bild des Lehrers in der Gesellschaft ist gekennzeichnet durch den steigenden Erwartungsdruck und eine Ausweitung des Aufgabenspektrums auf der einen Seite und fehlender Anerkennung auf der anderen Seite. Lehrer nehmen eine zentrale Rolle für die Zukunft der nachfolgenden Generationen ein, indem sie soziale Probleme korrigieren, Wissen vermitteln und die individuelle Entwicklung von Kindern und Jugendlichen fördern, genießen jedoch nicht die Anerkennung, die ihnen eigentlich zugestanden werden müsste (Barth, 1997). Das Ausmaß der ungerechtfertigt geringen öffentlichen Anerkennung des Lehrerberufs wird anhand eines Stoßstangenaufklebers aus den Vereinigten Staaten von Amerika mit dem Schriftzug "If you can read this, thank a teacher" deutlich. Eine Berufsgruppe die essenzielle Basisqualifikationen vermittelt wie Lesen, Schreiben und Rechnen, die eine wichtige Rolle in der Entwicklung und für den Fortbestand einer Kultur einnimmt, scheint in der Gesellschaft völlig ruhmfrei zu sein (Buski, Benson \& Sikorski, 2005). Bezeichnet man das Verhalten von jemandem als lehrerhaft, so ist diese Attribuierung eindeutig pejorativ (Tenorth, 2006). Auch Preusker, zitiert nach Arnhardt et al., 2000, S. 76) äußert sich unter Berufung auf Herder ähnlich über den Lehrerberuf und dessen Anerkennung: „Wessen Amt ist notwendiger und verdienstvoller für den Staat als das seinige, und welches ist, wie das seinige, so oft ohne Lohn und Ehre?"

Bisher liegen kaum Untersuchungen vor, die sich mit dem Zusammenhang von gesellschaftlich-kulturellen Bedingungen und deren belastenden Wirkungen auf Lehrer beschäftigen und nur vereinzelt werden solche Aspekte in wissenschaftlichen Erhebungen berücksichtigt (Krause, 2002). Czerwenka (1996) bezieht beispielsweise die von den Lehrern empfundene Belastung durch die abwertende Einschätzung ihrer Arbeit durch die Öffentlichkeit in seiner Untersuchung mit ein. Von einem Großteil der Lehrer wird das schlechte Bild, das in der Öffentlichkeit über den Berufsstand existiert, als belastend wahrgenommen. Etwa drei Viertel der Lehrer belastet es, dass ihnen nach-

\footnotetext{
${ }^{8}$ Weitere Ausführungen zum Ansehen der Lehrer in der Gesellschaft, in der Literatur, im Film und in der Musik siehe Kapitel 2.2.4.
} 
gesagt wird, wenig zu arbeiten und viel Freizeit zu haben. Analysen von Medienberichten, die sich mit Lehrkräften beschäftigen, ergaben, dass die meisten Berichte negativ geprägt sind und gängige Klischees bedient werden (Blömeke, 2005). Besonderes Belastungspotenzial birgt auch die Tatsache, dass offensichtlich in der Gesellschaft differenziert wird zwischen dem schwierigen Lehrerberuf und denjenigen, die den Beruf ausüben, zu denen überwiegend negative Attribuierungen vorliegen (siehe Kap. 2.2.4). Folglich geben $79,9 \%$ der Lehrer an, nie oder nur selten mit dem Status des Berufes zufrieden zu sein (Farber, 1991). Vorurteile, die in der Gesellschaft über Lehrer kursieren und die letztendlich auch zu den Bezeichnungen Halbtagsjobber oder faule Säcke führten, scheinen in der öffentlichen Meinung auf keinen Lehrer so zuzutreffen wie auf den Sportlehrer, in dessen Unterricht es ja offensichtlich mehr um Spaß und Spielen, als um schulisches Lernen geht (Thomann, 2006). Selbst in wissenschaftlichen Abhandlungen wird der Sportunterricht als ein Fach mit überwiegend niedrigem Belastungspotenzial gesehen, da keine Korrekturarbeit anfalle und Konflikte im Sportunterricht weit weniger ins Gewicht fallen würden als im Klassenzimmerunterricht (Wendt, 2001).

Sportlehrer? Immer braungebrannt, strahlend vor Energie und Tatkraft, nach der Schule schnell zum Tennis spielen (neuerdings auch Golf), und am letzten Tag vor den jeweiligen Ferien mit gepacktem Wohnmobil (Surfbretter auf dem Dach) morgens zum Unterricht, damit man bei Schulschluss sofort aufbrechen kann, bevor der große Stau kommt (Thomann, 2006, S. 206).

Von Sportlehrern werden solche pauschalisierten Ansichten, gerade aufgrund der zuvor in diesem Kapitel bereits dargestellten hohen Anforderungen, die teilweise sogar objektiv stark gesundheitsgefährdeten Charakter haben, als besonders belastend wahrgenommen. Selbstverständlich kann man ebenso wenig in die andere Richtung pauschal annehmen, dass keiner der Sportlehrer, dem in dem Zitat beschriebenen landläufigen Meinungsbild entspricht. Diejenigen, die jedoch ihren Beruf mit Einsatz, Liebe und Herzblut verfolgen, sehen die Prinzipien der Equity-Theorie, nach der Personen in sozialen Beziehung nach fairen Gegenleistung für ihren Einsatz trachten, als verletzt an. Resultat aus Engagement und Einsatz auf der einen Seite und ausbleibende Gratifikation auf der anderen Seite kann zu unterschiedlichen Reaktionen wie Motivationsverlust, Desengagement, Jetzt-erst-recht-Mentalität oder aber auch Zynismus, Burnout oder innere Kündigung führen (Heim \& Gerlach, 1998).

Neben dem schlechten Image und der fehlenden Anerkennung des Lehrerberufs sind die fehlenden Aufstiegsmöglichkeiten ein weiterer Faktor, der kennzeichnend für den Lehrerberuf ist. 35\% geben in einer Studie von an, „schlechte Erfahrungen mit berufli- 
chem Aufstieg" gemacht zu haben (Ulich, 1996, S. 77). Diese Tatsache ist nicht verwunderlich, da es sich beim Lehrerberuf um einen sogenannten Beruf ohne Karriere handelt, bei dem die Unterschiede in der Bezahlung aufgrund der Berufsjahre und nicht aufgrund der Leistungen zustande kommen und beruflicher Aufstieg sehr wenigen vorbehalten bleibt (Rothland \& Terhart, 2007, S. 16). 


\section{Bewältigung von Belastungen, Stressoren und Konflikten}

Nachdem bereits in Kapitel 3 die Begrifflichkeiten Stress, Belastung und Beanspruchung erläutert und bezogen auf den Lehrerberuf dargestellt wurden, geht es in Kapitel 4 schwerpunktmäßig um den individuellen Umgang mit diesen Störungen der Homöostase im Sinne von Bewältigungsmaßnahmen. Auf der Basis der Definition von Coping (Bewältigung) in Kapitel 4.2 wird ausgehend von den Situations- und Personenmerkmalen (siehe Kap. 4.4 und 4.5) in Kapitel 4.6 eine Übersicht über verschiedene Copingmodelle, Bewältigungsformen und -arten gegeben (siehe Kap. 4.7). Auf dieser theoretischen Grundlage werden verschiedene Messinstrumente zur Erfassung von Coping vorgestellt und das im Zentrum dieser Arbeit stehende evasive Bewältigungsverhalten sportunterrichtsspezifisch thematisiert (siehe Kap. 4.9) sowie Folgen unangemessener Belastung wie innere Kündigung und Burnout aufgearbeitet.

\subsection{Erste Überlegungen zu Bewältigungsprozessen - Beginn der Forschungsaktivitäten}

Die ersten populär gewordenen Überlegungen zu Coping (Bewältigung) stammen von Charles Darwin $(* 1809 ; \dagger 1881)$, der sich mit der Anpassung von Lebewesen an Umweltgegebenheiten beschäftigte. Die evolutionäre Entwicklung der Darwinfinken, der wohl berühmtesten Entdeckung Darwins, zeigt in basaler Art und Weise die Anpassung an ökologische Nischen durch Bewältigung gegebener Lebensumstände ${ }^{1}$.

In der Folgezeit prägten Untersuchungen im pathophysiologischen Bereich, vor allem in Form von Tierversuchen, die Forschung und das Wissen über Bewältigung. Spätestens seit den 60er Jahren des vergangenen Jahrhunderts, als Stress längst als ein Aspekt menschlichen Befindens anerkannt war, wurde klar, dass das Bewältigungsverhalten den großen Unterschied ausmacht, der im individuellen Stresserleben festzustellen ist. Aus einer unbedeutenden Fußnote entwickelte sich ein eigenständiges Forschungsgebiet, in dem man sich damit beschäftigte, Strategien der Bewältigung zu messen, zu kategorisieren, zu verbessern und zu optimieren. Ausgehend von der Klärung des Begriffs sollen in diesem Kapitel verschiedene Coping-Modelle, die Kategorisierung und Messung von Bewältigung und die bedeutsamen Charakteristika von Situationen und Personen dargestellt werden. Am Ende des Kapitels geht es vor allem darum, welche Auswirkungen unangemessene Strategien im Umgang mit Stress, Belas-

\footnotetext{
${ }^{1}$ Durch Selektionsdruck und geografische Separation kam es dazu, dass noch unbesetzte Nischen auf anderen Inseln des Galapagosarchipels von Finken der Gründerpopulation besetzt werden konnten. Dieser durch unterschiedliche Nahrungs- und Lebensbedingungen und räumliche Trennung verursachte Prozess der adaptiven Radiation führte letztendlich zur Bildung von 14 verschiedenen Arten.
} 
tungen und Beanspruchungen auf die Physis und Psyche von Individuen haben können. Neben Burnout wird in diesem Zusammenhang auch das Phänomen der inneren Kündigung behandelt, welches im Lehrerberuf von erheblicher Bedeutung ist und gleichermaßen wie Burnout die Lehrergesundheit und die Qualität des Unterrichts erheblich beeinflusst.

\subsection{Was ist Coping? (Definition)}

Das Gerundium Coping leitet sich vom englischen Verb für bewältigen bzw. überwinden to cope ab und beschreibt den Umgang mit Störungen in interaktiven Systemen mit dem Ziel, einen Gleichgewichtszustand wiederherzustellen (Krapp \& Weidenmann, 2001). Synonyme im deutschsprachigen Raum sind Stressbewältigung und Stressverarbeitung. Im angloamerikanischen Raum benutzt man Begriffe wie "struggling", "trying“, „managing" oder "dealing with a situation“ (Nusko, 1986, S. 13). Für die Definition von Coping bzw. Bewältigung ist es unerheblich, ob auch wirklich die angestrebten Ergebnisse mit dem Verhalten erzielt werden, denn bereits „das Bemühen, die Transaktion zu beenden, wird als Coping bezeichnet“ (van Dick, 2006, S. 49).

Neben der Forschungen um die Psyche des Menschen, existiert eine traditionsreiche Forschungshistorie mit Tiermodellen, in denen Coping als Kontrolle aversiver Umweltbedingungen zur Verhinderung bzw. Verminderungen psychophysischer Störungen definiert ist. Dies wird auch in der Definition von Miller (1980, zitiert nach Lazarus \& Folkman, 1984, S. 118), der Coping als "the learned behavioral responses that are successful in lowering arousal by neutralizing a dangerous or noxious condition" beschreibt, deutlich. In dieser Definition wird auch das Ergebnis, d.h. der Erfolg bzw. der Misserfolg mit eingeschlossen. Ähnlich wie bereits in Kapitel 3, im Zusammenhang mit der Begriffsbestimmung von Stress, wurde auch Coping diversen weiteren Definitionsversuchen, meist von der Forschergruppe rund um Lazarus, unterzogen, deren Hauptaspekte im Folgenden dargestellt werden sollen.

Lazarus und Folkman (1984, S. 118) sehen Coping als „realistic and flexible thoughts and acts that solve problems and thereby reduces stress. Coping is the process through which the individual manages the demands of the person-environment relationship that are appraised as stressful and the emotions they generate". Ferner definieren sie den Begriff Coping als "constantly changing cognitive and behavioral efforts to manage specific external and/or internal demands that are appraised as taxing or exceeding the ressources of a person " (Lazarus \& Folkman, 1984, S. 141). Diese Definition schließt neben der Wahrnehmung und der Bewertung auch das Denken einer Person über das Verhältnis zur Umwelt mit ein und geht damit über das hinaus, was zuvor lediglich aus 
Tiermodellen abgeleitet wurde. Weiterhin wird in dieser Definition deutlich, dass Coping mit einer Anstrengung auf Seiten des Bewältigenden verbunden ist und sich so von dem Prozess der reinen Anpassung abgrenzt. In einer weiteren Definition der Berkeley-Gruppe um Lazarus wird mit der Ungewissheit bzw. ungewohnten Situationen zusätzlich ein Aspekt mit einbezogen, der sich in der Folgezeit als bedeutsam herausstellen sollte (Lazarus \& Folkman, 1984). Bettet man Coping-Handlungen in das transaktionale Stressmodell (siehe Kap. 3.1.3) und damit in einen größeren Zusammenhang ein, werden Wechselwirkungen, die bereits in den frühen Definitionen angedeutet wurden deutlich: Coping dient dazu, mit Schaden, Bedrohung oder auch Herausforderungen umzugehen, wenn Routinen oder Automatismen nicht verfügbar sind bzw. nicht existieren. Pearlin und Schooler (1978) schreiben Coping zusätzlich eine protektive Funktion zu: „Coping refers to behavior that protects people from being psychologically harmed by a problematic social experience, a behavior that importantly mediates the impact that societies have on their members" (zitiert nach Nusko, 1986, S. 13) wenngleich das Ergebnis von Coping nicht zwangsläufig positiv sein muss (Kleinke, 2007).

\section{3 (Dispositionsähnlicher) Charakter von Coping!?}

Nachdem verschiedene Definitionen des Begriffs Coping vorgestellt wurden, soll nun geklärt werden, ob es sich bei der Bewältigung um eine Disposition handelt, die konstituierenden Einfluss ausübt, oder ob Stressverarbeitungsmaßnahmen eher situationsspezifisch und damit unabhängig von den Eigenschaften der Persönlichkeit zu sehen sind. Verschiedene Autoren beschäftigten sich mit der Beantwortung dieser Fragestellung und versuchten herauszufinden, ob es sich bei der Art und Weise der Bewältigung um eine stabile individuelle Disposition handelt, die zeit- und situationskonstant Anwendung findet, oder ob Bewältigung als situationsspezifische bzw. prozessimmanente Variable aufzufassen ist. Die Antwort auf diese Frage fällt jedoch keinesfalls eindeutig aus. Auf der einen Seite konnte in mehreren Studien gezeigt werden, dass Bewältigungshandlungen eine hohe Situationsspezifität aufweisen, sodass ein reiner dispositioneller Charakter scheinbar ausgeschlossen werden kann. Auf der anderen Seite jedoch stehen die interindividuellen Unterschiede im Stresserleben und Bewältigungshandeln, die eine ausschließliche Situationsspezifik höchst fraglich erscheinen lassen. Lazarus und seine Mitarbeiter gehen davon aus, dass der spezifische Copingprozess weder allein von Persönlichkeitsmerkmalen, noch ausschließlich durch Prozesskomponenten der jeweiligen Situation bestimmt wird, sondern in das übergreifende Modell der Person-Situation-Transaktion integriert ist und sich dementsprechend stets neu konstituiert (Jerusalem, 1990). Man spricht von spezifischen Coping- 
Stilen, die im Zusammenspiel mit den Situationsparametern konkrete CopingStrategien ergeben (Nusko, 1986). Das heißt, Personen reagieren in Situationen, in denen Copinghandlungen erforderlich sind, nicht per se mit derselben Strategie, verfügen aber über bevorzugte Handlungsweisen oder Stile, die sich gegenüber Veränderungen als relativ stabil erweisen (Carver, Scheier \& Weintraub, 1989).

Auch wenn auf die Frage zum Charakter von Coping-Reaktionen keine umfassende und generell gültige Antwort gegeben werden kann, ist anhand der methodischen Gestaltung der Untersuchungsfragestellung ableit- bzw. vorhersagbar, ob die im Rahmen eines konkreten Forschungsprojektes erhobenen Daten situationsspezifischen oder eher dispositionellen Charakter aufweisen. Während durch Instruktionen in einem Fragebogen, die sich auf bestimmte erlebte Episoden und Ereignisse beziehen, wie z.B. im "Ways of Coping Questionnaire“ (siehe Kap. 4.8) der situative Aspekt stärker betont wird, nehmen andere Instrumente, wie z.B. auch der im Zentrum dieser Arbeit stehende neu entwickelte Fragebogen (siehe Kapitel 6), personentypische, nicht auf ein bestimmtes Ereignis bezogene Verhaltensweisen in den Fokus. Durch entsprechende Instruktionen wird hier das Verhalten in situationskonstanterer Art und Weise erhoben. Bereits bei der Planung von Erhebungen sollte also, bedingt durch die im Zentrum stehende Fragestellung, bei der Konstruktion von Instrumenten bedacht werden, welche Fokussierung gewählt wird. Auf die verschiedenen Instrumente zur Erfassung von Coping-Verhalten bzw. Coping-Stilen wird in Kapitel 4.8 näher eingegangen.

\subsection{Formale Eigenschaften von Situationen}

Wie bereits in den vorangegangenen Kapiteln der Arbeit deutlich wurde, werden Situationskonstellationen von Individuen in unterschiedlicher Art und Weise wahrgenommen, bewertet und bewältigt. Neben der Persönlichkeit gehören jedoch auch verschiedene Eigenschaften der Situation zu den antezedenten Bedingungen, die Einfluss auf den Verlauf der im Hintergrund ablaufenden handlungs- und gefühlsgesteuerten kognitiven Prozesse haben (Kramis-Aebischer, 1996). Lazarus und Folkman (1984) erwähnen neben inhaltlichen Situationseigenschaften wie äußere Stressoren, Deprivation primärer Bedürfnisse, Leistungsstressoren und soziale Stressoren auch verschiedene formale Charakteristika, die dazu führen, dass Situationen als gefährlich, bedrohlich oder schädlich wahrgenommen werden. Während die inhaltlichen Eigenschaften von Situationen bereits in Kapitel 3 unter der Bezeichnung der objektiven Belastung bzw. der wahrnehmungsbezogenen Intensität der subjektiven Belastung dargestellt und theoretische und empirische Klassifikationsversuche vorgestellt wurden, werden im Folgenden die formalen Eigenschaften von Situationen skizziert. 
In Abgrenzung zu den inhaltlichen Situationseigenschaften stellen formale Situationseigenschaften keine Form der Kategorisierung dar, sondern ermöglichen eine elementare Charakterisierung von einzelnen Situationen anhand spezifischer Kriterien. Neuigkeit, Ereignisunsicherheit, zeitliche Bedingungen, Ambiguität sowie die Angemessenheit des Zeitpunktes beeinflussen die Bedrohlichkeit von Anforderungen und damit auch das sich anschließende Bewältigungshandeln des Individuums (Jerusalem, 1990). Im Anschluss sollen die einzelnen formalen Situationseigenschaften etwas genauer dargestellt werden.

Unter Neuigkeit einer Situation versteht man, dass die Situation in der aktuellen und akuten Erscheinungsform so noch nicht vorgekommen ist und daher auch keine Informationen über bereits in der Vergangenheit liegende Bewältigungshandlungen vorhanden sind. Obwohl keine negativen Attributionen vorliegen und die Situation vollkommen neuartig und wertfrei ist, entsteht dennoch oft Unsicherheit. Komplett neue Situationen kommen allerdings so gut wie gar nicht vor. Meist werden einzelne Facetten des Geschehens wiedererkannt und als Basis einer Interpretation mit sich anschließender wertender Einordnung genutzt (Lazarus \& Folkman, 1984). Als Lehrer wird man allein aufgrund der Tatsache, dass man Umgang mit einer hohen Anzahl von Schülern hat, die sowohl intraindividuell als auch interindividuell höchst verschiedene Verhaltensweisen untereinander, gegenüber der Lehrkraft und bezogen auf Vermittlungsprozesse und Inhalte an den Tag legen, mit an Sicherheit grenzender Wahrscheinlichkeit immer wieder in Situationen geraten, die durch Neuigkeit geprägt sind. Gerade im Sportunterricht existiert, durch den hohen Grad an sozialer Interaktion und der zusätzlichen motorischen Aktivität, eine höhere Situationsvariabilität als in anderen Schulfächern. Im Laufe des Berufslebens stehen Lehrern jedoch zunehmend Erfahrungen und Routinen zur Verfügung, sodass das Belastungspotenzial der meisten Situationen nicht primär durch Neuigkeit, sondern eher durch andere Eigenschaften bestimmt wird. Auch wenn neuartige Situationen auftreten, werden aufgrund von Erfahrungen aus anderen bzw. ähnlichen Konstellationen Interpretationen abgeleitet, die bei der Bewältigung genutzt werden. Im umgekehrten Fall können natürlich auch negativ geprägte Erfahrungen aus erfolglosen Bewältigungsversuchen dazu beitragen, dass neuartige Situationen automatisch unidirektional wahrgenommen werden und erfolgreiche bzw. angemessene Bewältigung hierdurch be- oder gar verhindert wird.

Als Ereignisunsicherheit bezeichnet man die subjektive Eintrittswahrscheinlichkeit eines Ereignisses, die im Wesentlichen das Stresserleben bestimmt. Bei geringer Eintrittswahrscheinlichkeit, d.h. wenn man nicht weiß, ob ein Ereignis eintritt oder nicht, ist präventives und antizipatives Coping nicht möglich und die Stresswirksamkeit damit 
erhöht. Sicherheit bezogen auf das Eintreten eines Ereignisses ist jedoch nicht gleichzusetzen mit erfolgreichem und vorausschauendem Coping, denn auch hier ist bei fehlender Kontrollierbarkeit eher mit Hilflosigkeit und Unsicherheit, anstatt mit effektivem und antizipativem Bewältigungshandeln zu rechnen (Jerusalem, 1990). Ähnlich wie bei der Neuartigkeit von Situationen ist der Beruf des Lehrers im Allgemeinen und wiederum der des Sportlehrers im Speziellen durch einen hohen Grad an Ereignisunsicherheit geprägt. Im Studium und Referendariat erlernen angehende Lehrer fachliche Inhalte und die entsprechenden Vermittlungswege, doch auch bei erfahrenen Lehrern führt der Faktor Schüler stets zu Verunsicherung. Im Unterricht ist das tatsächliche Eintreten von Geplantem keineswegs gesichert, da der Verlauf von Situationen im Rahmen der Interaktion von Schülern, Inhalten und der Lehrkraft meist unvorhersehbar ist.

Die Vorhersehbarkeit von Ereignissen ist demnach eine weitere Eigenschaft, die die Stresswirksamkeit von Situationen bestimmt. Wie sich herausstellte, werden vorhersehbare Stressereignisse aufgrund der Möglichkeit, antizipativ bzw. präventiv zu handeln meist als weniger intensiv wahrgenommen als Ereignisse, die ohne Vorankündigung und plötzlich eintreten. Im Umkehrschluss bedeutet die Vorhersehbarkeit von Ereignissen Sicherheit, d.h. ermöglicht Phasen der Entspannung, soweit diese nicht im Vorfeld bereits die Erwartung eines Scheiterns bzw. Hilflosigkeit entstehen lässt (Lazarus \& Folkman, 1984).

Zeitliche Faktoren, wie z.B. die Dauer bis zum Eintritt des Ereignisses oder die Dauer des Stressereignisses sowie zeitliche Unsicherheit prägen ebenfalls das Stresspotenzial von Situationen. Besonders intensiv erlebt man Situationen, die plötzlich, unerwartet und ohne Ankündigung eintreten. In diesem Fall fehlt die Zeit für Prozesse der Informationssuche und für das genaue Abwägen der Pro- und Kontra-Aspekte der zur Verfügung stehenden Handlungsalternativen (Lazarus \& Folkman, 1984). Eine ebenfalls erhebliche Belastung kann durch die Dauer des Wartens auf ein bestimmtes Geschehen entstehen, wobei hier die Intensität des Stresserlebens geringer ist. Insgesamt ermöglicht eine lange Dauer bis zum Eintreten eines Ereignisses komplexere Bewertungsvorgänge und damit verbundene positive oder negative Emotionen. Dauert ein Stressereignis sehr lange an, kann dies Erschöpfung und Resignation auslösen, es kann jedoch auch zu einem erfolgreichen Umgang, zu einer Gewöhnung, Distanzierung oder Vermeidung führen (Jerusalem, 1990). Als weitere Form nennen Lazarus und Folkman (1984) chronische, aber unterbrochene Verläufe von Ereignissen, die besondere Auswirkungen auf den Bewertungsprozess haben. Zeitliche Unsicherheit bezeichnet letztlich den Zustand, genau zu wissen, dass ein bestimmtes Ereignis eintreten wird, wann 
ist aber nicht bekannt. Die Folge ist eine höhere Wachsamkeit und eher vermeidungsorientiertes Coping-Verhalten (Lazarus \& Folkman, 1984). Die unter dem Begriff der zeitlichen Faktoren summierten Aspekte sind in der täglichen Unterrichtstätigkeit von Lehrern leicht wiederzufinden. Stressereignisse wie Konflikte zwischen Schülern, Motivations- und Disziplinprobleme oder Unfälle im Schulsport können als fast tagtägliche Wegbegleiter von Sportlehrkräften bezeichnet werden. Wann, wo und wie sie stattfinden bzw. wer beteiligt ist, ist jedoch unklar. Des Weiteren erstreckt sich ein chronisches Stressereignis, bedingt durch Faktoren wie Lärm, Orientierungs- und Handlungsdruck (siehe Kap. 3) über die gesamte Unterrichtszeit hinweg. Wie in Kapitel 4.9 deutlich wird, können neben Gewöhnung an lang andauernde bzw. wiederkehrende Stresserlebnisse auch habituelle Bewältigungshandlungen wie Vermeidung oder Akzeptanz die Folge sein, bei denen bestehende Verhältnisse ausgeblendet werden oder der Problemcharakter geleugnet wird. Auch Änderungen von Einstellungen und beruflichen Zielen kann die Folge dauerhafter Belastung sein, vor allem dann, wenn der Einfluss als gering eingeschätzt wird, die herrschenden Verhältnisse zu ändern bzw. etwas bewirken zu können.

Fehlen wichtige Informationen bei der Einschätzung einer Situation, spricht man von fehlender Eindeutigkeit oder Ambiguität. Wenn unbekannt ist, ob überhaupt ein Problem vorliegt, wie lange dies andauern wird und um was es dabei überhaupt geht, werden notwendige Urteilsprozesse erschwert. Differenzieren sollte man zwischen situationsbezogener Ambiguität und personenbezogener Unsicherheit, die durch personale Ressourcen konstituiert wird. Je größer die Ambiguität in Situationen ist, desto größeren Einfluss haben Persönlichkeitsmerkmale auf die Interpretation von Situationen (Lazarus \& Folkman, 1984). Abhängig von den individuellen Kompetenz- und Kontrollüberzeugungen entsteht ein mehr oder weniger hohes Stressniveau. Für Lehrkräfte mit ausgeprägten Selbstkompetenzen weisen Situationen, die durch einen hohen Grad an Unsicherheit geprägt sind, ein moderates Stressniveau auf, da sie auf ihre eigenen Stärken vertrauen und über genug Selbstsicherheit verfügen, um die Situation zu bewältigen. Für Lehrpersonen, die jedoch nur in geringem Maß von den eigenen Fähigkeiten und Kompetenzen überzeugt sind, verkörpern solche Situationen höchste Stressrelevanz. Da im Lehrerberuf Ambiguität in Form der zuvor bereits vorgestellten formalen Situationsmerkmale in hohem Maße ausgeprägt ist, kommt den konstituierenden Persönlichkeitsmerkmalen eine enorme Bedeutung zu. Individuelle Persönlichkeitsmerkmale und die damit in Verbindung stehenden Bewältigungshandlungen sind sowohl für die Erhaltung der Gesundheit als auch für die Erfüllung des Arbeitsauftrages maßgeblich verantwortlich. 
Eingebettet in eine übergreifende Lebensperspektive wird anhand eines Abgleichs mit normativen Setzungen die Angemessenheit des Zeitpunktes als weitere formale Eigenschaft von Situationen benannt (Jerusalem, 1990). Neben der Einordnung in das Leben des Betroffenen werden hiermit vor allem auch die Relationen zu anderen Stressoren gesehen. Dies führt zu der bereits in Kapitel 3 beschriebenen summativen und multiplikativen Wirkung von Stressereignissen. Wie in der Beschreibung des formalen Taxonomieansatzes deutlich wurde sind die Situationseigenschaften oft nicht klar voneinander zu isolieren und bedingen sich gegenseitig.

\subsection{Eigenschaften von Personen}

Nachdem bereits mit den formalen Eigenschaften von Situationen, die für alle im selben Umfeld sich befindenden bzw. arbeitenden Individuen identisch sind, begonnen wurde, sollen im Anschluss die Ich-Ressourcen als weitere wichtige Determinanten des Bewältigungsprozesses dargestellt werden.

In den individuellen Unterschieden der Persönlichkeit sind die Abweichungen im Verhalten und Erleben von objektiven Situationsanforderungen begründet. Nur durch die Individualität von Personen sind die unterschiedlichen Reaktionen und Beanspruchungen auf identische äußere Reizkonstellationen sowie die vorausgehenden Bewertungen zu erklären. Neben globalen Ressourcen stellen situationsspezifische Ressourcen den anderen Pol eines Kontinuums dar, bei dem die Grenzen fließend ineinander übergehen (Jerusalem, 1990). Wie bereits in Kapitel 4.3 dargestellt, ist es daher nicht immer eindeutig möglich, ein Verhalten einer stabilen Verhaltensdisposition zuzuschreiben oder aber ausschließlich situationsspezifische Parameter dafür verantwortlich zu machen.

Neben den im Anschluss vorgestellten eher subjektiven Selbsteinschätzungen und Überzeugungen in Form von Persönlichkeitseigenschaften sind selbstverständlich auch objektive Merkmale von Personen mit ausschlaggebend für die Resistenz bzw. Vulnerabilität gegenüber Stress. Wissen, Intelligenz, Gesundheit und spezifische Fähigkeiten, für den Lehrberuf d.h. vor allem fachdidaktische und pädagogische Kompetenzen stellen die tatsächlich vorhandenen objektiven Coping-Ressourcen dar. Für den Sportlehrerberuf sind dies ergänzend theoretische und praktische Kompetenzen in den verschiedenen sportlichen Aktivitäten sowie der persönliche Fitnesszustand. Diese objektiven Ressourcen sind jedoch oft nur mäßig korreliert mit den selbst perzipierten und damit auch ausschlaggebenden Coping-Ressourcen, die im Anschluss dargestellt werden (siehe Kap. 4.5.1 und 4.5.2). 
Eine weitere nicht zu vernachlässigende Komponente, die nicht direkt den subjektiven Selbsteinschätzungen und Überzeugungen zuzuordnen ist, aber dennoch erheblichen Einfluss auf den Umgang mit Stress und Belastung bzw. Beanspruchung aufweist, sind soziale Ressourcen. Soziale Unterstützung als multidimensionales Konstrukt hat eine lange Forschungstradition und wurde in verschiedensten Forschungsansätzen untersucht. Unter Begriffen wie soziales Netz, sozialer Rückhalt, soziale Integration oder soziale Unterstützung werden soziale Ressourcen in wissenschaftlichen Untersuchungen stets als bedeutsamer und zentraler Einflussfaktor auf die Stresswahrnehmung bzw. -bewertung, auf das Coping-Verhalten und letztendlich auf die Gesundheit gesehen (Herzog, 2007). Schaarschmidt (2005a) berichtet, dass intakte und intensive zwischenmenschliche Beziehungen sowie das Erleben sozialer Unterstützung die wichtigsten Entlastungsfaktoren im Berufsalltag darstellen. Neben der Möglichkeit, sich sowohl privat als auch im Arbeitsbereich mit nahestehenden Personen auszusprechen, stellen Freizeitaktivitäten, Hobbys oder Zeit, die man mit der Familie verbringt, wichtige Ausgleichsmöglichkeiten dar. Schulspezifisch sind das soziale Klima im Kollegium sowie die Unterstützung durch die Schulleitung relevante Faktoren. Vor allem die Führungskompetenz der Schulleitung stellt eine wichtige Einflussgröße sowohl für die Qualität der Schule als auch für das individuelle Befinden und die Gesundheit der Lehrkräfte dar. Miethling (2000) kritisiert, dass gerade Sportlehrkräfte in der jüngeren Entwicklung an Wertschätzung und Reputation eingebüßt haben. Er berichtet, dass vermehrt von Elternseite bedenkenlos gegen die Lehrkräfte Partei ergriffen wird. Neben individuumsbezogenen Interventionen sieht Schaarschmidt (2008) auch gerade im sozialen Klima im Kollegium einen entscheidenden Ansatzpunkt für eine Verbesserung der Arbeitsbedingungen und folglich auch der Gesundheit, was sich durch einen eigenständigen Bereich in dem Arbeitsbewertungscheck für Lehrkräfte (ABC-L) niederschlägt (Schaarschmidt \& Kieschke, 2006). Auch Barth (1997) sieht langfristig einen sehr hohen Wert in der kollegialen Unterstützung, um in vielfältiger Form unangenehme Gefühle abzubauen und neue Lösungen zu erarbeiten. Auch van Dick (2006) schreibt der wahrgenommenen sozialen Unterstützung protektive Funktion zu, indem er direkte Effekte auf verschiedene Arten von Beschwerden nachwies. Die Ergebnisse waren jedoch nur bedingt replizierbar und es zeigte sich, dass den in Kapitel 4.5.2 dargestellten Kompetenzerwartungen eine bedeutsamere Funktion zugeschrieben wird.

Wie in Kapitel 3 dargestellt wurde, wird im Rahmen der sekundären Bewertung des transaktionalen Stressmodells ein Abgleich mit den subjektiv perzipierten Ressourcen, die situationsübergreifend sind und dispositionsähnlichen Charakter aufweisen, vorgenommen. Neben den zuvor dargestellten objektiven Situationseigenschaften und den 
sozialen Ressourcen sind dies individuelle Eigenschaften von Personen, die die Widerstandsfähigkeit gegenüber Belastungssituationen bestimmen und damit auch die Grundlage für eine erfolgreiche und angemessene Auswahl und Durchführung von Bewältigungsmaßnahmen darstellen (Jerusalem, 1990). Lazarus und Folkman (1984) untergliedern die für die Bewertung, Verarbeitung und Bewältigung von Belastungssituationen verantwortlichen Eigenschaften von Personen in commitments und beliefs. Während unter commitments Motivationsstrukturen der Persönlichkeit zusammengefasst werden, stellen beliefs allgemeine und bereichsspezifische Überzeugungen dar, wie z.B. die Kontrollierbarkeit oder die Wahrnehmung von verfügbaren externalen Ressourcen.

\subsubsection{Commitments}

Commitments sind Eigenschaften von Personen, die die subjektive Bedeutsamkeit und damit gleichbedeutend auch die Stressrelevanz von Ereignissen bestimmen bzw. beeinflussen (Jerusalem, 1990). Sie drücken aus, was wichtig für Personen ist und steuern damit auch die Höhe des Einsatzes, mit dem bestimmte Ziele verfolgt werden. Commitments sind daran beteiligt, ob Situationen die herausfordernd oder bedrohlich sind, aufgesucht werden oder nicht. Je intensiver und tiefer die Commitments ausgeprägt sind, desto größer ist die verspürte potenzielle Herausforderung oder Bedrohung. Inwieweit diese Motivationsstrukturen das Verhalten beeinflussen, lässt sich sehr anschaulich anhand der Karriereorientierung aufzeigen. Während Personen mit dem Bestreben es beruflich möglichst weit zu bringen, eine Überprüfung und Bewertung ihrer Leistungen als bedeutsam wahrnehmen und folglich stets darum bemüht sind, die Erwartungen, die an sie von außen gestellt werden zu erfüllen bzw. wenn möglich sogar zu übertreffen, liegt das Stressniveau bei Personen ohne Aufstiegsorientierung deutlich niedriger (Lazarus \& Folkman, 1984). Im Lehrberuf, einem Beruf ohne Karriere, kann es aufgrund der fehlenden Aufstiegsmöglichkeiten, den lediglich sporadischen Leistungsüberprüfungen und den fehlenden Gütemaßstäben zu motivationalen Problemen kommen. Übertragen auf den konfliktreichen Alltag ist zu erwarten, dass Lehrkräfte, die weniger motiviert sind, Konflikte und Probleme beim Unterrichten mit größerer Gelassenheit verarbeiten als Personen, die einen hohen Anspruch an die Erträge des eigenen Tuns und damit auch eine hohe Ausprägung an Engagement aufweisen. Inwieweit sich jedoch eine große Motivation und die damit verbundene große potenzielle Herausforderung für besonders engagierte Lehrer auch zu einer Bedrohung entwickeln kann, zeigt Kapitel 4.9.5. 


\section{Leistungsmotivation}

Commitments beeinflussen die Bewältigung von Situationen durch zahlreiche verschiedene Mechanismen. Neben der Leistungsmotivation nehmen eine Reihe weiterer Moderatorvariablen Einfluss auf die Wahrnehmung, Widerspiegelung und Bewertung von Belastungen und die sich anschließende Bewältigung. Eine umfassende Schilderung weiterer Moderatorvariablen, die die subjektive Bedeutsamkeit und damit gleichbedeutend auch die Stressrelevanz beeinflussen, erlaubt der Rahmen dieser Arbeit jedoch nicht. Im methodischen Teil dieser Arbeit werden Motivationsstrukturen der Lehrkräfte unter anderem durch die Kurzversion der Skala zur Erfassung der Leistungsmotivation von Engeser (2004, entnommen aus Rheinberg, 2004) erfasst. Anhand der Leistungsmotivation wird exemplarisch der Einfluss von commitments auf das Verhalten und die daraus resultierenden interindividuellen unterschiedlichen Bewältigungshandlungen skizziert. Klinger (1975) stellte heraus, dass Personen, bei denen die Motivationsstrukturen stärker ausgeprägt sind, auch bei sich auftuenden Widerständen und Hindernissen an ihren Zielen festhalten und keine Umdeutungen von Situationen bzw. Vermeidungsstrategien Anwendung finden. Die Anreize der Zielerreichung können äußerst vielschichtig sein und reichen von Ergebnissen, die für die Person selbst oder das Umfeld gewinnbringend und nützlich sind, bis hin zu Stolz, etwas Schwieriges geschafft zu haben. Grundsätzlich strebt man in Situationen nach einem auf den eigenen Bemühungen basierenden Erfolgserlebnis, das für verschiedene Personen einen höchst unterschiedlich ausgeprägten Aufforderungscharakter aufweisen kann (Rheinberg, 2006). Für Lehrer kann so ein Erfolgserlebnis bereits die gelungene Vermittlung zwischen zwei Streithähnen im Unterricht sein, die durch das Eingreifen der Lehrperson eventuelles Fehlverhalten erkennen und Besserung geloben.

Leistungsmotiviert im psychologischen Sinn ist ein Verhalten nur dann, wenn es auf die Selbstbewertung eigener Tüchtigkeit zielt, und zwar in Auseinandersetzung mit einem Gütemaßstab, den es zu erreichen oder zu übertreffen gilt. Man will wissen, was in einem Aufgabenfeld gerade noch gelingt und was nicht, und strengt sich deshalb besonders an (Rheinberg, 2006, S. 60).

Das der Leistungsmotivation zugrunde liegende Leistungsmotiv, als bedürfnisähnlicher Faktor, stellt eine personenspezifische Konstante dar, die Einfluss auf die Wahrnehmung und Bewertung von Situationen hat und bestimmte Handlungstendenzen in Situationen, in denen Gütemaßstäbe anwendbar sind, bedingt (Lang \& Fries, 2006). Das Motiv als relativ stabile Determinante löst den mehr oder minder komplexen Prozess der Motivation aus (Thomae, 1983a). Am Beispiel der Persönlichkeit von Lehrkräften 
heißt das, dass Personen mit ausgeprägtem Leistungsmotiv Handlungssituationen eher wahrnehmen und diese verstärkt als anregend und bedeutsam einschätzen als Lehrkräfte mit geringer ausgeprägtem Leistungsmotiv. Die Leistungsmotivation als aktuelle Motivation ist letztlich abhängig von der Wechselbeziehung zwischen der vorliegenden Situation und dem Leistungsmotiv. Situationen, in denen Erfolg und Misserfolg in etwa gleichen Wahrscheinlichkeiten möglich sind, werden meist als Herausforderungen interpretiert, während extrem einfache bzw. schwere Aufgaben meist mit deutlich geringerem Engagement und weniger Lust angegangen werden (Rheinberg, 2006).

Antworten über den Zusammenhang von Erfolgswahrscheinlichkeit und Erfolgsanreiz liefert das Risikowahl-Modell von Atkinson (1957). Situationen mit einer Erfolgswahrscheinlichkeit von nahezu 100 Prozent sind zwar mit Sicherheit zu schaffen, lösen aber dennoch keine Leistungsmotivation aus, da jeglicher Erfolgsanreiz fehlt. Genauso ist es bei extrem schwierigen Situationen, in denen die Aussicht auf Erfolg schwindend gering ist (Rheinberg, 2006). Zwischen der Wahrscheinlichkeit und dem Anreiz des Erfolges besteht eine negativ-lineare Beziehung, sodass in einer graphischen Darstellung eine umgekehrte U-Funktion für die Leistungsmotivation resultiert (siehe Abb. 7).

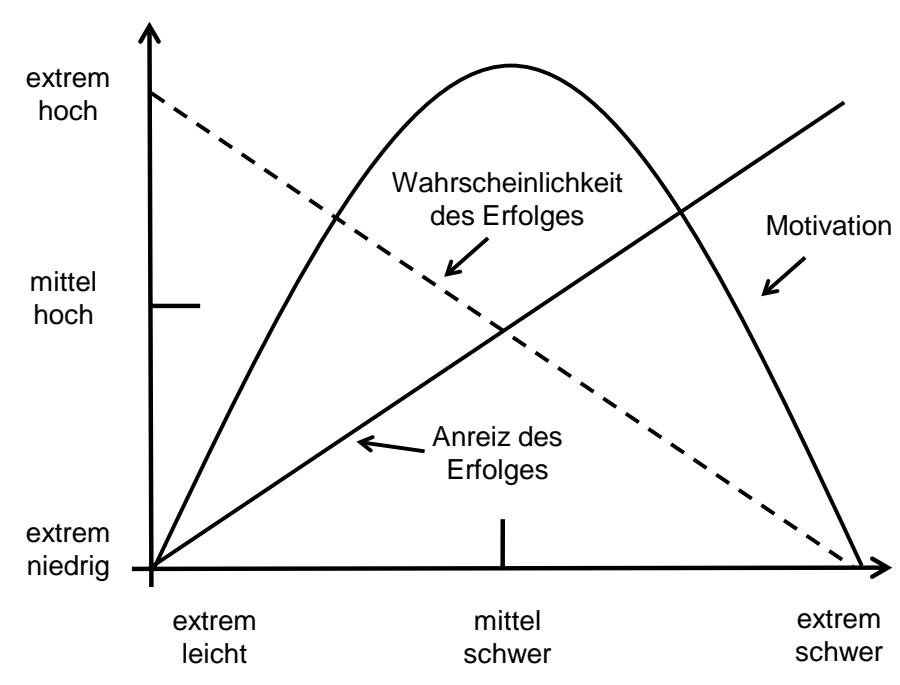

Subjektive Aufgabenschwierigkeit

Abb. 7: Kurvenartiger Verlauf der Leistungsmotivation im Risikowahl-Modell nach Atkinson (Rheinberg, 2006, S. 72)

Für das Verständnis des in Abbildung 7 dargestellten Kurvenverlaufes ist es wichtig, dass nicht die tatsächliche sondern die subjektive Aufgabenschwierigkeit als Bezugsgröße herangezogen wird. Z.B. würde eine Sprunghöhe eines Sportlers, der 1,80 Meter als Bestleistung im Hochsprung hat, von 1,78 Meter eine hohe Leistungsmotivation auslösen, da das Reißen bzw. Überspringen der Latte ungewiss und in starkem Maße 
von der eigenen Anstrengung abhängig ist. Je näher die Erfolgswahrscheinlichkeit an 50 Prozent, d.h. je größer die Ungewissheit über das Ergebnis ist, desto stärker ist die Motivation ausgeprägt (Atkinson, 1957). Im Umkehrschluss gilt: Je geringer die Ungewissheit des Erfolgs bzw. Misserfolgs ist, desto geringer wird auch die Motivation sein. Weder eine Sprunghöhe von 2,10 Meter noch 1,20 Meter würde zu einer motivierten Grundhaltung des Sportlers führen, da die Gewissheit des Scheiterns bzw. Meisterns jeweils stark ausgeprägt ist. Wenn freie Aufgabenwahl besteht, streben Personen mit hoher Leistungsmotivation verstärkt zu neuen und schwierigen Aufgaben. Werden sie in ihrer Wahl eingeschränkt oder behindert und beispielsweise nur mit extrem leichten oder extrem schwierigen Aufgaben konfrontiert, die fast unlösbar sind, führt dies zu einem schrittweisen Interessensverlust an der Aufgabe bzw. der Arbeit (Atkinson, 1957).

Neben der Stärke der Leistungsmotivation ist jedoch zudem auch deren Richtung handlungsleitend, die dazu führt, dass Situationen von Personen eher erfolgszuversichtlich oder misserfolgsmeidend angegangen werden. Das Leistungsmotiv lässt sich in seine Komponenten Erfolgsmotiv und Misserfolgsmotiv aufgliedern, die in Erhebungsinstrumenten getrennt als "Hoffnung auf Erfolg" und „Furcht vor Misserfolg" gemessen werden (Lang \& Fries, 2006). Die Richtung des Leistungsmotivs kann als „Netto-Hoffnung" durch Subtraktion der beiden Werte errechnet werden.

Die in Abbildung 7 dargestellte Kurve entspricht einer eher erfolgszuversichtlichen Person. Bei misserfolgsmotivierten Personen verläuft die Kurve genau umgekehrt: Die mittleren Schwierigkeitsgrade, an denen am deutlichsten die Tüchtigkeit und die eigenen Fähigkeiten offenbart werden, werden von ihnen als bedrohlich empfunden und daher verstärkt gemieden. Besonders leichte Aufgaben hingegen werden als attraktiv wahrgenommen, denn sie verschaffen zwar kein Erfolgserlebnis, ein Scheitern ist aber auch unwahrscheinlich. Extrem schwierige Aufgaben schrecken Misserfolgsmotivierte überraschenderweise nicht $a b$, da das Scheitern nicht auf das eigene Versagen, sondern vielmehr auf das „Nicht-zu-Schaffen-sein“ zurückgeführt wird (Rheinberg, 2006). In Ringwurfexperimenten, bei denen mit Gummiringen auf verschiedene Ziele geworfen werden muss, wurden diese theoretischen Annahmen bestätigt. Die teilnehmenden Kinder konnten die Entfernung zum Ziel selbst bestimmen. Erfolgsmotivierte Kinder wählten meist moderate und angemessene Entfernungen, während misserfolgsmotivierte Kinder entweder ein extrem geringes oder aber ein extrem hohes Anforderungsniveau wählten (Atkinson, 1957). Weiterhin neigen Personen, deren Motivation durch die Furcht vor Misserfolg bestimmt ist, dazu, vor allem bei ich-nahen 
Leistungen eher defensiv zu agieren und wenn möglich, kritische Situationen ganz zu vermeiden, um der Konfrontation mit Gütemaßstäben aus dem Weg zu gehen.

Neben dem bisher dargestellten klassischen Leistungsmotivationsansatz wird in neueren Ansätzen (Elliot \& Church, 1997; Elliot \& McGregor, 2001) versucht, zusätzlich motivationale Orientierungen in der Leistungsmotivationsforschung zu berücksichtigen. In der deutschsprachigen Literatur unterscheidet man zwischen Aufgaben- und EgoOrientierung. Aufgabenorientierung umfasst Bestrebungen von Personen, die das Ziel haben, Fertigkeiten zu verbessern bzw. besser steuern zu können, während es bei der Ego-Orientierung darum geht, die eigene Kompetenz zu demonstrieren und sich mit anderen zu messen. In ihrem hierarchischen Modell von 1997 schlagen Elliot und Church eine weitgehende Differenzierung in Aufgabenorientierung, aufsuchende und vermeidende Ego-Orientierung vor. 2001 differenzierten Elliot und McGregor zusätzlich auch die Aufgabenorientierung in eine aufsuchende und eine vermeidende Komponente. Die Orientierungen werden hierbei als Ziele verstanden, die durch die höher angesiedelten Motivdispositionen bestimmt werden (Elbe, Wenhold \& Müller, 2005). Wie man in Abbildung 8 erkennen kann, führt die Ausprägung der Hoffnung auf Erfolg (approaching success) zu einer aufsuchenden Aufgabenorientierung (masteryapproach goal) oder einer aufsuchenden Ego-Orientierung (performance-approach goal), während eine ausgeprägte Furcht vor Misserfolg (avoiding failure) eine vermeidende Ego-Orientierung (performance-avoidance goal) und nach dem neueren Ansatz eine vermeidenden Aufgabenorientierung (mastery-avoidance goal) bedingt (Elliot \& Church, 1997; Elliot \& McGregor, 2001).

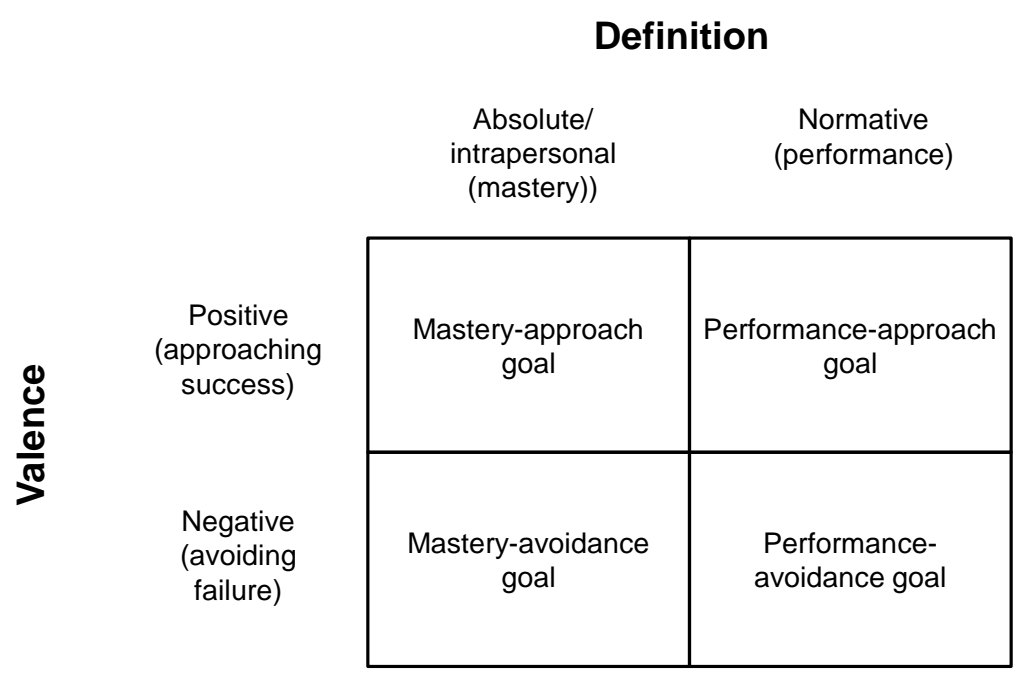

Abb. 8: $2 \times 2$ achievement goal framework (Elliot \& McGregor, 2001, S. 502) 
Bei der Ursachenforschung für die Ausprägung der Leistungsmotivation und dem damit verbundenen Verhalten stießen Forscher schnell auf die Kausalattribution von Erfolg und Misserfolg. Im Zentrum dieser Ursachenzuschreibung steht die Frage, ob internale oder externale Bedingungen für den Erfolg bzw. das Scheitern verantwortlich gemacht werden. Wird für einen Misserfolg die eigene Inkompetenz verantwortlich gemacht, sinkt die künftige Erfolgserwartung, da man beim erneuten Eintreten dieser Situation mit einem ähnlichen Ergebnis rechnet. Verbindet man hingegen das Scheitern mit mangelnder Anstrengung oder Pech, wird die Erfolgserwartung nicht oder nur in sehr geringem Maße beeinträchtigt. Misserfolgs- und erfolgsmotivierte Personen unterscheiden sich in der angesprochenen Attribution von Erfolg und Misserfolg. Erfolgsmotivierte erklären Erfolge durch die eigenen Fähigkeiten, während sie Misserfolge der mangelnden Anstrengung oder Pech zuschreiben, wodurch zukünftige Handlungssituationen motiviert angegangen werden. Misserfolgsmotivierte erklären Misserfolge durch mangelnde Fähigkeiten, für Erfolge hingegen wird Glück oder die Aufgabenleichtigkeit verantwortlich gemacht, sodass ein Erfolg minimalen Wert besitzt und meist keine nachhaltigen Folgen aufweist (Rheinberg, 2006). Der Zusammenhang der Ausprägung der Leistungsmotivation mit der individuellen Attribution von Erfolg und Misserfolg kann das Verhalten in Anforderungssituationen, wie z.B. bei der Bewältigung von beruflichen Stressoren, vor allem aber auch Handlungsweisen wie das Vermeiden, später als Evasion bezeichnet, zumindest partiell erklären. „Die aufsuchende Komponente kommt nur zustande, wenn eine misserfolgsängstliche Person überzeugt ist, die Aufgabe lösen zu können (Kompetenzerwartung)“ (Elbe, Wenhold \& Müller, 2005, S. 59). Es ist daher davon auszugehen, dass die Leistungsmotivation einen nicht unerheblichen Einfluss auf das Bewältigungsverhalten von Lehrkräften hat. Auch das Phänomen Burnout (siehe Kap. 4.9.5.1) als Konsequenz bzw. Beanspruchungsfolge kann durch den Erklärungsgehalt der Leistungsmotivation und der in diesem Zusammenhang stehenden Attributionsmuster besser nachvollzogen werden. Auf die mit der Leistungsmotivation offensichtlich in einem kausalen Verhältnis stehende Attribuierung wird im Kapitel 4.5.2.2 durch die Darstellung der Kompetenz- und Kontrollüberzeugungen näher eingegangen.

\subsubsection{Beliefs}

Beliefs stellen Überzeugungen und Erwartungen dar, die Einfluss auf die stressbezogenen Einschätzungsmaßnahmen nehmen. Sie umfassen vor allem die persönliche Kontrollierbarkeit von Situationen und damit die subjektiv perzipierten Fähigkeiten, Kompetenzen und Ressourcen. Die Ausprägung der Kompetenz- und Kontrollüberzeugungen werden für das Stresserleben als besonders bedeutsam angesehen, da sie an der 
intrapersonalen Lösung von Entscheidungskonflikten im Rahmen von ÜberzeugungsWert-Systemen, also an einem Zusammenspiel von kognitiven und motivationalen Variablen beteiligt sind (Thomae, 1983a).

Neben Überzeugungen, bezogen auf internale bzw. externale Kontrolle, sind unter beliefs mit Selbstwertgefühl, Kompetenz- und Konsequenzerwartung, Leistungsängstlichkeit und Depressivität weitere Personenmerkmale gefasst, die als eine Art Überzeugungssystem im Laufe des Lebens durch Generalisierung entstehen und auf neue Situationen angewendet werden (Thomae, 1983). Lazarus und Folkman (1984) differenzieren, ähnlich wie bei der Frage nach dem dispositionellen Charakter des Copingverhaltens, zwischen generalisierten und situationsspezifischen Überzeugungen. Nach Rotter (1975) sind situationsspezifische Überzeugungen und Erwartungen vor allem dann relevant, wenn man in bekannten und eindeutigen Situationen agiert, während generalisierte Überzeugungen und Kompetenzerwartungen vor allem dann bedeutsam sind und prognostische Qualitäten aufweisen, wenn Situationen neuartig, mehrdeutig und durch einen hohen Grad an Ungewissheit geprägt sind.

Im Rahmen der Erläuterung der commitments (siehe Kap. 4.5.1) wurde deutlich, dass neben der Attraktivität des Ergebnisses bzw. einer Aufgabe auch die Zielerreichbarkeit durch das eigene Handeln von zentraler Bedeutung ist. Erscheint eine Aufgabe unlösbar, ein Ergebnis unerreichbar, spricht man vom Vorliegen eines Wirksamkeitsdefizits, einem ernsthaften Motivationsproblem. In Situationen, die unlösbar scheinen, die weder Spaß machen, noch durch Fremdkontrolle eine Handlung erfordern, bleibt demzufolge Engagement aus. Im konkreten Fall werden also die Kompetenzen, die man zu besitzen glaubt, mit den Anforderungen verglichen, die die Ergebniserreichung erfordern und so eine Erwartung, ein bestimmtes Handlungsziel durch eigenes Handeln zu erreichen konstituiert (Rheinberg, 2004). Hier wird deutlich, dass commitments und beliefs in einem wechselseitigen Bedingungsgefüge stehen und beispielsweise Überzeugungen über die eigene Wirksamkeit unmittelbar die Leistungsmotivation beeinflussen können. Im Rahmen dieser Untersuchung wurden beliefs durch die Skala zur Erfassung der Lehrerselbstwirksamkeitserwartung von Schmitz und Schwarzer (2000), sowie den Fragebogen zu Kompetenz- und Kontrollüberzeugungen von Krampen (1991) als zentrale Elemente der aus Situation, Handlung, Ergebnis und Folgen bestehenden kognitiv repräsentierten Ereigniskette erfasst.

\subsubsection{Selbstwirksamkeitserwartung (self-efficacy)}

„Unter Selbstwirksamkeit versteht man die subjektive Gewissheit, eine neue oder schwierige Aufgabe auch dann erfolgreich bearbeiten zu können wenn sich Widerstände in den Weg stellen“ (Schmitz \& Schwarzer, 2000, S. 12). 
Das Konstrukt der Selbstwirksamkeit ist eingebunden in die sozial kognitive Theorie, in der sie eine zentrale Rolle spielt. Als Synonym wird auch der Begriff Selbstwirksamkeitserwartung verwendet. Selbstwirksamkeit ist zukunftsorientiert und mehr von der wahrgenommenen, anstatt der tatsächlich vorliegenden Kompetenz abhängig, d.h. sie weist einen stark subjektiven Charakter auf (Woolfolk Hoy \& Burke-Spero, 2005). Ursprünglich geht das Konzept der Selbstwirksamkeit auf Bandura (1977) zurück, der die subjektive Erwartung der persönlichen Wirksamkeit dafür verantwortlich macht, ob überhaupt eine Bewältigungshandlung initiiert wird oder nicht. Neben der Auswahl einer Handlung, bestimmt diese Erwartung auch die Entscheidung, wie viel Einsatz gezeigt wird und wie hartnäckig und ausdauernd man auch Hindernissen und Widrigkeiten trotzt, um Handlungserfolge zu erreichen. Wie zuvor bereits angeführt, wird die tatsächliche objektive Leistung nicht nur durch tatsächliche objektive Fähigkeiten, sondern auch in starkem Maße von diesen abweichenden subjektiven Überzeugungen der Wirksamkeit des eigenen Handelns beeinflusst (Schmitz \& Schwarzer, 2000). Die Ausprägung der Selbstwirksamkeit wird bestimmt durch Erfahrungen, soziale Unterstützung und die Persönlichkeit des Individuums (Schunk, 1995).

Bandura (1977) unterscheidet des Weiteren mit der Höhe (magnitude später level), der Stärke (strength) und der Übertragbarkeit (generality) drei Dimensionen der Selbstwirksamkeitserwartung. Während sich die Höhe auf den Schwierigkeitsgrad der Aufgabe, für die die Kompetenzvorstellung existiert, bezieht, beschreibt die Übertragbarkeit die Situationsgeneralität und die Stärke die Stabilität der Selbstwirksamkeit. Selbstwirksamkeitserwartungen sind je nach dem Grad der Generalität auf unterschiedlichen Stufen der Erwartungshierarchie angesiedelt. Ursprünglich sah die Theorie von Bandura lediglich bereichs- bzw. situationsspezifische Selbstwirksamkeitserwartungen vor. Sowohl Ergebnisse aus der Forschung als auch unterschiedliche methodische Herangehensweisen führten jedoch dazu, dass man auch von generalisierter Kompetenzerwartung spricht, die mit der situativen Erwartung in einem wechselseitigen und dynamischen Verhältnis steht (Jerusalem, 1990; Voltmann-Hummes, 2008).

Nach Bandura (1977) existieren mit der Wirksamkeitserwartung (self-efficacy expectation) und der Ergebniserwartung (outcome-expectation) zwei Erwartungskomponenten, die voneinander abgegrenzt werden müssen, auch wenn meist Korrelationen zwischen .30 und .60 bestehen (siehe Abb. 9). Die Ergebnis- bzw. Konsequenzerwartung beinhaltet die Annahme, dass eine Handlung ein bestimmtes Ergebnis herbeiführen kann, wohingegen die Wirksamkeitserwartung sich darauf bezieht, ob bzw. dass man sich in der Lage sieht, die entsprechende Handlung auch ausführen zu können (Rheinberg, 2006). Während es bei den Ergebniserwartungen um die allgemeingültige 
Gesetzmäßigkeiten bzw. um die direkte Abhängigkeit des Ergebnisses von der Handlung geht, sind Kompetenzerwartungen stets durch einen Selbstbezug charakterisiert (Jerusalem, 1990). Ist eine Person überzeugt, effektiv zu handeln, werden Bewältigungshandlungen initiiert, geht sie hingegen davon aus, dass die eigenen Fähigkeiten nicht ausreichen, um eine erfolgreiche Bewältigungshandlung durchzuführen, resultiert Verängstigung und eher vermeidungsorientiertes bzw. passives Verhalten (Bandura, 1977).

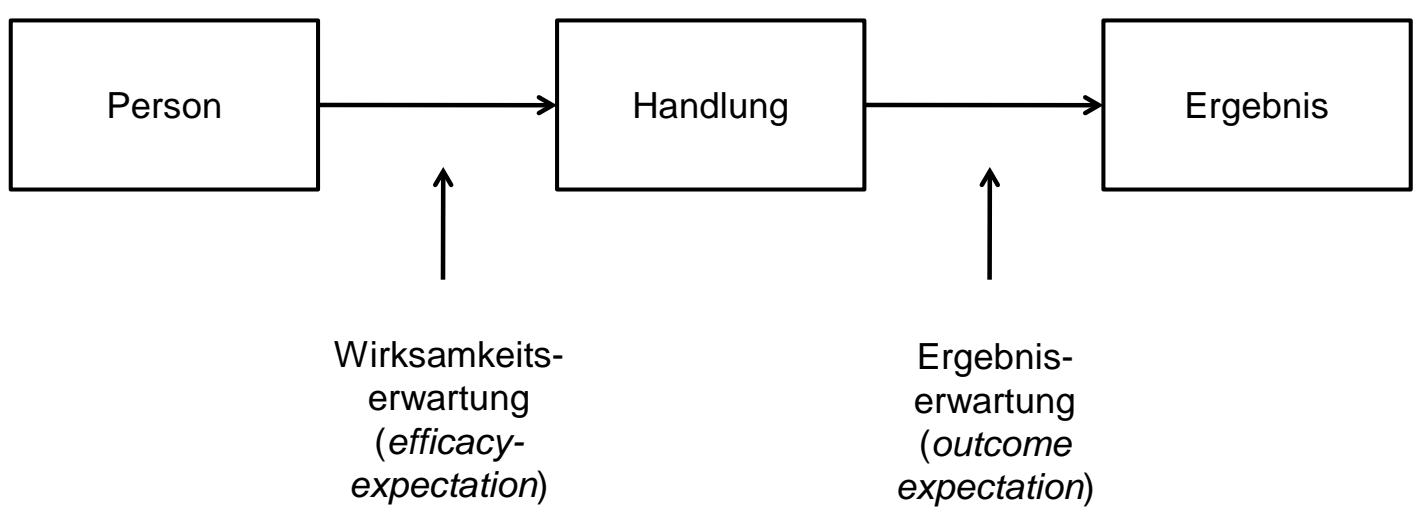

Abb. 9: Person-Handlung-Ergebnis: Wirksamkeits- und Ergebniserwartung nach Bandura (Rheinberg, 2006, S. 139).

Für die Entwicklung bzw. Ausprägung der Selbstwirksamkeitserwartung macht Bandura (1977) mit direkten Handlungserfahrungen, stellvertretenden Erfahrungen, sprachlichen Überzeugungen und körperlicher Erregung vier unterschiedliche Quellen verantwortlich. Direkte Handlungserfahrungen (mastery experience) durch eigenes aktives Handeln sowie das Erfahren der resultierenden Konsequenzen werden als besonderes einflussreich angesehen. Erfolgreiche Handlungen tragen zu einer positiven Entwicklung der Selbstwirksamkeitserwartung bei, während wiederholt auftretende Misserfolgserlebnisse negative Auswirkungen haben können. Ist bei einer Person die Selbstwirksamkeitserwartung deutlich ausgeprägt, ist der Einfluss gelegentlich auftretende Fehlschläge minimal. Im alltäglichen Wortgebrauch spricht man in diesem Zusammenhang oft von Selbstbewusstsein oder Selbstvertrauen. Bei stellvertretenden Erfahrungen (vicarious experience) resultiert die Einflussnahme auf die Erwartung der eigenen Wirksamkeit nicht auf eigenem aktivem Handeln, sondern auf modellhaften Beobachtungen. So kann z.B. das Verfolgen von anderen Personen, die in bedrohlichen Situationen erfolgreich und ohne negative Konsequenzen agieren, dazu führen, dass man denkt „wenn die das können, kann ich das schon lange“. Je größer die Ähnlichkeit zwischen der beobachteten Person und dem Selbst ist, desto einflussreicher sind die Erfahrungen nach dem Konzept der related attributes. Bei dieser Art der Erfahrung und 
der daraus resultierenden Stärkung bzw. Schwächung der eigenen Kompetenzerwartung werden jedoch die tatsächlichen Ressourcen weit weniger mit einbezogen als bei direkten Erfahrungen. Hierdurch gelten Veränderungen bezogen auf die eigene Wirksamkeitserwartung als weit weniger stabil. Sprachliche Überzeugungen (verbal persuasion) werden aufgrund ihrer leichten Hand- und stetigen Verfügbarkeit oft eingesetzt, sind jedoch noch einmal schwächer einzustufen als stellvertretende Erfahrungen, da sie nicht auf authentischen Begebenheiten basieren. Sowohl Selbstinstruktion als auch die Äußerungen Dritter können dazu dienen, Personen davon zu überzeugen, dass sie Situationen bewältigen können. Im umgekehrten Fall können natürlich auch sprachliche Überzeugungen dafür verantwortlich sein, dass Situationen mit negativer Grundeinstellung angegangen oder gar komplett gemieden werden. Körperliche Erregung (physiological arousal) dient als Indikator in bedrohlichen Situationen. Eine geringe Erregung kann als Anzeichen für eine erfolgreiche Bewältigung interpretiert werden, wohingegen starke Erregung abhängig vom individuellen Attributionsstil auf Probleme oder mangelnde Kompetenzen hindeuten können (Bandura, 1977; Jerusalem, 1990; Schmitz \& Schwarzer, 2000, Woolfolk Hoy \& Burke-Spero, 2005).

Tschannen-Moran, Wolfolk Hoy und Hoy (1998) entwickelten ein Modell (siehe Abb. 10), in dem Lehrer-Selbstwirksamkeit und deren Entwicklung in einem zyklischen Prozess eingebunden sind. Die bereits dargestellten Quellen der Selbstwirksamkeitserwartung dienen als Basis für kognitive Prozesse, die eine Bewertung der aktuellen Situation und den zur Verfügung stehenden Bewältigungsmöglichkeiten vornehmen.

Wie man anhand des Modells erkennen kann, beeinflusst die resultierende Selbstwirksamkeitserwartung die Planung und Durchführung von Unterricht. Bereits während der Planung von Unterricht versuchen Lehrkräfte mit einer geringen Ausprägung der Selbstwirksamkeit bewusst Inhalte und Methoden zu meiden, die ihre Fähigkeiten eventuell überschreiten könnten und zeigen sich, bezogen auf Unterrichtsziele und das Aspirationsniveau, eher zurückhaltend und vorsichtig. Im Unterricht neigen sie eher dazu, passiv oder ausweichend zu reagieren d.h. sie suchen alternative Möglichkeiten mit der Problematik umzugehen. Im Gegensatz hierzu führt eine hohe Selbstwirksamkeitserwartung dazu, dass z.B. Schwierigkeiten von Schülern eher beachtet werden und eine größere Beharrlichkeit und Ausdauer gezeigt wird. Lehrkräfte mit einer ausgeprägten Selbstwirksamkeit bemühen sich, den Schülern den Unterrichtsstoff in einer anderen, für die Schüler besser verständlichen Art und Weise zu vermitteln. Weiterhin investieren sie mehr Anstrengung in die Beschaffung von Unterrichtsmaterial, sind enthusiastischer, offen für neue Ideen und Methoden, nehmen Herausforderungen an und setzen alles daran, Schüler zum angestrebten Ziel zu führen, auch wenn Umwege 
und zusätzlicher Aufwand hierfür notwendig sind. Durch das Engagement der Lehrkraft werden die Leistungen der Schüler positiv beeinflusst und damit gleichzeitig in einer Art Rückkopplungsmechanismus auch die Selbstwirksamkeitserwartung der Lehrkraft. In Studien stellte sich heraus, dass die Lehrer-Selbstwirksamkeitserwartung ein signifikanter Prädiktor der Schülerleistung darstellt (Schunk, 1995; Woolfolk Hoy \& BurkeSpero, 2005). Zugleich ist die Selbstwirksamkeitserwartung ein protektiver Faktor für die Lehrkräfte, der vor Belastungserleben und langfristig sogar vor Burnout schützen kann (Abele \& Candova, 2007; Schmitz, 2001; Christ, Wagner \& van Dick, 2004; van Dick, 2006).

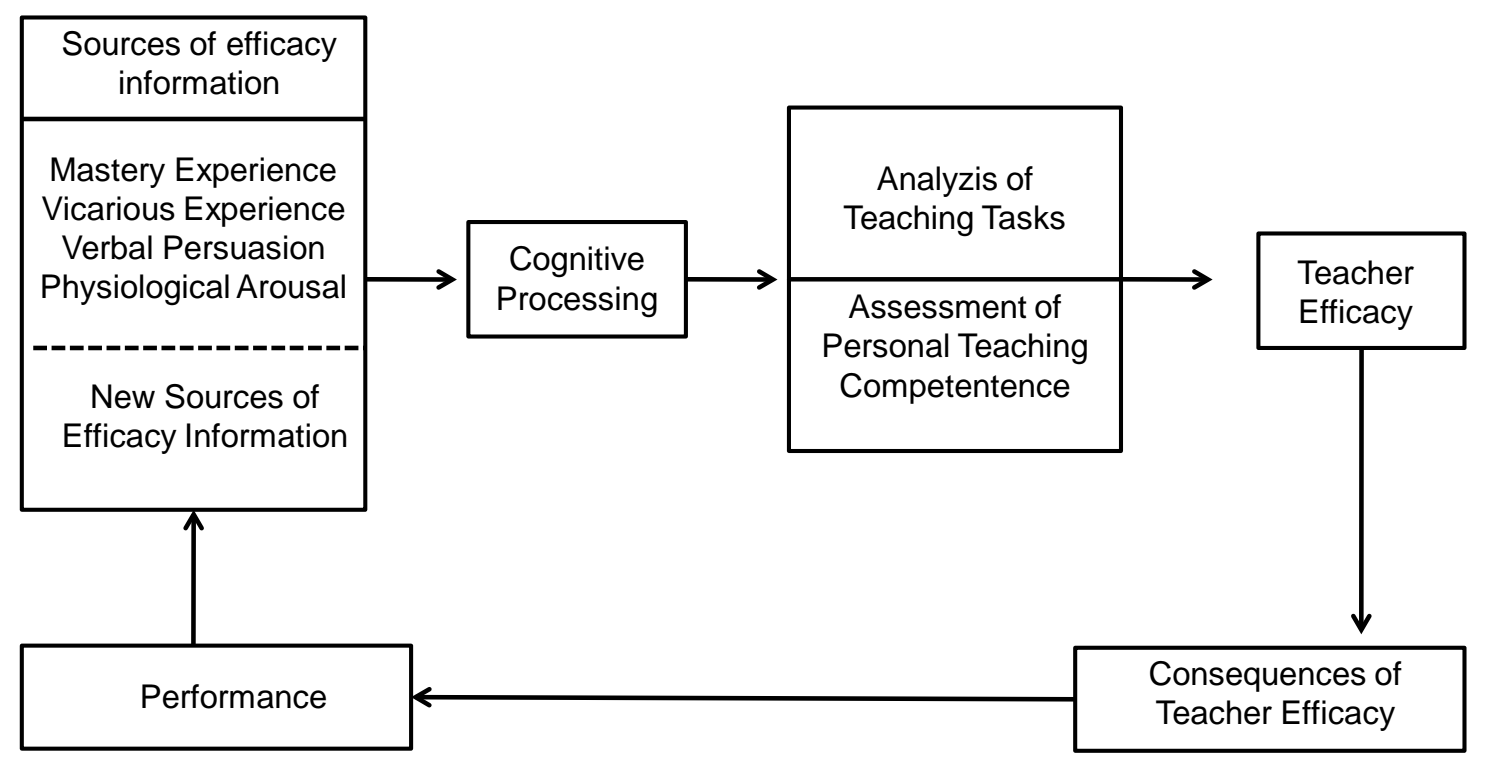

Abb. 10: Entwicklung der Selbstwirksamkeitserwartung von Lehrern (Tschannen- Moran, Woolfolk Hoy \& Hoy, 1998)

Egal ob im positiven oder im negativen Sinne: Handlungssituationen dienen durch Rückmeldungen über Erfolg bzw. Misserfolg als Quelle neuer Informationen für die kognitive Interpretation zukünftiger Ereignisse (Tschannen-Moran, Woolfolk Hoy \& Hoy, 1998). Der Einfluss auf die Selbstwirksamkeit wird jedoch nicht allein durch den tatsächlichen Handlungsverlauf bestimmt, sondern zu einem nicht unerheblichen Teil auch durch die Art und Weise, in der man die Zuschreibung von Erfolg und Misserfolg vornimmt. Schreibt man Erfolge den eigenen Fähigkeiten und Fertigkeiten zu, also internalen Faktoren, wird die Selbstwirksamkeitserwartung positiv beeinflusst. Geht man hingegen davon aus, dass der Erfolg durch Glück, Zufall oder das Mittun Dritter bedingt ist, wird der positive Einfluss geringer ausfallen. Solche Zusammenhänge werden im sich anschließenden Kapitel 4.5.2.2 näher beschrieben.

Setzt man Banduras Theorie bezogen auf den Lehreralltag um, muss also grundsätzlich unterschieden werden zwischen dem Wissen, mit welchen Handlungen man z.B. Kon- 
flikten mit Schülern begegnet, um sowohl den Erziehungs- als auch den Bildungsauftrag zu erfüllen (Ergebniserwartung) und der subjektiven Überzeugung, sich zu diesen Handlungen auch in der Lage zu sehen (Wirksamkeitserwartung). Beispielhaft für diese Unterscheidung kann der Umgang mit Motivations- und Disziplinproblemen von Schülern betrachtet werden. Diesen alltäglichen Problemen kann auf unterschiedliche Art und Weise begegnet werden. Gespräche mit Schülern oder Eltern können als wirksame Intervention das Verhalten nachhaltig beeinflussen und Probleme beheben. Über dieses Wissen sollten alle ausgebildeten Lehrkräfte verfügen. Die Überzeugung, sich selbst für diese Handlung (Wirksamkeitserwartung) als kompetent einzuschätzen, wird hingegen nicht automatisch bei jeder Lehrkraft in gleicher Art und Weise vorhanden sein. Um sinnvolle Aussagen über einen spezifischen Bereich wie z.B. den Lehrerberuf zu machen ist es notwendig, dass Items, die sich auf die Selbstwirksamkeit beziehen, inhaltliche Spezifität aufweisen. Schmitz und Schwarzer (2000) entwickelten eine auf der sozial-kognitiven Theorie basierende Skala zur Erfassung der Selbstwirksamkeitserwartung, die spezifisch an das Berufsfeld und die entsprechenden Kompetenzanforderungen adaptiert ist (siehe Kap. 6.6.8).

\subsubsection{Kontrollüberzeugungen (locus of control)}

Wie in Kapitel 4.5.2.1 angedeutet, nehmen selbstwirksamkeitsrelevante Informationen nicht direkt Einfluss auf die subjektive Kompetenzeinschätzung sondern, durchlaufen kognitive Prozesse, in denen objektive Informationen in Form von Informationsverarbeitungsprozessen selektiert, gewichtet, interpretiert oder gar verzerrt werden, bevor sie als subjektive Repräsentationen auf das Verhalten Einfluss nehmen. Im Zusammenhang mit der Selbstwirksamkeitserwartung ist also die Attribution von Leistungsergebnissen von besonderer Bedeutung (Jerusalem, 1990).

Nach Rotter und Mulry (1965) stellen Kontrollüberzeugungen (locus of control) generalisierte Erwartungen einer Person dar, ob Ereignisse im Leben beeinflussbar sind (internale Kontrollüberzeugung) oder nicht (externale Kontrollüberzeugung). Gerade in subjektiv neuen und mehrdeutigen Situationen, die nur in geringem Maße strukturiert werden können, nimmt der diagnostische Wert bereichsspezifischer Variablen wie der der Kontrollüberzeugungen zu. Krampen (1991) fasst im Gegensatz zu Rotter und Mulry Kontrollüberzeugungen nicht als unidimensional und bipolar auf, sondern als mehrdimensionales Konstrukt, indem er drei Aspekte unterscheidet. Unter Internalität ist die von der eigenen Person wahrgenommene Kontrolle über die Ereignisse in der Umwelt und das eigene Leben zusammengefasst. Externale Kontrollüberzeugungen differenziert Krampen in sozial bedingte Externalität und fatalistische Externalität. Während man bei ausgeprägter sozial bedingter Externalität davon überzeugt ist, dass 
wichtige Ereignisse im eigenen Leben durch andere mächtige Personen bestimmt werden, stellt die fatalistische Externalität die generalisierte Erwartung dar, dass der Lauf der Dinge durch Glück, Pech und vor allem das Schicksal gelenkt wird oder sogar vom Zufall abhängt.

Für die Arbeit des Lehrers scheint es demnach von Vorteil zu sein, wenn man davon überzeugt ist, sein eigenes Leben, und das schließt nun einmal auch den Beruf mit ein, selbst bestimmen zu können. Man vertritt die eigenen Interessen und Erfolg und Misserfolg scheinen von der eigenen Anstrengung abhängig zu sein. Gegenüber Personen, die sich durch eine hohe Externalität auszeichnen, sind Personen mit ausgeprägter Internalität eher zur Selbstaktualisierung fähig und in ihrem Urteilsvermögen unabhängiger. Lazarus und Folkman (1984) bringen Kontrollüberzeugungen in direkte Beziehung zur Bewältigung und bezeichnen externale Überzeugungen als ernsthaftes Hindernis problemorientierten Copings. Die Überzeugung, selbst die Kontrolle über den Ausgang von Situationen zu haben, bestimmt zusammen mit den Wirksamkeitserwartungen den Einsatz und die Hartnäckigkeit bei Bewältigungsmaßnahmen, während Externalität eher zu Hilflosigkeit führt. Anderson (1974) stellte fest, dass external Überzeugte Stress intensiver wahrnehmen und eher defensive Bewältigungsstrategien nutzen. Weiterhin konnte im Rahmen einer Längsschnittuntersuchung nachgewiesen werden, dass bei internal überzeugten Personen, die ihre Leistung zwischen den Messzeitpunkten steigern konnten, im Anschluss eine noch stärkere Ausprägung der internalen Kontrollüberzeugungen festzustellen war. Eine Leistungsverschlechterung der internalen Probanden hingegen führte zu keiner Veränderung der Ausprägung der Kontrollüberzeugungen. Externale, die sich in ihrer Leistung steigerten, zeigten eine leichte Tendenz dazu, noch etwas externaler zu denken, während Leistungsverschlechterungen zu einer starken Zunahme externaler Überzeugung führte. Extremausprägungen von Internalität können jedoch wie Borges et al. (1980) zeigten zu unrealistischen Erwartungen, Omnipotenzgefühlen, Selbstüberschätzung und Egozentrismus führen und damit ebenfalls negative Voraussetzungen für zukünftige Handlungssituationen darstellen (Krampen, 1991). Umgesetzt hat Krampen seine theoretischen Vorstellungen in der Entwicklung eines Fragebogens, der zunächst lediglich eine Weiterentwicklung des IPC-Fragebogens von Levenson (1972) darstellen sollte. Letztendlich führten die Arbeiten aber nicht zu einer Itemrevision, sondern zu einer differenzierten Neuentwicklung, die im Rahmen dieser Erhebung eingesetzt wurde und deren Struktur genauer in Kapitel 6.6.2 dargestellt wird. 


\subsection{Coping-Modelle}

Neben den physiologischen Modellen, in denen vor allem der Verlauf des Anpassungsvorgangs als Reaktion auf ein Stressereignis auf der Ebene verschiedener Aktivitäten des Organismus aufgeschlüsselt und beschrieben wird, kann Coping auch in Form von Phasen- bzw. Prozessmodellen dargestellt werden. In den biologisch-chemisch orientierten physiologischen Modellen werden hauptsächlich hormonale Wirkzusammenhänge beschrieben. Auf diese Konzeptionen wird in dieser Arbeit nicht eingegangen. In Phasen- und Prozessmodellen wird hingegen in vereinfachter und übersichtlicher Art und Weise versucht, die komplexen Abläufe und Zusammenhänge des Copingverhaltens zu abstrahieren und wissenschaftlich handhabbar darzustellen. Grundsätzlich unterscheidet man zwischen recht starren Phasenmodellen und variablen Prozessmodellen, die beide im Folgenden kurz dargestellt und beschrieben werden.

Bei der phasenhaften Darstellung des Bewältigungsvorgangs wird dieser als statisch gegliedert gesehen und die Abfolge der einzelnen Phasen ist festgelegt und unidirektional. Variabel sind lediglich die Dauer und die Intensität der Phasen, zudem sind Überschneidungen möglich. Die Anzahl der Phasen in den Modellen schwankt zwischen zwei im einfachsten und sechs im komplexesten Modell. In ZweiPhasenmodellen unterscheidet man zwischen einer initialen Schockphase, die meist stark emotional erlebt wird und einer Bearbeitungsphase, in der ein rationales Bewusstmachen einsetzt und eine schrittweise Integration ins Lebenskonzept erfolgt. Während in der Schockphase oft Abwehrmechanismen wie Verleugnung Anwendung finden, ist die Bearbeitungsphase durch aktives und bewusstes Bewältigen gekennzeichnet. Das am häufigsten beschriebene Phasenmodell ist das Drei-Phasenmodell, bei dem ebenfalls eine Schockphase mit Verleugnung und Vermeidung als initial gesehen wird. Durch das Abschirmen von der Realität wird das Ich vor einer Traumatisierung geschützt. In der zweiten Phase, die oft als Übergangsphase bezeichnet wird, findet ein Bewusstwerden statt, in der durch das Schwinden von Abwehrmechanismen eine schrittweise Integration erfolgt und die Realität akzeptiert wird. In der dritten Phase führt das Geschehen zu einer Reorganisation von Zukunftsplänen oder des Selbstkonzepts. Im Drei-Phasenmodell von Sutherland und Scherl (1970) ist nach der zweiten Phase, in der scheinbar Probleme durch eine Pseudoanpassung in Form von Verleugnung, Rationalisierung oder Suppression beseitigt sind, eine dritte Phase der Depression vorgesehen, in der die Gefühle der ersten Phase wieder auftauchen. Ein weiteres Drei-Phasenmodell stammt von Chodoff (1970) in dem aufgrund von extremen Gegebenheiten selbst in der dritten Phase noch Abwehrmechanismen vorherr- 
schen, da eine Bewältigung unmöglich erscheint und so eher von einer Langzeitanpassung gesprochen wird. In Vier-Phasenmodellen stellt Desorganisation die erste Phase dar, die geprägt ist durch Verwirrung, Einsamkeit, Schuldgefühle und Ambivalenz, bevor sich die Gefühle in der zweiten Phase in destruktiven, oberflächlichen oder antisozialen Verhaltensäußerungen manifestiert. In der dritten Phase wird versucht, den alten Lebensweg wieder zu erreichen und an die Zukunft zu denken. Letztendlich setzt eine Anpassung samt Integration ins Lebenskonzept in der vierten und letzten Phase ein. Fünf-Phasenmodelle zeichnen sich durch eine initiale Verneinung aus, die in aggressives Verhalten übergeht, bis letztendlich über Ausagieren und defensive Gefühlzustände die Stufe der Adaption erreicht wird. Die existierenden Sechs-Phasenmodelle weisen lediglich in der ersten und der letzten Phase Übereinstimmungen auf. Auf eine Schockphase, in der der Selbstschutz an oberster Stelle steht, folgen in unterschiedlicher Zahl Phasen, die von Trauer, Resignation und Abwehrmechanismen bestimmt sind, bevor an einem bestimmten Punkt Gegebenes akzeptiert wird und zukunftsbezogene Kognitionen das Denken zunehmend bestimmen (Nusko, 1986).

Wie in der zusammenfassenden Darstellung der Phasenmodelle deutlich wurde, ist die Einteilung in Phasen als relativ willkürlich zu sehen. Mit zunehmender Phasenanzahl kommt zusätzlich das Problem hinzu, die einzelnen Phasen voneinander abzugrenzen. Als relativ gesichert, da in allen Modellen enthalten, kann eine initiale Schock- und eine finale Adaptionsphase gesehen werden, während die Einteilung und Benennung der Phasen dazwischen von Modell zu Modell unterschiedlich ist.

In Prozessmodellen liegt keine konstante und unveränderliche Abfolge von phasenhaften Reaktionsmustern vor, sondern sie werden durch Interaktion oder Transaktion verschiedener Systeme bestimmt. Hierbei kann es zu jeder Zeit zu einer Reorganisation und Neuausrichtung kommen. In diesen Modellvorstellungen ist keine Kategorisierung in abhängige und unabhängige Komponenten möglich aufgrund des interdependenten Beziehungsgeflechts. Im Modell der Ich-Prozesse geht man davon aus, dass Beziehungen zwischen Coping-, Abwehr- und Fragmentierungsprozessen bestehen, in denen die zehn Hauptprozesse des Ichs $^{2}$ Ausdruck finden. Unter Coping wird dabei ein zweckhafter, unter Defense ein erzwungener und negierenden und unter Fragmentation ein automatischer und ritualisierter Prozess verstanden. Welcher Prozess in einer bestimmten Situation gewählt wird, beschreibt Haan (1963, zitiert aus Nusko, 1986, S. 44) folgendermaßen: „The person will cope if he can, defend if he must, and fragment if he is forced, but whichever mode he uses, it is still in the service of his attempt to

\footnotetext{
${ }^{2}$ Discrimination, detachment, means -end symbolization, delayed response, sensitivity, time reversion, selective awareness, diversion, transformation, restraint.
} 
maintain organisation“. Bei den Anforderungen wird zwischen normativen und nichtnormativen Formen unterschieden. Während bei der erstgenannten Form die Anpassung der Umwelt an die Bedürfnisse des Ichs (Assimilation) und die Anpassungen des Ichs an die Umwelt (Akkomodation) synchron verlaufen bzw. erfolgen, verläuft dies bei nicht-normativen Anforderungen asynchron. Bei den normativen Formen kommt es zu situationsadequaten und koordinierten Handlungen, während bei nicht-normativen Anforderungen unkoordiniert gehandelt wird und Affekte störenden Einfluss auf die Bewältigung ausüben. Durch Interaktion der Strukturen und Rückmeldung der Handlungseffekte ist eine individuelle Entwicklung im Rahmen solcher Prozessmodelle möglich (Nusko, 1986).

Neben dem Modell der Ich-Prozesse stellt das bereits in Kapitel 3 dargestellte transaktionale Stresskonzept von Lazarus das weitaus elaborierteste kognitive Prozessmodell dar. Im transaktionalen Stresskonzept beginnt der adaptive Prozess der Bewältigung mit einer stressrelevanten primären Bewertung und dem darauf folgenden komplexen Auswahlprozess der relevanten und adäquaten Form der Bewältigung im Rahmen der sekundären Bewertung. Sowohl bei der primären als auch bei der sekundären Bewertung spielen Neueinschätzungen eine bedeutende Rolle, sodass eine bedrohliche Situation durch das Vorliegen effizienter Bewältigungsressourcen bzw. -formen als weniger bedrohlich eingestuft wird. Dasselbe gilt natürlich auch für den umgekehrten Fall. Wie sich im sich anschließenden Kapitel 4.7 zeigen wird, unterscheiden Lazarus und Launier (1978) zwischen vier Formen der Bewältigung , die zu der Lösung von Problemen oder zumindest zu deren Linderung eingesetzt werden können und auf vergangene, gegenwärtige oder zukünftige Ereignisse gerichtet sind (Nusko, 1986).

\subsection{Kategorisierung von Bewältigungsformen und -arten}

Bei der Kategorisierung von Coping stellt meist eine dichotome Einteilung in aktiv gegen die Umwelt gerichtete Reaktionen und intrapsychischen Abwehrreaktionen das grundlegende Schema dar. Während Goldstein (1959) Versuchspersonen schlicht in Coper und Avoider einteilt, nehmen Lazarus und Folkman (1984) bei der Kategorisierung von Bewältigung grundsätzlich die verschiedenen Zielsetzungen, die angestrebt werden bzw. Funktionen die die Anstrengungen innehaben, in den Fokus. Auf der einen Seite identifizieren sie mit problem-focused coping das Bestreben, die Problemlage zu verbessern und auf der anderen Seite emotion-focused coping, welches dazu dient, die eigene emotionale Befindlichkeit durch die Regulation von Emotionen zu verbessern. Problemorientiertes Coping zielt auf eine Veränderung des gestörten Person- 
Umwelt-Verhältnis ab, ist daher eher als umgebungsbezogen zu bezeichnen, während bei dem emotionsorientierten Coping eher selbstbezogen agiert wird. Neben der Bezeichnung als emotionsorientiertes Coping, wird diese Verhaltensweise auch als palliatives Coping bezeichnet, da zwar an der Problemlage keine Änderungen bewirkt wird, durch die Regulierung und Kontrolle der aus der Transaktion hervorgegangenen somatischen und subjektiven emotionalen Reaktionen dennoch ein adaptiver Wert entstehen kann. Genauso kann jedoch palliatives Coping notwendige instrumentelle Bewältigungshandlungen verhindern oder verzögern (Jerusalem, 1990; Laux, 1983). Problemorientiertes Coping-Verhalten wird vor allem dann gezeigt, wenn die Situation kontrollierbar erscheint und nützliche bzw. sinnvolle Verhaltensweisen sich anbieten. Emotionsorientierte Bewältigungsmaßnahmen hingegen werden verstärkt dann einsetzt, wenn Personen das Gefühl haben, gegen einen Stressor nichts ausrichten zu können und diesen ertragen bzw. dulden müssen und daher eher intrapsychische Strategien einsetzen (Carver et al., 1989).

Im Rahmen des emotionsorientierten bzw. problemorientierten Coping-Prozesses werden verschiedene Arten der Bewältigung eingesetzt: Neben Informationssuche, einer direkten Handlung und dem Unterlassen einer Handlung, werden zudem intrapsychische Bewältigungsformen genannt. Unter Informationssuche wird eine Bewältigungsart verstanden, die sowohl genutzt wird um instrumentelle Versuche der Veränderung einer Transaktion zu unterstützen, als auch um palliative Funktion zu übernehmen. Zusätzliche Informationen über eine spezifische Person-Umwelt-Konstellation können dazu dienen, sich selbst zu beruhigen oder getroffene Entscheidungen zu rechtfertigen. Charakteristische Elemente des Stressereignisses werden herausgefiltert und können so zur Linderung oder Lösung des Problems beitragen. Wenn die sich anschließenden Coping-Aktivitäten auf die eigene Person bzw. die Umwelt gerichtet sind, spricht man von direkten Handlungen, die sowohl instrumentelle als auch emotionsregulierende Funktion z.B. in Form von Entspannungsübungen haben können. Auch das Unterlassen von Handlungen kann sinnvoll sein, um eine Verschlimmerung zu verhindern oder wenn Handlungen z.B. den allgemein anerkannten gesellschaftlichen Normvorstellungen widersprechen würden. Unter intrapsychischen Bewältigungsformen werden all diejenigen kognitiven Prozesse summiert, die der emotionalen Kontrolle und Regulation dienen. Abwehrmechanismen wie Verneinung, Intellektualisierung und Isolation können jedoch auch instrumentelle Funktionen erfüllen wie z.B. durch positive Selbstinstruktion (Laux, 1983).

Neben der zuvor dargestellten Taxonomie der Bewältigungsarten der Lazarusgruppe existiert in der wissenschaftlichen Literatur, aufbauend auf der grundsätzlichen Zwei- 
teilung in aktiv gegen die Umwelt gerichtete Reaktionen und intrapsychische Abwehrmechanismen eine verwirrende Fülle an Klassifikationsansätzen. Verwoerdt (1972) schlägt beispielsweise mit einer Einteilung in Rückzug, Verdrängung und Meisterung eine eher produktorientierte Gliederung vor, während Mitscherlich \& Mitscherlich (1967) die Untergliederung der Bewältigungsmechanismen stärker auf die Form und den Ort der Manifestation fokussiert. Er unterscheidet zwischen passiv-äußerer, passiv-innerer, aktiv-äußerer und aktiv-innerer Bewältigung (Nusko, 1986).

Perrez und Reicherts (1992) differenzieren das Bewältigungsverhalten in aktives, evasives und passives Verhalten. Im ersten Fall wird in direkter Art und Weise Einfluss auf die stressverursachenden Komponenten genommen. Bei evasiven Handlungen ist das Vermeiden bzw. die Flucht vor den Stressoren von primärer Bedeutung, während passive Verhaltensweisen Reaktionen wie Zögern, Abwarten und Unterlassen einschließen (Perrez \& Reicherts, 1992). Endler und Parker (1994) identifizierten zusätzlich mit avoidance, dem Vermeiden von Problemen, eine weitere Copingdimension, die sowohl aufgaben- bzw. problemorientierte als auch personen- bzw. emotionsorientierte Anteile beinhaltet.

Czerwenka (1996) greift in seinem theoretischen Ansatz die Differenzierung von Lazarus und Folkman (1984) in problembezogenes bzw. instrumentelles und emotionsbezogenes bzw. palliatives Coping auf und ergänzt diese beiden Arten subjektiver Begegnungsmöglichkeiten durch die jeweilige Richtung, in die sie abzielen (siehe Abb. 11).

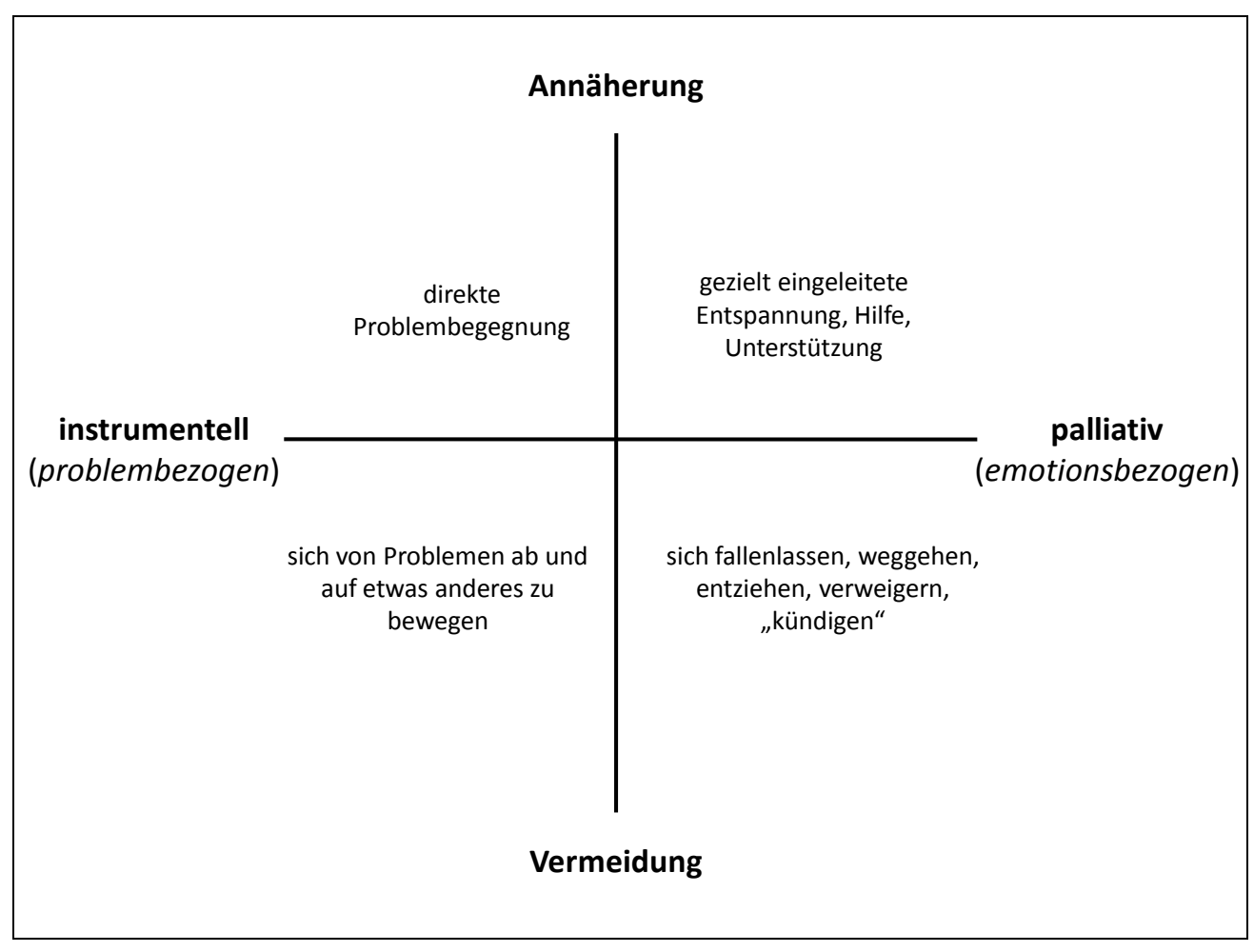

Abb. 11: Grundformen der Bewältigung (Czerwenka, 1996; mod. nach van Dick, 2006, S. 53) 
Während mit Annäherung ein direktes Zugehen auf den bzw. die Auslöser des Problems bzw. dessen spezifischen Symptome gemeint ist, stellt Vermeidung eher eine vom Problem bzw. den Symptomen abwendende Richtung dar. Die vier Grundformen der Bewältigung ließen sich auch empirisch durch faktorenanalytische Verfahren bestätigen.

Pines, Aronson und Kafry (1985) sehen ebenfalls eine zweidimensionale Taxonomie der Bewältigungsmaßnahmen vor. Auf der einen Seite unterscheiden sie in aktive, den Stressfaktor oder sich selbst verändernde Bewältigungsversuche und passive Maßnahmen, in der Stressereignisse vermieden, geleugnet oder sogar vor ihnen geflohen wird. Zusätzlich untergliedern sie in direkte und indirekte Maßnahmen, sodass vier unterschiedliche Typen (siehe Tab. 2) resultieren. Direkte Maßnahmen sind äußere Handlungsweisen, die sich gegen die umweltgegebenen Stressfaktoren richten, während indirekte Maßnahmen als innere Strategien zu verstehen sind. Die zwölf angegebenen Verhaltensweisen wurden in einer Untersuchung Beispielen aus dem Lehreralltag zugeordnet und in Form eines Fragebogens zum Aufdecken von Zusammenhängen mit Burnout genutzt.

Tab. 2: Vier Typen der Bewältigung einschließlich möglicher Verhaltensweisen für jeden Typ Pines, Aronson und Kafry (1985)

\begin{tabular}{|l|l|l|}
\hline & \multicolumn{1}{|c|}{ aktiv } & \multicolumn{1}{c|}{ inaktiv } \\
\hline \multirow{4}{*}{ direkt } & - die stresshafte Situation verändern & - die stresshaften Elemente der Situation \\
& - bestimmte Stressfaktoren beeinflussen & ignorieren \\
& - eine positive Einstellung einnehmen & - die stresshaften Elemente der Situation \\
& & vermeiden \\
& & - die Situation verlassen \\
\hline \multirow{3}{*}{ indirekt } & - über den Stress sprechen & - Trinken \\
& - Selbstveränderung & - krank werden \\
& - andere Tätigkeit aufnehmen & - zusammenbrechen \\
\hline
\end{tabular}

\subsection{Messinstrumente zur Erfassung von Coping - Differenzie- rung von Bewältigungsprozessen}

Verschiedene Wissenschaftler wollten sich jedoch nicht mit theoretischen Gliederungen abfinden und suchten auf empirischem Weg durch faktorenanalytisches Vorgehen nach evidenten Differenzierungen von Bewältigungsprozessen. Gleichzeitig wurden, die in diesem Zusammenhang entstehenden Messinstrumente dazu genutzt, Korrelationen von verschiedenen Bewältigungsstilen und Persönlichkeitseigenschaften sowie psychischer und physischer Gesundheit aufzudecken. Der von Folkman et al. (1986) entwickelte "Ways of Coping Questionnaire“ (WCQ) ist das am Weitesten verbreitete 
Instrument und wurde in vielen unterschiedlichen Kontexten eingesetzt (Parker, Endler \& Bagby, 1993). In der ersten Version aus dem Jahr 1980 wurde unter der Bezeichnung "Ways of Coping Scale" oder auch "Ways of Coping Checklist" (WCC) in 60 Items versucht, die aus den theoretischen Überlegungen stammende Differenzierung der Bewältigungsarten in Informationssuche, direkte Handlung, Unterdrückung von Handlung oder intrapsychische Prozesse in dichotomisierter Form zu erfassen. In der Folgezeit wurde der Fragebogen durch Ausschluss bzw. Hinzufügen von Items und Modifikation des Antwortformats hin zu einer vierstufigen Likertskalierung zu einem 66 Items umfassenden Instrument weiterentwickelt, das sie "Ways of Coping Questionnaire" (WCQ) nannten (Lazarus \& Folkman, 1987). Da sich nach Lazarus Coping erst durch die spezifische Person-Situation-Transaktion determinieren lässt, sieht die Instruktion des Fragebogens stets ein einzelnes spezifisches stressreiches Ereignis vor, auf das die Antworten bezogen werden sollen. Diesem situationsorientierten Ansatz steht der im Anschluss vorgestellte verallgemeinernde Ansatz gegenüber. Faktorenanalytische Untersuchungen des neu konstruierten Instruments führten zu einer achtfaktoriellen Struktur, bei der sechs Faktoren als emotionsorientiert und nur ein Faktor als problemorientiert zu bezeichnen ist. Ein weiterer Faktor erwies sich als eine Mischform aus emotionsorientiertem und problemorientiertem Coping dar (Parker, Endler \& Bagby, 1993). Neben konfrontativer Bewältigung (confrontative coping), kognitiver Distanzierung (distancing), Selbstkontrolle (self-control), Suche nach sozialer Unterstützung (seeking social support), Übernahme von Verantwortung (accepting responsibility), Flucht-Vermeidung (escape-avoidance) und problembezogenen Lösungsversuchen (planful problem-solving) gehören auch positive Neueinschätzungen (positive reappraisal) zu den faktorenanalytisch extrahierten Dimensionen der Bewältigung (Lazarus \& Folkman, 1987). In Tabelle 3 ist zu den einzelnen Dimensionen jeweils ein Beispielitem aufgeführt.

Tab.3: Beispielitems des „Ways of Coping Questionnaires" (WCQ) (zitiert nach van Dick, 2006)

\begin{tabular}{|l|l|}
\hline Dimension & Beispielitem \\
\hline Konfrontative Bewältigung & $\begin{array}{l}\text { „Ich drücke meinen Ärger gegenüber der Person aus, die die Probleme ver- } \\
\text { ursacht hat.“ }\end{array}$ \\
\hline Kognitive Distanzierung & "Ich versuche, die ganze Sache zu vergessen." \\
\hline Selbstkontrolle & $\begin{array}{l}\text { "Ich versuche, nicht zu hastig zu handeln oder meinen ersten Impulsen } \\
\text { nachzugeben." }\end{array}$ \\
\hline Suche nach sozialer Unterstützung & "Ich frage einen Verwandten oder Freund um Rat." \\
\hline Übernahme von Verantwortung & "Ich nehme mir vor, es beim nächsten Mal besser zu machen." \\
\hline Flucht-Vermeidung & "Ich hoffe auf ein Wunder." \\
\hline Problembezogene Lösungsversuche & "Ich mache mir einen Plan und handele danach." \\
\hline Positive Neueinschätzung & "Ich sage mir, dass ich aus dieser Erfahrung lernen kann." \\
\hline
\end{tabular}


In der Folgezeit wurde jedoch das entwickelte Instrument heftig kritisiert, da die faktorielle Struktur nicht replizierbar war. Aldwin und Revenson (1987) konnten zwar ebenfalls acht Faktoren im Rahmen einer Hauptkomponentenanalyse extrahieren, diese entsprachen aber nicht den von Folkman et al. (1986) eigentlich vorgesehenen Faktoren und auch die konfirmatorische Faktorenanalyse von Parker, Endler und Bagby (1993) können die vorgeschlagene multidimensionale Lösung nicht bestätigen. Weiterhin wird kritisiert, dass die Höhe der Interkorrelationen zwischen den Dimensionen nicht akzeptabel sind, da beispielsweise konfrontative Bewältigung deutlich mit kognitiver Distanzierung und Flucht-Vermeidung korreliert.

Endler und Parker (1994) machten es sich zur Aufgabe, einen angemessenen, reliablen und validen Fragebogen zu entwerfen, um ein klares Modell von Coping zu ermöglichen. Nach der Generierung, Analyse und Selektion von Items entstand das Instrument das als "Coping Inventory for Stressful Situations" (CISS) bezeichnet wird. Im Unterschied zu dem WCQ wird hier mithilfe einer fünfstufigen Likert-Skalierung das durchschnittliche Verhalten abgefragt, da Endler und Parker davon ausgehen, dass CopingStile als relativ stabile Verhaltensdisposition vorliegen. Bei der dem Fragebogen vorausgehenden Instruktion wird dies in der Frage deutlich, die nach dem typischerweise in stressreichen Situationen an den Tag gelegten Verhalten fragt. Die im Anschluss durchgeführten Faktorenanalysen deuteten auf eine dreidimensionale Struktur des Instruments hin, welche sich in weiteren Erhebungen bestätigen lies. Neben Aufgabenorientierung (Task-Orientation) und Emotionsorientierung (Emotion-Orientation) bildet Vermeidungsorientierung (Avoidance-Orientation) den dritten stabilen Faktor, der sich aus den Subskalen „Zerstreuungsorientiertes Coping“ (distraction) und "Sozialablenkungsorientiertes Coping" (social-diversion) zusammensetzt (Endler \& Parker, 1994). In Tabelle 4 sind die drei Dimensionen sowie Beispielitems aus dem „Coping Inventory for Stressful Situations" aufgeführt.

Tab. 4: Beispielitems des „Coping Inventory for Stressful Situations" (dt. Übersetzung von Semmer, Tschan \& Schade, 1991)

\begin{tabular}{|l|l|}
\hline Dimension & Beispielitems \\
\hline Aufgabenorientiertes Coping & $\begin{array}{l}\text { "Ich erarbeite mir einen Plan und führe ihn auch aus." } \\
\text { "Ich nutze die Gelegenheit, um zu beweisen, was in mir steckt." }\end{array}$ \\
\hline Emotionsorientiertes Coping & "Ich mache mir Vorwürfe, weil ich nicht weiß, was ich machen soll." \\
\hline Vermeidungsorientiertes Coping & "Ich sage mir, dass das gar nicht wahr ist." \\
& "Ich nasche oder gönne mir meine Lieblingsspeise." \\
\hline
\end{tabular}


Auch Janke, Erdmann und Kallus (1984) gehen in ihrem Stressverarbeitungsfragebogen (siehe Kap. 6.6.3) davon aus, dass es sich bei den von einer Person eingesetzten Bewältigungsmaßnahmen um habituelle Personenmerkmale handelt, die über die Zeit relativ stabil sind. Neben der Zeitkonstanz gehen sie zudem von einer Situationskonstanz aus, d.h. dass relativ unabhängig von der Art der Situation in gleicher bzw. ähnlicher Weise Bewältigungsstile eingesetzt werden. Das Coping-Verhalten wird im Stressverarbeitungsfragebogen, je nach Version in 13 bzw. 20 verschiedenen Dimensionen, hoch differenziert erfasst.

Carver, Scheier und Weintraub entwickelten 1989 ebenfalls einen Fragebogen zur Erfassung des individuellen Umgangs mit stresshaften Situationen. Mit dem insgesamt dreizehn Dimensionen umfassenden vierstufig likert-skalierten Fragebogen "COPE" wird genau wie im CISS und im Stressverarbeitungsfragebogen (SVF) versucht, Coping als stabile und situationsunabhängige Disposition zu erfassen, was sich deutlich in den, Instruktionen des Fragebogens zeigt:

We are interested in how people respond when they confront difficult or stressful events in their lives. There are lots of ways to try to deal with stress. This questionnaire asks you to indicate what you generally do and feel, when you experience stressful events. Obviously, different events bring out somewhat different responses, but think about what you usually do when you are under a lot of stress (Carver, Scheier \& Weintraub, 1989, S. 271).

Die grundsätzliche Strukturierung des „COPE" entspricht mit der Dreiteilung in emotionsorientiertes Coping (fünf Skalen), problemorientiertes Coping (fünf Skalen) und einer dritten Dimension (drei Skalen) mit „weniger nützlichen Bewältigungsreaktionen“ im weitesten Sinn der von Endler und Parker (1994) vorgesehenen Differenzierung. Die Subskalen und Beispielitems werden in Tabelle 5 aufgeführt.

Tab. 5: Beispielitems des "COPE" (1 = problemorientiertes Coping, $2=$ emotionsorientiertes

Coping, 3 = weniger nützliche Bewältigungsreaktionen) (Carver, Scheier \& Weintraub, 1989)

\begin{tabular}{|c|c|}
\hline Skala & Beispielitem \\
\hline Active Coping $^{1}$ & „I concentrate my efforts on doing something about it." \\
\hline Planning $^{1}$ & "I think hard about what steps to take." \\
\hline Suppression of competing activities ${ }^{1}$ & "I put aside other activities in order to concentrate on this." \\
\hline Restraint Coping $^{1}$ & "I force myself to wait for the right time to do something." \\
\hline Seeking social support - instrumental ${ }^{1}$ & "I ask people who have had similar experiences what they did." \\
\hline Seeking social support - emotional $\left.\right|^{2}$ & "I try to get emotional support from friends or relatives." \\
\hline Positive reinterpretation $^{2}$ & "I look for something good in what is happening." \\
\hline Acceptance $^{2}$ & "I learn to live with it." \\
\hline
\end{tabular}




\begin{tabular}{|l|l|}
\hline Turning to religion & "I seek God's help." \\
\hline Focus on \& venting of emotions & "I get upset and let my emotions out." \\
\hline Denial $^{3}$ & "I refuse to believe that it has happened." \\
\hline Behavioral disengagement $^{3}$ & "I give up the attempt to get what I want." \\
\hline Mental disengagament & $\begin{array}{l}\text { "I turn to work or other substitute activities to take my mind off } \\
\text { things. " }\end{array}$ \\
\hline
\end{tabular}

\subsection{Evasives Bewältigungsverhalten}

In Kapitel 4.9 wird erläutert, was im Rahmen dieser Arbeit unter evasivem Bewältigungsverhalten verstanden wird und welche charakteristischen Situationen und Eigenschaften des Sportunterrichts diese Verhaltensweise besonders zu Tage treten lässt. Neben Begründungszusammenhängen und Legitimationscharakteristika von Lehrkräften wird eine Einbettung in Stress- bzw. Verhaltensmodelle vorgenommen, die Manifestation der Verhaltensweise im Alltag von Lehrkräften sowie die verschiedenen Facetten des evasiven Bewältigens dargestellt und die Bedeutung eines sachgerechten Umgangs mit Konflikten erläutert.

Wie in Kapitel 3.3 angeführt wurde, ist der Sportunterricht durch einen hohen Grad an Ungewissheit, gleichzeitig auftretenden Mehrfachanforderungen sowie Handlungsund Orientierungsdruck gekennzeichnet. Des Weiteren ist der Alltag einer Sportlehrkraft von einer Vielzahl an Konflikten geprägt, die gewohnte Situationen und Abläufe verändern oder diese gar infrage stellen. Solche Veränderungen erzeugen bei den Beteiligten Unsicherheit, welcher in unterschiedlicher Art und Wiese begegnet werden kann (Potthoff \& Wolf, 1976). Neben dem Wunsch nach mehr Handlungssicherheit und Routine entwickelt sich bei Lehrkräften vor allem ein Bedürfnis nach klaren Orientierungsschemata, anhand derer ein Umgang mit den alltäglichen Überraschungen des Unterrichtens erfolgen kann. Kritisch hinterfragt werden muss, wenn die zweifellos notwendige Routinisierung und die damit einhergehende Handlungssicherheit zu Leitprinzipien des Unterrichtens avancieren und pädagogische und didaktische Aspekte nur noch flankierende Bedeutung aufweisen, wie dies bereits Lange in einem Aufsatz aus dem Jahr 1981 kritisch anmerkte.

Nachdem bereits in den vorangehenden Kapiteln verschiedene Formen der Bewältigung und deren Antezedenzbedingungen, bezogen auf die Situation und die Person, beschrieben und erläutert wurden, soll es in diesem Abschnitt um eine Art von Bewältigung gehen, die gerade angesichts der Kennzeichen des Berufsbildes und den charakteristischen biographischen Verläufen von höchstem Interesse ist. Die als Evasion bezeichnete Strategie stellt eine Form des Umgangs mit Berufsanforderungen dar, die entweder Element einer Überlebensstrategie ist, bewusst aus pädagogischen Gründen 
eingesetzt wird oder aber ein Kennzeichen von fehlendem Arbeitsengagement sein kann. Der Begriff evasiv bzw. die substantivierte Form Evasion sind abgeleitet von dem lateinischen Verb evadere, und bedeutet verlassen, entrinnen, Ausflüchte suchen, vermeiden oder ausweichen. Das Gegenteil ist die sogenannte Invasion. Grundsätzlich ist evasives Verhalten im Unterricht als wertneutral zu betrachtet, da ein vermeidendes Verhalten zeitweise angebracht sein kann, um das Erreichen von Unterrichtszielen nicht durch Kleinlichkeiten zu verzögern bzw. zu behindern. So kann es in harmlosen Situationen durchaus sinnvoller sein, nicht auf einen Konflikt, eine Provokation oder eine Unterrichtsstörung einzugehen, da bei entsprechenden Voraussetzungen der Lerngruppe nicht immer eine Reaktion der Lehrkraft zwingend erforderlich ist. Zudem kann zeitweiliges Ignorieren auch dazu genutzt werden, emotional geladene Situationen abkühlen zu lassen oder bewusst zu zeigen, dass man trotz Störungen und Hindernissen in der Lage ist, am Unterrichtskonzept festzuhalten. In manchen Situationen lässt sich durch evasives Verhalten auch Zeit gewinnen, um eine sinnvolle und langfristige Lösung des Konflikts herbeizuführen. Ausgeprägtes evasives Verhalten, das bewusst und aus hedonistischen Gründen eingesetzt wird, stellt jedoch ein Hindernis erfolgreicher Arbeit dar. Das Ablehnen von erzieherischer Verantwortung gefährdet die Erfüllung des Auftrags einer Lehrkraft und kann zudem je nach Persönlichkeit eine ernsthafte Gefahr für die psychische und physische Gesundheit darstellen.

Neben bewusst eingesetztem evasivem Verhalten kann die Vermeidungsreaktion auch unbewusst erfolgen. In Situationen, die eine schnelle Lösung erfordern und kein Aufschub möglich ist, werden zum Teil Handlungen durchgeführt, die auf affektiven Entscheidungen basieren, die von der unteren limbischen Ebene des Gehirns gesteuert werden. Diese unmittelbaren und spontan ablaufenden Reaktionen sind durch Erfahrung und den eigenen Willen wenig steuerbar. Stammesgeschichtlich verwurzelte Verhaltensweisen in Situationen mit Handlungsdruck sind Flucht, Abwehr und Verteidigung, Angriff, Erstarren oder Unterwerfen (Roth, 2008). Das rationale Denkvermögen ist in evolutionär jüngeren Hirnarealen lokalisiert und wird in Bedrohungssituationen weggeschaltet, sodass der Mensch in ursprünglichere Verhaltensweisen zurückfällt (Schwarz, 1997). Durch die Ausschüttung von Botenstoffen werden in Situationen mit Zeitdruck und hoher emotionalen Beteiligung schnelle urtümliche Reaktionen initiiert, die in Macht- oder Bedrohungssituationen zum Einsatz kamen und für heutige komplexe Handlungssituationen keine angemessenen Reaktionsweisen darstellen. Solche Verhaltensweisen treten vor allem dann zu Tage, wenn unerwartete Dinge geschehen und im Handlungsgedächtnis kein passendes Reaktionsmuster gespeichert ist (Roth, 2008). Genau diese urtümlichen Verhaltensweisen zeigen sich auch immer wieder bei 
dem Phänomen der Fahrerflucht. Nach dem Verursachen eines Unfalls neigen viele Menschen dazu, erst einmal vom Ort des Geschehens zu flüchten und gelangen erst später zu der Einsicht, dass ihr Verhalten fehlerhaft und vor allem wirkungslos im Sinne einer zufriedenstellenden Lösung der Situation war. Meist ist das ausweichende Verhalten jedoch nicht so leicht zu erkennen und kann in unterschiedlichen Formen wie "unter den Teppich kehren“, "auf die lange Bank schieben“, Ignorieren, Verleugnen oder Verdrängen auftreten. Wie bereits zuvor am Beispiel der Fahrerflucht dargestellt, kann evasives Verhalten kurzfristig, d.h. meist nur für die Dauer der akuten Situation zu einer Entlastung führen, langfristig jedoch die Vulnerabilität sogar noch verstärken. Janke, Erdmann und Kallus (1984) unterteilen Copinghandlungen in stresserniedrigende und stresserhöhende Maßnahmen, zu denen sie u.a. Vermeidung rechnen. Warum dennoch bewusst ausweichende Strategien im Unterricht genutzt werden, lässt sich mit fehlenden Handlungsalternativen, mangelnder Motivation, Selbstschutz oder anhand des Konzepts der bounded rationality von Selten (1990) erklären. Obwohl Menschen wissen, dass bestimmte Verhaltensweisen langfristig einen deutlich größeren Gewinn versprechen als andere, ziehen sie dennoch aus Risikoscheu und Bequemlichkeit oft einen naheliegenden, aber geringen Gewinn vor (Roth, 2008). In der Realität erscheint eine Vielzahl an kritischen Situationen durch evasives Verhalten lösbar, da man sich schnell aus Situation entziehen kann, es keinen wahren Verlierer gibt und die scheinbare Lösung schmerz- und risikofrei sowie energiesparend ist. Setzt man jedoch dauerhaft ausweichende Strategien im Umgang mit Konflikten ein, zeigt sich, dass die scheinbar gelösten Konflikte erneut auftreten, teils sogar in verschärfter Form, und eine individuelle Weiterentwicklung verhindert wird. Engagierte Lehrer, die bereit sind, in ihre berufliche Tätigkeit zu investieren und sich stetig weiterentwickeln, sind durch ihren zunehmenden Erfahrungsschatz auf dem besten Weg, leichter und länger in ihrem Beruf durchzuhalten. Im Gegensatz dazu stellt die auf den ersten Blick attraktiv erscheinende Aufwandsminimierung eine beträchtliche Gefahr dar, eine frühe Erschöpfung zu erleiden die sich letztendlich im Ausbrennen (siehe Kap. 4.9.5.1) manifestieren kann (Struck, 2007).

In der berufsbiographischen Forschung zeigte sich immer wieder, dass von dem zu Beginn einer Karriere ausgebildeten hohen Selbstanspruch nach einiger Zeit nicht mehr viel übrig bleibt. Das Bedürfnis nach Entlastung und Handlungssicherheit erzeugt, bezogen auf den pädagogischen und didaktischen Auftrag ein Spannungsverhältnis, dessen Lösung durch Persönlichkeitseigenschaften der Lehrkraft wie z.B. Motivation oder Kompetenzerwartungen beeinflusst wird (Lange, 1981). Huberman (1989) stellte fest, dass mit zunehmendem Alter das Desengagement von Lehrkräften zunimmt. Auch 
Miethling (2000) bestätigt das zunehmende Desengagement im Karriereverlauf von Sportlehrkräfte und stellte in seiner qualitativen berufsbiographischen Studie vermehrt fest, dass sich ein Wandel von einem zum Berufseinstieg ausgeprägten Zielbild eines methodisch perfektionierten Sportunterrichts, hin zu einem von höchst unterschiedlichen Überlebensstrategien geprägten Unterricht abzeichnet. Auch Woods (1977, zitiert nach Scarth, 1991), der sich mit Lehrerstrategien beschäftigte, vermutet, dass mit zunehmendem Druck Lehrer nicht nur lernen mit den Anforderungen umzugehen, sondern auch didaktische Vorstellungen revidieren und versuchen, situative Erfordernisse und persönliche Interessen und Bedürfnisse auszubalancieren. Die unterschiedliche Entwicklung von Lehrkräften kann anhand des im Anschluss dargestellten Schemas gut nachvollzogen werden.

Nach der Einteilung von Huberman (1989) (siehe Abb. 12), die auf der Grundlage einer Interviewstudie generiert wurde, ist die erste Phase gekennzeichnet durch mehr oder weniger starke Unsicherheit und Rollenüberlastung, bevor in der zweiten Phase eine Stabilisierung stattfindet, in der man sich zunehmend im Klassenzimmer wohlfühlt, seinen eigenen Unterrichtsstil ausgebildet hat und eigene Wege geht. Aus einer relativ gesicherten Position heraus, meist nach erfolgter Lebenszeitverbeamtung, beginnt nun für einen Teil der Befragten eine Phase, die geprägt ist durch Aktivismus, Experimentieren und dem Bemühen, tatsächlich etwas zu verändern bzw. die Schule, das Fach oder den eigenen Unterricht nach vorne zu bringen. Doch nicht alle Lehrer gestalten die Phase in dieser Art. Einsetzende Langeweile, Zweifel an der Berufswahl sowie negative Erfahrungen und Enttäuschungen mit bzw. durch Schüler, Kollegen, Eltern oder formellen Dingen führen zu Selbstzweifeln und einer Neubewertung der eigenen beruflichen Lage. Diese Berufsphase ist gekennzeichnet durch Bemühungen, die Krise zu überwinden und für sich selbst Überlebensstrategien zu finden. Je nach individuellem Verlauf kann es dazu kommen, dass die Probleme als systemisch und unveränderbar angesehen werden und eine durch Desengagement gekennzeichnete Phase der Resignation eintritt. Die zweite Möglichkeit besteht darin, dass die Lehrkräfte neuen Aufwind bekommen und die anstehenden Herausforderungen annehmen. Im Falle einer negativen Bewertung hingegen gehen Lehrer zu Konservativismus und Zynismus über, bevor in den letzten Berufsjahren ein Berufsabschnitt folgt, der sogar als Verbitterung beschrieben werden kann. Diejenigen, die die Sinnkrise positiv überwunden haben, gelangen jedoch ebenfalls nach einigen Jahren in eine Phase, die zwar nicht mehr den bedrohlichen Charakter aufweist wie die erste Krise, aber in der dennoch Gelassenheit und Zweckdenken Einzug erhält (Huberman, 1991). 
Berufsjahre

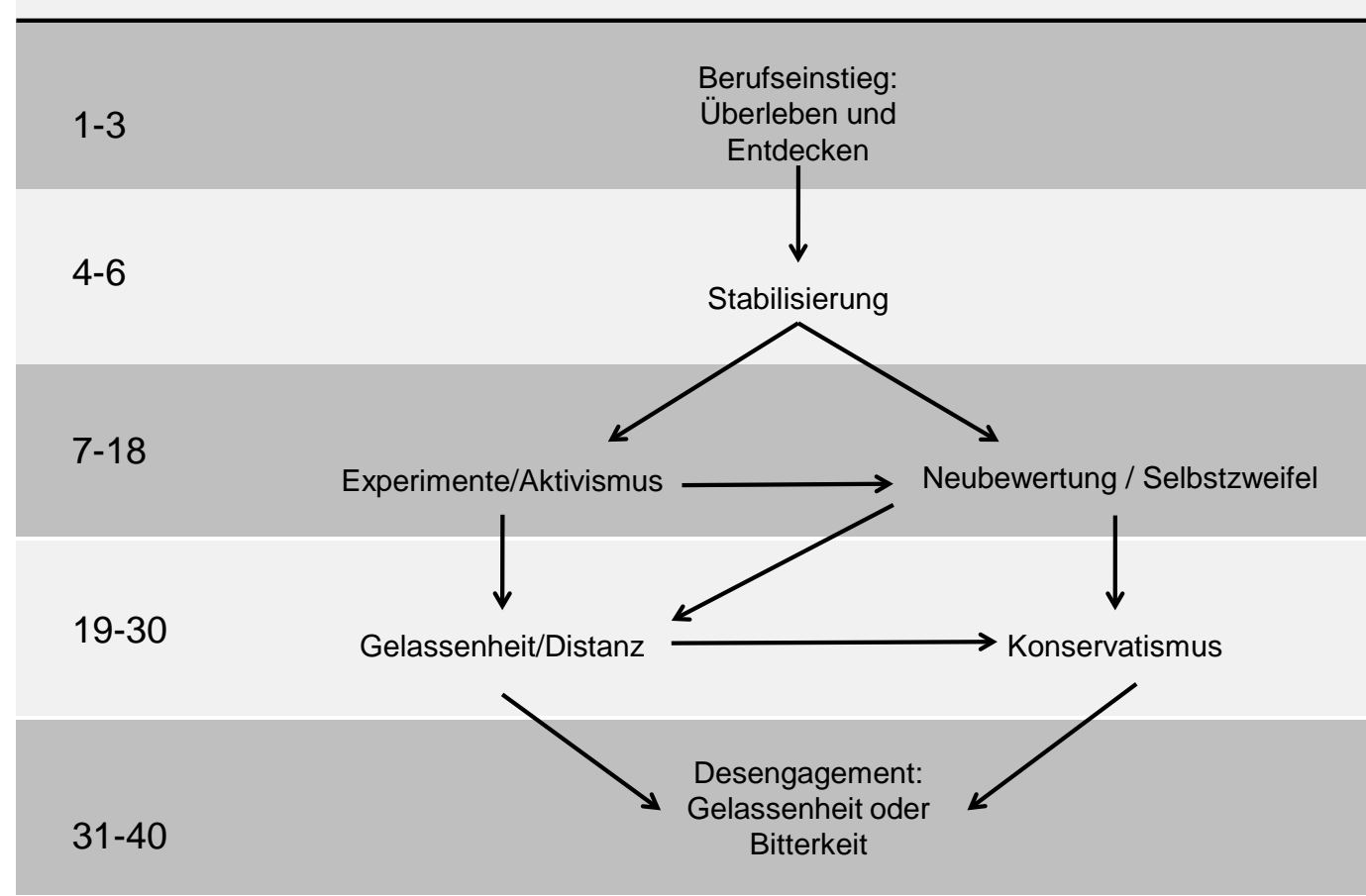

Abb. 12: Berufslaufbahn von Lehrern (Huberman, 1989; 1991, zitiert nach Miethling, 2000, S.47)

Aufgegriffen wurde das evasive Verhalten im Zusammenhang mit dem Sportunterricht im deutschsprachigen Raum zum ersten Mal von Kolb und Wolters 1999 im Rahmen eines Vortrages auf der Sektionstagung Sportpädagogik der Deutschen Vereinigung für Sportwissenschaft (DVS) in Regensburg. Ausgangspunkt waren Beobachtungen in Schulpraktika, die Kolb und Wolters unabhängig voneinander machten. Während bestimmte Unterrichtsinhalte gehäuft im Unterricht vertreten waren, wurden andere Inhalte im Schulalltag gemieden. In der Grundschule dominierten Bewegungslandschaften und in der Mittelstufe Sportspiele, die meist in Form eines freien Spiels organisiert waren. Auch wenn sich die Erkenntnisse auf eine sehr schmale Datenbasis bezogen, stellten die beiden dennoch eine deutliche Tendenz fest. Evasives Unterrichten stellt eine Bewältigungsstrategie dar, in der die Lehrkräfte versuchen Belastungen so gut wie möglich zu umgehen. Teilweise wird der Sportunterricht sogar als Auszeit im anstrengenden Unterrichtsalltag genutzt (Kolb \& Wolters, 2000). Auch in einem Zeitungsartikel der Rheinpfalz (2003) spricht Glasauer, Studiendirektor eines Gymnasiums und Schulsportdidaktiker, in einem Interview von einer zunehmenden Tendenz der evasiven Verhaltensweisen im Sportunterricht. Diese Feststellung leitet Glasauer aus Unterrichtsbeobachtungen und Befragungen aus 21 Berufsjahren ab, in denen er in seiner Funktion als Fachleiter für Sport an einem Studienseminar, jungen Sportlehr- 
kräften bei der Vorbereitung auf den Beruf zur Seite stand. Neben Sportlehrern, die nur zuschauten, was sich im Unterricht so ergibt, gibt es andere, die die Schüler einfach machen lassen, was sie wollen und wieder andere sind während des Unterrichts nicht mal in der Sporthalle, berichtet Glasauer.

Bei Betrachtung der verschiedenen Versuche das Bewältigungsverhalten zu klassifizieren (siehe Kap. 4.7), scheint das evasive Unterrichten am besten durch das Modell von Czerwanka (1996) (siehe Abb. 11) repräsentiert. Neben rein intrapsychischen palliativen Bewältigungsmaßnahmen sind hier auch instrumentelle Handlungen unter der Dimension Vermeidung vorgesehen. Neben Fluchtverhalten kann dies auch eine gezielte Veränderung der Problemlage sein, die zwar keine sinnvolle Lösung darstellt, aber der Lehrkraft Entlastung bietet. Weiterhin sind Ignorieren sowie das Verleugnen von Konflikten weitere Bewältigungsmaßnahmen, die unter dem Sammelbegriff evasives Unterrichten bündelbar sind. Grundsätzlich stellen evasive Handlungen im Unterricht also pragmatische Lösungen von problemhaften Situationen dar, die zu einem reibungslosen Ablauf des Unterrichts beitragen sollen.

Um sinnvoll und effektiv mit Konflikten umzugehen ist die Einsicht notwendig, einen Konflikt überhaupt als solchen anzuerkennen. Eine weit verbreitete Ansicht ist, dass wer Konflikte hat, nicht richtig zu führen weiß und die Kunst des Führens darin liege, Konflikte zu vermeiden. Diese Einschätzung, die man nicht nur in der Schule, sondern auch in anderen Organisationen, Firmen oder sozialen Gefügen findet, scheint gesellschaftlich bedingt zu sein (Schwarz, 1997).

\subsubsection{Einflussfaktoren auf das Unterrichten}

Sportlehrkräfte sind nicht nur mit den Anforderungen eines einzelnen Umweltfaktors konfrontiert, sondern agieren in einem diffus anmutenden Konglomerat verschiedenartiger Ansprüche und Erwartungen. Aus der Prozessperspektive betrachtet befindet sich die Lehrkraft mit ihren Persönlichkeitseigenschaften, Kenntnissen, Fähig- und Fertigkeiten im Zentrum des didaktischen Sterns, der eine Systematisierung der an die Lehrkräfte gestellten Anforderung ermöglicht (Scherler, 2004). Das Modell zeigt, dass Lehrer beim Unterrichten mit den zu vermittelnden Inhalten, den vorherrschenden Bedingungen und den interagierenden Schülern stets drei Dinge gleichzeitig berücksichtigen müssen. Aufgabe der Lehrkräfte ist es, in der Unterrichtspraxis eine Passung zwischen den verschiedenen Faktoren herzustellen. Konflikte, die der Lehrer als subjektive Belastungen verspürt, entstehen dann, wenn Erwartungen von einer Seite nicht erfüllt werden bzw. gegenläufige Interessen bestehen. Eine dezidierte Auflistung und Beschreibung von einzelnen belastenden Faktoren erfolgt an dieser Stelle nicht (siehe 
Kap. 3.3). Stattdessen sollen die verschiedenen Einfluss- bzw. Umweltfaktoren des Unterrichtens in übersichtlicher Art und Weise dargestellt werden.

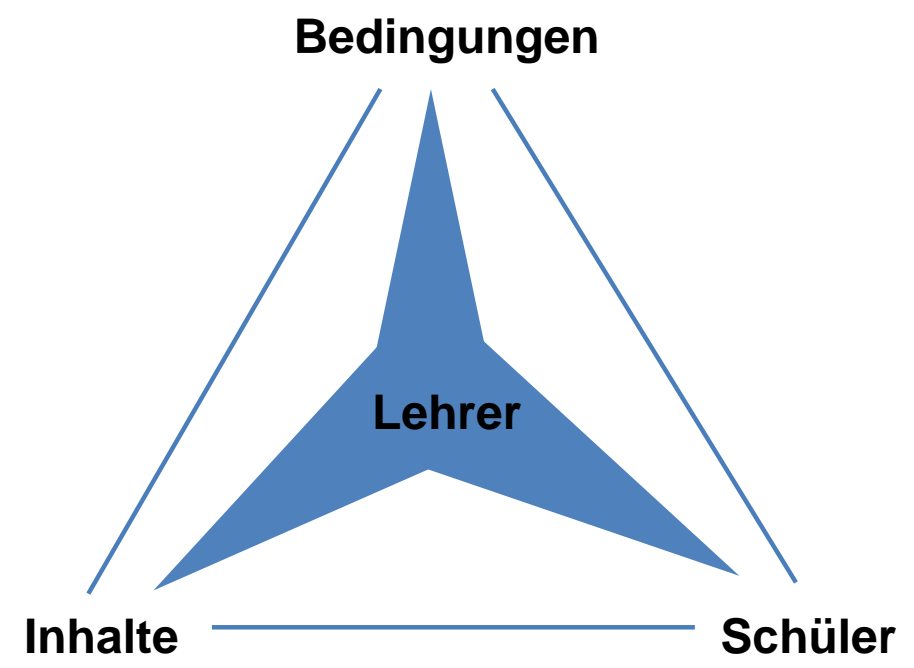

Abb. 13: Didaktischer Stern (Scherler, 2004, S. 18)

Der wohl am häufigsten thematisierte Bezug unterrichtlichen Handelns ist der Schülerbezug. Da es sich bei dem Lehrerberuf um einen sozialen Beruf handelt, bei dem ein beträchtlicher Teil der Arbeitszeit durch den intensiven Kontakt mit anderen Menschen gekennzeichnet ist, birgt dieser Bereich auch viele Probleme und Konflikte, die oft in zweideutigen oder unbefriedigenden Situationen münden (Barth, 1997). Dieser hauptsächlich sozial geprägte Einflussfaktor auf das Unterrichten schließt sowohl den Umgang mit Interessen und Bedürfnissen von Schülern, aber auch fachliche Leistungen und soziale Aspekte mit ein. Grundsätzlich ergeben sich bereits durch die vorgegebene Rollenverteilung und den strukturell verschiedenen Erwartungen von Lehrern und Schülern Reibungspunkte aufgrund gegenseitig erwartungswidrigen Verhaltens. Schulischer Unterricht findet in einer Gruppensituation statt, die sich nicht aus freien Stücken oder Sympathie gebildet hat, sondern ein zweck- und meist zwanghaftes Arbeitsbündnis darstellt. Der alltägliche Kontakt mit Schülern ist für Lehrer einerseits die Hauptquelle der beruflichen Belastung, andererseits bietet er jedoch auch die Möglichkeit, Zufriedenheit und Selbstbestätigung zu erlangen. Während eine unmittelbare Rückmeldung von Schülerseite selten gegeben wird und zudem stark durch das schulische Hierarchiegefüge beeinflusst ist, weisen positive und negative Beziehungserfahrungen eine zentrale Bedeutung für die Bewertung der eigenen Tätigkeit als Lehrer auf (Ulich, 1996).

Der Sportunterricht ist durch einen besonders hohen Grad an Interaktion zwischen den Schülern sowie zwischen der Lehrkraft und den Schülern gekennzeichnet und bietet damit hervorragende Voraussetzungen für das soziale Lernen. Voraussetzungscharak- 
ter für soziales Lernen hat jedoch neben der Interaktion der Schüler untereinander ein vom Lehrer geschaffener Rahmen, der sowohl Orientierung als auch Grenzen bietet. Während ihrer Unterrichtstätigkeit befinden sich Lehrkräfte demnach ständig in einem Spannungsverhältnis zwischen den Interessen und Bedürfnissen der Schüler, den auftretenden Motivations- und Disziplinkonflikten sowie den Konflikten zwischen Schülern und den vorgesehenen Lernzielen bzw. Kompetenzen (Kolb \& Wolters, 2000). Konkretisiert wurden die verschiedenen Spannungsverhältnisse, in denen sich die Lehrkräfte gegenüber Schülern befinden, bereits in Kapitel 2.2.3 und dem Schlagwort NäheDistanz-Antinomie.

Der bereits angesprochene Inhaltsbezug des Unterrichtens oder auch Sach- oder Themenbezug umfasst die Auswahl, Aufbereitung und methodische Umsetzung des Lehrstoffes. Der Sportunterricht kennzeichnet sich durch ein breites Inhaltsspektrum, um den Schülern möglichst vielfältige Erfahrungen zu ermöglichen. Sportlehrkräfte müssen, gemäß den Veränderungen der gesellschaftlichen Bewegungskultur, offen für neue Inhalte sein und zudem ihr Unterrichtskonzept orientiert an den aktuellen Bildungsplänen aktualisieren. Ein Beispiel aus der Vergangenheit ist die Einführung von Bildungsstandards und bewegungsfeldbezogenen Kerncurricula im Fach Sport in vielen Bundesländern (König, 2004). Belastungssituationen können vor allem dann entstehen, wenn Probleme bei der Umsetzung der curricularen Vorgaben auftreten und Unterstützung bzw. Anleitungen fehlen. Des Weiteren treten, durch den Aufbau von Gerätearrangements, Hilfe- und Sicherungsstellungen oder die Demonstration von Bewegungen, bei den verschiedenen Inhalten in unterschiedlichem Maße auch physische Belastungsmomente auf, die vor allem mit fortschreitendem Berufsalter zusätzliche Relevanz erlangen. Einen weiteren Aspekt stellt das bewegungsbedingte Verletzungspotential von Schülern, aber auch von Lehrkräften dar. Studien, die im Auftrag der Unfallkassen durchgeführt wurden, zeigen, dass erhebliche Unterschiede hinsichtlich der Risikofaktoren der einzelnen Sportarten bestehen. In Kombination mit Vorschriften und Erlassen, die dem sich anschließenden Bedingungs- oder Umweltbezug zuzuordnen sind, können hierdurch sowohl physische als auch psychische Belastungen resultieren (König, 2004).

Der dritte Einflussfaktor auf das Unterrichten stellt der Bedingungsbezug bzw. Umweltbezug dar. Hier werden Einflüsse auf das Unterrichten zusammengefasst, die nicht dem Umgang mit Schülern, den Inhalten oder dem Selbst der Lehrperson zuzuordnen sind. Äußere Rahmenbedingungen des Unterrichts wie z.B. die Geräte- oder Materialausstattung der Sportstätten gehören ebenso zu diesem Bereich wie der Umgang mit Eltern, Kollegen und der Schulleitung. Ein weiterer Bestandteil sind Vorschriften, Erlas- 
se und Gesetze, die einen Orientierungsrahmen, meist aber auch einen Belastungsfaktor darstellen. Als besonders belastend wird von Lehrern oft die Zusammenarbeit mit bzw. der Kontakt zu den Eltern beschrieben. Neben einer Quelle von Belastungen kann der Umgang mit Eltern jedoch auch einen protektiven Faktor darstellen. Durch einen kooperativen Umgang mit den Eltern ist es in vielen Fällen möglich, Erziehungsziele zu erreichen und gleichzeitig Alltagsbelastungen in Form von Konflikten mit Schülern zu reduzieren, sodass die investierte Zeit und das Engagement durch den Ertrag übertroffen werden (Struck, 1994). Durch besondere Brisanz ist das Verhältnis zwischen Lehrern und Eltern vor allem bezogen auf die Schulabschlusserwartungen gekennzeichnet, die in den letzten Jahrzehnten deutlich gestiegen sind. Oft bestehen erhebliche Diskrepanzen zwischen den Erwartungen, Hoffnungen und Wünschen der Eltern und der realen, durch die Lehrkräfte erlebten Leistungssituation in der Schule. Bedingt durch die Entscheidungsgewalt der Lehrer über die weitere schulische Karriere und dem mit dem Beruf verbundenen Bewertungsmonopol, kommt es zu Aversionen, teils sogar Aggressionen der Eltern gegenüber den Lehrern (Ulich, 1996). Das gespannte Verhältnis zwischen Eltern und Lehrer stellt jedoch trotz teilweise aktueller Entwicklungen, wie z.B. im Bezug auf gestiegene Bildungserwartungen, keine neue Erscheinung dar, sondern war auch in der Vergangenheit durch Konflikte geprägt, was das überraschend aktuell erscheinende Zitat aus dem Jahr 1908 von Matthias (zitiert nach Ulich, 1996, S. 132 f.) deutlich macht.

Das gegenwärtige Verhältnis von Schule und Haus gleicht vielfach einer recht unglücklichen Ehe: Von den schlechten Resultaten der Erziehung sucht ein Teil dem anderen die ganze Schuld zuzuschieben, statt dass jeder den Grund bei sich sucht.

Unterstützt wird die problematische Sicht der Eltern gegenüber den Lehrern durch negative Erfahrungen aus der eigenen Schulzeit, die durch den Schulbesuch der Kinder wieder aufgewärmt werden. Oft haben Vorbehalte von Eltern gegenüber Lehrern gar nichts mit der aktuellen Situation zu tun, sondern speisen sich aus am eigenen Leib Erlebtem (Struck, 2007). Auch Kompetenzkonflikte, die bereits durch Widersprüchlichkeiten zwischen dem Grundgesetz und den Schulgesetzen bedingt sind, sind prägend für die Beziehung zwischen Eltern und Lehrern. Während in Artikel 6 des Grundgesetzes die Erziehung als natürliches Recht der Eltern bezeichnet wird, enthält Artikel 7 die Aussage, dass das gesamte Schulwesen unter Aufsicht des Staates steht und damit auch die damit verbundene Erziehung. Weiter konkretisiert wird der staatliche Anspruch auf die Beteiligung an der Erziehung letztendlich in den Schulgesetzen, in denen 
ein gemeinsamer Erziehungsauftrag von Eltern und Lehrern formuliert ist (Deutscher Bundestag, 2009; Ulich, 1996).

Ebenso wie bei der Konstellation zwischen Klasse und Lehrer, setzen sich Lehrerkollegien nicht durch Freiwilligkeit oder gegenseitiger Anziehung zusammen sondern stellen zunächst erst einmal eine durch den Zufall bestimmte Zwangsgruppe dar, in der mehr oder weniger ausgeprägten Gemeinsamkeiten und Sympathien zwischen den einzelnen Mitgliedern besteht (Ulich, 1996). Statt persönlicher Passung zwischen Lehrkräften entscheiden fast immer Fächerkombinationen, Wartelistenpositionen, Mangelfächer oder Abschlussnoten über die Zusammensetzung von Lehrerkollegien (Struck, 1994). Das Kollegium, das eigentlich dem subjektiven Bedürfnis der Lehrer nach Kontakt, Solidarität und Verständnis nachkommen sollte, entwickelt sich stattdessen für viele Lehrer zu einem Ort des Einzelgängertums, der keine Ent- sondern eine Belastung darstellt. Neuere Bestrebungen, die bei der Personalauswahl den Schulen mehr Autonomie zuerkennen, setzen genau an diesem Punkt an, um eine Profilbildung und Prägung von Schulen zu unterstützen. Der Hauptgrund für ein schlechtes Klima im Kollegium wird meist jedoch in dem Verhalten der Schulleitung gesehen, die nichts zu einer Verbesserung beiträgt. Das Verhältnis zwischen Lehrern und der Schulleitung kennzeichnet sich dadurch, dass die Mitglieder der Schulleitung in Personalunion gleichzeitig Kollegen und Vorgesetzte sind, wodurch es zu unausweichlichen emotionsgeladenen Rollenkonflikten kommt wie z.B. bei dienstlichen Beurteilungen (Ulich, 1996).

\subsubsection{Konflikte und ihre Bedeutung}

Wie zuvor beschrieben können Konflikte auf verschiedenen Ebenen entstehen. Entscheidend für das Verhalten im Konfliktfall ist die grundsätzliche Überzeugung, mit der man einem Konflikt begegnet: Sieht man einen Konflikt generell eher als Panne, die es zu vermeiden gilt oder aber als eine Notwendigkeit für das Zusammenleben, die eine Chance für die Weiterentwicklung von Individuen und deren Beziehungen untereinander bietet (Schwarz, 1997). Fest steht, dass Konflikte unumgängliche Erscheinungen im Zusammenleben von Menschen darstellen, da nicht jeder zur gleichen Zeit auch das Gleiche will und so unterschiedlichste Interessen miteinander kollidieren. In der Schule treten Konflikte schon allein aufgrund der institutionell bedingten Grundeigenschaft auf, dass Schüler mehr oder weniger unfreiwillig an einem Ort sind, an dem die Lehrkraft auch noch stetig Forderungen an sie stellt (Glöckel, 2000).

Neben diesen eher sozial geprägten dialogischen Konflikten kennt man in der Psychologie zusätzlich intrapsychische Konflikte, bei denen einem Menschen zwei verschiedene Tendenzen innewohnen. Bei dieser Konfliktkategorie unterscheidet man zwischen Appetenz-Appetenz-Konflikten, Aversions-Aversions-Konflikten und Appetenz- 
Aversions-Konflikten. Bei Appetenz-Appetenz-Konflikten besteht eine Rivalität zwischen zwei als erstrebenswert angesehenen Zielverfolgungen, während bei AversionsAversions-Konflikten ein Entscheidungszwang zwischen zwei als unangenehm oder gefürchtet empfundenen Folgen besteht. Bei Appetenz-Aversions-Konflikten besteht Rivalität zwischen verlockenden und bedrohlichen Aspekten der Zieldimension (Potthoff \& Wolf, 1976). Im Rahmen von Kapitel 4.9.5 wird ein Rückbezug zu diesen intrapsychischen Konfliktkategorien bei der Aufarbeitung der Beanspruchungsreaktionen und Beanspruchungsfolgen in Form des Schonungs- und des Burnout-Musters hergestellt.

Bei der Klassifikation von Konflikten existiert eine Vielzahl von Ansätzen, die meist aufgrund von phänomenologischen oder ätiologischen Aspekten zustande kommen. Während bei phänomenologischen Klassifikationen die Konflikte nach dem Erscheinungsbild zusammengefasst werden, sind die Konfliktursachen und -bedingungen die Grundlage von ätiologischen Einteilungen. Glöckel (2000) hingegen schlägt eine Einteilung in alltägliche Bagatellschäden und personale Konflikte vor. Während Unbedachtheit und Unbeherrschtheit von Schülern als klassische unterrichtliche Bagatellstörungen bezeichnet werden, die nicht als absichtlich-bewusstes Verhalten zu interpretieren sind, werden personale Konflikte durch bewusstes Verhalten gegen vorgegebene Rahmungen und Normen ausgelöst. Bagatellstörungen, die aufgrund des besonderen Charakters des Sportunterrichts als Bewegungsfach besonders oft vorkommen, sind einzeln betrachtet kaum der Rede wert, können summiert jedoch als erhebliche Belastung wahrgenommen werden. Im eigentlichen Sinn stellen sie keine wahren Konflikte dar, sondern sind eher als deren Vorboten bzw. Auslöser zu sehen. Personale Konflikte stellen eine Sammelbezeichnung für Ungehorsam, Unterlassen von Tun oder Aufsässigkeit dar, die sich in aktiven Störungen des Unterrichts niederschlagen und bereits isoliert betrachtet als sehr belastend empfunden werden. Für den Umgang mit Konflikten empfiehlt Glöckel (2000, S. 103) folgende oberste Regel: „Den Anfängen wehren, aber nicht mit Kanonen auf Spatzen schießen!“ In dem Zitat wird deutlich, dass es auf das rechte Maß an Disziplinierungsmaßnahmen ankommt. Zwischen dem Fehlverhalten und der Häufigkeit an Regulierungen im Unterricht besteht ein kurvilinearer Zusammenhang: Sowohl ein Zuviel als auch ein Zuwenig an Maßregelungen bewirkt eine Zunahme an Fehlverhalten auf Seiten der Schüler.

Während viele Lehrkräfte denken, dass Konflikte im Berufsalltag am besten gar nicht auftreten sollten und lediglich die Manifestation von Fehlern darstellen, sieht man in Theorien des Konfliktmanagements Potenzial für die Entwicklung von Persönlichkeiten. Durch Konflikte werden Unterschiede zwischen Individuen deutlich und fruchtbar ge- 
macht, können aber auch die Einheitlichkeit von Gruppen wieder herstellen. Viele Dinge kommen erst in Konflikten ans Tageslicht und können dann dazu genutzt werden, Beziehung neu bzw. anders zu gestalten. Grundsätzlich ist es so, dass derjenige, der sich in seinen Bedürfnissen eingeschränkt sieht, früher oder später rebellieren wird. In der Schule stellen die durch Schüler oder andere Interaktionspartner ${ }^{3}$ initiierten Konflikte ein Ausdrucksmedium dar, durch das sich Unzufriedenheit oder aber auch Unteroder Überforderung ausdrücken lässt. Erst durch Konflikte fließen Aspekte und Dimensionen in menschliche Beziehungen mit ein, die Vielfalt in Gruppen und die dort ablaufenden Prozessen überhaupt erst ermöglichen. Gleichzeitig muss jedoch auch angeführt werden, dass es unmöglich ist, alle Einzelinteressen zu berücksichtigen. Die basale Voraussetzung für das Eingehen auf Einzelinteressen ist ein Grundkonsens, eine bestehende Einheit, auf der Entwicklungen und Neuerungen aufbauen können. Konflikte stellen auch den Motor für Veränderungen dar. Entwickelt sich eine Gruppe oder ein Individuum weiter, ist dies meist auf Konflikte zurückzuführen (Schwarz, 1997). Gerade Schüler benötigen Widerstände, an denen sie sich reiben können, gegen die sie aufbegehren und sich auflehnen können. Verweigert man ihnen diese wichtigen Erfahrungen im Umgang mit Widerständen, Schwierigkeiten und Barrieren, bleiben bedeutende Entwicklungsreize aus und es besteht die Gefahr, dass diese Reize an anderer unpassender Stelle zu einem unpassenden Zeitpunkt provoziert werden (Glöckel, 2000). Für Personen in Führungspositionen, wie es unbestreitbar auch Lehrer sind, deuten Konflikte und die dahinter verborgene Kritik, die Notwendigkeit einer reflexiven Überprüfung des eigenen Verhaltens an. Ebenso können sie aber auch das Bestehende erhalten, indem durch sie das „Böse“ oder „Falsche“ bzw. der „Sündenbock“ herausgestellt wird. Wie sich zeigt, sind Konflikte keinesfalls nur negativ zu sehen, sondern stellen wichtige Evaluations- und Reflexionselemente zwischenmenschlicher Interaktion und damit auch der Arbeit von Lehrern dar. Um jedoch das in Konflikten inne wohnende Potenzial nutzen zu können, sind sowohl eine gefestigte Persönlichkeit als auch ein konstruktiver Umgang der Lehrkraft mit auftretenden Hindernissen und Widrigkeiten wichtige Voraussetzungen, die im weiteren Verlauf genauer beschrieben und analysiert werden sollen.

\subsubsection{Einbettung von evasivem Verhalten in Stress- bzw. Verhal- tensmodelle}

In Kapitel 3.1.3 wurde anhand des transaktionalen Stressmodells von Lazarus (siehe Abb. 3) und des Modells des Lehrerstresses von Rudow (siehe Abb. 6) aufgezeigt, wie Belastungen wahrgenommen, bewertet und bewältigt werden bzw. welche Rückkopp-

\footnotetext{
${ }^{3}$ Eltern, Kollegen, Schulleitung.
} 
lungsprozesse und Folgen zu berücksichtigen sind. Abhängig von den subjektiven Fähigkeiten und Ressourcen der Persönlichkeit wird während der sekundären Bewertung ein individuelles Bewältigungsverhalten geplant und initiiert. Neben der Abhängigkeit von den zu diesem Zeitpunkt bedeutsamen Persönlichkeitsmerkmalen ist aber auch die primäre Bewertung für das Bewältigungsverhalten mit entscheidend. Bereits beim ersten Kontakt mit dem potenziellen Stressor laufen entscheidende Prozesse ab, die den weiteren Verlauf der Bewältigung beeinflussen. Während Situationen, die als herausfordernd eingeschätzt werden, verstärkt ein aktives Handeln nach sich ziehen, kann eine wahrgenommene Bedrohung auch zu Inaktivität, Ausweichen und Vermeiden führen. Situationsmerkmale werden von Personen mit unterschiedlichen Eigenschaften in spezifischer Art und Weise widergespiegelt. Eine weitere Besonderheit der Belastungsverarbeitung im transaktionalen Stressmodell von Lazarus ist, dass Situationen bezüglich ihres Einflusses auf das Wohlergehen ständig neu bewertet werden. In einem Abgleich der Situationseigenschaften und deren Entwicklung mit den persönlichen Ressourcen ist es möglich, dass sich aus einem anfangs problemorientierten und offensiven Bewältigen ein eher energiesparendes und ausweichendes Handeln entwickelt. Ebenso ist es auch möglich, dass durch eine Neubewertung der Situation erkannt wird, dass die Bedrohlichkeit nicht so hoch ist, wie in der primären Bewertung eingeschätzt und die eigenen adaptiven Mittel durchaus ein adäquates Handeln ermöglichen. In diesem Fall verschiebt sich im Prozess der Stressperzeption die Ausrichtung des Bewältigungshandelns von evasiv hin zu invasiv. Weitere moderierende Einflussgrößen im Rahmen der Bewältigung bzw. der sekundären Bewertung stellen Konsequenz- und Kompetenzerwartungen dar (siehe Kap. 4.5), die mit zunehmendem Berufsalter durch eigene Erfahrungen im Umgang mit Konflikten sowie durch Informationen über vergangene Erfolge und Misserfolge bei verschiedenen Bewältigungsversuchen beeinflusst werden. Im Schulkontext ist es durchaus denkbar, dass evasives Verhalten und die offensichtliche Folgenlosigkeit zu einer Verfestigung dieser Strategie im Handlungsrepertoire von Lehrkräften führt. Evasives Verhalten erscheint im stressbehafteten Berufsalltag als eine attraktive selbstschützende Strategie, die eingesetzt werden kann, ohne dass Sanktionen von Seiten der Schulleitung oder Eskalationen im Unterricht unmittelbar zu befürchten sind.

Allmer (1982) beschäftigte sich intensiver mit dem Handeln von Lehrern und den im Vorfeld dieser Handlungen ablaufenden Prozessen. In seinem Modell zum Lehrerverhalten (siehe Abb. 14) sieht er eine Aufwand- und Effektkalkulation vor, die die resultierende Handlungsplanung bestimmt. Während bei der Aufwandkalkulation geklärt wird, in welcher Art und Weise die Zielerreichung, z.B. die Bewältigung einer spezifi- 
schen Situation, Anstrengungen der Lehrperson erfordert, wird in der Effektkalkulation die Frage nach der Nützlichkeit gestellt. Sind die positiven Effekte, die durch die Zielerreichung intendiert sind, als unwahrscheinlich anzusehen, wird das Engagement der Lehrkraft, unter allen Umständen die gesetzten Ziele zu erreichen, gering sein. Moderierenden Einfluss in diesem durch Rückkopplung und Reflexion gekennzeichneten Modell haben das Selbstmodell der Lehrperson sowie das Außenweltmodell, das durch Schüler und den Unterricht geprägt wird (Allmer, 1982).

Eine sinnvolle Ergänzung des Modells stellt die Integration der aussagenlogischen Fragen des erweiterten kognitiven Motivationsmodells von Heckhausen und Rheinberg (1980) dar, das die Determinanten der Leistungsmotivation, den Anreiz und die Erfolgswahrscheinlichkeit, beinhaltet (siehe Kap. 4.5).

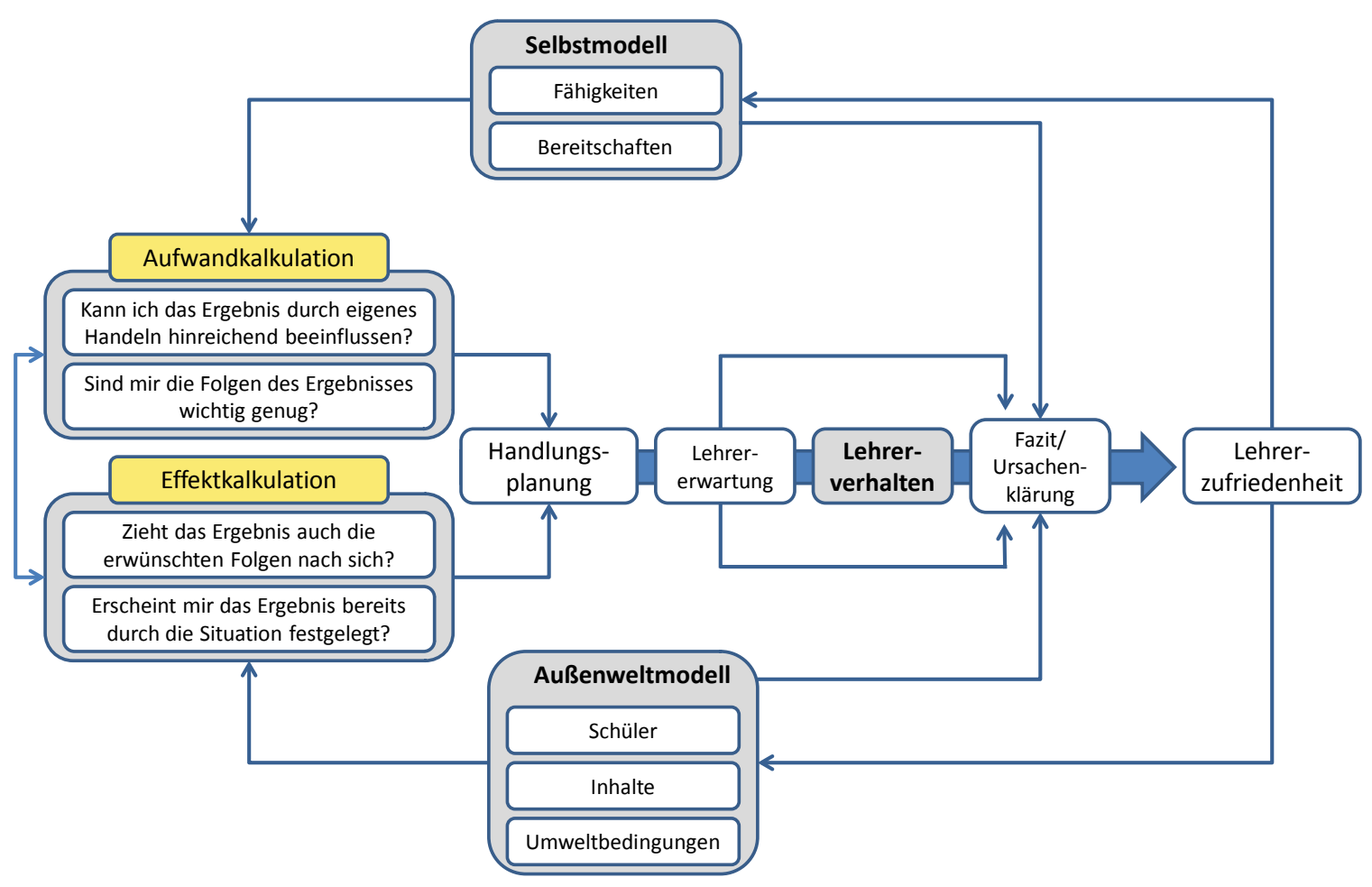

Abb. 14: Integration der aussagenlogischen Fragen des erweiterten kognitiven Motivationsmodells von Heckhausen und Rheinberg (1980) in das Modell des Lehrerverhaltens nach Allmer (1982)

Das ursprünglich zur Analyse von Schülerlernleistungen im Unterricht konzipierte aussagenlogische Modell ist auf die Initiierung von Lehrerhandlungen, im Sinne des Umgangs mit stresshaften Situationen, gut übertragbar. Lediglich wenn die Fragen der Aufwandkalkulation sowie die erste Frage der Effektkalkulation mit ja, die zweite Frage der Effektkalkulation mit nein beantwortet werden, erfolgt in einer konkreten Situation eine Handlungsplanung mit sich anschließender Umsetzung durch die Lehrkraft. Sobald in irgendeiner Art eine Abweichung von diesem Antwortmuster erfolgt, wird 
die Lehrkraft Passivität, Inaktivität oder eine andere evasive Reaktionsweise an den Tag legen. Wird eine Lehrkraft beispielsweise mit einer Situation konfrontiert, in der Schüler untereinander deutliches, vermutlich durch die familiale Sozialisation bzw. Erziehung bedingtes Fehlverhalten zeigen, ist es, durch den moderierenden Einfluss des Selbstmodells möglich, dass die Lehrperson aufgrund einer geringen Erwartung der eigenen Selbstwirksamkeit davon ausgeht, die Grundsituation durch eigenes Handeln nicht beeinflussen zu können. Entweder die Lehrkraft entzieht sich der Situation durch Inaktivität oder Flucht oder versucht in einer anderen, eher palliativen Art und Weise zumindest Schlimmeres zu verhindern bzw. zu vermeiden. In der konkreten Situation könnte die Lehrperson z.B. den Konflikt komplett ignorieren oder durch das Einbringen eines neuen attraktiven Unterrichtsinhalts die Schüler ablenken, um so einer aktiven Auseinandersetzung aus dem Weg zu gehen. Die Bearbeitung der zweiten Frage im Rahmen der Aufwandkalkulation ist eher abhängig von motivationalen Aspekten wie Engagement, Verausgabungsbereitschaft, Perfektionsstreben und Leistungsmotivation der Lehrkraft. In der Effektkalkulation eruiert die Lehrperson, ob durch eine erfolgreiche Zielerreichung der Handlung auch die intendierten Effekte eintreten. Die Lehrperson in der zuvor beschriebenen Beispielsituation wird, abhängig vom Ausmaß der Verhaltensauffälligkeiten der Schüler und dem Erziehungsstil der Eltern, unter Umständen die Wahrscheinlichkeit, durch eine konstruktive Lösung des Konflikts eine langfristigen Verhaltensänderung des Schülers zu erwirken, als gering einstufen. In diesem Fall ist entscheidend, inwieweit die Lehrkraft an sich selbst den Anspruch hat, die Situation nicht auf sich beruhen zu lassen und trotz der geringen Aussicht auf nachhaltige Effekte, eigene Kräfte Investiert. Hinzu kommt, dass eine Honorierung des persönlichen Einsatzes berufsbedingt äußerst fragwürdig ist, da weder von Seiten der Eltern eine positive Rückmeldung zu erwarten ist, noch in irgendeiner Art und Weise ein berufliches Fortkommen in Form einer Beförderung oder Ähnlichem in Aussicht steht. An diesem Punkt wird erneut das Missverhältnis von Aufwand und Ertrag der sozialen Berufe (Gratifikationskrise) deutlich. Sieht der Lehrer das Ergebnis bereits durch die Situation prädisponiert, unabhängig davon ob er nun handelt oder nicht, wird er eher dazu tendieren, seine Kräfte für aussichtsreichere Unternehmen aufzusparen.

Die Begriffe Aufwand- und Effektkalkulation in diesem theoretischen Modell legen nahe, dass in allen Situationen ein elaboriertes Zweckhandeln an den Tag gelegt wird, d.h. dass vor jeder Handlung bzw. Inaktivität Erwartungen, Folgen etc. genau abgewägt werden. Tatsächlich aber laufen einzelne kognitive Prozesse schnell und unbewusst ab, wenn sie erst einmal routinisiert sind, wovon im Lehreralltag, ab einer bestimmten Berufserfahrung auszugehen ist (Heckhausen \& Rheinberg, 1980). Wie man anhand 
des Modells erkennen kann, beeinflusst auch die durch Erfahrungen aus vergangenen Situationen bestimmte Lehrerzufriedenheit in Form von Rückkopplungsprozessen das Selbstmodell und damit zukünftige Kalkulationsprozesse. Bedeutsam bei der Verarbeitung der Rückmeldungen ist die Art und Weise der Attribution von Erfolg und Misserfolg bzw. der Vergleich von erwartetem und tatsächlichem Handlungsablauf. In der Attribution bzw. der Ursachenerklärung spielen Kompetenz- und Kontrollüberzeugungen eine maßgebliche Rolle (siehe Kap. 4.5.2).Während in der zuvor dargestellten Beispielsituation eine Lehrkraft mit ausgeprägter Internalität eine erfolgreiche Lösung des Konflikts durch eigenes aktives Problemlösen als Selbstbestärkung empfindet, ändert sich für external Überzeugte trotz des Erfolges nichts, da sie eher Zufall oder Glück als ursächlich betrachten. Im Falle eines Misserfolges würden die Rückkopplungsprozesse bei vorwiegend external attribuierenden Personen dazu führen, dass zunehmende Hilflosigkeit in Situationen verspürt wird, in denen der Einfluss auf eine erfolgreiche Lösung offensichtlich und jetzt auch nachgewiesenermaßen, scheinbar gering ist. Internal überzeugte Lehrkräfte sehen einen Misserfolg als Signal, sich beim nächsten Mal noch mehr anzustrengen um aus eigener Kraft Situationen wie diese zu bewältigen. Im Rahmen eines Gedankenexperiments kann man sich mit der Leistungsverbesserung von Schülern beschäftigen. Auch hier ist die Ursachenzuschreibung für die Zufriedenheit und die damit verbundenen Rückkopplungsprozesse von enormer Bedeutung. Schreibt man den Vermittlungserfolg den eigenen pädagogischen und didaktischen Fähigkeiten zu, wird man eine Verstärkung des eigenen Fähigkeitsmodells erfahren. Geht man davon aus, dass die Schüler die Verbesserung auch alleine, ohne die didaktischen und methodischen Hilfestellungen erreicht hätten, wird ein positiver Effekt auf das Selbstmodell ausbleiben. In besonders affekthaltigen Situationen kann es auch dazu kommen, dass einzelne Schritte des Verhaltensmodells übersprungen werden und es zu Impulshandlungen kommt, in denen verschiedene Aspekte unberücksichtigt bleiben und spontane, teils ursprüngliche Verhaltensweisen wie Angriff oder Flucht gezeigt werden (Heckhausen \& Rheinberg, 1980).

\subsubsection{Evasives Verhalten im Berufsalltag}

Bevor versucht wird, das evasive Verhalten im Bezug auf die in Kapitel 4.9.1 dargestellten, den Unterricht beeinflussenden Umweltfaktoren zu konkretisieren, soll zuvor ein Beispiel für ausweichendes und vermeidendes Verhalten im Unterricht gegeben werden. Anhand einer Szene aus der Verfilmung des von Heinrich Spoerl im Jahre 1933 veröffentlichten Romans "Die Feuerzangenbowle“ lässt sich in anschaulicher Art zeigen, wie verschiedene Beweggründe einen zunächst offensiven problemorientierten Bewältigungsversuch, in eine vermeidende und eher defensive Problembewältigung 
verwandeln können. Weiterhin lässt sich zeigen, dass die als Umweltfaktoren bezeichneten Einflüsse nicht als gänzlich voneinander isolierte Komponenten aufzugreifen sind, sondern untereinander Kohärenz aufweisen, teils sogar gekoppelt sind. Im sich anschließenden Auszug aus der Verfilmung des Romans „Die Feuerzangenbowle“ durch Helmut Weiss (1944) werden die Dialoge, sowie die Szenerie in der sich diese ereignen, dargestellt.

Der Schüler Ackermann befindet sich während dem Deutschunterricht am Gymnasium von Babenberg an der Tafel. Bei dem unterrichtenden Lehrer handelt es sich um Professor Crey, der unter den Schülern auch als „Schnauz" bekannt ist. An der Tafel stehen einige bekannte deutsche Literaten, samt deren Geburts- und Todesjahren. Als die Tafel voll ist, fordert Professor Crey den Schüler Ackermann auf, die Tafel umzudrehen. Ackermann antwortet: „Ja... das...das geht nicht, ich glaube die klemmt". Offensichtlich wenig überzeugt begibt sich Prof. Crey mit den Worten „Oh...zeigen Sie mal her" von seinem erhöhten Pult zu der Tafel und dreht diese, mit einem unterstützenden und wohl dosierten gezielten Fausthieb, ohne Probleme um. Als sich Professor Crey gerade abwenden will sieht er, was sich auf der Rückseite der Tafel befindet - eine Zeichnung einer nackten, offensichtlich weiblichen Person, die in einer Pose mit leicht gespreizten Schenkeln, mit Kreide skizziert wurde. Während Professor Crey die Zeichnung mit hinter dem Rücken verschränkten Armen inspiziert, ist bei den sich auf ihrem Platz befindlichen Schülern Unruhe, und vor allem auf dem Gesicht von Johannes Pfeiffer ein erfreutes Grinsen zu sehen. Professor Crey dreht sich um und lässt seinen Blick über die Klasse schweifen. Ackermann fragt: "Soll ich das da vielleicht wegwischen?". Professor Crey antwortet erzürnt: „Holen sie den Herrn Direktor?“. Nachdem als Überblendung eine ZeusStatue im Bild erscheint, die sich offensichtlich im Klassenzimmer befindet, erscheint das Gesicht von Direktor Knauer, der Stellung zum Vorfall nimmt: „Ich muss sagen, ich bin aufs Tiefste erschüttert, einen solchen Fall sittlicher Entgleisung in meiner Anstalt zu erleben - Pfui über euch! Bevor ich in die Untersuchung dieses Falls eintrete, will ich dem schuldigen Schüler die Gelegenheit geben, sich freiwillig zu melden. Nein! Nicht hier vor der Klasse, die Schande will ich ihm ersparen, er mag heimlich auf mein Zimmer kommen." Der Schüler Pfeiffer meldet sich. Direktor Knauer: „Was wollen sie Pfeiffer?“. Pfeiffer steht auf und spricht: „Also Herr Direktor, Sie können mich totschlagen, aber ich find das Bild ganz hübsch. Direktor Knauer (wild gestikulierend): „Also Pfeiffer, ein junges Mädchen, vielleicht aus bester Familie und ohne jegliche Bekleidung.“ 
Pfeiffer entgegnet: „Ja aber Herr Direktor, das seh ich doch überhaupt nicht ein, das ist doch gar kein Mädchen, das soll doch ein Junge sein." Der Schüler Pfeiffer kann nun sein Grinsen nicht mehr zurückhalten. „Wir sind mit der Zeichnung nur nicht ganz fertig geworden." Die Klasse ist in heller Aufregung aufgrund des offensichtlich genialen Schachzugs von Pfeiffer. Pfeiffer setzt sich und der Direktor überlegt mit an den Hals gelegter Hand. Er betrachtet die Zeichnung erneut und antwortet: "In der Tat, ich habe mir auch schon so etwas gedacht". Er reibt sich die Hände und fährt fort: „Na jedenfalls ist es mir außerordentlich lieb, dass dieser peinliche Vorfall eine so harmlose Aufklärung gefunden hat. Und nun wollen wir nicht mehr darüber sprechen. Ackermann wischen sie das aus!“. Der Direktor verlässt scheinbar zufrieden den Klassenraum (Weiss, 1944).

In diesem Auszug aus dem Film bzw. dem Roman „Die Feuerzangenbowle“ kann man exemplarisch Wirkzusammenhänge der verschiedenen Einflüsse und Umwelten aufzeigen, unter denen Lehrer aber auch Direktoren ihre tägliche Arbeit verrichten. Direktor Knauer verhält sich gegenüber seinen Schülern, nachdem er zuerst auf offensive Art und Weise einen Schuldigen finden wollte, evasiv. Bezogen auf das transaktionale Stresskonzept von Lazarus nimmt er im Verlauf des Konfliktes eine Neubewertung aufgrund der veränderten Ausgangslage vor. Direktor Knauer, der von den Schülern den Spitznamen "Zeus", wegen seinem lenkenden und ausgleichenden Verhalten in der Schule erhielt, zeichnet sich vor allem dadurch aus, dass er überflüssige Konflikte vermeidet. Nach seiner Ansicht sind jedoch alle Konflikte überflüssig (Grimm \& Rieger, 1999). Eine interpretative Deutung des Verhaltens von Direktor Knauer wird in der Beschreibung des evasiven Verhaltens im Bezug auf den Umgang mit Schülern sowie im Bezug auf äußere Rahmenbedingungen erfolgen. Die Darstellung des evasiven Verhaltens in Bezug auf Inhalte wird in Kapitel 4.9.4.2 allgemein, nicht orientiert am Filmbeispiel erfolgen.

4.9.4.1 Evasives Verhalten im Bezug auf den Umgang mit Schülerinnen und Schülern

Pädagogische Situationen sind stets geprägt durch Spannungszustände aufgrund voneinander abweichenden Verhaltensweisen, unterschiedlichen Entwicklungsstadien oder Differenzen im Können und Wissen sowie disparaten außerschulischen Einflüssen (Potthoff \& Wolf, 1976). Um sinnvolle Arbeit im Sportunterricht zu leisten, muss in angemessener Art und Weise mit Schülern umgegangen werden. Konflikte mit Schülern oder unter Schülern verlangen von Lehrkräften eine unmittelbare und angemessene Reaktion, um einerseits eine Lösung der aktuellen Situation herbeizuführen und 
um andererseits Schülern für die Zukunft einen Orientierungsrahmen zu bieten. Für die Praxis bedeutet dies, dass Lehrkräfte sich auf eine Gratwanderung begeben müssen zwischen konsequentem Handeln auf der einen und angebrachter Großzügigkeit auf der anderen Seite. Sportlehrkräfte stehen oft vor der Frage, wofür es sich überhaupt lohnt „Zeit und Nerven zu lassen“, da es aufgrund der Charakteristika des Berufes und des Faches unmöglich ist, alle Probleme und Konflikte aktiv anzunehmen und pädagogisch sinnvoll aufzulösen (Wolters, 2008, S. 5). Struck (2007) betont, dass gegenwärtige Investitionen durch die Lehrer erst langfristig zu einer Entlastung und gleichzeitig besseren Lernerfolgen der Schüler führen. Als evasiv würde man den Verhaltensstil einer Lehrkraft dann bezeichnen, wenn die Großzügigkeit gegenüber dem konsequenten Handeln deutlich überwiegt und keine didaktische Legitimierung zugrunde liegt, sondern Desengagement, Schonung oder Selbstschutz die handlungsbestimmenden Faktoren sind. Allmer (1982) sieht die Gründe für das Übersehen von Problemen und Konflikten weniger im motivationalen Bereich, als vielmehr in einer geringen Fähigkeitszuschreibung und dem damit verbundenen Fehlen jedweder aktiver Handlungsmöglichkeiten.

Probleme im Bezug auf den Umgang mit Schülern können im Bereich der Sach-, Rahmen- und Beziehungsanforderungen entstehen (Wolters, 2008). In diesem Zusammenhang wird deutlich, dass zwischen den verschiedenen Umweltfaktoren starke Interdependenzen bestehen und Entscheidungen auf der Inhaltsebene stets Einfluss auf den Umgang mit Schülern etc. nimmt.

Sportlehrkräfte können durch die Planung und Durchführung ihres Unterrichts beeinflussen, in welcher Art und Weise Schüler mit Sachanforderungen im motorischen oder konditionellen Bereich konfrontiert werden. Personen, die beabsichtigen, mit möglichst wenig Aufwand und Ärger im Sinne eines muddling through ${ }^{4}$ durch den Unterrichtstag zu kommen, werden ihren Unterricht so ausrichten, dass Konflikte mit enttäuschten Schülern aufgrund von erlebten Misserfolgen ausbleiben. Durch die Verschulung und damit auch Rahmung des Sportunterrichts sind Schüler zur Teilnahme verpflichtet und müssen auch unbeliebte Inhalte akzeptieren und sich mit ihnen Auseinandersetzen. Auch hier kann die Umsetzung durch die Lehrkräfte wieder stark differieren. Während es einer Lehrkraft, so lange kein nach außen auffallendes Fehlverhalten an den Tag gelegt wird, egal ist, dass einzelne Schüler sich aufgrund von Unzufriedenheit mit dem Fach generell oder dem aktuellen Inhalt hauptsächlich passiv verhalten, setzen andere Lehrkräfte ihre Energie ein, um möglichst alle Schüler am Unterrichtsgeschehen zu beteiligen. Den dritten Anforderungsbereich an die Schüler stellen

\footnotetext{
${ }^{4}$ Deutsche Übersetzung: „sich Durchwursteln“.
} 
die sozialen Beziehungen dar, die aus den Inhalten und den Rahmungen in dem diese stattfinden resultieren (Wolters, 2008). Auch hier sind von Seiten der Lehrkraft sowohl präventiv als auch situativ Bestrebungen möglich, die einen reibungslosen Ablauf garantieren, auch wenn dafür das Potenzial, das der Sportunterricht für soziales Lernen und das Sammeln von unmittelbaren Erfahrungen im Umgang mit anderen Menschen in sich trägt, nicht ausgeschöpft wird. Begünstig werden solche evasiven Verhaltenstendenzen durch die Charakteristik des Sportunterrichts, der Ausgleichs- und Ventilfunktion für Schüler aufweist. Durch weitestgehende Reglementierung in den anderen Schulfächern, vor allem bezogen auf den motorischen aber auch den sozialen Bereich, entwickelt sich im Sportunterricht schnell eine Eigendynamik, die von der Lehrkraft kaum noch steuerbar ist und auch ein Hinausschießen der Schüler über das Ziel keine Seltenheit darstellt (Miethling, 1989). Während in den Fächern, die im Klassenunterricht organisiert sind, Anzeichen von Aggressivität bzw. von Konflikten mehr oder weniger elegant unter den Teppich gekehrt werden können, liegt aufgrund der unmittelbaren Auseinandersetzung von Schülern miteinander im Sportunterricht eine Situation vor, die einen anderen Umgang mit Konflikten erfordert. Im Sportunterricht machen es sich Lehrkräfte zur Aufgabe, einzelne kritische Situationen zu beheben, ohne dabei unmittelbar die Ursachen bzw. den eigentlichen Kern des Problems, der z.B. durch das häusliche bzw. familiäre Umfeld gebildet wird, zu tangieren. So wie in anderen sozialen, pädagogischen oder helfenden Berufen stellt sich bald diese schmerzliche Erkenntnis des fast aussichtlosen Bemühens ein, die jedoch nicht zu einer resignative Grundhaltung führen sollte, die sich durch Bewältigungsmaßnahmen, wie Evasion kennzeichnet. Auch wenn das Ausweichen vor bzw. das Ignorieren von Konflikten und Problemen auf eine bequeme Art und Weise eine Scheinlösung offeriert, sollte dennoch versucht werden, die Entwicklung der Schüler zu unterstützen, um so auch dem gesellschaftlichen Erziehungsauftrag langfristig gerecht zu werden (Heine, 1981).

Im zuvor dargestellten Konfliktfall aus dem Film „Die Feuerzangenbowle“ zeigt der Direktor zunächst genau das Verhalten, das Professor Crey sich versprochen hat: Mit harter Hand die Aufklärung der sittlichen Entgleisung voranzutreiben. Das an einer Schule mit gutem Ruf höchst verachtenswerte Verhalten der Schüler erfordert nach Einschätzung des Direktors eine umgehende Aufklärung. Es stellt sich jedoch heraus, dass der Vorfall nicht so einfach zu handhaben ist, wie zunächst gedacht. Angeregt durch die Aussage von Pfeiffer, auch wenn die Zeichnung und die dahinterstehende Intention, vor allem durch das Schmunzeln von Pfeiffer ziemlich eindeutig sind, öffnet sich für den Direktor eine Hintertür. Die Situation, deren Lösung mehr zu erfordern scheint als eine Drohung, offeriert eine weitere Alternative im Rahmen derer zwar das 
Verhalten ungesühnt bleibt, der Direktor aber dennoch sein Gesicht wahren kann. Der Direktor lässt sich auf die hanebüchene Erklärung von Pfeiffer ein und beendet die ganze Situation schnellstmöglich, um nicht mit einer weiteren Schüleräußerung oder einer Rückfrage von Professor Crey konfrontiert zu werden. Neben diesen Motiven, die eher als Aufwandsvermeidung zu bezeichnen sind, weisen weitere Aspekte, die unter dem Bezug zu äußeren Rahmenbedingungen in Kapitel 4.9.4.3 konkretisiert werden, eine vielleicht sogar noch einflussreichere Bedeutung auf.

\subsubsection{Evasives Verhalten im Bezug auf Inhalte}

Bereits im Jahr 1982 stellte Allmer eine ähnliche inhaltliche Schwerpunktsetzung im Sportunterricht fest wie Kolb und Wolters (2000) in ihrer Unterrichtsbeobachtungen und lieferte hierfür auch Begründungszusammenhänge. Allmer vermutet, dass das Lehrerverhalten eng mit dem Fähigkeitsselbstkonzept verbunden ist und demzufolge Unterrichtsinhalte in denen eine Person sich selbst gute Fähigkeiten zuschreibt bzw. die Anforderungen der durchzuführenden Aktivität als gering ansieht, mit Vorliebe zum Dauermittelpunkt des Unterrichts avancieren, andere Inhalte hingegen gemieden werden. Mit zunehmendem Alter nimmt die Angst zu, den Ansprüchen, die von curricularer Seite gestellt werden, nicht mehr genügen zu können. Ein Ausweg aus diesem Dilemma stellen Fort- und Weiterbildungsmaßnahmen dar, die die Sorge um die Qualität des eigenen Unterrichts durch den Aufbau und die Aktualisierung der Fach- und Methodenkompetenz abpuffern können. Sportlehrer, die ihr individuelles Fortbilden vernachlässigen, befinden sich hingegen entweder tagtäglich in einer bedrückenden Situation, die durch Unsicherheit und Angst zu versagen geprägt ist oder aber organisieren ihren Unterricht fernab von jeglichem Anspruchsdenken in Form einer läppischen Spielerei, um fehlende Kompetenz zu verschleiern (Meusel, 1989). Da mit zunehmendem Alter auch die Einsatzbereitschaft ab- bzw. das Desengagement zunimmt, werden häufig alternative Auswege aus diesem Dilemma gesucht. Ein Rückzug oder gar ein Ausstieg aus dem Sportunterricht ist eine häufig beobachtete Tendenz. Bei dem Rückzug muss jedoch differenziert werden zwischen Lehrern, die diese Zielsetzung durch eine Reduzierung der Sportstundenzahl erreichen und denjenigen, die zwar weiter Sport unterrichten, aber ihren Arbeitseinsatz als Notlösung stark zurückfahren und mit evasivem Verhalten einen Weg der bewussten Entlastung einschlagen. Lehrplanänderungen werden dann nicht mehr rezipiert und umgesetzt und auch im außerunterrichtlichen Bereich wird kein Engagement mehr gezeigt. Konfliktträchtige Unterrichtsinhalte werden präventiv ausgeklammert, um stattdessen durch die Erfüllung von Schülerwünschen den Weg des geringsten Widerstands zu gehen (Kastrup \& Neu- 
mann, 2008). Dieses als „innere Kündigung“ bezeichnete Verhalten wird in Kapitel 4.9.5.2 genauer erläutert.

Das Resultat von Problemen bei der Realisierung von Inhalten ist oft eine Reduzierung auf das Machbare. Hierbei werden im Gewand einer Minimalorientierung diejenigen Inhalte durchgeführt, die aufgrund des Verhaltens von Schülern sowie den eigene Fähig- und Fertigkeiten als relativ problemlos umsetzbar erscheinen (Lange, 1981). Zur Konfliktvorbeugung im Unterricht erfolgt außerdem eine Reduktion motorischer Anforderungen auf ein Mindestmaß, sodass von Seiten der Lehrkraft keine großen Vermittlungstätigkeiten notwendig sind und die Schüler in Form einer Selbstbetätigung möglichst eigenständig und ohne Eingriffe von außen tätig werden können. Komplexere Unterrichtsinhalte werden aufgrund des höheren organisatorischen Aufwands und den notwendigen vorbereitenden technischen und taktischen Unterrichtsanteilen gemieden (Kolb \& Wolters, 2000). In der Praxis stellt gerade Gerätturnen einen Unterrichtsinhalt dar, der trotz unbestrittenem Wert, bezogen auf die Entwicklung von motorischen Fertigkeiten und der Ausbildung von Körperbeherrschung und Gleichgewicht, aus verschiedenen Gründen von Lehrkräften gemieden wird. Neben dem relativ aufwendigen Aufbau von Großgeräten mit den dazugehörigen Sicherungen durch Matten etc. stellen die meisten turnerischen Übungen durch Hilfe- und Sicherungsstellungen zusätzliche Anforderungen an die Lehrkräfte. Weiterhin werden gerade hier eigene Defizite schnell aufgedeckt und das Verletzungsrisiko fälschlicherweise als hoch eingeschätzt ${ }^{5}$. Bei genauer Betrachtung der Feststellungen von Kolb und Wolters (2000) zu den schwerpunktmäßig durchgeführten Inhalten lassen sich die Einschätzungen bezüglich des Unfallrisikos bestätigen. Während in der Grundschule Bewegungslandschaften morgens in der ersten Stunde aufgebaut und in den folgenden Stunden meist in Form des freien Turnens genutzt werden, scheint auch das Volleyballspiel nicht nur aufgrund des didaktischen oder gesundheitlichen Werts in solcher Ballung als Unterrichtsinhalt gewählt zu werden. Das Volleyballspiel zeichnet sich dadurch aus, dass aufgrund der räumlichen Trennung der beiden Mannschaften kein direkter Kontakt zwischen den Schülern zustande kommt und damit auch das Potenzial für Schülerkonflikte geringer ist. Des Weiteren zeichnet sich das Volleyballspiel durch einen in Studien zum schulsportlichen Unfallgeschehen berechneten unterdurchschnittlichen Risikofaktor aus, der das relative Unfallrisiko der Sportart beschreibt (Hübner \& Pfitzner, 2001; 2003).

\footnotetext{
${ }^{5}$ Die Auswertungen der Schulsportunfälle durch Hübner und Pfitzner $(2001 ; 2003)$ in NordrheinWestfalen und Bayern zeigen, dass der Risikofaktor beim Gerätturnen, der die relative Unfallhäufigkeit angibt, sogar leicht unterdurchschnittlich ist.
} 


\subsubsection{Evasives Verhalten im Bezug auf äußere Rahmenbedin-} gungen

Grundsätzlich sind Lehrkräfte dazu verpflichtet, ihren Unterricht gemäß gesetzlicher Vorschriften und Erlasse durchzuführen. Neben der Einhaltung der Zeitordnung sowie genau geregelten Bestimmungen zur Aufsichtspflicht sind eine Reihe weiterer institutioneller Vorschriften zu beachten. Diese werden von Sportlehrern in unterschiedlichem Maß als Belastung empfunden (Lange, 1981; Kolb \& Wolters, 2000). Während zu Beginn der Berufskarriere Sportlehrkräfte ihren Unterricht oft in schülerorientierter Art und Weise planen und durchführen, wandelt sich dies nach einigen Jahren zu einer eher institutsorientierten Ausrichtung. Der breite Katalog an didaktischen und pädagogischen Zielen, die im Referendariat verfolgt wurden, wird abgelöst durch eine reine Sachorientierung, die von Pragmatismus geprägt ist. Diese reine Ablauforientierung wird unterstützt durch das auch heute noch weit verbreitete Klischee, dass ein guter Pädagoge seine Klasse im Griff hat und sein Unterricht problem- und reibungslos verläuft (Lange, 1981). Es gilt als nicht schicklich, im Lehrerzimmer über Probleme zu reden und das Vermeiden von Gesprächen über schwierige Schüler und Probleme im Umgang mit ihnen ist genauso ritualisiert wie die Sitzordnung im Lehrerzimmer (Struck, 2007). In den Gruppennormen, die sich aufgrund von Handlungsräumen bilden, die aus sozialen und persönlichen Gründen unsicher sind, spiegeln sich Abwehrformen wider, die mehrheitlich unter den Sammelbegriffen Konfliktvermeidung sowie gegenseitige Diskretion und Imagepflege zusammenzufassen sind. Dies trifft gerade auf den Sportunterricht zu, der sich, wie in Kapitel 2.3 beschrieben, in einem stetigen Legitimationsprozess zu befinden scheint und durch die Körperlichkeit und das Modelllernen mehr noch als andere Fächer von der gesamten Persönlichkeit des Unterrichtenden geprägt ist. Dementsprechend werden Probleme in der praktischen Unterrichtsdurchführung oft mit individueller Inkompetenz verknüpft, wodurch die Persönlichkeit der Lehrkraft schnell negativer beurteilt wird, als dies in anderen Fächern der Fall ist.

Hat sich aber ein solches Normenpaar erst einmal etabliert, so hat das Folgen: Man kennt sich untereinander mit den persönlichen Schwächen und Eigenheiten zwar genau, Gründe für Ärger durch reale Übergriffe und persönliche Beeinträchtigungen gibt es nach wie vor in Hülle und Fülle. Aufgrund der allseits akzeptierten Normen geht man aber einfach „nicht“ damit um: man tut so, als wäre nichts. Da ein Austausch über die verschiedenen Formen der Wirklichkeitsverarbeitung als tabuisierte Konfliktaustragung oder Indiskretion negativ sanktioniert wird, bleibt - und damit schließt sich der Kreis zu der festen Verteilung von Gruppenpositionen - je- 
der auf seine spezielle Form der Differenz gegenüber den anderen fixiert und lebt sie - je nachdem: „freizeitlich“ oder „unterrichtlich“ - privatisierend aus (Kern, 1986, S. 217).

Kommunikation im Kollegium wird in Untersuchungen von Lehrern meist als gemischt bis eher negativ eingeschätzt, was nicht zuletzt durch das Verschweigen von Unterrichtsproblemen und der damit verbundenen fehlenden Offenheit bedingt ist (Ulich, 1996). Aufgrund der mangelnden Kommunikation und versteckten subkutanen Machtauseinandersetzungen resultieren aus fixen Rollenzuweisungen innerhalb des Kollegiums letztendlich Majoritäts- und Minoritätsgruppen, die durch maximale Konfliktvermeidung bei hohem Konfliktpotenzial zur Selbstvergiftung des Kollegiums führen können (Kern, 1986).

Auch die Notengebung ist ein Bereich, aus dem Konflikte mit Schülern, vor allem aber auch mit ihren Eltern, entstehen können. Welcher Lehrer will schon wegen der Sportnote Ärger mit den Eltern, da gibt man doch lieber gute Noten und erspart sich diesen. Im Sportunterricht wird oft eine weiche Benotung vorgenommen, die sowohl die Gleichwertigkeit des Faches Sport mit den übrigen Fächern gefährdet, als auch bezogen auf die Bedeutsamkeit für die sportliche Selbsteinschätzung der Schüler kritisch zu betrachten ist. Selbstverständlich ist ein gewisses Fingerspitzengefühl von Nöten bei der Vergabe von Noten im Sportunterricht, da viel mehr als in anderen Fächern die Schüler sich selbst, mit ihrem gesamten Körper als Be- und teils Verurteilte fühlen, was den Lehrer jedoch nicht von der Aufgabe entbindet, erbrachtes Engagement und Leistungen zu bewerten. Während in anderen Fächern die Note „befriedigend“ oder „ausreichend" auf der Tagesordnung steht, bekommen die meisten Kinder und Jugendlichen im Fach Sport ein "sehr gut" oder zumindest ein "gut" für ihre Leistung attestiert. Wer nur ein „befriedigend“ am Ende des Schul- bzw. Halbjahres erhält, scheint nun wirklich gänzlich unbegabt oder hat sich zu oft mit dem Lehrer angelegt: „Noten schlechter als befriedigend gibt es sowieso nicht“ (Thomann, 2006, 206). In der SPRINT-Studie, in die Daten von knapp 9.000 Schülerinnen und Schülern aus verschiedenen Bundesländern einbezogen wurden, stellte sich heraus, dass die Durchschnittszensuren im Fach Sport deutlich besser ${ }^{6}$ sind als in Mathematik oder Deutsch. Ebenfalls zeigte sich, dass das Notenspektrum von sehr gut bis befriedigend reicht und schlechtere Noten nur sehr selten gegeben werden ${ }^{7}$ (Gerlach et al., 2006)

\footnotetext{
${ }^{6}$ Um ca. 0,75 Notenpunkte.

${ }^{7}>94 \%$ zwischen sehr gut und befriedigend, $<5 \%$ ausreichend, $<1 \%$ mangelhaft und ungenügend (Gerlach et al. 2006).
} 
Das bereits im Bezug zu dem Verhalten gegenüber den Schülern erläuterte evasive Verhalten von Direktor Knauer in "Die Feuerzangenbowle" ist in starkem Maße auch bestimmt durch äußere Rahmenbedingungen. Während der Maßregelung der Klasse, wächst in Direktor Knauer das Bewusstsein, welch schlechtes Licht auf die Schule und seine Führung fällt, wenn an die Öffentlichkeit gerät, welche Unsitten sich im Unterricht ereignen. Diese im Rahmen der Effektkalkulation vorgenommenen antizipativen Gedanken zu der Frage, ob das Ergebnis auch die gewünschten Folgen nach sich zieht, muss Direktor Knauer deutlich verneinen. Sobald ein Schuldiger gefunden und damit auch die ganze peinliche Situation als wahrhaftige Tatsache verfestigt wird, droht ein Verlust des Ansehens und schlechtes Gerede über die Arbeit des Direktors und die Schule. Er ist grundsätzlich auf der Wahrung seines Ansehens bedacht und vermeidet daher Ärger mit Behörden und ranghöheren Personen. Würde er der Tat nachgehen und einen Schuldigen finden, zöge dies auch eine Mitteilung an die Eltern und vielleicht sogar ein Gespräch mit diesen nach sich, das für ihn ebenfalls unangenehm verlaufen könnte. Die anhand des Beispiels dargestellte Situation kann ebenso auch auf normale Lehrkräfte bezogen werden, die aufgrund der Angst vor Kritik durch Kollegen, Eltern und Vorgesetzte den einen oder anderen Störfall lieber für sich behalten, um letztendlich nicht eine Teilschuld an den Vorfällen zugewiesen zu bekommen (Ulich, 1996).

Die schlechte Ausstattung von Sportstätten ist eine Thematik, die bereits seit längerer Zeit und wiederholt in fachwissenschaftliche Diskussionen auftaucht (z.B. Oesterreich, 2008) und mehr als moderierender Faktor in diesem Zusammenhang zu sehen ist. Da die räumlichen und materiellen Bedingungen an vielen Schulen nicht optimal sind, ist der Sportunterricht oft mit Unannehmlichkeiten und zusätzlichem Aufwand für die Sportlehrkräfte verbunden. Wege zu Trainings- und Sportstätten, Transport von Material oder gar schlechte hygienische Bedingungen in Umkleiden etc. können dazu führen, dass Engagement ausbleibt. Zum Teil ist sogar eine resignative oder trotzige Grundhaltung bei Sportlehrkräften zu sehen, die es nicht einsehen, die Leidtragenden der stets zunehmenden Sparpolitik der Länder zu sein bzw. die irgendwann nicht mehr in der Lage sind, durch eigenes Engagement kompensatorisch zu wirken. ${ }^{8}$ Ausweichendes und auf Schonung bedachtes evasives Verhalten ist in diesem Zusammenhang teils eine notwendige, wenn auch meist ungewollte Verhaltensstrategie, die dem Überleben als Sportlehrkraft dient.

\footnotetext{
${ }^{8}$ Bei einem Vortrag und der sich anschließenden Diskussion auf dem Kongress des Deutschen Sportlehrerverbands im Jahr 2008 wurde deutlich, inwieweit sich Sportlehrkräfte im Stich gelassen fühlen und aufgrund schlechter Rahmenbedingungen den unterrichtlichen Ertrag, vor allem aber auch ihre eigene Gesundheit in Gefahr sehen.
} 


\subsubsection{Beanspruchungsreaktionen und Beanspruchungsfolgen resul- tierend aus evasivem Verhalten}

Wie bereits in Kapitel 3.1 und 3.2 dargestellt, sind Stressereignisse und die daraufhin inszenierten Bewältigungsmaßnahmen mit kurzfristigen Reaktionen und teils langfristigen Folgen verbunden. Während sich die kurzfristigen Beanspruchungsreaktionen unmittelbar auf aktuelle Ereignisse beziehen und mit psychophysischen, kognitiven, affektiven oder verhaltensbezogenen Reaktionen einhergehen, sind die Beanspruchungsfolgen nur bedingt reversibel und treten erst mittel- bis langfristig auf. Im Rahmen der Darstellung von evasivem Verhalten sollen mit Burnout und Innerer Kündigung vor allem zwei Folgeerscheinungen dauerhafter, sich wiederholender unangemessener Bewältigungshandlungen skizziert werden. Dass es sich bei evasivem Verhalten meist um eine unangemessene Form der Bewältigung handelt, macht bereits Sigmund Freud deutlich, der in der Verdrängung einen Grundmechanismus sieht, der zwar strukturell am einfachsten, für die Krankheitsentwicklung jedoch am bedeutendsten ist (Nusko, 1986).

\subsubsection{Burnout}

Bereits im Jahr 1911 berichtet der „Oberpfälzer Schulanzeiger" von einer für den Lehrerberuf typischen Nervenkrankheit, der Neurasthenie, die aufgrund ungünstigen Arbeitsbedingungen und Überforderung entsteht und sich in einem relativ umfangreichen Krankheitsbild äußert. Neben allgemeiner Nervenerschöpfung ist auch die Rede von Schlafstörungen, schneller Ermüdbarkeit, Überempfindlichkeit, verminderter Leistungsfähigkeit, Angst, Reizbarkeit und gedrückter, niedergeschlagener Stimmung (Barth, 1997). Im Verlauf dieses Kapitels wird sich zeigen, dass das beschriebene Phänomen große Schnittmengen zu dem aufweist, was heutzutage als Burnout bezeichnet wird.

Trotz der Tatsache, dass bereits zur damaligen Zeit offenbar ein nicht unerheblicher Teil der Lehrer an solchen Störungen litt, dauerte es bis ins Jahr 1974, bis Freudenberger den Begriff des Burnouts ins Zentrum des wissenschaftlichen Interesses rückte und damit bildlich gesprochen den Startschuss für unzählige Forschungsvorhaben, Datenerhebungen und Publikationen abgegeben hat. Alle bisherigen Bemühungen konnten jedoch meist nur widersprüchliche Ergebnisse liefern (Schmitz, 2004). Da Burnout ein mehrdimensionales Konstrukt mit verschiedenen Facetten psychischen Verhaltens und Erlebens darstellt und dementsprechend in mehreren Bereichen verwurzelt ist, stellen sich die wissenschaftlichen Publikationen zu dem theoretischen Konzept sowie die zugehörige Forschungsrichtung als relativ uneindeutig heraus. Im deutschsprachigen Raum beschäftigen sich Forscher seit 1983 mit Burnout als Phänomen, das hauptsäch- 
lich in sozialen Berufen auftritt. Der Begriff Burnout stammt aus dem englischen Sprachraum und wurde ursprünglich im technischen Bereich eingesetzt und bedeutet wörtlich übersetzt ausbrennen oder durchbrennen (Barth, 1997). Es bezeichnet vorrangig negative Folgen von Arbeitsbeanspruchungen von psychosozialen Berufsgruppen, die aus fehlgeschlagener Bewältigung hervorgehen (Kramis-Aebischer, 1996). Sosnowsky-Waschek (2010) bezeichnet Burnout als Mischung aus Vielem und Nichts. Eine spontane Assoziation mit Burnout ist die Vorstellung von einer Person, die am Ende einer leidvollen und anstrengenden Zeit, die zunehmend von Hilflosigkeit geprägt war, resigniert und erschöpft in sich zusammenbricht, da sie sich aufopferte und dabei vergaß, auf sich selbst zu achten (Sosnowsky, 2007). Sinnvoll in diesem durch Unschärfe und inflationäre Nutzung des Burnout-Begriffs in der Gesellschaft geprägten Forschungsfeld ist es, die Vielzahl an teils diskrepanten Definitionsansätzen in Form einer Meta-Analyse aufzuarbeiten, wie dies von Schaufeli und Enzmann (1998) umgesetzt wurde:

Burnout ist ein andauernder negativer, arbeitsbezogener psychischer Zustand, ,normaler' Personen, die primär durch Erschöpfung gekennzeichnet ist und von Überforderung (distress), dem Gefühl verminderter Wirksamkeit, abnehmender Motivation sowie der Entwicklung dysfunktioneller Einstellungen und Verhaltensweisen begleitet wird. Dieser psychische Zustand entwickelt sich langsam, kann aber von den Betroffenen lange unbemerkt bleiben. Er resultiert aus einem Missverhältnis (misfit) von Intentionen der Arbeitswirklichkeit. Oft wird er durch inadäquate Bewältigungsstrategien aufrechterhalten (zitiert nach Schmitz, 2004, S. 52).

Während in der obigen Definition von Schaufeli und Enzmann schwerpunktmäßig eine Beschreibung des Zustands vorgenommen wird, sind in der Definition von Pines, Aronson und Kafry (1992) zusätzlich kausale Erklärungen enthalten. Sie beschreiben Burnout als Zustand physischer, emotionaler und mentaler Erschöpfung, verursacht durch lang anhaltende Einbindung in emotional fordernde Situationen und beschränken damit in ihrem Ansatz Burnout nicht auf soziale und helfende Berufe, wie dies bis in die 1990er Jahre hinein der Fall war, sondern dehnten die Anwendung des Begriffs auf weitere Bereiche aus. Von der Weltgesundheitsorganisation (WHO) wurde das Burnout-, Syndrom“ zwar in die Internationale Klassifikation psychischer Störungen (ICD-10) aufgenommen, jedoch wurde ihm nicht der Status „Diagnose“ zuerkannt, sodass es im medizinischen Sinn keine Krankheit darstellt. Für wissenschaftliche Forschungsansätze ist diese Einschränkung relativ unbedeutend, für Betroffene hingegen hat es insoweit Bedeutung, dass Krankenkassen sich bei der Kostenübernahme an den Vorgaben der 
WHO orientieren und dementsprechend nicht zwangsläufig zu Zahlungen verpflichtet sind (Schmitz, 2004).

Die Verbreitung von Burnout unter Lehrern wird in verschiedenen Studien (Barth, 1997; Kramis-Aebischer, 1996; Schaarschmidt, 2005a) meist mit einem Anteil zwischen 15 und 36 Prozent beziffert, wobei einige der Studien nicht repräsentativ sind und daher eher als Indizien und nicht als Widerspiegelung der Realität zu sehen sind. Bei der Altersverteilung der Lehrkräfte, die von Burnout betroffen sind, stellte sich heraus, dass jüngere Lehrer häufiger betroffen sind als ältere. Man kann jedoch nicht mit Sicherheit ausschließen, dass diese Befundlage dadurch zustande kommt, dass die Ausgebrannten in fortgeschrittenen Karriereabschnitten bereits zum Großteil den Beruf verlassen haben oder aber, dass ältere Lehrer, die unter Burnout leiden, seltener die Fragebogen ausfüllen. Aufgrund der beiden genannten Einschränkungen, die auch durch zum Teil geringe Stichproben verschärft werden, kann man nicht mit Sicherheit sagen, ob ältere Lehrkräfte auch wirklich gesünder sind als diejenigen, die sich gerade in der Phase des Praxisschocks befinden bzw. diesen gerade überwunden haben (Schmitz, 2004). Byrne (1999) fasst in ihrem Überblick ebenfalls altersbezogene Ergebnisse zu dem Auftreten von Burnout zusammen und beschreibt eine eher inkonsistente Befundlage.

Bei den geschlechtsspezifischen Auswertungen zeigt sich immer wieder, dass Frauen häufiger unter Burnout leiden als Männer (Schmitz, 2004). Eine Begründung hierfür ist einerseits die Doppelbelastung von Beruf und Familie, es zeigt sich, dass Männer durchschnittlich deutlich höhere Angaben bei der wöchentlichen Freizeit angeben. Andererseits tendieren Frauen eher zu emotionaler Labilisierung und Erschöpfung, Selbstzweifel und Resignation, während Männer eher mit Dehumanisierung oder motivationalem Disengagement auf Belastungssituationen reagieren. Allerdings gibt es auch Untersuchungen, in denen sich keine signifikanten Geschlechtsunterschiede herausstellten, sodass auch hier lediglich von Indizien gesprochen werden kann (Byrne, 1999; Schaarschmidt, 2005a). Ungeachtet eventueller methodischer Unstimmigkeiten stellt Burnout sowohl von seiner volkswirtschaftlichen als auch seiner menschlichen Bedeutung ein zentrales Thema der Psychologie, Erziehungswissenschaft und gesundheitsbezogener Fachrichtungen dar, gerade weil in Studien vermehrt ein Ansteigen der Häufigkeit, verbunden mit der Ausweitung der Arbeitsaufgaben von Lehrern und den damit einher gehenden Anforderungen festzustellen ist (Barth, 1997).

Einigkeit besteht heute darin, dass der Verlauf von Burnout einen schleichenden Prozess beschreibt und es sich nicht um einen Zustand handelt, der durch einzelne traumatische Erlebnisse ausgelöst wird. Die Forschungsergebnisse deuten übereinstim- 
mend daraufhin, dass es sich bei Burnout um eine kontinuierliche Variable handelt, die in unterschiedlicher Ausprägung vorliegen kann, wobei die Festlegung eines Cut-offWerts, der das individuelle Eintreten des Erschöpfungssyndroms symbolisiert, problematisch und kaum möglich ist (Barth, 1997). Dies wird unter anderem auch in der Definition von Maslach \& Jackson (1981) deutlich, die emotionale Dauerbelastung als Grund für emotionale Erschöpfung sehen, aus der wiederum durch Bewältigungsversuche Gefühle der Dehumanisierung resultieren. Mit Dehumanisierung ist hierbei eine nicht-mitfühlende und herzlose Verhaltensweise gegenüber Schülern oder Kollegen gemeint, die von Zynismus geprägt ist (Barth, 1997). Erweisen sich auch die weiteren Versuche, die fortwährenden Belastungen zu bewältigen, als nicht erfolgreich, treten Beschwerden auf, zu denen letztendlich noch das Gefühl verminderter Leistungsfähigkeit hinzukommt. Betroffene fühlen sich immer weniger kompetent, die Arbeitsbelastungen bewältigen zu können (Schmitz, 2004). Die Burnout-bedingte Veränderung des Verhaltens von Lehrern und Schüler verstärkt zu kritisieren, den Unterricht in geringerem Maß vorzubereiten und generell die Involviertheit in die Klassenraumaktivitäten zu reduzieren, führt auf Schülerseite zu geringeren Kompetenzgefühlen, geringerer intrinsischer Motivation und weniger Engagement. Letztendlich machen sich die Verhaltensänderungen der Lehrkraft in einer geringeren Tiefe des Lernens, d.h. einer verminderten Durchdringung des Lernstoffes deutlich. Auf lange Sicht führt das distanzierte und unengagierte, von Zynismus geprägte Verhalten der Lehrer zu einer DeIdentifizierung der Schüler mit der Lehr-Lern-Situation, was umgangssprachlich als das Verlieren von Lust am Lernen und der Schule bezeichnet wird (Huberman \& Vandenberghe, 1999).

Vielfach wurde in der Vergangenheit versucht, Burnout prozesshaft in Phasenmodellen abzubilden. Die Einteilung in die Phasen basiert bei den Modellen jedoch nicht auf empirischen Studien, sondern stellen meist intuitive Typisierungen dar, wo auch das Auslassen einer oder mehrerer Phasen oder eine Änderung der Reihenfolge der Phasen möglich ist. Ein populärer Ansatz stammt von Cherniss (1980), der sich auf das transaktionale Stressmodell von Lazarus, auf die Theorie der erlernten Hilflosigkeit von Seligman (1979) und auf die Ausführungen von Kahn et al. (1964) zu Rollenkonflikten und Ambiguität bezieht. Burnout beginnt nach Cherniss mit Berufsstress, der durch das Verhältnis von Anforderungen und Ressourcen bestimmt wird und Stressreaktionen, Spannungsgefühle, Reizbarkeit etc. in der Phase des Stillstands hervorruft (siehe Abb. 15). 


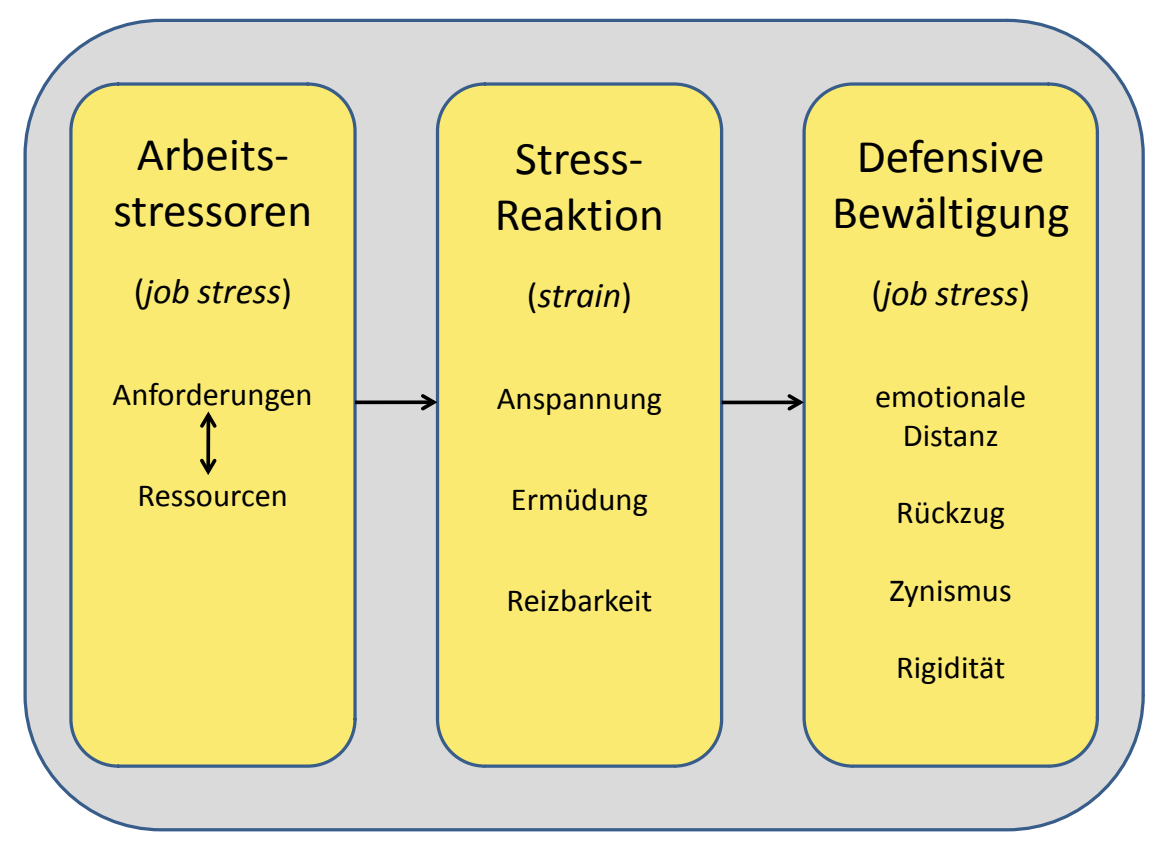

Abb. 15: Transaktionale Burnout-Definition von Cherniss (1980, zitiert nach Enzmann \& Kleiber, 1989, S. 43)

Entscheidend in dieser Phase ist die Art der Bewältigung die ausgewählt wird, um mit den Stressoren umzugehen. Zunächst werden Personen aktive direkte Problemlösungen anstreben. Sind diese Strategien nicht von Erfolg gekrönt, wird versucht durch indirekte aktive Maßnahmen, wie Stress-Management-Techniken ${ }^{9}$ die Spannung zu vermindern. Gelingt dies nicht und fühlen sich Personen gegenüber den Anforderungen der Arbeit hilflos, setzen sie eher defensive Formen der Bewältigung wie Distanzierung, Vermeidung, Herabsetzen der Ansprüche oder das Verantwortlichmachen von anderen ein, um sich selbst durch das Zurückziehen aus der direkten Konfrontation mit dem Stressereignis zu schützen. Burnout wird also nicht als Folge von Stressereignissen gesehen, die z.B. im Lehrerberuf unvermeidbar sind, sondern als Resultat von „unbewältigtem" Stress aufgrund ineffektiver und unangemessener Bewältigung (Barth, 1997; Kramis-Aebischer, 1996). Auch Vertreter der Theorie der conservation of ressources von Antonovsky sehen in unangemessener Bewältigung von als bedrohlich bewerteten Anforderungen den Beginn von Burnout, der sich zunächst in einer Verknappung der Ressourcen äußert. Erweisen sich auch in der Folge aktive und problemorientierte Bewältigungsmaßnahmen als ineffektiv, reagieren Burnout-Gefährdete dazu, aufzugeben, zu resignieren und in Zukunft verstärkt defensive Verhaltensweisen an den Tag zu legen. Endform eines solchen Prozesses stellt nicht selten eine Depression dar (Schmitz, 2004).

\footnotetext{
${ }^{9}$ Umbewertung der Situation oder meditative Entspannungsübungen.
} 
Angelehnt an Cherniss gibt es eine Reihe weiterer prozesshafter Definitionen von Burnout, die zwischen zwei und mehr als zehn Phasen aufweisen und als mehr oder weniger verbindlich in ihrer Abfolge gesehen werden können. Burisch (2006) unterscheidet beispielsweise sieben verschiedene Stufen mit jeweils zugeordneten Symptomen. Je mehr Symptome auftreten bzw. je intensiver diese sind, desto fortgeschrittener ist der Verlauf des Burnout-Prozesses. Eine festgelegte Abfolge der Einzelphasen ist nicht zwingend vorgesehen, sodass diese sogar parallel zueinander auftreten können (siehe Abb. 16).
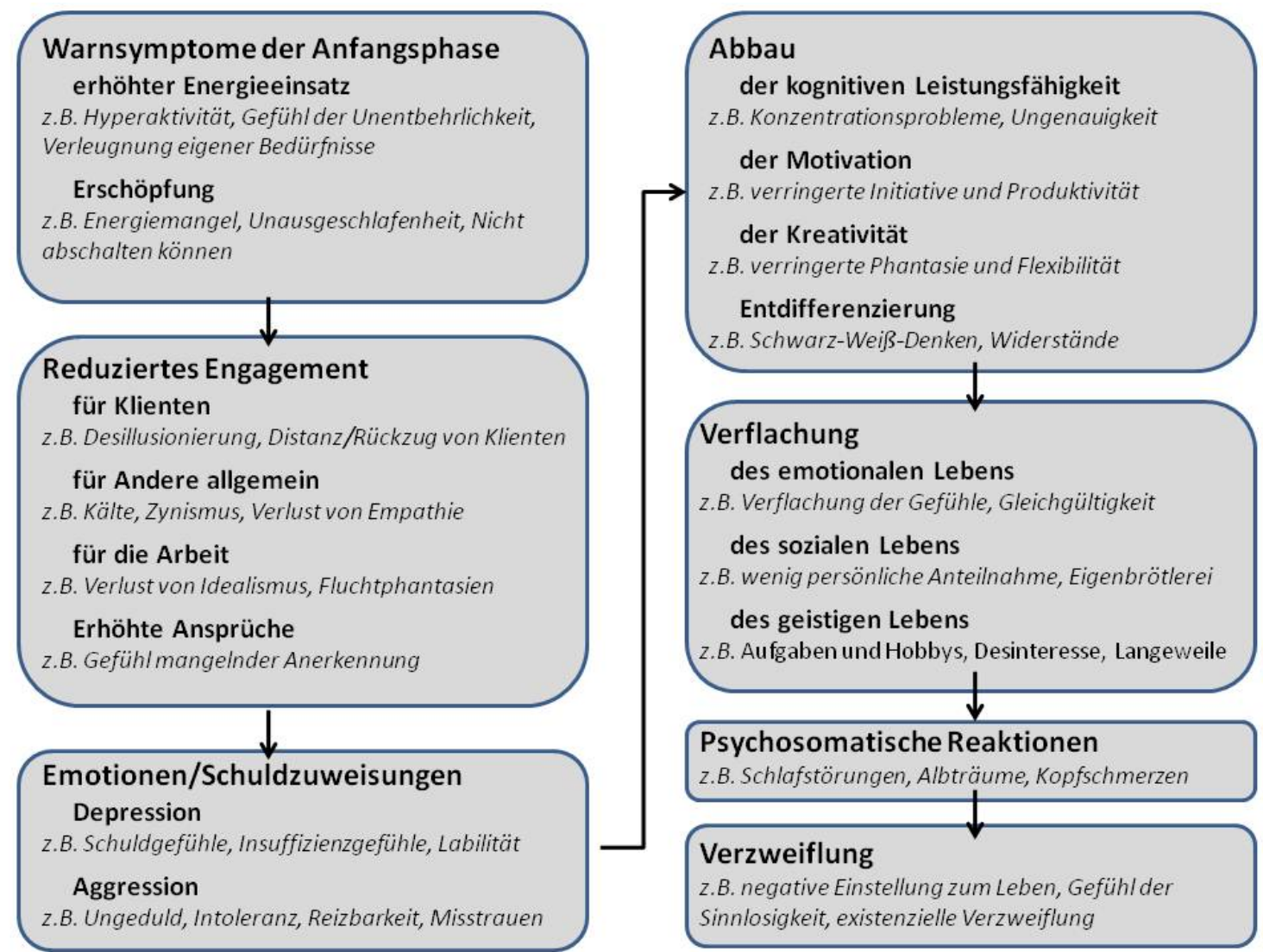

Abb. 16: Burnout-Phasen nach Burisch (2006) (zit. n. Sosnowsky, 2007, S. 128).

Bei den Voraussetzungen für Burnout bzw. den Indikatoren aus dem Arbeitsumfeld stellen subjektiv erlebte Beanspruchungen bedeutsamere Einflussgrößen dar, als die realen objektiv messbaren Belastungen. Konkret werden in Lehrerstichproben vor allem die Klassengröße, unklare Informationswege, mangelnde Anerkennung und fehlende Erfolgsrückmeldungen sowie Rollenkonflikte und mangelnde Kollegialität im Zusammenhang mit der Entstehung von Burnout genannt (Schmitz, 2004). Barth (1997) nennt weiterhin Isolation, Autonomiemangel, öffentliche Missverständnisse über die Natur der Arbeit, verwaltungsmäßige Unstimmigkeiten sowie exzessive Anforderungen an die Produktivität, bei ungenügender Vorbereitung auf die Arbeit, als den Sozialberu- 
fen gemeinsame Bedingungen, die im Zusammenhang mit dem Ausbrennen zu sehen sind. Weiter gefasst beruht das Auftreten eines Phänomens wie Burnout auf der Individualisierung, Entfremdung, Anonymität, Unpersönlich- und Beziehungslosigkeit unserer modernen Gesellschaft, sodass Gemeinsinn, sozialer Zusammenhalt und kollegiale Unterstützung als Puffer in schwierigen Situationen und Lebensphasen in geringerem Maß zur Verfügung stehen. Im engeren Umfeld sind soziale Unterstützungssysteme, sowohl privat als auch beruflich in Form technischer und emotionaler Unterstützung von großer Bedeutung. Soziale Unterstützung kann vor dem Ausbrennen schützen bzw. Symptome lindern und Wege zurück in ein glücklicheres Berufsleben aufzeigen (Barth, 1997).

In einer Reihe von Studien setzte man sich mit den Persönlichkeitseigenschaften auseinander, die das Auftreten von Burnout begünstigen. Mangelnde Widerstandsfähigkeit, geringe Selbstwertschätzung, Selbstvertrauen, Kompetenzgefühle, Ängstlichkeit und Neurotizismus stellen mit Burnout korrelierende Variablen dar, was jedoch keine Kausalität bedeuten muss, da meist reziproke Beziehungen vorliegen (Schmitz, 2004). Auch die Art der Attribuierung bzw. die Ausprägung der Kontrollüberzeugungen stehen in Verbindung mit der Entstehung von Burnout. Während externale Kontrollüberzeugungen zu einer höheren Anfälligkeit für emotionale Erschöpfung beitragen, wirkt sich die Überzeugung, den Verlauf der Dinge selbst bestimmen zu können, wie dies bei ausgeprägter Internalität der Fall ist, protektiv aus (Byrne, 1999). Als Bewältigungsmaßnahmen, die mit dem Auftreten von Burnout in Verbindung gebracht werden sind Flucht, Abwehr und Vermeidung zu nennen, protektiv wirken problembezogene Strategien (Schmitz, 2004).

Frühe Ergebnisse der Forschung zu Burnout in den 1970er Jahren und selbst die Untersuchungen von Barth in den 1990er Jahren sehen in beruflichem Idealismus die Grundlage des Ausbrennens. Nur wer einmal entflammt war, könne ausbrennen. „Es sind die allerbesten Leute bestimmter Berufsgruppen, deren Arbeitseffizienz nachlässt" (Pines, Aronson \& Kafry (1985, zitiert nach Barth, 1997, S. 17). Cherniss wandte sich bereits 1982 gegen die Auffassung, dass Ausbrennen eine Reaktion auf Überengagement ist und konnte dies anhand einer Studie mit Schwestern eines katholischen Ordens zeigen, die sieben Tage in der Woche ohne jegliche Gratifikation geistig Behinderte pflegten. Er stellte fest, dass die Schwestern trotz höchstem Engagement, Idealismus und Hingabe nicht ausbrannten, da sie Rückhalt und Bestätigung im Glauben zu Gott bzw. der Ordensgemeinschaft fanden (Kramis-Aebischer, 1996; Sosnowsky, 2007). Nach dem heutigen Forschungsstand geht man davon aus, dass nicht die Begeisterung für den Beruf, sondern vielmehr das Maß an Diskrepanz zwischen den Erwartungen und 
der Realität die Burnout-begünstigende Variable darstellt. Überhöhte oder unklare berufliche Zielsetzungen führen dazu, dass die empfundene Diskrepanz zwischen Anspruch und Wirklichkeit nicht kompensiert werden kann und zu einer geringerer Motivation, Resignation oder einem Verlieren an Interesse am Beruf führt (Schmitz, 2004). In dieselbe Richtung deuten auch die Ergebnisse der Langzeitstudie von Rauin (2007; 2010), der feststellte, dass diejenigen, die besonders über Belastungen im Beruf klagen und sich den beruflichen Belastungen nicht gewachsen fühlen, nie „gebrannt" haben, und schon im Studium weniger motiviert und stärker überfordert waren als die idealistischeren Lehramtsstudenten.

Schwierig ist es, eine klare Symptomatik für Burnout zu definieren, da es unzählige mit Burnout in Verbindung stehende Symptome bzw. Folgen gibt, wobei die Unterscheidung bzw. die Kausalität oft unklar ist. Grundsätzlich unterscheidet man aber zwischen psychisch-mentalen Merkmalen (chronische Ermüdung, depressive Stimmung, Hilfund Hoffnungslosigkeit, Angst, Nutzlosigkeit, Erschöpfung der emotionalen Ressourcen etc.), körperlichen Merkmalen (Kopf- und Rückenschmerzen, Schlaflosigkeit, Übelkeit, Störungen der Sensomotorik, Hypertonie, Magen-Darm-Probleme etc.), Verhaltensmerkmalen (unkonzentriertes Verhalten, Rückzug von sozialen Kontakten, gesteigerte Unruhe etc.) und Veränderungen der Einstellung (Gefühllosigkeit, Gleichgültigkeit, Langeweile, Zynismus, Verlust an Eifer, Interesse und Hingabe, geringe Arbeitsmoral, Absentismus etc.) (Schmitz, 2004). Es muss jedoch deutlich gesagt werden, dass diese Aufzählung nicht so zu verstehen ist, dass alle diese Symptome bei einem Burnout auftreten, sondern höchst individuelle Ausprägungen und Kombinationen denkbar sind, was die Diagnose und die sich anschließende Behandlung zu einer schwierigen Aufgabe für Ärzte und Therapeuten macht. Des Weiteren werden oft Burnout und depressive Tendenzen in einem Atemzug genannt. Die Unterschiede zwischen einer „echten“ Depression und Burnout bestehen in der Bereichs- und Situationsspezifität. Während von Burnout betroffene Personen sich außerhalb der Arbeit meist relativ wohl fühlen umfasst bei depressiven Personen die negative Stimmung oft alle Bereiche des Lebens (Schmitz, 2004).

\subsubsection{Innere Kündigung}

Der Begriff der inneren Kündigung wurde zum ersten Mal im Jahr 1982 verwandt und Selbst-Pensionierung als synonymer Begriff dazu angegeben (Jehle \& Schmitz, 2007). Ursprünglich wurde das Phänomen zunächst nur für Beamte aus staatlichen Verwaltungsorganisationen beschrieben, empirische Daten belegen jedoch mittlerweile, dass innere Kündigung in allen Wirtschaftsbereichen auftreten kann und mit einer generellen Arbeitsunzufriedenheit und deren Bedingungen einhergeht. Im Volksmund ist solch 
ein Verhalten auch als Dienst nach Vorschrift unter einem relativ neutralen Ausdruck geläufig. Weitere synonym verwendete Begriffe sind innere Verweigerung, innere $A b$ wanderung, unausgesprochene Kündigung oder Selbstbeurlaubung (Brinkmann \& Stapf, 2005). Innere Kündigung stellt im Gegensatz zu einer offenen Kündigung eine nicht sanktionierbare Verweigerung von Leistungen dar, zu denen man laut Arbeitsvertrag nicht dezidiert verpflichtet ist (Schmitz, Jehle \& Gayler, 2004). Innere Kündigung ist dabei ein „zeitlich relativ stabiles Verhaltensmuster bzw. eine zeitlich andauernde Verhaltensintention, die mit einer ablehnenden später auch depressiv-resignativen Grundhaltung gegenüber der Arbeitssituation in Verbindung steht" (Brinkmann \& Stapf, 2005, S. 17 f.).

Nicht nur in der Schule, auch in der freien Wirtschaft sind die Schäden durch innere Kündigung beachtlich, jedoch nicht ohne Weiteres abzuschätzen. Das Zitat des ehemaligen amerikanischen Präsidenten Abraham Lincoln (zitiert nach Brinkmann \& Stapf, 2005, S.31): „Ein Mann, der nicht mehr leistet als das, wofür er bezahlt wird, leistet so wenig, dass er nicht wert ist, was er bekommt“, macht deutlich, dass eine exakte Rechnung von Aufwand und Ertrag und eine darauf folgende persönliche Aufwandsminimierung und Nutzenmaximierung, eine für Organisationen und Gesellschaften nicht anzustrebende Einstellung darstellt. Bevor der Begriff der inneren Kündigung aufkam, war die reale Fluktuation das einzige Anzeichen für ein gutes oder schlechtes betriebliches Klima (Brinkmann \& Stapf, 2005).

Begründet ist diese Form des Zurücknehmens von Engagement und Eigeninitiative, das im Rahmen einer inneren Kündigung erfolgt, meist in einem Bruch des psychologischen Arbeitsvertrages, der eine psychische Repräsentation von gegenseitigen Verpflichtungen, Erwartungen, Hoffnungen und Wünschen der Vertragspartner darstellt, die nicht formal-vertraglich, sondern nur informell festgelegt sind. Konzeptionell basiert dieser Vertrag auf der Einsicht, dass die Motivation von Mitarbeitern und damit die Höhe ihrer Leistung durch Anreiz und Belohnung seitens des Arbeitgebers bzw. des Vorgesetzten aufrecht erhalten werden muss. Dieses Geben und Nehmen stellt einen komplizierten Austauschprozess dar, der aus vielfältigen gegenseitigen Erfahrungen besteht (Brinkmann \& Stapf, 2005). Psychologische Verträge existieren auch zwischen Lehrern und Schulleitern bzw. der Bildungsverwaltung, Lehrern und Schülern sowie Lehrern und Eltern. Von Schulleitern erwarten Lehrer, dass diese sie bei wichtigen Entscheidungen mit einbeziehen, sich ihnen gegenüber objektiv verhalten, vor Über- und Unterforderung schützen, Handlungs- und Entscheidungsspielraum gewähren sowie die Arbeit und das Engagement lobend anerkennen. Insgesamt erhoffen sich Lehrer einen kooperativen Führungsstil mit fachlicher Einbindung und der Gewährung von 
individuellen Frei- und Gestaltungsäumen. Schulleiter hingegen haben neben der Erwartung der Ein- und Unterordnung des Lehrers in vorhandene Strukturen und unbedingter Loyalität sogenannte Extra-Rollenverhaltens-Erwartungen an Lehrer, die meist zusätzliche Verpflichtungen an die Lehrer umfassen, sodass diese oft wenig kompatibel mit den Wünschen und Vorstellungen der Lehrer sind und schnell die subjektive Wahrnehmung eines Vertragsbruchs aufkommt (Schmitz, Jehle \& Gayler, 2004). Ein solcher Bruch ist bedingt durch die reziproke Eigenschaft sozialer Interaktion: Wie du mir, so ich dir! Verstöße gegen das Reziprozitätsprinzip ziehen in der Regel Ungerechtigkeitserlebnisse nach sich und der sich anschließenden Antwort des enttäuschten Vertragspartners (Brinkmann \& Stapf, 2005).

Der Bruch eines solchen Vertrages wird meist nicht durch ein einmaliges Erlebnis ausgelöst, sondern verläuft schrittweise. Stellt man zwischen dem derzeitigen Zustand und dem Sollzustand eine Diskrepanz fest, wird eine Handlungstendenz ausgelöst, diese Diskrepanz zu minimieren. Nach einer Zeit wird die Situation, konform des transaktionalen Stressmodells von Lazarus, einer Neubewertung unterzogen. Besteht aufgrund dieser Neubewertung Hoffnung auf Erfolg, werden erneut Versuche initiiert, die Diskrepanzen zwischen Soll- und Ist-Wert auszugleichen. Sind die Anstrengungen zur Reduzierung der Diskrepanz offensichtlich effektlos und es besteht keine Hoffnung auf Erfolg, werden die Soll-Vorstellungen zum Großteil aufgegeben und das Engagement drastisch reduziert, oft erfolgt auch ein Rückzug ins Privatleben (Schmitz, 2004). Dieser Rückzug stellt einen Schutzmechanismus da, durch den sich die Betroffenen vor drohenden Hilflosigkeits- und Ohnmachtsgefühlen schützen wollen (Brinkmann \& Stapf, 2005). Inwieweit die Nicht-Erfüllung, der individuell durch den Lehrer formulierten Soll-Vorstellungen durch widrige Bedingungen oder aber überhöhte Erwartungen bedingt ist, ist von Fall zu Fall verschieden (Jehle \& Schmitz, 2007). Die Lehrkraft als der eine Vertragspartner sieht es als eine Art Verpflichtung der anderen Partei an, auf eine erbrachte Leistung mit einer gleichwertigen Gegenleistung oder zumindest mit Anerkennung zu reagieren (Jehle \& Schmitz, 2007). Die innere Kündigung letztendlich stellt eine kognitiv-emotionale und behaviorale Reaktion auf die Störung des Gleichgewichts von Aufwand und Ertrag dar. Lehrer sehen in dieser Unausgewogenheit der Kosten und Nutzen ein Überschreiten des Vertragspartners über ein subjektives grenzwertiges Maß hinaus, sodass der Gegenstand des Vertrages als unerfüllt angesehen wird und man sich dementsprechend diesem auch nicht mehr verpflichtet fühlen muss. Die gestörte Reziprozität kann man nach Schwere und Dauer differenzieren. Leichte Störungen führen meist nicht zu einer Aufkündigung des Vertrages, während bei stärkeren Störungen bereits Gespräche mit dem Vertragspartner oder weitere Hilfsmaßnahmen 
notwendig sind, um im Sinne des abgeschlossenen Vertrages weiterarbeiten zu können. Können sich die Vertragspartner nicht einigen oder ist generell keine kooperative Lösung möglich, kann dies dazu führen, dass durch die Reduktion des Engagements versucht wird, das Gleichgewicht im psychologischen Vertrag wieder herzustellen. Liegen günstige finanzielle Rahmenbedingungen vor, kann auch eine formale Kündigung erfolgen (Schmitz, 2004). Angst vor einer Veränderung, finanzielle Einbußen sowie die Ungewissheit darüber, ob die fachliche Eignung oder das Alter die Suche nach einer neuen Position überhaupt erlauben, führt in Kombination mit der relativen Alternativlosigkeit des Lehrerberufs zu der pragmatischen und nutzenorientierten Entscheidung, im Beruf zu verbleiben, denn bereits in Wallensteins Tod äußert Schiller die Maxime: "Nur vom Nutzen wird die Welt regiert" (Schiller, 1799, zitiert nach Brinkmann \& Stapf, 2005. S. 27).

Ebenso wie zwischen Lehrern und Schulleitern bestehen auch zwischen Lehrern und Schülern psychologische Verträge, die Lehrer meist als belastet sehen, da Schüler sich nicht so verhalten, wie es „die Lehrer wegen ihrer Investition an persönlichen Kosten und Mühen erwarten (Zitat einer Lehrerin, die wegen Burnout in klinischer Behandlung war: ,Ich habe mir so viel Mühe gegeben und wollte doch nur ihr Bestes, aber sie waren so böse zu mir" " (Schmitz Jehle \& Gayler, 2004, S. 71). In Abbildung 17 ist ein Strukturmodell zur inneren Kündigung bei Lehrern dargestellt, das auf der einen Seite Commitment-Faktoren, die letztendlich gemeinsam die berufliche Identität, das Anspruchsdenken und die Verwirklichungstendenzen in der pädagogischen Arbeit umfassen und auf der anderen Seite die relevanten Mangelfaktoren einschließen.

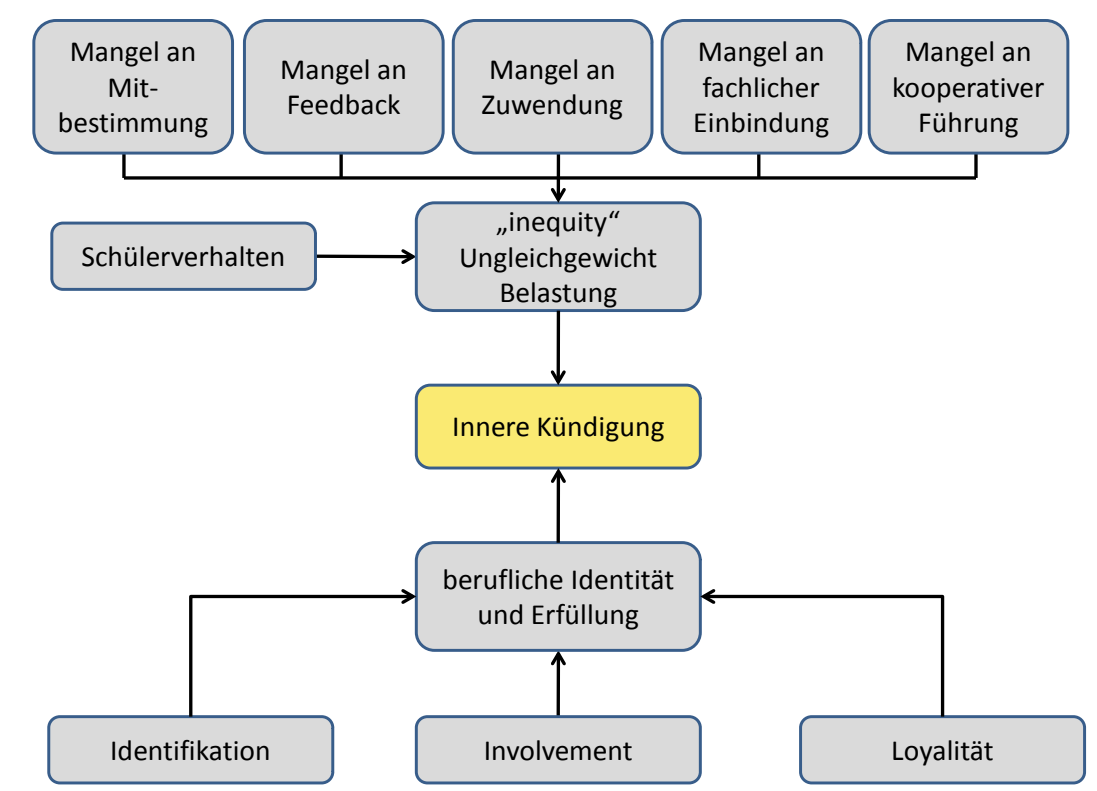

Abb. 17: Strukturmodell der inneren Kündigung bei Lehrern (Schmitz et al., 2004, S. 77) 
Eine eindeutige Diagnose einer inneren Kündigung stellt sich als schwierig heraus, da sie ein sehr diffuses und vielschichtiges Gebilde darstellt und sich im Rahmen eines langsamen, meist unmerklichen und mehrstufigen Prozess entwickelt (Brinkmann \& Stapf, 2005). Bei den Anzeichen bzw. Indikatoren von innerer Kündigung kann man unterscheiden zwischen Verhaltensweisen, die im Unterricht bzw. der Arbeit in der Schule an den Tag gelegt werden und objektiven Kriterien. Beim Verhalten fällt Mitläufertum und eine ausgeprägte Zustimmungstendenz auf, die begründet ist in verlorengegangenem Interesse an Auseinandersetzungen im Kollegium. Innerlich Gekündigte befinden sich bei Entscheidungen stets bei der Mehrheit und haben sich zu angepassten Konformisten entwickelt. Sie sind im Umgang sehr angenehm und zum Teil überfreundlich (Brinkmann \& Stapf, 2005). Weiterhin werden Eingriffe in den eigenen Zuständigkeitsbereich kommentarlos hingenommen, Kritik und Vorschläge werden nicht mehr eingebracht und die eigenen Kompetenzen nicht ausgeschöpft (Schmitz, Jehle \& Gayler, 2004). Leistungen über die reguläre Unterrichtszeit hinweg werden vermieden und zusätzliche Aufgaben werden nicht übernommen (Jehle \& Schmitz, 2007). Innerlich Gekündigte beachten jedoch, dass sie sich mit ihrem Verhalten stets unterhalb der Grenze der Auffälligkeit bewegen. Auffällig hingegen sind die objektiven Indikatoren wie die hohe Absentismusrate, das gering ausgeprägte Fortbildungsengagement sowie das mangelnde Engagement bei der Gestaltung von Schulleben oder der Übernahme von Sonderaufgaben oder Vertretungsstunden. Dieses Verhalten nehmen Kollegen und Schulleiter als unkollegial wahr, was nicht selten zu Folgekonflikten, Sanktionen und Ärgernissen führt, die eine weitere Belastung des psychologischen Vertragsverhältnisses darstellt (Schmitz, Jehle \& Gayler, 2004).

In einer Interviewstudie von Kastrup und Neumann (2008) mit Sportlehrkräften stellte sich heraus, dass innere Kündigung sich durch einen Rückzug in unterschiedliche Bereiche manifestiert. Neben der Ablehnung von Lehrplanrevisionen, die sich in der Abwertung neuer Methoden, Konzeptionen und Inhalte zeigt, beteiligen sich innerlich Gekündigte nicht mehr an der Fachschaftsarbeit und auch die Umsetzung von schulinternen Curricula erfolgt nicht bzw. nur teilweise. Zusätzliche Aufgaben wie das Führen einer Arbeitsgemeinschaft mit dem Schwerpunkt Sport oder die Organisation bzw. Betreuung von Schülern auf Sportwettkämpfen werden nicht übernommen und pädagogische Ansprüche bezogen auf Inhalte und soziale Interaktion drastisch reduziert.

Wie zuvor bereits angedeutet ist eine strukturierte phasenhafte Beschreibung des Verlaufs einer inneren Kündigung schwer zu erstellen, da die Ursache, Erscheinung und Entwicklung höchst unterschiedlich sein kann und zwischen vollem Engagement und innerer Kündigung viele Zwischenstufen existieren (Schmitz; Jehle \& Gayler, 2004). Die 
innere Kündigung vollzieht sich auf leisen Sohlen, die Entwicklung ist meist nicht merklich und stellt das Endresultat eines langwierigen Prozesses dar. Grundsätzlich besteht bei innerlich Gekündigten ein Missverhältnis zwischen den an der Arbeit gebotenen Befriedigungsmöglichkeiten und den eigenen Bedürfnissen. Ist man nicht in der Lage, dieses Missverhältnis zu bewältigen, entsteht Stress, dem innerlich Gekündigte aufgrund von Erfahrungen über die scheinbare Beziehungslosigkeit zwischen eigenen Anstrengungen und der Arbeitssituation durch Absenkung ihrer Ansprüche und damit einhergehend einer Reduzierung der kognitiven Dissonanzen entgegentreten. Die sogenannte erlernte Hilflosigkeit etabliert sich im Denken. Aufgrund wiederholter fehlender Zielerreichung geht man nun davon aus, auch zukünftig erfolglos zu sein. Betroffene Personen reagieren tendenziell mit Inaktivität und sich bietende Chancen auf Veränderung werden nicht als solche wahrgenommen. Sieht man diese Unfähigkeit etwas zu verändern in der eigenen Persönlichkeit, kommt es zu der evolutionär sinnvollen Reaktion, keine weitere Energie sinnlos zu verbrauchen und Apathie bzw. Resignation setzt ein. Werden hingegen die Situationseigenschaften als verantwortlich für das Nicht-Greifen von Interventionsmaßnahmen gesehen, stellt ein Entziehen aus der Situation die folgerichtige Reaktion dar (Brinkmann \& Stapf, 2005).

Bei der inneren Kündigung kann man zusätzlich zwischen einer aktiven und einer passiven Form der inneren Kündigung unterscheiden. Bei der aktiven Form werden Probleme systematisch ausgeblendet und der Job zur eigenen Zufriedenheit umgestaltet bzw. gerechter gemacht, während bei der passiven Form reaktive emotionale Erschöpfung und Hilflosigkeit kennzeichnend sind (Schmitz, Jehle \& Gayler , 2004). Hierbei findet ein bewusster Rückzug aus dem Job statt, der auch im Vordergrund steht. Aus Erfahrungen wissen die betroffenen Personen, dass eine Leistungszurückhaltung keine negativen Konsequenzen hat und sich sogar positiv auswirken kann. Paradoxerweise nehmen manche Vorgesetzte das Verhalten von innerlich gekündigten Mitarbeitern als positiv wahr, da endlich das ewige Nörgeln und Nein-Sagen aufhört (Brinkmann \& Stapf, 2005). Eine weitere Form der inneren Kündigung stellt die informelle HalbPensionierung dar, bei der der Rückzug ausschließlich auf die Schuladministration bezogen ist, mit der keine weitere Zusammenarbeit erfolgt. Im Bezug auf die Lehrtätigkeit und den Umgang mit Schülern gehen diese Personen ihrem Beruf mit unreduziertem Engagement weiter nach. Als letzte Gruppe sollen auch diejenigen nicht verschweigen werden, die das Schonungsverhalten lediglich aus niederen Motiven an den Tag legen, um das eigene Wohlergehen zu steigern (Jehle \& Schmitz, 2007). Innere Kündigung kann, muss aber nicht das Ende der beruflichen Entwicklung darstellen, denn ebenso kann sie auch ein Zwischenschritt auf dem Weg zu einer Neuorientierung 
sein. Als Ausweg zwischen Ausbrennen und Hilflosigkeit kann sie eine geglückte Anpassung einer Zwangslage darstellen, die die Basis für das Schöpfen von neuem Mut, das Entwickeln neuer Ideen und die Veränderungen von Einstellungen und Arbeitsabläufen liefert (Brinkmann \& Stapf, 2005).

Innere Kündigung führt in vielen Fällen jedoch nicht zu der erwarteten Entspannung und Ausgeglichenheit, sondern hat zum Teil erhebliche Folgen. Die Innere Kündigung eines Lehrers und das damit verbundene Verhalten sind für Schulleiter, Schüler und Kollegen deutlich und erfahrbar. Fehlendes Engagement der Lehrkraft wird von Schülern schnell bemerkt, hat negativen Einfluss auf deren Lernmotivation und folglich der Leistung, stört kollegiale Beziehungen und problematisiert die Arbeitsatmosphäre. In der Interviewstudie von Kastrup \& Neumann (2008) wird gerade in den Kollegien, in denen der Anteil sich innerlich Zurückgezogener besonders hoch ist, das soziale Klima als schlecht beschrieben (Kastrup \& Neumann, 2008). Doch nicht nur im sozialen Bereich sind Folgen der inneren Kündigung spürbar, sondern es ist auch eine überdurchschnittliche Anhäufung psychosomatischer Beschwerden festzustellen. In einer Untersuchung zeigte sich, dass bei fast allen innerlich Gekündigten im Vergleich zu den nicht innerlich Gekündigten signifikant höhere Werte psychosomatischer Beschwerden vorzufinden waren (Jehle \& Schmitz, 2007). Mit innerer Kündigung werden in der Forschungsliteratur immer wieder Symptome wie Niedergeschlagenheit, Konzentrationsprobleme, Magen- und Verdauungsprobleme, zunehmend schlechter Gesundheitszustand, Schlafstörungen und andere psychosomatische Störungen in Verbindung gebracht (Jehle \& Schmitz. 2007). Die innere Abkehr von der Arbeit kann zu psychischen Problemen führen, die bis hin zu Depressionen reichen, deren Entwicklung neben den Parametern des Arbeitsumfelds auch durch Persönlichkeitseigenschaften und ökonomischen und sozialen Lebensbedingungen beeinflusst wird (Brinkmann \& Stapf, 2005).

Das Datenmaterial zur Verbreitung von innerer Kündigung ist sehr dürftig. Schätzungen gehen davon aus, dass $40 \%$ der arbeitenden Bevölkerung in den alten und weit über $50 \%$ in den neuen Bundesländern betroffen sind. In Großbetrieben in der Schweiz rechnet man mit ca. 20\%, bei Banken zwischen 32 und 35\% und bei Behörden mit immerhin 42\% (Brinkmann \& Stapf, 2005). In Studien zu innerer Kündigung bei Lehrern, gab immerhin knapp die Hälfte an, zumindest ab und an schon einmal innerlich gekündigt zu haben bzw. darüber nachgedacht zu haben (Schmitz, Jehle \& Gayler, 2004).

Im Vergleich stellen Burnout und innere Kündigung zwei unterschiedliche Reaktionsweisen bzw. Belastungsfolgen dar, die ein Resultat der Diskrepanzen zwischen den Erwartungen und der alltäglichen Realität offenbaren. Während man bei einsetzendem Burnout weiterhin innerlich an den Soll-Vorstellungen und Ansprüchen an sich selbst 
festhält, werden diese Soll-Vorstellungen im Sinne der Kündigung des psychologischen Arbeitsvertrages bei der inneren Kündigung über Bord geworfen. Personen, die ihre innere Kündigung vollzogen haben, ziehen sich aus der Verantwortung am Arbeitsplatz zurück und verhindern dadurch Zusammenbrüche und Sinnkrisen, die bis zur Selbstaufgabe führen können. Wie bei Burnout ist es auch bei der inneren Kündigung schwierig, Aussagen über Antezedenz- und Moderatorvariablen zu machen, von Gesetzmäßigkeiten des Ablaufs bzw. der Prozesshaftigkeit ganz zu schweigen. Zwischen der inneren Kündigung und der Gratifikationskrise nach Siegrist werden immer wieder Bezüge hergestellt. Auch die caregiver-recepient"-Beziehung, die im Rahmen der Equity-Theorie aussagt, dass Menschen auf ein ausgeglichenes Verhältnis des Gebens und Nehmens achten, scheint ein verwandtes Konstrukt zu sein. Ist dieses Verhältnis unausgeglichen und gibt man mehr als zu nehmen, ist oft eine Verminderung der emotionalen Ressourcen die Folge (Schmitz, 2004). Während unter Burnout leidende Lehrer nicht das leisten können was sie gerne möchten, sind innerlich Gekündigte meist zu mehr Leistung in der Lage, verweigern diese aber. Ein weiterer Unterschied stellt die Instanz dar, die hauptsächlich für die Entstehung der beiden Folgeerscheinungen verantwortlich ist. Während die innere Kündigung sich meist auf Probleme mit einer höheren Hierarchieebene zurückführen lässt, wie die Schulleitung oder die Schulverwaltung, ist Burnout meist durch die Beziehung zu Rezipienten wie Schüler, Patienten oder Klienten bedingt (Schmitz, Jehle \& Gayler, 2004). Gemein ist den beiden Beanspruchungsfolgen aber, dass sowohl die Innere Kündigung als auch Burnout oft mit psychosomatischen Beschwerden einhergehen und bei beiden die Unterrichtsqualität negativ beeinflusst wird. 


\section{Ableitung der Fragestellungen und Hypothesenbildung}

In Kapitel 5 dieser Arbeit soll, basierend auf dem voranstehenden Theorieteil, eine Ableitung der im Ergebnisteil bearbeiteten Fragestellungen (siehe Kap. 7) erfolgen. Weiterhin soll jeweils ein hypothetischer Erwartungshorizont angeführt werden, in dem die grundlegenden Erwartungen an den jeweiligen Fragenkomplex formuliert werden.

Wie im Laufe der theoretischen Aufarbeitung des Berufsfeldes von Sportlehrkräften und in der Darstellung der Belastungs-Beanspruchungsreaktion im Rahmen der Bewältigung deutlich wurde, stellt evasives Bewältigungsverhalten eine sowohl die psychophysische Gesundheit der Lehrkräfte als auch die Qualität von Bildung und Erziehung gefährdende Bewältigungsstrategie dar. Evasives Unterrichten, in diesem speziellen Fall bezogen auf das Verhalten von Lehrkräften im Sportunterricht, fällt in den Bereich der psychologisch orientierten Schulforschung und ist bis auf den Ansatz von Kolb und Wolters aus dem Jahr 2000 bisher unbeachtet geblieben. Eine grundlegende wissenschaftliche Aufarbeitung, trotz der unbestrittenen Bedeutsamkeit für die Qualität von Unterricht und die psychische und physische Gesundheit von Lehrkräften, blieb bisher aus.

Grundlage für die Beantwortung aller im Anschluss formulierten empirischen Fragestellungen, die sich um das evasive Bewältigen drehen, setzt das Vorhandensein eines geeigneten Messinstruments voraus, das, basierend auf den theoretischen Überlegungen zum evasiven Unterrichten, das ausweichende Verhalten in seinen verschiedenen Facetten (siehe Kap. 4.9.4) erfassen kann. Da die zum Zeitpunkt der Untersuchung zur Verfügung stehenden Instrumente, sowohl in Bezug auf die Erfassung des relativ breit gefächerten evasiven Verhaltens als auch bezogen auf die spezielle Situation in der Schule ungeeignet erschienen, war es notwendig, ein neues Instrument zu erstellen, zu erproben und einzusetzen (siehe Kap. 6), um auf die in diesem Kapitel abgeleiteten Fragestellungen geeignete Antworten geben zu können (siehe Kap. 7).

Neben der Erstellung eines Instrumentes zur Messung von evasiven Verhaltenstendenzen soll zudem das evasive Bewältigungsverhalten anhand einer breiten Datenbasis genauer beschrieben werden, hierzu wurden zusätzlich eine Vielzahl von persönlichen und berufsbiographischen Daten abgefragt und psychologischsozialwissenschaftliche Instrumente eingesetzt. Die Zusammenhänge zwischen dem erhobenen evasiven Verhaltenspotenzial und persönlichen und berufsbiographischen Daten soll in insgesamt drei Untersuchungskomplexen Aufschluss über diese spezielle Form der Bewältigung liefern sowie erste Rückschlüsse auf deren Auswirkungen 
ermöglichen, einerseits bezogen auf die Erfüllung des schulischen Erziehungs- und Bildungsauftrages und andererseits in Bezug auf die Wahrung der individuellen Gesundheit der Sportlehrer. Während im ersten Untersuchungskomplex vor allem persönliche und berufsbiographische Daten herangezogen werden, steht im zweiten Fragenkomplex die unterrichtliche Charakteristik und im dritten Fragenkomplex das Verhalten und Erleben im bzw. von Sportunterricht im Mittelpunkt des Forschungsinteresses.

\subsection{Untersuchungskomplex 1: Persönliche und berufsbiogra- phische Merkmale - „Wer nutzt evasive Strategien im Un- terricht?"}

Im ersten Untersuchungskomplex werden verschiedene personelle Faktoren und Ressourcen auf Zusammenhänge mit der Ausprägung der evasiven Verhaltenstendenzen im Sportunterricht überprüft. Bezogen auf die Frage „Wer nutzt evasive Strategien im Unterricht?" können persönliche Daten und berufsbezogene Angaben Hinweise liefern, in welchen Gruppen bzw. mit welchen Eigenschaften evasives Verhalten korrelative Bezüge aufweist. Durch die Erkenntnisse dieses Untersuchungskomplexes ist es möglich genauer aufzuschlüsseln, mit welchen Personenmerkmalen evasive Verhaltenstendenzen zusammenhängen, was bei der Auswahl von Personal aber auch bei der Personalentwicklung hilfreich sein kann.

\section{Folgende Teilfragen werden im Rahmen dieses ersten Komplexes bearbeitet:}

1. Gibt es Zusammenhänge zwischen der Ausprägung von evasivem Verhalten und dem Alter bzw. der Berufserfahrung?

- Hypothese 1: Mit zunehmendem Alter bzw. mit zunehmender Berufserfahrung sinken die Ansprüche an das eigene Unterrichten und es wird verstärkt zu ausweichenden Handlungen tendiert.

- Hypothese 2: Aufgrund von mangelnder Erfahrung und fehlender Routine neigen vor allem junge Lehrer mit geringer Berufserfahrung dazu, Konflikten im Sportunterricht aus dem Weg zu gehen.

- Hypothese 3: Das evasive Potenzial stellt eine stabile Verhaltensdisposition dar und weist keinerlei Zusammenhänge zu Alter oder Berufserfahrung auf.

2. Bestehen im Geschlechtervergleich Unterschiede in der Ausprägung von evasivem Verhalten? 
- Hypothese 1: Sportlehrer verhalten sich aufgrund ihres im Vergleich stärker ausgeprägten zynischen und desengagierten Reaktionsmusters auf Belastungen (siehe Kap. 3.3.3) signifikant evasiver als ihre Fachkolleginnen.

- Hypothese 2: Sportlehrerinnen verhalten sich deutlich evasiver als ihre Fachkollegen, da sie aufgrund von emotionaler Labilisierung und Resignation weniger problemorientiert und verstärkt situativ belastungsreduzierende Handlungsweisen an den Tag legen.

- Hypothese 3: Es bestehen keine statistisch überzufälligen Unterschiede zwischen den Geschlechtern in der Ausprägung des evasiven Verhaltenspotenzials.

3. Beeinflusst die Studienwahl (extrinsisch versus intrinsisch) die Ausprägung der evasiv-invasiv Polarität?

- Hypothese 1: Sportlehrkräfte, die aufgrund von extrinsischen Motiven den Beruf ergriffen haben, zeigen verstärkt evasive Tendenzen, da sie nicht bereit bzw. nicht dazu in der Lage sind, Konflikte in ihrem Unterricht als Entwicklungschance anzunehmen und zu nutzen, da hedonistische Überzeugungen das Berufsbild prägen.

- Hypothese 2: Vor allem Sportlehrer, die den Beruf aufgrund von intrinsisch-altruistischen Motiven ergriffen haben, zeigen evasive Tendenzen, da sie sich an ihren eigenen, teils überhöhten Zielsetzungen "aufgerieben“ haben und mittlerweile aufgrund der Diskrepanz von Anspruch und Wirklichkeit kapitulieren müssen.

- Hypothese 3: Die Berufswahlmotivation hat keinen Einfluss auf die Ausprägung der evasiv-invasiv Polarität.

4. Inwieweit stellt die individuelle praktische und theoretische Vermittlungskompetenz in den einzelnen Schulsportarten eine relevante Einflussgröße auf die Ausprägung des evasiven Potenzials dar?

- Hypothese 1: Sportlehrkräfte mit einer geringeren praktischen und theoretischen Vermittlungskompetenz weisen vermehrt evasive Tendenzen auf, da bei innen die Unsicherheit im sportlichen und unterrichtlichen Handeln stärker ausgeprägt ist.

- Hypothese 2: Die praktische und theoretische Vermittlungskompetenz stellt keine relevante Einflussgröße auf das evasive Verhaltenspotenzial dar, da dies eine höher angesiedelte Verhaltensdisposition repräsentiert.

- Hypothese 3: Lediglich die theoretische Vermittlungskompetenz stellt eine relevante Einflussgröße auf die Ausprägung des evasiven Potenzials dar, da evasive Handlungen bereits im Vorfeld des Unterrichts geplant werden. 
- Hypothese 4: Lediglich die praktische Vermittlungskompetenz stellt eine relevante Einflussgröße auf die Ausprägung des evasiven Potenzials dar, da nur in der konkreten Drucksituation im Unterricht evasive Handlungen zustande kommen.

5. Stellt die individuelle Sportaffinität (Fitnesszustand, Sportaktivität in der Freizeit, Vereinstrainerschaft, aktuell oder in der Vergangenheit betriebener Wettkampfsport, Stellenwert von Sport im Privatleben) eine die Bewältigung beeinflussende Variable dar?

- Hypothese 1: Sportlehrkräfte mit einer geringeren Sportaffinität weisen vermehrt evasive Tendenzen auf, da ihre Verbindung zu sportlicher Betätigung und damit auch das bereichsbezogene Anspruchsdenken geringer ausgeprägt ist.

- Hypothese 2: Die individuelle Sportaffinität stellt keine relevante Einflussgröße auf das evasive Verhaltenspotenzial dar, da dies eine höher angesiedelte Verhaltensdisposition repräsentiert.

6. Inwieweit wirkt sich die Kombination von Fachrichtungen bzw. Haupt- und Nebenfächern auf die meidende bzw. aufsuchende Bewältigung aus?

- Hypothese 1: Sportlehrkräfte, die neben Sport in einem Hauptfach unterrichten, weisen im Sportunterricht verstärkt evasive Tendenzen auf, da sie in dem jeweiligen anderen Fach ihre Hauptaufgabe und im Sport nur ein ergänzendes Beifach sehen.

- Hypothese 2: Die Fachkombinationen spielen keine Rolle bei der Ausprägung der ausweichenden Verhaltenstendenzen im Sportunterricht, da Persönlichkeitseigenschaften und nicht die Berufsinfrastruktur den entscheidenden Einflussfaktor darstellen.

7. Bestehen Zusammenhänge zwischen der Gesamtstundenverpflichtung, der Sportstundenzahl bzw. dem prozentualen Anteil des Sportunterrichts an der Gesamtlehrverpflichtung und der Ausprägung von evasivem Verhalten?

- Hypothese 1: Lehrkräfte mit einer hohen Gesamtstundenverpflichtung weisen verstärkt evasive Verhaltenstendenzen auf, da es ihnen aufgrund der Belastungssummation nicht möglich ist, mit den Konflikte in dem Maß problemorientiert umzugehen, wie dies Kollegen mit einer geringen Stundenverpflichtung tun.

- Hypothese 2: Lehrkräfte, die eine hohe wöchentliche Sportstundenzahl unterrichten, weisen aufgrund des hohen fachbedingten Belastungspotenzials, stärkere evasive Verhaltenstendenzen auf, als Sportlehrer, die weniger Sport pro Woche unterrichten. 
- Hypothese 3: Durch eine hohe wöchentliche Sportstundenzahl erlangen Sportlehrkräfte zusätzliche Sicherheit und Routine und weisen daher ein geringeres evasives Potenzial auf als diejenigen, die weniger Sport unterrichten.

- Hypothese 4: Sportlehrkräfte mit einer geringen wöchentlichen Sportstundenanzahl weisen stärkere evasive Tendenzen auf als ihre Kollegen mit einer höheren Sportstundenanzahl, da sie aufgrund unangemessener Bewältigungsversuche und der damit einhergehenden höheren Belastung eine Stundenreduzierung/Umverteilung beantragt haben.

8. Bestehen korrelative Zusammenhänge zwischen dem fächerübergreifenden, dem fachspezifischen und dem privaten Fortbildungsengagement und evasivem Unterrichten?

- Hypothese 1: Durch den Ausbau und die Aktualisierung der eigenen Fachkompetenz sind Sportlehrkräfte verstärkt in der Lage, im Unterricht aktiv und invasiv zu handeln und Konfliktsituationen so fruchtbar zu machen.

- Hypothese 2: Fort- und Weiterbildungsmaßnahmen haben keinen Einfluss auf das evasive Potenzial, da es sich um eine stabile Verhaltensdisposition handelt, die durch die Aktualisierung von Wissen und Lehrmethoden nicht beeinflusst wird.

\subsection{Untersuchungskomplex 2: Unterrichtliche Charakteristik: „In welchen Aspekten unterscheidet sich der Unterricht von eher evasiv und eher invasiv Unterrichtenden?"}

Der zweite Untersuchungskomplex, der im Rahmen dieser Arbeit bearbeitet wird, umfasst die Aufdeckung einer eventuellen speziellen unterrichtlichen Charakteristik von eher evasiv unterrichtenden Lehrkräften. Die Frage: „In welchen Aspekten unterscheidet sich der Unterricht von eher evasiv und eher invasiv Unterrichtenden?" wird anhand von Angaben der Sportlehrkräfte beantwortet, die sie selbst zu Planung, Durchführung und Auswertung bzw. Reflexion ihres Sportunterrichts machten. Selbstverständlich sind diese Angaben, wie alle anderen Daten, die die empirische Grundlage dieser Arbeit darstellen, Selbstauskünfte der Lehrkräfte und daher anfällig im Sinne einer überpositiven Antworttendenz, die auch als soziale Erwünschtheit bezeichnet wird.

\section{Folgende Teilfragen werden im Rahmen dieses zweiten Komplexes bearbeitet:}

1. Bestehen Zusammenhänge zwischen der Ausprägung von evasivem Bewältigungsverhalten und der Bedeutsamkeit von Kriterien für die Inhaltswahl? 
- Hypothese 1: Sportlehrkräfte mit ausgeprägtem evasivem Potenzial wählen Inhalte verstärkt anhand von Aspekten aus, die individuelle Belastungen bzw. den Aufwand reduzieren bzw. minimieren.

- Hypothese 2: Es bestehen keine Zusammenhänge zwischen der Ausprägung des evasiven Potenzials und der individuellen Bedeutsamkeit der Kriterien für die Inhaltswahl.

2. Unterscheidet sich die Durchführungshäufigkeit verschiedener Sportarten von eher evasiv und eher invasiv unterrichtenden Sportlehrkräften?

- Hypothese 1: Sportlehrkräfte mit ausgeprägtem evasivem Potenzial wählen vermehrt Sportarten aus, die sich durch ein geringes Verletzungsrisiko und ein geringes Konfliktpotenzial zwischen Schülern auszeichnen.

- Hypothese 2: Zwischen der Durchführungshäufigkeit verschiedener Sportarten und der Ausprägung des evasiven Potenzials bestehen keine Zusammenhänge.

3. Bestehen Zusammenhänge zwischen der Ausprägung des evasiven Bewältigungspotenzials und der Bedeutsamkeit von verschiedenen Vermittlungsaspekten im Sportunterricht?

- Hypothese 1: Eher evasiv und eher invasiv Unterrichtende unterscheiden sich in der Bedeutsamkeit von verschiedenen konfliktträchtigen Vermittlungsaspekten wie z.B. Kondition, soziales Lernen und Disziplin.

- Hypothese 2: Zwischen der Ausprägung des evasiven Verhaltenspotenzials und der Bedeutsamkeit unterschiedlicher Vermittlungsaspekte bestehen keine Zusammenhänge.

4. Unterscheiden sich eher evasiv und eher invasiv unterrichtende Sportlehrkräfte in der Häufigkeit der aktiven Teilnahme am Sportunterricht?

- Hypothese 1: Zwischen der Ausprägung des evasiven Verhaltenspotenzials und der aktiven Teilnahme am Sportunterricht besteht ein negativer Zusammenhang, da durch die Nichtteilnahme „vermeintlich“ zusätzliche Belastungen vermindert werden.

- Hypothese 2: Zwischen der Ausprägung des evasiven Verhaltenspotenzials und der aktiven Teilnahme am Sportunterricht besteht kein bzw. ein positiver Zusammenhang.

5. Gibt es einen Zusammenhang zwischen der Ausprägung des evasiven Verhaltens im Unterricht und dem Arbeitsaufwand für Vor- und Nachbereitung für das Fach Sport?

- Hypothese 1: Sportlehrkräfte, die ihren Sportunterricht intensiv vor- und nachbereiten, weisen aufgrund der so entstehenden Planungssicherheit 
eine geringere Tendenz zu evasivem Verhalten auf als jene Sportlehrkräfte, die nur wenig Zeit in die Vor- und Nachbereitung investieren.

- Hypothese 2: Das evasive Verhalten stellt eine übergeordnete Verhaltensdisposition dar, die in keinem Zusammenhang zu der investierten Zeit für die Vor- und Nachbereitung steht. Es handelt sich um eine Persönlichkeitseigenschaft, die durch methodisch-didaktische Vorstrukturierungen nur sehr bedingt beeinflussbar ist.

6. Unterscheiden sich die individuelle Lehrweise und die unterrichtliche Organisationsform von eher evasiv und eher invasiv Unterrichtenden?

- Hypothese 1: Während eher evasiv Unterrichtende aufgrund der vermeintlichen höheren Planungssicherheit verstärkt deduktiv-lehrerzentrierte Unterrichtsarrangements bevorzugen, findet man unter den eher invasiv Unterrichtenden deutlich mehr Lehrkräfte, die induktiv-schülerorientierte Vorgehensweisen im Unterricht bevorzugen.

- Hypothese 2: Zwischen der Ausprägung des evasiven Verhaltenspotenzials und der unterrichtlichen Organisationsform sowie der individuellen Lehrweise bestehen keine Zusammenhänge.

7. Bestehen Zusammenhänge zwischen dem technisch-taktischen bzw. dem konditionellen Anforderungsniveau und der Ausprägung von evasivem Verhalten im Unterricht?

- Hypothese 1: Sportlehrkräfte mit ausgeprägtem evasivem Potenzial legen ein statistisch überzufällig geringeres Anforderungsniveau für ihren Unterricht im technisch-taktischen bzw. konditionellen Bereich fest als eher invasiv Unterrichtende.

- Hypothese 2: Der Unterricht von Sportlehrkräften mit ausgeprägtem evasiven Potenzial kennzeichnet sich durch ein signifikant höheres Anforderungsniveau, wodurch zwangsläufig auch die Konflikthäufigkeit und dem folgend die wahrgenommene Belastung sowie die Tendenz, Problemen auszuweichen, erhöht ist.

- Hypothese 3: Zwischen der Ausprägung des evasiven Verhaltenspotenzials und dem technisch-taktischen und konditionellen Anspruchsniveau im Unterricht bestehen keine Zusammenhänge.

8. Bestehen Unterschiede zwischen eher evasiv und eher invasiv Unterrichtenden in der subjektiven Einschätzung des Stellenwerts des Sportunterrichts im Fachvergleich?

- Hypothese 1: Der Stellenwert des Sportunterrichts wird von Sportlehrkräften, die eher evasiv agieren im Fächervergleich als geringer eingestuft, sodass hierdurch eine Legitimation des eigenen Handelns erfolgt. 
- Hypothese 2: Eher evasiv und eher invasiv Unterrichtende unterscheiden sich bezüglich ihrer subjektiven Einschätzung des Stellenwerts des Sportunterrichts im Fachvergleich nicht.

\subsection{Untersuchungskomplex 3: Verhalten und Erleben im bzw.} von Sportunterricht "Welche Merkmale sind in Bezug auf das Verhalten und Erleben im bzw. von Sportunterricht kennzeichnend für Personen mit ausgeprägtem evasiven Potenzial?"

In einem dritten Untersuchungskomplex sollen die Zusammenhänge zwischen dem vermeidenden Bewältigen und dem Verhalten und Erleben im bzw. von Sportunterricht ergründet werden. Die Frage "Welche Merkmale sind in Bezug auf das Verhalten und Erleben im bzw. von Sportunterricht kennzeichnend für Personen mit ausgeprägtem evasiven Potenzial?" wird anhand von verschiedenen Teilfragen beantwortet. Neben dem individuellen Belastungsempfinden wird überprüft, ob Zusammenhänge zwischen dem Bewältigungsverhalten und den Beanspruchungsfolgen wie innere Kündigung und Burnout sowie deren konstituierenden Merkmale wie z.B. der Arbeitszufriedenheit, dem Spaß am Unterrichten oder der Atmosphäre im Kollegium bestehen. Weiterhin wird überprüft, inwieweit sich die zum Verhalten und Erleben in einem interdependenten Verhältnis stehenden beliefs und commitments bei eher evasiv und eher invasiv Unterrichtenden unterscheiden.

\section{Folgende Teilfragen werden im Rahmen dieses dritten Komplexes bearbeitet:}

1. Gibt es Zusammenhänge zwischen dem Erleben von beruflicher Belastung und der Ausprägung des evasiven Verhaltens im Sportunterricht?

- Hypothese 1: Sportlehrkräften mit ausgeprägtem evasiven Potenzial gelingt es, durch ihr Verhalten Ressourcen zu schonen und demzufolge empfinden sie den Sportunterricht als geringer belastend im Vergleich mit eher invasiv agierenden Sportlehrkräften.

- Hypothese 2: Sportlehrkräfte mit ausgeprägter evasiver Tendenz nehmen den Sportunterricht subjektiv als belastender im Vergleich zu den eher invasiv Agierenden wahr, da Konflikte meist nur vordergründig behoben werden und im späteren Unterrichtsverlauf erneut, teils in verschärfter Form auftreten.

2. Inwieweit unterscheiden sich die von Schaarschmidt (1996) entwickelten arbeitsbezogenen Verhaltens- und Erlebensmerkmalen, bezogen auf die Tendenz, evasive Verhaltensweisen im Sportunterricht an den Tag zu legen? 
- Hypothese 1: Aufgrund der geringen Ausprägung der Bedeutsamkeit der Arbeit, des Perfektionsstrebens, des beruflichen Ehrgeizes und der Verausgabungsbereitschaft neigen vor allem Sportlehrkräfte des Schonungsmusters zu evasivem Verhalten.

- Hypothese 2: Aufgrund von mangelnden Ressourcen neigen vor allem Sportlehrkräfte, die dem Burnout-Muster zugeordnet werden dazu, evasive Verhaltensweisen an den Tag zu legen bzw. die unangemessene Form der Bewältigung führt durch die nur vordergründige Lösung von Konflikten überhaupt erst zur Ausprägung des Burnouts.

- Hypothese 3: Sportlehrkräfte, die dem Muster Gesundheit zugeordnet werden, erreichen diese gesundheitsfördernde Variante des arbeitsbezogenen Verhaltens und Erlebens der Arbeit durch evasives Bewältigungsverhalten im Alltag.

3. Inwieweit bestehen Zusammenhänge zwischen evasivem Verhalten im Unterricht und der Ausprägung von berufsbedingter Erschöpfung und arbeitsbezogenem Disengagement?

- Hypothese 1: Zwischen berufsbedingter Erschöpfung sowie dem arbeitsbezogenem Disengagement und der Ausprägung von evasivem Potenzial besteht ein positiver Zusammenhang, da durch ausweichendes Verhalten keine Belastungsreduzierung erreicht wird.

- Hypothese 2: Zwischen berufsbedingter Erschöpfung sowie dem arbeitsbezogenem Disengagement und der Ausprägung von evasivem Potenzial besteht ein negativer Zusammenhang, da das evasive Verhalten der Belastungsreduzierung dient.

- Hypothese 3: Zwischen berufsbedingter Erschöpfung sowie dem arbeitsbezogenem Disengagement und der Ausprägung von evasivem Potenzial besteht kein Zusammenhang.

4. In welchem Zusammenhang steht die Ausprägung von evasivem Bewältigungsverhalten mit der Arbeitszufriedenheit im Allgemeinen und im Speziellen (Zufriedenheit mit der Stundenverteilung, Spaß am Unterrichten, wahrgenommene Atmosphäre im Gesamt- bzw. Sportkollegium, Gefühle mit der bisherigen Karriere, Gefühle für die Zukunft, Wechsel- bzw. Ausstiegsgedanken aus dem Beruf, eine erneute Berufswahl)?

- Hypothese 1: Zwischen der Arbeitszufriedenheit und der Ausprägung des evasiven Verhaltenspotenzials bei Sportlehrkräften besteht ein negativer Zusammenhang, da durch die unangemessene Bewältigung das Belastungspotenzial erhöht ist und damit auch die Zufriedenheit mit der Arbeit und dem Beruf absinkt. 
- Hypothese 2: Zwischen der Arbeitszufriedenheit und der Ausprägung des evasiven Verhaltenspotenzials bei Sportlehrkräften besteht ein positiver Zusammenhang, da durch das ausweichende Verhalten Konflikten aus dem Weg gegangen werden kann und so die Zufriedenheit steigt.

- Hypothese 3: Zwischen der Arbeitszufriedenheit und der Ausprägung des evasiven Verhaltenspotenzials bei Sportlehrkräften besteht kein Zusammenhang.

5. Inwieweit bestehen Zusammenhänge zwischen Persönlichkeitseigenschaften wie beliefs, commitments und der Ungewissheitstoleranz und der Ausprägung des evasiven Bewältigungsverhaltens?

- Hypothese 1: Sportlehrkräfte, die von ihrer eigenen Wirksamkeit in geringerem Maße überzeugt sind, weisen ein höheres evasives Verhaltenspotenzial auf als Sportlehrkräfte, die von der Wirksamkeit ihres Tuns überzeugt sind.

- Hypothese 2: Sportlehrkräfte, die davon überzeugt sind, den Lauf der Dinge selbst in der Hand zu haben und weniger vom Einfluss des Schicksals und anderer Personen überzeugt sind, verhalten sich im Sportunterricht verstärkt invasiv.

- Hypothese 3: Sportlehrkräfte, die sich durch eine ausgeprägte Furcht vor Misserfolg und eine geringe Hoffnung auf Erfolg auszeichnen agieren im Sportunterricht evasiv.

- Hypothese 4: Sportlehrkräfte, die sich tolerant gegenüber Ungewissheit zeigen, agieren in Situationen, die sich durch Neuartigkeit und Ereignisunsicherheit auszeichnen, verstärkt invasiv.

- Hypothese 5: Zwischen Persönlichkeitseigenschaften wie beliefs, commitments und der Ungewissheitstoleranz und der Ausprägung des evasiven Verhaltenspotenzials bestehen keine Zusammenhänge.

6. In welchem Zusammenhang steht evasives Verhalten mit der krankheitsbedingten Absentismusrate?

- Hypothese 1: Zwischen dem evasiven Potenzial und der krankheitsbedingten Absentismusrate besteht ein positiver Zusammenhang, da durch die unangemessene Bewältigung zusätzliche Belastungen entstehen, die zu psychophysischen Krankheiten und dementsprechend höheren Fehlzeiten führen können.

- Hypothese 2: Zwischen dem evasiven Potenzial und der krankheitsbedingten Absentismusrate besteht ein negativer Zusammenhang, da durch das ausweichende Verhalten wichtige Ressourcen geschont und so Krankheiten verhindert werden. 
- Hypothese 3: Es bestehen keinerlei Zusammenhänge zwischen dem evasiven Bewältigungsverhalten und der Absentismusrate, da lediglich Faktoren wie z.B. eine gesunde Lebensführung oder das soziale Klima im Kollegium Einfluss auf den Gesundheitszustand aufweisen. 


\section{Methodische Umsetzung}

\subsection{Konstruktion des Erhebungsinstruments evasiver Sportun- terricht (EESU)}

\subsubsection{Zielsetzung und theoretische Grundlagen}

Um die vorliegenden Fragestellungen bezüglich des Problemfeldes (siehe Kap. 5) des evasiven Unterrichtens im Sportunterricht beantworten zu können, war zunächst wichtig zu entscheiden, mit welcher Methode dies erfolgen soll. Während das Führen von Interviews im Vergleich zu einer schriftlichen Erhebung durch Fragebogen den Vorteil hat, dass individuelle Begründungszusammenhänge in einer größeren Genauigkeit erschlossen werden können, sprachen dennoch einige Gründe dafür, von solch einem Verfahren Abstand zu nehmen. Ausweichendes Verhalten stellt, wie bereits in Kapitel 4.9 dargelegt, durchaus eine notwendige Bewältigungsstrategie dar, ist aber dennoch auch bei Sportlehrern negativ attribuiert, d.h. sehr anfällig für Verzerrungen bzw. positive Verfälschung der Antworten. Da es sich bei dem Untersuchungsgegenstand um ein sensibles Thema handelt, ist die als soziale Erwünschtheit beschriebene Antworttendenz nicht zu unterschätzen. Durch eine schriftlich-anonymisierte Erhebung ist hingegen ein ehrlicheres Antworten zu erwarten. Des Weiteren sind deutlich größer Stichprobengrößen möglich, was sowohl bezogen auf die Ökonomie der Erhebung als auch im Rahmen eines Forschungsvorhabens ohne vorliegende empirische Basisdaten vorteilhaft ist.

Wie bereits im Kapitel 5 angeführt und erläutert, war es unerlässlich, ein neues Erhebungsinstrument zu gestalten, um das evasive Potenzial der Sportlehrkräfte möglichst umfassend abbilden zu können. Bereits existierende Instrumente wie beispielsweise der von Janke, Erdmann und Kallus (1984) entwickelte "Stressverarbeitungsfragebogen $120 "$ (SVF 120) und einige weitere der in Kapitel 4.8 genannten Instrumente weisen zwar Schnittmengen mit der in Kapitel 4.9.5 skizzierten Bewältigungsstrategie des evasiven Unterrichtens auf, stellen jedoch keine geeigneten Instrumente für die angestrebte Untersuchung dar. Einige wenige Fragen haben in modifizierter Form jedoch Einzug in das neu konstruierte Erhebungsinstrument gefunden. Ein weiteres verwandtes Instrument stellt der "Coping Inventory for Stressful situations“ (CISS) von Endler und Parker (1990), in der deutschen Übersetzung von Semmer, Tschan und Schade (1991), sowie die Kurzformen von Kälin (1995) dar. Hier liegt eine Subskala zum vermeidungsorientierten Coping vor, die jedoch für den Einsatz bei Lehrpersonen ungeeignet ist, was an den lehrberufsinadequaten Formulierungen der Items liegt. Antwor- 
ten wie "Ich mache einen Einkaufsbummel", "Ich versuche zu schlafen", "Ich gehe essen" oder "Ich besuche eine(n) Freund(in)" auf die Frage: „Was tun Sie typischerweise, wenn Sie in einer schwierigen, stressvollen oder ärgerlichen Situation sind?" können kaum als für stressvolle Unterrichtssituationen geeignete und vor allem realisierbare Bewältigungsmaßnahmen angesehen werden. Mit „Ich wende mich von dem Problem $a b$ und lasse es erst mal gut sein" ist von den insgesamt 13 vermeidungsorientierten Items nur eines auf die konkrete Situation im Unterricht anwendbar. Ein weiteres verwandtes Instrument ist der „Ways of Coping Questionnaire“. Der von der Gruppe um Lazarus konstruierte Fragebogen misst in acht Dimensionen das Bewältigungsverhalten (Lazarus \& Folkman, 1987). Es zeigte sich jedoch wie in Kapitel 4.8 bereits dargestellt, dass sich die faktorielle Struktur des Instruments nicht replizieren ließ und man vielmehr nur zwischen problemorientiertem und emotionsorientiertem Coping unterscheiden konnte (Steger, 2006). Wie sich anhand der kurzen Analyse der bereits existierenden verwandten Konstrukte zeigt, war eine Neukonstruktion unumgänglich.

Da Lehrer mit einer Vielzahl von Anforderungen, Wünschen und Interessen konfrontiert werden, soll dies auch im zu konstruierenden Fragebogen berücksichtigt werden. Nach dem didaktischen Modell von Scherler (2004) lassen sich neben dem Sportlehrenden selbst, mit den Schülerinnen und Schülern, den Inhalten und den äußeren Bedingungen drei Facetten unterscheiden, die Einfluss auf das Unterrichten haben. Diese Unterscheidung, die im Rahmen des didaktischen Modells vorgenommen wird, soll sich auch im Design des Erhebungsinstruments widerspiegeln, um der Zielsetzung, evasives Verhalten differenziert in Bezug auf die verschiedenen Umweltfaktoren abzubilden, nachkommen zu können. Neben der Differenzierung im Bezug auf die Umweltfaktoren bzw. Facetten des evasiven Unterrichtens sollen weiterhin die verschiedenen Beweggründe für evasives Handeln integriert werden. Neben Herausforderung/Motivation, Handlungssicherheit und -aufschub soll zudem der Aspekt Prävention Berücksichtigung finden. Im weiteren Verlauf des Kapitels werden die einzelnen Schritte von der Erstellung der ersten Version des Fragebogens für die erste Pilotierungsphase bis hin zur Endversion beschrieben.

\subsubsection{Skalenkonstruktion und -modifikation}

In einer ersten Pilotierungsphase wurde ein Itempool unter Berücksichtigung der verschiedenen Einflussfaktoren auf das Unterrichten nach Scherler ${ }^{1}$ (2004) sowie den Beweggründen ${ }^{2}$ generiert. Der erste Testentwurf (siehe Anhang I, Frage 27), der insgesamt 29 Items enthielt, wurde mit einer vierstufigen Likert-Skala von trifft nicht $z u$ bis

\footnotetext{
${ }^{1}$ Schülerinnen und Schüler, Inhalte, äußere Rahmenbedingungen.

${ }^{2}$ Herausforderung/Motivation, Handlungssicherheit und -aufschub, Prävention.
} 
trifft $z u$ in Form einer Verhaltensselbsteinschätzung durch insgesamt 32 Sportlehramtsstudentinnen und -studenten mit Lehrerfahrung der Universität Kassel getestet. Außerdem wurde eine Reihe weiterer Instrumente, die der Validierung dienen sollten, eingesetzt. Es wurden ausschließlich Studenten ausgewählt, die bereits ihr Fachpraktikum absolviert haben, damit die Stichprobeneigenschaften eine möglichst große Ähnlichkeit zu einer Lehrerstichprobe aufweist. Die Studenten füllten den Fragebogen nach Anleitung des Seminarleiters aus, dem Hinweise und Informationen zum Ausfüllen des Fragebogens in schriftlicher Form vorlagen. Die Bearbeitungszeit betrug ca. 30 Minuten. In jedem Item dieses ersten Testentwurfs war, gemäß der in Kap. 6.1.1 gesetzten Zielsetzung, in irgendeiner Art und Weise eine Spezifikation enthalten, die eine Zuordnung zu einem Umweltfaktor ermöglichen sollte und die eine inhaltliche Fokussierung, bezogen auf einen der Beweggründe für evasives Verhalten, beinhaltete.

Anhand der Ergebnisse dieser ersten Pilotierung, die eine ausgeprägte Heterogenität der Items deutlich machte, konnten die psychometrischen Gütekriterien für eine angestrebte Auswertung nach Facetten bzw. Beweggründen des evasiven Verhaltens nicht erfüllt werden. Ein Einsatz des Testentwurfs wäre lediglich als Gesamtskala ohne weitere Differenzierungen bezüglich der zugrunde liegenden Struktur möglich gewesen. Weder die angestrebte Auswertung nach Einzelfacetten noch die Identifikation der Beweggründe waren realisierbar, wodurch Aufwand und Ertrag dieses Instruments in keinem Verhältnis zueinander stehen und eine umfassende Revision unabdingbar war.

In der zweiten Pilotierungsphase wurde eine grundlegende Modifikation des Erhebungsinstruments zur Erfassung evasiven Verhaltens im Sportunterricht vorgenommen. In Anlehnung an das differenzielle Selbstkonzept-Gitter von Rost und Sparfeldt (2002) die eine Gitterversion zur Erfassung des fachspezifischen Selbstkonzepts in verschiedenen Schulfächern entwickelten und validierten, wurde eine Neukonzeption des Instruments vorgenommen. Die Ergebnisse bei Rost und Sparfeldt zeigten, dass der Einsatz von Items, die in identischer Form für verschiedene Facetten ${ }^{3}$ beantwortet werden, unter ökonomischen Aspekten vorteilhaft und teststatistisch nicht mit Nachteilen behaftet ist. Bei Rost und Sparfeldt ist der Einsatz der identischen Itemformulierungen problemlos in Form einer Gitterversion möglich, denn die inhaltliche Abgrenzung der Einzelfacetten, in denen das Selbstkonzept gemessen werden soll, ist für die Schüler problemlos bewältigbar, da zwischen den Fächern kaum sich überschneidende Bereiche existieren. Bezogen auf das evasive Verhalten im Sportunterricht liegt eine etwas andere Lage der Dinge vor: Unterrichtliches Handeln ist stets ein komplexes Konstrukt, bei dem wechselseitige Interdependenz zwischen allen Faktoren

\footnotetext{
${ }^{3}$ In diesem Fall Schulfächer.
} 
bestehen, die in irgendeiner Art und Weise Teil des Unterrichts sind bzw. diesen beeinflussen. Der Grad an Komplexität und damit auch die Problematik einer klaren Abgrenzung, hier konkret das Verhalten im Unterricht bezogen auf den Umgang mit Schülern, Inhalten und äußeren Bedingungen ist deutlich höher bzw. schwieriger als die differenzierte Einschätzung des Selbstkonzepts in verschiedenen Schulfächern. Aufgrund der dementsprechend zweifellos deutlich komplexeren Anforderungsstruktur wurde bei der Konstruktion des Instruments zum evasiven Sportunterricht auf eine Verdichtung, wie dies bei einer Gitterstruktur der Fall ist, verzichtet. Durch eine räumliche Separation der Itemfortsetzungen, die für die verschiedenen Facetten (Itembasen) beantwortet werden sollen, wird eine gedanklich-inhaltliche Abgrenzung der Bereiche untereinander erleichtert. In Tabelle 6 sind die drei Itembasen dargestellt, die in Kombination mit den Itemfortsetzungen, das Verhalten der Sportlehrkraft im Bezug auf die Einflussfaktoren des Unterrichts erfassen sollen.

Tab 6: Darstellung der Itembasen

\begin{tabular}{|l|}
\hline Itembasen der Facetten (Einflussfaktoren auf das Unterrichten) \\
\hline Im Bezug auf den Umgang mit Schülerinnen und Schülern im Sportunterricht... \\
\hline Im Bezug auf Inhalte im Sportunterricht... \\
\hline $\begin{array}{l}\text { Im Bezug auf äußere Bedingungen...(Vorschriften und Erlasse, Beziehungen zum Kollegium/zur Schulleitung, } \\
\text { Zeitordnungen, Notengebung, materielle Ausstattung) }\end{array}$ \\
\hline
\end{tabular}

Diese dargestellte Grundstruktur wird in Fragebogenform durch die Itemfortsetzungen komplettiert (siehe Tab. 7), die so formuliert werden mussten, dass sie sowohl für den Umgang mit Schülerinnen und Schülern, mit Inhalten als auch mit äußeren Bedingungen zu beantworten sind. Im Rahmen eines Seminars des Instituts für Sportwissenschaften an der Georg-August-Universität Göttingen zur Belastungsproblematik von Sportlehrenden beantworteten insgesamt 102 Sportstudentinnen und Sportstudenten ${ }^{4}$, insgesamt 26 Items in identischer Form gebündelt für jede der drei Facetten(siehe Anhang II, Fragen 9-11). Des Weiteren wurde erneut eine Reihe weiterer Instrumente zu Validierungszwecken eingesetzt.

Für die Hauptuntersuchung wurde eine umfangreiche Itemanalyse durchgeführt, um das Instrument, bei gleichzeitiger Beibehaltung der inhaltlichen Ansprüche, zu ökonomisieren. Auf Einzelitemebene wurden die Verteilungen, also die Maße der zentralen Tendenz sowie Dispersion, die Schwierigkeit und die Trennschärfe analysiert und zur Eliminierung von Items genutzt, die den Ansprüchen nicht genügten. Ziel war es, ein

\footnotetext{
${ }^{4}$ Überwiegend mit Lehrerfahrung.
} 
Instrument zu generieren, das möglichst kurz ist, bezogen auf Reliabilität und Validität und dennoch den Mindestansprüchen genügt und inhaltlich die gesamte Bandbreite der verschiedenen Fokussierungen evasiven Verhaltens repräsentiert. Besonders schwierig stellte sich die Itemselektion aufgrund der durch das Design des Instruments bedingten Situation dar, nämlich dass die einzelnen Items die gesetzten Ansprüche, Kriterien und Vorgaben für alle drei Facetten erfüllen mussten, um wirklich sowohl im Bezug auf den Umgang mit Schülerinnen und Schülern, mit Inhalten als auch mit äußeren Bedingungen reliable und vor allem aber aussagekräftige Ergebnisse zu erzielen, um so eine Vergleichbarkeit der Facetten untereinander zu ermöglichen. In der folgenden Tabelle sind die Itemfortsetzungen aufgeführt, die letztendlich in der Hauptuntersuchung in Kombination mit den drei Itembasen (siehe Tab. 6) eingesetzt wurden.

Tab. 7: Itemfortsetzungen

\begin{tabular}{|l|l|}
\hline \multicolumn{2}{|l|}{ Itemfortsetzungen } \\
\hline Item 1 & $\begin{array}{l}\text {...schlage ich lieber mir vertraute Wege ein, bevor ich bei Neuem das Risiko eingehe zu schei- } \\
\text { tern. }\end{array}$ \\
\hline Item 2 & $\ldots$ suche ich mir umgehend eine größere Herausforderung, wenn ich ein Ziel erreicht habe. \\
\hline Item 3 & $\ldots$ lasse ich ein Problem erst einmal „,vor sich hinköcheln“, bevor ich es anpacke. \\
\hline Item 4 & $\ldots$ nehme ich schwierige Situationen, in denen viel von mir abhängt, gerne an. \\
\hline Item 5 & $\ldots$ achte ich bewusst darauf, Schwierigkeiten zu vermeiden. \\
\hline Item 6 & $\ldots$ suche ich mir gern Herausforderungen und gehe dafür auch Risiken ein. \\
\hline Item 7 & $\ldots$..gehe ich lieber auf Nummer sicher, um Ärger zu vermeiden. \\
\hline Item 8 & $\ldots$ habe ich das Ziel, nicht anzuecken. \\
\hline
\end{tabular}

\subsubsection{Faktorenanalyse}

Im Rahmen der Validierung neu entwickelter Konstrukte werden häufig multivariate Verfahren wie die exploratorische oder die konfirmatorische Faktorenanalysen eingesetzt, um die Dimensionalität des Instruments zu überprüfen bzw. Korrelationen der Items mit den Faktoren zu identifizieren. Im Rahmen dieser Verfahren werden Items anhand ihrer Testkennwerte nach ihrer korrelativen Ähnlichkeit sortiert. Primäres Ziel ist es, eine ordnende Struktur in einem größeren Satz an Variablen zu erzeugen, indem Variablen, die gemeinsame Informationen erfassen, in Gruppen klassifiziert werden (Bortz, 2005). Während die exploratorische Faktorenanalyse ein strukturaufklärendes Verfahren darstellt, bei dem man aufgrund der erhobenen Daten die zugrunde liegende Faktoren ermittelt, ist die konfirmatorische Faktorenanalyse ein strukturprüfendes Verfahren, bei dem theoretisch oder empirisch fundierte Modelle auf ihre Modellgüte hin überprüft werden (Bühner, 2004). Zum Einsatz kommt die erstgenannte Art der Faktorenanalyse dann, wenn das neue Konstrukt nicht auf einer bereits vorliegenden 
Theorie basiert und demzufolge keine Hypothesen über die Art und Anzahl der enthaltenen Faktoren vorliegen (Moosbrugger \& Schermelleh-Engel, 2007). Bei der konfirmatorischen Faktorenanalyse wird bereits vor der Berechnung ein Modell spezifiziert, d.h. dass klare Vorhersagen über die Art und Anzahl der Faktoren und deren Beziehungen zueinander vorliegen (Bühner, 2004; Gorsuch, 1997). Im vorliegenden Fall der Messung des evasiven Bewältigungsverhaltens handelt es sich um eine Novität, d.h. dass das Instrument auf keinem Modell und keiner bereits existierenden Theorie basiert. Im Vorfeld ist unbestimmt, welche Variablen zu einem Faktor zusammengefasst werden können. Aufgrund der zuvor genannten Tatsache stellt die exploratorische Faktorenanalyse das angemessene Verfahren dar, um die faktorielle Struktur des Instruments aufzuklären (Arminger, 1979; Bühner, 2004; Field, 2005; Moosbrugger \& Schermelleh-Engel, 2007). Perspektivisch, in einem weiteren Schritt hin zu einer Normierung des Instruments, könnte durch eine zusätzliche Erhebung, die in dieser Arbeit aufgedeckte Faktorenstruktur durch eine konfirmatorische Faktorenanalyse überprüft werden (Floyd \& Widaman, 1995). Eine Normierung bzw. Überprüfung der Faktorenstruktur ist jedoch nicht Zielsetzung der vorliegenden Arbeit.

Ebenso wie bei der Itemanalyse (siehe Kap. 6.1.2) stellt das Design des Instruments besondere Anforderungen an die Faktorenanalyse, da in allen drei Facetten die gleiche faktorielle Struktur vorliegen sollte. Zielsetzung der exploratorischen Faktorenanalyse der drei Facetten des „Erhebungsinstruments evasiver Sportunterricht“ (kurz: EESU) ist es, jeweils die Dimensionalität d.h. die faktorielle Struktur des Messinstruments aufzuklären, um im Anschluss mit eventuellen Subskalen weitere Analysen durchzuführen. Neben dem Vorliegen von Daten im Intervall- bzw. quasi-Intervallskalenformat sind hierzu Linearität, das Vorliegen substanzieller Korrelationen der Variablen, eine ausreichende Stichprobengröße bezogen auf die Itemanzahl sowie eine Normalverteilung der Daten die notwendigen Voraussetzungen.

\subsubsection{Voraussetzungen der Faktorenanalyse und Umgang mit fehlenden Werten}

Grundsätzlich ist ein Intervallskalen- bzw. Quasi-Intervallskalenniveau ${ }^{5}$ eine Voraussetzung für die Durchführung einer exploratorischen Faktorenanalyse. Ob die erhobenen Daten für die Durchführung einer Faktorenanalyse geeignet sind, spiegelt sich in der Korrelationsmatrix wider. Für die Überprüfung der Eignung der Daten stehen verschiedene Kriterien zur Verfügung.

\footnotetext{
${ }^{5}$ Das evasive Potenzial wird im vorliegenden Erhebungsinstrument durch eine fünfstufige LikertSkalierung gemessen, die üblich als (quasi-)intervallskaliert aufgefasst wird (Floyd \& Widaman, 1995).
} 
Mit einer Stichprobengröße von N=705 liegen beste Voraussetzungen für die Durchführung einer Faktorenanalyse vor, denn die Mindestanforderung an die Größe der Stichprobe in statistischen Veröffentlichungen, die mit dem vier- bis zehnfachen der Itemzahl angegeben wird, ist deutlich übertroffen (de Vet et al., 2005). Sind Faktoren schwach besetzt und die Kommunalitäten eher gering, werden 500 Teilnehmer als Minimalstichprobengröße empfohlen (Bühner, 2004). Auch dieses Kriterium wird erfüllt.

Ein weiteres Problem bei exploratorischen Faktorenanalysen stellen fehlende Werte dar. Das Maximum an fehlenden Werten für die Durchführung einer Faktorenanalyse wird bei $25 \%$ gesehen. In der vorliegenden Stichprobe beträgt der Ausfall auf Einzelitemebene maximal 3,4\% und stellt damit eine unbedeutende Störgröße dar. Insgesamt kennzeichnet sich das Datenmaterial durch eine hohe Konsistenz. Fehlende Werte werden daher durch listenweisen Fallausschluss eliminiert.

Das Vorliegen substanzieller Korrelationen wird durch die Berechnungen der KaiserMeyer-Olkin-Koeffizienten (KMO) der drei Facetten sowie der Measure of sample adequacy (MSA) auf Einzelitemebene überprüft. Der KMO gibt Aufschluss darüber, ob die vorliegenden Variablen für die Durchführung einer Faktorenanalyse geeignet sind und wird durch die Division des gemeinsamen Varianzanteils der Variablen miteinander und durch den gemeinsamen Varianzanteil der Variablen miteinander plus den quadrierten Partialkoeffizienten berechnet (Bühner, 2004). Der KMO variiert zwischen 0 und 1; je höher die Werte liegen, desto besser sind die Daten für eine Analyse der Faktorstruktur geeignet. Für die potenzielle Durchführbarkeit sieht man einen Grenzwert von $>.5$ als maßgeblich ${ }^{6}$. Um zu prüfen ob die Voraussetzungen für die Durchführung einer Faktorenanalyse gegeben sind, wurde der Kaiser-Meyer-Olkin-Koeffizient einzeln für jede Facette berechnet. Mit Werten zwischen .793 und .836 liegen mittelmäßig bis gute Koeffizienten vor. Auch auf Einzelitemebene (vgl. Tab. 9) sind die Voraussetzungen, ausgedrückt durch den MSA-Wert, erfüllt, um eine Faktorenanalyse durchzuführen. Zusätzliche Bestätigung liefern zudem die Ergebnisse des Bartlett-Tests auf Sphärizität, $\chi^{2}(28)=1019,835$ (EESU-S), $p<.01 ; 1376,373$ (EESU-I), $p<.01$; 1687,266 (EESU-B), $p<.01$ (Field, 2005; Bühner, 2004).

Auf Einzelebene, ausgewiesen in der Hauptdiagonalen der Anti-ImageKorrelationsmatrix, werden zur Einschätzung der Eignung die MSA-Werte angegeben. Die Einschätzung der Werte erfolgt nach dem identischen Raster wie beim KMO. Alle Items stellen sich, bezogen auf ihre MSA als mindestens mittelprächtige adäquat, meist sogar als noch deutlich besser geeignet für die Durchführung einer Faktorenana-

\footnotetext{
${ }^{6}$ Das komplette Bewertungsraster für den KMO ist in der Überschrift von Tabelle 8 aufgeführt.
} 
lyse dar, sodass alle Items in die statistische Auswertung mit einbezogen werden können.

Tab. 8: Kaiser-Meyer-Olkin-Koeffizient der Einzelfacetten (EESU-S; EESU-I; EESU-B) und der Gesamtskala (EESU-SIB). Bewertungsraster ${ }^{7} 0,9$ bis 1,0 fabelhaft (marvelous); 0,8 bis unter 0,9 recht gut (meritorious); 0,7 bis unter 0,8 mittelprächtig (middling); 0,6 bis unter 0,7 mäßig (mediocre); 0,5 bis unter 0,6 schlecht (miserable); unter 0,5 inakzeptabel (unacceptable)

\begin{tabular}{lc}
\hline Facette & KMO \\
\hline EESU-S &, 793 \\
EESU-I &, 815 \\
EESU-B &, 836 \\
\hline EESU-SIB & $\mathbf{8 9 0}$ \\
\hline
\end{tabular}

Tab. 9: Measure of sample Adequacy (MSA) der Einzelitems nach Facetten

\begin{tabular}{lccc}
\hline & EESU-S & EESU-I & EESU-B \\
\hline Item 1 & .873 & .893 & .911 \\
Item 2 & .757 & .803 & .812 \\
Item 3 & .839 & .860 & .900 \\
Item 4 & .830 & .816 & .822 \\
Item 5 & .865 & .868 & .863 \\
Item 6 & .760 & .793 & .829 \\
Item 7 & .755 & .778 & .796 \\
Item 8 & .745 & .773 & .822 \\
\hline
\end{tabular}

\subsubsection{Faktorenextraktion}

Durch die Hauptkomponentenanalyse, die streng betrachtet keine Faktorenanalyse im eigentlichen Sinne ist, sollen die Variablen, die eine hohe Ladung auf eine Hauptkomponente bzw. einen Faktor aufweisen, in einem Sammelbegriff bzw. einer Komponente zusammengefasst werden (Fabrigar et al., 1999). Aus den ursprünglichen Daten soll möglichst viel Information in möglichst wenigen Faktoren aggregiert werden, d.h. dass möglichst viel Varianz aufgeklärt wird (Bortz, 2005). Für die Extraktion der in einem Instrument enthaltenen latenten Merkmalsdimensionen können verschiedene Methoden herangezogen werden, die zu mehr oder weniger eindeutigen Antworten führen können. Neben dem Kaiser-Guttman-Kriterium gehören der Scree-Test nach Cattell und die Parallelanalyse nach Horn zu den am meistverwendeten Verfahren. Auch ein vorher festgelegter Prozentsatz an aufgeklärter Varianz kann als Abbruchkriterium herangezogen werden. Zusätzlich zu den genannten Kriterien sind die extrahierten Faktoren jedoch stets auch auf inhaltliche Plausibilität zu prüfen.

\footnotetext{
${ }^{7}$ Entnommen aus Brosius (2004).
} 
Im Rahmen der durchgeführten Hauptkomponentenanalyse stellte sich die Ausprägung der Eigenwerte als wenig hilfreich als Extraktionskriterium heraus. Nach dem Kaiser-Kriterium sind Faktoren mit einem Eigenwert größer als 1 als bedeutend anzusehen und dementsprechend zu berücksichtigen (Janssen \& Laatz, 2007). Im vorliegenden Ergebnis der Hauptkomponentenanalyse deutet sich eine zweifaktorielle Struktur in allen drei Facetten an, die jedoch aufgrund der geringen Eigenwerte des zweiten Faktors von $\lambda=1,159\left(\right.$ EESU-S $\left.^{8}\right), \lambda=1,226\left(\right.$ EESU-I $\left.^{9}\right)$ und $\lambda=1,197\left(\right.$ EESU-B ${ }^{10}$ ) keine eindeutige Bestimmung der Faktorenzahl zulässt. Da die Bestimmung der Anzahl der Faktoren durch dieses Kriterium häufig die tatsächliche Anzahl an eigenständigen Faktoren überschätzt, soll dieser Methode der Extraktion nicht allein Vertrauen geschenkt werden und weitere Extraktionskriterien herangezogen werden (Floyd \& Widaman, 1995; Bühner, 2004). Bortz (2005, S. 544) empfiehlt sogar, nur in Ausnahmefällen „alle Faktoren mit $\lambda>1$ zu akzeptieren bzw. für die Rotation vorzusehen“.

Wie in den Abbildungen 18 bis 20 zu sehen, liefert jedoch auch das sogenannte Ellbogenkriterium des Scree-Tests nach Cattell (1966), bei dem die Eigenwerte in eine Rangreihe gebracht und in einem Diagramm dargestellt werden, keine klare Antwort auf die Frage nach der Anzahl der Faktoren. Die Lokalisation des Ellbogens, also dem bedeutsamen Knick, ab dem sich der Verlauf der Kurve asymptotisch der Abszisse annähert, ist nicht zweifelsfrei zu bestimmen. Wie in den Abbildungen ersichtlich ist, befindet sich ein mehr oder weniger deutlich erkennbarer Knick bei dem zweiten Eigenwert, was nach Moosbrugger und Kelava (2007) auf eine einfaktorielle Lösung hindeutet.

Da jedoch ab dem Knick beim zweiten Eigenwert noch kein eindeutiger asymptotischer Verlauf festzustellen ist, liefert auch dieses Abbruchkriterium kein klares Ergebnis. Aufgrund der Uneindeutigkeit dieser beiden Faktorextraktionsmethoden wurde zusätzlich eine Parallelanalyse nach Horn durchgeführt, die von verschiedenen Autoren als das beste Extraktionskriterium angesehen wird (Fabrigar et al., 1999). Bei diesem Verfahren werden normalverteilte Zufallsvariablen durch die von O'Connor (2000) verfasste SPSS-Syntax erstellt, aus denen anschließend Eigenwerte gebildet und als Vergleichswerte zu den Daten der Stichprobe herangezogen werden. Als bedeutsam werden Faktoren angesehen, wenn sich ihre Eigenwerte vor dem Schnittpunkt der beiden Verlaufskurven befinden, d.h. über den aus Zufallsvariablen erzeugten Eigenwerten liegen. Wie man anhand der Abbildungen 18 bis 20 erkennt, deuten die Ergebnisse der Parallelanalyse auf einen starken ersten Faktor und einen knapp überzufälligen zweiten Faktor hin, der in der weiteren Auswertung mit einbezogen wird, da die Anzahl der

\footnotetext{
${ }^{8}$ Facette: Umgang mit Schülerinnen und Schülern.

${ }^{9}$ Facette: Inhalte im Sportunterricht.

${ }^{10}$ Facette: äußere Bedingungen.
} 
durch die Parallelanalyse extrahierten Faktoren nur selten überschätzt wird (Bühner, 2004).

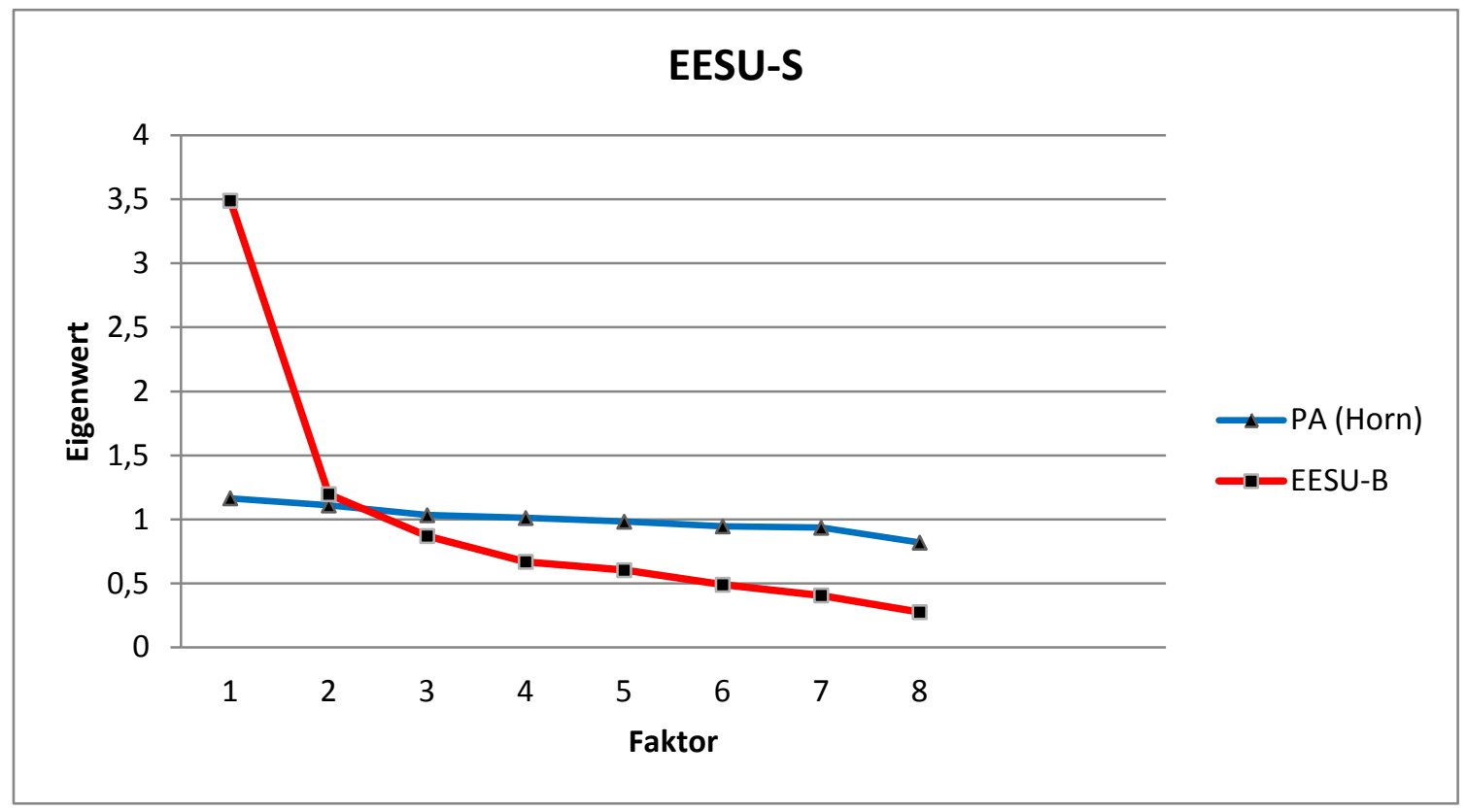

Abb. 18: Darstellung des Eigenwertverlaufs der Facette EESU-S in der Parallelanalyse nach Horn (1965) zu Zufallsdaten als Kriterium für die Faktorenextraktion

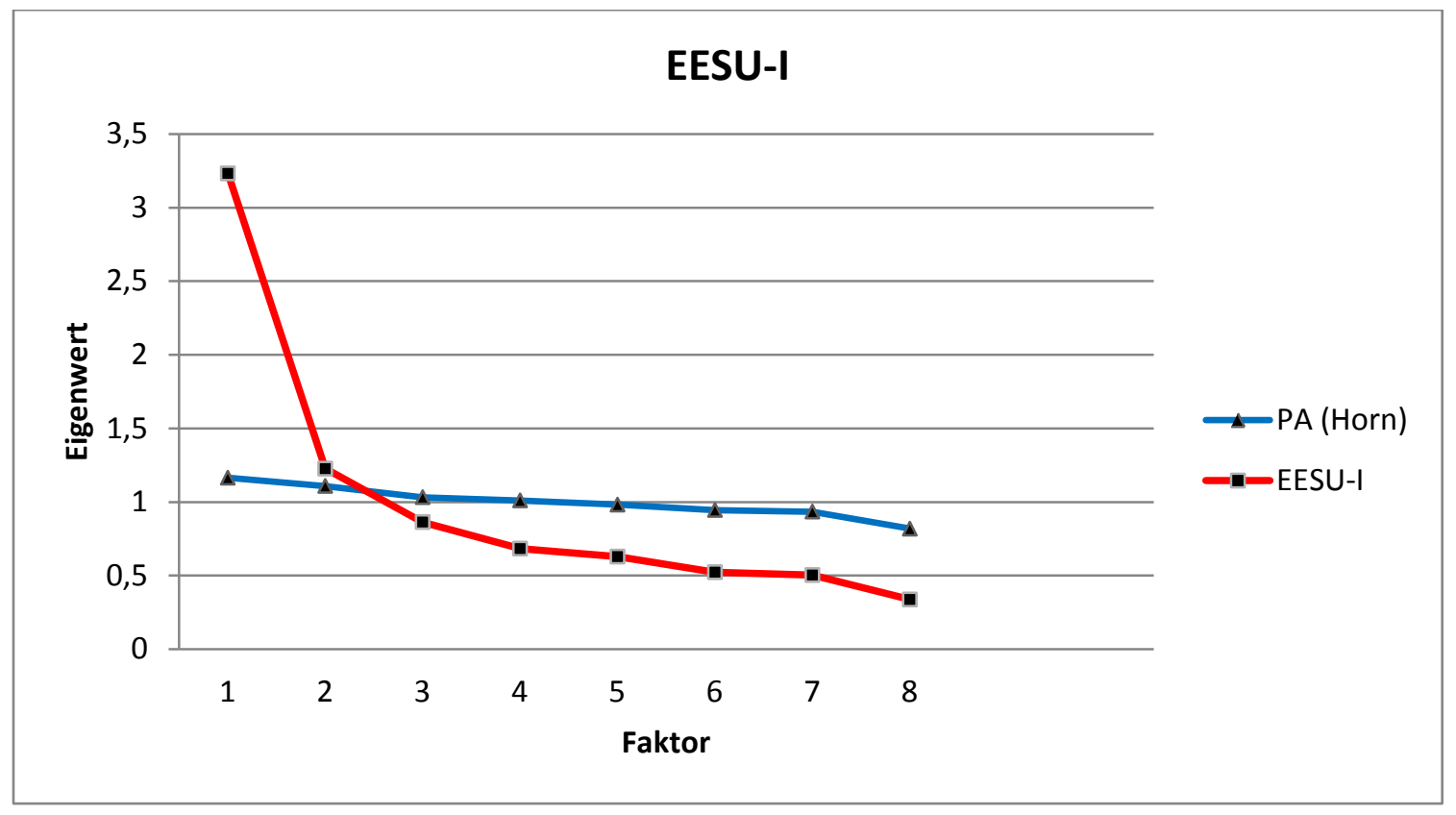

Abb. 19: Darstellung des Eigenwertverlaufs der Facette EESU-I in der Parallelanalyse nach Horn (1965) zu Zufallsdaten als Kriterium für die Faktorenextraktion 


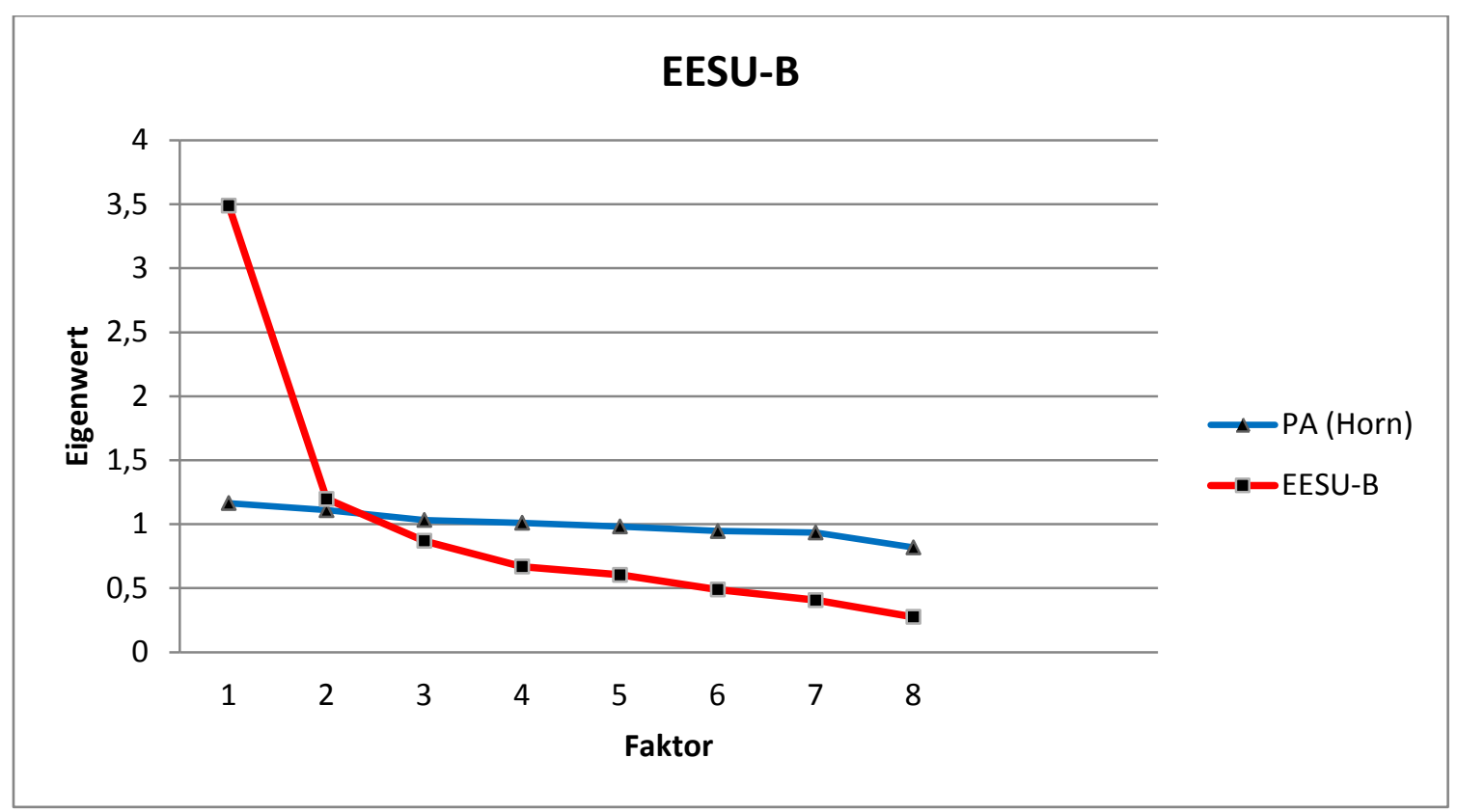

Abb. 20: Darstellung des Eigenwertverlaufs der Facette EESU-B in der Parallelanalyse nach Horn (1965) zu Zufallsdaten als Kriterium für die Faktorenextraktion

Ein weiteres eher pragmatisches Kriterium für die Extraktion stellt die durch die Faktoren aufgeklärte Varianz dar. Ein Faktor bzw. die Faktoren sollten mindestens $50 \%$ der Gesamtvarianz bzw. 80\% der geschätzten gemeinsamen Varianz aufklären soll (Floyd \& Widaman, 1995). Aufgrund der geringen Varianzaufklärung mit nur einem Faktor (EESU-S: 36,318\%; EESU-I: 40,402\%; EESU-B: 43,613\%) und den vorliegenden Indizien der zuvor aufgeführten Extraktionsverfahren, wird eine zweifaktorielle Struktur der drei Facetten des Erhebungsinstruments evasiver Sportunterricht angenommen. Die aufgeklärte Varianz liegt dann im annehmbaren Bereich (EESU-S: 55,921\%; EESU-I: 57,668\%; EESU-B: 56,947\%) Gestützt wird diese Entscheidung von Fabrigar et al. (1999), die eine Überfaktorisierung im Vergleich zu einer Unterfaktorisierung als unproblematischer bezeichnen. Auch Field (2005) empfiehlt, lieber zu viele als zu wenige Faktoren zu extrahieren. Ergänzend muss jedoch hinzugefügt werden, dass nahezu alle Items untereinander Korrelationen aufweisen und daher gemeinsam einen starken inhaltlichen Faktor prägen, der aufgrund seiner komplexen Struktur jedoch nicht in befriedigendem Maß die Daten repräsentiert. Der zweite Faktor kann in seiner Bedeutsamkeit nicht mit dem ersten Faktor gleichgesetzt werden, da durch ihn deutlich weniger Varianz aufgeklärt wird.

\subsubsection{Faktorenrotation}

Mathematische Kriterien, die zur Bestimmung der Faktoren angewendet werden, führen meist nicht zu inhaltlich interpretierbaren Faktoren, da durch die Zielsetzung, maximale Varianz aufzuklären, meist viele Variablen auf dem ersten Faktor laden (Bortz, 
2005). Um eine inhaltliche Interpretation der extrahierten Faktoren zu ermöglichen, ist es meist notwendig, ein Rotationsverfahren anzuwenden, bei dem die Faktorladungen durch eine Drehung des Faktorraumes optimiert werden, um eindeutigere Ladungsmuster im Sinne einer Einfachstruktur zu erhalten. Eine Einfachstruktur liegt dann vor, wenn Items lediglich auf einem Faktor eine hohe Ladung aufweisen (Moosbrugger \& Schermelleh-Engel, 2007). Die Varianz der aus der Extraktion hervorgegangenen Faktoren wird auf die rotierten Faktoren umverteilt (Bortz, 2005). Auch bei der Faktorenrotation unterscheidet man zwischen verschiedenen Verfahren, die sich im Wesentlichen in die Gruppen der orthogonalen bzw. rechtwinkligen und obliquen bzw. schiefwinkligen Techniken unterteilen lassen. Während man bei orthogonalen Rotationsverfahren von der Unkorreliertheit der Faktoren ausgeht, um unabhängig voneinander interpretierbare Faktoren zu erhalten, werden bei den obliquen Verfahren Korrelationen der Faktoren untereinander zugelassen (Moosbrugger \& Schermelleh-Engel, 2007).

Wie bereits bei der Faktorenextraktion deutlich wurde, liegen miteinander korrelierende Faktoren vor, die aufgrund ihrer geringen Anzahl einer graphischen Rotation, mit der Absicht eine Einfachstruktur per Hand zu erzielen, unterzogen wurden. Aufgrund der Korrelation zwischen den beiden Faktoren, wird von einer orthogonalen Rotation Abstand genommen und mit Direct Oblimin eine oblique Technik eingesetzt, bei der die beiden Achsen schiefwinklig zueinander stehen. Nach Arminger (1979) gibt es kein Patentrezept bei der Wahl von $\delta$ (Delta), das den Grad der Schiefwinkligkeit bestimmt. Man kann jedoch von der Faustregel ausgehen, dass man bei starken Korrelationen zwischen den Faktoren maximal einen Wert von 0.8 wählen sollte, liegen niedrige Korrelationen vor, sollte man einen Wert von minimal -4.0 wählen, was schon fast einer orthogonalen Stellung entspricht (Janssen \& Laatz, 2007). Neben den korrelativen Betrachtungen spielen vor allem aber auch inhaltliche Überlegungen eine Rolle bei der Faktorenrotation und der Festlegung von $\delta$.

Die unrotierten Ladungsdiagramme deuten auf korrelative Beziehungen der beiden Faktoren hin, denen durch eine oblique Rotation entsprochen wird. Anhand der Ladungsdiagramme der Hauptkomponentenanalyse kann man erkennen, inwieweit eine Modifikation des Achsenkreuzes notwendig ist, um eine bessere Repräsentation der Variablen durch die Faktoren zu errreichen. Zielsetzung ist, dass sich möglichst viele Punkte bzw. Variablen in maximal möglicher Nähe der Achsen befinden. Um den Grad der Schiefwinkligkeit zu bestimmen, wurde im konkreten Fall ein mittleres Korrelationsniveau angenommen und zusätzlich wurde je Faktor eine Leitvariable ${ }^{11}$ gewählt bzw. eine Ausrichtung am Punktecluster vorgenommen (Bortz, 2005; Bühner, 2004).

\footnotetext{
${ }^{11}(\mathrm{v} 18.2 \& \mathrm{v} 18.7, \mathrm{v} 19.2 \& \mathrm{v} 19.7, \mathrm{v} 20.2 \& \mathrm{v} 20.7)$
} 
Durch einen $\delta$-Wert von -2,2 wird der vorliegenden Korrelation entsprochen und die ausgewählten Leitvariablen werden am besten durch die beiden Faktoren repräsentiert, was man in den Abbildungen 21 bis 23 an der Nähe zu der den Faktor repräsentierenden Linien sehen kann. Wie man anhand der Abbildungen erkennen kann, wurde jeweils Item 2 für Faktor 1 und Item 7 für den zweiten Faktor als Leitvariable gewählt. Während in der ersten Facette (Abb. 21) die Punktewolken im Komponentendiagramm noch relativ weit streuen, verdichten sich die Faktoren in den beiden anderen Facetten zu kompakteren und leichter interpretierbareren Faktoren (siehe Abb. 22 und 23).

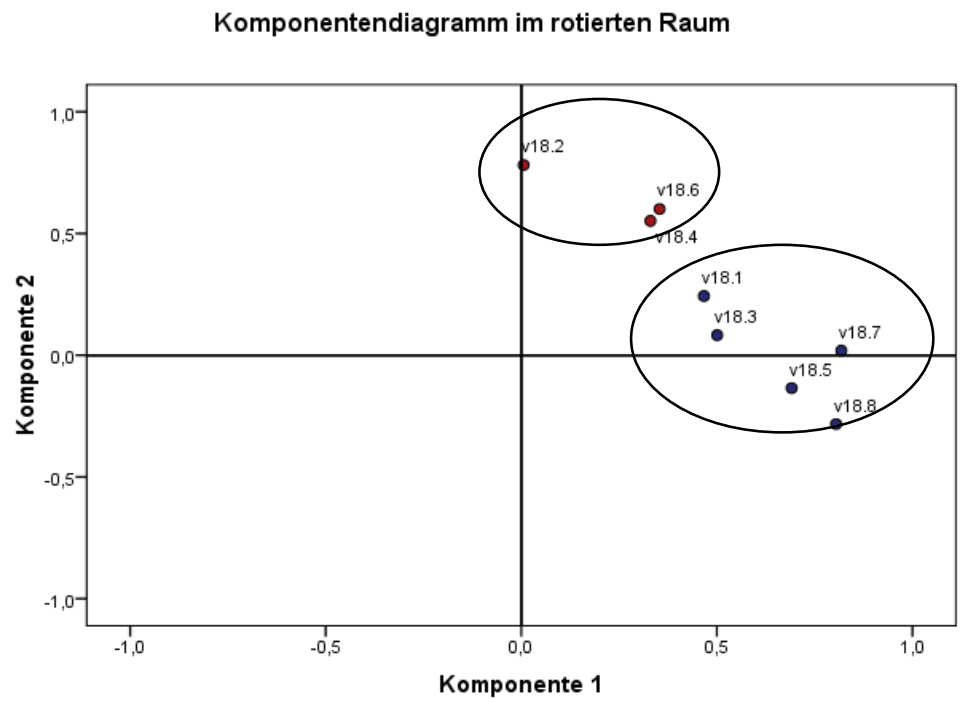

Abb. 21: Ladungsdiagramm EESU-S: Direct-Oblimin (Delta $=-2,2$ )

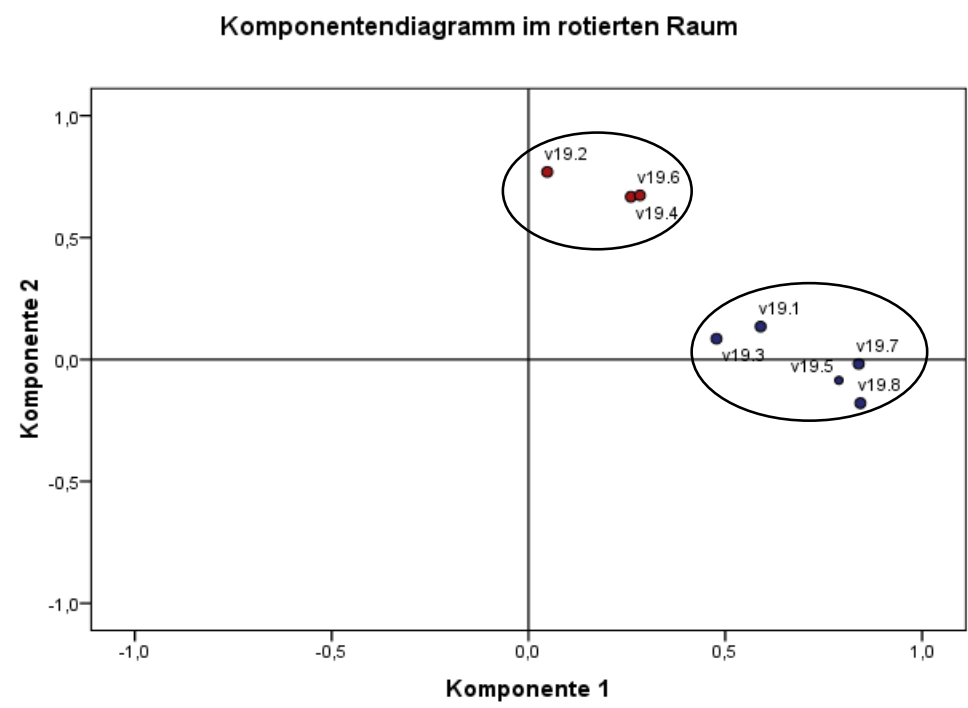

Abb. 22: Ladungsdiagramm EESU-I: Direct-Oblimin (Delta $=-2,2)$ 
Komponentendiagramm im rotierten Raum

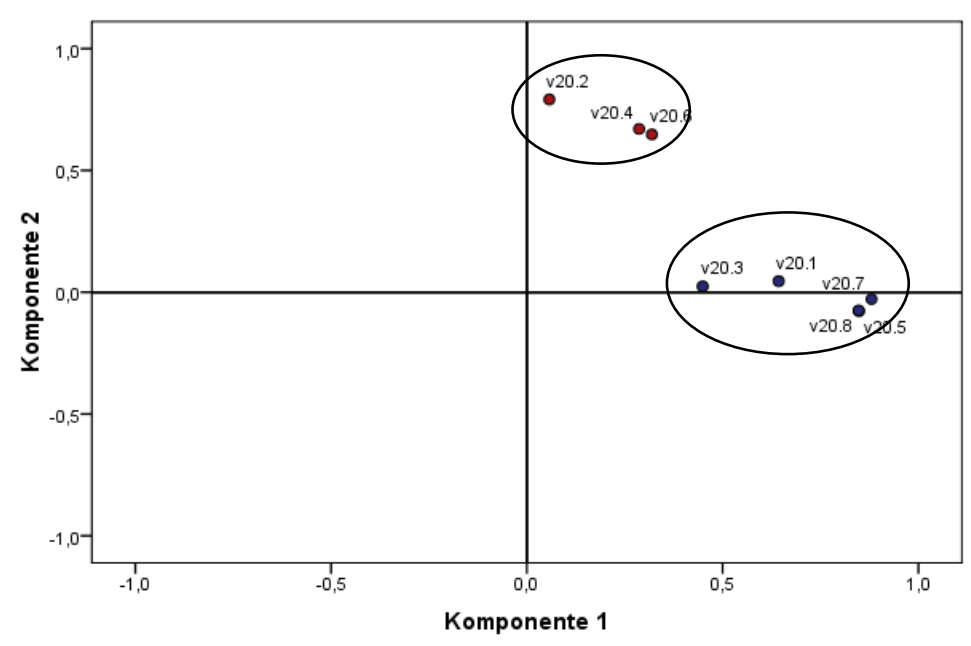

Abb. 23: Ladungsdiagramm EESU-B: Direct-Oblimin (Delta $=-2,2$ )

In Tabelle 10 sind die Faktorladungen der rotierten Lösung sowie die Kommunalitäten der Items der Einzelfacetten ${ }^{12}$ zusammengefasst. In einer Stichprobe dieser Größenordnung sind bereits Faktorladungen zwischen .091 und .105 als signifikant anzusehen. Aufgrund der Fehlerwahrscheinlichkeit sollten diese Ad-hoc-Werte jedoch verdoppelt werden, wodurch ein Grenzwert zwischen .182 und .210 als Mindestmaß resultiert. Als statistisch bedeutend wird trotz der deutlich milderen stichprobengrößenabhängigen kritischen Mindestwerte meist der Wert von .300 (z.B. Kline, 1999) oder der von Stevens (1992) vorgeschlagene Wert von .400 sowohl statistisch als auch psychologisch signifikant angesehen (Bühner, 2004; Field, 2005). Für diese Untersuchung wurden dementsprechend Ladungen von .400 als Mindestwert angenommen. Niedrigere Ladungen werden in der Tabelle nicht dargestellt, um eine größere Übersichtlichkeit zu gewährleisten. Wie sich in der Tabelle 10 zeigt, führt dieses Kriterium, über dir drei Facetten evasiven Unterrichtens hinweg, zu eindeutigen Verteilungen der Ladungen auf die beiden Faktoren.

\footnotetext{
${ }^{12}$ S: Im Bezug auf den Umgang mit Schülerinnen und Schülern, I: Im Bezug auf Inhalte im Sportunterricht, B: Im Bezug auf äußere Rahmenbedingungen... (Vorschriften und Erlasse, Beziehungen zum Kollegium/zur Schulleitung, Zeitordnungen, Notengebung, materielle Ausstattung).
} 
Tab. 10: Faktorladungen, Kommunalitäten für die Items des EESU: Extraktionsmethode: Hauptkomponentenanalyse. Rotationsmethode: Oblimin mit Kaiser-Normalisierung (Delta=-2,2). A) Die Rotation ist in 22 Iterationen konvergiert. B) Die Rotation ist in zwölf Iterationen konvergiert. C) Die Rotation ist in zehn Iterationen konvergiert. Faktorladungen unter 400 werden der Übersichtlichkeit halber in der Tabelle nicht abgebildet.

\begin{tabular}{|c|c|c|c|c|}
\hline \multirow{2}{*}{\multicolumn{2}{|c|}{ Itemfortsetzung }} & \multicolumn{2}{|c|}{ Faktoren } & \multirow{3}{*}{$\begin{array}{c}h^{2} \\
.329\end{array}$} \\
\hline & & \multirow{2}{*}{$\frac{1}{.467}$} & \multirow[t]{2}{*}{2} & \\
\hline \multirow{3}{*}{$\begin{array}{l}\text {...schlage ich lieber mir vertraute Wege ein, bevor ich bei Neuem das Risiko eingehe zu } \\
\text { scheitern. }\end{array}$} & $\mathrm{S}$ & & & \\
\hline & 1 & .590 & & .405 \\
\hline & B & .643 & & .430 \\
\hline \multirow{3}{*}{$\begin{array}{l}\text {...suche ich mir umgehend eine größere Herausforderung, wenn ich ein Ziel erreicht } \\
\text { habe. }\end{array}$} & $\mathrm{S}$ & & .781 & .612 \\
\hline & 1 & & .769 & .612 \\
\hline & B & & .791 & .651 \\
\hline \multirow{3}{*}{...lasse ich ein Problem erst einmal „vor sich hinköcheln“, bevor ich es anpacke. } & $\mathrm{S}$ & .501 & & .276 \\
\hline & 1 & .478 & & .255 \\
\hline & B & .449 & & .207 \\
\hline \multirow{3}{*}{...nehme ich schwierige Situationen, in denen viel von mir abhängt, gerne an. } & $\mathrm{S}$ & & .552 & .496 \\
\hline & 1 & & .667 & .597 \\
\hline & B & & .670 & .625 \\
\hline \multirow{3}{*}{...achte ich bewusst darauf, Schwierigkeiten zu vermeiden. } & $\mathrm{S}$ & .691 & & .453 \\
\hline & 1 & .789 & & .597 \\
\hline & B & .848 & & .693 \\
\hline \multirow{3}{*}{...suche ich mir gern Herausforderungen und gehe dafür auch Risiken ein. } & $\mathrm{S}$ & & .601 & .582 \\
\hline & 1 & & .673 & .626 \\
\hline & B & & .648 & .623 \\
\hline \multirow{3}{*}{...gehe ich lieber auf Nummer sicher, um Ärger zu vermeiden. } & $\mathrm{S}$ & .818 & & .677 \\
\hline & 1 & .839 & & .697 \\
\hline & B & .880 & & .764 \\
\hline \multirow{3}{*}{...habe ich das Ziel, nicht anzuecken. } & $\mathrm{S}$ & .805 & & .624 \\
\hline & 1 & .843 & & .670 \\
\hline & B & .847 & & .692 \\
\hline
\end{tabular}

\subsection{Interpretation der beiden Faktoren}

Anhand der exploratorischen Faktorenanalyse stellte sich heraus, dass es sich bei dem EESU um ein zweidimensionales Konstrukt handelt, bei dem der erste Faktor deutlich dominiert. Einschränkend muss hinzugefügt werden, dass diese Struktur zwar stabil in allen drei Facetten vorliegt, eine einfaktorielle Lösung jedoch aufgrund der nicht eindeutigen Faktorenextraktion und der korrelativen Zusammenhänge der beiden Faktoren nicht völlig ausgeschlossen ist. Der erste Faktor, der von insgesamt fünf Items gebildet wird (Items 1, 3, 5, 7 und 8) ist gekennzeichnet durch Variablen, die den inhaltlichen Fokussierungen der Prävention, der Handlungssicherheit und dem -aufschub entsprechen. In diesen Items wird hauptsächlich die Tendenz deutlich, die an anderer Stelle schon als „muddling through", das „sich durchwursteln“ beschrieben wurde. Es wird 
die Neigung erfasst, den Unterricht präventiv so auszulegen, dass man bereits in der Planung möglichst alles Neue und Risikobergendes ausspart und lieber zu Altbewährtem greift. Im Unterricht wird versucht, Schwierigkeiten bewusst zu vermeiden. Tauchen dennoch Probleme in dem bewusst auf Handlungssicherheit und Reibungslosigkeit ausgelegten Unterricht auf, werden diese in der Hoffnung ignoriert, dass sie sich von alleine lösen.

Der zweite Faktor, der von insgesamt drei Items gebildet wird (Items: 2, 4 und 6) erfasst die Bereitschaft, herausfordernde Situationen anzunehmen und stellt damit eher eine motivationale Komponente dar. In diesem Faktor wird die Tendenz erfasst, mit welchem Anspruch und Engagement Lehrer an ihren eigenen Unterricht gehen. Er drückt neben dem individuellen Entwicklungsstreben durch das Aufnehmen neuer Ziele nach Teilerfolgen auch das Eingehen von Risiken für lohnenswerte unterrichtliche Aktivitäten (Inhalte, Methoden etc.) aus. Zudem wird erfasst, inwieweit Lehrkräfte dazu bereit sind, herausfordernde Situationen anzunehmen, deren Erfolg bzw. Scheitern im Wesentlichen von dem eigenen Einsatz, dem Engagement und dem individuelles Können und Wissen abhängig ist.

\subsection{Reliabilität}

Für die Validierung eines Fragebogens ist es neben der Faktorenanalyse sinnvoll und notwendig, die Reliabilität(en) des Instrumentes bzw. dessen Bestandteilen zu bestimmen. Die Reliabilität gibt die Genauigkeit bzw. die Zuverlässigkeit der erhobenen Daten an, indem berechnet wird, wie genau im Durchschnitt der gemessene Wert dem wahren Wert entspricht (Bortz, 2005; Janssen \& Laatz, 2007). Die Schätzung der inneren Konsistenz, wie sie in dieser Untersuchung vorgenommen wurde, wird durch den Koeffizienten Cronbachs Alpha angegeben. Liegen wie im Fall des neu konstruierten Erhebungsinstruments zur Erfassung evasiver Tendenzen im Sportunterricht mehrere Subskalen vor, sind für diese separat Reliabilitätswerte zu bestimmen. In Tab. 11 bis 13 sind neben Cronbachs Alpha für jede der drei Facetten zusätzlich die Trennschärfen der Einzelitems angeben. 
Tab. 11. Trennschärfen der Einzelitems, Reliabilitäten der Facette EESU-S

\begin{tabular}{|c|c|c|c|}
\hline Im Bezug auf den Umgang mit Schülerinnen und Schülern... & $r_{i, t}$ & $r_{i, t(F 1)}$ & $r_{i, t(F 2)}$ \\
\hline $\begin{array}{l}\text {...schlage ich lieber mir vertraute Wege ein, bevor ich bei Neuem das Risiko eingehe zu } \\
\text { scheitern. }\end{array}$ & .407 & .354 & --- \\
\hline ...suche ich mir umgehend eine größere Herausforderung, wenn ich ein Ziel erreicht habe. & .293 & --- & .373 \\
\hline ...lasse ich ein Problem erst einmal „vor sich hinköcheln“, bevor ich es anpacke. & .367 & .351 & --- \\
\hline ...nehme ich schwierige Situationen, in denen viel von mir abhängt, gerne an. & .452 & --- & .424 \\
\hline ....achte ich bewusst darauf, Schwierigkeiten zu vermeiden. & .409 & .421 & --- \\
\hline ...suche ich mir gern Herausforderungen und gehe dafür auch Risiken ein. & .498 & --- & .467 \\
\hline ...gehe ich lieber auf Nummer sicher, um Ärger zu vermeiden. & .628 & .623 & --- \\
\hline ...habe ich das Ziel, nicht anzuecken. & 427 & .487 & --- \\
\hline & & $\alpha=69,0$ & $\alpha=61,2$ \\
\hline & & \multicolumn{2}{|c|}{$\alpha$ (gesamt) $=74,1$} \\
\hline
\end{tabular}

Tab. 12. Trennschärfen der Einzelitems, Reliabilitäten der Facette EESU-I

\begin{tabular}{|c|c|c|c|}
\hline Im Bezug auf Inhalte... & $r_{i, t}$ & $r_{i, t(F 1)}$ & $r_{i, t(F 2)}$ \\
\hline $\begin{array}{l}\text {...schlage ich lieber mir vertraute Wege ein, bevor ich bei Neuem das Risiko eingehe zu } \\
\text { scheitern. }\end{array}$ & .488 & .462 & --- \\
\hline ...suche ich mir umgehend eine größere Herausforderung, wenn ich ein Ziel erreicht habe. & .348 & --- & .452 \\
\hline ...lasse ich ein Problem erst einmal „vor sich hinköcheln“, bevor ich es anpacke. & .368 & .356 & --- \\
\hline ...nehme ich schwierige Situationen, in denen viel von mir abhängt, gerne an. & .484 & --- & .502 \\
\hline ...achte ich bewusst darauf, Schwierigkeiten zu vermeiden. & .544 & .574 & --- \\
\hline ...suche ich mir gern Herausforderungen und gehe dafür auch Risiken ein. & .506 & --- & .530 \\
\hline ...gehe ich lieber auf Nummer sicher, um Ärger zu vermeiden. & 642 & .656 & --- \\
\hline ...habe ich das Ziel, nicht anzuecken. & .539 & .604 & --- \\
\hline & & $\alpha=76,1$ & $\alpha=68,1$ \\
\hline & & \multicolumn{2}{|c|}{$\alpha$ (gesamt) $=78,6$} \\
\hline
\end{tabular}

Tab. 13. Trennschärfen der Einzelitems, Reliabilitäten der Facette EESU-B

\begin{tabular}{|c|c|c|c|}
\hline $\begin{array}{l}\text { Im Bezug auf äußere Bedingungen...(Vorschriften und Erlasse, Beziehungen zum } \\
\text { Kollegium/zur Schulleitung, Zeitordnungen, Notengebung, materielle Ausstat- } \\
\text { tung) }\end{array}$ & $r_{i, t}$ & $r_{i, t(F 1)}$ & $r_{i, t(F 2)}$ \\
\hline $\begin{array}{l}\text {...schlage ich lieber mir vertraute Wege, ein bevor ich bei Neuem das Risiko eingehe zu } \\
\text { scheitern. }\end{array}$ & .500 & .487 & --- \\
\hline ...suche ich mir umgehend eine größere Herausforderung, wenn ich ein Ziel erreicht habe. & .360 & --- & .464 \\
\hline ...lasse ich ein Problem erst einmal „vor sich hinköcheln“, bevor ich es anpacke. & .329 & .316 & --- \\
\hline ...nehme ich schwierige Situationen, in denen viel von mir abhängt, gerne an. & .508 & --- & .534 \\
\hline ...achte ich bewusst darauf, Schwierigkeiten zu vermeiden. & .635 & .676 & --- \\
\hline ...suche ich mir gern Herausforderungen und gehe dafür auch Risiken ein. & .522 & --- & .538 \\
\hline ...gehe ich lieber auf Nummer sicher, um Ärger zu vermeiden. & .708 & .736 & --- \\
\hline ...habe ich das Ziel, nicht anzuecken. & .632 & .668 & --- \\
\hline & & $\alpha=79,4$ & $\alpha=69,6$ \\
\hline & & \multicolumn{2}{|c|}{$\alpha$ (gesamt)= 81,1 } \\
\hline
\end{tabular}


Field (2005) sieht einen Mindestwert für die Trennschärfe von .300 vor, der bei 23 der 24 Variablen erreicht wird. Für den Gesamtfragebogen mit den drei Facetten wird eine interne Konsistenz mit einem Cronbachs Alpha von 90,8 erreicht. Die Trennschärfen insgesamt bzw. der beiden Faktoren $\left(r_{i, t(F)}\right.$ und $\left.r_{i, t\left(F_{2}\right)}\right)$ liegen zwischen $r_{i, t}=.293$ und $r_{i, t}$ =.736. Auch die Reliabilitäten der beiden Faktoren liegen in allen drei Facetten im stabil befriedigenden Bereich, wobei hier die erste Facette (EESU-S) deutlich schlechtere Werte erreicht als die beiden anderen Facetten (EESU-I und EESU-B), was sich auch in der Gesamtfacetten-Reliabilität mit einem Cronbachs Alphavon 74,1 gegenüber 78,6 (EESU-I) und 81,1 (EESU-B) zeigt.

\subsection{Validität}

In diesem Abschnitt wird, nachdem zuvor die faktorielle Struktur sowie die Messgenauigkeit überprüft wurden, geklärt, ob das neu entwickelte Instrument überhaupt das misst, was es messen soll. Die Kriterien der Inhaltsvalidität sollen durch die theoretische Herleitung in Kapitel 4 gewährleistet werden. Ferner wurden in Form einer kommunikativen Validierung durch Stipendiaten des Graduiertenkollegs etwaige Hindernisse und Widersprüchlichkeiten in dem Konstrukt im Vorfeld beseitigt. Praktisch erfolgt die Überprüfung der Konstruktvalidität durch die Berechnung von Korrelationen zwischen den Testwerten des Erhebungsinstruments zur Erfassung evasiver Strategien im Sportunterricht und anderen erhobenen Variablen, zwischen denen begründete Annahmen über Höhe und Richtung der Zusammenhänge bestehen. Bei hohen Zusammenhängen spricht man von konvergenter Validität, liegen keine bzw. nur geringe Korrelationen vor, spricht man von diskriminanter Validität (Hartig, Frey, Jude, 2007). Zur Bestimmung der Konstruktvalidität erfolgte die Berechnung der Korrelationskoeffizienten zwischen dem zu validierenden Konstrukt und verschiedenen Instrumenten bzw. Subskalen. In der zweiten Pilotierungsphase wurden Skalen eingesetzt, die aus untersuchungsökonomischen Gründen in der Hauptuntersuchung nicht erneut genutzt wurden, die dennoch zu Validierungszwecken herangezogen werden sollen.

Als bedeutsam für die Validität des neuen Instruments werden vor allem die eingesetzten Subskalen des Stressverarbeitungsfragebogens (SVF 120) von Janke, Erdmann und Kallus (1984) angesehen. Während bei den konstruktfernen Skalen „Bagatellisierung", "Schuldabwehr"13 und "Ablenkung" zu erwarten ist, dass keine bzw. nur geringe Zusammenhänge vorliegen, werden bei den Skalen Flucht, Resignation und Vermeidung bedeutsame Korrelationen erwartet. Bei den Subskalen des AVEM (Arbeitsbezogene Verhaltens- und Erlebensmuster) von Schaarschmidt \& Fischer (1996) werden substan-

\footnotetext{
${ }^{13}$ Die Skalen Bagatellisierung und Schuldabwehr wurden nur in der zweiten Pilotierung und nicht in der Hauptuntersuchung eingesetzt.
} 
zielle Korrelationen zwischen dem neuen Instrument und den Skalen "offensive Problembewältigung" und "Resignationstendenz bei Misserfolg" erwartet. Weiterhin ist davon auszugehen, dass evasives Verhalten im Unterricht mit der Überzeugung, durch das eigene Verhalten etwas Erwünschtes zu erreichen, negativ korreliert. Zudem ist zu erwarten, dass bei Personen mit geringer Selbstwirksamkeitserwartung es unwahrscheinlicher ist, dass ein Handlungsversuch unternommen wird, wodurch deutliche Zusammenhänge zu der Selbstwirksamkeitserwartung, erfasst durch die Skala "WIRKLEHR" von Schmitz und Schwarzer (2000) bedingt sind. In Tabelle 14 sind die Zusammenhänge zwischen den drei Facetten des Erhebungsinstruments zur Erfassung des evasiven Verhaltens sowie der Gesamtskala mit den ausgewählten Instrumenten bzw. Skalen aufgeführt.

Tab. 14: Produkt-Moment Korrelationskoeffizienten zur Validierung des EESU (Zusammenhänge ab einem mittleren Korrelationskoeffizienten fett markiert)

\begin{tabular}{|c|c|c|c|c|}
\hline & EESU-S & EESU-I & EESU-B & EESU-SIB \\
\hline SVF 120 - Resignation & $.420 * *$ & $.400 * *$ & $.348 * *$ & $.429 * *$ \\
\hline SVF 120 - Bagatellisierung & -.080 & -.110 & -.076 & -.081 \\
\hline SVF 120 - Schuldabwehr & .148 & .015 & -.053 & .050 \\
\hline SVF 120 - Vermeidung & $.353^{* *}$ & $.384^{* *}$ & $.352^{* *}$ & $.405 * *$ \\
\hline AVEM - Resignationstendenz bei Misserfolg & $.322^{* *}$ & $.338 * *$ & $.318 * *$ & $.361 * *$ \\
\hline AVEM - Offensive Problembewältigung & $-.433 * *$ & $-.400 * *$ & $-.398 * *$ & $-.462 * *$ \\
\hline
\end{tabular}

In Tabelle 14 wird deutlich, dass die Datenlage im weitesten Sinne den Erwartungen entspricht. Als bedeutsam sind neben den Skalen „Resignation“, „Flucht" und „Vermeidung" des Stressverarbeitungsfragebogens von Janke, Erdmann \& Kallus (1984) die Skala „Offensive Problembewältigung“ des AVEM-Fragebogens von Schaarschmidt \& Fischer (1996) und die Skala zur Erfassung der "Lehrerselbstwirksamkeitserwartung“ von Schmitz und Schwarzer (2000). Die Korrelationen mit den Skalen „Flucht", „Vermeidung", "Resignation", "offensive Problembewältigung“ und „Lehrerselbstwirksamkeitserwartung" sind nach der Orientierungshilfe zur Interpretation von Korrelationskoeffizienten von Brosius (2004) als mittlere Zusammenhänge zu bezeichnen. Die konstruktfernen Subskalen „Bagatellisierung" und "Schuldabwehr" des Stressverarbeitungsfragebogens weisen erwartungsgemäß keine statistisch bedeutsamen Zusammenhänge, die Dimension „Ablenkung“ allenfalls sehr schwache Korrelationen auf. Neben der zuvor beschriebenen konvergenten Validität ist damit auch diskriminante Validität des neuen Konstrukts gegeben. 
Einen weiteren klaren Hinweis auf die Validität des Instruments liefern die signifikanten Mittelwertunterschiede der AVEM-Muster im Bezug auf die beiden durch das Instrument erfassten Faktoren. Beim ersten Faktor, der eher auf die Prävention von Problemen und der Wahrung von Handlungssicherheit ausgerichtet ist, ergaben sich überzufällige Unterschiede im evasiven Verhalten zwischen dem Muster Gesundheit und Burnout sowie Anstrengung, nicht aber zum Muster Schonung. D.h. dass Angehörige des Musters Gesundheit signifikant weniger evasiv im Sinne von Konfliktprävention und Wahrung an Handlungssicherheit agieren, als die Angehörigen der Muster Burnout und Anstrengung. Die Analyse des zweiten Faktors, der motivationale Aspekte des evasiven Verhaltens erfasst, zeigt statistisch bedeutsame Unterschiede zwischen den Angehörigen des Musters Gesundheit und des Musters Burnout sowie zwischen dem Muster Gesundheit und Schonung, nicht aber zum Muster Anstrengung (sieh Tab. 15).

Tab. 15: EESU-Summenscore-Differenzen der AVEM-Muster nach Faktoren

\begin{tabular}{|c|c|c|c|c|c|c|c|}
\hline \multirow{12}{*}{$\begin{array}{l}\text { EESU-SIB } \\
\text { (Faktor 1) }\end{array}$} & Schonung & Burnout & $-5,33^{*}$ & \multirow{12}{*}{$\begin{array}{l}\text { EESU-SIB } \\
\text { (Faktor 2) }\end{array}$} & \multirow[t]{3}{*}{ Schonung } & Burnout & $-2,65^{*}$ \\
\hline & & Anstrengung & $-1,22$ & & & Anstrengung & 1,40 \\
\hline & & Gesundheit & 2,44 & & & Gesundheit & $2,42 *$ \\
\hline & \multirow[t]{3}{*}{ Burnout } & Schonung & $5,33^{*}$ & & Burnot & Schonung & $2,65^{*}$ \\
\hline & & Anstrengung & $4,10^{*}$ & & & Anstrengung & $4,06^{*}$ \\
\hline & & Gesundheit & $7,76^{*}$ & & & Gesundheit & $5,07 *$ \\
\hline & \multirow[t]{3}{*}{ Anstrengung } & Schonung & 1,22 & & Anstrengung & Schonung & $-1,40$ \\
\hline & & Burnout & $-4,10^{*}$ & & & Burnout & $-4,06 *$ \\
\hline & & Gesundheit & $3,66^{*}$ & & & Gesundheit & 1,01 \\
\hline & \multirow[t]{3}{*}{ Gesundheit } & Schonung & $-2,44$ & & Gesundheit & Schonung & $-2,42 *$ \\
\hline & & Burnout & $-7,76^{*}$ & & & Burnout & $-5,07^{*}$ \\
\hline & & Anstrengung & $-3,66^{*}$ & & & Anstrengung & $-1,01$ \\
\hline
\end{tabular}

\subsection{Durchführung der Hauptuntersuchung}

Die Hauptuntersuchung fand in Form einer postalischen Befragung statt. Um auf möglichst schnelle Art und Weise an Informationen über Schulen und die dort unterrichtende Sportlehreranzahl zu gelangen, wurde im Rahmen einer Online-Recherche die jeweilige Größe des Sportkollegiums ermittelt. Dies geschah entweder durch absolute Angaben seitens der Schule oder indirekt anhand von strukturellen Daten, wie Schüleroder Gesamtlehrerzahl, die eine Schätzung der Anzahl der Sportlehrkräfte ermöglichte. Nach einer Vorankündigung der Studie per E-Mail an die ausgewählten Gymnasien in Niedersachsen wurden Informationsfaltflyer (siehe Anhang III) in der zuvor ermittelten Anzahl an die Schulen zur Weitergabe an die Sportlehrkräfte gesendet. In einem Begleitschreiben an die Schulleitung bzw. den Schulsportkoordinator wurde das weitere 
Vorgehen erläutert und es wurde um Mithilfe gebeten. In dem Informationsflyer wurden die Sportlehrkräfte über Sinn und Zweck sowie den zeitlichen Ablauf der Untersuchung informiert, außerdem wurde über eine individuelle Rückmeldung der Ergebnisse informiert, die optional angeboten wurde. Diese Rückmeldung konnte dann anhand eines sechsstelligen Codes auf dem Fragebogen anonym ermöglicht werden.

Insgesamt wurden etwa 2.000 Fragebögen an die 190 ausgewählten Gymnasien in Niedersachsen gesendet, wobei pro Schule je ein bis zwei Fragebögen mehr als die ermittelte Sportlehrerzahl beigelegt wurden, um eine Nichtteilnahmen aufgrund von fehlendem Untersuchungsmaterial zu verhindern. Insgesamt besitzt die Stichprobe also ein Potenzial von ca. 1.600 Rückmeldungen. Die Fragebogen wurden gebündelt an die einzelnen Schulen samt Begleitschreiben und vorfrankiertem Rückumschlag versandt, sodass die Rücksendung zentral über die Schulen erfolgte. Selbstverständlich wurde den Sportlehrkräften auch ermöglicht, ihren Fragebogen separat direkt an die Universität zurückzuschicken.

Nach zweimaligem Versenden von Erinnerungsmails an die Schulleiter bzw. Schulsportkoordinatoren konnten insgesamt 705 Fragebögen in die Datenauswertung einbezogen werden, was einer Rücklaufquote von rund $44 \%$ entspricht. Aufgrund des Umfangs des Fragebogens ist die Rücklaufquote als gut einzustufen.

Im Anschluss an die Eingabe und umfassende Auswertung der Daten erfolgte eine individuelle Ergebnisrückmeldung an die einzelnen Lehrkräfte, die ihren Fragebogen mit einem Code versehen haben. Die teilnehmenden Schulen erhielten zudem einen kurzen Ergebnisbericht, der an die Schulleitung versandt wurde.

\subsection{Weitere eingesetzte Instrumente}

Im weiteren Verlauf dieses Kapitels werden die Instrumente vorgestellt, die im Rahmen dieser Untersuchung zusätzlich zu dem neukonstruierten Erhebungsinstrument zum Einsatz kamen. Neben dem AVEM-Fragebogen von Schaarschmidt und Fischer (1996) sind der Fragebogen zu Kontroll- und Kompetenzüberzeugungen (FKK) von Krampen (1991), der Stressverarbeitungsbogen (SVF 120) von Janke, Erdmann und Kallus (1994), die Ungewissheitstoleranzskala (UGTS) von Dalbert (1999) der Fragebogen Berufliche Belastung (BEL) von Enzmann und Kleiber (1989), die Achievement-MotiveScale (AMS-kurz) von Engeser (2004), das Oldenburger-Burn-Out-Inventar (OLBI) von Demerouti et al. (2003), die Skala zur Lehrerselbstwirksamkeitserwartung (WIRKLEHR) von Schmitz und Schwarzer (2000) sowie weitere persönliche, berufsbiographische und didaktisch-pädagogische Daten, Einstellungen und Überzeugungen der Lehrkräfte zu nennen. 


\subsubsection{Arbeitsbezogene Verhaltens- und Erlebensmuster (AVEM)}

Ausgangspunkt für die Entwicklung des Fragebogens zur Erfassung der Arbeitsbezogenen Verhaltens- und Erlebensmuster war die Tatsache, dass berufliche Tätigkeiten und Anforderungen unter vergleichbaren Bedingungen von verschiedenen Personen in unterschiedlicher Art und Weise wahrgenommen und erlebt werden. Dependent von der zuvor beschriebenen Tatsache werden auch unterschiedliche Reaktionsweisen an den Tag gelegt. Diese auf Gefühle, Einstellungen und Kompetenzen zurückgehende Auseinandersetzung mit beruflichen Anforderungen bezeichnet man typischerweise als Bewältigung. Ziel der Potsdamer Arbeitsgruppe um Schaarschmidt war die Konstruktion eines diagnostischen Instruments zur Identifizierung verschiedener Muster der Bewältigung beruflicher Belastung. Schaarschmidt und seinen Kollegen ging es einerseits darum, die relativ konstanten und übergreifenden Verhaltens- und Erlebensmerkmale als Folgen vergangener Beanspruchungen abzubilden, andererseits aber auch darum, die individuellen Voraussetzungen für die Bewältigung zukünftiger Anforderungen zu identifizieren. Die Überlegungen basieren auf der Ressourcen-Theorie von Antonovsky und dem transaktionalen Stress- und Coping-Konzept von Lazarus (Schaarschmidt \& Fischer 1996; 2001). Mit dem AVEM-Fragebogen wurde ein diagnostisches Verfahren entwickelt, bei dem mittels Selbsteinschätzung die individuelle Wahrnehmung von Arbeit und Beruf erfasst werden kann sowie durch Profilvergleiche bestimmte Risikogruppen identifiziert werden können (van Dick, 2001).

\subsubsection{Komponenten des AVEM}

In den Fragebogen finden drei verschiedene Aspekte der Bewältigung Einzug. Diese aus einer faktorenanalytischen Strukturierung hervorgehenden Sekundärfaktoren sind neben dem beruflichen Engagement und der Widerstandskraft gegenüber Belastungen auch konkomitierende Emotionen. Im Fragebogen wird die Bewältigung durch insgesamt elf Dimensionen konkretisiert, die sich wiederum aus insgesamt 66 Items zusammensetzen und gesundheitsrelevante Merkmale berücksichtigen.

\section{Berufliches Engagement}

Um den inhaltlichen Bereich des beruflichen Engagements möglichst genau abzubilden, greifen Schaarschmidt und Fischer (1996) auf Dimensionen zurück, die erfassen, inwieweit wohldosiertes Engagement als Voraussetzung für Erfolgserleben im Beruf zu sehen ist und damit eine gesundheitsförderliche Bedingung vorliegt. Durch Engagement wird in starkem Maße Sinnerleben und eine aktive Lebenseinstellung ausgedrückt (Schaarschmidt, 2005a). 
Folgende Dimensionen werden im Rahmen dieses Sekundärfaktors erfasst:

- Subjektive Bedeutsamkeit der Arbeit

- Beruflicher Ehrgeiz

- Verausgabungsbereitschaft

- Perfektionsstreben

- Distanzierungsfähigkeit

Während bei der Dimension "Subjektive Bedeutsamkeit der Arbeit" der Stellenwert der Arbeit im persönlichen Leben erfasst wird, spiegelt sich im „Beruflichen Ehrgeiz" das Streben nach Zielen und Weiterkommen im Beruf wider. Die Frage nach der Bereitschaft, die persönlichen Kräfte für die Erfüllung von Arbeitsaufgaben einzusetzen, ist Inhalt der Dimension "Verausgabungsbereitschaft", während der Anspruch an die eigene Arbeitsleistung bezüglich Sorgfalt, Güte und Zuverlässigkeit durch die Dimension „Perfektionsstreben“ erfasst wird. Inwieweit die Fähigkeit und Bereitschaft ausgeprägt ist, sich nach der Arbeit auch von dieser distanzieren zu können, macht die Dimension „Distanzierungsfähigkeit" deutlich.

\section{Widerstandskraft gegenüber Belastungen}

Merkmale, die unter dem Sekundärfaktor „Widerstandsfähigkeit gegenüber Belastungen“ zusammengefasst werden, weisen selbstverständlich Relevanz für die Bewältigung beruflicher Belastung, vor allem im Bezug auf die Gesundheit, auf. Auch diesem Sekundärfaktor ist, jedoch in negativer Polung, die "Distanzierungsfähigkeit“ zuzurechnen. Die aufgeführten Merkmale bzw. Dimensionen dieses Sekundärfaktors stellen persönliche Ressourcen dar, die dazu dienen, Stresserscheinungen zu mildern und die seelische Gesundheit zu schützen bzw. zu verbessern (Schaarschmidt \& Fischer, 1996).

Folgende Dimensionen werden im Rahmen dieses Sekundärfaktors erfasst:

- Resignationstendenz bei Misserfolg

- Offensive Problembewältigung

- Innere Ruhe und Ausgeglichenheit

- Distanzierungsfähigkeit (negative Polung)

Personen, die eine geringe Resignationstendenz bei Misserfolg haben, zeichnen sich generell durch eine optimistische Lebenshaltung aus und haben eine gering ausgeprägte Neigung, sich mit Misserfolgen abzufinden und leicht aufzugeben. In diesem Zusammenhang ist auch die offensive Bewältigung von Problemen zu nennen, da Personen mit einer ausgeprägten Widerstandsfähigkeit gegenüber Belastungen von Grund auf eine aktive und optimistische Haltung gegenüber Herausforderungen und auftretenden Problemen aufweisen. Um eine maximale Ausprägung dieses belastungsmin- 
dernden Sekundärfaktors zu erzielen, ist zudem ein hohes Maß an innerer Ruhe und Ausgeglichenheit notwendig, die ein ausgeprägtes Erleben psychischer Stabilität und des inneren Gleichgewichts inkludiert (Schaarschmidt \& Fischer, 2001).

\section{Berufsbegleitende Emotionen}

Die diesen Sekundärfaktoren prägenden Merkmale kennzeichnen den Hintergrund, vor dem sich die Bewältigung der beruflichen Anforderungen abspielt. Diese Dimensionen stellen einen psychologischen Schutzfaktor gegenüber kritischen Situationen dar. Die enthaltenen drei Dimensionen spiegeln nicht das komplette Spektrum an Emotionen wider, sondern lediglich den Bereich, der bezogen auf die Zufriedenheit und Geborgenheit beim Umgang mit beruflichen Anforderungen relevant ist. Die erfassten Merkmale sind sowohl Voraussetzungen als auch Folgen des Bewältigungsprozesses.

Folgende Dimensionen werden im Rahmen dieses Sekundärfaktors erfasst:

- Erfolgserleben im Beruf

- Lebenszufriedenheit

- Erleben sozialer Unterstützung

In der Dimension Erfolgserleben im Beruf spiegelt sich die Zufriedenheit mit dem beruflich Erreichten wider, während Lebenszufriedenheit die generelle Zufriedenheit mit der gesamten, und damit auch privaten Lebenssituation ausdrückt. Unter dem Erleben sozialer Unterstützung ist das Vertrauen in die Unterstützung durch nahstehende Menschen und dem damit verbundenen Gefühl der sozialen Geborgenheit zusammengefasst (Schaarschmidt \& Fischer, 1996).

\subsubsection{Musterstruktur}

Um die Möglichkeiten des AVEM vollständig zu nutzen, werden über die einzelnen Dimensionen hinausgehend Dependenzen und Independenzen in Form komplexer Profile erstellt. Basierend auf den elf Dimensionen wurde im Rahmen einer Clusteranalyse nach homogenen Gruppen gesucht, die sich in den Ausprägungen der Subskalen entsprachen. Die Analysen wurden nach der Methode Quick-Cluster mit fallweisem Ausschluss der Datensätze und Aktualisierung der Clusterzentren durch gleitende Mittelwertbildung nach jeder Fallzuordnung durchgeführt. Die Clusterlösung mit vier Mustern wurde reklassifiziert und kreuzvalidiert (siehe Abb. 24) und erwies sich in der Validierungsstudie von van Dick (2001) bis auf einige wenige Einschränkungen als replizierbar (Sarges, 2000). 


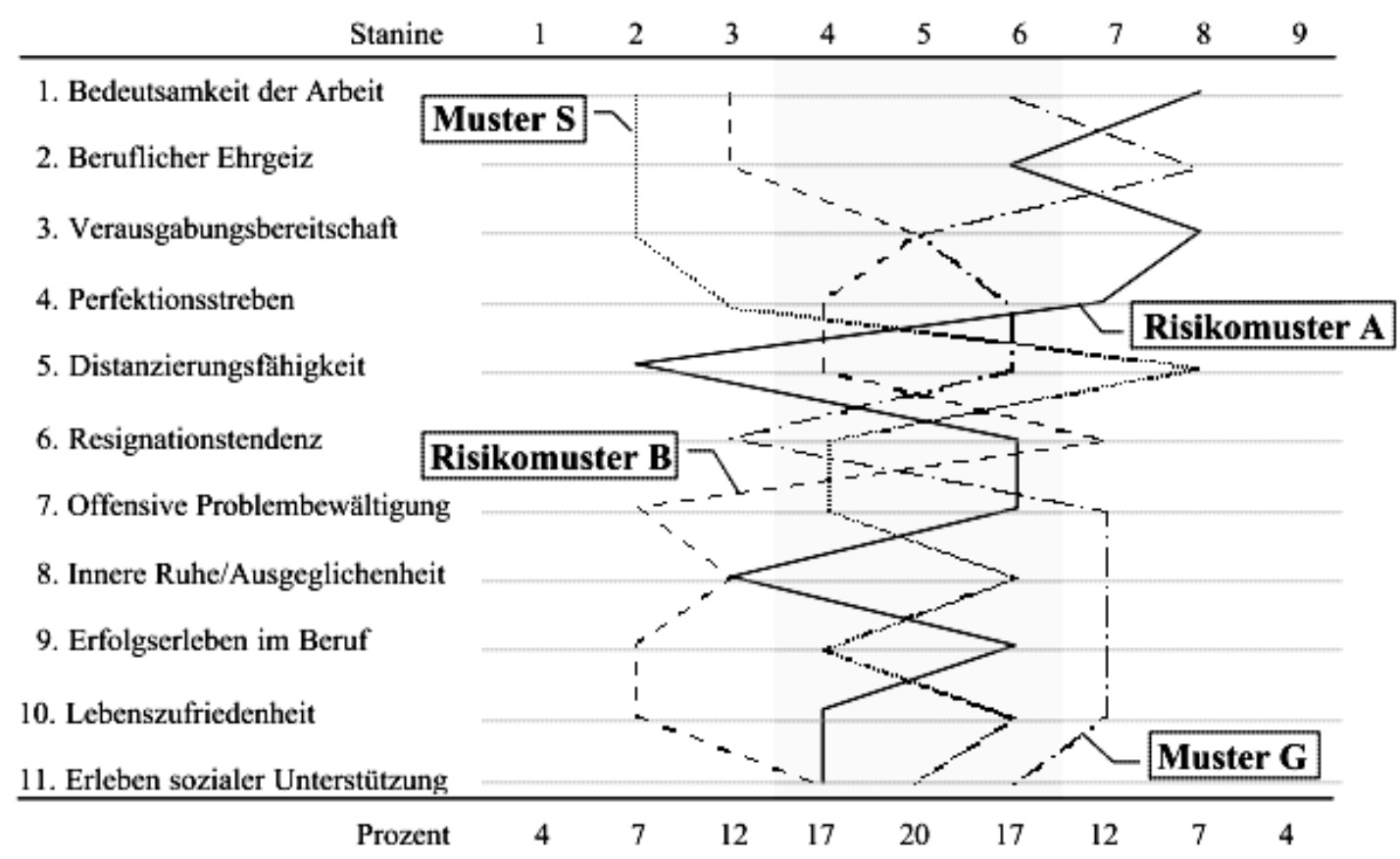

Abb. 24: Profilverlauf der vier Bewältigungsmuster (in der oberen Zeile ist eine Stanine-Skala abgetragen, anhand der unteren Zeile kann man ablesen, mit welcher prozentualen Häufigkeit bestimmte Skalenwerte vorkommen) (Schaarschmidt, 2005a, S. 24)

\section{Muster G - Gesundheit}

Schaarschmidt (2005a) bezeichnet dieses Muster als die gesundheitsfördernde Variante des arbeitsbezogenen Verhaltens und Erlebens des Berufs. In den Dimensionen, die dem arbeitsbezogenen Engagement zuzuordnen sind, können hohe, wenn auch nicht extreme Werte festgestellt werden, wobei der berufliche Ehrgeiz am stärksten ausgeprägt ist. Hier findet sich eine Passung im Bezug auf die Ausgewogenheit von Engagement und der trotzdem vorliegenden Distanzierungsfähigkeit. Im zweiten Sekundärfaktor, der Widerstandsfähigkeit gegenüber Belastungen, liegen ebenfalls positive Ausprägungen der einzelnen Merkmale, mit einem geringen Maß an Resignationstendenz bei Misserfolg, den höchsten Werte in der offensiven Problembewältigung, verbunden mit innerer Ruhe und Ausgeglichenheit vor. Höchstwerte werden im Bereich der berufsbegleitenden Emotionen erreicht. Angehörige des Musters $\mathrm{G}$ haben ein ausgeprägtes berufliches Erfolgserleben, sind zufrieden mit ihrem Leben und weisen hohe Werte im Erleben sozialer Unterstützung durch Personen des privaten Umfelds auf. Man kann zusammenfassen, dass Personen, die diesem Muster angehören, berufliches Engagement und die notwendige Regeneration durch das Ziehen von Grenzen, dem Verfolgen realistischer Zielsetzungen und dem Haushalten mit den eigenen Kräften gesundheitsdienlich ausbalancieren (Heyse, 2008). 


\section{Muster S - Schonung}

Bereits mit der Bezeichnung dieses Musters wird das Verhältnis der Zugehörigen zu ihrer Arbeit gekennzeichnet. Angehörige dieses Musters weisen niedrige Werte in der Bedeutsamkeit der Arbeit, der Verausgabungsbereitschaft und dem Perfektionsstreben auf (Schaarschmidt \& Fischer, 1996). Angehörige dieses Musters sind meist diejenigen, die das negative öffentliche Bild des Berufsstandes Lehrer prägen. Höchste Werte liegen im Bereich Distanzierungsfähigkeit vor, Klagen über berufsbezogene Probleme sind daher meist weit weniger ausgeprägt als bei den anderen Mustern, solange die Zurückhaltung nicht aus Verbitterung oder Frustration resultiert (Heyse, 2008). Sowohl geringe Resignationstendenz, hohe Ausprägungen der inneren Ruhe und Ausgeglichenheit als auch Lebenszufriedenheit deuten auf ein durchweg positives Lebensgefühl hin. Meist werden die Schwerpunkte im Privaten d.h. im Freizeitbereich gesetzt und die Arbeit hat nur nachgeordneten Stellenwert. Das berufliche Engagement beschränkt sich meist auf den sogenannten Dienst nach Vorschrift. Dieses Verhalten kommt entweder aufgrund des Bruches des psychologischen Arbeitsvertrages (siehe Kap. 4.9.5) oder fehlenden beruflichen Herausforderungen zustande. Neben der Persönlichkeit der Individuen können auch institutionelle Ursachen wie z.B. defizitäre Arbeitsbedingungen zu der Ausprägung des Verhaltens führen (Schaarschmidt, 2005a) Mit diesem Muster kann am ehesten von einer wie in Kapitel 4.9.5 beschriebenen inneren Kündigung gesprochen werden.

\section{Muster A- Anstrengung}

In dieser Musterausprägung ist vor allem das überhöhte Engagement auffallend. In der Bedeutsamkeit der Arbeit, dem Perfektionsstreben und der Verausgabungsbereitschaft werden Werte erreicht, wie in keinem anderen Muster. Zum Risikomuster wird dieses Profil durch die fehlende Distanzierungsfähigkeit, d.h. das Vermögen, Abstand von Arbeitsproblemen zu gewinnen, um Erholungsprozesse zu ermöglichen. Verbunden mit dem ausgeprägten Engagement geht aus den Profilverläufen dieses Risikomusters eine verminderte Widerstandsfähigkeit gegenüber Belastungen hervor sowie eine niedrige Ausprägung in der Lebenszufriedenheit und dem Erleben sozialer Unterstützung, sodass den hohen beruflichen Anstrengungen offensichtlich keine entsprechende Anerkennung gezollt wird (Schaarschmidt, 2005a). Siegrist (1991) bezeichnet dieses Missverhältnis aus Aufwand und Ertrag als Gratifikationskrise.

\section{Muster B - Burnout}

Der zweite als Risikomuster bezeichnete Profilverlauf kennzeichnet einen Typus, der im Bereich Engagement geringe Ausprägungen aufweist, besonders in der subjektiven Bedeutsamkeit der Arbeit und dem beruflichen Ehrgeiz. Im Bereich Engagement be- 
stehen also keine bedeutenden Unterschiede zwischen dem Burnout-Muster und dem eben bereits beschriebenen Schonungstyp. Unterschiedlich ist aber in großem Maße die Ausprägung der Distanzierungsfähigkeit von Arbeitsproblemen. Während für die Angehörigen des Schonungsmusters private und außerberufliche Dinge höhere Priorität haben, schlagen sich diejenigen, die dem Profilverlauf Burnout zugeordnet werden, den ganzen Tag mit den Problemen und Sorgen der Arbeit herum. Die negative Grundeinstellung schlägt sich in einer hohen Resignationstendenz bei Misserfolg, der eher defensiven Problembewältigung, einer generellen Lebensunzufriedenheit sowie einem Mangel an sozialem Rückhalt deutlich nieder (Schaarschmidt \& Fischer, 1996). Hoffnungslosigkeit sowie ein Gefühl von Niedergeschlagenheit zählen zu den häufig beobachteten Anzeichen dieses Musters. Um auch dem präventiven Anliegens des AVEM nachzukommen ist zu beachten, dass dieses Muster und auch das Muster $A$ in engem Zusammenhang mit körperlichen, vor allem aber auch psychischen Störungen und Erkrankungen zu sehen ist (Schaarschmidt, 2005a).

\subsubsection{Fragebogen zu Kontroll- und Kompetenzüberzeugungen (FKK)}

Per Definition sind Kontrollüberzeugungen generalisierte Erwartungen einer Person darüber, ob durch eigenes Verhalten Ereignisse im Leben beeinflusst werden können oder nicht. Einzug in die psychologische Forschung fanden die Kontrollüberzeugungen in bipolarer Ausrichtung durch Rotters soziale Lerntheorie in den 60er Jahren des 20. Jahrhunderts (Rotter \& Mulry, 1965). Während man im deutschsprachigen Raum meist von internaler und externaler Kontrolle spricht lautet die englische bzw. amerikanische Bezeichnung generalized expectancy for internal versus external control of reinforcement oder kurz locus of control. Ein von Levenson (1972) vorgelegter Fragebogen (IPC) zur Erfassung von drei Aspekten generalisierter Kontrollüberzeugungen, wurde möglichst wortgetreu in die deutsche Sprache übersetzt, konnte jedoch aufgrund mangelnder Reliabilität, seiner Lebensferne, adressatenspezifischer partieller Nichtanwendbarkeit sowie der fehlenden Anbindung an grundlegende Theorien den wissenschaftlichen Ansprüche nicht genügen und regte zu einer Weiter- bzw. Neuentwicklung an (Krampen, 1991). Der daraufhin von Krampen (1991) konstruierte Fragebogen zu Kompetenz- und Kontrollüberzeugungen (FKK) basiert auf dem handlungstheoretischen Partialmodell der Persönlichkeit, da sowohl allgemeinpsychologische als auch persönlichkeitspsychologische Ansätze theoretisch fundiert integriert werden. Der Fragebogen stellt somit eine Weiterentwicklung und Differenzierung der sozialen Lerntheorie von Rotter dar (Rotter \& Mulry, 1965). Die Zielsetzung des Verfahrens ist es, eine generalisierte Kompetenz- und Kontingenzerwartungen zu erfassen und situationsunspezifisch relativ stabil abzubilden. Krampen (1991) hält den Einsatz des FKK zu 
Diagnosezwecken dann für sinnvoll, wenn sich Personen in Handlungs- und Lebenssituationen befinden, die sich nur in geringem Ausmaß kognitiv strukturieren lassen und für die Person mehrdeutig, neu oder unklar sind und daher keine spezifische habitualisierte Handlungsweisen ausgeprägt sind. Durch das Verfahren können Vorhersagen über Handlungs- und Verhaltenstendenzen getroffen werden.

\section{Darstellung der Skalen}

Zu den bereits im IPC enthaltenen Skalen „Internalität", „Externalität durch Fatalismus" und "Externalität durch subjektive Machtlosigkeit" kommt im FKK mit dem "Selbstkonzept eigener Fähigkeiten" aufgrund faktorenanalytischer Befunde eine zusätzliche Skala hinzu, sodass insgesamt vier Skalen (siehe Tab. 16) mit jeweils acht Items vorliegen. Neben der jeweiligen Primärskala mit je acht Items können zudem zwei Sekundärskalen mit jeweils 16 Items abgeleitet werden. Aus den Skalen „Selbstkonzept eigener Fähigkeiten“ und „Internalität" wird die Skala „Selbstwirksamkeit" und aus "Sozialer Externalität" und "fatalistischer Externalität" die Sekundärskala „Externalität" gebildet. Schlussendlich ist als letzter Schritt eine Tertiärskala vorgesehen, bei der alle Items im Sinne „Internalität versus Externalität" zusammengefasst werden. Die Items werden mittels einer sechsstufigen Likert-Skala von sehr falsch bis sehr richtig eingeschätzt.

Tab. 16: Überblick über die Skalen des FKK

\begin{tabular}{|c|c|c|}
\hline Kurzbezeichnung & Benennung der Skala & Itemzahl \\
\hline FKK-SK & $\begin{array}{l}\text { Selbstkonzept eigener Fähigkeiten } \\
\text { (generalisiertes Selbstkonzept eigener Fähigkeiten) } \\
\text { Die generalisierte Erwartung, dass in Handlungs- und Lebenssituationen zumin- } \\
\text { dest eine Handlungsmöglichkeit zur Verfügung steht }\end{array}$ & 8 \\
\hline FKK-I & $\begin{array}{l}\text { Internalität } \\
\text { (Internalität in generalisierten Kontrollüberzeugungen) } \\
\text { Subjektiv bei der eigenen Person wahrgenommene Kontrolle über das eigene } \\
\text { Leben und über Ereignisse der personenspezifischen Umwelt. }\end{array}$ & 8 \\
\hline FKK-P & $\begin{array}{l}\text { Soziale Externalität } \\
\text { (sozial bedingte Externalität in generalisierten Kontrollüberzeugungen; „powerful } \\
\text { others control“) } \\
\text { Generalisierte Erwartung, dass wichtige Ereignisse im Leben vom Einfluss anderer } \\
\text { („,mächtiger") Personen abhängen. }\end{array}$ & 8 \\
\hline FKK-C & $\begin{array}{l}\text { Fatalistische Externalität } \\
\text { (fatalistisch bedingte Externalität in generalisierten Kontrollüberzeugungen } \\
\text { "chance control" } \\
\text { Generalisierte Erwartung, dass das Leben und Ereignisse vom Schicksal, Glück, } \\
\text { Pech und dem Zufall abhängen. }\end{array}$ & 8 \\
\hline \multicolumn{3}{|l|}{ Sekundärskalen } \\
\hline FKK-SKI & $\begin{array}{l}\text { Selbstwirksamkeit } \\
\text { (generalisierte Selbstwirksamkeitsüberzeugungen) }\end{array}$ & 16 \\
\hline FKK-PC & $\begin{array}{l}\text { Externalität } \\
\text { (generalisierte Externalität in Kontrollüberzeugungen) }\end{array}$ & 16 \\
\hline \multicolumn{3}{|l|}{ Tertiärskala } \\
\hline FKK-SKI-PC & $\begin{array}{l}\text { Internalität versus Externalität } \\
\text { (generalisierte Internalität versus Externalität in Kontrollüberzeugungen) }\end{array}$ & 32 \\
\hline
\end{tabular}




\section{Bedeutung der Skalenwerte}

Da die Kurzbeschreibungen, die die Einzelskalen repräsentieren nur ein sehr undifferenziertes Bild bezüglich der psychologischen und diagnostischen Bedeutung der Ausprägungen der Skalenwerte liefern, soll an dieser Stelle die Bedeutung verschiedener Skalenausprägung auf Individuumsebene dargestellt werden. Personen, die einen hohen Wert im „Selbstkonzept eigener Fähigkeiten“ aufweisen, sehen in Problemsituationen sich selbst als handlungsfähig, wählen eine angemessene Handlungsmöglichkeit aus mehreren vorliegenden Alternativen aus und erleben sich als selbstsicher, aktiv, tatkräftig und ideenreich. In neuartigen Situationen fühlen sie sich selbstsicher, was sich unter anderem durch hohes Selbstvertrauen ausdrückt. Personen, die einen niedrigen Wert in dieser Skala erreichen, stellen sowohl in der Selbstsicherheit, dem Selbstvertrauen, dem Ideenreichtum und den vorliegenden Handlungsalternativen den Gegenpol zu den Verhaltens- und Persönlichkeitseigenschaften der Personen mit einem hohen Wert dar. Hohe Werte in der Primärskala "Internalität" symbolisieren die Ansicht des Individuums, Situationen im Leben selbst erfolgreich beeinflussen zu können, die eigenen Interessen zu vertreten und so das gewünschte bzw. angestrebte Ziel zu erreichen. Erfolg und Misserfolg in wichtigen Dingen im Leben werden als abhängig vom eigenen Handeln und Tun gesehen und somit genau wie soziale Interaktionsprozesse als regulierbar erlebt. Ist der Bereich Internalität hingegen in geringem Maß ausgeprägt, wird der eigene Einfluss auf den Erfolg in wichtigen Lebensereignissen als nachgeordnet angesehen und die eigenen Handlungen werden als wenig effektiv erlebt, da hierdurch in der eigenen Wahrnehmung selten das Geplante und Gewünschte erreicht wird. Wenn Personen einen hohen Wert in der Skala "Soziale Externalität" aufweisen, sehen sie ihr Leben als im hohen Maße abhängig vom Einfluss anderer Menschen und sind demzufolge auch stark emotional anhängig vom bzw. durch das Verhalten Dritter. Sie sind weniger durchsetzungsfähig und Gefühle der Ohnmacht und Hilflosigkeit bestimmen den Alltag. Geringe Ausprägungen hingegen deuten auf durchsetzungsfähige Persönlichkeiten hin, die ihren Alltag selbst bestimmen und sich nicht durch andere beeinträchtigen lassen und sich nicht von ihnen abhängig fühlen. Weisen Personen einen hohen Wert in der Skala "fatalistische Externalität" auf, sehen sie ihr Leben als im großen Maße vom Schicksal abhängig und gehen davon aus, sich nicht vor Pech und Unglück schützen zu können. Stellen sich Erfolge ein, werden diese als vom Glück abhängig eingestuft und nicht der eigenen Leistung zugeschrieben. Menschen mit einer deutlichen Ausprägung in diesem Teilbereich der Persönlichkeit denken wenig rational und sind schicksalsgläubig. Individuen, die einen niedrigen Wert in der Skala erzielen, glauben nicht an Schicksal und Zufall und sehen Möglichkeiten, sich vor 
Pech zu schützen und eigene Ziele durch eigene Anstrengung zu erreichen. Glück und Unglück spielen in der Attribuierung kaum eine Rolle und das Handeln weist stark rationalen Charakter auf (Krampen, 1991).

Im Bezug auf die Frage, ob bestimmte Ausprägungen bestimmter Variablen als erstrebenswert anzusehen sind, kann gefolgert werden, dass Personen mit einem ausgeprägten Selbstkonzept eigener Fähigkeiten, einer hohen Internalität sowie einer geringen Ausprägung der externalen Überzeugungen als psychisch stabiler und unabhängiger im Urteilsvermögen sind als Personen mit umgekehrt ausgeprägten Kompetenzund Kontrollüberzeugungen. Menschen, die eher dem ungünstigen Ausprägungsprofil zuzuordnen sind, nehmen den persönlichen Handlungsspielraum und die Selbstwirksamkeit als gering wahr, fühlen sich ihrem Schicksal oder anderen Menschen ausgeliefert und suchen oft nicht einmal nach Möglichkeiten, eine effektive Problemlösung zu erzielen. Weiterhin muss jedoch bedacht werden, dass extreme Ausprägungen sowohl von Internalität als auch von Externalität zu unrealistischen Erwartungen führen wie Omnipotenzgefühle und Selbstüberschätzung auf der einen und Hilflosigkeits- und Ohnmachtsgefühle auf der anderen Seite. Erstrebenswert sollte es daher sein, ein realistisches Selbstbild zu entwickeln, das durch einen Fundus an situationsangemessenen Strategien gesichert wird.

\subsubsection{Stressverarbeitungsfragebogen (SVF 120)}

„Der SVF120 dient der Erfassung der individuellen Tendenz, unter Belastung verschiedene Stressverarbeitungsweisen einzusetzen. Diese Verarbeitungsweisen sind durch 20 Subtests mit jeweils 6 Feststellungen (Items) definiert" (Janke \& Erdmann, 1997, S. 4). Der Stressverarbeitungsbogen von Janke, Erdmann und Kallus (1984) ist ein Verfahren, das von der Theorie von Lazarus (1966) und den darin formulierten Zusammenhängen zwischen Coping und den Reaktionen bzw. Folgen der Belastung inspiriert wurde. Grundlage für die Messung der Verarbeitung von Stress ist die Annahme, dass es sich bei den eingesetzten Maßnahmen um habituelle Personenmerkmale handelt, d.h. dass eine gewisse Zeitkonstanz gegeben ist und dass die Stressverarbeitungsstrategien einer Person insoweit bewusst sind, dass sie diese in Fragebogenform verbalisieren bzw. einschätzen kann (Janke, Erdmann \& Kallus, 1984).

Einleitend durch einen Satz, der eine verallgemeinernde Belastungssituation konstruiert, ${ }^{14}$ wird in jeweils sechs Items pro Subtest auf einer fünfstufigen Likert-Skalierung von gar nicht bis sehr wahrscheinlich eingeschätzt, inwieweit die im Item angegebene Art zu reagieren dem eigenen Verhalten entspricht. Die 20 Subtests werden eingeteilt

\footnotetext{
${ }^{14}$ Wenn ich durch irgendetwas oder irgendjemand beeinträchtigt, innerlich erregt oder aus dem Gleichgewicht gebracht worden bin...
} 
in Positiv-Strategien, Negativ-Strategien und singuläre Strategien. Im Rahmen der vorliegenden Untersuchung wurden insgesamt sechs Subtetsts des SVF120 eingesetzt, die aus den drei zuvor genannten Bereichen entstammen. "Schuldabwehr", "Bagatellisierung" und "Ablenkung" werden den Positiv-Strategien zugerechnet, da sie zur Stressreduktion eingesetzt werden und prinzipiell dazu auch geeignet sein können. Während "Schuldabwehr" eine Abwehrstrategie darstellt, bei der die Verantwortung abgelehnt wird, versucht man bei „Bagatellisierung“ in Form einer Selbstinstruktion die Bedeutsamkeit der Ereignisse für sich selbst zu minimieren. „Ablenkung“ beschreibt die Tendenz, sich von der Belastung abzulenken um auf diese Art und Weise das Stresspotenzial zu vermindern. Diese Strategien sind zwar nicht als offensive Problembewältigung zu bezeichnen, eignen sich jedoch prinzipiell dazu, Belastungen zu reduzieren. Wie bereits in Kapitel 6.1.5 beschrieben, bestehen zwischen diesen Skalen und dem evasiven Verhalten, wenn überhaupt, nur sehr geringe Korrelationen. Die NegativStrategien "Flucht" und "Resignation“ sind ein Anzeichen von mangelnder Bewältigungskompetenz und führen eher zu einer Verstärkung des Stressereignisses, anstatt dieses abzumildern. In der Fluchttendenz sind einerseits resignative Elemente enthalten, die auf das Entkommen aus der Situation abzielen, andererseits fehlt jedoch auch die Bereitschaft bzw. die Fähigkeit sich mit dem Stressereignis aktiv auseinanderzusetzen. Bei dem Subtest „Resignation“ wird erfasst, inwieweit die Gefühle Hilf- und Hoffnungslosigkeit im Angesicht von Belastungssituationen verspürt werden, die zum Aufgeben von Bewältigungsbemühungen führt. Letztendlich wurde noch die Skala „Vermeidung" eingesetzt, die Janke und Erdmann (2002) den singulären Strategien zuordnet. Grundsätzlich umfasst die Skala die Tendenz bzw. den Vorsatz, Belastungen bzw. eine erneute Konfrontation mit ähnlichen Situationen zu vermeiden. Ist eine Belastungssituation durch das Individuum nicht zu kontrollieren, kann das Vermeiden eine positive Verhaltensweise darstellen, wird der Belastung jedoch aus anderen Grünen lediglich ausgewichen, führt dieses Verhalten kurz- oder längerfristig zu negativen Belastungsfolgen.

\subsubsection{Ungewissheitstoleranzskala (UGTS)}

Ungewissheit entsteht dann, wenn Personen zu wenig bzw. keine Informationen über die Entwicklung einer Situation haben. „Dies ist etwa der Fall, wenn der Eintritt zukünftiger Ereignisse ungewiss ist oder wenn der richtige Umgang mit der Situation ungewiss ist" (Dalbert, 1999, S. 4). Friedel und Dalbert (2003) fassen die Ungewissheitstoleranz als bereichsübergreifendes Persönlichkeitsmerkmal auf, durch das ein intraindividueller Umgang mit ungewissen Situationen erklärt werden kann. Personen, die sich gegenüber Ungewissheit tolerant zeigen, fassen solche Ereignisse eher als Her- 
ausforderung auf, während Ungewissheitsintolerante schnell eine Bedrohung verspüren. Im Zusammenhang damit wird auch erwähnt, dass ungwissheitsintolerante Personen dazu neigen, ungewisse Situation präventiv zu meiden bzw. zu beenden, während ungewissheitstolerante Personen durch aufsuchende Tendenzen charakterisiert sind. Dalbert (1999) entwickelte eine Skala, die anders als bisher existierende Instrumente versucht, die Ungewissheit bereichsübergreifend und ökonomisch zu erfassen. Mit acht Items, die mithilfe einer sechsstufigen Likert-Skala von stimmt genau bis stimmt überhaupt nicht reichen, stellt die Skala ein überaus ökonomisches Instrument dar, das besonders für diese Erhebung geeignet erscheint, da die Lehrsituation, vor allem im Sportunterricht, durch ein extrem hohes Maß an Ungewissheit gekennzeichnet ist.

\subsubsection{Berufliche Belastung (BEL)}

Das Instrument „Berufliche Belastung“ wurde von Enzmann und Kleiber (1989) entwickelt, um die Belastungen der konkreten beruflichen Tätigkeit zu erfassen. Insgesamt 15 Items ergeben als Gesamtskala einen Wert, der die berufliche Gesamtbelastung ausdrückt. Eine weitere Differenzierung in die Subskalen „Arbeitsüberforderung" (sechs Items), „Kontrolliertheitserleben" (drei Items) und "Arbeitsunzufriedenheit" (sechs Items) ist möglich. Die Einschätzung der formulierten Aussagen erfolgt anhand einer fünfstufigen Likert-Skalierung von trifft gar nicht zu bis trifft zu. Während in den Subskalen „Arbeitsüberforderung" und „Kontrolliertheitserleben“ ein konkreter Situationsbezug zur tagtäglichen Arbeit hergestellt wird, ist der Charakter der Skala „Arbeitsunzufriedenheit" allgemeiner gehalten und bezieht sich auf Einschätzungen des Individuums, die durch das komplette Berufsleben gekennzeichnet sind.

\subsubsection{Achievement-Motive-Scale (AMS-kurz)}

Bei der Kurzskala von Engeser (2004) handelt es sich um einen Fragebogen zur Erfassung eines motivationalen Selbstbildes in Form der Leistungsmotivation. In dieser Form der Erhebung mittels Fragebogen werden im Gegensatz zu den projektiven Verfahren selbst zugeschriebene explizite Motive, die in der eigenen Selbstwahrnehmung vorliegen, gemessen (Rheinberg, 2004). Die Skala von Engeser (2004) basiert auf der insgesamt 30 Items enthaltenden Skala von Gjesme und Nygard aus dem Jahre 1970, die 1980 von Göttert und Kuhl ins Deutsche übersetzt wurde. Motiviert durch die meist unbefriedigende Lösungsstabilität der Faktorenanalyse erstellte Engeser (2004) eine optimierte Kurzversion mit insgesamt zehn Items, die in dieser Arbeit zum Einsatz kommt. Die mit der Langversion hoch korrelierte Kurzversion liefert eine stabile zweifaktorielle Lösung, wobei, wie bei Gjesme und Nygard, ein Faktor (fünf Items) die „Hoffnung auf Erfolg“ und ein Faktor (fünf Items) die „Furcht vor Misserfolg“ erfasst. 
Die Einschätzung erfolgt anhand einer vierstufigen Likert-Skalierung von trifft gar nicht auf mich zu bis trifft völlig auf mich zu. Von besonderem Interesse in dieser Untersuchung ist die Ausprägung der „Furcht vor Misserfolg" (vgl. hierzu auch Kap. 4.5.1), die bei starker Ausprägung maladaptive Vermeidungsstrategien, wie z.B. in Form von evasivem Verhalten, erwarten lassen (Rheinberg, 2004).

\subsubsection{Oldenburger Burn-Out-Inventar (OLBI)}

Das Oldenburger Burn-Out-Inventar wurde von Demerouti et al. (2001) erstellt. Demerouti entwickelte den Fragebogen, da das bis zu diesem Zeitpunkt eingesetzte Instrument, das "Mas/ach-Burnout-Inventory" (1986) bzw. "Maslach Burnout-Inventory General Survey" (1996) von Maslach und Jackson (1986) in allen Skalen entweder einseitig negativ („Exhaustion“ und „Cynism“) oder einseitig positiv formuliert („Personal accomplishment“) waren. Aus einem psychometrischen Blickwinkel sind solche Skalen jenen unterlegen, die sowohl positiv als auch negativ gepolte Items enthalten. Immer wieder berichtete Schwächen des $\mathrm{MBI}^{15}$ führten zur Entwicklung des Oldenburger Burnout-Inventars, das sich aus zwei Subskalen („Exhaustion“ und "Disengagement from work“) zusammensetzt. „Exhaustion“ ist dabei definiert als die Konsequenz von körperlicher, emotionaler und kognitiver Anstrengung in Form einer Beanspruchungsfolge. ${ }^{16}$ Personen mit einer hohen Ausprägung fühlen sich innerlich leer, überlastet von der Arbeit, haben ein ausgeprägtes Gefühl nach einer Auszeit vom Beruf und verspüren auch im körperlichen Bereich eine Überbeanspruchung. In der Skala „Disengagement from work" wird die innere emotionale Distanzierung von der Arbeit und das Verspüren von begleitenden negativen Gefühlen gemessen. Damit verbunden sind oft motivationalen Einstellungen, z.B. dass die Arbeit uninteressant und nicht herausfordern ist, sodass der Arbeitsauftrag lediglich mechanisch abgeleistet wird. Es liegt keine bzw. nur eine gering ausgeprägte Identifikation mit dem Beruf vor. Des Weiteren sind sowohl die Items der Skala „Exhaustion“ (acht Items) als auch die der Skala „Disengagement" (acht Items), die jeweils auf einer vierstufigen Likert-Skalierung von stimmt nicht bis stimmt eingeschätzt werden, sowohl positiv als auch negativ gepolt (Demerouti et al., 2001).

\footnotetext{
${ }^{15}$ Unter anderem stellten Schwarzer und Jerusalem (1999) fest, dass die dreifaktorielle Struktur des Inventars nicht replizierbar ist und die Itemkennwerte zum Teil nicht befriedigend sind.

${ }^{16} \mathrm{Im} \mathrm{MBI}$ bzw. MBI-GS wird nur die emotionale Erschöpfung konzeptualisiert. In dieser Untersuchung (Sportlehrkräfte) ist jedoch auch die physische und die kognitive Erschöpfung von Belang, dadurch ist der Einsatz des OLBI legitimiert.
} 


\subsubsection{Skala zu Lehrerselbstwirksamkeitserwartung (WIRKLEHR)}

Auf die allgemeine Selbstwirksamkeitserwartung und die Abgrenzung zur Ergebniserwartung wurde bereit in Kapitel 4.5.2.1 ausführlich eingegangen. Da die Erwartungen der eigenen Wirksamkeit bereichsspezifisch sind (Harter, 1981), war es notwendig auch in dieser Untersuchung ein speziell auf den Lehrerberuf abgestimmtes Instrument einzusetzen. Die Skala zur Erfassung der Lehrerselbstwirksamkeitserwartung (WIRKLEHR) wurde von Schmitz und Schwarzer (1999) entwickelt und erfasst die Lehrerselbstwirksamkeitserwartung als berufsspezifische Persönlichkeitsvariable mit insgesamt zehn Items, die anhand einer vierstufigen Likert-Skalierung von stimmt nicht bis stimmt eingeschätzt werden. In dem Fragebogen von Schmitz und Schwarzer sind die Items in Bezug auf die Bereiche, die Kompetenzanforderungen an die Lehrkräfte stellen, formuliert. Neben der beruflichen Leistung sind dies die berufliche Weiterentwicklung, die soziale Interaktion mit Schülern, Eltern und Kollegen und der Umgang mit Berufsstress (Rudow, 1995). Die Items weisen mit der subjektiven Gewissheit, etwas tun zu können, auch wenn eine Barriere im Weg steht, eine eindeutige semantische Struktur auf.

\subsubsection{Persönliche, berufsbiographische und didaktisch-pädagogische Daten, Einstellungen und Überzeugungen der Lehrkräfte}

Neben dem neu konstruierten Instrument und den in Kapitel 6.6 .1 bis 6.6 .8 beschriebenen Verfahren wurden eine Reihe weitere persönliche und berufsbiographische Daten sowie didaktische Einstellungen und Überzeugungen der Lehrkräfte im Rahmen der Hauptuntersuchung abgefragt.

Neben dem Geschlecht und dem Alter sollten die Sportlehrerinnen und Sportlehrer außerdem angeben, wie lange sie bereits im Beruf tätig sind. Desweiteren wurde erfragt, ober eher extrinsische Gründe für die Wahl des Lehrerberufs vorlagen oder ob die Befragten sich zum Lehrerdasein berufen fühlten. Auch die Frage, ob die Befragten, wenn sie heute noch einmal vor der Berufsentscheidung stünden, erneut den Lehrerberuf wählen würden, ist in diesem Zusammenhang interessant. Das Beifach neben Sport, die Gesamtlehrverpflichtung und die Sportstundenanzahl stellen weitere Eckdaten dar, die im allgemeinen Bereich erhoben wurden.

Im Bereich Unterrichtsorganisation wurden die Sportlehrenden nach der Bedeutsamkeit von verschiedenen Kriterien für die Inhaltswahl gefragt, die anhand einer vierstufigen Likert-Skalierung von trifft nicht $z u$ bis trifft zu für den eigenen Unterricht eingeschätzt werden sollten. Um einen genaueres Bild vom jeweiligen Unterricht zu bekommen wurde die Durchführungshäufigkeit der populärsten Unterrichtsinhalte 
(Sportarten) erfragt und die Lehrweise ${ }^{17}$ sowie die Art der Unterrichtsorganisation ${ }^{18}$ erhoben. Außerdem schätzten die Lehrer das in ihrem Unterricht von den Schülerinnen und Schülern abverlangte technisch-taktische und konditionelle Anforderungsniveau ein. Um über Ziele und Prioritäten im Unterricht Aufschluss zu erhalten, wurden die Sportlehrer gebeten, die Bedeutsamkeit von verschiedenen Vermittlungsaspekten anzugeben. In einem weiteren Bereich wurde die Belastung bzw. die Zufriedenheit mit der Arbeit durch verschiedene Variablen erhoben. Neben der Einschätzung des individuellen Belastungspotenzials von insgesamt 16 Faktoren wurden zusätzlich Indikatoritems für die generelle und spezielle Arbeitszufriedenheit eingesetzt. Erfragt wurde die Zufriedenheit mit der Sportstundenzahl, der Spaß am Unterrichten, die erlebte Atmosphäre im Kollegium, aber auch die Gefühle und Erfahrungen aus der bisherigen Karriere sowie die Gefühle und Erwartungen, die die Lehrer haben wenn sie an ihr zukünftiges Berufsleben denken. Außerdem wurde erhoben, an wie vielen Tagen die Lehrerinnen und Lehrer krankheitsbedingt gefehlt haben und ob sie in näherer Zukunft einen Berufswechsel bzw. einen Ausstieg planen.

Des Weiteren wurde durch verschiedene Items die Affinität zu Sport erhoben. Neben der Einschätzung der sportartspezifischen theoretischen und praktischen Vermittlungskompetenz von (sehr) schlecht bis (sehr) gut, wird zu diesem Bereich auch die Häufigkeit der aktiven Teilnahme am eigenen Unterricht und die individuelle Bedeutsamkeit des Sportunterrichts im Vergleich zu anderen Fächern gezählt. Ergänzend wurden die Lehrer danach gefragt, ob sie früher bzw. derzeitig Wettkampfsport betrieben haben bzw. betreiben und ob sie einmal als Vereinstrainer tätig waren bzw. noch tätig sind. Neben einer Einschätzung des eigenen Fitnesszustandes und der eigenen privaten wöchentlichen sportlichen Aktivität, sollte zudem auch der Stellenwert von Sport im Privatleben eingeschätzt werden.

In einem letzten Bereich wurden noch einige Informationen zu dem beruflichen Engagement erhoben, indem die Sportlehrkräfte sowohl Angaben zu ihrem zeitlichen Aufwand für die wöchentliche Vor- und Nachbereitung des Sportunterrichts als auch zum Fortbildungsengagement machten.

\footnotetext{
${ }^{17}$ Induktiv oder deduktiv.

${ }^{18}$ Von stark lehrerzentriert bis schülerorientiert (fünfstufig likert-skaliert).
} 


\section{Darstellung der Ergebnisse}

In diesem Kapitel erfolgt zunächst eine deskriptive Darstellung der Stichprobe und sportunterrichtsspezifischer Aspekte, bevor die verschiedenen Variablen, Skalen und Konstrukte in den drei Untersuchungskomplexen (siehe Kap. 5) zu den Facetten des evasiven Verhaltens in Beziehung gesetzt werden. Da in dieser Arbeit erste Basisdaten zum evasiven Verhalten im Sportunterricht geliefert werden sollen, wird auf eine differenzierte Darstellung bezüglich der beiden in Kapitel 6 identifizierten Faktoren verzichtet. ${ }^{1}$ Während im ersten Untersuchungskomplex hauptsächlich personelle Faktoren und Ressourcen sowie Angaben zur Berufsbiographie und deren Zusammenhang mit der Ausprägung des evasiven Bewältigungsverhaltens herausgearbeitet werden, stellt die unterrichtliche Charakteristik und deren Korrelation mit der Ausprägung der evasiven Verhaltenstendenz den zweiten Schwerpunkt dar. Im Rahmen eines weiteren Untersuchungskomplexes werden die Zusammenhänge zwischen dem Verhalten und Erleben im bzw. von Sportunterricht im Zentrum der Aufmerksamkeit stehen, wobei auch die in Kapitel 4.9.5 dargestellten Beanspruchungsfolgen erneut fokussiert werden. Im Anschluss an die Ergebnisdarstellung der drei Untersuchungsschwerpunkte werden die Erkenntnisse jeweils in Form einer interpretativen Zusammenfassung in Beziehung zueinander gesetzt und Begründungszusammenhänge aufgedeckt.

\subsection{Stichprobe}

Die Untersuchungsstichprobe setzt sich aus insgesamt 705 Gymnasiallehrerinnen und -lehrern mit Fakultas Sport aus dem Bundesland Niedersachsen zusammen. Mit insgesamt 152 sich beteiligenden Schulen stammen die Lehrer aus einem relativ breiten Schulspektrum: Neben großen vielzügigen Gymnasien nahmen auch viele kleinere Gymnasien teil, die etwa gleichverteilt sowohl in städtischen als auch in ländlichen Gebieten angesiedelt waren.

Bei dem Geschlechterverhältnis ist eine leichte Überrepräsentanz der männlichen Lehrkräfte ( $n=379 ; 55,5 \%$ ) gegenüber den weiblichen Lehrkräften ( $n=304 ; 44,5 \%)^{2}$ festzustellen, was den vom Statistischen Bundesamt (2007a) ermittelten Daten für Lehrkräfte an Gymnasien entspricht. Auch hinsichtlich des Alters, das im Durchschnitt bei 46,06 Jahren ( $S D=11,051$ ) liegt, besteht Konformität zu den repräsentativen Daten des Statistischen Bundesamtes (2007a). In der Altersverteilung fällt auf, dass aufgrund der

\footnotetext{
${ }^{1}$ Eine Darstellung aller Ergebnisse für jeden der beiden Faktoren würde den Rahmen dieser Arbeit sprengen und würde, aufgrund der vorliegenden Korrelationen der beiden Faktoren untereinander wahrscheinlich nur einen geringen zusätzlichen Erkenntnisgewinn ermöglichen.

${ }^{2}$ Zum Umgang mit fehlenden Werten siehe Kap. 6.
} 
großen Anzahl an neu eingestellten Lehrkräften in den 70er und frühen 80er Jahren des vergangenen Jahrhunderts, die Gruppe der heute über 50-Jährigen deutlich überproportional stark vertreten ist. Insgesamt stellt sich die Altersverteilung (siehe Abb. 25) zweigipflig dar. Diese, etwas sonderbare Verteilung ergibt sich aus der geringen Anzahl von Lehrern, die aufgrund des Einstellungsstopps in den späten 1980er bzw. frühen 1990er Jahren, zum Untersuchungszeitpunkt zwischen 40 und 50 Jahre alt waren. Auffällig ist weiterhin, dass sich nur eine sehr geringe Anzahl Sportlehrer in der Phase unmittelbar vor der Pensionierung befindet. Diese Tatsache ist zum Teil dadurch zu erklären, dass trotz der Verschärfung der Bedingungen von Frühpensionierungen durch die Einführung von Abschlägen bei Dienstunfähigkeit vor dem 63. Lebensjahr aus dem Jahr 2001, kaum Lehrer tatsächlich bis zum regulären Pensionierungsalter arbeiten. ${ }^{3}$ Eine weitere Möglichkeit, die aber eher untersuchungsmethodischen Charakter aufweist, stellt, aufgrund der Freiwilligkeit der Teilnahme an der recht umfangreichen Erhebung, eine geringere Antworthäufigkeit älterer, eventuell weniger motivierter Lehrkräfte dar. Sportlehrer, die sich im letzten Abschnitt ihrer Berufskarriere befinden, könnten weniger daran interessiert sein, an Studien teilzunehmen, da eventuelle Konsequenzen und Verbesserungen, die sich aufgrund der Erhebung ergeben könnten, für sie selbst keine Relevanz mehr aufweisen. Vermutlich spielen beide Gründe zusammen eine Rolle für die geringe Antworthäufigkeit der über 60-Jährigen.

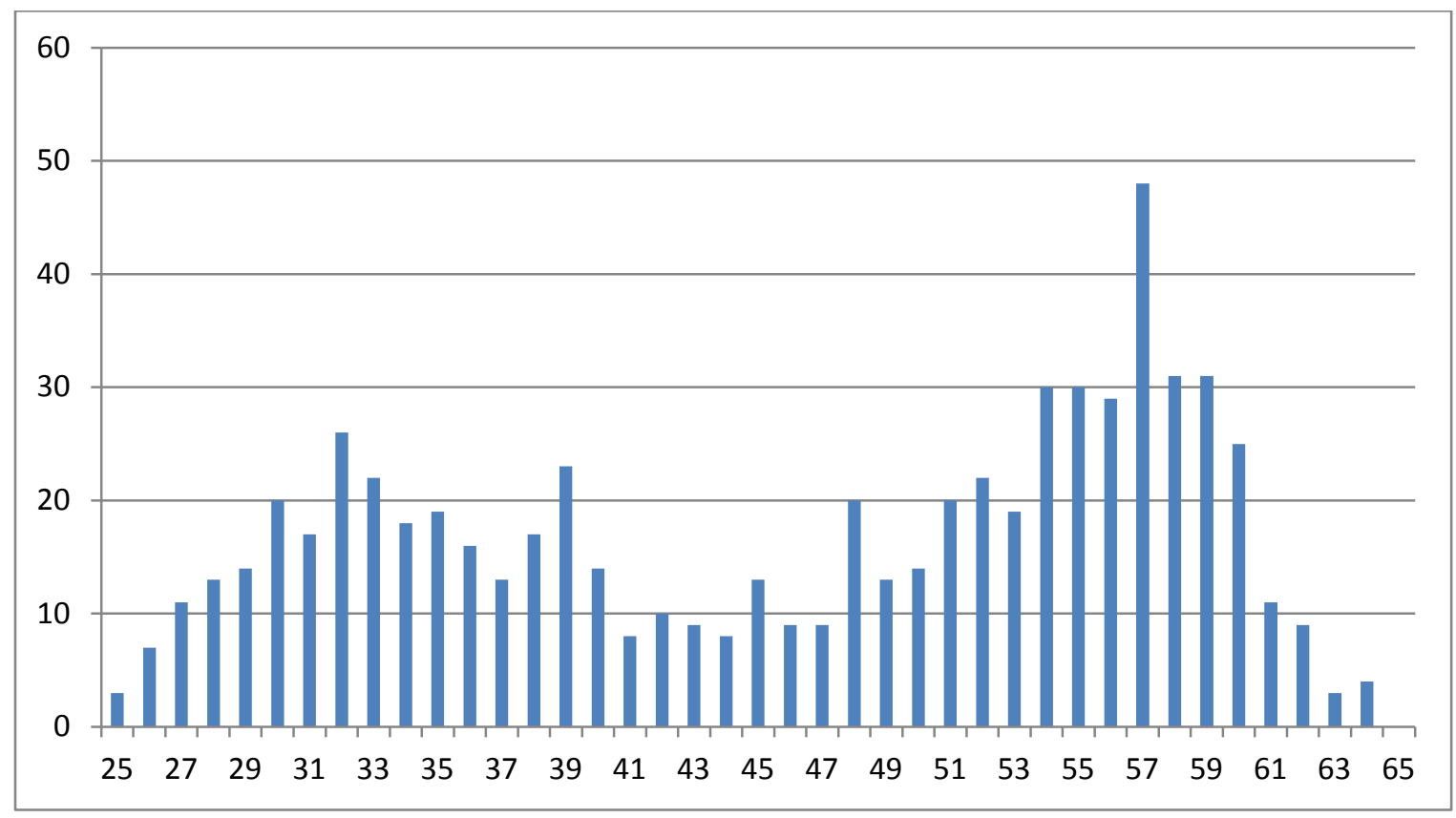

Abb. 25: Histogramm der Altersverteilung ( $n=679)$

\footnotetext{
${ }^{3}$ Das Statistische Bundesamt $(2005 ; 2007 b)$ ermittelte nach der verschärften Regelung ein durchschnittliches Pensionierungsalter von 62 Jahren. Zuvor lag das durchschnittliche Pensionierungsalter bei 59 Jahren.
} 
Die Berufserfahrung stellt im Lehrerberuf, proportional gesehen, ein mit dem Alter nahezu verlaufsidentisches Merkmal dar. Die durchschnittliche Berufserfahrung der Teilnehmenden $(n=700)$ liegt bei 17,03 Jahren $(S D=12,725){ }^{4}$

In Bezug auf die grundlegende Motivation, den Lehreberuf zu ergreifen, waren die Sportlehrkräfte aufgefordert anzugeben, ob intrinsisch-altruistische Beweggründe, also eine Art Berufung für die Berufsentscheidung ausschlaggebend war oder aber, ob extrinsisch hedonistische Motive dominierten. Zu der zweitgenannten Kategorie zählen Argumente wie z.B. ein sicherer Arbeitsplatz, ein gutes und vor allem sicheres Gehalt, lange Ferienzeiten, fehlende Alternativen oder die Studienentscheidung bzw. der Einfluss von Freundinnen und Freunden. Mit 71,6\% $(n=499)$ macht ein Großteil der Sportlehrer intrinsisch-altruistische Beweggründe für ihre Studien- bzw. Berufswahl verantwortlich, während 28,4\% ( $n=199)$ der Sportlehrkräfte extrinsisch-altruistische Gründe für ihre Berufswahl angaben.

Bei der Frage nach der Ausbildungsinstitution ergab sich ein relativ eindeutiges Bild. In 92,1\% ( $n=643)$ aller Fälle schlossen die Teilnehmer der Studie die erste Phase ihrer Ausbildung zum Lehrer an einer Universität ab. 4,3\% $(n=30)$ absolvierten diesen ersten Ausbildungsabschnitt an einer Fachhochschule, 2,7\% $(n=19)$ besuchten eine pädagogische Hochschule und nur 0,9\% $(n=6)$ gaben an, an einem Institut für Lehrerbildung ausgebildet worden zu sein. ${ }^{5}$

Die Frage, ob die Teilnehmerinnen und Teilnehmer, wenn sie heute erneut vor der Studien- bzw. Berufswahl stehen würden, sich erneut für den Lehrerberuf entscheiden würden, stellt einen Indikator für die Zufriedenheit im Beruf dar: Mit 79,5\% ( $n=552)^{6}$ gaben die meisten Sportlehrkräfte an, dass sie erneut den Lehrerberuf wählen würden, sodass insgesamt eine relativ hohe Zufriedenheit mit dem Beruf vorzuliegen scheint. Genauer aufgeschlüsselt würden nur 5,0\% ( $n=35)$ den Beruf nicht wieder wählen und 15,5\% ( $n=108)$ eher nicht. 33,7\% ( $n=234)$ tendieren mit der Angabe eher ja zu einer erneuten Wahl des Lehrerberufs während sich 45,8\% ( $n=318)$ sehr sicher sind und auf diese Frage mit einem uneingeschränkten ja antworteten.

Bei der Auswertung der arbeitsbezogenen Verhaltens- und Erlebensmuster, die anhand des AVEM-Fragebogens von Schaarschmidt und Fischer (1996) berechnet wurden, stellte sich heraus, dass 42,8\% ( $n=296)$ der Lehrkräfte dem Schonungsmuster zuzuord-

\footnotetext{
${ }^{4}$ Auf eine Darstellung in Form eines Histogramms wird verzichtet, da der Verlauf nahezu deckungsgleich zum Histogramm der Altersverteilung verläuft.

${ }^{5}$ Da die Verteilung sehr eindeutig ist und die Studieneinrichtungen neben der Universität stark unterrepräsentiert sind, wird dieser Aspekt im weiteren Verlauf der Arbeit nicht weiter berücksichtigt.

${ }^{6}$ Hierfür wurden die Angaben ja und eher ja addiert.
} 
nen sind. Mit 30,1\% ( $n=201)$ stellt das Muster Burnout die zweithäufigste Gruppe in der Stichprobe dar. Während 14,3\% ( $n=99)$ dem Muster Gesundheit angehören, werden $12,9 \%(n=89)$ dem Muster Anstrengung zugeordnet. Betrachtet man die einzelnen Muster bezüglich der Verteilung von Sportlehrerinnen und Sportlehrern fällt auf, dass die Verteilung stark voneinander abweicht. Während 46,1\% ( $n=171)$ der Männer ins Schonungsmuster fallen, sind dies auf der Frauenseite nur 38,4\% ( $n=116)$. 37,7\% $(n=114)$ der weiblichen Teilnehmer gehören dem Muster Burnout an, was einem deutlich größeren Anteil entspricht als der Anteil unter den männlichen Teilnehmern $(23,7 \%, n=88)$. Während der Anteil des Musters Anstrengung bei den Sportlehrerinnen und Sportlehrern fast gleich ist, werden Männer mit 18,1\% ( $n=67)$ etwa doppelt so häufig in das Gesundheitsmuster eingeordnet als ihre weiblichen Kolleginnen (9,6\%, $n=27)$.

Neben dem Fach Sport unterrichten die an der Erhebung Teilnehmenden meist zusätzlich ein weiteres Fach, dass zu 52,1\% ( $n=342$ ) ein Hauptfach und zu 47,9\% ( $n=315)$ ein Nebenfach ist. Segregiert in die verschiedenen Fachrichtungen ist die Kombination mit dem Bereich Mathematik und Naturwissenschaften (38,6\%; $n=221$ ) knapp vor den Sprachen (31,5\%; $n=180)$ am häufigsten vertreten. 19,8\% $(n=113)$ der Sportlehrkräfte unterrichten neben dem Fach Sport in einer Gesellschaftswissenschaft bzw. in dem Fach Wirtschaft und 10,1\% ( $n=58)$ in sogenannten "weichen Fächern" wie Kunst, Musik, textilem Gestalten/Werken oder darstellendem Spiel/Theater. Bei der geschlechtsspezifischen Betrachtung fallen einige Besonderheiten auf: Sportlehrerinnen unterrichten mit 56,9\% ( $n=157)$ häufiger zusätzlich in einem Hauptfach als in einem Nebenfach (43,1\%; $n=119)$. Das zweite Fach der männlichen Kollegen hingegen ist mit 52,1\% ( $n=188$ ) öfter ein Nebenfach als ein Hauptfach (47,9\%; $n=173)$. Bei der Verteilung auf die Fachrichtungen zeigt sich ebenfalls ein geschlechtsspezifisch unterschiedlich geprägtes Bild. Wie man anhand von Tabelle 17 ablesen kann, sind die Unterschiede in der Verteilung auf Haupt- und Nebenfach der beiden Geschlechter durch die Überrepräsentation der Sportlehrer in den Gesellschaftswissenschaften bzw. dem Fach Wirtschaft, die beide zu den Nebenfächern gerechnet werden, bedingt. Weiterhin ist auffällig, dass die „weichen Fächer" viel häufiger von Sportlehrerinnen als von Sportlehrern unterrichtet werden.

Tab. 17: Geschlechtsspezifische Verteilung auf die Fachrichtungen neben dem Fach Sport

\begin{tabular}{|l|l|l|l|l|}
\hline $\begin{array}{l}\text { Ge- } \\
\text { schlecht }\end{array}$ & $\begin{array}{c}\text { Mathematik u. Na- } \\
\text { turwissenschaften }\end{array}$ & $\begin{array}{c}\text { Sprachwissen- } \\
\text { schaften }\end{array}$ & $\begin{array}{c}\text { Wirtschafts- und Gesell- } \\
\text { schaftswissenschaften }\end{array}$ & $\begin{array}{c}\text { "weiche } \\
\text { Fächer" }\end{array}$ \\
\hline weiblich & $33,1 \%(n=79)$ & $39,3 \%(n=94)$ & $9,6 \%(n=23)$ & $18,0 \%(n=43)$ \\
\hline männlich & $43,0 \%(n=136)$ & $25,6 \%(n=81)$ & $27,5 \%(n=87)$ & $3,8 \%(n=12)$ \\
\hline
\end{tabular}


Im Durchschnitt beträgt die Unterrichtszeit der Lehrerinnen und Lehrer der Stichprobe 21,08 Wochenstunden ( $S D=5.15)$, wobei mit 9,23 Stunden ( $S D=5.18$ ) der prozentuale Anteil des Sportunterrichts an der Gesamtlehrverpflichtung bei $44,58 \%$ liegt. Sportlehrerinnen unterrichten mit 19,99 Stunden ( $S D=5.20)$ zwar insgesamt weniger als ihre männlichen Kollegen ( $M=21,93 ; S D=4.97)$, was jedoch auf das zweite Fach zurückgeht, da sie im Fach Sport mit durchschnittlich 9,41 Wochenstunden (SD=5.64) sogar etwas mehr unterrichten als ihre männlichen Kollegen $(M=9,21 ; S D=4.85)$. Prozentual zur Gesamtlehrverpflichtung nimmt der Sportunterricht bei den Lehrerinnen 47,91\% $(S D=26,29)$ ein, während die Sportlehrer nur 42,51\% (SD=20.36) ihrer Unterrichtszeit im Sportunterricht eingesetzt sind.

$62,1 \%(n=413)$ der Sportlehrkräfte sind mit der Stundenverteilung auf die meist zwei Fächer, die sie unterrichten, zufrieden. 11,0\% $(n=73)$ der Lehrerinnen und Lehrer wünschen sich, dass der Anteil des Sportunterrichts an der Gesamtlehrverpflichtung reduziert wird, 26,9\% ( $n=179)$ wünschen sich einen höheren Anteil des Sportunterrichts.

Anhand mehrerer Angaben, die die Sportlehrkräfte im Fragebogen gemacht haben, kann daraus eine mehr oder weniger starke Affinität zu Bewegung, Spiel und Sport gefolgert werden. Bei der Einschätzung des eigenen Fitnesszustands geben lediglich $6,6 \%(n=46)$ an, dass dieser eher schlecht bzw. (sehr) schlecht ist. 32,6\% $(n=226)$ geben ein mittleres, 47,6\% ( $n=330)$ ein eher gutes Fitnessniveau an. 13,1\% $(n=91)$ bezeichnen ihren eigenen Fitnesszustand sogar als sehr gut. Des Weiteren waren die Sportlehrer aufgefordert anzugeben, in welchem Umfang sie in ihrer Freizeit privat Sport treiben. Nur ein geringer Anteil der Sportlehrkräfte von 1,7\% $(n=12)$ betätigt sich privat nicht sportlich, 22,1\% ( $n=154)$ sind in einem Zeitrahmen von bis zu zwei Stunden pro Woche aktiv. 39,0\% ( $n=272)$ der Befragten geben einen Zeitrahmen von zwei bis vier Stunden an, $25,8 \%(n=180)$ verbringen zwischen vier und sechs Stunden pro Woche mit Sport in ihrer Freizeit. 11,3\% $(n=79)$ sind sogar pro Woche mehr als sechs Stunden privat sportlich aktiv.

Neben reinem Freizeitsport üben 23,1\% ( $n=159)$ zum Zeitpunkt der Befragung aktiv Wettkampfsport aus, 66,5\% ( $n=458)$ waren zumindest in der Vergangenheit an wettkampfmäßig organisierter Sportaktivität beteiligt, 10,4\% ( $n=71)$ kamen mit solch organisiertem Sport als Aktiver bisher nicht in Kontakt. In einem weiteren Teilbereich der Affinität zu Sport wurden die Sportlehrer befragt, ob sie sich in den letzten fünf Jahren als Übungsleiter, Vereins -oder Kadertrainer engagiert haben. Immerhin 36,3\% ( $n=253)$ der Teilnehmenden begleiteten eines der zuvor genannten Ämter in dem angegebenen Zeitraum. 
Abschließend wurden die Sportlehrkräfte gebeten, den Stellenwert von Sport in ihrem Privatleben auf einer fünfstufigen Likert-Skala von (sehr) gering bis (sehr) hoch einzuschätzen. Nur drei Personen $(0,4 \%)$ gaben an, dass Sport in ihrem Privatleben nur eine sehr geringe Bedeutung hat. 34 Sportlehrkräfte $(4,9 \%)$ bewerten den Stellenwert des Sports in ihrem Privatleben als eher gering, 177 (25,6\%) geben eine mittlere Bedeutsamkeit an. Der Großteil der teilnehmenden Sportlehrkräfte schätzt jedoch den Stellenwert im Privatleben als (eher) hoch $(44,4 \% ; n=307)$ bzw. (sehr) hoch $(24,6 \% ; n=170)$ ein.

\subsection{Deskriptive Darstellung sportunterrichtsspezifischer Aspekte}

Neben den zuvor dargestellten Angaben zur eigenen Person enthielt der Fragebogen zudem Variablen, die die individuelle Gestaltung des Unterrichts, bezogen auf inhaltliche sowie pädagogisch-didaktische Entscheidungen, genauer beschreiben. Neben den Kriterien für die Inhaltswahl, der Durchführungshäufigkeit von unterschiedlichen Sportarten sowie die Bedeutsamkeit von verschiedenen Vermittlungsaspekten, waren dies zudem die individuelle Lehr- und Organisationsform des Unterrichts und das technisch-taktische Aspirationsniveau, an dem der eigene Unterricht ausgerichtet wird. Im Anschluss werden die Angaben der Lehrkräfte bezüglich der individuellen sportartspezifischen theoretischen und praktischen Vermittlungskompetenz, bezüglich der aktiven Teilnahme am Sportunterricht und dem Spaß am Unterrichten sowie den als belastend empfundenen Aspekten des Unterrichtsalltags dargestellt. Abschließend werden mit dem Zeitaufwand für die Vor- und Nachbereitung des Unterrichts und dem Fortbildungsengagement eher motivational geprägte Aspekte zusammengefasst sowie das individuelle Fühlen und Erleben im bzw. des Berufes beschrieben.

\subsubsection{Kriterien der Inhaltswahl}

Die erste bedeutsame Entscheidung, die eine Sportlehrkraft im Unterricht bzw. bei dessen Planung treffen muss, ist, welcher Inhalt zu welchem Zeitpunkt im Laufe des Schuljahres durchgeführt werden soll. Durch die Revision der Lehrpläne hin zu Kerncurricula wird nur noch ein grober inhaltlicher Rahmen vorgegeben, den die Lehrer individuell bzw. schulintern z.B. in Fachgruppen mit konkreten Unterrichtsvorhaben bzw. Inhalten zu füllen haben (siehe Kap. 2.3).

Auf die Frage, anhand welcher Aspekte die Sportlehrkräfte die Inhalte für ihren Sportunterricht auswählen, wurde den Vorgaben, die durch den Lehrplan bzw. das Kerncurriculums als Orientierungshilfe gegeben werden, die größte Bedeutsamkeit beigemessen (siehe Tab. 18). Als einflussreich auf die Wahl der Inhalte wird zudem die Ausstat- 
tung der Sportstätte angesehen, die sich, wie bereits in mehreren anderen Studien deutlich wurde (Altenberger et al., 2005; Deutscher Sportbund, 2006), häufig als limitierender Faktor erweist. Das Interesse der Schüler folgt in der Rangfolge auf dem nächsten Platz und auch der eigenen Vermittlungs- und Praxiskompetenz sowie den persönlichen Vorlieben wird durchaus ein bedeutsamer Einfluss auf die Inhaltswahl bescheinigt. Weniger bedeutsam hingegen werden Kriterien eingeschätzt, die hauptsächlich auf einen reibungslosen Ablauf des Unterrichts gerichtet sind, wie ein geringes Verletzungsrisiko, der mit dem Inhalt verbundene Organisations- und Aufbauaufwand sowie ein geringes Konfliktpotenzial zwischen den Schülern.

Tab. 18: Kriterien der Inhaltswahl im Sportunterricht

\begin{tabular}{|l|c|c|}
\hline Kriterien & M & SD \\
\hline Curriculare Vorgaben & 3,47 & .662 \\
\hline Ausstattung der Sportstätte & 3,25 & .771 \\
\hline Schülerinteresse & 2,93 & .697 \\
\hline Vermittlungskompetenz in der Sportart & 2,89 & .796 \\
\hline Eigene Vorlieben & 2,77 & .801 \\
\hline Praxiskompetenz in der Sportart & 2,66 & .835 \\
\hline Geringes Verletzungsrisiko & 2,22 & .831 \\
\hline Organisations- und Aufbauaufwand & 2,12 & .802 \\
\hline Geringes Konfliktpotenzial zwischen Schülern & 1,75 & .796 \\
\hline
\end{tabular}

\subsubsection{Durchführungshäufigkeit verschiedener Inhalte}

Seit dem Jahr 2007 bestimmt in Niedersachsen ein kompetenzorientiertes Kerncurriculum für die Grund- und Förderschulen sowie die Sekundarstufe I die Ausrichtung und Organisation des Schulsports. Für die Sekundarstufe II und die Berufs- bzw. Berufsfachschulen gelten bis zur Einführung eines Kerncurriculums noch die grundlegenden Regelungen der Rahmenrichtlinien. Neben dem prozessbezogenen Kompetenzbereich, der eine kognitive Auseinandersetzung mit Bewegung, Spiel und Sport umfasst, werden in Erfahrungs- und Lernfeldern inhaltsbezogene Kompetenzen vermittelt. Mit der Einführung vom Bewegungs- und Lernfeldern sind zwar die Sportarten wie Fußball, Leichtathletik oder Turnen von der ersten Gliederungsebene verschwunden, sind aber in der konkreten Ausgestaltung in verschiedenen Kontexten und Akzentuierungen weiterhin essenzieller Bestandteil der übergreifenden Rahmung. Wie man in Tab. 19 ablesen kann, führen Sportlehrkräfte am häufigsten kleine Spiele im Unterricht durch, die oft auch als Aufwärm- oder Abschlussspiele eingesetzt werden. Mit Leichtathletik, Basketball und Volleyball folgen auf den nächsten Plätzen verstärkt eher körperlose Spiele. In der zweiten Hälfte sind vor allem die körperbetonten Sportarten vertreten oder sol- 
che, die besondere Anforderungen an die Sportlehrkraft oder die Sportstätte bzw. materielle Ausstattung richten wie Schwimmen, Trendsportarten und Hockey.

Tab. 19: Durchführungshäufigkeit verschiedener Inhalte im Sportunterricht

\begin{tabular}{|l|c|c|}
\hline Inhalte & M & SD \\
\hline Kleine Spiele & 3,32 & .690 \\
\hline Leichtathletik & 3,13 & .558 \\
\hline Basketball & 3,01 & .624 \\
\hline Volleyball & 2,91 & .667 \\
\hline Turnen & 2,84 & .690 \\
\hline Konditionstraining & 2,80 & .734 \\
\hline Badminton & 2,53 & .794 \\
\hline Fußball & 2,53 & .732 \\
\hline Handball & 2,46 & .701 \\
\hline Schwimmen & 2,40 & .891 \\
\hline Trendsportarten & 2,09 & .764 \\
\hline Hockey & 2,06 & .782 \\
\hline Gymnastik/Tanz & 2,03 & .866 \\
\hline
\end{tabular}

\subsubsection{Bedeutsamkeit von Vermittlungsaspekten}

Um herauszufinden, welche Zielsetzungen schwerpunktmäßig von den Sportlehrenden im Unterricht verfolgt werden, wurden die Vermittlungsaspekte hinsichtlich ihrer Bedeutsamkeit im eigenen Sportunterricht eingeschätzt. Die Spitzenposition und damit das als am wichtigsten eingeschätzte Anliegen der Sportlehrer ist es, Schülern Freude an Bewegung zu vermitteln (siehe Tab. 20). Dieser Aspekt schließt sowohl das gegenwärtige Tun, d.h. die freudvolle Auseinandersetzung mit Bewegung, Spiel und Sport im Unterricht als auch das Heranführen an das lebenslange Sporttreiben im Sinne des Doppelauftrags des Schulsports ein. Das in der Rangfolge der Bedeutsamkeit folgende soziale Lernen stellt einen weiteren Aspekt dar, auf dem, wie bereits in Kapitel 2.3 deutlich wurde, neuere Legitimationen des Faches aufbauen. Der Sportunterricht bietet wie kein anderes Fach Erfahrungs- und Lernmöglichkeiten im Umgang miteinander, wodurch diesem Vermittlungsaspekt auch für die Zukunft, unabhängig von den Entwicklungen der Sportkultur, enorme Bedeutung zukommt. Fachliche Kenntnisse und Taktikschulung, die beide eher im kognitiven Bereich anzusiedeln sind, werden als weniger wichtig eingeschätzt. Die in den 1970er und 1980er Jahren propagierte Leistungserziehung nimmt den letzten Platz in dem „Bedeutsamkeitsranking" ein. Ergänzend muss hinzugefügt werden, dass Disziplin trotz der Minderbewertung der Leistungserziehung dennoch einen mittleren Stellenwert einnimmt, d.h. dass zwar reines Leistungsdenken und -streben keine primäre Zielsetzung darstellt, Disziplin und Erzie- 
hung aber dennoch als wichtig angesehen werden und dementsprechend im Unterrichtsalltag Berücksichtigung finden bzw. ihnen Bedeutsamkeit beigemessen wird.

Tab. 20: Vermittlungsaspekte

\begin{tabular}{|l|c|c|}
\hline Vermittlungsaspekte & M & SD \\
\hline Freude an Bewegung & 3,76 & .443 \\
\hline Soziales Lernen & 3,54 & .545 \\
\hline Spielen & 3,52 & .511 \\
\hline Fitness & 3,49 & .565 \\
\hline Koordinative Fähigkeiten & 3,46 & .540 \\
\hline Körpererfahrung & 3,42 & .605 \\
\hline Selbstständigkeit & 3,36 & .557 \\
\hline Disziplin & 3,20 & .566 \\
\hline Kondition & 3,09 & .646 \\
\hline Technik & 3,06 & .530 \\
\hline Körperbildung & 2,98 & .700 \\
\hline Kreativität & 2,97 & .627 \\
\hline Fachliche Kenntnisse & 2,83 & .615 \\
\hline Taktik & 2,80 & .564 \\
\hline Leistungserziehung & 2,63 & .680 \\
\hline
\end{tabular}

\subsubsection{Lehrweise und Organisationsform}

$54,1 \%(n=290)$ der Sportlehrer gaben an, eher eine induktive Lehrweise im Unterricht zu verfolgen, d.h. dass sie eher prozessorientiert vorgehen, verschiedene Lernwege ausprobiert werden können und die Auseinandersetzung bzw. die Entwicklung einer Strategie ein Problem zu lösen, einen wichtigen Teil des Unterrichts ausmacht. 45,9\% $(n=246)$ verfahren eher deduktiv. Beim deduktiven Unterrichten werden die Lernwege vorgegeben, um ein möglichst effizientes Lernen von Bewegungen, Techniken und Sportarten zu gewährleisten. Weiterhin wurden die Sportlehrkräfte befragt, in welcher Art und Weise sie ihren Unterricht organisieren, d.h. ob sie eher selbst den Ablauf im Sinne einer Lehrerzentrierung bestimmen oder aber, ob eine Schülerorientierung eher ihrem Unterrichtsstil entspricht. Die Mehrheit der befragten Sportlehrer (56,8\%; $\mathrm{n}=396$ ) gaben an, dass sie ihren eigenen Unterricht weder als ausschließlich lehrerzentriert noch als durchweg schülerorientiert sehen, sondern dass sie ein ausgewogenes Verhältnis dieser beiden Pole anstreben und umsetzen. Im Vergleich von Lehrerzentrierung und Schülerorientierung scheint die erstgenannte Variante leicht zu überwiegen (siehe Abb. 26). Im Vergleich der Geschlechter stellt sich heraus, dass Sportlehrer ihren Unterricht etwas deduktiver und lehrerzentrierter einschätzen als Sportlehrerinnen, die im Vergleich eher induktiv und schülerorientiert vorgehen. 


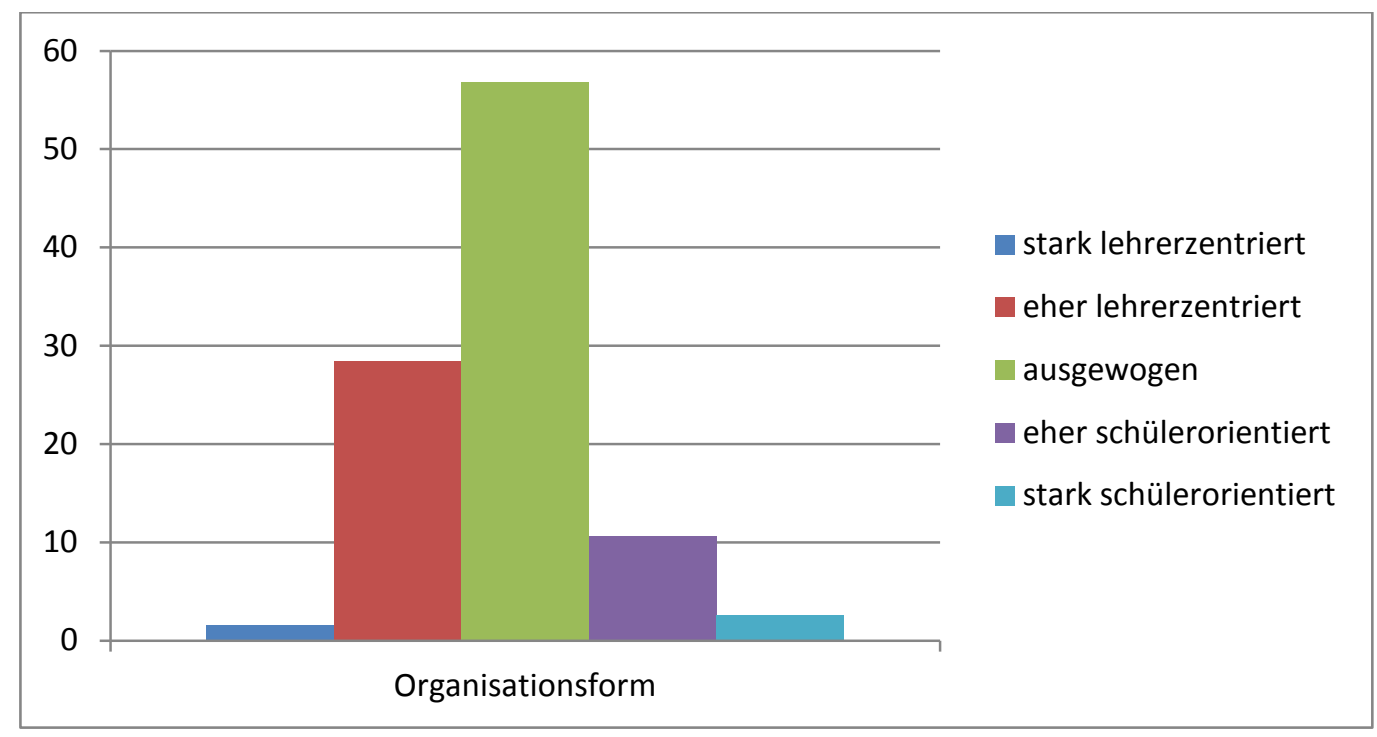

Abb. 26: Prozentuale Verteilung der Organisationsformen des Sportunterrichts $(n=697)$

\subsubsection{Technisch-taktisches und konditionelles Aspirationsniveau}

In diesem Fragenaspekt geht es um das Anspruchsniveau an die Schüler, das die Sportlehrkräfte in ihrem Unterricht verfolgen. Die Einschätzung erfolgte differenziert für den technisch-taktischen und den konditionellen Bereich. Bei dem technisch-taktischen Bereich geht es vor allem um die Umsetzung von Spielkonzeptionen in den Sportspielen, aber auch um die technisch korrekte Bewegungsausführung in allen Inhaltsbereichen. Das konditionelle Aspirationsniveau ist ein Produkt aus dem Belastungsumfang und der Intensität im Sportunterricht. Die Anforderungen, die Sportlehrer an ihre Schüler stellen, schätzen die Befragten meist als mittel bzw. eher hoch ein, was sich in dem Mittelwerten von 3,39 (SD=.615) für den technisch-taktischen und in dem Mittelwert von 3,35 (SD=.738) für den konditionellen Bereich zeigt. Nur jeweils eine einzige Person für den technisch-taktischen sowie für den konditionellen Bereich gab an, dass ihr/sein Unterricht durch niedrige Anforderungen an die Schüler geprägt ist. Wie man anhand von Abbildung 27 erkennen kann, wurde mit 4,9\% ( $n=34)$ im technischtaktischen und 10,9\% ( $n=76)$ im konditionellen Bereich auch die Bewertung eher niedrig nur von sehr wenigen Lehrerinnen und Lehrern gewählt. Die Mehrheit mit 52,9\% ( $n=368)$ im technisch-taktischen und 47,2\% ( $n=329)$ im konditionellen Bereich gaben an, in ihrem Unterricht mittlere Anforderungen an die Schüler zu stellen. Sogar als eher hoch schätzen 40,4\% ( $n=281)$ der Befragten den Anspruch im technisch-taktischen Bereich ein, im konditionellen Bereich zu 37,3\% ( $n=260)$. Immerhin zwölf (1,7\%) bzw. 31 $(4,4 \%)$ Lehrkräfte bezeichnen in den beiden Bereichen ihre Anforderungen als hoch. 


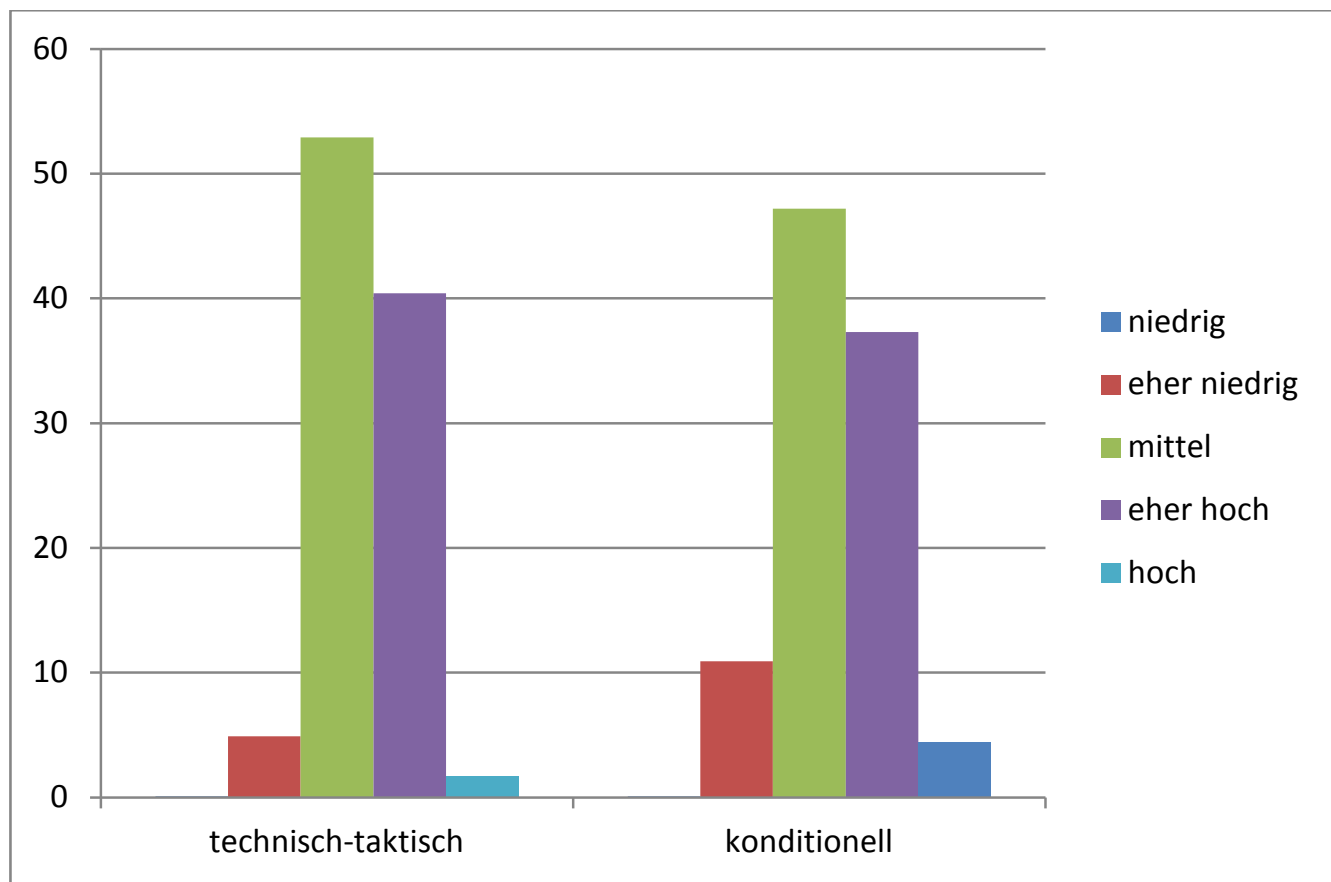

Abb. 27: Technisch-taktisches und konditionelles Aspirationsniveau ( $n=696)$

Im Geschlechtervergleich zeigte sich, dass Sportlehrerinnen sowohl im technischtaktischen als auch im konditionellen Bereich ein etwas geringeres Anspruchsniveau in ihrem Sportunterricht angeben als Sportlehrer. Während die Unterschiede im konditionellen Bereich keine statistische Signifikanz aufweisen ${ }^{7}$, zeigen die Ergebnisse des t-Tests bei dem technisch-taktischen Anforderungsniveau überzufällige Unterschiede $(t(652,393)=-5,125 ; p<.01)$ zwischen männlichen $(M=3,49 ; S D=.620)$ und weiblichen Sportlehrkräften $(M=3,25 ; S D=.592)$ an, was einem kleinen Effekt von $d^{8}=.40$ entspricht.

\subsubsection{Sportartspezifische Praxis- und Theoriekompetenz der Lehrkräfte}

Betrachtet man die Einschätzung der eigenen Kompetenz bezogen auf das Praxis- und Theoriekönnen in den einzelnen Schulsportarten, stellt man schnell fest, dass die Mittelwerte (siehe Tab. 21) und die daraus resultierende Reihung mit einigen Ausnahmen stark der Tabelle zur Durchführungshäufigkeit verschiedener Inhalte im Sportunterricht (siehe Tab. 19) ähnelt. Auffällig ist zudem, dass das individuelle Leistungsvermögen durchgängig in allen Sportarten für den praktischen und den theoretischen Bereich

\footnotetext{
${ }^{7}$ Männlich ( $\left.M=3,39, \mathrm{SD}=.787\right)$, weiblich ( $\left.M=3,31, \mathrm{SD}=.673\right)$.

${ }^{8}$ Das Effektstärkemaß $d$ wurde mit dem Programm G-Power 3.0 berechnet. $d$ stellt als Effektstärkemaß die standardisierte Distanz zwischen zwei Mittelwerten dar (Rasch et al., 2006). Da d einen beliebigen Wert annehmen kann und kein prozentuales Maß für den Effekt darstellt, sind Konventionen zur Einschätzung der Werte notwendig. Nach Cohen (1988) entspricht $d=0,20$ einem kleinen, $d=0,50$ einem mittleren und $d=0,80$ einem großen Effekt.
} 
nahezu gleich eingeschätzt wird. Betrachtet man die Angaben geschlechtsspezifisch, fallen auf den ersten Blick die typischen Charakteristika auf. Am auffälligsten sind die Unterschiede in der Einschätzung der eigenen Kompetenz im Bereich Fußball zugunsten der Männer und im Bereich Gymnastik und Tanz zugunsten der Frauen.

Tab. 21: Praxis- und Theoriekompetenz in den verschiedenen Sportarten

\begin{tabular}{|l|c|c|c|c|}
\cline { 2 - 5 } \multicolumn{1}{c|}{} & \multicolumn{2}{c|}{ Praxis } & \multicolumn{2}{c|}{ Theorie } \\
\hline Inhalte & M & \multicolumn{1}{c|}{ SD } & M & \multicolumn{1}{c|}{ SD } \\
\hline Volleyball & 3,31 & .696 & 3,35 & .648 \\
\hline Kleine Spiele & 3,30 & .595 & 3,35 & .576 \\
\hline Konditionstraining & 3,19 & .682 & 3,29 & .634 \\
\hline Badminton & 3,10 & .765 & 3,13 & .698 \\
\hline Leichtathletik & 3,06 & .619 & 3,23 & .558 \\
\hline Basketball & 3,03 & .670 & 3,13 & .638 \\
\hline Schwimmen & 2,97 & .793 & 3,09 & .703 \\
\hline Turnen & 2,79 & .814 & 3,01 & .695 \\
\hline Handball & 2,75 & .798 & 2,93 & .703 \\
\hline Fußball & 2,66 & .928 & 2,83 & .807 \\
\hline Trendsportarten & 2,54 & .773 & 2,56 & .733 \\
\hline Hockey & 2,44 & .825 & 2,63 & .783 \\
\hline Gymnastik/Tanz & 2,27 & 1,025 & 2,37 & 1,009 \\
\hline
\end{tabular}

7.2.7 Aktive Teilnahme am Sportunterricht und Spaß am Unterrichten

Da das Vorleben einer aktiven Einstellung zum Leben, Bewegung und Sport eine immense Bedeutung hat (siehe Kap. 2.3), kann ein „Mitmachen“ der Sportlehrkräfte dazu führen, dass Schüler mit größerem Interesse und einer höherer Motivation am Unterricht teilnehmen. Wie man anhand von Abbildung 28 erkennen kann, sehen dies die an der Studie beteiligten Lehrkräfte genauso, da Nicht- $(2,4 \% ; n=17)$ bzw. Seltenteilnehmer $(12,2 \% ; n=85)$ nur in geringer Anzahl in der vorliegenden Stichprobe zu finden sind. Die meisten Lehrkräfte (48,6\%; $\mathrm{n}=339)$ geben an, zumindest $a b$ und zu aktiv am Sportunterricht teilzunehmen, während etwas mehr als ein Viertel der befragten Lehrerinnen und Lehrer angeben, oft $(28,9 \% ; n=202)$ aktiv am Sportunterricht teilzunehmen. 7,9\% $(n=55)$ der Lehrkräfte beteiligen sich sehr oft/immer aktiv am Sportunterricht. Auch bei dieser Fragestellung offenbaren sich erneut Unterschiede zwischen den Geschlechtern. Sportlehrer beteiligen sich mit einem Mittelwert von 3,41 (SD=.905) deutlich häufiger aktiv am Sportunterricht $(t(668,493)=-4,365, p<.01)$ als ihre weiblichen Kollegen mit einem Wert von 3,12 (SD=.795), was einem Effekt von $d=.340$ entspricht. 


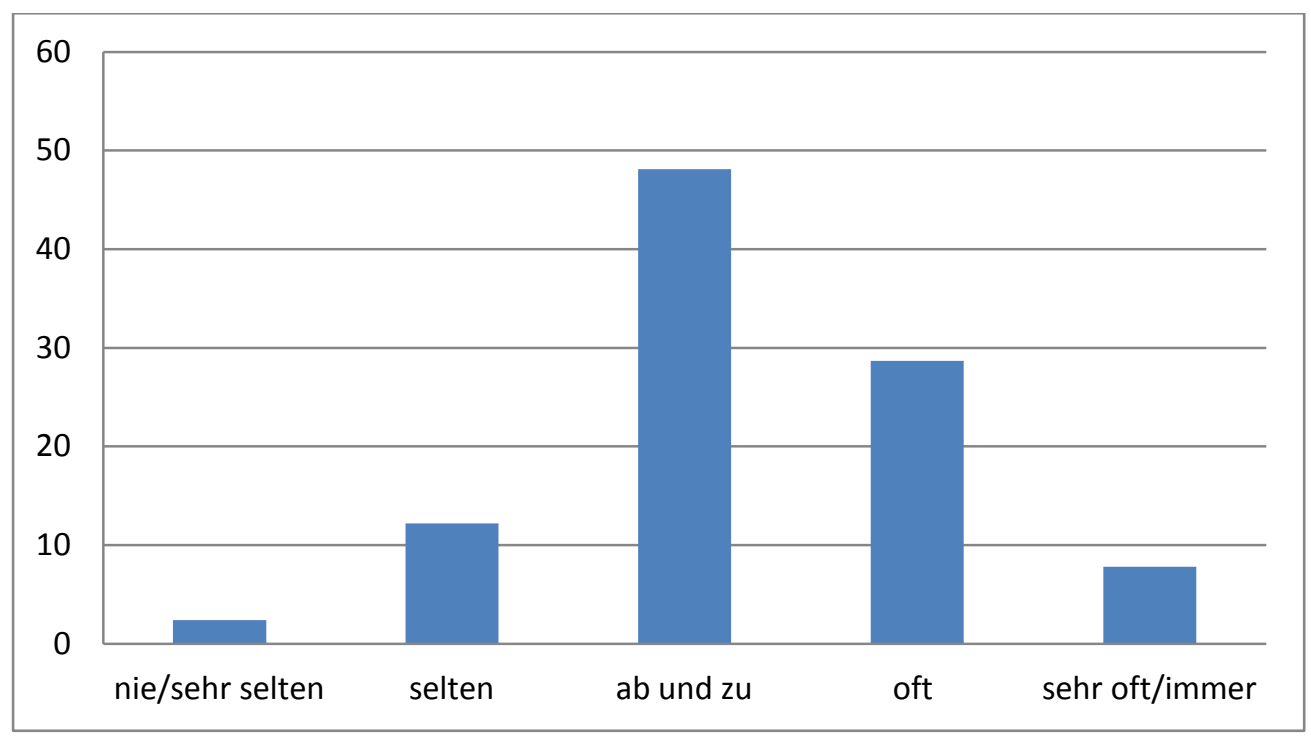

Abb. 28: Aktive Teilnahme am Sportunterricht ( $n=698)$

Noch deutlicher fällt die Verteilung der Antworten auf die Frage, wie oft die Sportlehrkräfte selbst Spaß am Unterrichten haben aus (siehe Abb. 29). Insgesamt gaben nur sechs Teilnehmer $(0,8 \%)$ an, nie/sehr selten bzw. selten Spaß am Unterrichten zu haben. 11,2\% ( $n=76)$ der Lehrkräfte macht das Unterrichten des Faches Sport zumindest ab und zu Spaß, während 59,5\% ( $n=405)$ Lehrkräfte oft und 28,5\% ( $n=194)$ sogar sehr oft/immer Spaß bei der Verrichtung ihrer Arbeit in der Sporthalle haben. Insgesamt schätzen die Befragten diese fünfstufig Likert-skalierte Frage durchschnittlich mit einem Wert von 4,15 (SD=.645) ein, sodass sich ein überaus positives Bild im Bezug auf die Freude am Unterrichten (siehe Kap. 2.2.2) abzeichnet.

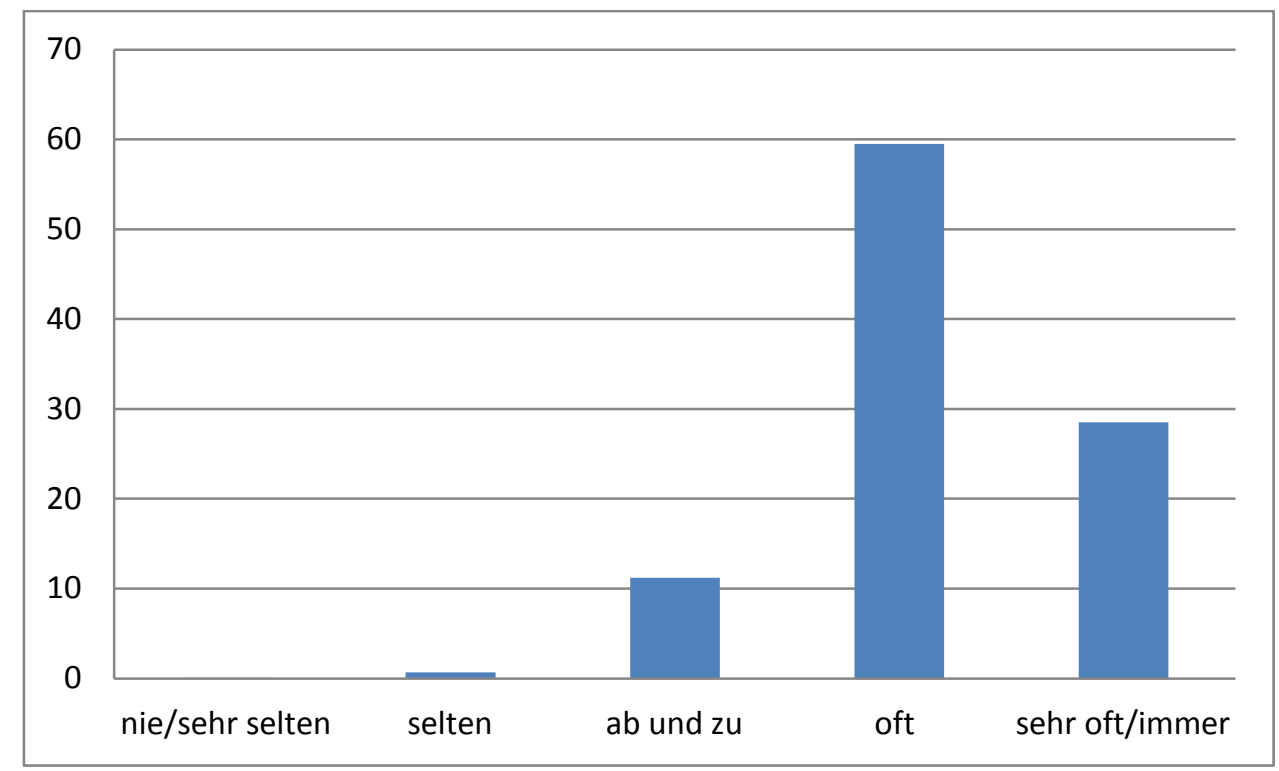

Abb. 29: Spaß am Unterrichten $(n=681)$ 


\subsubsection{Belastungsfaktoren im Sportunterricht}

Im Fragebogen wurden die Sportlehrkräfte aufgefordert, insgesamt 17 verschiedene potenzielle Faktoren bezüglich der individuell erlebten subjektiven Belastung einzuschätzen. Neben fachspezifischen Faktoren, die meist nur im Sportunterricht auftreten, wie körperliche Beanspruchung, das Leisten von Hilfestellung, die erhöhte Unfallgefahr oder die Demonstration von Bewegungen, wurden ebenfalls allgemeine, jeglichen Unterricht beeinflussende Faktoren, wie die Klassengröße oder auftretendes Schülerfehlverhalten, bewertet. Bei der Auswertung in dieser Kategorie zeigte sich, dass weniger die Belastungsfaktoren, die für den Sportunterricht als Bewegungsfach charakteristisch sind, als besonders belastend wahrgenommen werden, sondern vielmehr die Faktoren, die den organisatorischen Rahmen des Unterrichts betreffen, wie die Klassengröße, der hohe Lärmpegel und geringe bzw. fehlende Pausen- und Erholungszeiten im Unterrichtsalltag (siehe Tab. 22).

Tab. 22: Belastungsfaktoren im Sportunterricht

\begin{tabular}{|l|r|r|}
\hline Belastungsfaktoren & M & SD \\
\hline Klassengröße & 3,34 &, 722 \\
\hline Lärmpegel & 3,08 &, 785 \\
\hline Geringe Pausen- bzw. Erholungszeiten & 2,63 &, 824 \\
\hline Schlechte räumlich-materielle Bedingungen & 2,58 &, 945 \\
\hline Vielzahl von gleichzeitig auftretenden Aufgaben & 2,57 &, 824 \\
\hline Unangemessene curriculare Vorgaben & 2,48 &, 727 \\
\hline Soziales Schülerfehlverhalten und Motivationsprobleme & 2,44 &, 775 \\
\hline Außerunterrichtliche Aktivitäten (z.B. Sportfeste, Bundesjugend-Spiele) & 2,28 &, 737 \\
\hline Auf- und Abbau & 2,23 &, 738 \\
\hline Stetig latente Unfallgefahr & 2,21 &, 796 \\
\hline Körperliche Beanspruchung (allgemein) & 2,21 &, 774 \\
\hline Hilfestellungen & 2,21 &, 782 \\
\hline Häufig wechselnder Ordnungsrahmen im Unterricht & 2,19 &, 734 \\
\hline Erhöhter Aufwand zur Erhaltung der eigenen Fitness/Erlernen neuer Sportarten & 2,08 &, 742 \\
\hline Rechtfertigungsdruck gegenüber dem Kollegium (nicht Sport) & 1,93 &, 798 \\
\hline Demonstration von Bewegungen & 1,89 &, 653 \\
\hline Schwierigkeiten im Umgang mit Eltern, Kollegen, Schulleitung & 1,74 &, 635 \\
\hline
\end{tabular}

Das Fehlverhalten sowie die Motivationsprobleme von Schülern, die in populärwissenschaftlichen Aufsätzen und Berichten oft als die Belastungen schlechthin im Berufsalltag von Lehrkräften beschrieben werden, nehmen lediglich einen Platz im Mittelfeld ein. Selbstverständlich muss ergänzend angefügt werden, dass die einzelnen Belastungsfaktoren miteinander in Beziehung stehen und sich gegenseitig beeinflussen und bedingen. So wirkt die Klassengröße beispielsweise auf nahezu alle anderen Faktoren 
als bedeutende Einflussgröße, löst Probleme und Störungen überhaupt erst aus, verschärft diese oder lässt sie sichtbar werden. Das soziale Umfeld in dem sich der Sportunterricht bzw. die Arbeit der Sportlehrkraft abspielt, vermag hingegen kaum ein Problem darzustellen. Weder scheint ein besonderer Rechtfertigungsdruck gegenüber Kollegen anderer Fachdisziplinen zu bestehen, noch stellt der Umgang mit Dritten, d.h. Eltern, Kollegen oder der Schulleitung ein bedeutender Belastungsfaktor dar. Im Vergleich der Geschlechter fällt auf, dass sich Sportlehrer ( $M=2,28 ; S D=.39)$ insgesamt etwas geringer belastet fühlen $(t(680)=6,117, p<.01)$ als Sportlehrerinnen $(M=2,46$; $\mathrm{SD}=.38)^{9}$, was mit $d=.47$ nahezu einem mittleren Effekt entspricht.

\subsubsection{Zeitaufwand für Vor- und Nachbereitung}

Auch im Sportunterricht ist ein mehr oder weniger großer Zeitaufwand notwendig, um den Unterricht vor- und nachzubereiten. In dem Fragebogen wurden die Teilnehmer aufgefordert anzugeben, welche Zeit sie neben der regulären Unterrichtsverpflichtung pro Woche zusätzlich investieren. Die Angaben der Lehrer schwanken zwischen null Stunden, d.h. dass sie keine zusätzliche Zeit für die Vor- oder Nachbereitung des Unterrichts investieren, bis hin zu 20 Stunden.

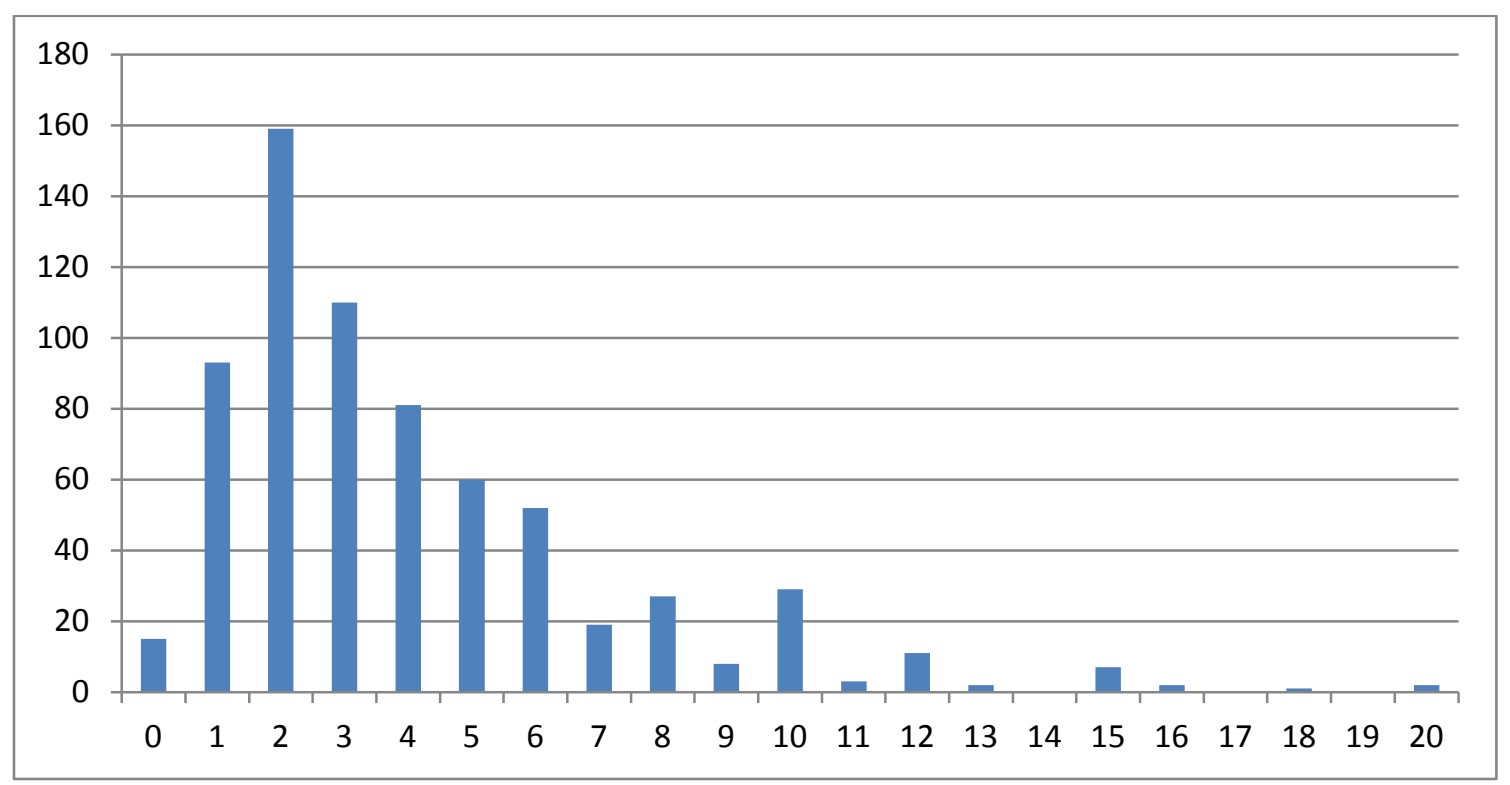

Abb. 30: Häufigkeiten des wöchentlichen Aufwandes für Vor- und Nachbereitung des Sportunterrichts $(n=682)$

Im Durchschnitt wenden die Sportlehrer 4,19 Stunden $(S D=4,29)$ zusätzlich für die Vorund Nachbereitung des Sportunterrichts auf. Setzt man diesen Wert in Beziehung zu

\footnotetext{
${ }^{9}$ Dies entspricht den Ergebnissen von Dallermassen aus dem Jahr 1986, der sich ebenfalls mit den Belastungen im Berufsalltag von Sportlehrkräften auseinandersetzte. Abele \& Candova (2007) stellten in einer Stichprobe, die sich aus allen Fachrichtungen zusammensetzte, keine geschlechtsspezifischen Unterschiede im Belastungserleben fest.
} 
der wöchentlichen Sportstundenzahl ergibt sich eine Vor- bzw. Nachbereitungszeit von etwas mehr als 27 Minuten pro Unterrichtsstunde. In Abbildung 30 sind die absoluten Häufigkeiten dargestellt. Man kann erkennen, dass die Spannweite der Antworten zwar sehr breit ist, der wöchentliche Zeitaufwand aber meist zwischen einer und sechs Stunden pro Woche beträgt.

\subsubsection{Fortbildungsengagement}

Neben dem Unterrichten und der Vor- und Nachbereitung gehört es auch zu den Aufgaben von Lehrkräften, sich stetig selbst fort- und weiterzubilden, um neue Erkenntnisse aus Forschung und Wissenschaft in der eigenen Lehre umzusetzen und den eigenen Unterricht stetig reflexiv zu hinterfragen. Im Durchschnitt absolvierten die Sportlehrkräfte 3,7 Fortbildungstage $(S D=5,40 ; n=689)$ im letzten Jahr, wovon ca. 2,1 Tage $(S D=3,95 ; n=690)$ für sportunterrichtspezifische Veranstaltungen und 1,6 Tage $(S D=3,02 ; n=689)$ für fächerunspezifische Veranstaltungen aufgewendet wurden. Überraschend bei der Beantwortung dieser Frage ist, dass 52,0\% ( $n=358)$ keine fächerübergreifende und $38,7 \%(n=267)$ keine sportunterrichtsspezifische Fortbildungsveranstaltung im letzten Jahr besucht haben. 23,2\% ( $n=160)$ haben im letzten Jahr weder eine sportunterrichtsspezifische noch eine fächerunspezifische Fortbildung absolviert.

Auf die Frage nach dem privaten Fortbilden mithilfe von Fachzeitschriften und -büchern antworteten 2,2\% $(n=15)$ der teilnehmenden Sportlehrkräfte, dass sie sich sehr selten bzw. nie privat fortbilden, 9,9\% $(n=68)$ gaben an, dies selten zu tun. 43,3\% $(n=299)$ bilden sich $a b$ und $z u, 36,4 \%(n=251)$ oft und 8,3\% $(n=57)$ sogar sehr oft eigenverantwortlich bzw. privat fort.

\subsubsection{Individuelles Fühlen und Erleben im bzw. von Sportunterricht}

In diesem deskriptiven Teil geht um das Fühlen und Erleben der Sportlehrkräfte im Unterricht bzw. ihrem schulischen und gesellschaftlichen Umfeld.

Die erste Dimension, die eine Aussage über das Fühlen und Erleben und vor allem über das Wohlfühlen erlaubt, ist die durch die Lehrkräfte eingeschätzte Atmosphäre im Kollegium. Im Rahmen des Fragebogens wurde unterschieden in "Atmosphäre im Gesamtkollegium" und "Atmosphäre im Sportkollegium", um das soziale Arbeitsumfeld möglichst differenziert zu erfassen. Wie man anhand der Abbildung 31 zur Atmosphäre im Kollegium erkennen kann, bewerten Sportlehrkräfte das Klima in der Fachschaft Sport als geringfügig positiver im Vergleich zu dem Klima im Gesamtkollegium. Die Bewertung der Zusammenarbeit bzw. des sozialen Klimas als (sehr) schlecht wurde nur von je 0,4\% $(n=3)$ vergeben, als eher schlecht bezeichnen 6,0\% $(n=42)$ die Atmosphäre im Gesamtkollegium und 5,4\% ( $n=38)$ im Sportkollegium. Mit Mittelwerten von 3,72 
( $S D=.762 ; n=695)$ für das Gesamtkollegium und 3,90 (SD=.816; $n=699)$ für das Sportkollegium ergibt sich für das soziale Klima jedoch insgesamt ein gutes Gesamturteil, was in der Abbildung 31 vor allem in der Ausprägung der beiden Balken der Bewertung eher gut deutlich wird.

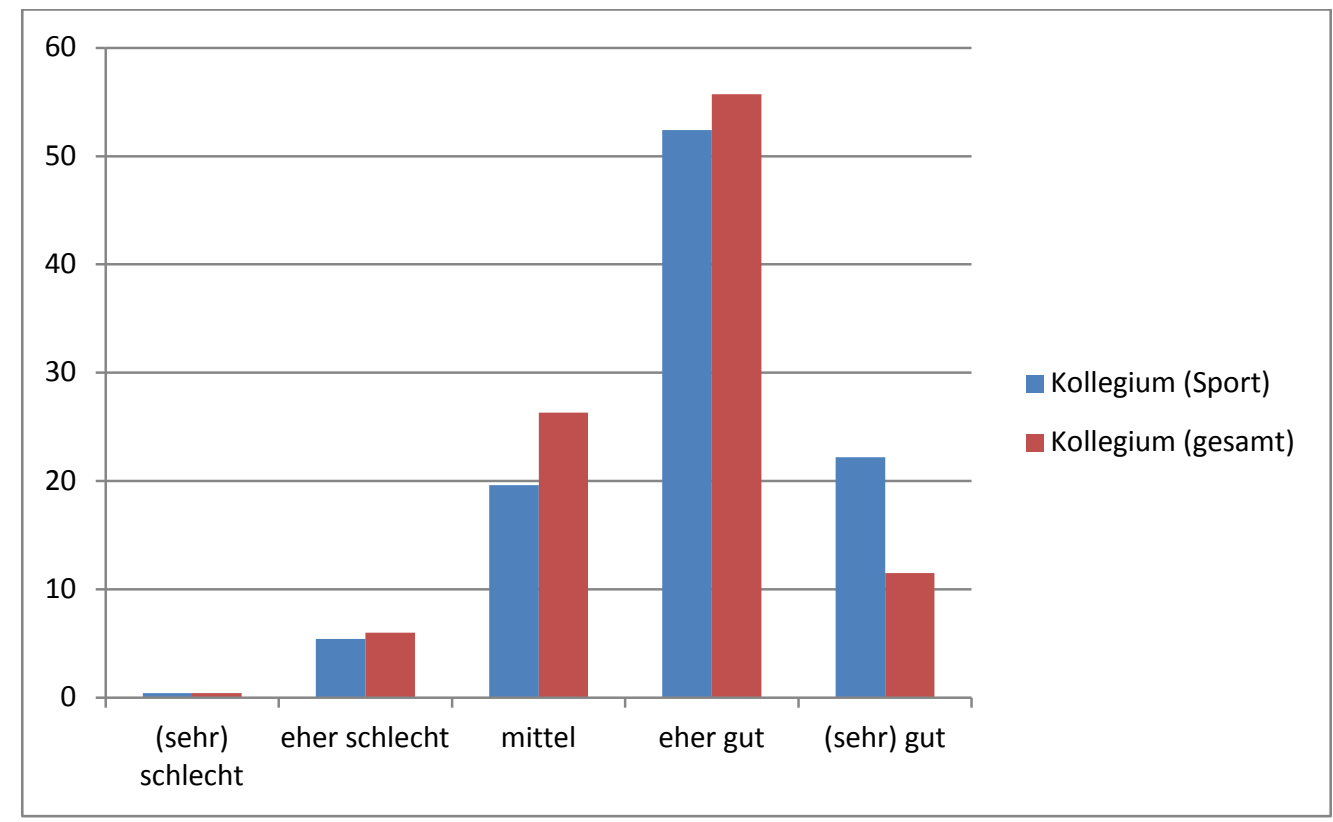

Abb. 31: Atmosphäre im Kollegium/Sportkollegium

Wie bereits in Kapitel 2.2.4 dargestellt, wird Sportunterricht in der Literatur und im gesellschaftlichen Meinungsbild oft nicht als ein gleichwertiges Fach angesehen. Der Rechtfertigungsdruck bzw. die Legitimationsprobleme stellen keine bedeutsamen Belastungsquellen dar (siehe Kap. 7.2.8), daher wurden die Sportlehrer zusätzlich befragt, wie sie selbst den Stellenwert des Faches im Vergleich zu den anderen Fächern sehen. Bei dieser Einschätzung gaben nur 12,2\% ( $n=85)$ an, dass der Sportunterricht ihrer Einschätzung nach einen geringeren Stellenwert aufweist. Die meisten Lehrkräfte (66,3\%; $\mathrm{n}=463$ ) gaben an, dass es sich beim Sportunterricht um ein gleichwertiges Fach handelt, das weder eine geringere noch eine höhere Bedeutsamkeit gegenüber anderen Fächern aufweist. 22,5\% ( $n=150)$ sehen den Sportunterricht sogar als ein Fach mit einer höheren Bedeutsamkeit an.

In Abbildung 32 ist die Bewertung der Lehrkräfte bezogen auf Gefühle und Erfahrungen, die sie mit ihrer bisherigen Karriere verbinden, sowie eine Prognose zukünftiger Erwartungen des Verlaufs des weiteren Berufslebens, dargestellt. Auffällig ist hierbei, dass die Erwartungen an die Zukunft deutlich negativer $(M=3,70 ; S D=.867)$ geprägt sind, als die Erfahrungen und Gefühle der Vergangenheit $(M=4,06 ; S D=.719)$. Diese etwas negativere Einschätzung der Zukunft führt jedoch nicht in letzter Konsequenz zum frühzeitigen Aussteigen aus dem Beruf, was nur 3,8\% ( $n=27)$ schon bald und 
$11,3 \%(n=78)$ auf längere Sicht planen. Hierbei ist jedoch nicht in allen Fällen klar, ob eventuell auch Lehrkräfte, die kurz vor der regulären Pensionierung stehen, hier ein Aussteigen aus dem Beruf angegeben haben und das Adjektiv frühzeitig in der Itemformulierung nicht berücksichtigt wurde.

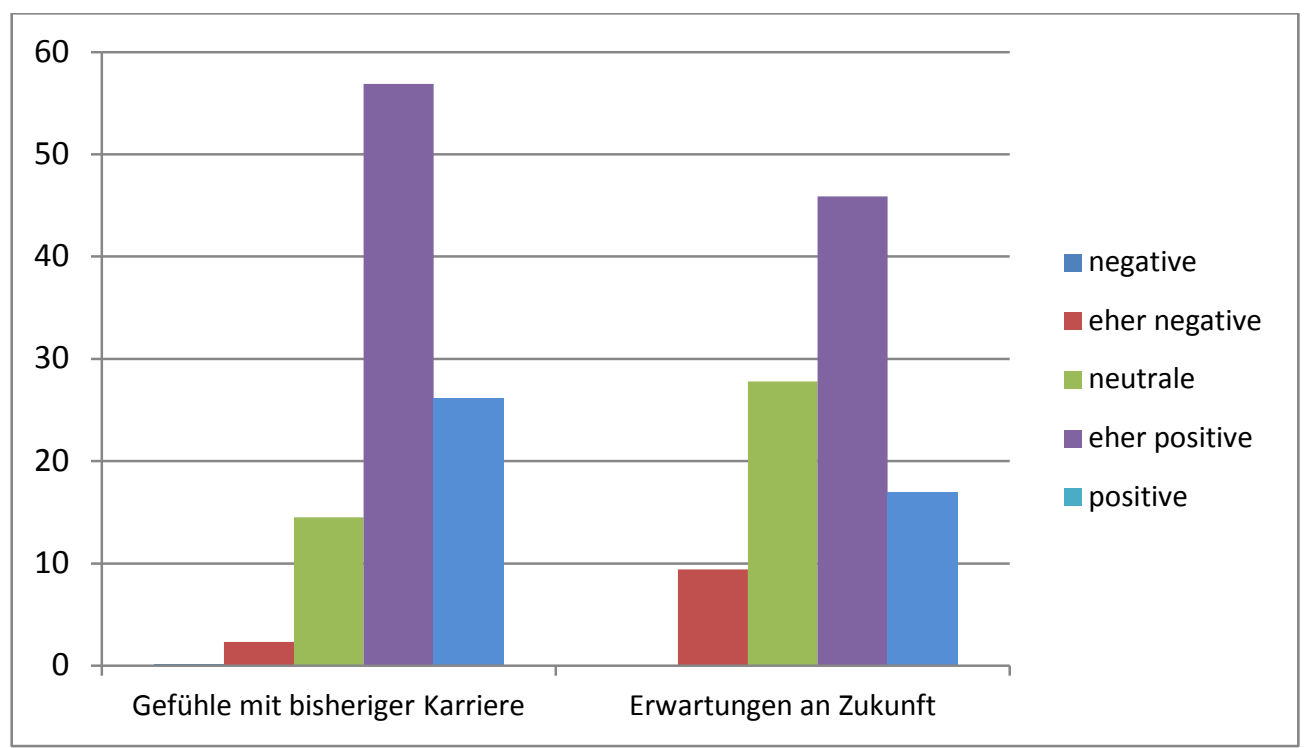

Abb. 32: Gefühle mit der bisherigen Karriere; Erwartungen an die Zukunft im Berufsleben

Ein weiterer Indikator, der letztendlich auch durch das Fühlen und Erleben im bzw. von Sportunterricht beeinflusst wird, ist die Absentismusrate. Die Sportlehrkräfte wurden gebeten anzugeben, an wie vielen Tage sie im vergangenen Kalenderjahr krankheitsbedingt fehlten. Im Durchschnitt gaben Sportlehrkräfte an, dass sie an 5,1 Tagen ( $N=690 ; S D=8.53$ ) krankheitsbedingt gefehlt haben. Die krankheitsbedingten Fehlzeiten sind damit gerade einmal halb so hoch wie der Wert, der für Lehrerinnen und Lehrer aller Fachrichtungen erhoben wurde (IKK-Bundesverband, 2007). Auffällig ist, dass Sportlehrerinnen mit 6,62 Tagen (SD=11.06) deutlich häufiger krank waren $(t(420,893)=3,776 ; p<.01)$ als ihre männlichen Kollegen $(M=3,95 ; S D=5,72)$. Dieser statistisch signifikante Unterschied der Mittelwerte entspricht einem Effekt von $d=.30$. Des Weiteren gaben 29,9\% ( $n=206)$ der Sportlehrkräfte an, an keinem einzigen Tag im zurückliegenden Kalenderjahr krankheitsbedingt gefehlt zu haben, nur $11 \%(n=76)$ fehlten an mehr als zehn Tagen im Jahr. 


\subsection{Untersuchungsschwerpunkt 1: Persönliche und berufsbio- graphische Merkmale und evasives Unterrichten: „Wer nutzt evasive Strategien im Unterricht"?}

Im Rahmen dieses Untersuchungsschwerpunkts wird der Frage nachgegangen, inwieweit persönliche und berufsbiographische Merkmale im Zusammenhang mit dem Vermeiden und Ausweichen von Konflikten im Sportunterricht stehen. Neben Angaben wie dem Alter, der Berufserfahrung, dem Geschlecht oder den Beweggründen für die Wahl des Berufs werden sowohl die praktische und theoretische Vermittlungskompetenz als auch die Affinität zu Bewegung Spiel und Sport in Beziehung zur Ausprägung des evasiven Verhaltenspotenzials gesetzt. Des Weiteren werden berufsbezogene Daten wie die Stunden- bzw. Lehrverpflichtung, das Fortbildungsengagement oder die Unterschiede bezüglich verschiedener Fachkombinationen herangezogen, die letztendlich eine Antwort auf die Frage: „Wer nutzt evasive Strategien?" liefern sollen. Die Antworten können, wie bereits in Kapitel 5 angedeutet, dabei helfen, "die Richtigen" für den Beruf zu finden. Neben einer Steigerung der Qualität der Lehrer können so zudem Enttäuschungen und berufsbedingte Erschöpfung vermieden werden.

\subsubsection{Alter und Berufserfahrung ${ }^{10}$}

Mit dem Alter und der Berufserfahrung werden an dieser Stelle zwei Merkmale in Beziehung zu der Ausprägung des evasiven Potenzials in seinen drei Facetten sowie zum Gesamtsummenwert gesetzt. Zu diesem ersten Untersuchungsaspekt konnte anhand der theoretischen Abhandlung keine klare und eindeutige Hypothese vorab formulierbar werden (vgl. Kap. 5.1). Sowohl ein Absinken des evasiven Potenzials mit zunehmender Lebens- und Berufserfahrung durch Routinisierung und den Gewinn an Sicherheit als auch eine schrittweise Reduzierung eigener Ansprüche im Laufe der Zeit, gemäß dem Schema von Huberman (1989) (siehe Kap. 4.9), sind denkbar. Ebenso ist es nicht ausgeschlossen, dass keinerlei Zusammenhang zwischen dem Vermeiden von Konflikten und dem Alter bzw. der Berufserfahrung besteht.

Die Korrelationen von EESU-S $(r=.119, p<.01)$ sowie EESU-I $(r=.130, p<.01)$ und dem Alter sind nach Cohen (1988) als gering zu bezeichnen. Mit zunehmendem Alter ist also ein leichtes Ansteigen des evasiven Verhaltenspotenzials in Bezug auf den Umgang mit Schülerinnen und Schülern sowie den Inhalten des Sportunterrichts festzustellen. Zu der Facette EESU-B sowie zur Gesamtskala EESU-SIB bestehen hingegen keine korrelativen Zusammenhänge.

\footnotetext{
${ }^{10}$ Da die Berufserfahrung und das Alter der Sportlehrkräfte sehr hoch miteinander korrelieren, wird an dieser Stelle lediglich der Zusammenhang zwischen dem evasiven Potenzial und dem Alter dargestellt.
} 
Da aufgrund von immer wieder stattfindenden Reformen in der Lehrerausbildung nicht auszuschließen ist, dass sich einzelne Altersgruppen von den anderen unterscheiden, wurde zudem eine Gruppierung in Altersstufen vorgenommen. Möglicherweise weicht die berufsbiographische Entwicklung von Hubermans Schema (1989) ab und es kommt es zu einem atypischen Verlauf, bei dem auf eine motivierte Berufseinstiegsphase eine Stagnationsphase mit dem Absinken des eigenen Anspruchs folgt, die wiederum von einer erneuten Motivationsphase abgelöst wird. In solch einem Fall kann eine einfache Überprüfung auf korrelative Zusammenhänge keine weiteren Aufschlüsse liefern. Um die Zusammenhänge zwischen dem Alter und dem evasiven Verhaltenspotenzial etwas genauer aufzuschlüsseln, wurde im Rahmen einer einfaktoriellen Varianzanalyse (ANOVA) überprüft, ob zwischen den in Bereiche klassierten Altersstufen ${ }^{11}$ und dem evasiven Potenzial in den verschiedenen Facetten signifikante Unterschiede bestehen. In der Facette EESU-S ist zwar von Stufe zu Stufe eine Zunahme des Einsatzes evasiver Strategien festzustellen, jedoch nicht in signifikantem Maß, $F(3,667)=2.511, p=.058$. Bei der Facette-EESU-I hingegen liegen signifikant Unterschiede bezüglich der Evasionswerte, $F(3,654)=4.715, p<.01$, zwischen den verschiedenen Altersstufen vor. Altersstufe $3(M=21,12 ; S D=4.39)$ und $4(M=21,01 ; S D=4.65)$ unterscheiden sich signifikant gegenüber Altersstufe $1(M=19,73 ; S D=4.75)$ und $2(M=19,70 ; S D=4.31)$, d.h. dass mit zunehmendem Alter die Tendenz, Konflikten auszuweichen, zunimmt. Vergleicht man die Mittelwertdifferenz zwischen Altersstufe 2 und 3, ergibt Cohens $d$ einen kleinen Effekt $\left(d_{2-3}=0.32\right)$, genau wie bei dem Vergleich von Altersstufe 1 und 3 sowie 1und 4. Die Ergebnisse deuten an, dass bezogen auf die Inhalte der Anstieg im evasiven Potenzial lediglich auf den deutlichen Anstieg von Altersstufe 2 zu Altersstufe 3 zurückzuführen sind. Der Übergang von Altersstufe 3 zu 4 bringt keinen weiteren Anstieg des evasiven Potenzials mit sich. Die Facette EESU-B weist im Bezug auf die verschiedenen Altersstufen keine signifikanten Unterschiede auf, zeigt jedoch tendenziell, dass mit zunehmendem Alter vermeidendes Verhalten in geringerem Maß als zu Beginn der Karriere eingesetzt wird. Eine mögliche Erklärung, ist die aufgebaute Routine und Erfahrung, die Lehrerinnen und Lehrern im letzten Karriereabschnitt zu einer offensiveren Haltung im Umgang mit Kollegen, der Schulleitung und den Eltern führt. Probleme werden in diesem Altersbereich weniger oft übergangen, sondern die eigenen Interessen und Zielsetzungen verstärkt vertreten. In Abbildung 33 sind die Ausprägungen der verschiedenen Facetten des evasiven Potenzials, differenziert nach den unterschiedlichen Altersgruppen, abgebildet.

\footnotetext{
${ }^{11}$ Altersstufe 1=25-34 Jahre, Altersstufe 2=35-44 Jahre, Altersstufe 3=45-54 Jahre, Altersstufe 4=55-64 Jahre.
} 


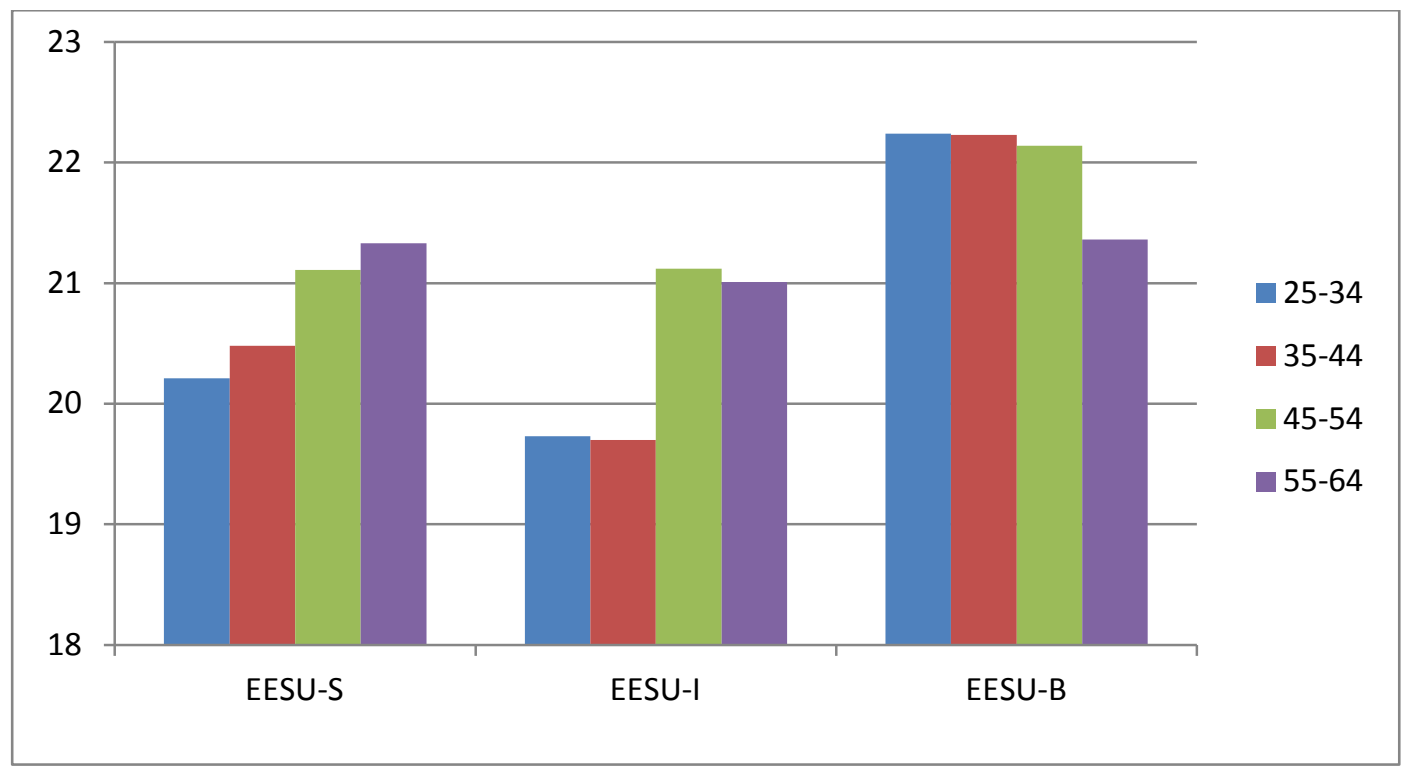

Abb. 33. Evasives Potenzial der drei Facetten in den vier Altersklassifikationen

Betrachtet man den Summenwert (EESU-SIB) aller drei Facetten (siehe Abb. 34) kann man anhand der Altersgruppen 1 ( $M=62,21 ; S D=11.61), 2(M=62,81 ; S D=12.17)$ und 3 ( $M=64,29 ; S D=12.88$ ) einen tendenziellen altersbedingten Anstieg des evasiven Verhaltenspotenzials erkennen, bevor im Alterssegment 4, d.h. bei den 55- bis 64-Jährigen der Wert wieder absinkt ( $M=63,61 ; S D=12.99)$. Jedoch sind die Unterschiede lediglich als Tendenz zu interpretieren, da sie statistisch nicht signifikant sind.

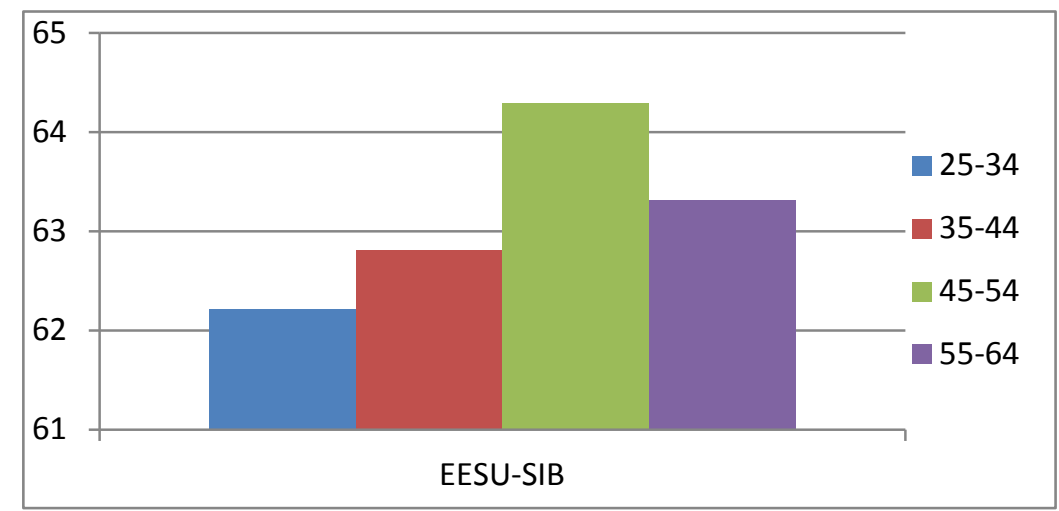

Abb. 34: Evasives Potenzial (Gesamtskala EESU-SIB) in den vier Altersklassifikationen

\subsubsection{Geschlechtsvergleich}

In diesem Abschnitt wird überprüft, ob es signifikante Unterschiede zwischen Sportlehrerinnen und Sportlehrern bezüglich des evasiven Potenzials gibt. Auch hier werden die Unterschiede wieder jeweils für die drei Facetten sowie den Gesamtwert berechnet. Auch in diesem Aspekt waren unterschiedliche Ausprägungen bzw. das Nichtvorhandensein von Korrelationen zu dem evasiven Potenzial denk- und begründbar (siehe Kap. 5.1). 
In der Facette EESU-S bestehen zwischen männlichen und weiblichen Sportlehrkräften keine signifikanten Unterschiede im evasiven Potenzial. Frauen weisen mit einem mittleren Summenwert von 21,01 (SD=4.36) einen minimal höheren Wert als ihre männlichen Kollegen mit 20,68 (SD=4.30) auf. Signifikante Unterschiede bestehen hingegen in den Facetten EESU-I und EESU-B sowie dem Gesamtsummenwert EESU-SIB. Bezogen auf die Inhalte verhalten sich Frauen $(M=20,94 ; S D=4.58)$ signifikant $(t(658)=2,431$; $p<.05)$ häufiger evasiv als Männer $(M=20,07 ; S D=4.55)$. Ebenso wie bei den Inhalten verhalten sich Frauen ( $M=22,48 ; S D=5.02)$ auch im Bezug auf den Umgang mit äußeren Rahmenbedingungen signifikant $(t(652)=2,44 ; p<.05)$ evasiver als Männer $(M=21,53$; $\mathrm{SD}=4.83)$ wodurch sich auch die signifikanten $(t(653)=2.335 ; p<.05)$ Unterschiede zwischen Lehrinnen ( $M=64,56 ; S D=12.60)$ und Lehrern $(M=62,26 ; S D=12.30)$ im Gesamtsummenwert des evasiven Verhaltens ergeben. Die vorliegenden Effekte sind jedoch sowohl in der Facette EESU-I mit $d=0.196$, EESU-B mit $d=0.190$ als auch bezogen auf den Gesamtsummenwert EESU-SIB mit $d=0.186$ als klein zu bezeichnen.

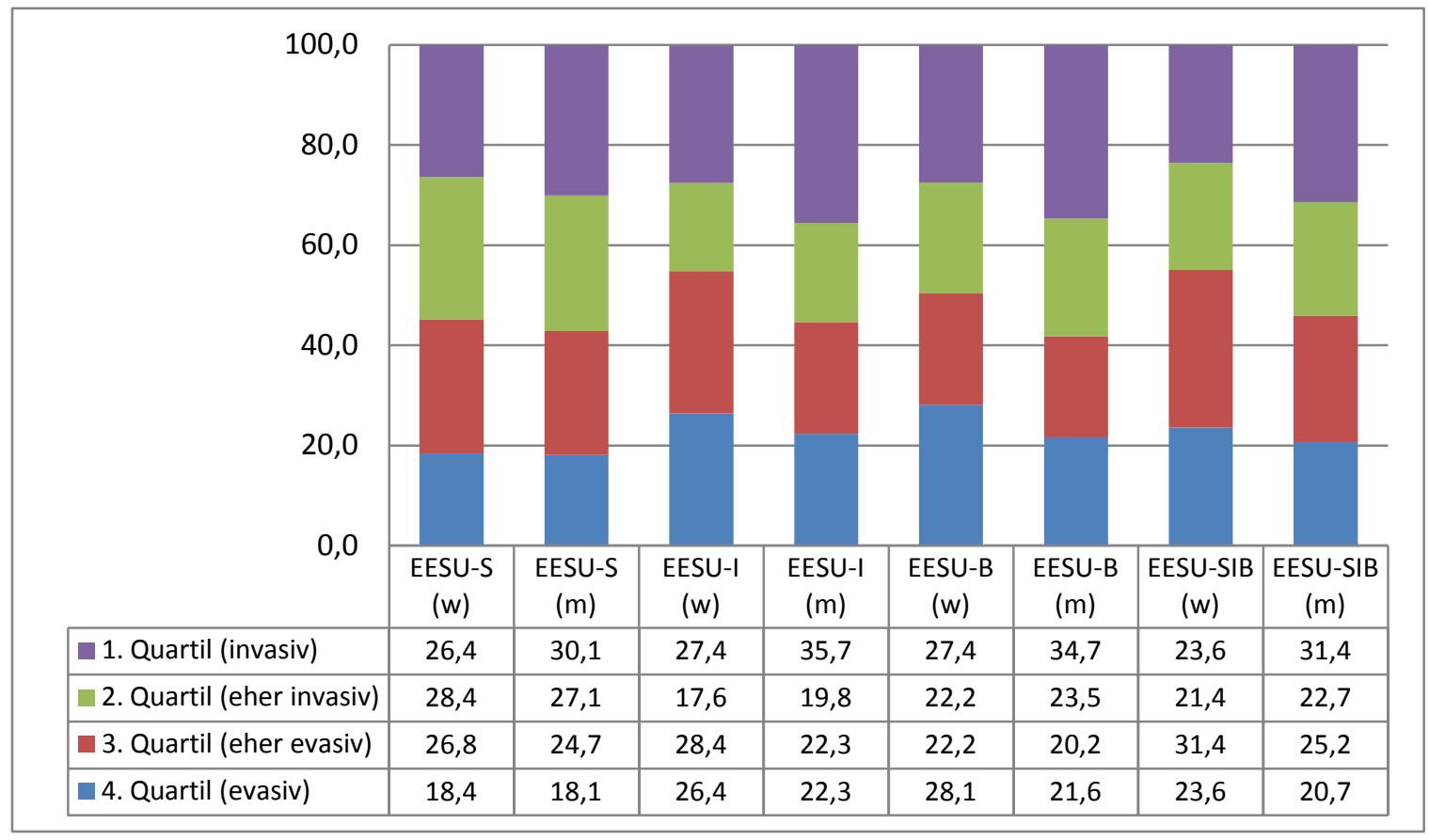

Abb. 35: Geschlechtsspezifisches evasives Potenzial der drei Facetten sowie dem Gesamtsummenwert (jeweils eingeteilt in Quartile)

Unterteilt man das evasive Potenzial in vier Quartile, sodass in Quartil 1 und 2 die invasiv und eher invasiv agierenden Lehrkräfte und in Quartil 3 und 4 die eher evasiv und evasiv unterrichtenden Sportlehrkräfte zusammengefasst werden, können die Unterschiede zwischen Sportlehrerinnen und Sportlehrern auch graphisch sichtbar gemacht werden. Die Verteilung der Lehrkräfte auf das in vier Quartile eingeteilte evasive Potenzial zeigt in den drei Facetten als auch bei dem Gesamtscore jeweils höhere Anteile 
im dritten und vierten Quartil für die weiblichen Lehrkräfte, sodass diese insgesamt etwas evasiver agieren als ihre männlichen Kollegen. Die in die Abbildung 35 integrierte Tabelle zeigt die Prozentwerte der Zuordnungen zu den Quartilen an.

\subsubsection{Studien- und Berufswahl (extrinsisch versus intrinsisch)}

In diesem Abschnitt wird der Frage nachgegangen, inwieweit Zusammenhänge zwischen den Gründen für die Studien- und Berufswahl und der Ausprägung der evasivinvasiv Polarität bestehen. Neben der Möglichkeit, dass aufgrund hedonistischer Motive, die in Konflikten enthaltenen Entwicklungschancen bei den extrinsisch Motivierten verkannt werden, ist es ebenso denkbar, dass vor allem die intrinsisch-altruistisch Motivierten aufgrund überhöhter Ansprüche und den resultierenden Enttäuschungen vermehrt evasive Tendenzen aufweisen. Ebenso ist es denkbar, dass keinerlei Zusammenhänge vorliegen, da es sich um eine höher angesiedelte Persönlichkeitseigeneschaft handelt.

Aufgrund der Nichterfüllung der Voraussetzungen für die Anwendung eines $t$-Tests, wurden die unterschiedlichen Mittelwerte im Rahmen eines Mann-Whitney-U-Test auf Signifikanzen hin untersucht. Wie sich in der Auswertung zeigte, weisen Lehrkräfte, die sich aus intrinsisch-altruistischen Motiven für ein Lehramtsstudium und den Beruf entschieden haben, in allen drei Facetten signifikant geringere Evasionswerte (EESU-S: $\mathrm{U}=47749 ; p<.05$; EESU-I: $U=41175,5 ; p<.01$; EESU-B: $U=37400,5 ; p<.001)$ auf, als diejenigen, die extrinsisch-hedonistische Gründe angaben, wie fehlende Alternativen, Studienwahl von Freunden, lange Ferienzeiten oder einen sicheren Arbeitsplatz (siehe Abb. 36).

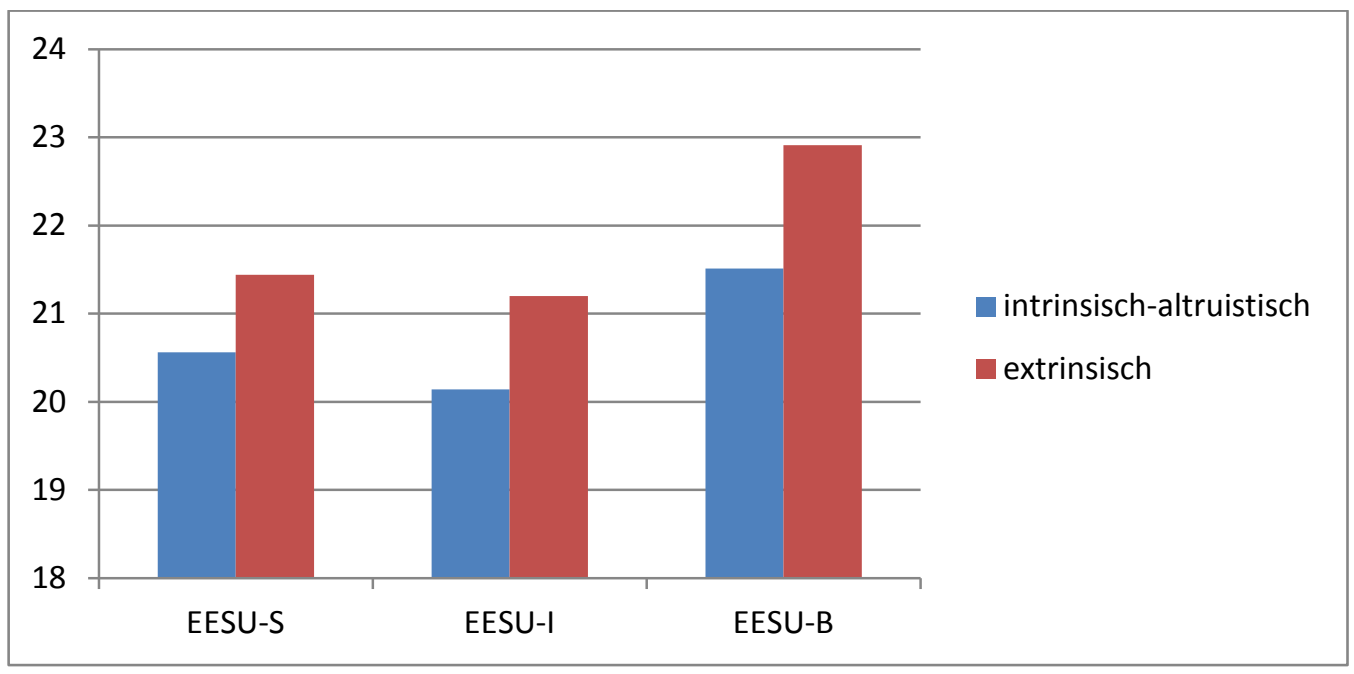

Abb. 36: Evasives Potenzial der unterschiedlichen Berufswahlmotivationen (intrinsischaltruistisch/extrinsisch) 
Folglich ergibt sich auch für die Gesamtskala EESU-SIB für intrinsisch-altruistisch Motivierte ( $M=62,23 ; S D=11.94)$, die im Beruf eher eine Berufung sehen, ein deutlich geringerer EESU-SIB-Wert ( $U=36679 ; p<.005)$ als durch andere Gründe extrinsisch Motivierte $(M=65,54 ; S D=13,27)$. Die Effektstärke dieser Unterschiede erweist sich jedoch sowohl in den drei Facetten $r_{\mathrm{EESU}-\mathrm{S}}=-.094, r_{\mathrm{EESU}-\mathrm{I}}=-.086, r_{\mathrm{EESU}-\mathrm{I}}=-.138$ als auch im Gesamtsummenwert $r_{\mathrm{EESU}-\mathrm{SIB}}=-.117$ als gering.

\subsubsection{Praktische- und theoretische Vermittlungskompetenz}

Anhand der Angaben, die die Sportlehrer zu ihrer sportartspezifischen Praxis- und theoretischen Vermittlungskompetenz machten, wurden ein individueller Summenwert für diese beiden Kompetenzen und ein Gesamtkompetenzwert errechnet. Zwischen allen drei Facetten sowie für den Gesamtsummenwert des evasiven Potenzials bestehen mittlere Korrelationen zu der sportartspezifischen Praxis-, der theoretischen Vermittlungs- sowie der Gesamtkompetenz (siehe Tab. 23). Auffällig ist zudem, dass die theoretische Vermittlungskompetenz eine, bezogen auf die offensive bzw. vermeidende Problemlösung, einflussreichere Größe darstellt als das Praxiskönnen in den verschiedenen Sportarten. An dieser Stelle soll nicht verschwiegen werden, dass auch zwischen dem Alter und der theoretischen und praktischen Vermittlungskompetenz Zusammenhänge bestehen. Junge, frisch ausgebildete Sportlehrkräfte verspüren weniger körperliche Beeinträchtigungen und verfügen über aktuelleres Vermittlungswissen im Vergleich zu ihren älteren Kollegen. Aufgrund der deutlich geringeren Zusammenhangsmaße zwischen dem Alter und der Vermittlungskompetenz ( $r_{\text {theoretische }}$ Vermittlunskompetenz $=$-.138; $\left.r_{\text {praktische Vermittlunskompetenz }}=-.216 ; r_{\text {Gesamtkompetenz }}=-.190\right)$ kann aber dennoch von einem nicht zu vernachlässigenden altersunabhängigen Zusammenhang zwischen der Vermittlungskompetenz und dem evasiven Potenzial ausgegangen werden.

Tab. 23: Korrelationsmatrix für die Praxis-, theoretische Vermittlungs- und Gesamtkompetenz und dem evasiven Potenzial in den drei Facetten sowie dem Gesamtscore $\left({ }^{* *}=p<.01\right)$

\begin{tabular}{|l|l|l|l|}
\cline { 2 - 4 } \multicolumn{1}{c|}{} & \multicolumn{1}{c|}{ Praxiskompetenz } & $\begin{array}{c}\text { theoretische Vermitt- } \\
\text { lungskompetenz }\end{array}$ & \multicolumn{1}{c|}{$\begin{array}{c}\text { Gesamtkompetenz } \\
\text { (Praxis \& Theorie) }\end{array}$} \\
\hline EESU-S & $-.311^{* *}$ & $-.432^{* *}$ & $-.405^{* *}$ \\
\hline EESU-I & $-.329 * *$ & $-.406^{* *}$ & $-.399^{* *}$ \\
\hline EESU-B & $-.265^{* *}$ & $-.345^{* *}$ & $-.332^{* *}$ \\
\hline EESU-SIB & $-.335^{* *}$ & $-.430^{* *}$ & $-.417^{* *}$ \\
\hline
\end{tabular}


7.3.5 Individuelle Sportaffinität (Fitnesszustand, Sportaktivität in der Freizeit, Vereinstrainerschaft, aktuell oder in der Vergangenheit betriebener Wettkampfsport, Stellenwert von Sport im Privatleben) Im Rahmen der Frage: „Inwieweit beeinflusst die individuelle Affinität zu Sport das evasive Verhalten im Sportunterricht?" werden anhand verschiedener Angaben zu der sportlichen Aktivität bzw. dem Engagement im Bereich Sport Zusammenhänge zum ausweichenden Verhalten überprüft. In Kapitel 5.1 wurde neben der Hypothese, dass die Sportaffinität einen prädiktiven Wert für die Ausprägung des evasiven Potenzials aufweist, ebenso in Betracht gezogen, dass keinerlei Zusammenhänge bestehen, da höher angesiedelte Verhaltensdimensionen auf Eben der Persönlichkeit entscheidenden Einfluss ausüben.

Generell zeigen die Antworten der Sportlehrkräfte, dass diejenigen Lehrer, bei denen Bewegung, Spiel und Sport, in welcher Form auch immer, einen hohen Anteil bzw. Stellenwert in der Freizeit einnimmt, sich im Unterricht in geringerem Maße evasiv verhalten als diejenigen, die mit Spiel, Sport und Bewegung eigentlich nur in der Schule in Kontakt kommen. Die Korrelationen zwischen den die Sportaffinität beschreibenden Variablen und dem evasiven Potenzial bewegen sich alle durchweg im niedrigen Bereich. Auffällig ist, dass das Verhalten im Bezug auf äußere Rahmenbedingungen (EESU-B) aufgrund der entfernteren Beziehung zur Praxis des Sportunterrichts erwartungsgemäß in geringerem Maß bzw. in keiner Beziehung zu den die private Sportaffinität beschreibenden Variablen steht. Grundsätzlich kann man jedoch die Aussage treffen, dass je schlechter der eigene Fitnesszustand, je geringer die Sportaktivität in der Freizeit und der Stellenwert von Sport im Privatleben ist, desto eher werden evasive Bewältigungsstrategien im Sportunterricht angewendet (siehe Tab. 24). Im Umkehrschluss scheint also Sportaffinität ein Faktor zu sein, der mit offensiven Bewältigungsmaßnahmen im Unterricht in Verbindung steht.

Tab. 24: Korrelationen zwischen verschiedenen, die Sportaffinität beschreibenden Variablen und dem evasiven Potenzial $\left({ }^{*}=p<.05 ;{ }^{* *}=p<.01\right)$

\begin{tabular}{|l|l|l|l|l|}
\cline { 2 - 5 } \multicolumn{1}{c|}{} & \multicolumn{1}{c|}{ EESU-S } & \multicolumn{1}{c|}{ EESU-I } & \multicolumn{1}{c|}{ EESU-B } & \multicolumn{1}{c|}{ EESU-SIB } \\
\hline Eigener Fitnesszustand & $-.154^{* *}$ & $-.168^{* *}$ & $-.107^{* *}$ & $-.160^{* *}$ \\
\hline Sportaktivität in der Freizeit & $-.107^{* *}$ & $-.122^{* *}$ & $-.092^{*}$ & $-.122^{* *}$ \\
\hline Stellenwert von Sport im Privatleben & $-.129 * *$ & $-.122^{* *}$ & -.070 & $-.109 * *$ \\
\hline
\end{tabular}

Ein weiteres Merkmal, das unter dem Sammelbegriff Sportaffinität gefasst wird, stellt die Einbindung in Wettkampfsport in der Vergangenheit bzw. Gegenwart dar. In Kapitel 7.1 wurde bereits dargestellt, dass $66,5 \%(n=458)$ der Sportlehrer in der Vergan- 
genheit, 23,1\% ( $n=159)$ noch aktuell aktiv am wettkampfmäßig organisierten Sport partizipieren bzw. partizipierten. Auch in Bezug auf diese Angabe wurde der Datensatz nach Zusammenhängen untersucht. Zwischen Sportlehrkräften, die aktuell bzw. in der Vergangenheit Wettkampfsport betrieben haben und denjenigen, die nie am wettkampfmäßig organisierten Sport als Aktive beteiligt waren, bestehen hinsichtlich des evasiven Potenzials keinerlei statistisch verwertbaren Unterschiede.

Im Gegensatz dazu ergibt die Auswertung des EESU für Lehrkräfte, die innerhalb der letzten fünf Jahre als Vereins- oder Kadertrainer aktiv waren bzw. noch sind, einen deutlich geringeren Wert in der Ausprägung des evasiven Verhaltens im Vergleich zu denen, die in dieser Zeit kein zusätzliches Vereinsmandat inne hatten (siehe Abb. 37). In der Facette EESU-S unterscheiden sich die Vereins- oder Kadertrainer ( $M=19,89$; $S D=4.01)$ gegenüber denjenigen, die kein Amt innehaben bzw. -hatten $(M=21,37$; $\mathrm{SD}=4.36)$ in signifikanter Art und Weise $(t(686)=-4.386 ; p<.01)$, was einem Effekt von $d=0.349$ entspricht. Ebenso entsprechen auch die Mittelwertunterschiede in der Facette EESU-I, $t(672)=-4.209, p<.001\left(\mathrm{M}_{1}=19.51, \mathrm{SD}_{1}=4.17, \mathrm{M}_{2}=21.02, \mathrm{SD}_{2}=4,65\right)^{12}$ einem kleinen Effekt von $d=0.341$. Ebenso kleine Effekte von $d=0.292$ und $d=0.372$ liegen für die Facette EESU-B, $t(668)=-3.620, p<.001 \quad\left(\mathrm{M}_{1}=21,00, \quad \mathrm{SD}_{1}=4.75, \quad \mathrm{M}_{2}=22.41\right.$, $\left.\mathrm{SD}_{2}=4,94\right)^{13}$ und den Gesamtsummenwert EESU-SIB, $t(651)=-4.523, p<.001\left(\mathrm{M}_{1}=60,33\right.$, $\left.\mathrm{SD}_{1}=11.34, \mathrm{M}_{2}=64,81, \mathrm{SD}_{2}=12.70\right)$ vor. Zusammenfassend lässt sich sagen, dass die Sportaffinität eine nicht zu unterschätzende Einflussgröße auf die Ausprägung des evasiven Potenzials ist.

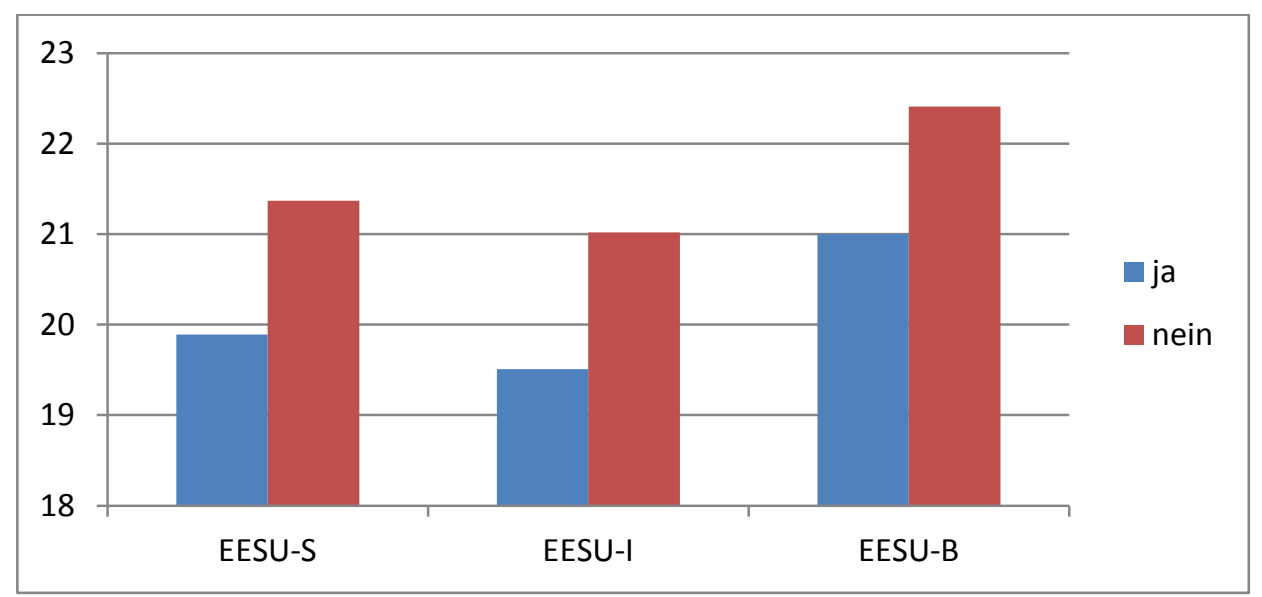

Abb. 37: Ausprägung des evasiven Potenzials (als Summenscore) im Zusammenhang mit der Angabe über eine Vereins- oder Kadertrainertätigkeit in den letzten fünf Jahren

\footnotetext{
${ }^{12} M_{1}$ \& $S_{1}=$ Vereins- oder Kadertrainer in den letzten fünf Jahren; $M_{2}$ \& $S_{2}=$ kein Vereins- oder Kadertrainer in den letzten fünf Jahren.

${ }^{13} M_{1}$ \& SD $=$ =Vereins- oder Kadertrainer in den letzten fünf Jahren; $M_{2} \& S D_{2}=$ kein Vereins- oder Kadertrainer in den letzten fünf Jahren.
} 


\subsubsection{Kombination von Fachrichtungen bzw. Haupt- und Nebenfächern}

Neben dem Fach Sport unterrichten die Lehrkräfte der Stichprobe meist zusätzlich mindestens in einem weiteren Fach. In diesem Abschnitt wird überprüft, inwieweit Unterschiede zwischen den Lehrkräften der verschiedenen Fachrichtungen und der Ausprägung des evasiven Potenzials bestehen. Die angegebenen Fächer wurden hierzu in die bereits in Kapitel 7.1 vorgestellten Fachrichtungen eingruppiert. Neben mathematisch-naturwissenschaftlichen, sprachlichen und gesellschaftwissenschaftlichen Fächern stellt die Bezeichnung „weiche Fächer" eine Sammelbezeichnung für Fächer wie Textiles Gestalten, Kunst, Musik oder darstellendes Spiel dar. Im Rahmen einer einfaktoriellen Varianzanalyse (ANOVA) wurden signifikante Unterschiede bezüglich des evasiven Potenzials der verschiedenen Fachrichtungen auf Signifikanzen überprüft. Signifikant divergierende Mittelwerte $F(3,563)=4.003, p<.01$ bestehen im Bezug auf die Facette EESU-S, die im Wesentlichen auch für die Range in dem in Abbildung 38 abgebildeten Gesamtscore (EESU-SIB) verantwortlich ist. Vergleicht man die Mittelwerte von EESU-S der Lehrkräfte der sprachwissenschaftlichen Fächer ( $M=19,90$; $S D=4.08)$ der mathematisch-naturwissenschaftlichen Fächer $(M=21,16 ; S D=4.08)$ und den "weichen Fächern" ( $M=21,69 ; \mathrm{SD}=3.97)$ ergeben sich statistisch signifikante Unterschiede. Auffällig ist, dass die sprachwissenschaftlichen Fächer im Vergleich zu den anderen beiden Fachrichtungen eine deutlich geringere evasive Verhaltenstendenz im Umgang mit Schülerinnen und Schüler aufweisen. Cohens $d$ deutet in beiden Fällen auf einen kleinen Effekt ( $\left.d_{\text {Sprachen-Mathem./Nawi }}=0.308 ; d_{\text {Sprachen-weiche Fächer }}=0.445\right)$ hin. In den Facetten (EESU-I) und (EESU-B) bestehen keine signifikanten Unterschiede bezüglich des evasiven Verhaltenspotenzials zwischen den verschiedenen Fachrichtungen. Anhand von Abbildung 38, in der die Gesamtsummenwerte EESU-SIB der einzelnen Fachrichtungen dargestellt sind, erkennt man, dass Lehrer, deren Zweitfach eine Sprache ist, die geringsten Werte $(M=61,63 ; S D=13.43)$ im evasiven Verhalten zeigen, wohingegen, die Lehrer der weichen Fächer ( $M=65,70 ; S D=11.95)$ den evasivsten Unterrichtsstil der vier Fachrichtungen aufweisen. Diese Mittelwertdifferenz erwies sich zwar in der Varianzanalyse als statistisch nicht signifikant $F(3,528)=1,994, n s$, dennoch liegt in diesem Fall ein kleiner Effekt von $d=0.320$ vor. Die gesellschaftswissenschaftlichen $(M=62,51 ; S D=12.70)$ und mathematisch-naturwissenschaftlichen Fächer $(M=64,0$; $\mathrm{SD}=11.70$ ) liegen zwischen den beiden zuvor genannten Fachrichtungen. Diese Unterschiede im Gesamtwert für das evasive Verhalten im Sportunterricht sind sowohl untereinander als auch im Bezug auf die beiden zuvor genannten Fachrichtungen nicht statistisch signifikant und daher nur als Tendenz zu interpretieren. 


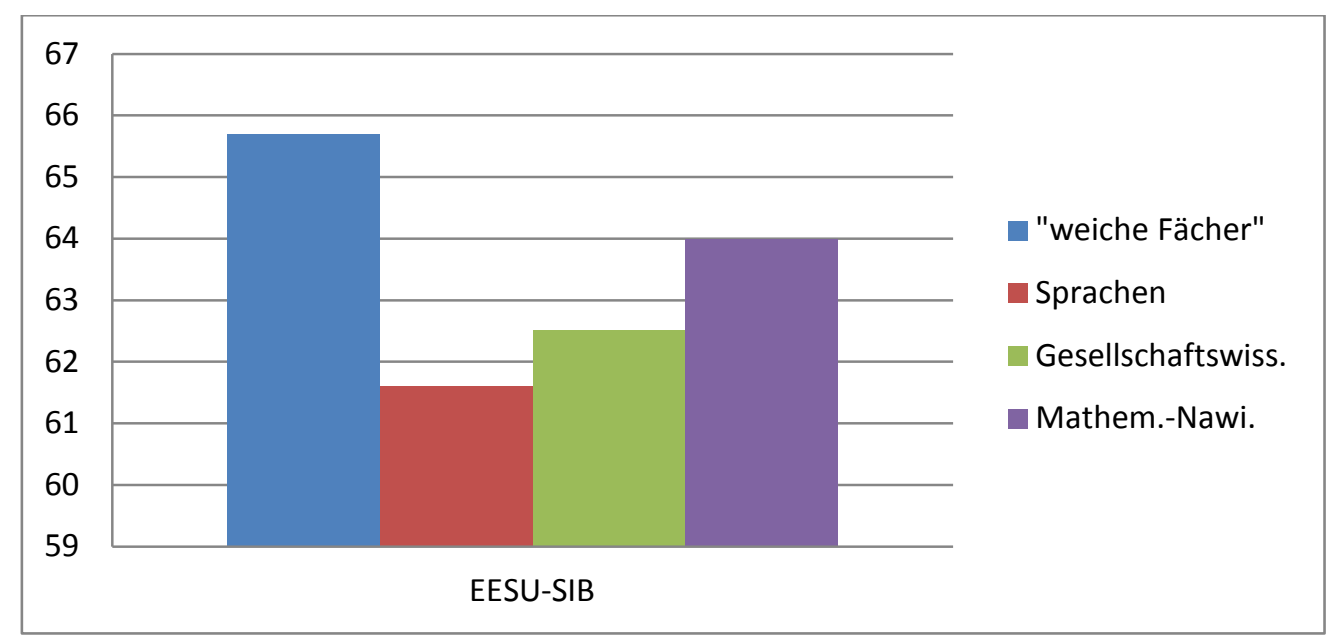

Abb. 38: Vergleich des Gesamtscore des evasiven Verhaltenspotenzial (EESU-SIB) der verschiedenen Fachrichtungen

Unterteilt man die Zweitfachangaben in Haupt- und Nebenfächer, lassen sich weder in den einzelnen Facetten noch im Gesamtsummenwert bedeutsame Mittelwertunterschiede im evasiven Potenzial feststellen. Eine Erklärung hierfür stellt die zugrunde liegende Berechnung der Werte für die Neben- und Hauptfächer dar. Die „evasiven weichen Fächer" wurden mit den weniger evasiv agierenden gesellschaftswissenschaftlichen Fächern zu den Nebenfächern gezählt und die eher invasiv agierenden sprachlichen Fächer wurden mit einem Großteil der eher evasiv unterrichtenden mathematisch-naturwissenschaftlichen Fächern zusammengerechnet, wodurch sich die zuvor dargestellten Unterschiede in den Fachrichtungen fast vollständig nivellieren.

\subsubsection{Gesamtstundenverpflichtung, Sportstundenzahl und prozentualer}

\section{Anteil des Sportunterrichts an der Gesamtlehrverpflichtung}

In Kapitel 7.1 wurden bereits die Messwerte der wöchentlichen Gesamtstundenverpflichtung, die Sportstundenanzahl sowie der prozentualen Anteil des Sportunterrichts an der Gesamtlehrverpflichtung vorgestellt. An dieser Stelle wird überprüft, ob sich zwischen der Gesamtlehrverpflichtung sowie der Sportstundenzahl und der Ausprägung der evasiven Verhaltenstendenz Zusammenhänge finden lassen.

Zwischen der Gesamtlehrverpflichtung und dem evasiven Potenzial im Bezug auf Inhalte (EESU-I) $(r=-0.109 ; p<.01)$ besteht ebenso wie zum Gesamtsummenwert (EESU-SIB) $(r=-.083 ; p<.05)$ eine geringe signifikante negative Korrelation. Weitere signifikante negative Korrelationen $(p<.01)$ bestehen zwischen der wöchentlichen Sportstundenanzahl und den Facetten EESU-S ( $r=-.122 ; p<.01)$, EESU-I $(r=-.110 ; p<.01)$ sowie zum Gesamtsummenscore EESU-SIB ( $r=-.111 ; p<.01)$. Diese korrelativen Zusammenhänge deuten an, dass mit zunehmender Gesamtlehrverpflichtung die Tendenz, eher defensive und ausweichende Bewältigungsmaßnahmen zu nutzen, abnimmt. Lehrer, die eine 
geringere Stundenzahl, z.B. in Form einer halben Stelle, unterrichten, weisen also ein höheres evasives Potenzial auf, als diejenigen, die gemäß dem vollen Stellenumfang unterrichten. Dies gilt in ähnlichem Maße für die Sportstundenzahl. Auch hier weisen Lehrkräfte, die eine geringere Wochenstundenzahl unterrichten, eine höhere evasive Verhaltenstendenz auf als Lehrerinnen und Lehrer, die mehr Sportunterricht geben.

Ebenso wie die wöchentlich Gesamtlehrverpflichtung und die Sportstundenanzahl steht auch der prozentuale Anteil des Sportunterrichts an der Gesamtlehrverpflichtung zum Teil in einem negativen Korrelationsverhältnis zum evasiven Unterrichten. Zwischen dem prozentualen Anteil des Sportunterrichts an der Gesamtlehrverpflichtung, bezogen auf die Facette EESU-S besteht ein statistisch bedeutsamer negativer Zusammenhang ( $r=-.094 ; p<.05)$. Zwischen den Facetten EESU-I, EESU-B sowie dem Gesamtsummenwert EESU-SIB und dem prozentualen Anteil des Sportunterrichts an der Gesamtlehrverpflichtung bestehen keine Zusammenhänge. Als zusammenfassende Aussage kann man folgern, dass mit zunehmender wöchentlichen Gesamt- und Sportstundenanzahl sowie mit zunehmendem Anteil des Sportunterrichts an der Gesamtstundenzahl in geringerem Maß evasive Tendenzen im Unterricht das Verhalten bestimmen.

\subsubsection{Fächerübergreifendes, fachspezifisches und privates Fortbil- dungsengagement}

Eine in der Literatur oft als bedeutsam bezeichnete Einflussgröße auf die Qualität von Unterricht stellt die Aktualisierung von Wissen und Können im Rahmen von organisiertem oder privatem Fortbilden dar. Im Rahmen der durchgeführten Untersuchung wurden die Lehrkräfte zu ihrem fächerübergreifenden und sportunterrichtsspezifischen Fortbildungsengagement sowie zu dem Umfang der Bemühungen im privaten Bereich befragt. In diesem Abschnitt wird überprüft, inwieweit das Engagement sich fortzubilden, sei es organisiert oder privat, in einem Zusammenhang zur Ausprägung des evasiven Unterrichtsstils steht. Die Ergebnisse der Korrelationsberechnung zwischen dem Fortbildungsengagement und evasiven Verhaltenstendenzen sind relativ eindeutig zu interpretieren: Sportlehrkräfte, die sich häufiger fortbilden zeigen in geringeren Maße Vermeidungstendenzen im Sportunterricht, wie sich an den in Tabelle 25 ablesbaren Werten zeigt. 
Tab. 25: Korrelationsmatrix: Fortbildungsengagement $-\operatorname{EESU}\left({ }^{*}=p<.05 ;{ }^{* *}=p<.01\right)$

\begin{tabular}{|l|l|l|l|l|}
\cline { 2 - 5 } \multicolumn{1}{c|}{} & EESU-S & EESU-I & \multicolumn{1}{c|}{ EESU-B } & $\begin{array}{c}\text { EESU- } \\
\text { SIB }\end{array}$ \\
\hline Fächerübergreifendes Fortbildungsengagement & $-.116^{* *}$ & $-.101^{* *}$ & $-.150^{* *}$ & $-.139^{* *}$ \\
\hline Sportunterrichtsspezifisches Fortbildungsengagement & $-.113^{* *}$ & $-.098^{*}$ & -.064 & $-.102^{* *}$ \\
\hline Gesamt-Fortbildungsengagement & $-.148^{* *}$ & $-.129^{* *}$ & $-.131^{* *}$ & $-.153^{* *}$ \\
\hline Privates Fortbildungsengagement & $-.217^{* *}$ & $-.195^{* *}$ & $-.106^{* *}$ & $-.184^{* *}$ \\
\hline
\end{tabular}

Anhand von Tabelle 25 wird zudem deutlich, dass der Einfluss von Fortbildungen auf den Umgang mit Schülern am größten ist. Konform den Erwartungen steht die Ausprägung von EESU-B vor allem im Zusammenhang mit dem fächerübergreifenden Fortbilden, im Rahmen derer auch Reflexion und Weiterbildung bezogen auf das weitere schulische Umfeld erfolgt, das unter der Facette EESU-B zusammengefasst wird. Sportunterrichtsspezifische Fortbildungen stehen in keinem Zusammenhang mit der Facette EESU-B, da hier meist fachliche Schwerpunkte wie z.B. die didaktisch-methodische Aufbereitung von traditionellen Sportarten oder die Einführung von Trendsportarten behandelt werden. Als grundsätzliche Aussage kann man anhand der vorliegenden Daten jedoch formulieren, dass mit zunehmendem Fortbildungsengagement die Tendenz ausweichend im Sportunterricht zu agieren sinkt, bzw. evasiv agierende Lehrkräfte ein geringeres Fortbildungsengagement aufweisen.

Sinn und Zweck der verschiedenen Arten der Fortbildungen, egal ob es fächerübergreifende oder sportunterrichtsspezifisch organisierte Fort- und Weiterbildungsveranstaltungen sind, ist die Aktualisierung und der Ausbau von Fachkompetenz. Im Rahmen der durchgeführten Untersuchung wurden 16 Sportarten bezüglich der individuellen theoretischen Vermittlungskompetenz und der Praxiskompetenz eingeschätzt, zu Gesamtwerten verrechnet sowie in Beziehung zur Ausprägung des evasiven Verhaltenspotenzials gesetzt (siehe Kap. 7.1 und 7.3.4). Im Folgenden wird überprüft inwieweit das Fortbildungsengagement tatsächlich Auswirkungen auf die Vermittlungskompetenz im Fach Sport aufweist. Während sowohl die theoretische Vermittlungskompetenz $(r=.241 ; p<.01)$ als auch die Praxiskompetenz $(r=.227 ; p<.01)$ einen korrelativen Bezug zum privaten sportunterrichtsspezifischen Fortbildungsengagement durch Fachzeitschriften und Fachliteratur aufweisen, bestehen zum fächerübergreifenden und sportunterrichtsspezifischen „organisierten“ Fortbilden keine bzw. nur sehr geringe Zusammenhänge. Während das fächerübergreifende Fortbildungsengagement keinerlei korrelativen Bezüge zur theoretischen Vermittlungskompetenz und Praxiskompetenz aufweist, besteht zwischen dem sportunterrichtsspezifisch organisierten Fortbil- 
den lediglich in einem geringen Maß ein Zusammenhang zur Praxiskompetenz ( $r=.81$; $p<.05)$, nicht jedoch zur theoretischen Vermittlungskompetenz.

\subsubsection{Zusammenfassende Interpretation der Ergebnisse}

Im Verlauf dieses Kapitels konnten in vielfältiger Weise Zusammenhänge zwischen persönlichen und berufsbiografischen Merkmalen und der Ausprägung des evasiven Verhaltenspotenzials festgestellt werden. In diesem Abschnitt soll der Antwort auf die Frage „Wer nutzt evasive Strategien?" etwas näher gekommen werden. Neben einigen Zusammenhängen, die in gewissem Maß eine Charakterisierung des Typus „evasiv unterrichtende Sportlehrkraft" ermöglichen, erlauben andere Bereiche keine klare Zuschreibung von Ursache und Auswirkung. So zeigt sich z.B., dass die Studien- und Berufswahl und die dahinterstehenden Motive ganz deutliche Auswirkungen auf das Verhalten im Sportunterricht haben. Anhand der Daten wird zudem ersichtlich, dass die Erfüllung des beruflichen Auftrags eines Lehrers mehr erfordert als rationale und hedonistische Überzeugungen, was sich im Umgang mit Konflikten im Alltag widerspiegelt. Altruistische Überzeugungen sind notwendig, um auch nach Enttäuschungen, Gratifikationsdefiziten oder mangelnder Wirksamkeit des eigenen Handelns weiterhin an Erziehungs- und Bildungszielen festzuhalten und Konflikte problemorientiert anzunehmen. Während hier, allein durch die zeitliche Eindeutigkeit Klarheit darüber besteht, was als Ursache und was als Wirkung zu bezeichnen ist, liegen bei dieser Untersuchungsfrage auch Ergebnisse vor, die nicht so einfach zu interpretieren sind. Sowohl bei der Stundenverteilung als auch dem Fortbildungsengagement ist es nicht möglich, ein eindeutiges Ursache-Wirkungsgefüge zu formulieren. In Kapitel 7.3.7 wurde dargestellt, dass das evasive Potenzial mit steigender Gesamtlehrverpflichtung, Sportstundenzahl und prozentualem Anteil des Sportunterrichts an der Gesamtlehrverpflichtung absinkt. Lehrkräfte, die weniger (Sport) unterrichten, verhalten sich im Umkehrschluss beim Auftreten von Konflikten und Problemen deutlich evasiver als diejenigen mit einer höheren Lehrverpflichtung. Eine eindeutige Erklärung hierfür ist jedoch schwer zu formulieren. Einerseits ist es möglich, dass die Lehrkräfte mit einer niedrigeren Stundenverpflichtung weniger Erfahrung im alltäglichen Unterrichtsgeschäft haben, wodurch eine unterschwellige Unsicherheit resultiert, die sich auf die Problembewältigung auswirkt. Lehrkräfte mit einer hohen Lehrverpflichtung hingegen bilden möglicherweise Routinen und Mechanismen aus, die einen effektiven Umgang mit Belastungen fördern bzw. ermöglichen. Denkbar ist es ebenso, dass Sportlehrkräfte mit einer hohen Stundenverpflichtung sich kein ausweichendes Konfliktverhalten „leisten" können, um für sich selbst im Unterricht angemessene Arbeitsbedingungen zu gewährleisten. Als dritte Möglichkeit ist in Betracht zu ziehen, dass aufgrund von Überlastung, 
bedingt durch einen unangemessenen Bewältigungsstils und dem daraus resultierenden Wiederauftreten der Probleme, die Stundenverpflichtung bewusst als Selbstschutz reduziert wurde. Bezogen auf die Zusammenhänge zwischen dem prozentualen Anteil der Sportstundenzahl an der Gesamtlehrverpflichtung könnte es auch sein, dass Sport bei Lehrkräften mit einem geringen Sportstundenanteil als ergänzendes Beifach gesehen wird und daher Motivation und Anspruchsdenken nur mäßig ausgeprägt sind. Auch wenn, wie bereits in Kapitel 7.2.8 und 7.2.11 beschrieben, die Atmosphäre im Kollegium und der fachspezifische Legitimationsdruck nicht als vorrangige Belastungsquelle gesehen werden, kann auch aus eigener Erfahrung berichtet werden, dass fachfremde Kollegen die Bedeutsamkeit des Sportunterrichts eher gering einschätzen und mehr freizeitliche Attribute mit dem Fach verbinden, als dass sie tatsächliche Erziehungs- und Bildungsarbeit in dem für sie fremden Unterrichtssetting vermuten. Möglicherweise führen solche Begegnungen in Lehrerzimmern dazu, dass manche Sportlehrkräfte selbst die Ansichten übernehmen und ihr Anspruchsdenken senken.

Beim Fortbildungsengagement und der Ausprägung des ausweichenden Verhaltens fällt die Zuschreibung von Ursache und Wirkung ebenfalls schwer. Hier ist zwar naheliegend, dass evasives Verhalten ein Resultat von fehlendem Fortbildungsengagement ist, ebenso können jedoch auch der evasive Unterrichtsstil und die daraus resultierenden Belastungen dafür verantwortlich sein, dass das Fortbilden als zusätzliche, nicht bewältigbare Belastung empfunden, und daher vernachlässigt wird.

Eindeutiger zu interpretieren als die Zusammenhänge zwischen dem evasiven Potenzial und der Stundenverteilung bzw. dem Fortbildungsengagement lassen sich die korrelativen Beziehungen zur zuvor bereits angesprochenen Studien- und Berufswahl, dem Alter, dem Geschlecht, der praktischen und theoretischen Vermittlungskompetenz, der Sportaffinität sowie den Fachrichtungen, denen die Teilnehmer neben Sport angehören. Wie sich in Kapitel 7.3.1 herausstellte, unterscheiden sich die verschiedenen Altersgruppen im Ausmaß des Einsatzes von evasiven Strategien im Sportunterricht. In Kapitel 4.9 und 5.1 wurde gemutmaßt, dass evasives Verhalten entweder vor allem zu Berufsbeginn aufgrund fehlender Erfahrung und mangelnder Routine eingesetzt wird oder aber sich im Laufe des Berufslebens mit der Zeit entwickelt, da Ansprüche entwicklungsbedingt sinken und zunehmend Ablauforientierung das Handeln bestimmt. Anhand der vorliegenden Daten konnte gezeigt werden, dass mit zunehmendem Alter und zunehmender Berufserfahrung die Tendenz steigt, Konflikten und Problemen mit Schülern sowie mit Inhalten aus dem Weg zu gehen bzw. diese zu vermeiden. Mit zunehmendem Alter scheint aber auch der Anspruch bzw. die Widerstandsfähigkeit zu sinken, aktiv und offensiv mit Konflikten umzugehen. Im Umgang mit äußeren Rah- 
menbedingungen wurde bei der Einteilung in Altersquartile hingegen ein leichter Trend festgestellt, der den zuvor getroffenen Aussagen widerspricht. Im Umgang mit Kollegen, der Schulleitung sowie Vorschriften und Erlassen ist eine leicht invasivere Verhaltenstendenz der Lehrkräfte im letzten Altersquartil festzustellen. Im letzten Berufsabschnitt gehen Sportlehrkräfte also Probleme, die ihren Ursprung eher im weiteren Umfeld des Unterrichts haben, offensiver oder zumindest nicht evasiver an als ihre jüngeren Kolleginnen und Kollegen. Der höhere Evasionswert bezogen auf den Umgang mit Inhalten mit zunehmendem Alter könnte im Sinne von Meusel (1989) als Kompensation von eigenen Schwächen interpretiert werden, die nur durch stetiges Fort- und Weiterbilden verhindert werden können. In der Untersuchungsstichprobe stellte sich jedoch heraus, dass zwischen dem Alter und dem sportunterrichtsspezifischen Fortbildungsengagement ein negativer Zusammenhang $(r=-.083 ; p<.05)$ besteht, sodass die von Meusel formulierte Hypothese durchaus der Realität entsprechen könnte.

Die in Kapitel 7.3.2 dargestellten Unterschiede der Geschlechter bezüglich des evasiven Verhaltens sollen nicht ins Zentrum etwaiger Folgerungen und Interpretationen gestellt werden, da sie einerseits nur knapp unter dem festgelegten Signifikanzniveau liegen und andererseits aus Studien bekannt ist, dass Männer generell dazu neigen, sich selbst besser wahrzunehmen und einzuschätzen, als dies Frauen tun.

Die Zusammenhänge und Mittelwertunterschiede, die in Kapitel 7.3.5 dargestellt wurden, lassen sich mit der Aussage zusammenfassen, dass diejenigen, die eine höhere Affinität zu Bewegung, Spiel und Sport haben, auch ein geringeres evasives Verhaltenspotenzial aufweisen. Berührungspunkte sowie eine aktive Einstellung zu sportlichen Betätigungsformen führen scheinbar dazu, dass Lehrpersonen im Sportunterricht mit mehr Selbstvertrauen und Zuversicht auftreten und eher offensive Strategien im Umgang mit Konflikten präferieren. Außerdem zeigt sich, dass allein das wettkampfmäßige Betreiben einer Sportart keine zusätzliche Handlungssicherheit und daraus resultierend offensive Problembewältigung bewirkt. Anders als zu dem Merkmal „Vereins- bzw. Kadertrainer", "eigener Fitnesszustand“, "Sportaktivität" sowie "Stellenwert von Sport im Privatleben“ liegen keine Korrelationen zum evasiven Verhaltenspotenzial vor. Relativ ausgeprägt stellen sich die Zusammenhänge zwischen der Selbsteinschätzung der theoretischen und praktischen Vermittlungskompetenz und dem evasiven Bewältigen heraus (siehe Kap. 7.3.4), wobei hierbei Kompetenzen, die die theoretische Planung von Unterricht beeinflussen, einen höheren Stellenwert aufweisen als Kompetenzen, die die praktische Umsetzung der Unterrichtsvorhaben betreffen. Eine theoretisch fundierte Grundlage in den einzelnen Unterrichtsinhalten sowie Kompetenzen, 
die für die praktischen Unterrichtsdurchführung notwendig sind, scheinen einen nicht zu unterschätzenden Einfluss auf die Problembewältigung aufzuweisen, sodass die grundsätzliche Eignung für den Beruf sowie Aspekte der Ausbildung neben den im sich anschließenden Untersuchungsschwerpunkt behandelten Persönlichkeitsmerkmalen ebenso kontextspezifische Relevanz aufweisen.

Insgesamt zeigte sich in diesem ersten Untersuchungsschwerpunkt, dass die Ausprägung des evasiven Bewältigungspotenzials ein Merkmal ist, dass mit zunehmendem Alter an verhaltensbestimmendem Charakter gewinnt und zudem recht stark durch die berufsbezogene Motivation der Lehrkräfte und deren individuellen Kompetenzen beeinflusst wird. Dies gilt in starkem Maße für die Basismotivation der Lehrer, die in diesem Fall als Studien- und Berufswahlmotivation bezeichnet wird, aber auch für die Motivation, die eigene Kompetenzen und Fähigkeiten durch Fortbilden zu aktualisieren und zu erweitern. Bereits in Kapitel 2.3 wurde angesprochen, dass Sportlehrkräfte einen Vorbild- und Modellcharakter für die Schüler übernehmen sollten. Ergänzend kann nach der Auswertung dieses ersten Schwerpunkts bestätigt werden, dass auch in Bezug auf das Bewältigungshandeln eine Nähe zu dem Fach sowohl im kognitiven als auch im körperlichen Bereich einen unterstützenden Faktor darstellt.

\subsection{Untersuchungsschwerpunkt 2: Unterrichtliche Charakte- ristik: „In welchen Aspekten unterscheidet sich der Unter- richt von eher evasiv und eher invasiv Unterrichtenden?"}

Im zweiten Untersuchungsschwerpunkt werden unterrichtliche Merkmale herausgearbeitet, in denen sich eher evasiv und eher invasiv unterrichtende Sportlehrkräfte unterscheiden. Neben den Kriterien für die Auswahl der Inhalte, die Häufigkeit der Durchführung von verschiedenen Sportarten und der individuellen Bedeutsamkeit von verschiedensten Vermittlungsaspekten kann auch die Lehrweise und die Organisationsform des Unterrichts dazu herangezogen werden, um den Unterricht von eher evasiv und eher invasiv Unterrichtenden zu vergleichen. Auch die aktive Teilnahme der Sportlehrkräfte am Unterricht, der Arbeitsaufwand für das Fach Sport für Vor- und Nachbereitung sowie der subjektive Stellenwert des Faches Sport im Vergleich stellen indirekte Parameter der Unterrichtsrealität dar. Als weiterer Aspekt wird unter diesem Untersuchungsschwerpunkt das technisch-taktische und konditionelle Aspirationsniveau im Zusammenhang mit der Ausprägung des evasiven Potenzials betrachtet. Hierbei wird geprüft, ob sich die Anforderungen unterscheiden, die eher evasiv und eher invasiv Unterrichtende von ihren Schülern abverlangen. In einer abschließenden zusammenfassenden Interpretation der Ergebnisse soll eine Antwort auf die Frage: „In welchen 
Aspekten unterscheidet sich der Unterricht von eher evasiv und eher invasiv Unterrichtenden?" gegeben werden.

\subsubsection{Kriterien der Inhaltswahl}

In diesem Abschnitt wird überprüft, ob Zusammenhänge zwischen der Ausprägung des evasiven Potenzials und der individuellen Einschätzung der Bedeutsamkeit von verschiedenen Kriterien für die Inhaltswahl vorliegen. Wie bereits in Kapitel 7.2.1 beschrieben, haben die Sportlehrkräfte verschiedene Kriterien bezüglich deren Bedeutung im individuellen Entscheidungsprozess bei der Inhaltswahl im Unterricht eingeschätzt. Das bedeutendste Kriterium, die curricularen Vorgaben, steht in einem negativen Korrelationsverhältnis ( $r=-.132 ; p<.01)$ zum Gesamtsummenwert des Erhebungsinstruments zur Erfassung des evasiven Potenzials. Sowohl zu der Facette EESU-S $(r=-.155 ; p<.01)$ als auch zu den Facetten EESU-S $(r=-.148 ; p<.01)$ sowie EESU-B $(r=-.086 ; p<.05)$ bestehen negative Zusammenhänge, d.h. dass je größer das individuelle evasive Potenzial ausgeprägt ist, desto weniger stellen curriculare Vorgaben ein Entscheidungskriterium für die Inhaltswahl dar. Die Ausstattung der Sportstätte, als eine die Inhaltswahl beeinflussende Größe, steht in einem geringen positiven Zusammenhang $(r=.097 ; p<.05)$ zum evasiven Verhaltensstil. D.h. je evasiver das Bewältigungsverhalten der Lehrkräfte geprägt ist, desto eher stellen materielle und infrastrukturelle Vorgaben und Bedingungen ein entscheidendes Kriterium für oder gegen einen bestimmten Inhaltsbereich dar. Zwischen dem Kriterium Schülerinteresse und den eigenen Vorlieben und dem evasiven Potenzial bestehen hingegen keine Zusammenhänge. Sehr wohl bestehen jedoch Korrelationen zwischen dem erwarteten Konfliktpotenzial zwischen den Schülern bei den verschiedenen Inhalten und der Ausprägung des evasiven Potenzials. Ein mittlerer Zusammenhang von $r=.302$, $p<.01$ zum Gesamtsummenwert EESU-SIB deutet darauf hin, dass das erwartete Konfliktpotenzial bei Personen, die im neu entwickelten Fragebogen EESU einen höheren Wert erreichen, eine deutlich größere Rolle spielt als bei Personen mit geringerem Evasionswert. Ebenso stellt das Verletzungsrisiko einen weiteren Faktor dar, der Sportlehrkräfte mit evasiver Verhaltenstendenz bei der Entscheidung für bzw. gegen verschiedene Inhalte stärker beeinflusst $(r=.290 ; p<.01)$ als invasiv Agierende. Der Organisations- und Aufbauaufwand $(r=.262 ; p<.01)$, die eigene theoretische Vermittlungskompetenz $(r=.183 ; p<.01)$ sowie die Praxiskompetenz $(r=.172 ; p<.01)$ stellen ebenfalls Attribute dar, die in einem positiven Zusammenhang mit dem evasiven Unterrichten stehen. Wie die Auswertung zeigt, agieren evasiv Unterrichtende bereits bei der Auswahl der Inhalte, d.h. im Vorfeld des Unterrichts, präventiv ausweichend. Dies zeigt sich daran, dass vor allem Aspekte, die 
Einfluss auf das Auftreten von Konflikten haben, Korrelationen zur Ausprägung des evasiven Potenzials aufweisen.

\subsubsection{Durchführungshäufigkeit von Sportarten}

Im Rahmen der Fragestellung: „Bestehen Zusammenhänge zwischen der Durchführungshäufigkeit der verschiedenen Sportarten und der Ausprägung des evasiven Potenzials?" soll überprüft werden, ob das Bewältigungsverhalten tatsächlich Auswirkungen auf die konkrete Ausgestaltung des Unterrichts aufweist. Bei der Durchführungshäufigkeit verschiedener Sportarten ist auffällig, dass Personen mit einer stärkeren Ausprägung von evasivem Verhalten bei fast allen Sportarten einen niedrigeren Wert für die Durchführungshäufigkeit angaben. Signifikante Korrelationen, die alle negativer Natur sind bestehen zwischen dem evasiven Potenzial und der Durchführungshäufigkeit von Leichtathletik ( $r=-.130 ; p<.01)$, Handball ( $r=-.125 ; p<.01)$, Turnen $(r=-.142 ; p<.01)$, Badminton $(r=-.084 ; p<.05)$, Konditionstraining $(r=-.106 ; p<.01)$, Basketball $(r=-.086 ; p<.05)$, Schwimmen ( $r=-.088 ; p<.05)$, Hockey $(r=-.127 ; p<.05)$ und den Trendsportarten $(r=-$ $.207 ; p<.01)$. Keine Korrelationen bestehen zwischen dem evasiven Potenzial und der Durchführungshäufigkeit von Fußball, Gymnastik/Tanz, kleine Spiele sowie Volleyball.

Zieht man in diesem Zusammenhang die in Studien zum Unfallgeschehen (Hübner \& Pfitzner, 2001; 2003) im Schulsport errechneten Risikofaktoren der einzelnen Sportarten heran, stellt sich heraus, dass die Sportarten offensichtlich entgegen den Zusammenhängen, die sich durch die Korrelation zu dem Verletzungsrisiko als Kriterium für die Inhaltswahl in 7.4.1 andeuteten, nicht aufgrund der Unfallhäufigkeit ausgewählt werden. Mit Fußball und der mit der Sammelbezeichnung kleine Spiele bestehen zu zwei Inhaltsbereichen, die sich durch einen überdurchschnittlich hohen Risikofaktor auszeichnen, keine statistisch verwertbaren Zusammenhänge. Die Sportarten, die von Sportlehrern mit höherem evasivem Verhaltenspotenzial weniger oft durchgeführt werden wie Turnen, Konditionstraining, Leichtathletik und Schwimmen stellten sich in den Studien zum Unfallgeschehen sogar als unterdurchschnittlich risikobehaftet heraus, sodass dieses Kriterium insgesamt als eher nachrangig in der Bedeutsamkeit im Zusammenhang mit der Ausprägung des evasiven Potenzials zu sehen ist.

\subsubsection{Bedeutsamkeit von Vermittlungsaspekten im Sportunterricht}

In der Rangfolge der Bedeutsamkeit von Vermittlungsaspekten (siehe Kap. 7.2.3) nahm die "Vermittlung von Freude an Bewegung, Spiel und Sport" sowie "soziales Lernen" die ersten beiden Plätze in der Rangfolge ein. "Fachliche Kenntnisse", "Taktik" und "Leistungserziehung" bildeten den Schluss dieser Liste und werden als weniger bedeutsam eingeschätzt. 
Von großem Interesse im Hinblick auf den Untersuchungsschwerpunkt „Unterrichtliche Charakteristik“ ist, ob Zusammenhänge zwischen der Bedeutsamkeit verschiedener Vermittlungsaspekte und der Tendenz, sich im Sportunterricht evasiv zu verhalten, existieren. Hierzu wurden die 15 Aspekte, die in Kapitel 7.2.3 bereits aufgeführt wurden, im Bezug zu der Ausprägung der drei Facetten des evasiven Unterrichtens sowie dem Gesamtsummenwert auf Korrelationen überprüft.

Genau wie bei der Durchführungshäufigkeit der Sportarten gaben Lehrkräfte mit stärker ausgeprägtem evasivem Potenzial auch bei der Bedeutsamkeit von Vermittlungsaspekten generell geringere Bewertungen ab. Als signifikant stellten sich diese Minderbewertungen vor allem in Vermittlungsaspekten heraus, die in neueren Lehrplänen bzw. Kerncurricula die Kernargumente für die Legitimation des Sportunterrichts im Fächerkanon sowie die Anspruch- und Zielsetzung in neueren Unterrichtskonzeptionen repräsentieren (vgl. Kap. 2.3.2).

Neben der Vermittlung von taktischen Fähigkeiten $(r=-.154 ; p<.01)$ und einer allgemeinen Spielfähigkeit $(r=-.135 ; p<.01)$ sind es vor allem die Korrelationen zwischen dem evasiven Verhaltenspotenzial und der Bedeutsamkeit des sozialen Lernens ( $r=-.196$; $p<.01)$, der Förderung der Kreativität $(r=-.147 ; p<.01)$, der Vermittlung von Freude an der Bewegung mit dem Ziel, zu einem lebenslangen Sporttreiben zu motivieren ( $r=-.109 ; p<.01)$, sowie die Förderung der Selbstständigkeit $(r=-.166 ; p<.01)$, die auf Unterschiede in der Schwerpunktsetzung im Bezug auf die Vermittlungsaspekte hindeuten. Des Weiteren bestehen Korrelationen zwischen der Bedeutsamkeit der Leistungserziehung $(r=-.151 ; p<.01)$, der koordinativen Fähigkeiten $(r=-.137 ; p<.01)$ und der Körpererfahrung $(r=-.188 ; p<.01)$. Zwischen der Vermittlung von fachlichen Kenntnissen, also den kognitiven Unterrichtsaspekten und dem evasiven Verhalten bestehen lediglich geringfügige Zusammenhänge $(r=-.086 ; p<.05)$. Bei der Bedeutsamkeit von traditionelleren Vermittlungsaspekten wie Fitness, Kondition, Körperbildung, Disziplin und Technikvermittlung bestehen keine korrelativen Zusammenhänge zum Gesamtsummenwert des evasiven Verhaltens.

\subsubsection{Aktive Teilnahme am Sportunterricht}

Wie in Kapitel 2.3.3 erläutert, ist es nicht nur die Aufgabe der Sportlehrkräfte, verschiedenste Inhalte zu präsentieren, aufzubereiten und für die Schüler erfahrbar zu machen sondern sie stellen auch selbst durch ihre Person bzw. Persönlichkeit eine Einflussgröße dar, die aus motivationstheoretischer Perspektive betrachtet durchaus Relevanz aufweist. Selbstverständlich kann die aktive Teilnahme eines Sportlehrers am Unterricht nicht allein als entscheidendes Kriterium für die Beurteilung der Güte des Unterrichts herangezogen werden, sie stellt aber durchaus ein Indiz für die Einstellung 
der Lehrkraft dar, sich selbst als Vorbild wahrzunehmen und einen aktiven und gesundheitsbewussten Lebensstil zu verkörpern. Zwischen dem evasiven Verhaltensstil und der Häufigkeit der aktiven Teilnahme am Sportunterricht bestehen negative Zusammenhänge, d.h. dass mit zunehmendem evasivem Potenzial die aktive Teilnahme am Unterricht seltener erfolgt als bei Lehrkräften, die sich im Unterricht Problemen gegenüber eher offensiv verhalten bzw. invasiv agieren. Am größten ist der korrelative Zusammenhang erwartungsgemäß zwischen der aktiven Teilnahme am Sportunterricht und dem evasiven Verhalten in den Facetten EESU-S $(r=-.244 ; p<.01)$ und EESU-I ( $r=-$ .237, $p<.01)$, wohingegen der Zusammenhang zur Facette EESU-B $(r=-.192 ; p<.01)$ erwartungsgemäß niedriger ausfällt. Der Zusammenhang zum Gesamtsummenwert EESU-SIB liegt bei $r=-.244, p<.01$.

Nutzt man bei dem Gesamtsummenwert EESU-SIB die bereits im Laufe des Ergebnisteils dargestellte Aufteilung des evasiven Potenzials in Quartile, stellt sich heraus, dass das „invasive“ Quartil 1 ( $M=3,52 ; \mathrm{SD}=0.897)$ im Vergleich zu dem „evasiven“ Quartil 4 $(M=3,01 ; S D=0.815)$ deutlich häufiger aktiv am Sportunterricht teilnimmt. Die aufgeführte Mittelwertdifferenz entspricht einem großen Effekt ( $d_{\text {Quartil1-Quartil4 }}=1.19$ ).

\subsubsection{Arbeitsaufwand für Vor- und Nachbereitung}

Eine weitere Variable, die hilft, den Charakter des Unterrichts bzw. die Auffassung der Lehrkräfte zu erfassen, stellt der Arbeitsaufwand dar, den Sportlehrer zusätzlich zu ihrer regulären Stundenverpflichtung wöchentlich für das Fach Sport investieren. Wie bereits in Kapitel 7.2.9 berichtet, beträgt der durchschnittliche außerunterrichtliche Zeitaufwand für das Fach Sport 4,19 Stunden (SD=4,29; $n=682$ ) was einer Vorbereitungszeit von etwa 27 Minuten pro Unterrichtsstunde Sport entspricht. Zwischen defensiven ausweichenden Verhaltensstrategien und dem Arbeitsaufwand bestehen lediglich in der Facette EESU-S minimale negative Zusammenhänge ( $r=-.092 ; p<.05)$. Zwischen den Facetten EESU-I und EESU-B sowie dem Gesamtsummenwert EESU-SIB und dem Zeitaufwand für die Vorbereitung bestehen keine Zusammenhänge. Auch eine varianzanalytische Überprüfung auf Mittelwertunterschiede der einzelnen Quartile ergab keine signifikanten Abweichungen in der Zeit für Vor- und Nachbereitung des Unterrichts. Schlussfolgernd investieren Lehrkräfte, die evasiv unterrichten nicht mehr oder weniger Zeit für die Vor- und Nachbereitung ihres Unterrichts als invasiv Unterrichtende, was vor allem in Bezug zu den arbeitsbezogenen Verhaltens- und Erlebensmuster von Schaarschmidt und Fischer (1996) interessant ist (siehe Kap. 6.3.1) und in Kapitel 8 erneut aufgegriffen werden soll. 


\subsubsection{Lehrweise und unterrichtliche Organisationsform}

Die Lehrweise und die Organisationform sind zwei weitere Unterrichtsmerkmale, die bereits in Kapitel 7.2.4 für die Gesamtstichprobe beschrieben wurden und das Unterrichtskonzept und den Lehrstil von Lehrkräften etwas genauer beschreiben. Auch in diesem Fall soll überprüft werden, ob sich bei der Lehrweise in der Ausprägung des deduktiven bzw. induktiven Vorgehens im Unterricht Zusammenhänge mit der Ausprägung des evasiven Potenzials nachweisen lassen. Wie sich herausstellte, bestehen signifikante Mittelwertdifferenzen im evasiven Potenzial zwischen denjenigen, die eher induktiv unterrichten und denen, die eine deduktive Lehrweise bevorzugen. In der Facette EESU-S verhalten sich Lehrkräfte, die sich eher mit einer induktiven Lehrweise identifizieren $(M=20,38 ; S D=4.40)$ in signifikant geringerem Maße $t(528)=-2.41, p<.05$ evasiv im Vergleich zu den eher deduktiven Unterrichtenden ( $M=21,29 ; S D=4.30)$. Auch in der Facette EESU-B sind die Mittelwertunterschiede zwischen induktiv ( $M=21,58$; $\mathrm{SD}=4.95)$ und deduktiv Unterrichtenden ( $\mathrm{M}=22.60 ; \mathrm{SD}=5.07)$ überzufällig $t(517)=-2.31$, $p<.05$. Der Gesamtsummenwert unterscheidet sich letztendlich ebenfalls signifikant $t(506)=-2.35, p<.05$ zwischen den induktiv $(M=62,28 ; S D=12.62)$ und den deduktiv $(M=64,89 ; S D=12.39)$ unterrichtenden Lehrkräften. Die Effekte dieser Mittelwertdifferenzen sind jedoch mit $d_{\text {EESU-S }}=0.21, d_{E E S U-B}=0.20$ und $d_{\text {EESU-SIB }}=0.21$ als gering einzuschätzen. In Abbildung 39 ist exemplarisch die Verteilung der induktiv bzw. deduktiv unterrichtenden Lehrkräfte auf die unterschiedlichen Quartile des evasiven Potenzials der Facette EESU-S dargestellt.

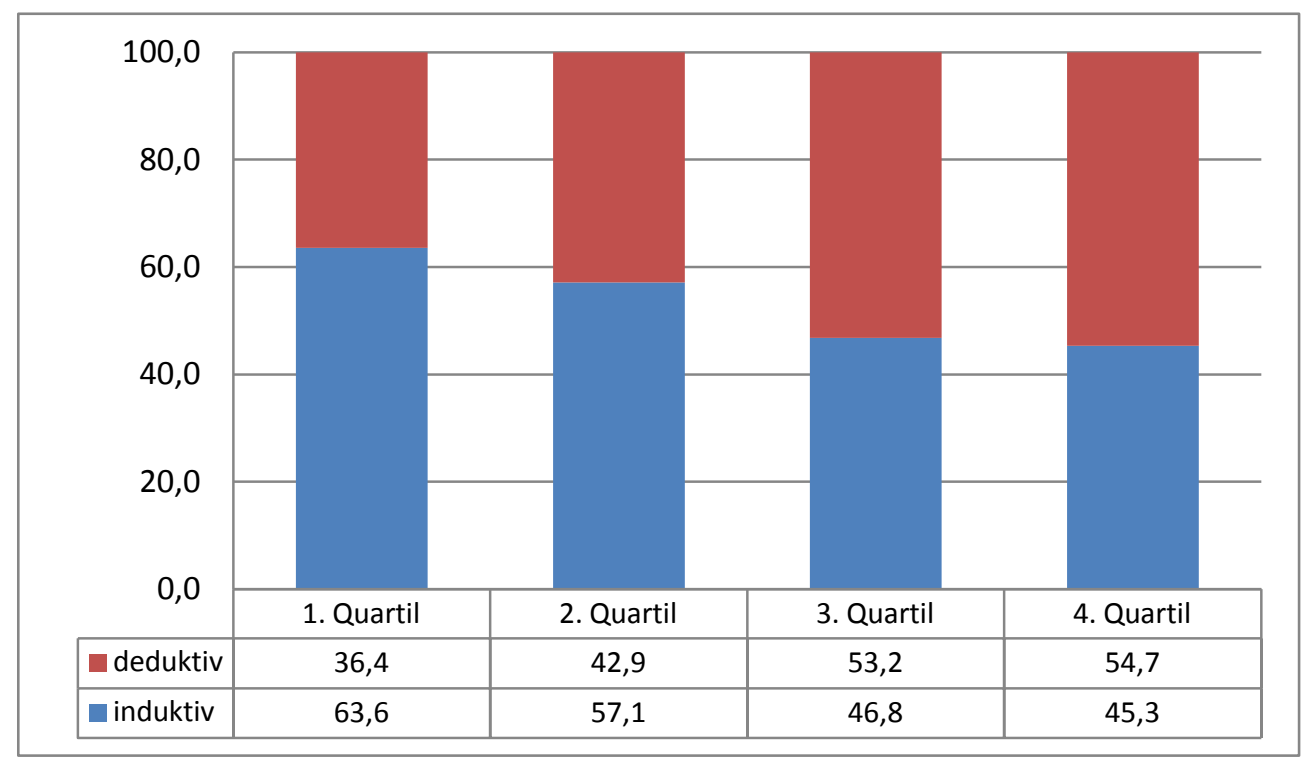

Abb. 39: Lehrweise (deduktiv/induktiv) differenziert nach dem evasiven Potenzial der Facette EESU-S (in Quartilen) 
Anhand von Abbildung 39 kann man erkennen, dass mit zunehmendem evasivem Potenzial in der Facette EESU-S, in diesem Fall dargestellt durch die vier Quartile, auch der Anteil der Sportlehrkräfte abnimmt, die induktiv unterrichten. Im vierten Quartil bevorzugen mehr als die Hälfte der in diese Stufe eingeordneten Lehrpersonen eine deduktive Lehrweise, wohingegen das 1. Quartil viel stärker durch einen induktiven Lehrstil geprägt ist. Die Verteilungen für die Facetten EESU-I, EESU-B und den Gesamtsummenwert entsprechen im Wesentlichen denen im oben beispielhaft dargestellten Diagramm der Facette EESU-S (siehe Abb. 39), sodass selbiges stellvertretend für die weiteren Facetten bzw. den Gesamtsummenwert steht.

Bei der Organisationsform des Unterrichts haben die Sportlehrkräfte ihren eigenen Unterricht zwischen den beiden Polen starke Lehrerzentrierung und Schülerorientierung eingeschätzt (siehe Kap. 7.2.4). Zwischen der Ausprägung des evasiven Potenzial und der Organisationsform des Unterrichts besteht in allen drei Facetten ( $r_{\text {EESU-s }}=.-187$, $\left.p<.01 ; r_{\mathrm{EESU}-\mathrm{I}}=.-149, p<.01 ; r_{\mathrm{EESU}-\mathrm{B}}=.-146, p<.01 ; r_{\mathrm{EESU}-\mathrm{SIB}}=.-183, p<.01\right)$ ein geringer negativer Zusammenhang, d.h. dass mit zunehmender evasiver Verhaltenstendenz die Lehrerzentrierung im Unterricht ebenfalls zunimmt. Insgesamt zeigte sich also, dass evasiv Unterrichtende verstärkt lehrerzentriert-deduktive Unterrichtsarrangements bevorzugen, da sie sich so vermutlich ein höheres Maß an Handlungssicherheit versprechen.

\subsubsection{Technisch-taktisches und konditionelles Aspirationsniveau}

Wie bereits in Kapitel 7.2.5 berichtet, sehen die Sportlehrkräfte das von ihnen festgelegte Anforderungsniveau auf einem relativ hohen Niveau, sowohl bezogen auf Technik und Taktik als auch im konditionellen Bereich. In Beziehung gesetzt zum evasiven Verhaltenspotenzial ergaben sich negative korrelative Zusammenhänge, d.h. je ausgeprägter das evasive Verhalten ist, desto geringer wird das Anforderungsniveau im Unterricht gewählt. Dies deutet darauf hin, dass Sportlehrkräfte, die dazu neigen, Konflikten eher defensiv bzw. vermeidend entgegenzutreten, ein geringeres Anspruchsniveau in ihrem Unterricht verfolgen. Bei der genauen Betrachtung fällt auf, dass der negative Zusammenhang zwischen dem evasiven Potenzial und dem technisch-taktischen Aspirationsniveau ( $r=-.281 ; p<.01$ ) deutlich stärker ausgeprägt ist als im Vergleich zum konditionellen Anspruch ( $r=-.147 ; p<.01)$. Auffällig bei den Antworten war zudem, dass Sportlehrerinnen $(M=3,25 ; S D=0.592)$ im technisch-taktischen Bereich ein signifikant geringeres Anspruchsniveau $t(673)=-5.099, p<.001$ als Sportlehrer $(M=3,49 ; S D=0.620)$ angeben, was einem Effekt von $d=0.40$ entspricht. Im konditionellen Bereich bestehen keine signifikanten Unterschiede. 


\subsubsection{Subjektive Einschätzung des Stellenwerts des Sportunterrichts im Fachvergleich}

Die Auswertung der Einschätzung des Stellenwerts des Fachs Sport von Sportlehrern ergab, dass die meisten Sportlehrkräfte den Sportunterricht im Fachvergleich als ein gleichwertiges Fach betrachten (siehe Kap. 7.2.11). Unterschiede bestehen jedoch wieder bezogen auf die Einschätzung des Stellenwertes des Sportunterrichts in Abhängigkeit von der Ausprägung des evasiven Potenzials. Während diejenigen, die dem Sportunterricht einen deutlich geringeren Stellenwert beimessen als den anderen Fächern mit einem Gesamtsummenwert (EESU-SIB) von 81,20 (SD=11.08) eine stark überdurchschnittliche evasive Tendenz aufweisen, erreichen diejenigen, die den Sportunterricht als ein Fach sehen, dass eine höhere Bedeutung als andere Fächer aufweist, einen Gesamtsummenwert von 58,95 ( $S D=13.29)$. Umgekehrt formuliert schätzen Sportlehrkräfte mit zunehmendem evasivem Potenzial das Fach als deutlich geringer bedeutsam ( $r=-.177 ; p<.01)$ ein als andere im schulischen Fächerkanon vertretene Fachdisziplinen.

Die Antworten eher geringer $(M=68,58 ; S D=11.70)$, gleichwertig $(M=62,78 ; S D=12.30)$ und eher höher $(M=61,93 ; S D=11.44)$ liegen mit ihrem Gesamtsummenwert (EESU-SIB) für das evasive Verhalten zwischen den zuvor aufgeführten Polen. Für die Berechnung der Mittelwertunterschiede und der Effektstärke wurden die Einschätzungen deutlich geringer und geringer sowie eher höher und höher jeweils zusammengefasst, um statistisch besser verwertbare Ergebnisse zu erhalten. Der Mittelwert der Lehrkräfte, die den Sportunterricht als deutlich geringer bzw. als eher geringer als die anderen Fächer sehen, ist mit einem Gesamtsummenwert (EESU-SIB) von $M=69.38$ ( $S D=12.00)$ signifikant $t(220)=4.888, \mathrm{p}<.01$ höher als bei denjenigen, die den Stellenwert des Sportunterrichts als eher höher und höher einschätzen ( $M=61,15 ; S D=11.98)$. Diese Mittelwertdifferenz entspricht einem mittleren Effekt von $d=0.69$.

\subsubsection{Zusammenfassende Interpretation der Ergebnisse}

Durch die Ergebnisse, die im Rahmen des zweiten Untersuchungsschwerpunkts dargestellt wurden, kann evasives Verhalten als Phänomen mit speziellem Blickwinkel auf die Gestaltung des Unterrichts genauer beschrieben werden. Durch das Zusammensetzen der einzelnen Ergebnisse lässt sich ein Bild des evasiven Unterrichts erstellen, dass durch bereits existierende Musterprofile, wie z.B. die von Schaarschmidt und Fischer (1996) (AVEM) oder Janke, Erdmann und Kallus (1984) (SVF120) nicht möglich sind. In fast allen Items, die in diesem Untersuchungsaspekt untersucht wurden, stellt sich die Ausprägung des evasiven Potenzials als eine bedeutsame Einflussgröße auf den Unterricht heraus. 
Bereits im Vorfeld des Unterrichts unterscheiden sich evasive und invasive Lehrertypen. Während die Vorgaben, die durch Lehrpläne, Rahmenrichtlinien oder sonstige Curricula gemacht werden, für evasiv Unterrichtende weniger bedeutsam sind als für invasiv Unterrichtende, sind das Verletzungsrisiko der Sportart sowie der Aufwand für die Organisation und den Aufbau für eher ausweichend Unterrichtende entscheidendere Einflussfaktoren. Während sich vermeidungsorientierte Sportlehrkräfte bei der Inhaltswahl stärker von den äußeren Rahmenbedingungen beeinflussen lassen, um dadurch im Vorfeld Problemen vorzubeugen, bemühen sich die invasiv Agierenden darum, die Gegebenheiten zu akzeptieren und möglichst gut mit ihnen umzugehen. Dies ist vor allem von Bedeutung, da der Sportunterricht durch den schlechten Zustand bzw. dem mangelndem Platzangebot von Sportstätten und fehlendem oder minderwertigem Material an vielen Schulen beeinträchtigt ist. Auch die Zusammenhänge zwischen dem Konfliktpotenzial zwischen den Schülern als Kriterium für die Inhaltswahl und dem ausweichenden Verhalten unterstützen diese These des präventiven Konfliktvermeidens. Bei dem angesetzten Aspirationsniveau zeigen sich die Differenzen, die durch den evasiven Unterrichtsstil von Sportlehrkräften bedingt sind. Vor allem im technisch-taktischen Bereich legen evasiv Unterrichtende einen anderen Maßstab an als Lehrkräfte mit stärker ausgeprägter invasiver Verhaltenstendenz. Gerade bei der Vermittlung von Technik und Taktik besteht die Gefahr, dass Schüler in eher kognitiv beanspruchenden Unterrichtssituationen, die ausdauernde Konzentration erfordern, Konflikte untereinander, aber vor allem gegenüber der Lehrkraft eingehen bzw. provozieren. Diese Unterrichtssituationen konfligieren oft mit den Interessen der Schüler, was zu Missmut und Unzufriedenheit führen kann und damit ein höheres Konfliktpotenzial aufweisen kann als konditionell anspruchsvolle Inhalte, zu denen in geringerem Maße Zusammenhänge zur Ausprägung des evasiven Unterrichts bestehen.

Bei der Durchführungshäufigkeit von Inhalten wäre anhand der Einschätzung der Kriterien zur Inhaltswahl zu erwarten gewesen, dass aufgrund der Bedeutsamkeit des Verletzungsrisikos von evasiv unterrichtenden Sportlehrkräften vor allem die "riskanten“ Sportarten gemieden werden. Wie sich jedoch herausstellte, kann kein klares Muster anhand der angegebenen Durchführungshäufigkeit abgeleitet werden. Während erwartungsgemäß das hohe Anforderungen an die Lehrkräfte stellende Gerätturnen ${ }^{14}$ statistisch überzufällig seltener von evasiv Unterrichtenden durchgeführt wird, ergaben sich zu Sportarten mit überdurchschnittlichem Risikofaktor keine Zusammenhänge. Ebenso überraschten die negativen Zusammenhänge zu den Sportarten mit unterdurchschnittlichen Risikofaktoren wie Leichtathletik, Schwimmen und Konditionstrai-

\footnotetext{
${ }^{14}$ Durch den Aufbau der Geräte und das Geben von Hilfestellung.
} 
ning. Möglicherweise werden diese Inhalte verstärkt gemieden, da sie bei den Schülern meist unbeliebt sind und höhere Anforderungen an Durchhaltevermögen und Disziplin stellen und damit ein höheres Konfliktpotenzial aufweisen als Sportarten, die bei Schülern erfahrungsgemäß weniger Widerstände auslösen. Wenig überraschend ist in diesem Zusammenhang, dass zwischen Gymnastik/Tanz und Volleyball und der Ausprägung des evasiven Potenzials keine Korrelationen bestehen, da es sich um Sportarten mit unterdurchschnittlichem Risikofaktor handelt, die sich durch keinen bzw. nur geringen Körperkontakt auszeichnen und denen ein geringes Konfliktpotenzial innewohnt. Auch bei diesem Fragekomplex ist, genau wie bei der Bedeutsamkeit der Kriterien für die Inhaltswahl, eine generelle Minderbewertung der Einzelitems festzustellen, was auf eine allgemeine Unentschlossenheit bzw. Unsicherheit rückschließen lässt.

Im Verlauf dieser Arbeit wurde an mehreren Stellen bereits die Einsatzbereitschaft der Lehrkräfte angesprochen, die unter anderem ausschlaggebend für Erziehungs- und Lernerfolge ist. Ein Indikator für die Einstellung zur Arbeit stellt der Arbeitsaufwand für Vor- und Nachbereitung des Unterrichts dar. Während Zugehörige des Schonungsmusters sich durch niedrige Werte in der Ausprägung in der Verausgabungsbereitschaft und dem Perfektionsstreben auszeichnen (siehe Kap. 6.6.1.2), ist diese Tendenz bei dem evasiven Unterrichtsstil nicht festzustellen. Die investierte Zeit für die Vor- und Nachbereitung des Unterrichts unterscheidet sich nicht in signifikanter Art und Weise von der der invasiv Unterrichtenden, sodass nicht von einem Bruch des psychologischen Arbeitsvertrages mit anschließendem Dienst nach Vorschrift auszugehen ist, wie dies im Rahmen einer inneren Kündigung (siehe Kap. 4.9.5.2) charakteristisch ist.

Anknüpfungspunkte an das Schonungsmuster bieten sich hingegen bei der Einschätzung der Bedeutsamkeit des Sportunterrichts im Fachvergleich. Lehrpersonen mit ausgeprägtem evasivem Bewältigungsverhalten sehen im Vergleich zu den invasiv Unterrichtenden das Fach Sport als weit weniger bedeutsam an. Durch diese Minderbewertung des Sportunterrichts ergibt sich folglich auch eine geringere Bedeutsamkeit der Arbeit, zumindest bezogen auf den Sportunterricht, wie dies auch im Schonungsmuster der Fall ist. Für einige Lehrkräfte stellt der Sportunterricht offensichtlich lediglich ein nebensächliches Beifach neben ihrem anderen Unterrichtsfach dar. Es ist nicht verwunderlich, dass gerade diese Lehrkräfte öfter einen eher vermeidenden Umgang im Bezug auf Konflikte an den Tag legen, da das Ansehen und die Wichtigkeit des Faches sowie die Bedeutsamkeit von Inhalten und Zielen für sie eine aktive und problemorientierte Auseinandersetzung nicht unbedingt erforderlich machen. Grundsätzlich kann es jedoch auch zu dieser Minderbewertung des Faches durch negative Erfahrungen und die erlebte Unwirksamkeit von pädagogischen Interventionen im eigenen Unterricht 
kommen. Ebenso könnte die Geringschätzung des Faches auch durch den eigenen evasiven Verhaltensstil und den daraus resultierenden negativen Folgeerscheinungen wie z.B. das stetige Wiederauftreten von ungelösten Konflikten bedingt sein.

In der konkreten Unterrichtsdurchführung konnten mehrere Aspekte aufgeklärt werden, in denen sich evasiv und invasiv Unterrichtende unterscheiden. Während evasiv Unterrichtende eher lehrerzentriert unterrichten und deduktive Lernwege bevorzugen, setzen invasiv Unterrichtende verstärkt auf Schülerorientierung und induktive Lernmöglichkeiten. Des Weiteren scheint eine unterschiedliche Rollenauffassung vorzuliegen, die sich in der aktiven Teilnahme am Sportunterricht zeigt, die von invasiv Agierende deutlich häufiger angegeben wurden. Insgesamt ist der evasive Sportlehrertypus als eher konservativ in der Ausrichtung des Sportunterrichts zu bezeichnen, was sich neben den zuvor dargestellten Merkmalen bezüglich der didaktischen Ausrichtung des Unterrichts vor allem in der Bedeutsamkeit der Vermittlungsaspekte bemerkbar macht. Wie bereits in Kapitel 2.3 dargestellt, ist der Auftrag des Sportunterrichts äußerst vielfältig und aufgrund gesellschaftlicher Veränderungen in einem stetigen Wandlungsprozess. Gerade das soziale Lernen wird in neueren Konzeptionen des Sportunterrichts als unverzichtbarer und bedeutsamer Bestandteil des Unterrichts herausgehoben und stellt neben dem erfahrungsorientierten Lernen und der Förderung der Kreativität einen Aspekt dar, der durch die gesellschaftlich bedingte veränderte Kindheit auch weiterhin an Wichtigkeit gewinnen wird. Gerade diese Bereiche werden von Sportlehrkräften mit ausgeprägtem evasivem Potenzial, im Vergleich mit eher invasiv Unterrichtenden als weniger bedeutsam eingeschätzt, was die eher konservative Grundeinstellung erneut unterstreicht.

\subsection{Untersuchungsschwerpunkt 3: Verhalten und Erleben im} bzw. von Sportunterricht: „Welche Merkmale sind in Bezug auf das Verhalten und Erleben im bzw. von Sportunterricht kennzeichnend für Personen mit ausgeprägtem evasiven Potenzial?"

In diesem Kapitel wird der Frage: „Welche Merkmale sind in Bezug auf das Verhalten und Erleben im bzw. von Sportunterricht kennzeichnend für Personen mit ausgeprägtem evasiven Potenzial?" nachgegangen. Anhand von verschiedenen Variablen, Skalen und Instrumenten, die eine Beschreibung verschiedener Verhaltens- und Erlebensdimensionen liefern, sollen weitere Erkenntnisse zur gemessenen evasiven Verhaltenstendenz geliefert werden. Während in den vorangegangenen Untersuchungsschwerpunkten persönliche und berufsbiographische bzw. unterrichtsspezifische Aspekte Be- 
rücksichtigung fanden, werden in diesem Kapitel neben den Persönlichkeitseigenschaften vor allem das individuelle Befinden und die Wahrnehmung der Unterrichtsrealität in Abhängigkeit des Bewältigungsverhaltens betrachtet.

\subsubsection{Erleben von beruflicher Belastung}

In Kapitel 7.2.8 wurden die subjektiven Bewertungen verschiedener unterrichtlicher Belastungsfaktoren bereits in zusammengefasster Form vorgestellt. An dieser Stelle wird überprüft, inwieweit das Belastungserleben und das evasive Verhalten im Sportunterricht miteinander in Beziehung stehen. Im Rahmen der Bildung von Hypothesen zum Zusammenhang zwischen dem individuellen Belastungserleben und der Ausprägung des evasiven Verhaltens (siehe Kap. 5.3) wurde bereits herausgestellt, dass man aufgrund dieser Angaben Rückschlüsse darüber ziehen kann, inwieweit das vermeidende Verhalten im Konfliktfall belastungsmindernd wirken kann oder nicht. Der aus den verschiedenen Belastungsfaktoren berechnete Summenwert der Gesamtbelastung weist signifikante Korrelationen $(p<.01)$ mittlerer Stärke zu den verschiedenen Facetten des evasiven Unterrichtens auf. Sowohl im Bezug auf das Verhalten gegenüber den Schülerinnen und Schülern (EESU-S) $(r=.318)$, Inhalten (EESU-I) $(r=.312)$, äußeren Rahmenbedingungen (EESU-B) ( $r=.298)$ und folglich auch zu dem Gesamtscore (EESU-SIB) $(r=.344)$ liegen deutliche Zusammenhänge vor. Die Zusammenhänge deuten darauf hin, dass je ausgeprägter das evasive Verhaltenspotenzial ist, desto mehr fühlen sich Sportlehrkräfte durch die unterschiedlichen Einflussfaktoren belastet. Zwischen den einzelnen Faktoren, die als belastend wahrgenommen werden und dem evasiven Unterrichten bestehen erhebliche Unterschiede bezogen auf das jeweilige Zusammenhangsmaß, die in der Tabelle 26 aufgeführt sind. Während das Verletzungsrisiko der Sportarten bereits bei der Auswahl der Inhalte (siehe Kap. 7.4.1) ein für evasiv unterrichtende Sportlehrkräfte im Vergleich zu Personen mit eher invasiver Verhaltenstendenz bedeutenderes Entscheidungskriterium darstellt, fühlen sich Lehrkräfte mit ausgeprägtem evasiven Potenzial durch den Faktor "stetig latente Unfallgefahr" in überzufälligem Maß stärker belastet. Je stärker evasive Tendenzen das Verhaltensrepertoire prägen, desto mehr wird auch das Unfallrisiko im Sportunterricht als Belastungsfaktor wahrgenommen. Weiterhin bestehen deutliche korrelative Beziehungen zwischen der wahrgenommenen Belastung der Lehrkräfte durch Fehlverhalten sowie Motivationsprobleme von Schülern und der Ausprägung des evasiven Potenzials. Erwartungsgemäß liegt hier der größte Zusammenhang zur Facette EESU-S vor. Die nächstkleinere Korrelationen bestehen zu den Belastungen, die durch einen „häufig wechselnden Ordnungsrahmen" und die "Vielzahl an gleichzeitig auftretenden Aufgaben" im Unterricht auftreten. Diese beiden Belastungsfaktoren, genau wie die erstgenannten, gehö- 
ren nicht zu den in Kapitel 7.2.8 berechneten Hauptbelastungen und sind eher als dem Sportunterricht genuine Eigenschaften zu bezeichnen. Die in der Rangreihe folgenden Korrelationen (siehe Tab. 26) betreffen Belastungsfaktoren, die dem physischen Bereich zuzuordnen sind. Neben der Demonstration von Bewegung, dem Aufwand zum Erhalt der Fitness, der allgemeinen körperlichen Beanspruchung, dem Lärmpegel, ist auch das Geben von Hilfestellung und der Auf- und Abbau im Unterricht diesem Bereich zuzurechnen.

Tab. 26: Korrelationen der einzelnen Belastungsfaktoren und der evasiven Verhaltenstendenz (geordnet absteigend nach dem Zusammenhangsmaß des Gesamtsummenwerts)

\begin{tabular}{|c|c|c|c|c|}
\hline Belastungsfaktoren & EESU-S & EESU-I & EESU-B & EESU-SIB \\
\hline Stetig latente Unfallgefahr & $.252^{* *}$ & $.265^{* *}$ & $.253^{* *}$ & $.285 * *$ \\
\hline $\begin{array}{l}\text { Soziales Schülerfehlverhalten und Motivations- } \\
\text { probleme }\end{array}$ & $.270 * *$ & $.227^{* *}$ & $.243^{* *}$ & $.280 * *$ \\
\hline $\begin{array}{l}\text { Häufig wechselnder Ordnungsrahmen im Unter- } \\
\text { richt }\end{array}$ & $.255^{* *}$ & $.217^{* *}$ & $.227^{* *}$ & $.255^{* *}$ \\
\hline Vielzahl von gleichzeitig auftretenden Aufgaben & $.215^{* *}$ & $.196 * *$ & $.230 * *$ & $.242 * *$ \\
\hline Demonstration von Bewegungen & $.229 * *$ & $.235^{* *}$ & $.164 * *$ & $.239 * *$ \\
\hline $\begin{array}{l}\text { Erhöhter Aufwand zum Erhalt der eigenen Fit- } \\
\text { ness/Erlernen neuer Sportarten }\end{array}$ & $.190 * *$ & $.238^{* *}$ & $.156^{* *}$ & $.211^{* *}$ \\
\hline Körperliche Beanspruchung (allgemein) & $.196 * *$ & $.219 * *$ & $.142^{* *}$ & $.204 * *$ \\
\hline Hoher Lärmpegel & $.158^{* *}$ & $.128 * *$ & $.181^{* *}$ & $.175^{* *}$ \\
\hline Auf- und Abbau & $.146^{* *}$ & $.138^{* *}$ & $.113^{* *}$ & $.155^{* *}$ \\
\hline Hilfestellungen & $.147^{* *}$ & $.163^{* *}$ & $.083^{*}$ & $.148 * *$ \\
\hline Klassengröße & $.114^{* *}$ & .073 & $.118^{* *}$ & $.114 * *$ \\
\hline Geringe Pausen- bzw. Erholungszeiten & $.096^{*}$ & .072 & $.115^{* *}$ & $.111^{* *}$ \\
\hline $\begin{array}{l}\text { Schwierigkeiten im Umgang mit Eltern, Kollegen, } \\
\text { Schulleitung }\end{array}$ & .074 & $.102 * *$ & $.107^{* *}$ & $.107^{* *}$ \\
\hline Unangemessene curriculare Vorgaben & $.106^{* *}$ & $.113^{* *}$ & .043 & $.091^{*}$ \\
\hline $\begin{array}{l}\text { Rechtfertigungsdruck gegenüber dem Kollegium } \\
\text { (nicht Sport) }\end{array}$ & .048 & $.084^{*}$ & $.116^{* *}$ & $.088^{*}$ \\
\hline Schlechte räumlich-materielle Bedingungen & .028 & .043 & $.100 * *$ & $.079 *$ \\
\hline $\begin{array}{l}\text { Außerunterrichtliche Aktivitäten (z.B. Sportfeste, } \\
\text { Bundesjugend-Spiele) }\end{array}$ & .072 & .068 & .068 & .074 \\
\hline
\end{tabular}

Die Korrelationen zu den in Kapitel 7.2.8 als besonders belastend herausgestellten Items wie die „Klassengröße“ oder die „geringen Pausen- und Erholungszeiten“ stehen in keinem erwähnenswerten Zusammenhang zur Art des Bewältigungsverhaltens. Ebenso bestehen kaum Zusammenhänge zwischen der Ausprägung der wahrgenommenen Belastung durch "Rechtfertigungsdruck gegenüber dem Kollegium (nicht Sport)“, Schwierigkeiten im Umgang mit Eltern, Kollegen, Schulleitung“, „Unangemes- 
sene curriculare Vorgaben“, "schlechte räumlich-materielle Bedingungen" und "außerunterrichtliche Aktivitäten (z.B. Sportfeste, Bundesjugendspiele)" und der Ausprägung des evasiven Verhaltens. Insgesamt deuten die Ergebnisse daraufhin, dass die Belastungswahrnehmung von evasiv und invasiv Unterrichtenden unterschiedlich ist. Sportlehrkräfte mit ausgeprägtem evasiven Potenzial nehmen verstärkt Faktoren als belastend wahr, die sich direkt auf den konkreten Unterricht beziehen, während sich bei Faktoren, die nicht direkt im Unterrichtsgeschehen anzusiedeln sind keine signifikanten Unterschiede zwischen den Gruppen zeigen.

Neben diesen konkreten unterrichtlichen Belastungsfaktoren wurde, wie bereits in Kapitel 6.6.5 dargestellt, der Fragebogen von Enzmann und Kleiber (1989) zur Erfassung der beruflichen Belastung (BEL) eingesetzt, der sich aus den Skalen "Kontrolliertheitserleben", „Arbeitsunzufriedenheit" und "Arbeitsüberforderung" zusammensetzt ${ }^{15}$. An dieser Stelle soll ergänzend zu den Belastungsfaktoren das Kontrolliertheitserleben als weiterer belastender Faktor berücksichtigt werden. Während im Bezug auf den Umgang mit Schülern nur sehr geringe korrelative Zusammenhänge bestehen ( $p<.05 ; r=.094)$ verhalten sich Personen mit zunehmendem Kontrolliertheitsempfinden sowohl im Bezug auf Inhalte $(p<.01 ; r=.125)$ als auch vor allem im Bezug zu äußeren Rahmenbedingungen ( $p<.01 ; r=.181)$ evasiver als Personen, die sich in geringerem Maße bei ihrer Arbeit kontrolliert fühlen.

\subsubsection{Arbeitsbezogene Verhaltens- und Erlebensmuster}

In Kapitel 7.1 wurden bereits die Ergebnisse des Fragebogens von Schaarschmidt und Fischer (1996) zur Messung der „Arbeitsbezogenen Verhaltens- und Erlebensmuster" (AVEM) in der vorliegenden Stichprobe vorgestellt. An dieser Stelle soll nun überprüft werden, ob Zusammenhänge zwischen den einzelnen Subskalen bzw. Musterstrukturen und der Ausprägung des evasiven Verhaltenspotenzials vorliegen. In Tabelle 27 sind die Korrelationen der drei Facetten des evasiven Verhaltens sowie des Gesamtsummenwertes mit den einzelnen Subskalen des AVEM dargestellt. Wie sich in Tabelle 27 zeigt, entsprechen die Korrelationen nur in geringem Maß den im Vorfeld formulierten Erwartungen. Mit Ausnahme der Korrelation zwischen der Ausprägung der „offensiven Problembewältigung" und dem evasiven Verhaltenspotenzial entsprechen die Ergebnisse nicht unbedingt dem, was zu erwarten war. Mit Blick auf den von Schaarschmidt \& Fischer (1996) als "Schonungs-Typ" bezeichneten Lehrer, zu dem eine Verwandtschaft mit dem evasiven Verhaltensmuster denkbar wäre, sind vor allen Dingen ausgeprägte Korrelationen zu Skalen wie "Subjektive Bedeutsamkeit der Arbeit“, „Ver-

\footnotetext{
${ }^{15}$ Die Skalen „Arbeitsüberforderung“ und „Arbeitsunzufriedenheit“ werden in Kapitel 7.5.4.1 unter dem Aspekt „Berufliche Zufriedenheit“ in Beziehung zur Ausprägung des evasiven Potenzials gesetzt.
} 
ausgabungsbereitschaft" und „Perfektionsstreben“ zu erwarten gewesen. Die genannten Skalen stehen jedoch in keinem bzw. nur in sehr geringem Zusammenhang zu der Ausprägung des evasiven Potenzials. Anhand der Ausführungen bezüglich der inneren Kündigung (siehe Kap. 4.9.5.2) wären deutliche Korrelationen hier keine Überraschung gewesen. Doch offensichtlich scheint evasives Verhalten keine Bewältigungsstrategie zu sein, die aufgrund von Enttäuschungen, in Form des Bruchs des psychologischen Arbeitsvertrages zustande kommt. Betrachtet man nun die Skalen des AVEM, die mit den Ausprägungen des evasiven Verhaltens in den verschiedenen Facetten korrelieren, stellt man schnell fest, dass mit "Resignationstendenz bei Misserfolg“, „Erfolgserleben im Beruf", "Innere Ruhe und Ausgeglichenheit", „Erleben sozialer Unterstützung" und „Lebenszufriedenheit" offensichtlich statt den ressourcenschonenden Merkmalen eher das Selbst bedrohende Verhaltens- und Erlebensmerkmale mit ausweichendem Verhalten in Verbindung zu setzen sind.

Tab. 27: Korrelationen der einzelnen Skalen des AVEM-Fragebogen mit den Facetten der evasiven Verhaltenstendenz

\begin{tabular}{|l|c|c|c|c|}
\cline { 2 - 5 } \multicolumn{1}{c|}{} & EESU-S & EESU-I & EESU-B & EESU-SIB \\
\hline Subjektive Bedeutsamkeit der Arbeit & -.072 & -.065 & $-.081^{*}$ & $-.086^{*}$ \\
\hline Beruflicher Ehrgeiz & $-.239^{* *}$ & $-.218^{* *}$ & $-.222^{* *}$ & $-.260^{* *}$ \\
\hline Verausgabungsbereitschaft & -.075 & -.059 & -.063 & -.067 \\
\hline Perfektionsstreben & -.005 & -.038 & -.006 & -.009 \\
\hline Distanzierungsfähigkeit & -.067 & $-.078^{*}$ & -.070 & $-.084^{*}$ \\
\hline Resignationstendenz bei Misserfolg & $.322^{* *}$ & $.338^{* *}$ & $.318^{* *}$ &. $.361^{* *}$ \\
\hline Offensive Problembewältigung & $-.433^{* *}$ & $-.400^{* *}$ & $-.398^{* *}$ &.$- .462^{* *}$ \\
\hline Innere Ruhe und Ausgeglichenheit & $-.148^{* *}$ & $-.201^{* *}$ & $-.119^{* *}$ & $-.174^{* *}$ \\
\hline Erfolgserleben im Beruf & $-.358^{* *}$ & $-.317^{* *}$ & $-.327^{* *}$ & $-.369^{* *}$ \\
\hline Lebenszufriedenheit & $-.259^{* *}$ & $-.299^{* *}$ & $-.251^{* *}$ & $-.304^{* *}$ \\
\hline Erleben sozialer Unterstützung & $-.247^{* *}$ & $-.224^{* *}$ & $-.189^{* *}$ & $-.236^{* *}$ \\
\hline
\end{tabular}

Weiteren Aufschluss in diesem Zusammenhang liefert die einfaktorielle Varianzanalyse (ANOVA) in der die vier von Schaarschmidt und Fischer (1996) identifizierten Muster (Gesundheit, Anstrengung, Burnout und Schonung) auf Unterschiede in der Ausprägung des evasiven Bewältigungsverhaltens untersucht werden. Es zeigte sich, dass die EESU-Werte für die vier Muster in allen drei Facetten sowie dem Gesamtsummenwert in statistisch signifikantem Maß divergieren, $F_{\text {EESU-S }}(3,680)=25.049, p=.000 ; F_{\text {EESU-I }}(3$, $667)=28.530, p=.000 ; F_{\text {EESU-B }}(3,662)=22.213, p=.000 ; F_{\text {EESU-SIB }}(3,646)=31.979, p=.000$. Tabelle 28 zeigt die Mittelwerte der verschiedenen Facetten des EESU in den verschiedenen Musterausprägungen. Auffällig ist vor allem die Ausprägung des evasiven Potenzials des Musters Burnout, das in allen drei Facetten, aber auch im Gesamtsum- 
menwert deutlich stärker ausgeprägt ist als bei den anderen drei arbeitsbezogenen Verhaltens- und Erlebensmustern. Ebenso ist es überraschend, dass nicht die teils durch extreme Verausgabungsbereitschaft auffallenden Muster-A Lehrkräfte, sondern die dem Gesundheitsmuster entsprechenden Sportlehrer am invasivsten im Unterricht agieren.

Tab. 28: Mittelwerte der Facetten des evasiven Verhaltens der vier AVEM-Muster

\begin{tabular}{|c|c|c|c|c|c|c|c|c|c|}
\hline & & \multicolumn{2}{|c|}{ EESU-S } & \multicolumn{2}{|c|}{ EESU-I } & \multicolumn{2}{|c|}{ EESU-B } & \multicolumn{2}{|c|}{ EESU-SIB } \\
\hline \multirow{2}{*}{ Gesundheit } & $M$ & 18,70 & \multirow{2}{*}{$\mathrm{n}=98$} & 18,20 & \multirow{2}{*}{$\mathrm{n}=98$} & 19,74 & \multirow{2}{*}{$n=97$} & 56,54 & \multirow{2}{*}{$n=96$} \\
\hline & SD & 3.89 & & 4.28 & & 4.73 & & 11.51 & \\
\hline \multirow{2}{*}{ Schonung } & $M$ & 20,32 & \multirow{2}{*}{$n=292$} & 19,82 & \multirow{2}{*}{$n=284$} & 21,39 & \multirow{2}{*}{$n=283$} & 61,57 & \multirow{2}{*}{$n=273$} \\
\hline & SD & 4.20 & & 4.32 & & 4.90 & & 12.07 & \\
\hline \multirow{2}{*}{ Burnout } & $M$ & 22.67 & \multirow{2}{*}{$n=206$} & 22,62 & \multirow{2}{*}{$n=201$} & 24,03 & \multirow{2}{*}{$n=197$} & 69,45 & \multirow{2}{*}{$n=194$} \\
\hline & SD & 3.99 & & 4.20 & & 4.42 & & 11.06 & \\
\hline \multirow{2}{*}{ Anstrengung } & $M$ & 20,16 & \multirow{2}{*}{$n=88$} & 20,01 & \multirow{2}{*}{$n=88$} & 21,06 & \multirow{2}{*}{$n=89$} & 61,08 & \multirow{2}{*}{$n=87$} \\
\hline & SD & 4.15 & & 4.33 & & 4.72 & & 11.48 & \\
\hline \multirow{2}{*}{ Gesamt } & $M$ & 20.78 & \multirow{2}{*}{$\mathrm{N}=684$} & 20,45 & \multirow{2}{*}{$N=671$} & 21,88 & \multirow{2}{*}{$N=666$} & 63,11 & \multirow{2}{*}{$N=650$} \\
\hline & SD & 4.30 & & 4.54 & & 4.93 & & 12.42 & \\
\hline
\end{tabular}

In Tabelle 29 sind die Ergebnisse der Post-hoc-Tests (Tukey-HSD) in komprimierter Form dargestellt. ${ }^{16}$ Es wird deutlich, dass vor allem das Muster Burnout, wie man auch anhand der absoluten Werte in Tabelle 28 ablesen konnte, sich von allen anderen Mustern erheblich durch ein deutlich stärker ausgeprägtes evasives Potenzial abhebt. Die zweite Auffälligkeit betrifft das Muster Gesundheit, das sich im Gesamtsummenwert (EESU-SIB) von allen drei anderen Mustern durch signifikant geringere Evasionswerte unterscheidet. Zwischen den Mustern Anstrengung und Schonung bestehen keine signifikanten Mittelwertdifferenzen. Die Mittelwertdifferenz des Gesamtsummenwerts (EESU-SIB) zwischen dem Muster Gesundheit und dem Muster Burnout entspricht einem großen Effekt von $d_{\mathrm{G}-\mathrm{B}}=1.14$. Die Differenz der Mittelwerte der Gesamtsummenwerte vom Muster Gesundheit und Schonung $\left(d_{\mathrm{G}-\mathrm{S}}=0.43\right)$ entspricht ebenso wie die Unterschiede zwischen Gesundheit und Anstrengung $\left(d_{\mathrm{G}-\mathrm{A}}=0.39\right)$ einem Effekt geringer Stärke. Die Mittelwerte im Summenwert von den Mustern Schonung und

\footnotetext{
${ }^{16}$ Anhand der Tabelle kann man ablesen, dass das Muster Gesundheit sich in allen drei Facetten sowie in dem Gesamtsamtsummenwert in signifikanten Maß vom Muster Schonung unterscheidet $(* *=p<.01$; $\left.{ }^{*} \mathrm{p}<.05\right)$. Anhand der Operatoren „+“ und „-" in den Klammern kann man ablesen, ob der jeweilige Mittelwert, in diesem Fall des Musters Gesundheit, signifikant höher oder niedriger ist als der Vergleichswert. Die erste Zeile zeigt also, dass sich das Muster Gesundheit in hoch signifikantem Maß vom Muster Schonung in der Facette EESU-S unterscheidet. Das Muster Gesundheit weist einen geringeren Wert in dieser Facette auf, verhält sich also in geringerem Ausmaß evasiv.
} 
Burnout repräsentieren mit $d_{S-B}=0.68$ ebenso wie die Unterschiede zwischen Anstrengung und Burnout mit $d_{\mathrm{A}-\mathrm{B}}=0.74$ einen mittleren Effekt.

Tab. 28: Post-Hoc-Tests (Tukey-HSD): Evasives Verhaltenspotenzial der AVEM-Muster

\begin{tabular}{|c|c|c|c|c|c|}
\hline & & Gesundheit & Schonung & Burnout & Anstrengung \\
\hline \multirow{4}{*}{ Gesundheit } & EESU-S & & $* *(-)$ & $* *(-)$ & \\
\hline & EESU-I & & $* *(-)$ & $* *(-)$ & $*(-)$ \\
\hline & EESU-B & & $*(-)$ & $* *(-)$ & \\
\hline & EESU-SIB & & $* *(-)$ & $* *(-)$ & $*(-)$ \\
\hline \multirow{4}{*}{ Schonung } & EESU-S & $* *(+)$ & & $* *(-)$ & \\
\hline & EESU-I & $* *(+)$ & & $* *(-)$ & \\
\hline & EESU-B & $*(+)$ & & $* *(-)$ & \\
\hline & EESU-SIB & $* *(+)$ & & $* *(-)$ & \\
\hline \multirow{4}{*}{ Burnout } & EESU-S & $* *(+)$ & $* *(+)$ & & $* *(+)$ \\
\hline & EESU-I & $* *(+)$ & $* *(+)$ & & $* *(+)$ \\
\hline & EESU-B & $* *(+)$ & $* *(+)$ & & $* *(+)$ \\
\hline & EESU-SIB & $* *(+)$ & $* *(+)$ & & $* *(+)$ \\
\hline \multirow{4}{*}{ Anstrengung } & EESU-S & & & $* *(-)$ & \\
\hline & EESU-I & $*(+)$ & & $* *(-)$ & \\
\hline & EESU-B & & & $* *(-)$ & \\
\hline & EESU-SIB & $*(+)$ & & $* *(-)$ & \\
\hline
\end{tabular}

\subsubsection{Berufsbedingte Erschöpfung und arbeitsbezogenes (Dis-) Engagement}

Im Rahmen dieses Abschnitts soll überprüft werden, inwieweit berufsbedingte Erschöpfung und arbeitsbezogenes Disengagement in einem Korrelationsverhältnis zur Ausprägung des evasiven Potenzials stehen. Neben der Skala "Disengagement", enthält das Oldenburger Burnout Inventar mit „Exhaustion“ eine weitere Skala, die die berufsbedingte Erschöpfung misst. Zwischen dieser Skala und den drei Facetten sowie dem Gesamtsummenwert des EESU bestehen negative Korrelationen. d.h., je evasiver das Verhaltenspotenzial der Sportlehrkräfte ist, desto höher schätzen die Lehrkräfte ihre individuelle Erschöpfung ein. Die Zusammenhänge ergeben sich sowohl im Bezug auf den Umgang mit Schülerinnen und Schülern ( $r=-.284 ; p<.01)$, auf Inhalte $(r=-.279$; $p<.01)$ als auch im Umgang mit äußeren Rahmenbedingungen $(r=-.244 ; p<.01)$. Die Unterschiede in der Erschöpfung zwischen dem 1. Und dem 4. Quartil des evasiven Verhaltens entspricht einem Effekt von $d_{\text {Quartil1-Quartil4 }}=0.81$.

Nachdem bereits in Kapitel 7.5.2 der berufliche Ehrgeiz und die Verausgabungsbereitschaft aus dem AVEM als Skalen, die das arbeitsbezogene Engagement in unterschiedlicher Art und Weise beschreiben, in Beziehung zur Ausprägung des evasiven Potenzi- 
als gesetzt wurden, wird an dieser Stelle die Skala „Disengagement" aus dem Oldenburger Burnout Inventar in Beziehung zu den drei Facetten evasiven Verhaltens und dem Gesamtsummenwert gesetzt. Zu allen drei Facetten bestehen negative Korrelationen (EESU-S: $r=-.384, \quad p<.01$; EESU-I: $r=-.383, \quad p<.01$; EESU-B: $r=-.312$; EESU-SIB: $r=-.400, p<.01)$, d.h. dass bei einer höheren evasiven Verhaltenstendenz das Disengagement in Form einer inneren emotionale Distanzierung von der Arbeit zunimmt. Durch das Verspüren von negativen, die Motivation beeinflussende Gefühlen, wird die Arbeit als uninteressant wahrgenommen, was letztendlich zu einer gering ausgeprägten Identifikation mit dem Beruf führt. Die Unterschiede im Disengagement zwischen dem 1. Und dem 4. Quartil des evasiven Verhaltens entspricht einem Effekt von $d_{\text {Quartil1-Quartil4 }}=1.08$.

Wie bereits in Kapitel 6.6.1.1 dargestellt, sieht Schaarschmidt und Fischer (1996) als weitere Möglichkeit, die Angaben des AVEM-Fragebogens auszuwerten die Verrechnung zu sogenannten Sekundärfaktoren vor. Im Rahmen des Sekundärfaktors „Berufliches Engagement" werden die Skalen "Subjektive Bedeutsamkeit der Arbeit", „Beruflicher Ehrgeiz", "Verausgabungsbereitschaft", „Perfektionsstreben" und "Distanzierungsfähigkeit" zusammengerechnet um in umfassenderem Sinn eine Einschätzung über die Dosierung des Engagements als Voraussetzung für Erfolgserleben im Beruf unter gleichzeitiger Berücksichtigung gesundheitsförderlicher Aspekte vornehmen zu können. Wie die Auswertung der von den Sportlehrkräften gemachten Angaben ergab, besteht eine negative Korrelation $(r=-.170 ; p<.01)$ zwischen dem "gesunden“ Engagement und der Ausprägung des evasiven Verhaltenspotenzials. Es zeigt sich, dass evasives Bewältigungsverhalten keine angemessene Art der Bewältigung darstellt, da sowohl die berufsbedingte Erschöpfung als auch das Disengagement in negativer Ausprägung vorliegen.

\subsubsection{Berufliche Zufriedenheit}

In diesem Teil der Arbeit soll überprüft werden, ob Zusammenhänge zwischen der Zufriedenheit und dem Spaß am bzw. im Arbeitsleben und dem Potenzial, sich in unterrichtlichen Konfliktsituationen evasiv zu verhalten, existieren. Weiterhin wird überprüft ob die soziale Atmosphäre im Kollegium und speziell im Sportkollegium in Beziehung zu der Ausprägung des evasiven Verhaltens steht. Anschließend werden ebenjene Zusammenhänge für die Folgen der Zufriedenheit mit dem Arbeitsleben in Form von Ausstiegsambitionen, Gefühlen für die Zukunft etc. überprüft. 


\subsubsection{Arbeitsunzufriedenheit und Arbeitsüberforderung}

Nachdem bereits in Kapitel 7.5.1 die Skala „Kontrolliertheitserleben“ aus dem Fragebogen „Berufliche Belastung" (BEL) von Enzmann und Kleiber (1989) in die Auswertung mit einbezogen wurde, werden im Zusammenhang mit der Zufriedenheit im Beruf die Skalen „Arbeitsunzufriedenheit" und "Arbeitsüberförderung" auf Zusammenhäng zum evasiven Verhalten überprüft.

Wie Tabelle 30 zeigt, bestehen zwischen der Skala „Arbeitsunzufriedenheit" und den drei Facetten sowie dem Gesamtsummenwert Zusammenhänge mittleren Niveaus, wohingegen die Zusammenhänge zur Skala „Arbeitsüberforderung“ etwas geringer ausfallen und nur in dem Gesamtsummenwert im mittleren Bereich liegen. Die Ergebnisse deuten an, dass Personen, die sich, aus welchen Gründen auch immer, im Unterricht evasiv verhalten, deutlich unzufriedener sind und sich zudem in stärkerem Maße durch die Arbeit überfordert fühlen als invasiv Agierende.

Tab. 30: Korrelationen der Skalen „Arbeitsunzufriedenheit" und „Arbeitsüberforderung" aus dem Fragebogen BEL von Enzmann und Kleiber (1989) und den Facetten des evasiven Verhaltens

\begin{tabular}{|c|c|c|c|c|}
\cline { 2 - 5 } \multicolumn{1}{c|}{} & EESU-S & EESU-I & EESU-B & EESU-SIB \\
\hline Arbeitsunzufriedenheit & $.336^{* *}$ & $.331^{* *}$ & $.332^{* *}$ & $.370^{* *}$ \\
\hline Arbeitsüberforderung & $.278^{* *}$ & $.285^{* *}$ & $.284^{* *}$ & $.318^{* *}$ \\
\hline
\end{tabular}

\subsubsection{Zufriedenheit mit der Stundenverteilung}

In Kapitel 7.1 wurden bereits die Ergebnisse der Frage zur Zufriedenheit mit der Stundenverteilung vorgestellt. Im Folgenden soll überprüft werden, ob es Zusammenhänge zwischen der Zufriedenheit mit der Stundenverteilung im Bezug auf den Anteil des Sportunterrichts und der Ausprägung des evasiven Potenzials gibt. Wie man anhand von Tabelle 31 erkennen kann, ist das evasive Potenzial am geringsten bei denjenigen Lehrkräften ausgeprägt, die sich einen noch höheren Anteil des Sportunterrichts an der Gesamtehrverpflichtung wünschen. Die Mittelposition nehmen die „Zufriedenen“ ein. Am deutlichsten sind evasive Tendenzen im Verhalten der Sportlehrkräfte ausgeprägt, die sich eine Reduzierung des Anteils des Sportunterrichts wünschen. Statistisch gesehen unterscheiden sich diese Sportlehrkräfte signifikant $t(621)=3,431 p<.05$ bezüglich des evasiven Potenzials von denjenigen, die gerne mehr Sport unterrichten möchten.

Tab. 31: Evasives Potenzial (EESU-SIB) der Zufriedenheitsangaben mit der Stundenverteilung

\begin{tabular}{|l|c|c|}
\cline { 2 - 3 } \multicolumn{1}{c|}{} & $\mathbf{n}$ & EESU-SIB \\
\hline Gerne mehr Sportunterricht & 164 & 61,72 \\
\hline Zufrieden & 389 & 63,37 \\
\hline Gerne weniger Sportunterricht & 69 & 66,39 \\
\hline
\end{tabular}


An dieser Stelle muss ergänzend hinzugefügt werden, dass der prozentuale Anteil des Sportunterrichts an der Gesamtlehrverpflichtung bei den drei in der Tabelle aufgeführten Gruppen nicht auf einem Niveau liegt. Während diejenigen, die sich eine Reduzierung der Sportstundenzahl wünschen zu 66,9\% (SD=21.88) ihrer Gesamtlehrverpflichtung das Fach Sport unterrichten, liegt dieser Wert bei denjenigen, die sich gerne mehr Sport im Stundenplan wünschen lediglich bei 26,9\% (SD=13.34). Die Sportlehrer, die mit der Verteilung der Stunden auf den Sportunterricht und das weitere Fach/die weiteren Fächer zufrieden sind, weisen einen ausgeglichenen prozentualen Anteil des Sportunterrichts von 47,5\% (SD=20.78) auf. Diese Ungleichverteilung hat jedoch keinen Einfluss auf die durch die Tabelle 31 ausgedrückten statistischen Evidenzen, da zwischen dem prozentualen Anteil des Sportunterrichts an der Gesamtlehrverpflichtung und dem evasiven Potenzial keine korrelativen Beziehungen bestehen. Insgesamt steigt mit zunehmendem evasivem Potenzial also die „Ablehnungstendenz" des Faches Sport, unabhängig von dem tatsächlich unterrichteten Umfang des aktuell abgeleisteten Unterrichts im Fach.

\subsubsection{Spaß am Unterrichten}

In Kapitel 7.2.7 wurde bereits ausgeführt, dass ein Großteil der befragten Sportlehrer zumeist Spaß am Unterricht im Fach Sport hat. In Bezugnahme auf das evasive Verhalten im Unterricht fällt auf, dass je ausgeprägter evasive Verhaltenstendenzen sind, desto weniger Spaß macht den Lehrkräften das tagtägliche Unterrichten. Diese korrelativen Zusammenhänge existieren sowohl für die drei Einzelfacetten (EESU-S: $r=-.413$, $p<.01$; EESU-I: $r=-.360, p<.01$; EESU-B: $r=-.333, p<.01$ ) als auch für den Gesamtsummenwert (EESU-SIB: $r=-.406 ; p<.01)$. Umgekehrt kann man ebenso formulieren, dass je weniger Spaß die Lehrkräfte am Unterrichten haben, desto häufiger verhalten sie sich im Unterricht evasiv und weichen auftretenden Konflikten lieber aus, als eine problemorientierte Lösung anzustreben.

\subsubsection{Wahrgenommene Atmosphäre im Sport- und Gesamt kollegium}

In Kapitel 7.2.11 wurde bereits dargestellt, dass die befragten Sportlehrer die Atmosphäre im Sportkollegium als etwas besser bewerten als die Atmosphäre im Gesamtkollegium. An dieser Stelle soll nun überprüft werden, ob eine gute Atmosphäre als soziales Unterstützungssystem evasiven Tendenzen vorbeugt bzw. unterstützt oder aber ob es keinen Einfluss auf die Art der Bewältigung ausübt. Anhand von Tabelle 32 kann man ablesen, dass das Sportkollegium im Vergleich zum Gesamtkollegium eine bedeutsamere Einflussgröße auf den Unterricht bzw. die Bewältigung darstellt. Wäh- 
rend bis auf in der Facette EESU-I zwischen der Atmosphäre im Gesamtkollegium und den Evasionswerten keine Zusammenhänge festzustellen sind, bestehen zwischen allen drei Facetten sowie dem Gesamtsummenwert und der Atmosphäre im Sportkollegium negative Korrelationen. D.h. dass je schlechter die Atmosphäre im Sportkollegium wahrgenommen wird, desto eher prägen konfliktvermeidende Strategien das tägliche Handeln und Tun.

Tab. 32: Korrelationen des evasiven Potenzials mit den Angaben zur Atmosphäre im Sport- und Gesamtkollegium

\begin{tabular}{|l|c|c|c|c|}
\cline { 2 - 4 } \multicolumn{1}{c|}{} & EESU-S & EESU-I & EESU-B & EESU-SIB \\
\hline Atmosphäre im Gesamtkollegium & -.019 & $-.078^{*}$ & -.071 & -.070 \\
\hline Atmosphäre im Sportkollegium & $-.111^{* *}$ & $-.106^{* *}$ & $-.142^{* *}$ & $-.138^{* *}$ \\
\hline
\end{tabular}

7.5.4.5 Gefühle mit der bisherigen Karriere, Gefühle für die Zukunft

Bei den Gefühlen, Erfahrungen und Erwartungen, die die Sportlehrkräfte mit der Vergangenheit sowie der Zukunft verbinden stellte sich in Kapitel 7.2.11 heraus, dass die Vergangenheit generell besser eingeschätzt wird als die Zukunft. Die Zusammenhänge zwischen den drei Facetten und dem Gesamtsummenwert des neu konstruierten Fragebogens EESU und diesen beiden Einschätzungen sind negativer Natur, wobei die Zusammenhänge mit den Gefühlen der bisherigen Karriere (EESU-S: $r=-.335, p<.01$; EESU-I: $r=-.382, p<.01$; EESU-B: $r=-.321, p<.01$; EESU-SIB: $r=-.387, p<.01$ ) etwas bedeutsamer sind im Vergleich mit den Erwartungen an die Zukunft (EESU-S: $r=-.312, p<.01$; EESU-I: $r=-.344, p<.01$; EESU-B: $r=-.263, p<.01$; EESU-SIB: $r=-.343, p<.01$ ). Diese Korrelationen deuten an, dass die Sportlehrkräfte, die eine ausgeprägtere evasive Verhaltenstendenz aufweisen, sowohl ihre Vergangenheit negativer einschätzen als auch eine niedrigere Erwartungshaltung an die Zukunft aufweisen, sodass erneut indirekt der Einfluss des Bewältigungsverhaltens auf die Berufszufriedenheit nachgewiesen ist.

\subsubsection{Erneute Berufswahl}

In dieser Frage werden die verschiedenen Aspekte der Zufriedenheit mit dem Beruf an sich und die Erlebnisse und Erfahrungen, die man im Karriereverlauf gemacht hat bzw. welche Entwicklung man sich für die Zukunft ausmalt aggregiert. Wie bereits in Kapitel 7.1 erwähnt würden die meisten Sportlehrer, wenn sie heute noch einmal vor der Studien- bzw. Berufswahl stehen würden, sich erneut für den Lehrerberuf entscheiden. Vergleicht man die Mittelwerte des EESU-Gesamtsummenwerts, ergibt sich eine klar abzulesende Tendenz: Diejenigen, die den Beruf nicht erneut wählen würden, weisen mit 69,94 (SD=11.86) den höchsten Wert auf, diejenigen, die den Beruf auf jeden Fall 
noch einmal wählen würden mit 60,53 (SD=11.93) den niedrigsten Evasionswert auf. Mit einem Wert von 65,55 (SD=13.88) bei denjenigen, die den Beruf eher nicht noch einmal wählen würden und einem Wert von 64,69 (SD=11.75) bei denen, die den Lehrerberuf vermutlich nochmal wählen würden liegen diese beide Abstufungen zwischen den erstgenannten Polen bezüglich der Ausprägung des evasive Potenzials.

\subsubsection{Persönlichkeitseigenschaften: Lehrerselbstwirksamkeitserwar- tung, Kompetenz- und Kontrollüberzeugungen, Leistungsmotiva- tion, Ungewissheitstoleranz}

Die Überzeugungen und Erwartungen, die vor allem subjektive Fähigkeiten, Kompetenzen und Ressourcen einschließen, wurden bereits unter dem Sammelbegriff „beliefs" in Kapitel 4.5.2 zusammenfassend erörtert und mithilfe der in Kapitel 6 vorgestellten Instrumenten erfasst. In diesem Abschnitt soll nun überprüft werden, ob Zusammenhänge zwischen den verschiedenen Konstrukten und den Evasionswerten bestehen. „Commitments" beschreiben wie anhand von 4.5.1 deutlich wurde Motivationsstrukturen, die daran beteiligt sind ob bestimmte Situationen und Ereignisse als herausfordernd oder bedrohlich aufgefasst werden bzw. ob sie überhaupt aufgesucht werden oder nicht. In diesem Abschnitt werden mit der Leistungsmotivation und deren Komponenten "Hoffnung auf Erfolg" und "Furcht vor Misserfolg" sowie dem aus insgesamt fünf Einzelskalen berechneten Sekundärfaktor „Berufliches Engagement" aus dem AVEM-Fragebogen von Schaarschmidt und Fischer (1996) verschiedenen Quellen, die Motivation messen herangezogen, um Zusammenhänge zwischen der Tendenz evasiv im Unterricht zu agieren und Motivationsstrukturen aufzuklären. Neben der Ungewissheitstoleranz wird zudem in diesem Kapitel die krankheitsbedingte Absentismusrate auf Zusammenhänge mit dem evasiven Verhalten überprüft.

\subsubsection{Lehrerselbstwirksamkeitserwartung}

Die Selbstwirksamkeitserwartung als subjektive Erwartung der persönlichen Wirksamkeit stellt ein zukunftsbezogenes Konstrukt dar, das mit dafür verantwortlich ist, dass eine Handlung, wie z.B. die problemorientierte Auseinandersetzung mit Konflikten überhaupt initiiert wird. Des Weiteren wird durch die Ausprägung der Lehrerselbstwirksamkeitserwartung bestimmt, mit welcher Hartnäckigkeit auch bei Widrigkeiten an den vorab formulierten Zielvorstellungen festgehalten wird. Im Rahmen dieses Abschnitts soll überprüft werden, ob diese relativ stabile und auf zukünftiges Handeln gerichtete Persönlichkeitseigenschaft Zusammenhänge zur Ausprägung des evasiven Verhaltenspotenzials aufweist. 
Wie die Auswertung der vorliegenden Daten ergab, bestehen zwischen der Lehrerselbstwirksamkeitserwartung und den verschiedenen Facetten des evasiven Verhaltens negative Korrelationen, d.h. dass je geringer die Selbstwirksamkeitserwartung bei den Lehrern ausgeprägt ist, desto höher ist der Wert auf den Evasionsskalen. Mit Werten von $r=-.430, p<.01$ (EESU-S), $r=-.379, p<.01$ (EESU-I), $r=-.412, p<.01$ (EESU-B) und $r=-$ .456, $p<.01$ (EESU-SIB) liegen die Korrelationen auf nahezu mittlerem Niveau. Bei der einfaktoriellen Varianzanalyse (ANOVA) zeigten die Quartile des evasiven Verhaltens signifikant divergierende Mittelwerte $(F(3,634)=42,72, p<.01)$ in der Ausprägung der Lehrerselbstwirksamkeitserwartung. Die Unterschiede zwischen dem evasiven Quartil $(M=26,53, S D=3.85)$ und dem invasiven Quartil $(M=31,03, S D=3.32)$ entsprechen einem großen Effekt von $d_{\text {Quartil1-Quartil4 }}=1.25$.

\subsubsection{Kompetenz- und Kontrollüberzeugungen}

Gerade im Lehrerberuf, der durch viele subjektiv neue und mehrdeutige Situationen gekennzeichnet ist, nehmen generalisierte Erwartungen und Überzeugungen, Unterrichtsereignisse beeinflussen zu können, einen hohen Stellenwert ein. Im Anschluss soll überprüft werden, inwieweit diese Persönlichkeitseigenschaften Einfluss auf das Bewältigungsverhalten von Sportlehrkräften haben.

Das „Selbstkonzept eigener Fähigkeiten“ (FKK-SK), also die Erwartung, dass in Handlungssituationen zumindest eine Handlungsmöglichkeit zur Verfügung steht, korreliert, wie sich in Tabelle 33 zeigt, negativ mit den drei Facetten und dem Gesamtsummenwert des EESU. Auch die Skala „Internalität“ (FKK-I), also die subjektiv von der Person wahrgenommene Kontrolle über die Ereignisse des eigenen Lebens korreliert negativ mit den Evasionswerten. D.h. je geringer die Ausprägungen des Selbstkonzepts und die Überzeugung den Verlauf von Ereignissen selbst bestimmen zu können, desto größer ist die Tendenz sich in konfliktträchtigen Situationen vermeidend und ausweichend zu verhalten. Positive Korrelationen liegen vor zwischen den Skalen soziale Externalität (FKK-P) und fatalistische Externalität (FKK-C). D.h. je mehr die Sportlehrkräfte davon überzeugt sind, dass der Verlauf der Dinge durch andere Personen bzw. den Zufall oder das Schicksal bestimmt wird, desto mehr wenden sie evasive Strategien an. Es zeigt sich anhand der Ergebnisse, dass die Kompetenz-und Kontrollüberzeugungen als generalisierte Erwartungen wie bereits in Kapitel 4.5.2.2 beschrieben, vor allem in ungewissen und neuartigen Situationskonstellationen, wie sie im Sportunterricht häufig auftreten von Bedeutung sind. 
Tab. 33: Zusammenhänge zwischen den Skalen des Fragebogen zu Kontroll- und Kompetenzüberzeugungen (FKK) und den Skalen des EESU

\begin{tabular}{|l|c|c|c|c|}
\hline & EESU-S & EESU-I & EESU-B & EESU-SIB \\
\hline Selbstkonzept eigener Fähigkeiten (FKK-SK) & $-.425^{* *}$ & $-.428^{* *}$ & $-.381^{* *}$ & $-.456^{* *}$ \\
\hline Internalität (FKK-I) & $-.242^{* *}$ & $-.230^{* *}$ & $-.181^{* *}$ & $-.242^{* *}$ \\
\hline Soziale Externalität (FKK-P) & $.308^{* *}$ & $.275^{* *}$ & $.331^{* *}$ & $.338^{* *}$ \\
\hline Fatalistische Externalität (FKK-C) & $.271^{* *}$ & $.278^{* *}$ & $.280^{* *}$ & $.308^{* *}$ \\
\hline
\end{tabular}

\subsubsection{Leistungsmotivation}

Wie bereits in Kapitel 4.5.1 beschrieben, können sowohl die Ausprägungen der Erfolgshoffnung als auch das Niveau der Furcht vor Misserfolg zu einem Fernbleiben bzw. Verlassen von Situationen führen. Ob tatsächlich auch Zusammenhänge zur Leistungsmotivation bestehen wird im Anschluss überprüft.

Wie man anhand von Tabelle 34 ablesen kann, bestehen zwischen dem Summenwert der Skala "Hoffnung auf Erfolg“ und den Skalen des Erhebungsinstruments evasiver Sportunterricht negative Zusammenhänge, d.h. je geringer das individuelle Hoffen auf Erfolg ausgeprägt ist, desto eher ist das Verhalten durch evasive Anteile geprägt. Zwischen der Furch vor Misserfolgen und dem evasiven Verhalten bestehen hingegen erwartungsgemäß positive Korrelationen in den drei Facetten und dem Gesamtsummenwert, d.h. dass je höher die Furcht vor Misserfolg ausgeprägt ist, desto mehr ist auch das Verhalten durch Evasion gekennzeichnet. Die Nettohoffnung, die sich durch Subtraktion der Furcht vor Misserfolg von der Hoffnung auf Erfolg ergibt, gibt die Richtung des Leistungsmotivs an und korreliert folgerichtig negativ mit den Evasionswerten.

Tab. 34: Zusammenhänge zwischen der Leistungsmotivation und den Skalen des EESU

\begin{tabular}{|l|c|c|c|c|}
\cline { 2 - 5 } \multicolumn{1}{c|}{} & EESU-S & EESU-I & EESU-B & EESU-SIB \\
\hline Hoffnung auf Erfolg (HE) & $-.447^{* *}$ & $-.436^{* *}$ & $-.382^{* *}$ & $-.476^{* *}$ \\
\hline Furcht vor Misserfolg (FM) & $.402^{* *}$ & $.445^{* *}$ & $.390^{* *}$ & $.461^{* *}$ \\
\hline Nettohoffnung (HE-FM) & $-.507^{* *}$ & $-.529 * *$ & $-.457^{* *}$ & $-.556^{* *}$ \\
\hline
\end{tabular}

Anschaulicher als die Korrelationstabelle ist jedoch die Abbildung, in der die mittleren Ausprägungen der Nettohoffnung der vier Evasions-Quartile ${ }^{17}$ dargestellt sind. Wie man in Abbildung 40 sieht, dominiert bei dem invasiv agierenden Quartil 1 deutlich die Erfolgsorientierung gegenüber der Misserfolgsorientierung. Auch die Quartile 2 und 3 zeichnen sich durch eine deutlich stärkere Hoffnung auf Erfolg im Vergleich zu der

\footnotetext{
${ }^{17}$ Unterteilt man das evasive Potenzial in vier Quartile, sodass in Quartil 1 und 2 die invasiv und eher invasiv agierenden Lehrkräfte und in Quartil 3 und 4 die eher evasiv und evasiv unterrichtenden Sportlehrkräfte zusammengefasst werden, können die Unterschiede zwischen Sportlehrerinnen und Sportlehrern auch graphisch sichtbar gemacht werden.
} 
Furcht vor Misserfolg aus. Bei dem evasivsten Quartil 4 sind sie beiden Größen nahezu ausgeglichen sodass ein relativ neutraler Wert entsteht und man hier nicht mehr von einer klaren Erfolgsmotivation sprechen kann. Der Unterschiede in den mittleren Nettohoffnung zwischen dem 1. Quartil und dem 4. Quartil entspricht einem großen Effekt von $d_{\text {Quartil1-Quartil4 }}=1.48$.

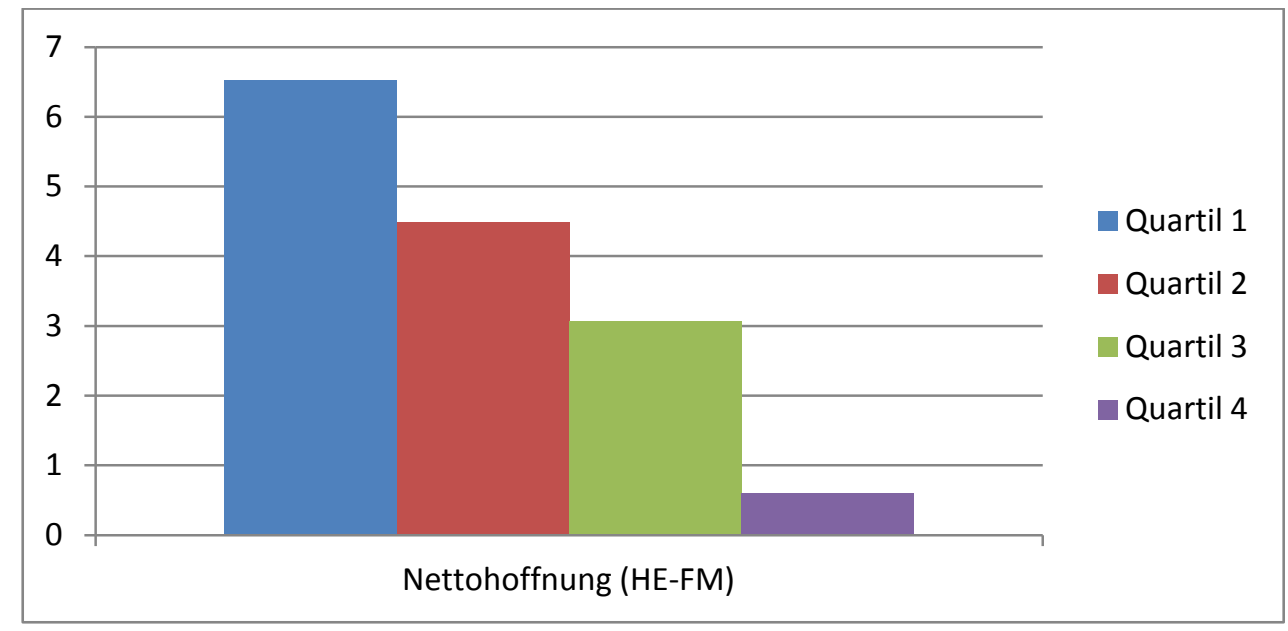

Abb. 40: Nettohoffnung der vier Quartile des evasiven Verhaltenspotenzials

\subsubsection{Ungewissheitstoleranz}

Schulische Handlungssituationen sind, wie bereits mehrfach beschrieben wurde, durch Neuigkeit und Ereignisunsicherheit sowie Ambiguität bzw. Ungewissheit geprägt, sodass eine gewisse Toleranz gegenüber Neuem und Ungewissen erforderlich scheint. Auch hier besteht ein negativer Zusammenhang zwischen der Ausprägung des evasiven Verhaltenspotenzials und der Toleranz gegenüber Ungewissheit. Je ungewissheitsintoleranter Personen sind, desto evasiver verhalten sich die Lehrkräfte im Sportunterricht (EESU-S: $r=-.360, p<.01$; EESU-I: $r=-.384, p<.01$; EESU-B: $r=-.335, p<.01$; EESU-SIB: $r=-.401, p<.01)$.

\subsubsection{Krankheitsbedingte Absentismusrate}

In Kapitel 7.2.11 wurden bereits die Ergebnisse der Angaben der Sportlehrkräfte zu den krankheitsbedingten Fehltagen im letzten Kalenderjahr vorgestellt. An dieser Stelle soll überprüft werden, ob evasives Unterrichten und das krankheitsbedingte Fernbleiben von der Arbeit in einem Zusammenhang stehen. In der Korrelationsüberprüfung zwischen der Absentismusrate und dem evasiven Verhalten ergaben sich keinerlei Zusammenhänge. Auch in der einfaktoriellen Varianzanalyse (ANOVA), in der das krankheitsbedingte Fernbleiben vom Unterricht mit der Ausprägung des evasiven Verhaltens in Quartile eingeteilten Gruppen verglichen wurde, ergaben sich keine überzufälligen Mittelwertunterschiede, sodass ein statistisch feststellbarer Einfluss von aus- 
weichendem Bewältigungsverhalten auf die Absentismusrate für die vorliegende Stichprobe nicht vorliegt.

\subsubsection{Zusammenfassende Interpretation der Ergebnisse}

Im Rahmen dieses Untersuchungsschwerpunkts, stellt sich am deutlichsten heraus, dass es sich bei evasiven Bewältigungsmaßnahmen um eine unangemessene Art des Umgangs mit Belastungen handelt. Es handelt sich um eine Strategie, die Belastungen und Konflikte mindern soll, im Idealfall, wie dies im Rahmen des Schonungstyps von Schaarschmidt und Fischer (1996) beschrieben ist: geringes berufliches Engagement bei gleichzeitig ausgeprägter Distanzierungsfähigkeit, die dazu führt, dass der Arbeitsalltag als wenig belastend wahrgenommen wird.

Im Rahmen des zuvor aufgearbeiteten dritten Untersuchungsschwerpunkt, bei dem sich alles um das Verhalten und Erleben im bzw. von Spotunterricht drehte, stellte sich heraus, dass evasives Verhalten keine entlastende, sondern eine das Belastungspotenzial sowie die berufliche Unzufriedenheit förderliche Bewältigungsstrategie darstellt.

Bereits der erste Untersuchungsaspekt, die wahrgenommene Belastung, macht deutlich, dass Sportlehrkräfte, deren Verhalten verstärkt durch evasive Strategien gekennzeichnet ist, subjektiv eine stärkere Gesamtbelastung verspüren als Kollegen, die eher invasiv agieren, d.h. Probleme offensiv angehen. Bei der genaueren Analyse der Korrelationen stellte sich zudem heraus, dass vor allem Belastungsfaktoren, die aus den Besonderheiten des Sportunterricht resultieren (siehe Kap. 2.3) von evasiv Agierenden in höherem Maße als belastend wahrgenommen werden und zum Teil durch Ergebnisse der beiden zuvor bearbeiteten Untersuchungsschwerpunkte erklärt werden können: In dem ersten Untersuchungsschwerpunkt stellte sich heraus, dass die Sportlehrkräfte mit ausgeprägtem evasiven Potenzial ihre eigene Kompetenz, besonders im theoretischen Bereich, als schlechter einschätzten als ihre invasiv agierenden Fachkollegen. Zudem weisen sie ein geringeres Fortbildungsengagement auf und zeichnen sich generell durch eine geringere Sportaffinität aus, die sich auch in einem schlechteren Fitnesszustand manifestiert. All diese Gründe sorgen dafür, dass z.B. die stetig latente Unfallgefahr, die Demonstration von Bewegungen oder das Leisten von Hilfestellung im Sportunterricht als belastender wahrgenommen wird, als dies bei Kollegen der Fall ist, die dafür sorgen, dass ihre Fähigkeiten den Zielen des Faches nicht entgegenstehen. An zweiter Stelle des „Korrelationsrankings“ rangiert die empfundene Belastung durch soziales Fehlverhalten von Schülern sowie Motivationsprobleme. An dieser Stelle deutet sich an, dass das Ignorieren bzw. bewusst präventive Vermeiden von Konflikten mit Schülern tatsächlich zu einer Verschärfung der Situation führt. Langfristig er- 
weist sich das evasive Verhalten, wie die Unterschiede in der Belastungswahrnehmung deutlich machen, nicht als belastungsreduzierend, sondern als belastungssteigernd.

Die Ergebnisse bezüglich des Kontrolliertheitserlebens können in der Begründung der Manifestation des ausweichenden Verhaltens ein wenig Licht ins Dunkel bringen. Aufgrund der Zusammenhänge zwischen der Ausprägung dieser Skala von Enzmann und Kleiber (1989) und dem evasiven Verhaltenspotenzial deutet sich an, dass die in Lehrerzimmern verbreitete Ansicht ein guter Lehrer hat keine Probleme (siehe Kap. 4.9.4.3) scheinbar tatsächlich einen gewissen Wahrheitsgehalt aufweist, denn je beobachteter, je kontrollierter eine Lehrkraft sich bei der Ausführung der Arbeit fühlt, desto stärker sind die konfliktmeidenden Strategien im Handlungsrepertoire verankert. Diese Tatsache steht nicht zuletzt in Beziehung zu den Erkenntnissen bezüglich der in Kapitel 7.5.5 zusammengefassten Informationen zu Persönlichkeitseigenschaften der Sportlehrer. Angst vor Misserfolg bei gleichzeitiger geringen Hoffnung auf Erfolg, ein geringes Selbstkonzept eigener Fähigkeiten und damit verbunden eine geringe Erwartung der Wirksamkeit des eigenen Handelns führen dazu, dass präventiv unabhängig von den konkreten Situationseigenschaften verstärkt evasiv agiert wird. Weitere Zusammenhänge zeigten sich bei der Ungewissheitstoleranz, d.h. je ausgeprägter die Ungewissheitsintoleranz ist, desto evasiver agieren Lehrkräfte im Unterricht. Auch bezüglich der Attribuierung des Einflusses auf Ereignisse unterscheiden sich eher evasiv und eher invasiv Unterrichtende. Während internale Überzeugungen bei evasiv Unterrichtenden in geringerem Maße ausgeprägt sind prägen mit zunehmender ausweichender Verhaltenstendenz fatalistische und soziale Überzeugungen das Denken. Mit den vorliegenden Ergebnissen wird der diagnostische Wert, der in Kapitel 4.5.2.2 beschriebenen generalisierten Erwartungen, Ereignisse im Leben selbst bestimmen zu können, bestätigt. Scheinbar erfolgt bei evasiv unterrichtenden Personen eine selektive Gewichtung der objektiven Informationen, die zu einer subjektiven Kompetenzeinschätzung führt. Gerade in mehrdeutigen Situationen entsteht so Unsicherheit, da keine klare Strukturierung zwischen beeinflussbaren und nicht beeinflussbaren Komponenten vorgenommen werden kann, wodurch es zum Ausbleiben einer Handlung bzw. vermeidendem Verhalten kommt,

Keine Zusammenhänge bestehen zur krankheitsbedingten Absentismusrate. Betrachtet man das Bild des "faulen Sacks" und des "Halbtagsjobbers" (siehe Kap. 2.2.4) bzw. des "Schonungstyps" (siehe Kap. 6.6.1.2) würde man erwarten, dass bei einem Anflug von Schnupfen schnell der individuelle Genesungsprozess der Arbeitsverpflichtung vorgezogen wird. Wie man anhand der Zusammenhänge zwischen den Ergebnissen des AVEM-Fragebogens und den Ergebnissen des EESU erkennen konnte, deuten die 
Korrelationen darauf hin, dass nicht Schonung und Belastungsreduzierung, sondern eher das Selbst gefährdende Tendenzen festzustellen sind. Auch bei der Formulierung der Hypothesen (siehe Kap. 5) zu diesem Untersuchungsaspekt wurden verschiedene Varianten in Betracht gezogen. Eine der Hypothesen war, dass die Lehrkräfte, die nach Schaarschmidt in das Schonungsmuster fallen, sich im Unterrichtsalltag durch eine besonders ausgeprägte evasive Verhaltenstendenz auszeichnen. Aufgrund der geringen Verausgabungsbereitschaft und der wenig ausgeprägten Bedeutsamkeit der Arbeit könnten Konflikte möglicherweise ignoriert und vermieden werden, bei einer insgesamt hohen Lebenszufriedenheit. Anhand der Daten stellte sich jedoch heraus, dass nicht der Schonungstyp, sondern Lehrkräfte, die dem Muster Burnout zugerechnet werden, in verstärktem Maße evasive Verhaltensweisen an den Tag legen.

Zwar deuten die Zusammenhänge zwischen der Ausprägung des evasiven Verhaltens und dem beruflichen Ehrgeiz auf das Schonungsmuster hin, was jedoch durch die die Ausprägungen der Skalen Distanzierungsfähigkeit, Resignationstendenz bei Misserfolg, innere Ruhe und Ausgeglichenheit, Perfektionsstreben und Lebenszufriedenheit relativiert wird. Zu all jene Skalen, die beim Schonungstypen protektiv wirken, bestehen ungünstig gerichtete Korrelationen: Je höher das evasive Potenzial ausgeprägt ist, desto schlechter können sich die Lehrkräfte von den Problemen der Arbeit distanzieren, desto weniger sind sie innerlich ruhig und ausgeglichen und sind insgesamt deutlich unzufriedener mit ihrem Leben im Allgemeinen, verfolgen aber dennoch dieselben beruflichen Ziele, wie sich in der Ausprägung der Skala Perfektionsstreben zeigt. Während das Gesundheitsmuster die geringste Ausprägung des evasiven Verhaltens zeigt, weisen das Schonungs- und das Anstrengungsmuster mittlere, das Burnout-Muster ausgeprägte evasive Tendenzen auf.

Das Verspüren von negativen Emotionen bei der Arbeit, die daraus folgende innere Distanzierung samt negativen motivationalen Einstellungen ${ }^{18}$ bzw. die berufsbedingte Erschöpfung wurden zudem durch die Korrelationen des evasiven Verhaltens zu den beiden Skalen des Oldenburger Burnout-Inventars und dem Sekundärfaktor aus dem AVEM-Fragebogen bestätigt.

Auch in den weiteren eingesetzten Skalen, die mehr oder weniger direkt ein Abbild der Zufriedenheit bei bzw. mit der Arbeit ausdrücken, zeichnet sich ein eindeutiges Bild ab. Je stärker das evasive Verhaltenspotenzial ausgeprägt ist, desto weniger Spaß haben die Lehrkräfte beim Kerngeschäft des Berufs, dem Unterrichten, desto schlechter sind die Gefühle in Bezug auf die Vergangenheit bzw. für die Zukunft und desto überforderter sind die Lehrkräfte. Des Weiteren zeigte sich, dass mit zunehmender evasiver Ver-

\footnotetext{
${ }^{18}$ Skala: Disengagement.
} 
haltenstendenz auch der Kreis derjenigen abnimmt, die den Beruf erneut wählen würden, was nicht zuletzt an der wahrgenommen Atmosphäre im Kollegium liegt. Je besser die Atmosphäre im Sportkollegium eingeschätzt wird, desto geringer ist die Ausprägung des evasiven Verhaltenspotenzials, da scheinbar soziale Unterstützungssysteme auch für die Bewältigung ausschlaggebend sind. Bezüglich der Zufriedenheit mit der Stundenverteilung ist mit steigender Sportstundenzahl ein Anstieg der Ablehnungstendenz festzustellen, d.h. diejenigen, die gerne weniger Sport unterrichten würden, weisen das höchste evasive Potenzial auf.

Anhand des dritten Untersuchungsschwerpunkts wurde an mehreren Stellen deutlich, dass evasives Verhalten als ressourcenschonende Laissez-faire-Strategie gänzlich unwirksam und sogar schädigend wirkt, aber generell nicht aus hedonistischen Gründen eingesetzt wird, sondern eher eine Strategie ist, die als letzte Rettung genutzt wird, als Pufferinstrument um Schäden der eigenen seelischen und körperlichen Gesundheit zu verhindern, wodurch jedoch wie in einem Teufelskreis genau das Gegenteil eintritt. 


\section{Diskussion}

In diesem Kapitel erfolgt zunächst eine zusammenfassende Diskussion der Ergebnisse, die in Bezug zu den Aspekten gesetzt werden, die im theoretischen und methodischen Teil dieser Arbeit im Vorfeld zusammengestellt und erarbeitet wurden. ${ }^{1}$ Zudem sollen die im Vorfeld der Erhebung formulierten Fragestellungen erneut in den Blick genommen werden und jeweils möglichst umfassend und exakt beantwortet werden. Im zweiten Teil dieses Kapitels sollen abgeleitet aus den Ergebnissen dieses eher im Grundlagenbereich angesiedelten Forschungsprojekts weitergehende Fragestellungen formuliert und die Perspektiven bzw. das Entwicklungspotenzial in verschiedenen Bereichen, wie z.B. im Rahmen der Lehrerausbildung, deutlich gemacht werden. Im Rahmen eines kurzen reflexiven Fazits sollen zudem die Durchführung als auch die Ergebnisse der Untersuchung kritisch hinterfragt werden.

\subsection{Zusammenfassende Diskussion der Ergebnisse}

Während bereits das in der Einleitung angeführte plakative Beispiel aus dem Alltag einer Beziehung aufzeigte, wie vielfältig die Denkprozesse im Entscheidungshandeln in nicht eindeutigen Situationen sind und wie weitreichend oder aber unbedeutend die Folgen sein können, wurde im Verlauf dieser Arbeit zunehmend deutlich, wie komplex das Bewältigungshandeln in der Schule, speziell im Sportunterricht ist. Wie in der Einleitung und in Kapitel 4.9.5 herausgestellt, ist in einem Fach wie dem Sportunterricht eine richtige Mischung aus offensiver Problembewältigung und Selbstregulierung von Konflikten und Problemen durch die Schüler notwendig, wodurch soziale Lernprozesse ermöglicht werden. Nur so kann ein angemessener und selbstständiger Umgang mit Konflikten und Problemen entwickelt werden.

Bevor man sich abschließend in differenzierter Art und Weise mit der Manifestation und den Besonderheiten des evasiven Bewältigungsverhaltens beschäftigt, musste zunächst geklärt werden, ob in der vorliegenden Sportlehrerstichprobe überhaupt ausweichend-meidende Verhaltenstendenzen vorliegt. Anhand der Daten zeigte sich, dass evasive Tendenzen im Sportunterricht durchaus vertreten sind, aber im Vergleich zu aktiven Prozessen der Problemlösung eine leicht geringere Bedeutung einnehmen. Dies zeigt sich am Mittelwert des evasiven Verhaltens aller drei Facetten von 2,63

\footnotetext{
${ }^{1}$ Aufgrund der interpretativen Zusammenfassungen der Ergebnisse der drei Untersuchungskomplexen in Kapitel 7 sind einige der Darstellungen redundant, was jedoch aufgrund der notwendigen Herstellung von Gesamtzusammenhängen zwischen den einzelnen Komplexen untereinander sowie mit theoretischen Aspekten nicht zu vermeiden war.
} 
$(S D=.56){ }^{2}$ Letztendlich bewertet werden kann die in der Stichprobe vorliegende Ausprägung jedoch nicht, da weder Vergleichswerte weiterer Sportlehrerstichproben noch Vergleichsdaten von Lehrern anderer Fächer oder aber anderer Berufsgruppen vorliegen. Sehr wohl kann jedoch angeführt werden, dass überzufällige Zusammenhänge zwischen der Ausprägung des evasiven Verhaltenspotenzials und berufsbiographischen und persönlichen Daten der Lehrkräfte, der Ausgestaltung des Unterrichtsalltags als auch zu Aspekten der beruflichen Zufriedenheit und Gesundheit vorliegen.

Nachdem die Fragestellung „Ist evasives Verhalten im Sportunterricht ein verbreitetes Problem bzw. tritt es in einer bedeutsamen Häufigkeit auf? aufgrund fehlender Vergleichsdaten nur unzureichend beantwortet werden konnte, brachte der erste Untersuchungskomplex zumeist zu erwartende Ergebnisse, er enthielt aber auch Überraschungen. Betrachtet man die Ergebnisse dieses ersten Komplexes (siehe Kap. 7.3) deutet sich vor allem Relevanz im Zusammenhang mit den in Kapitel 2 ausführlich dargestellten Besonderheiten des (Sport-) Lehrerberufs bzw. der Bedeutung der Berufswahlmotive für dessen Ausübung an. Anhand einiger Untersuchungsergebnisse, die im Ergebnisteil vorgestellt wurden, könnte man provokant fragen: „Werden die Falschen Lehrer?" Der Lehrerberuf, der Beruf, der im Zentrum der Gesellschaft steht und von manchen sogar die Verantwortung für die Rettung ebenjener aufgebürdet bekommt, ausgeübt von Ungeeigneten oder Unwilligen? In dem zugehörigen Theoriekapitel wurden u.a. Ergebnisse von Watt und Richardson (2007) vorgestellt, die herausfanden, dass Personen, die aus intrinsisch-altruistischen Beweggründen den Beruf wählten im weiteren Berufsleben höhere Werte im Arbeitsengagement erreichen. Ebenso wurde dargestellt, dass sich die besten Schüler eines Abiturjahrgangs aufgrund der fehlenden Attraktivität des Berufes meist nicht für den Lehrerberuf entscheiden, sondern andere Wege einschlagen (Wößmann, 2009) sowie abhängig vom Status des Elternhauses extrinsische Motive, wie z.B. die Sicherheit des Beamtenstatus, unterschiedlich große Bedeutsamkeit für die Berufswahl aufweisen (Weiner, 1993). Außerdem wurde über einen recht hohen Anteil an Studenten berichtet, für die der Lehrerberuf eine Negativauswahl aufgrund fehlender Alternativen darstellt und oft das Ergebnis einer Ad-hocEntscheidung von spätentschlossenen Abiturienten ist (Schwänke, 1988; Struck, 1994). Wie sich in Kapitel 7.1 zeigte, ist der Anteil der extrinsisch Motivierten mit 28,4\% tatsächlich in der Stichprobe nicht unbedeutend und auch bezogen auf die Ausprägung des evasiven Verhaltens besteht durchaus Relevanz: Zwischen den extrinsischhedonistisch Motivierten und den intrinsisch-altruistisch Motivierten bestehen signifi-

\footnotetext{
${ }^{2}$ Ein Wert von 1 entspräche einem extrem offensiven Bewältigungsstil, während ein Wert von 4 einem extrem ausgeprägten evasiven Verhaltenspotenzials entspräche.
} 
kante Unterschiede in der Ausprägung des evasiven Verhaltenspotenzials (siehe Kap. 7.3.3). Spranger (1963) spricht in diesem Zusammenhang von pädagogischer Liebe zum Kind, die eine elementare Voraussetzung für die Wahl des Berufs und dessen angemessener Ausführung ist. Er sieht in der pädagogischen Liebe eine grundsätzliche Einstellung, die naturgegeben und nicht erlernbar ist. In dieser Arbeit zeigen sich zusätzlich Parallelen bezogen auf die Liebe bzw. Affinität zum Sport, die für den Sportunterricht, genau wie die Liebe zum Kind für den Lehrberuf allgemein, eine wichtige Voraussetzung darstellt. Bereits in Kapitel 2.3 wurde die besondere Bedeutung der Person bzw. Persönlichkeit für die Rolle des Sportlehrers herausgestellt, der sowohl physischmotorisch als auch von seiner Einstellung und seinem Verhalten im Bezug auf Bewegung, Spiel und Sport eine wichtige Funktion im Modelllernen für die Schüler hat und in diesem Bereich eine Vorbildfunktion übernehmen soll. Des Weiteren bietet ein gewisses Niveau an Sportaffinität, sowohl bezogen auf Einstellungen und Ansichten als auch bezogen auf die eigene Körperlichkeit und einhergehend mit den damit verbundenen Kompetenzen eine Basis, die je nach ihrer Ausprägung stabilisierende und unterstützende Funktion im Arbeitsalltag übernehmen. Gröbe (2006) stellte hierbei heraus, dass beim sportlich-motorischen Können ein breites solides Fundament vorteilhaft im Vergleich zum Spezialistentum ist, bei dem kein ausgewogenes sportartbezogenes Kompetenzprofil vorliegt. Komprimiert man die Grunderkenntnisse aus Kapitel 7.3.4 und 7.3.5, kommt man zu der These, dass für einen angemessenen Umgang mit Belastungen bzw. Konflikten im Sportunterricht eine gewisse Sportlichkeit und Fitness und neben der Liebe zum Kind auch die Liebe zum Sport positive Voraussetzungen sind. Durch die Nähe zum Sport, die sich auch in dem eigenen motorischen Können widerspiegelt wird die Vermittlung erleichtert, da das Demonstrieren und Erklären sowie das anschließende Analysieren mit geringeren Anstrengungen verbunden sind, wodurch sowohl Unterrichtsziele leichter erreicht werden, als auch psychische und physische Belastungen besser kompensiert werden können.

Eben jene Affinität zum Sport in Kombination mit der Freude an der Arbeit mit Kindern und Jugendlichen könnte auch dafür verantwortlich sein, dass Lehrer mit geringerem evasiven Potenzial eine signifikant höhere Stundenverpflichtung sowohl insgesamt als auch im Fach Sport haben. ${ }^{3}$ Begründung hierfür ist vermutlich der ausgeprägte Spaß bzw. die Freude, die von diesen Lehrkräften im Vergleich zu Lehrern mit ausgeprägten evasiven Verhaltenstendenzen beim Unterrichten verspürt wird (siehe Kap. 7.5.4.3). Insgesamt zeigte sich, dass trotz des hohen Belastungspotenzials des Sportunterrichts die Zufriedenheit mit dem Beruf sehr hoch ist, was man aus den Antworten zum Spaß

\footnotetext{
${ }^{3}$ Für sie kommt vermutlich aufgrund des ausgeprägten Spaßes bei der Arbeit bzw. der Erfüllung eine Reduzierung der Stunde- bzw. der Sportstundenanzahl seltener infrage.
} 
am Unterrichten ableiten kann (siehe Kap. 7.2.7). 88\% der Befragten gaben an oft bzw. sehr oft/immer Freude am Unterrichten $\mathrm{zu}$ haben, was $\mathrm{zu}$ der „Wiederholungstäterquote ${ }^{4 \prime}$ von 79,5\% passt. Anhand der vom Institut für Demoskopie Allensbach im Jahr 2012 erhobenen Daten ${ }^{5}$ (Süßlin, 2012) zeigt sich, dass der Sportlehrerberuf trotz des hohen Belastungspotenzials, auch im Vergleich mit den anderen Schulfächern, durchaus einen attraktiven Charakter aufweist. ${ }^{6}$ Für eine bessere Rekrutierung von Junglehrern gilt es, auch nach außen hin selbstbewusst aufzutreten und zu zeigen, dass man im Lehrerberuf weder ein tyrannischer Sadist noch ein weltfremder Pauker ist bzw. wird (siehe Kap. 2.2.4), sondern dass der Beruf tatsächlich für viele eine Erfüllung darstellt, für die Ausübung aber auch gewisse Überzeugungen, Einstellungen sowie Fähig- und Fertigkeiten notwendig sind. Gelingt es, ein attraktives aber auch professionelles Bild des Lehrerberufs nach außen hin zu verkörpern, ist es möglich, dass sich mehr geeignete Personen für den als unattraktiv verschrienen Beruf entscheiden und gleichzeitig die Zahl der "Notlösungen" reduziert werden. Auch bezüglich des Erreichens der beiden Zielsetzungen, die im Rahmen des Doppelauftrags des Sportunterrichts in Kapitel 2.3 formuliert wurden, ist es besonders wichtig, die Richtigen in den Beruf zu bekommen, denn nur so kann sowohl in als auch zu Bewegung, Spiel und Sport erzogen werden, was vor allem aufgrund der veränderten Lebensbedingungen, die gekennzeichnet sind durch Individualität, Bewegungsarmut und Erfahrungsmangel, auch zukünftig weiter an gesellschaftlicher Bedeutung gewinnen wird.

Neben pädagogischem und didaktischem Geschick sowie der Affinität zu Sport deuten die Ergebnisse aus Kapitel 7.3.6 zudem darauf hin, dass die Ausprägung an kommunikativer Kompetenz ebenfalls ein beeinflussendes Element des Bewältigungshandelns ist. Geht man davon aus, dass sprachbegabte Personen häufiger eine Sprache unterrichten fällt auf, dass gerade diese Personen effektiver mit Problemen umgehen als andere. Doch auch unabhängig von weiteren unterrichteten Fächern sind Personen bezüglich ihrer kommunikativen Kompetenz naturgegeben unterschiedlich begabt. Sollte sich in weiteren Studien herausstellen, dass die kommunikativen Fähigkeiten eine entscheidende Einflussgröße für das Bewältigungshandeln ist, wäre dies ein erster Hinweis für Modifikationen von Fort- und Weiterbildungsmaßnahmen, aber auch für die Ausbildung von Lehrern, bei denen Kommunikationstraining und die Vermittlung von Strategien im Umgang mit Schülern einen größeren Stellenwert einnehmen sollte.

\footnotetext{
${ }^{4}$ Die Quote gibt an, dass Sportlehrkräfte, die, wenn sie heute erneut vor der Studien- bzw. Berufswahl stehen würden, ein weiteres Mal den bereits beschrittenen Weg einschlagen würden.

${ }^{5}$ In der Studie des Instituts für Demoskopie Allensbach gaben 63\% der Lehrer an, dass sie derzeit Freude am Beruf haben (Süßlin, 2012).

${ }^{6}$ In der Studie des Instituts für Demoskopie Allensbach gaben 52\% der Lehrer an, dass sie ihren Beruf als attraktiv wahrnehmen (Süßlin, 2012).
} 
Eine regelmäßige Aktualisierung und Entwicklung der eigenen Kompetenzen, ist laut den Maßgaben des Deutschen Bildungsrates (1972) bzw. der Kultusministerkonferenz (2004) im Rahmen des Kompetenzbereichs Innovieren als einer der Aufgabenbereiche von Lehrern vorgesehen bzw. vorgeschrieben. In der vorliegenden Untersuchung stellte sich heraus, dass mit zunehmendem Fortbildungsengagement, unabhängig ob allgemeindidaktisch oder sportunterrichtsspezifisch, verstärkt offensiv-invasiv agiert wurde. Zudem konnte die Erkenntnis gewonnen werden, dass sich nicht alle Sportlehrkräfte so intensiv weiterbilden, wie dies von der Bildungsverwaltung vorgesehen ist. Während das private Fortbildungsengagement deutlich ausgeprägter ist, besteht beim organisierten Fortbilden durchaus Nachholbedarf. Anhand der Daten konnte zudem festgestellt werden, dass die Ausprägung des Fortbildungsengagements neben dem Einfluss auf das Bewältigungshandeln auch die theoretische und praktische Vermittlungskompetenz beeinflusst (siehe Kap. 7.3.8). Überraschend dabei ist, dass weniger das organisierte Fortbilden, ${ }^{7}$ sondern vor allem das privat-eigenverantwortliche Fortund Weiterbilden durch Fachbücher und Zeitschriften Einfluss auf die zuvor genannten Kompetenzen hat. Die Frage nach der Sinnhaftigkeit und der Gestaltung von Fortbildungsveranstaltungen von Lehrerbildungsverbänden, Schulämtern und Kultusministerien drängt sich dabei natürlich auf. Fortbildungen, die überwiegend am Wochenendende stattfinden oder aber zu Problemen mit der Schulleitung im Hinblick auf Unterrichtsvertretung oder -ausfall führen können, sind entweder mit negativen Attributen besetzt und Inhalte werden nicht angenommen oder aber die Veranstaltungen sind generell nicht zielführend bzw. behandeln für die Sportlehrkräfte irrelevante Aspekte. Lipowsky (2010, S. 54) konnte zeigen, dass „ein Minimum an Akzeptanz eine notwendige, wenngleich eben keine hinreichende Voraussetzung für einen erfolgreichen Lernund Transferprozess darstellt". All diese Gründe können dazu führen, dass solche Veranstaltungen komplett gemieden werden, was den recht hohen Anteil an Lehrkräften, die sich im Jahr vor der Erhebung gar nicht organisiert fortbildeten erklärt (siehe Kap. 7.3.8). Neben der Frage, wie man die Sportlehrer dazu bringt, sich in größerem Umfang fortzubilden, ist damit vor allen Dingen die Ausrichtung von organisierten Fort- und Weiterbildungsveranstaltungen für Sportlehrer zu hinterfragen, die zwar meist praxisorientiert sind, aber dennoch keinen Einfluss auf die tatsächliche Vermittlungskompetenz der Lehrkräfte haben. Bezogen auf die im Rahmen dieser Untersuchung gewonnenen Erkenntnisse muss es in solchen Veranstaltungen neben einem Aufräumen mit althergebrachten Klischees, wie z.B. dass ein guter Lehrer keine Probleme im Unterricht hat (siehe Kap. 4.9.4.3), vor allem darum gehen, wie man für sich selbst einen

\footnotetext{
${ }^{7}$ Das organisierte sportunterrichtsspezifische Fortbildungsengagement weist lediglich einen geringen Zusammenhang zur Ausprägung der Praxiskompetenz auf.
} 
professionelleren Umgang mit Problemen und Konflikten entwickeln kann. Aufgrund der Individualität eines jeden Sportlehrers soll es dabei nicht um einen Masterplan gehen, den man uniform in der Praxis umsetzen kann, sondern vielmehr um eine Sensibilisierung für die Problematik des Bewältigungshandelns. Den Sportlehrkräften muss deutlich vor Augen geführt werden, dass durch ein Vermeiden, Ignorieren, Verschleiern und Herunterspielen von Konflikten und Problemen in der konkreten Situation die Belastung zwar kurzfristig reduziert werden kann, langfristig durch das Wiederauftreten derselben Konflikte und den fehlenden Einsichten von Schülern das Belastungspotenzial jedoch deutlich ansteigt. Ein paar richtige Entscheidungen und konsequentes Handeln können bei einer neuen Lerngruppe dazu führen, dass in Zukunft Probleme und Konflikte in deutlich verminderter Zahl auftreten und damit auch das Belastungspotenzial nachhaltig sinkt, die berufliche Zufriedenheit steigt und wichtige Ressourcen geschont werden. Elementar in diesem Zusammenhang ist es, den Lehrkräften für die Besonderheiten des Sportunterrichts (siehe Kap. 2.3) unterschiedliche Strategien und Verhaltensweisen an die Hand zu geben, die unnötige Konflikte, wie z.B. durch mangelnde Unterrichtsorganisation, verhindern und beim Auftreten von Konfliktsituationen einen effektiveren Umgang mit Unterrichtsproblemen ermöglichen. Hierbei sollte vor allen Dingen das Verhalten in Drucksituationen im Mittelpunkt stehen, d.h. wie komme ich unter Handlungsdruck zu einer angemessenen Entscheidung. So kann es nach der Beobachtung eines Schülerkonflikts bereits ausreichen, die beiden Beteiligten zu dem Problem Stellung nehmen zu lassen, um sich durch diesen Zeitpuffer über mögliche Handlungsmöglichkeiten und Konsequenzen klar zu werden. Genau an dieser Stelle spielen vermutlich auch die zuvor angesprochenen kommunikativen Kompetenzen eine Rolle, die manche Sportlehrer von Grund auf besitzen, andere hingegen erst entwickeln müssen. Durch den reflexiven Umgang mit Konflikten, aber auch dem eigenen Verhalten bzw. der eigenen Reaktion ist es so möglich, das eigene Bewältigungsverhalten stetig weiterzuentwickeln, wobei sowohl erzieherische Ansprüche als auch die eigene Gesundheit im Zentrum der Aufmerksamkeit stehen sollten. Auf diese Art und Weise kann jeder Lehrer seinen eigenen individuellen Bewältigungsstil ausbilden und bleibt dabei in seinem Handeln authentisch. Selbst erprobte bzw. entwickelte Verhaltensweisen können so zukünftig auch in Drucksituationen automatisiert abrufbar sein, sodass nach einer Phase, die der Erprobung von geeigneten Vorgehensweisen dient, in der auch Scheitern dazugehört, eine Entlastung im Alltag möglich ist. Findet eine solche individuelle Weiterentwicklung des Bewältigungshandelns hingegen nicht statt, wird sich, wie bereits von Huberman (1989) formuliert, mit zunehmendem Alter verstärkt Desengagement in Form von Gelassenheit oder Bitterkeit entwickeln. Neben 
der eigenen Unzufriedenheit wird dabei auch der Erziehungs- und Bildungsauftrag vernachlässigt. Anhand der erhobenen Daten zeigt sich, dass in der Stichprobe mit zunehmendem Alter auch das evasive Verhaltenspotenzial ansteigt (siehe Kap. 7.3.1). Zudem zeigte sich, dass je stärker das evasiven Verhalten ausgeprägt ist, desto niedriger ist das vom Lehrer im Unterricht angelegte Anforderungsniveau (siehe Kap. 7.4.7), sodass die von Meusel (1989) beschriebene läppische Spielerei zur Verschleierung fehlender Kompetenz Realität wird (siehe Kap. 4.9.4.2).

Bei dem zweiten Komplex war die Fragestellung auf die Gestaltung des Unterrichts gerichtet. Anhand verschiedener Aspekte wurde versucht, Unterschiede in der Planung und Durchführung des Unterrichts in Abhängigkeit des Bewältigungshandelns darzustellen (siehe Kap. 7.4). Während zuvor die Unterschiede und Besonderheiten der Lehrkräfte im Fokus standen, soll nun versucht werden, die Auswirkungen bzw. die Manifestation von evasivem Verhalten für die Gestaltung des beruflichen Alltags in Form einer überspitzten eindimensionalen Darstellung zu erläutern: Bereits zu Hause am Schreibtisch beginnt der Kampf mit Konflikten und Problemen. Welche Inhalte eignen sich, um möglichst unbeschadet durch den Tag zu kommen? Während in Kapitel 2.3 aufgezeigt wurde, welche wichtige Aufgabe der Sportunterricht für jedes einzelne Kind und dessen geistige, soziale und motorische Entwicklung hat, bestimmen bei ausgeprägt evasiv Unterrichtenden andere Gedanken den Prozess der Inhaltswahl. Während Lehrkräfte und viele Bildungswissenschaftler davon reden, dass wichtige Erfahrungen aufgrund von Individualisierung und Mediatisierung des Alltags sowie der Verinselung von Lebensräumen zur Mangelware werden, bestimmen hier vermehrt Ichbezogene Aspekte das Denken und Handeln. Curricula und Lehrpläne, eigentlich als Entscheidungshilfen gedacht, die stets aktualisiert werden, um gesellschaftliche und bildungstheoretische Veränderungsprozesse aufzugreifen, verlieren mit Blick auf die konkrete Umsetzung in der Unterrichtspraxis an Bedeutung. Größeres Gewicht bei der Inhaltswahl hat hingegen vor allem das erwartete Konfliktpotenzial. Warum jenen Inhalt durchführen, bei dem es sowieso nur Ärger und Unzufriedenheit gibt oder gar noch ein hohes Risiko für Verletzungen vorliegt. Einige andere Inhalte sind nur mit großem Aufbau- und Organisationsaufwand möglich oder erfordern Kreativität und Einsatz der Lehrkraft aufgrund der schlechten räumlich-materiellen Bedingungen auch diese Inhalte werden überzufällig stark gemieden. Inhalte, in denen man selbst nicht so kompetent ist, werden ausgeblendet, andere Inhalte avancieren zum Dauermittelpunkt des Unterrichts. Die Ergebnisse zu der Durchführungshäufigkeit verschiedener Inhalte relativieren jedoch die zuvor gemachten Aussagen ein wenig und zeigen, dass neben dem Verletzungsrisiko tatsächlich noch weitere Aspekte deutlichen Einfluss 
ausüben. Obwohl einige Sportarten wie z.B. Turnen, Konditionstraining, Leichtathletik und Schwimmen einen unterdurchschnittlichen Risikofaktor aufweisen, werden sie von Lehrkräften mit ausgeprägtem evasivem Potenzial vermehrt gemieden. Neben organisatorischen Hindernissen wie z.B. der Aufbau von Gerätearrangements und die Sicherstellung der Hilfestellung im Gerätturnen, scheint auch das Ablehnungsverhalten von Schülern und die damit einhergehende Konflikthäufigkeit mitentscheidend zu sein. Durch die so entstehende inhaltliche Begrenztheit wird die in Lehrplänen und Curricula geforderte Mehrperspektivität und Vielfältigkeit von Bewegung, Spiel und Sport (siehe Kap. 2.3.2) nur in begrenztem Maß umgesetzt. Ebenso verhält es sich bezüglich der didaktisch-pädagogischen Ausrichtung des Unterrichts, die durch die Unterschiede bei der Bedeutsamkeit von Vermittlungsaspekten im Sportunterricht für die Lehrkraft deutlich wird (siehe Kap. 7.4.3). Wie sollen Schüler ihren Horizont erweitern, wenn bereits diejenigen, die diese Prozesse anregen sollen, in ihrem Handeln und Denken durch Schranken und Hindernisse beeinflusst werden. Das Erreichen der Zielsetzung, Kinder und Jugendlichen neue Perspektiven und Zugänge zu Bewegung, Spiel und Sport anzubieten (siehe Kap. 2.3), scheint durch die signifikante Minderbewertung der Vermittlungsaspekte Kreativität, Körpererfahrung sowie Vermittlung von Freude an der Bewegung mit dem Ziel zu einem lebenslangen Sporttreiben zu motivieren äußerst fraglich. Auch der zweite Teil des Doppelauftrags scheint mit zunehmendem evasiven Verhaltenspotenzials aus dem Blick zu geraten. Aspekte wie soziales Lernen, Persönlichkeitsentwicklung oder die Förderung der Selbstständigkeit stellen in neueren Legitimationsansätzen (dvs, DSLV, DOSB \&FSW, 2008) wichtige Eckpfeiler dar, werden aber gerade von den eher traditionell geprägten evasiv Unterrichtenden als weniger bedeutsam für den eigenen Unterricht bewertet. Einige weitere Ergebnisse dieses Komplexes deuten an, dass sich mit zunehmendem evasivem Verhaltenspotenzial Tendenzen, die vermutlich der Belastungsreduzierung dienen sollen, im Unterricht wiederfinden. Neben dem zuvor bereits angeführten geringeren technisch-taktischen und konditionellen Anspruchsniveau im Unterricht, wodurch die Konflikthäufigkeit mit disziplinlosen Schülern gesenkt werden soll, beteiligen sich eher evasiv Unterrichtende deutlich seltener selbst aktiv im Unterricht. Über die genauen Hintergründe dieser Besonderheit lässt sich nur spekulieren, naheliegend ist jedoch, dass die Lehrkräfte denken, durch die eigene sportliche Aktivität neben der physischen Belastung zudem das Geschehen aus den Augen zu verlieren und dadurch weitere Probleme zu provozieren. Doch gerade das Mitmachen eines Sportlehrers, die unverschleierte Begeisterung für Bewegung, Spiel und Sport, fördert dessen Anerkennung bei den Schülern, die ihm seine Einstellung abkaufen, was ein wesentliches Element für das im Sportunterricht 
höchst bedeutsame Modelllernen darstellt (Bandura, 1979; Heine, 1981; Zimmer \& Volkamer, 1981). Dieselben Sicherheitsgedanken scheinen sich auf bei der weiteren Organisation des Unterrichts widerzuspiegeln, der mit zunehmendem evasivem Verhaltenspotenzial eine verstärkt deduktiv-lehrerzentrierte Ausrichtung aufweist.

Den Einstieg der Betrachtung des dritten und letzten Untersuchungskomplexes soll anhand von zwei Aspekten erfolgen, die inhaltlich eigentlich zu dem vorherigen Komplex gehören. Durch diese beiden Aspekte wird das Dilemma, indem sich evasiv Unterrichtende bezüglich ihrer Arbeit befinden, erfahrbar. Schaut man sich im Vorfeld der Untersuchung die verschiedenen von Schaarschmidt und Fischer (1996) identifizierten Musterstrukturen an und sucht Parallelen zum evasiven Unterrichten, stößt man schnell auf den Schonungstyp (siehe Kap. 6.6.1): Dies ist ein Lehrer, der bei hoher Lebenszufriedenheit und bester Gesundheit seinen beruflichen Auftrag sehr frei interpretiert und sich hauptsächlich auf sein Privatleben konzentriert. Sein Arbeitstag beginnt und endet meist mit dem Läuten der Schulglocke. Vertreter dieser Musterstruktur schätzen den Stellenwert des Faches Sport im Vergleich mit den anderen Schulfächern, genau wie die eher evasiv Unterrichtenden, als geringer ein. Überraschend ist jedoch, dass mit zunehmendem evasivem Verhaltenspotenzial die investierte Zeit für Vor- und Nachbereitung des Unterrichts nicht bzw. nur marginal absinkt. Letztendlich wird dadurch deutlich, dass Lehrkräfte, die evasive Verhaltenstendenzen aufweisen, nicht grundsätzlich unmotiviert sind und versuchen, durch schonendes Laissez-faireVerhalten, wie der Schonungstyp es anwendet, durch den Tag zu kommen. Evasives Verhalten stellt eine das Selbst bedrohende Bewältigungsstrategie dar, bei der trotz vorhandener Verausgabungsbereitschaft und Streben nach Perfektion ausweichende Tendenzen vermehrt handlungsleitende Funktion übernehmen. Zum Problem für die eigene psychische und physische Gesundheit entwickelt sich das Verhalten durch die mangelnde Distanzierungsfähigkeit von Problemen der Arbeit. Durch immer wiederkehrende Belastungen sinkt die Zufriedenheit mit dem eigenen Berufsalltag und dem eigenen Leben stark ab, was letztendlich auch zu einer ausgeprägten Resignationstendenz und Disengagement führt. All diese Eigenschaften von evasiv Unterrichtenden führen zu einer deutlichen Übereinstimmung mit dem von Schaarschmidt und Fischer (1996) skizzierten Burnoutmuster. Auch die beiden Skalen aus dem Oldenburger Burnout Inventar bestätigen diese Zusammenhänge. Neben einer allgemeinen Unzufriedenheit mit der Arbeit fühlen sich eher evasiv Unterrichtende zudem in signifikant höherem Maße durch die Arbeit überfordert und wünschen sich daher auch häufiger eine Reduzierung des Anteil des Sportunterrichts an der Gesamtlehrverpflichtung (siehe Kap. 7.5.4.2). Ergänzend kommt hinzu, dass ihnen das tagtägliche Unterrichten deut- 
lich weniger Spaß macht als ihren Kollegen, die Probleme und Konflikte offensiv angehen (siehe Kap. 7.5.4.3).

Betrachtet man das Modell des Lehrerverhaltens von Allmer (1982) mit den integrierten aussagenlogischen Fragen des erweiterten kognitiven Motivationsmodells von Heckhausen und Rheinberg (1980) (siehe Abb. 14, Kap. 4.9.3.4), kann das Denken und Handeln von ausweichend agierenden Lehrkräften nachvollzogen werden. Wie sich im dritten Untersuchungskomplex zeigte, unterscheiden sich evasiv Unterrichtende Sportlehrer bezüglich ihrer Persönlichkeitseigenschaften in einem hohen Maß von ihren invasiver agierenden Kollegen. Neben einer deutlich geringer ausgeprägten Erwartung, mit dem eigenen Handeln etwas an der Situation ändern zu können, sind sie zudem davon überzeugt, dass nicht sie, sondern das Schicksal oder andere Personen entscheidenden Einfluss auf den Verlauf von Situationen haben. Kommen zudem Motivationsprobleme hinzu, ${ }^{8}$ führt dies dazu, dass neben den beiden Fragen der Effektkalkulation und der ersten Frage der Aufwandkalkulation auch die zweite Frage in dieser Kategorie ${ }^{9}$ ungünstig beantwortet wird und eine Handlung ausbleibt. Neben einer ungünstigen Ausprägung von bestimmten Persönlichkeitseigenschaften, wie die Selbstwirksamkeitserwartung oder Kompetenz- und Kontrollüberzeugungen, können auch misslungene Bewältigungsversuche dazu führen, dass nach mehreren Durchläufen von Bewertungs-Bewältigungsprozessen eine Chronifizierung von Stress mit den damit einhergehenden Folgen eintritt, die letztendlich in Desengagement und Hilflosigkeit münden können. In einem Rückkopplungsprozess werden zukünftige Widerspiegelungsvorgänge beeinflusst und so mehr und mehr Routinen ausgebildet, die zu einer Verschärfung der unbefriedigenden Situation führt. Während Situationsparameter, d.h. die Eigenschaften der eine Handlung erfordernden Unterrichtssituation, an Bedeutung verlieren, übernehmen zunehmend prädisponierte Handlungsweisen bzw. Stile die Führung im Copingprozess (siehe Kap. 4.3). Abschließend soll, wie bereits in Kapitel 7.5.1 und 7.5.7 gezeigt werden konnte, eine der Haupterkenntnisse dieser Arbeit herausgestellt werden: Beim evasiven Verhalten handelt es sich um eine unangemessene Bewältigungsstrategie, die das Belastungspotenzial des Sportunterrichts, statt es zu senken, signifikant erhöht. Hierbei zeigte sich die Besonderheit, dass mit zunehmendem evasivem Bewältigungsverhalten vor allen Dingen die Faktoren als belastend beschrieben wurden, die genuin für den Sportunterricht sind. Einerseits wird dadurch das besondere Belastungspotenzial des Faches bestätig, andererseits wird erneut deutlich,

\footnotetext{
${ }^{8}$ Die z.B. durch eine Minderbewertung des Stellenwertes des Faches, grundsätzlichen Motivationsprobleme, die schon zu Berufsbeginn vorlagen oder aber durch ein Resignieren aufgrund wiederholter Misserfolge im Unterrichtsalltag bedingt sind.

${ }^{9}$ "Sind mir die Folgen des Ergebnisses wichtig genug?"
} 
dass man sich für den Beruf und das Fach tatsächlich aufgrund der Liebe bzw. Affinität zu Bewegung, Spiel und Sport entscheiden sollte und nicht aufgrund mangelnder Alternativen einen Beruf bzw. ein Fach wählt, bei dem geringe Widerstände bei angenehmen Arbeitsbedingungen erwartet werden.

\subsection{Ausblick, Desiderata, Fazit}

Insgesamt zeigte sich in dieser Arbeit, dass der Sportunterricht ein Unterrichtsfach ist, das besonders hohe Anforderungen an die Sportlehrkräfte stellt. Bereits Schaarschmidt (2005c) stellte eine besorgniserregende Verteilung auf die einzelnen arbeitsbezogenen Verhaltens- und Erlebensmuster fest, die in dieser Arbeit Bestätigung findet. $42,8 \%$ der Lehrkräfte ${ }^{10}$ in der vorliegenden Stichprobe gehören dem Schonungsmuster an, 12,9\% dem Muster Anstrengung und nur 14,3\% dem Gesundheitsmuster. ${ }^{11}$ Besorgniserregend ist der Wert von 30,1\% der Lehrkräfte, ${ }^{12}$ die ins Burnout-Muster fallen. Im Vergleich zu den bereits durchgeführten Erhebungen unter Lehrern (Schaarschmidt, 2005a; 2005c) zeigt sich eine deutliche Überrepräsentation der Muster Schonung und Burnout und eine Unterrepräsentation des Gesundheitsmusters. Einerseits besteht durch diese Musterverteilung die Gefahr, dass der Erziehungs- und Bildungsauftrag verfehlt wird, andererseits eine erhebliche Gefährdung für die seelische und körperliche Gesundheit der Lehrkräfte. Diese besondere Situation erfordert auf der einen Seite, dass Bemühungen unternommen werden, die Zahl der „Richtigen“ im Beruf zu erhöhen und die „Falschen“ frühzeitig zu beraten. Zu Überprüfen wäre, ob die Lehrkräfte vielleicht mit Sport nur das falsche Fach unterrichten, denn wie zuvor bereits dargelegt, werden vor allem die sportunterrichtsspezifischen Belastungen von den evasiv Unterrichtenden als bedeutsamer wahrgenommen, wohlmöglich verhalten Sie sich in ihrem anderen Fach ganz anders!

Während Rauin (2007) nachweisen konnte, dass das fehlende Engagement und Kompetenz zu Beginn der Berufskarriere mit verstärktem Belastungserleben korreliert und dies in der Aussage, dass diejenigen die ausgebrannt sind, nie gebrannt haben, konkretisierte, wurden auch in dieser Studie bezüglich des evasiven Verhaltens ähnliche Erkenntnisse gewonnen. Die Auswahl an Personal nimmt im Lehrerberuf eine entscheidende Weichenposition ein, die auch bezüglich des Verhaltens im Konfliktfall vorbestimmenden Einfluss hat. Tritt die Liebe zum Kind oder aber die Liebe zum Sport hinter extrinsische Gründe bei der Berufswahl zurück, sinkt die Bereitschaft oder aber es fehlen wichtige Voraussetzungen und Ressourcen für einen angemessenen Umgang mit

\footnotetext{
${ }^{10}$ Bei den männlichen Sportlehrkräften liegt dieser Wert sogar bei 46,1\%.

${ }^{11}$ Nur 9,6\% der weiblichen Sportlehrkräfte werden dem Gesundheitsmuster zugeordnet.

${ }^{12}$ Bei den weiblichen Sportlehrkräften liegt dieser Wert sogar bei 37,7\%.
} 
auftretenden Konflikten und Problemen. In Kapitel 2.1 wurde beschrieben, dass aufgrund wirtschaftlicher Schwankungen eine Tendenz zu einem verstärkten Sicherheitsdenken von Jugendlichen festzustellen ist und auch in der Studie des Instituts für Demoskopie Allensbach stellte sich heraus, dass für Lehrer, die in den ersten fünf Berufsjahren sind, der Beamtenstatus eine deutlich größere Bedeutung hat als für Lehrkräfte, die schon länger im Beruf sind (Süßlin, 2012). Für die Zukunft des Lehrerberufs bedeutet dies, dass einerseits das generelle Image verbessert werden muss, damit dem Beruf nicht wie bisher viele gute Kräfte verloren gehen (Denzler, Fiechter \& Wolter, 2005), gleichzeitig aber auch vor der Berufswahl sowie zu Beginn des Studiums deutlicher kommuniziert werden muss, dass einige Vorrausetzungen essenziell sind und der Beruf als Notlösungs-Alternative gänzlich ungeeignet ist. Ob Eignungsprüfungen in betrieblichen Assessment-Centern oder aber speziell entwickelte Fragebogen geeignete Verfahren der Personalauswahl darstellten ist stark infrage zu stellen, denn die wahre Eignung ist nur im Praxisalltag selbst festzustellen. Die verstärkte Integration von Praxisanteilen zu Beginn der Ausbildung von Lehrern stellt einen ersten wichtigen Schritt für eine sinnvollere Personalauswahl dar. Zusätzlich müssen jedoch die „Falschen“ auch tatsächlich zu einem frühen Zeitpunkt beraten werden, damit sie sich später nicht in der in Kapitel 2.1 beschriebenen beruflichen Einbahnstraßensituation wiederfinden. Sowohl im eigenen Lehramtsstudium als auch im Referendariat konnte ich die Erfahrung machen, dass Kommilitonen und Kollegen, trotz großer, teils unüberwindbarer Problemen von Professoren, Dozenten und Ausbildern bis zum Ende der jeweiligen Ausbildungsstufe "durchgewunken" wurden und dann in Prüfungen scheiterten oder aber im Berufsalltag stark überfordert sind. Diese negativen Erfahrungen und Lebenssituationen sollten den Schülern, vor allem aber den Studenten, Referendaren und Lehrern erspart werden.

Auch die Aus-, Fort- und Weiterbildung ist ein Feld, dem durchaus noch Entwicklungspotenzial innewohnt. Wie erst jüngst in der Studie vom Institut für Demoskopie Allensbach wieder bestätigt wurde, fühlen sich die meisten Lehrer auf ihre beruflichen Aufgaben nicht richtig vorbereitet, jeder fünfte Berufseinsteiger spricht sogar von einem wahren Praxisschock (Süßlin, 2012). Auch in der durchgeführten Untersuchung zeigte sich Entwicklungspotenzial unter anderem durch die Ablehnung bzw. Unwirksamkeit von Fortbildungsveranstaltungen.

Seitdem die Popularität von Studien über Belastungen und Burnout im Lehrerberuf gestiegen ist, haben diese Themen zwar auch Einzug in Aus- und Weiterbildungsveranstaltungen erhalten, erfordern jedoch aufgrund ihrer hohen Bedeutung höhere Beachtung als bisher. Wie bereits in Kapitel 8.1 angeführt gilt es angehende Lehrkräfte, aber 
auch bereits im Berufsleben stehende Lehrer für das Thema Bewältigungshandeln zu sensibilisieren, zu beraten und vielleicht sogar individuell zu schulen. Neben der Vermittlung allgemeiner Informationen müsste ein System installiert werden, bei dem Lehrkräften Handlungs- und Kommunikationsstrategien vermittelt werden, aus denen jede Lehrkraft für sich in einem selbstreflexiven Prozess einen zu den Fähigkeiten und Fertigkeiten passenden professionellen und angemessenen Umgang mit Belastungen im Alltag entwickeln kann.

Bezogen auf den entwickelten Fragebogen EESU sowie dem theoretischen Konzept bzw. den Begründungszusammenhängen des evasiven Unterrichtens konnte durch die Arbeit wichtige Grundlagenarbeit geleistet werden. Wie im Ergebnisteil deutlich wurde, bietet eine Untersuchung, die im Querschnittdesign angelegt ist, zwar eine Vielzahl an Daten, die jedoch an vielen Stellen die Frage nach Ursache und Wirkung aufwerfen und unbeantwortet lassen. So konnte beispielsweise gezeigt werden, dass sich Personen mit ausgeprägtem evasiven Verhaltenspotenzial deutlich seltener fortbilden als invasiv unterrichtende Lehrkräfte. Anhand der Daten konnte jedoch nicht belegt werden, ob diese Personen verstärkt evasiv handeln, weil sie die eigene Fort- und Weiterbildung vernachlässigt haben oder aber ob aufgrund des evasiven Verhaltens und dem dadurch gesteigerten Belastungsempfinden Fort- und Weiterbildungsmaßnahmen als zusätzliche Belastungsquelle aus dem Alltag eliminiert wurden. Untersuchungsmethodisch wäre zur Beantwortung solcher Fragestellungen eine im Längsschnitt angelegte Erhebung eine angemessene Vorgehensweise. Nach einer ersten Erhebung und der Auswahl von Personen mit verschiedenen Ausprägungen des evasiven Verhaltens könnte durch regelmäßiges Nacherheben ein Verlaufsprofil des evasiven Verhaltens erstellt werden, in dem Begründungszusammenhänge deutlich werden. Des Weiteren könnte im Rahmen eines solchen Vorgehens eine genauere Beschreibung der Genese des evasiven Verhaltens erstellt werden: Handelt es sich um eine Disposition, d.h. eine überdauernde Persönlichkeitseigenschaft, mit der Lehrer bereits in den Beruf einsteigen oder aber können Parallelen zu Phasen- bzw. Prozessmodellen, wie sie z.B. für den Verlauf eines Burnouts existieren (siehe Kap. 4.9.5.1), festgestellt werden. Eine weitere Forschungsmöglichkeit wäre anhand von Interviews, basierend auf der Datenlage dieser Erhebung, eine Konkretisierung der Begründungsmuster für das evasive Bewältigungsverhalten aufzudecken und auf diese Art die hinter dem Verhalten stehenden Denkstrukturen zu identifizieren.

Wie bereits zu Beginn von Kapitel 8.1 angedeutet, würde eine Einordnung der Ausprägung des evasiven Verhaltens im Vergleich zu anderen Schulfächern weitere wichtige Erkenntnisse in diesem noch frischen Forschungsbereich liefern. Aus den Vergleichsda- 
ten könnten unter Beachtung der Besonderheiten des Faches (siehe Kap. 2.3) weitere Entwicklungsprozesse angestoßen werden, die neben der Ausbildung von Sportlehrern, vor allem aber auch die Gestaltung der Infrastruktur des Arbeitsalltags betrifft. Bestrebungen in Form von Arbeitszeitmodellen, bei denen Lehrer von Korrekturfächern entlastet und Sportlehrkräfte zusätzlich belastet werden, sind aufgrund des immer wieder empirisch bestätigten ausgeprägten Belastungspotenzials zu modifizieren. Wie sich in der Interviewstudie von Kastrup \& Mylius (2012) zeigte, bewerten die befragten Sportlehrer die Bestrebungen die Korrekturfächer zu entlasten zwar als richtig, eine zusätzliche Belastung der Sportfachlehrer hingegen als nicht bewältigbar. Neben der direkten Belastung durch die Mehrarbeit fühlen sich die Lehrkräfte zudem aufgrund der fehlenden Anerkennung ihrer tagtäglichen Leistung herabgewürdigt, was zu Rückzugserscheinungen wie z.B. in Form der in Kapitel 4.9.5.1 beschriebenen inneren Kündigung führen kann.

Eine weitere Möglichkeit, das evasive Verhalten genauer zu beschreiben, wäre eine ReAnalyse bezüglich der beiden in Kapitel 6 identifizierten Faktoren. Während die in dieser Untersuchung betrachteten drei Facetten des evasiven Unterrichtens sich mehr oder weniger auf die verschiedenen Interaktionspartner bzw. die Umsetzung des Unterrichts beziehen und bei der Erstellung eines differenzierten Profils der Lehrkraft hilfreich sind, werden durch die beiden Faktoren eher die Begründungsmuster für die Evasion in den Blick genommen, die auf der einen Seite mit Prävention, Handlungssicherheit und Handlungsaufschub sowie auf der anderen Seite die Bereitschaft, herausfordernde Situationen anzunehmen, recht breit angesiedelt sind.

Abschließend kann man zusammenfassen, dass es sich bei dem Beruf des Sportlehrers entgegen der Vorurteile um eine Beschäftigung mit einem hohen Belastungspotenzial handelt, das durchaus Einfluss auf die Gesundheit haben kann. Nicht zuletzt bestätigen Korrelationen zwischen der krankheitsbedingten Absentismusrate und der wöchentlichen Sportstundenzahl bzw. dem prozentualen Anteil des Sportunterrichts ${ }^{13}$ an der Gesamtlehrverpflichtung diese Aussage ${ }^{14}$ und machen die Bedeutsamkeit eines angemessenen Bewältigungsverhalten in dem besonderen Lehrsettings des Sportunterrichts unabdingbar.

\footnotetext{
${ }^{13}$ Unterteilt man den prozentualen Anteil des Sportunterrichts in zwei Gruppen, ergeben sich für diejenigen, die bis maximal 50\% ihrer Lehrverpflichtung im Sportunterricht erfüllen eine signifikant geringere Absentismusrate $(p<.01)$. Die Sportlehrkräfte die bis maximal 50\% ihrer Gesamtstundenzahl das Fach Sport unterrichten fehlten im letzten Jahr an 4,29 Tagen (SD=6.18) krankheitsbedingt, während Sportlehrkräfte, die mehr als 50\% Sportunterrichtsanteil aufweisen an 6,60 Tagen (SD=11.03) im Jahr krankheitsbedingt dem Unterricht fernblieben. Diese Mittelwertdifferenz entspricht einem kleinen Effekt von $d=0.26$.

${ }^{14}$ Zwischen der krankheitsbedingten Absentismusrate und der Gesamtlehrverpflichtung besteht hingegen keine Korrelation.
} 
Insgesamt liefern die Angaben, die durch einen Fragebogen der überwiegend Fragen im geschlossenen Format enthält, bezüglich eines so komplexen Phänomens wie es das evasive Verhalten zweifellos darstellt, ein begrenztes, aber grundlegendes Bild. Es zeigte sich, nicht zuletzt anhand der recht hohen Rücklaufquote sowie dem ausgeprägten Interesse an einem individuellen Feedback bzw. dem Kurzergebnisbericht, dass die Thematik eine hohe Relevanz für die Sportlehrkräfte aufweist und vielleicht auch schon erste Reflexionsprozesse des eigenen Handelns angestoßen wurden. 


\section{Literatur}

Abele, A. E. \& Candova, A. (2007). Prädiktoren des Belastungserlebens im Lehrerberuf. Befunde einer 4-jährigen Längsschnittstudie. Zeitschrift für pädagogische Psychologie, 21 (2), 107-118.

Adrion, K. \& Schneider, K. (1979). Von Beruf Lehrer. Möglichkeiten der Selbstverwirklichung im konfliktreichen Alltag. Freiburg: Herder.

Aldwin, C. M., \& Revenson, T. A. (1987). Does coping help? A reexamination of the relation between coping and mental health. Journal of Personality and social Psychology, 53, 337-348.

Allmer, H. (1982). Zur psychischen Beanspruchung des Lehrers im Sportunterricht. In: H. Allmer \& J. Bielefeld (Hrsg.), Sportlehrerverhalten (S. 27-46). Schorndorf: Hofmann.

Allmer, H. \& Bielefeld, J. (Hrsg.), (1982). Sportlehrerverhalten. Schorndorf: Hofmann.

Altenberger, H., Erdnüss, S., Fröbus, R., Höss-Jelten, C., Oesterhelt, V., Siglreitmaier, F., Stefl, A. (2005). Augsburger Studie zum Schulsport in Bayern. Ein Beitrag zur Qualitätssicherung und Schulsportentwicklung. Donauwörth: Auer.

Altrichter, H., Gather Thurler, M. \& Heinrich, M. (2005). Editorial. Journal für Schulentwicklung, 9 (2), 4-9.

Anderson, C. R. (1974). Locus of control, coping behaviors, and performance in a stress setting: A longitudinal study. Journal of Applied Psychology, 62 (4), 446-451.

Arminger, G. (1979). Faktorenanalyse. Stuttgart: Teubner Studienskripten.

Arnhardt, G., Hofmann, F. \& Reinert, G.-B. (2000). Der Lehrer. Bilder und Vorbilder. Donauwörth: Auer.

Aschebrock, H. \& Stibbe, G. (2008). Standards, Kerncurricula und schuleigene Lehrpläne. Sportpädagogik, 57 (3), 4-13.

Atkinson, J. W. (1957). Motivational determinants of risk-taking behavior. Psychological Review, 64, 359-372.

Atkinson, J. W., Bastian, J. R., Earl, R. W. \& Litwin, G.H. (1960). The Achievement Motive, Goal Setting And Propability Pereferences. Journal of Abnormal and Social Psychology, 60 (1), 27-36.

Balster, K. (1990). Darum ist Lehrerfortbildung notwendig! Sportpraxis, 31 (1), 22. 
Balz, E. (2008). Welche Standards für den Schulsport? Eine Präzisierung sportpädagogischer Ansprüche. Sportpädagogik, 57 (3), 14-18.

Balz, E. \& Kuhlmann, D. (2004). Sportpädagogik. Ein Lehrbuch in 14 Lektionen. Aachen: Meyer \& Meyer.

Bandura, A. (1977). Self-efficacy: Toward a unifying theory of behavioral change. Psychological Review, 84 (2), 191-215.

Bandura, A. (1979). Lernen am Modell. Stuttgart: Klett.

Barth, A.- R. (1997). Burnout bei Lehrern. Göttingen: Hogrefe.

Bastick, T. (2000). Why teacher trainees choose the teaching profession: Comparing trainees in metropolitan and developing countries. Internationale Zeitschrift für Erziehungswissenschaft, 46 (3/4), 343-349.

Bastian, J. \& Combe, A. (2003). Angriffe auf den Lehrerberuf. Pädagogik, 55 (3), 6-10.

Bauer, J. (2004). Die Freiburger Schulstudie. Zugriff am 24.03 .2009 unter http://www.psychotherapie-prof-bauer.de/.

Bauer, J. (2008). Lob der Schule. Sieben Perspektiven für Schüler, Lehrer und Eltern. München: Heyne.

Baumert, J., Cortina, K. S. \& Leschinsky, A. (2005). Grundlegende Entwicklungen und Strukturprobleme im allgemein bildenden Schulwesen. In: K. S. Cortina, J. Baumert, A. Leschinsky, K. U. Mayer \& L. Trommer (Hrsg.), Das Bildungswesen der Bundesrepublik Deutschland. Strukturen und Entwicklungen im Überblick (S. 52147). Reinbek bei Hamburg: Rowolth.

Baumert, J. \& Kunter, M. (2006). Stichwort: Professionelle Kompetenz von Lehrkräften. Zeitschrift für Erziehungswissenschaft, 9 (4), 469-520.

Baumert, J. (2009, 30. April). Gute Lehre bitte! Die Lehrerausbildung an Universitäten muss gestärkt werden. Die Zeit, S. 72.

Behrens-Tönnies, U. \& Tönnies, S. (1986). Die Bedeutung des Kollegiums bei psychosozialer Belastung und Stress von Lehrern. In: H. Heyse (Hrsg.), Erziehung in der Schule - Eine Herausforderung für die Schulpsychologie. (S. 146-152). Bonn: Deutscher Psychologen Verlag.

Belz, C. \& Schmitt, K. (2008, Mai). Belastende Situationen im Sportunterricht. Vortrag auf dem Bundeskongress des Deutschen Sportlehrerverbandes (DSLV), Köln.

Berndt, J. ,Busch, D. \& Schönwälder, H.-G. (Hrsg.), (1988). Schulstreß - Schülerstreß Elternstreß. Bremen: Universität Bremen. 
Berufsverband Deutscher Psychologinnen und Psychologen (2008). Psychische Gesundheit am Arbeitsplatz in Deutschland. Zugriff am 25.04.2008 unter $\quad$ http://www.bdp-verband.org/aktuell/2008/bericht/BDPGesundheitsbericht-2008.pdf.

Blömeke, S. (2005). Das Lehrerbild in den Printmedien. Inhaltsanalyse von "Spiegel“ und „Focus“-Berichten seit 1990. Deutsche Schule, 97, 24-39.

Böhm, W. (1989). Das Bild des Lehrers im Wandel. In: H. Gröschel (Hrsg.), Die Bedeutung der Lehrerpersönlichkeit für Erziehung und Unterricht (S. 9-22). München: Ehrenwirth.

Böhm-Kasper, O. (2004). Schulische Belastung und Beanspruchung. Münster: Waxmann.

Borges, M. A., Roth, A., Nichols, G. T. \& Nichols, B. S. (1980). Effects of gender, locus of control, and self-esteem on estimates of college grades. Psychological Reports, 47, 831-837.

Bortz, J. (2005). Statistik für Human- und Sozialwissenschaftler. Heidelberg: Springer.

Bosse, D. (2004a). Umgang mit Heterogenität in der gymnasialen Oberstufe. In D. Bosse (Hrsg), Unterricht der Schülerinnen und Schüler herausfordert (S. 177-189). Bad Heilbrunn: Klinkhardt.

Bosse, D. (Hrsg.). (2004b). Unterricht, der Schülerinnen und Schüler herausfordert. Bad Heilbrunn: Klinkhardt.

Bräutigam, M. (2003). Sportdidaktik. Ein Lehrbuch in 12 Lektionen. Aachen: Meyer \& Meyer.

Brinkmann, R. D \& Stapf, K. H. (2005). Innere Kündigung. Wenn der Job zur Fassade wird. München: C. H. Beck.

Brosius, F. (2004). SPSS 12. Bonn: Mitp-Verlag.

Brühwiler, C., Spychiger, M. (1997). Subjektive Begründungen für die Wahl des Lehrberufes. Beiträge zur Lehrerbildung, 15 (1), 49-58.

Bühner, M. (2004). Einführung in die Test- und Fragebogenkonstruktion. München: Pearson Studium.

Bundesanstalt für Arbeitsschutz und Arbeitsmedizin (2009). DIN Norm EN ISO 1075. Zugriff am 19.12.2009 unter http://www.baua.de/de/Themen-von-AZ/Psychische-Fehlbelastung-Stress/ISO10075/FAQ/02/FAQ-2.html? nnn=true. 
Burisch, M. (2006). Das Burnout-Syndrom. Theorie der inneren Erschöpfung. Heidelberg: Springer.

Buski, W., Benson, T. \& Sikorski, J. F. (2005). The call to teach. Journal of Social and Clinical Psychology, 24 (1), 111-122.

Byrne, B. M. (1999). The nomological network of teacher burnout: A literature review and empirically validated Model. In: R. Vandenberghe \& A. M. Huberman (Hrsg.), Understanding and preventing teacher burnout. A sourcebook of international research and practice (S. 15-37). Cambridge: Cambridge University Press.

Carver, C. S., Scheier, M. F. \& Weintraub, J. K. (1989). Assessing coping strategies. A theoretically based approach. Journal of Personality and Social Psychology, 56 (2), 267-283.

Cattell, R. B. (1966). The Scree test for the numbers of factors. Multivariate Behavioral Research, 1, 245-276.

Cherniss, C. (1980). Staff Burnout. Job stress in the human services. Beverly Hills: Sage.

Chodoff, P. (1970). The german concentration camp as a psychological stress. Archieves of general psychiatry, 22, 78-87.

Christ, O., Wagner, U. \& van Dick, R. (2004). Belastung und Beanspruchung bei Lehrerinnen und Lehrern in der Ausbildung. In : A. Hillert \& R. Schmitz (Hrsg.), Psychosomatische Erkrankungen bei Lehrerinnen und Lehrern (S. 113-119). Stuttgart: Schattauer.

Cohen, J. (1988). Statistical power analysis fort he behavioral sciences. Hillsdale: Erlbaum.

Cohen, J. (1992). A Power Primer. Quantitative methods in psychology, 112 (1), 155159.

Cooper, C. L. \& Dewe, P. (2007). Stress: A Brief History from the 1950s to Richard Lazarus. In: A. Monat, R. S. Lazarus \& G. Reevy (Eds.), The Praeger Handbook on Stress and Coping (S. 7-32). Westport, Connecticut: Praeger.

Cortina, K.S., Baumert, J., Leschinsky, A., Mayer, K.U. \& Trommer, L. (Hrsg.), (2005). Das Bildungswesen in der Bundesrepublik Deutschland. Reinbek bei Hamburg: Rowohlt.

Czerwenka, K. (1996). Belastungen im Lehrerberuf und ihre Bewältigung. Bildung und Erziehung, 49 (3), 295-315. 
Dalbert, C. (1999). Die Ungewissheitstoleranzskala: Skaleneigenschaften und Validierungsbefunde. Hallesche Berichte zur Pädagogischen Psychologie 1. Halle: Martin-Luther-Universität.

Dallermassen, K. (1986). Schwierige Unterrichtssituationen für Leibeserzieher. Leibesübungen - Leibeserziehung, 40 (3), 67-71.

Dann, H.-D. \& Lechner, T. (2001). Berufswahlmotive Nürnberger Lehramtsstudierender. Mitteilungen der Erziehungswissenschaftlichen Fakultät. Universität Erlangen-Nürnberg, 1-3.

Demerouti, E., Bakker, A. B., Nachreiner, F. \& Schaufeli, W.B. (2001). The job demandsressources model of Burnout. Journal of Applied Psychology, 86, 499-512.

Demerouti, E., Bakker, A.B., Vardakou, I. \& Kantas, A. (2003). The convergent validity of two burnout instruments: A multitrait-multimethod analysis. European Journal of Psychological Assessment, 19, 12-23.

Demerouti, E., \& Nachreiner, F. (1999, Oktober). Bumout as an outcome of job demands, job resources and specific short-term consequences of strain. (Paper presented at the Fifth European Conference on Organizational Psychology and Health Care). Gent, Belgium.

Demmer, M. (2003). Vom faulen Sack zum armen Schwein...? Oder: Kann die geplante OECD-Lehrerstudie die Lehrerinnen und Lehrer bei der Professionalisierung unterstützen? Pädagogik, 55 (3), 20-24.

Denzler, S., Fiechter, U. \& Wolter, C. (2005). Die Lehrkräfte von morgen. Eine Untersuchung der Bestimmungsfaktoren des Berufswunsches bei bernischen Gymnasiasten. Zeitschrift für Erziehungswissenschaften, 8 (4), 502-520.

Deutscher Bundestag (Hrsg.), (2009). Das Grundgesetz der Bundesrepublik Deutschland. Berlin: Referat Öffentlichkeitsarbeit.

Deutscher Sportbund (Hrsg.), (2006). DSB-SPRINT-Studie. Eine Untersuchung zum Schulsport in Deutschland. Aachen: Meyer \& Meyer.

De Vet, H.C.W., Adèr, H.J., Terwee, C.B. \& Pouwer, F. (2005). Are factor analytical techniques used appropriately in the validation of health status questionnaires? A systematic review on the quality of factor analysis of the SF-36. Quality of Life Research, 14, 1203-1218.

Dordel, S. (2000). Kindheit heute: Veränderte Lebensbedingungen = reduzierte motorische Leistungsfähigkeit. Sportunterricht, 49 (11), 341-349. 
Dorsemagen, C., Lacroix, P. \& Krause, A. (2007). Arbeitszeit an Schulen: Welches Modell passt in unsere Zeit. Kriterien zur Gestaltung schulischer Arbeitsbedingungen. In: M. Rothland (Hrsg.), Belastung und Beanspruchung im Lehrerberuf. Modelle, Befunde, Interventionen (S. 227-247).Wiesbaden: Verlag für Sozialwissenschaften.

dvs, DSLV, DOSB \& FSW (2008). Memorandum zum Schulsport. Entwurfsfassung vom 19. Mai 2008. Zugriff am: 28.10.2008 unter http://www.dslv.de/memoData/ downloads/memorandum schulsport 200805 19.pdf.

Elbe, A.-M., Wenhold, F. \& Müller, D. (2005). Zur Reliabilität und Validität der Achievement Motives Scale-Sports. Zeitschrift für Sportpsychologie, 12 (2), 57-68.

Elliot, A. J. \& Church, M. A. (1997). A hierarchical model of approach and avoidance achievement motivation. Journal of personality and social psychology, 72 (1), 218-232.

Elliot, A. J. \& McGregor, H. A. (2001). A $2 \times 2$ achievement goal framework. Journal of personality and social psychology, 80 (3), 501-519.

Endler, N. S. \& Parker, J. D. A. (1990). Coping Inventory for Stressful Situations (CISS). Toronto: Multi Health Systems Inc.

Endler, N. S. \& Parker, J. D. A. (1994). Assessment Of Multidimensional Coping: Task, Emotion and Avoidance Strategies. Psychological Assessment, 6 (1), 50-60.

Engeser, S. H. (2004). Lernmotivation und volitionale Handlungssteuerung: Eine Längsschnittsuntersuchung beim Statistik Lernen im Psychologiestudium. Unveröffentlichte Dissertation. Universität Potsdam.

Enzmann, D. \& Kleiber, D. (1989). Helfer-Leiden: Stress und Burnout in psychosozialen Berufen. Heidelberg: Asanger.

Eurydice (2002). Der Lehrerberuf in Europa: Profil, Tendenzen und Anliegen. Bericht II: Angebot und Nachfrage. Allgemein Bildender Sekundarbereich I. Brüssel: Eurydice.

Eurydice (2003). Der Lehrerberuf in Europa: Profil, Tendenzen und Anliegen. Bericht III: Beschäftigungsbedingungen und Gehälter. Brüssel: Eurydice.

Evans, H. (1993). The choice of teaching as a career. Social and Economic Studies, 42 (2/3), 225-242. 
Fabrigar, L. R., Wegener, D. T., MacCallum, R. C., \& Strahan, E. J. (1999). Evaluating the Use of Exploratory Factor Analysis in Psychological Research. Psychological Methods, 4 (3), 272-299.

Farber, B. A. (1991). „Burnout“ bei Lehrern: Annahmen, Mythen, Probleme. In: E. Terhart (Hrsg.), Unterrichten als Beruf. Neuere amerikanische und englische Arbeiten zur Berufskultur von Lehrern und Lehrerinnen (S. 217-230). Köln: Böhlau.

Feindt, A., Klaffke, T., Röbe, E., Rothland, M., Terhart, E. \& Tillmann, K.-J. (Hrsg.), (2010). Friedrich-Jahresheft 2010. Seelze: Friedrich-Verlag.

Feuß, H. (1998). Angst - (k)ein Thema für Sportlehrer?! Lehrhilfen für den Sportunterricht, 47 (12), 187-192.

Field, A. (2005). Discovering Statistics Using SPSS. London: Sage.

Firley-Lorenz, M. (1994). Sportlehrerinnen in der Schule - ein kritischer Beitrag zu einem vernachlässigten Thema. Sportunterricht, 43, 148-157.

Floyd, F. J. \& Widaman, K. F. (1995). Factor Analysis in the Development and Refinement in Clinical Assessment Instruments. Psychological Assessment, 7 (3), 286299.

Folkman, S., Lazarus, R. S., Dunkel-Schetter, C., DeLongis, A. \& Gruen, R. J. (1986). Dynamics of a stressful encounter: Cognitive appraisal, coping, and encounter outcomes. Journal of Personality and Social Psychology, 50, 992-1003.

Freudenberger, H. (1974). Staff Burn-Out. Journal of Social Issues, 30 (1), 159-165.

Friedel, A. \& Dalbert, C. (2003). Belastung und Bewältigung bei Grundschullehrerinnen. Die Auswirkungen einer Versetzung an eine Förderstufe und der Einfluss der Ungewissheitstoleranz. Zeitschrift für pädagogische Psychologie, 17 (1), 55-68.

Frommel, H. (2006). Belastung und Beanspruchung von Sportlehrer/-innen in der Schule. Teil 2: Was Sportlehrkräfte leisten. Sportunterricht, 55 (8), 242-245.

Funke-Wienecke, J. (2007). Grundlagen der Bewegungs- und Sportdidaktik. Hohengehren: Schneider Verlag.

Gehmacher, E. (1980). Die Schule im Spannungsfeld von Schülern, Eltern und Lehrern. Wien: Österreichischer Bundesverlag Jugend und Volk.

Gerlach, E., Kussin, U., Brandl-Bredenbeck, H. P. \& Brettschneider, W.-D. (2006). In: Deutscher Sportbund (Hrsg.), DSB-SPRINT-Studie. Eine Untersuchung zum Schulsport in Deutschland (S. 115-152). Aachen: Meyer \& Meyer 
GEW-Sportkommission (2005). Gegen die Ausweitung der Arbeitszeit! Für die Gleichwertigkeit der Fächer! Zugriff am 17.06.2009 unter http://www.gewsportkommission.de/arbeitsbelastungen04.html.

Giesecke, H. (2001). Was Lehrer leisten. Portrait eines schwierigen Berufs. Weinheim: Juventa.

Ginzberg, E., Ginsburg, S., Axelrad, S. \& Herma, J. C. (1951). Occupational Choice. New York: Columbia University Press.

Gjesme, T. \& Nygard, R. (1970). Achievement-related motives: Theoretical considerations and construction of a measuring instrument. Unpublished Manuscript, University of Oslo.

Glöckel, H. (2000). Klassen führen - Konflikte bewältigen. Bad Heilbrunn: Klinkhardt.

Gold, A. \& Rauin, U. (2007). Lehrerberuf: Warum Studierende oft die falsche Wahl treffen. Forschung Frankfurt, (3), 83-87.

Goldstein, M. J. (1959). The relationship between coping and avoiding behavior and response to fear-arousing propaganda. Journal of Abnormal and Social Psychology, 58 (2), 233-251.

Gordon, J. E. (1957). Interpersonal predictions of repressors and sensitizers. Journal of Personality, 25 (6), 686-698.

Gorsuch, R. L. (1997). Exploratory Factor Analysis. It's role in item analysis. Journal of Personality Assessment, 68 (3), 532-560.

Göttert, R. \& Kuhl, J. (1980). LM-Fragebogen: Deutsche Übersetzung der AMS-Scale von Gjesme und Nygard. Unveröffentlichtes Manuskript, Ruhr Universität Bochum.

Grimm, E. \& Rieger, U. (1999). Das Lehrerbild in Heinrich Spoerls Die Feuerzangenbowle. In: H. - U. Grunder (Hrsg.), Der Kerl ist verrückt! Das Bild des Lehrers und der Lehrerin in der Literatur und der Pädagogik (S. 63-73). Zürich: Pestalozzianum.

Gröbe, R. (2006). Belastung und Beanspruchung von Sportlehrer/innen in der Schule. Teil 3: Belastungen und Beanspruchungen, die beim Kompetenzerwerb, bei der Erhaltung und beim Nachweis, dem Unterrichten, auftreten. Sportunterricht, 55 (10), 304-307.

Gröschel, H. (1980). Die Bedeutung der Lehrerpersönlichkeit für Erziehung und Unterricht. München: Ehrenwirth.

Größing, S. (2007). Einführung in die Sportdidaktik. Wiebelsheim: Limpert. 
Grunder,H.-U. (Hrsg.), (1999a). Der Kerl ist verrückt! Das Bild des Lehrers und der Lehrerin in der Literatur und der Pädagogik. Zürich: Pestalozzianum.

Grunder. H.-U. (1999b). Der Lehrer als Hungerleider und andere Lehrerbilder. In: H.-U. Grunder (Hrsg.), Der Kerl ist verrückt! Das Bild des Lehrers und der Lehrerin in der Literatur und der Pädagogik (S. 160-167). Zürich: Pestalozzianum.

Grupe, O. (2000). Sporterziehung und Schulsportkultur. Sportunterricht, 49 (1), 14-19.

Grupe, O. \& Krüger, M. (Hrsg.), (1997). Einführung in die Sportpädagogik. Schorndorf: Hofmann.

Gudjons, H. (Hrsg.), (1993). Entlastung im Lehrerberuf. Hamburg: Bergmann \& Helbig.

Gudjons, H. (2007). Beruf: LehrerIn. Wandlungen - Widersprüche - Wunschbilder. Pädagogik, 59 (9), 6-10.

Günzel, W. \& Laging, R. (Hrsg.), (1999). Neues Taschenbuch des Sportunterrichts. Band 1. Grundlagen und pädagogische Orientierungen. Hohengehren: Schneider.

Gutte, R. (1994). Lehrer-Ein Beruf auf dem Prüfstand. Reinbek bei Hamburg: Rowohlt.

Häbler, H. \& Kunz, A. (1985). Qualität der Arbeit und Verkürzung der Arbeitszeit in Schule und Hochschule. München: IMU-Institut.

Häcker, T. \& Zopfi, S. (2006). Wer soll Lehrer oder Lehrerin werden? bbaktuell, 180.

Harter, S. (1981). A model of mastery motivation in children: Individual differences and developmental changes. In: W. A. Collings (Ed.), Aspects of the development of competence: The Minnesota symposia on child psychology (S. 215-255). Hillsdale, NJ: Erlbaum.

Hartig, J., Frey, A. \& Jude, N. (2007). Validität. In: H. Mossbrugger \& A. Kelava (Hrsg.), Testtheorie und Fragebogenkonstruktion (S. 136-163). Heidelberg: Springer.

Hasselhorn, M. \& Gold, A. (2006). Pädagogische Psychologie. Stuttgart: Kohlhammer.

Heckhausen, H. \& Rheinberg, F. (1980). Lernmotivation im Unterricht, erneut betrachtet. Unterrichtswissenschaft, 8, 7-47.

Heim, R. \& Gerlach, E. (1998). Burnout - Auch ein Thema im Sportlehrerberuf? Körpererziehung, 10, 330-337.

Heim, R. \& Klimek, G. (1999). Arbeitsbelastungen im Lehrerberuf. Entwicklung eines Instruments zur Erfassung fachunterrichtlicher Stressoren. Psychologie und Sport, 6 (2), 35-45.

Heine, E. (1981). Kochrezepte zum Lehrerverhalten. Sportpädagogik, 5 (6), 24-30. 
Helmke, A. (2003). Unterrichtsqualität erfassen, bewerten, verbessern. Seelze: Kallmeyer.

Helsper, W. (1996). Antinomien des Lehrerhandelns in modernisierten pädagogischen Kulturen. Paradoxe Verwendungsweisen von Autonomie und Selbstverantwortlichkeit. In: A. Combe \& W. Helsper (Hrsg.), Pädagogische Professionalität. Untersuchungen zum Typus pädagogischen Handelns (S. 521-569). Frankfurt am Main: Suhrkamp.

Helsper, W. (2000). Antinomien des Lehrerhandelns und die Bedeutung der Fallrekonstruktion - Überlegungen zu einer Professionalisierung im Rahmen universitärer Lehrerbildung. In: E. Cloer, D. Klika \& H. Kunert (Hrsg.), Welche Lehrer braucht das Land? Notwendige und mögliche Reformen der Lehrerbildung (S. 142-177). Weinheim: Juventa.

Helsper, W. \& Böhme, J. (Hrsg.), (2004). Handbuch der Schulforschung. Wiesbaden: VS Verlag für Sozialwissenschaften.

Herrlitz, H.-G., Hopf, W., Titze, H. \& Cloer, E. (2005). Deutsche Schulgeschichte von 1800 bis zur Gegenwart. Eine Einführung. Weinheim: Juventa.

Herzog, S. (2007). Beanspruchung und Bewältigung im Lehrerberuf. Münster: Waxmann.

Hesse, H. (1972). Unterm Rad. Frankfurt am Main: Suhrkamp.

Hessischer Landtag (2005). Hessisches Schulgesetz in der Fassung vom 14. Juni 2005 (GVBI. I S. 441). Zugriff am 25.07.2008 unter http://www.hessen.de ¿irj/HKM Internet?cid=c1f7ee3ac049d51fa14df6f30a1b156a.

Hessisches Kultusministerium (2010). Lehrplan Sport. Gymnasialer Bildungsgang. Jahrgangsstufen 5G bis 9G und gymnasiale Oberstufe. Zugriff am 12.04.2011 unter http://www.kultusministerium.hessen.de/iri/HKM Internet?cid=fed0f69a1 3c4fa385a6d2a571d7d7e51.

Heyse, H. (Hrsg.), (1986). Erziehung in der Schule - Eine Herausforderung für die Schulpsychologie. Bonn: Deutscher Psychologen Verlag.

Heyse, H. (2004). Lehrergesundheit - eine gemeinsame Aufgabe von Lehrkräften, Kollegien und Schulleitungen. Lehren und Lernen, 31 (8/9), 39-53.

Heyse, H. (2008). Psychische Gesundheit im Lehrerberuf. In: Berufsverband Deutscher Psychologinnen und Psychologen (Hrsg.), Psychische Gesundheit am Arbeitsplatz in Deutschland. Zugriff am 25.04.2008 unter http://www.bdpverband.org/aktuell/2008/bericht/BDP-Gesundheitsbericht-2008.pdf. 
Hillert, A. \& Schmitz, R. (2004). Psychosomatische Erkrankungen bei Lehrerinnen und Lehrern. Stuttgart: Schattauer.

Horn, J. L. (1965). A rationale and test for the number of factors in factor analysis. Psychometrika 30 (2), 179-185.

Huberman, M. (1989). The Professional Life Cyle of Teachers. Teachers College Record, 91, 31-57.

Huberman, M. (1991). Der berufliche Lebenszyklus von Lehrern: Ergebnisse einer empirischen Untersuchung. In: E. Terhart (Hrsg.), Unterrichten als Beruf. Neuere amerikanische und englische Arbeiten zur Berufskultur von Lehrern und Lehrerinnen (S. 249-267). Köln: Böhlau.

Huberman, M. \& Vandenberghe, R. (1999). Understanding and preventing teacher burnout. Cambridge: Cambridge University Press.

Hübner, H. \& Pfitzner, M. (2001). Das schulsportliche Unfallgeschehen in NordrheinWestfalen. Münster: LIT.

Hübner, H. \& Pfitzner, M. (2003). Schulsportunfälle im Freistaat Bayern. Ergebnisse zum Unfallgeschehen im Schuljahr 2002/03. Münster: LIT.

Hübner, P. \& Werle, M. (1997). Arbeitszeit und Arbeitsbelastung Berliner Lehrerinnen und Lehrer. In: S. Buchen, U. Carle, P. Döbrich, H.-D. Hoyer \& H.-G. Schönwälder (Hrsg.), Jahrbuch für Lehrerforschung (S. 203-226). Weinheim: Juventa.

Hundeloh, H. (2005). Sportunfall - ein Stressor im Arbeitsalltag von Sportlehrkräften. Sportunterricht, 54 (2), 142-145.

IKK-Bundesverband (Hrsg.), (2007). Der IKK-Impuls. Berufsreport Lehrerinnen und Lehrer. Zugriff am 3.12.2008 unter: http://www.ikk.de/ikk/generator/ikk/fuerarbeitgeber/download-service/109308.pdf.

Institut für Demoskopie Allensbach (2008). Berufsprestigeskala 2008. Zugriff am 16.10.2009 unter http://www.ifd-allensbach.de/news/prd 0802.html.

Janke, W. \& Erdmann, G. (1997). Stressverarbeitungsfragebogen (SFV 120) nach W. Janke, G. Erdmann, K. W. Kallus und W. Boucsein. Kurzbeschreibung und grundlegende Kennwerte. Göttingen: Hogrefe.

Janke, W. \& Erdmann, G. (2002). SVF 78. Eine Kurzform des Stressverarbeitungsfragebogens SVF 120. Göttingen: Hogrefe.

Janke, W., Erdmann, G. \& Kallus, W. (1984). Stressverarbeitungsfragebogen (SVF) nach W. Janke, G. Erdmann und W. Boucsein. Handanweisung. Göttingen: Hogrefe. 
Janssen, J. \& Laatz, W. (2007). Statistische Datenanalyse mit SPSS für Windows. Heidelberg: Springer

Jehle, P., Schmitz, E. (2007) Innere Kündigung und vorzeitige Pensionierung von Lehrpersonen. In: M. Rothland (Hrsg.), Belastung und Beanspruchung im Lehrerberuf. Modelle, Befunde, Interventionen (S. 160-184). Wiesbaden: Verlag für Sozialwissenschaften.

Jerusalem, M. (1990). Persönliche Ressourcen, Vulnerabilität und Stresserleben. Göttingen: Hogrefe.

Kahn, R. L., Wolfe, D. M. Quinn, R. P., Snoek, J. D. \& Rosenthal, R. A. (1964), (Hrsg.), Organizational stress: Studies in role conflict and ambiguity. New York: Wiley.

Kaiser, A. (2006). Wie Kinder sich ihre Zukunft vorstellen. Einblicke, Forschungsmagazin der Carl von Ossietzky Universität Oldenburg, 43, 10-11.

Kälin, W. (1995). Deutsche 24-Item Kurzform des „Coping Inventory for Stressful Situations" (CISS) von N. S. Endler \& J. D. A. Parker. Basierend auf der Übersetzung von N. Semmer, F. Tschan \& V. Schade (unveröffentlichter Fragebogen). Bern: Universität, Institut für Psychologie.

Kapustin, P. (1979). Problem- und Konfliktsituationen im Sportunterricht. In: J. Recla \& H. Recla (Hrsg.), Sportunterricht im Aufriss. Bad Homburg: Limpert.

Kastrup, V., Dornseifer, A. \& Kleindienst-Cachay, C. (2008). Belastungswahrnehmung von Sportlehrkräften verschiedener Schulformen. Eine empirische Studie zur Belastungswahrnehmung von Sportlehrkräften in Abhängigkeit von Schulform, erteilter Sportstundenzahl, Alter und Geschlecht. Sportunterricht, 57 (10), 307313.

Kastrup, V. \& Mylius, E. (2012). Arbeitszeitmodelle im Lehrerberuf - Und wie stehen Sportlehrkräfte dazu? Sportunterricht, 61 (3), 66-71.

Kastrup, V. \& Neumann, P. (2008). Von Rückzug bis Ausstieg. Wie ältere Sportlehrkräfte auf die Belastungen des Faches reagieren. Auszüge aus einer Interviewstudie. Sportpädagogik, 57 (4/5), 84-87.

Kenyon, G. S. (1968). Six scales for assessing attitude towards physical activity. Research Quarterly, 39, 566-574.

Kern, B. (1986). Neuerungen im Schulalltag - Initiativen entfalten und aushandeln statt Vorschriften studieren und klagen! Die Deutsche Schule, 78 (2), 212-222. 
Kiel, E., Pollak, G., Eberle, T., Braune, A. \& Schliessleder, M. (2007). Lehrer werden ist nicht schwer...?! Die problematische Studienwahl von Lehramtsstudierenden. Pädagogik, 59 (9), 11-15.

Kirchem, A. (2002). Sportlehrer an allgemeinbildenden Schulen. Einstellungen und Selbsteinschätzungen zum Schulsport. In: G. Friedrich (Hrsg.), Sportpädagogische Forschung. Konzepte - Ergebnisse - Perspektiven. Jahrestagung der dvsSektion Sportpädagogik vom 14.-16.6.2001 in Münster (S. 268-273). Hamburg: Czwalina.

Kleinke, C. L. (2007). What Does It Mean to Cope. In: A. Monat, R. S. Lazarus \& G. Reevy (Eds.), The Praeger Handbook on Stress and Coping (Vol. 2) (S. 289-308). Westport, Connecticut: Praeger

Kline, P. (1999). The handbook of psychological testing. London: Routledge.

Klinger, E. (1975). Consequences of commitment to and disengagement from incentives. Psychological Review, 82, 1-25.

Klusmann, U., Kunter, M., Trautwein, U. \& Baumert, J. (2006). Lehrerbelastung und Unterrichtsqualität aus der Perspektive von Lehrenden und Lernenden. Zeitschrift für Pädagogische Psychologie, 20 (3), 161-173.

Kniveton, B. H. (2004). The influences and motivations on which students base their choice of career. Research in Education, 72, 47-59.

Kolb, M. \& Wolters, P. (2000). Evasives Unterrichten - Beobachtungen und Interpretationen zu aktuellen Inhalten des Sportunterrichts. In: E. Balz \& P. Neumann (Hrsg.), Anspruch und Wirklichkeit des Sports in Schule und Verein (S. 209-221). Hamburg: Czwalina.

König, S. (2004). Belastungen für Lehrkräfte im Sportunterricht. Ein Beitrag zur empirisch-analytischen Sportunterrichtsforschung. Sportwissenschaft, 34 (2), 152165.

König, S. (2008). Unterrichtsbelastungen für Sportlehrerinnen und Sportlehrer. Eine Analyse unter besonderer Berücksichtigung der Ausbildung. Sportunterricht, 57 (9), 289-293.

Konrad, F.-M. (2007). Geschichte der Schule. Von der Antike bis zur Gegenwart. München: C. H. Beck. 
Köppe, G. (1999). Lehrerhandeln im Schulsport und Schulleben. Vom Bild des Turnlehrers zum Sportlehrer(innen)handeln. In: W. Günzel \& R. Laging (Hrsg.), Neues Taschenbuch des Sportunterrichts. Band 1. Grundlagen und pädagogische Orientierungen. Hohengehren: Schneider.

Kramis-Aebischer, K. (1996). Stress, Belastungen und Belastungsverarbeitung im Lehrberuf. Bern: Paul Haupt.

Krampen, G. (1991). Fragebogen zu Kompetenz- und Kontrollüberzeugungen (FKK). Handanweisung. Göttingen: Hogrefe.

Krapp, A. \& Weidenmann, B. (Hrsg.), (2001). Pädagogische Psychologie. Weinheim: Beltz.

Krause, A. (2002). Psychische Belastungen im Unterricht - Ein aufgabenbezogener Untersuchungsansatz. Analyse der Tätigkeit von Lehrerinnen und Lehrern. Unveröffentlichte Dissertation. Universität Flensburg.

Krause, A. (2003). Lehrerbelastungsforschung - Erweiterung durch ein handlungspsychologisches Belastungskonzept. Zeitschrift für Pädagogik, 49 (2), 254-273.

Krause, A. \& Dorsemagen, C. (2007). Ergebnisse der Lehrerbelastungsforschung: Orientierung im Forschungsdschungel. In: M. Rothland (Hrsg.), Belastung und Beanspruchung im Lehrerberuf. Modelle, Befunden, Interventionen (S. 52-81). Wiesbaden: Verlag für Sozialwissenschaften.

Krüger, M. (2005). Einführung in die Geschichte der Leibeserziehung und des Sports. Teil 2: Leibeserziehung im 19. Jahrhundert. Turnen für Vaterland. Schorndorf: Hofmann.

Ksienzyk, B. \& Schaarschmidt, U. (2005). Beanspruchung und schulische Arbeitsbedingungen. In: U. Schaarschmidt (Hrsg.), Halbtagsjobber? Psychische Gesundheit im Lehrerberuf - Analyse eines veränderungsbedürftigen Zustandes (S. 72-87). Weinheim: Beltz.

Kugelmann, C. \& Klupsch-Sahlmann, R. (2000). Sportlehrerinnen und Sportlehrer heute und morgen. Sportpädagogik, 24 (1), 4-12.

Kühn, L. (2005). Das Lehrerhasserbuch. München: Knaur.

Kühne, S. (2006). Das soziale Rekrutierungsfeld der Lehrer. Zeitschrift für Erziehungswissenschaft, 9 (4), 617-631. 
Kultusministerkonferenz (2000). Aufgaben von Lehrerinnen und Lehrern heute - Fachleute für das Lernen. Gemeinsame Erklärung des Präsidenten der Kultusministerkonferenz und des Vorsitzenden der Bildungs- und Lehrergewerkschaften sowie ihrer Spitzenorganisationen. Beschluss der Kultusministerkonferenz vom 5.10.2000. Zugriff am 12.07.2008 unter http://www.kmk.org/fileadmin/ veroeffentlichungen beschluesse/2000/2000 10 05-Bremer-Erkl-Lehrerbildung.pdf.

Kultusministerkonferenz (2005). Standards für die Lehrerbildung. Beschluss der Kultusministerkonferenz vom 16.12.2004. Zugriff am 11.09.2008 unter www.kmk.org/doc/beschl/standards lehrerbildung.pdf.

Kurz, D. (2000). Die pädagogische Grundlegung des Schulsports in NordrheinWestfalen. In: Landesinstitut für Schule und Weiterbildung (Hrsg.), Erziehender Schulsport. Pädagogische Grundlagen der Curriculumrevision in NordrheinWestfalen (S. 9-55). Bönen: Kettler.

Kurz, D. (2008). Der Auftrag des Schulsports. Sportunterricht, 57 (7), 211-218.

Kyriacou, C. \& Sutcliffe, J. (1978). A model of teachers stress. Educational Studies, 4, 1-6.

Laging, R. \& Klupsch-Sahlmann (2001). Schulen in Bewegung. Sportpädagogik, 25 (2), 4-10.

Laging, R. (2004a). Sportlehrer. In: P. Röthig \& R. Prohl (Hrsg.), Sportwissenschaftliches Lexikon (S. 518f.). Schorndorf: Hofmann.

Laging, R. (2004b). Sportunterricht. In: P. Röthig \& R. Prohl (Hrsg.), Sportwissenschaftliches Lexikon (S. 546f.). Schorndorf: Hofmann.

Landert, Charles (1999). Die Arbeitszeit der Lehrpersonen in der Deutschschweiz. Ergebnisse einer einjährigen Erhebung bei 2500 Lehrerinnen und Lehrer verschiedener Schulstufen und Kantone. Zürich: Dachverband Schweizer Lehrerinnen und Lehrer.

Landesinstitut für Schule und Weiterbildung (Hrsg.), (2000). Erziehender Schulsport. Pädagogische Grundlagen der Curriculumrevision in Nordrhein-Westfalen: Bönen: Kettler.

Lange, A. \& Melches, A. (1999). Vorurteile gegenüber der Lehrkraft im Roman. Unterm Rad (Hermann Hesse) und Der Club der toten Dichter (N. H. Kleinbaum). In: H.G. Grunder (Hrsg.), Der Kerl ist verrückt! Das Bild des Lehrers und der Lehrerin in der Literatur und der Pädagogik (S. 17-23). Zürich: Pestalozzianum. 
Lang, J. W. B. \& Fries, S. (2006). A Revised 10-Item Version of the Achievement Motives Scale. European Journal of Psychological Assessment, 22 (3), 216-224.

Lange, J. (1981). Der Sportlehrer im Schulalltag. Sportpädagogik, 5 (6), 10-17.

Lange, H. \& Sinning, S. (2008). Handbuch Sportdidaktik. Balingen: Spitta.

Laux, L. (1983). Psychologische Stresskonzeptionen. In: H. Thomae (Hrsg.), Theorien und Formen der Motivation. Enzyklopädie der Psychologie: Themenbereich C, Serie IV. Motivation und Emotion. Band 1 (S. 453-535). Göttingen: Hogrefe.

Lazarus, R. S. (1966). Psychological stress and the coping process. New York: McGrawHill.

Lazarus, R. S. \& Folkman, S. (1984). Stress, Appraisal and Coping. New York: Springer.

Lazarus, R. S. \& Folkman, S. (1987). Transactional theory and research on emotion and coping. European Journal of Personality, 1 (1), 141-169.

Lazarus, R. S. \& Launier R. (1978). Stress-related transactions between person and environment. In: L. A. Pervin \& M. Lewis (Eds.), Perspectives in interactional psychology (S. 287-327). New York: Plenum.

Leuschner, G. (1979). Belastungsbedingungen im Lehrerberuf. Zeitschrift für gesamte Hygiene, 25, 18-21.

Levenson, H. (1972). Distinctions within the concept of internal-external control: Development of a new scale. Proceedings of the 80th Annual Convention of the American Psychological Association, Volume 7, 261-262.

Lipowsky, F. (2010). Empirische Befunde zur Wirksamkeit von Lehrerfortbildungen. In: F. H. Müller, A. Eichenberger, M. Lüders \& J. Mayr (Hrsg.), Lehrerinnen und Lehrer lernen - Konzepte und Befunde zur Lehrerfortbildung (S. 51-72). Münster: Waxmann.

Malanowsky, J. R. \& Wood, P. H. (1984). Burnout and self-actualization in public school teachers. The Journal of Psychology, 117, 23-26.

Maslach, C. \& Jackson, S. E. (1981). The measurement of experienced burnout. Journal of Occupational Behaviour, 2, 99-113.

Maslach, C. \& Jackson, S. E. (1986). Maslach Burnout Inventory. Manual. Palo Alto: Consulting Psychologists Press.

Matzig, G. (2005). Vati, ich will Spießer werden! Zugriff am 17.04.2008 ter http://www.sueddeutsche.de/kultur/frauen-und-maenner-neuesteermittlungen-im-krisengebiet-vati-ich-will-spiesser-werden-1.428076. 
Mclntyre, T. C. (1983). The effects of class size on perceptions of burnout by special education teachers. Mental Retardation and Learning Disability Bulletin, 11, 142-145.

Meier, E. (1983). Blinde Flecken in der Lehrerausbildung. Die Deutsche Schule, 5 (4), 357-367.

Meusel, H. (1989). Wenn Sportlehrer/innen älter werden - "Graue Welle" erreicht Sportunterricht. Sportpraxis, 30 (3), 15-17.

Miethling, W. D. (1989). Belastungssituationen und ihre Bewältigung bei Sportlehrern. SportPraxis, 30 (1), 3-5.

Miethling, W. D. (2000). Zwischen Traum und Albtraum. Zur beruflichen Entwicklung von Sportlehrern: Ergebnisse einer Längsschnittstudie. Sportpädagogik, 24 (1), 41-47.

Miethling, W. D. (2001). Lust und Frust von Sportlehrern- Biographische Entwicklungen im Schulalltag. In: R. Zimmer (Hrsg.), Erziehen als Aufgabe. Sportpädagogische Reflexionen. Festschrift anlässlich der Emeritierung von Meinhardt Volkamer (S. 162-178). Schorndorf: Hofmann.

Miethling, W. D. \& Brand, R. (2004). Stressoren im Sportunterricht und psychische Widerstandressourcen bei Sportlehrerinnen und Sportlehrern in der ersten Berufsphase- Spectrum der Sportwissenschaften, 16 (1), 48-67.

Miethling, W. D. \& Sohnsmeyer, J. (2009, Juni). Belastungsmuster von Sportlehrerlnnen. Vortrag auf der dvs-Jahrestagung - Sektion Sportpädagogik in Hildesheim.

Monat, A., Lazarus, R. S. \& Reevy, G. (Eds.), (2007). The Praeger Handbook on Stress and Coping. Westport, Connecticut: Praeger.

Moosbrugger H. \& Kelava, A. (2007). Testtheorie und Fragebogenkonstruktion. Heidelberg: Springer.

Moosbrugger H. \& Schermelleh-Engel (2007). Exploratorische (EFA) und Konfirmatorische Faktorenanalyse. In: H. Mossbrugger \& A. Kelava (Hrsg.), Testtheorie und Fragebogenkonstruktion (S. 307-324). Heidelberg: Springer.

Müller-Limmroth, W. (1980). Arbeitszeit-Arbeitsbelastung im Lehrerberuf. Frankfurt am Main: GEW.

Mummert \& Partner (1999). Untersuchung zur Ermittlung, Bewertung und Bemessung der Arbeitszeit der Lehrerinnen und Lehrer im Land Nordrhein-Westfalen. Zusammenfassung . Hamburg: Autor. 
Neumann, P (2003). Zeitkonflikte im Sportunterricht. Sportpädagogik, 27 (6), 39-41.

Niedersächsischer Landtag (1998). Niedersächsisches Schulgesetz (NSchG), in der Fassung vom 3. März 1998 (Nds.GVBI. S. 137). Zugriff am 25.07.2008 unter http://www.schure.de/nschg/nschg/nschg.htm.

Niedersächsisches Kultusministerium (2007). Kerncurriculum für die Schulformen des Sekundarbereichs 1. Schuljahrgänge 5-10. Zugriff am 24.03.2008 unter http://www.nibis.de/nli1/gohrgs/kerncurricula nibis/kc 2007/kc07 sekl/kc sek1 sport 07 nib.pdf.

Nusko, G. (1986). Coping. Bewältigungsstrategien des Ich im Zusammenhang von Kontext-, Person- und Situationsmerkmalen. Frankfurt am Main: Peter Lang.

O'Connor, B. P. (2000). SPSS and SAS programs for determining the number of components using parallel analysis and Velicer's MAP test. Behavior Research Methods, Instrumentation, and Computers, 32, 396-402.

Oesterreich, C. (2008). Arbeitsbedingungen von Sportlehrkräften. Sportunterricht, 57 (9), 282-288.

Oesterreich, C. \& Heim, R. (2006). Der Sportunterricht in der Wahrnehmung der Lehrer. In: Deutscher Sportbund (Hrsg.), DSB-SPRINT-Studie. Eine Untersuchung zur Situation des Schulsports in Deutschland (S. 153-180). Aachen: Meyer \& Meyer.

Oesterreich, D. (1987). Die Berufswahlentscheidung von jungen Lehrern. Studien und Berichte 46. Berlin: Max-Planck-Institut für Bildungsforschung.

Parker, J. D. A., N. S. Endler \& R. M. Bagby (1993). If It changes, It Might Be Unstable: Examaining the Factor Structure of the Ways of Coping Questionnaire. Psychological Assessment, 5 (3), 361-368.

Paulsen, F. (1885). Geschichte des gelehrten Unterrichts auf den deutschen Schulen und Universitäten vom Ausgang des Mittelalters bis zur Gegenwart. Leipzig: von Veit \& Comp.

Pearlin, L. \& Schooler, C. (1978). The structure of coping. Journal of health and social behavior, 42, 704-715.

Perrez, M. \& Reicherts, M. (1992). Stress, Coping, and Health. A Situation-Behavior Approach Theory, Methods, Applications. Seattle: Hogrefe \& Huber.

Pines, A. M., Aronson, E. \& Kafry, D. (1985). Ausgebrannt. Vom Überdruss zur Selbstentfaltung. Stuttgart: Klett-Cotta. 
Potthoff, A. \& Wolf, A.(1976). Pädagogische Konflikte in der Schule. Analysen und Hilfen für die Praxis. Freiburg: Herder.

Prohl, R. (2006). Grundriss der Sportpädagogik. Wiebelsheim: Limpert.

Prohl, R. (2008). Erziehung mit dem Ziel der Bildung: Der Doppelauftrag des Sportunterricht. In: H. Lange \& S. Sinning (Hrsg.), Handbuch Sportdidaktik (S. 40-53). Balingen: Spitta.

Pühse, U. (1994). „Miteinander“ als sportliche Sinnperspektive. Ein Plädoyer für einen erziehenden Sportunterricht. In: U. Pühse (Hrsg.), Soziales Handeln im Sport und Sportunterricht (S. 125-145). Schorndorf: Hofmann.

Pühse, U. (1999). Soziale Lernprozesse im Sportunterricht. In: W. Günzel \& R. Laging (Hrsg.), Neues Taschenbuch des Sportunterrichts. Band 1. Grundlagen und pädagogische Orientierungen (S. 215-234). Hohengehren: Schneider.

Rasch, B., Friese, M., Hofmann, W. \& Naumann, E. (2006). Quantitative Methoden (Band 1). Heidelberg. Springer Medizin Verlag.

Rauin, U. (2007). Im Studium wenig engagiert - im Beruf schnell überfordert. Forschung Frankfurt, 03/2008, 60-64.

Rauin, U. (2010). Wer wird eigentlich Lehrer? Über Eignung und Berufswahl. In: A. Feindt, T. Klaffke, E. Röbe, M. Rothland, E. Terhart \& K.-J. Tillmann (Hrsg.), Friedrich Jahresheft 2010 (S. 8-10). Seelze: Friedrich-Verlag.

Recla J. \& Recla, H. (Hrsg.), (1979). Sportunterricht im Aufriss. Bad Homburg: Limpert.

Rehfuss, W. D. (1995). Bildungs-Not. Hat die Pädagogik versagt. Stuttgart: Klett-Cotta.

Reicherts, M. (1988). Diagnostik der Belastungsverarbeitung. Neue Zugänge zu StressBewältigungs-Prozessen. Bern: Huber.

Rheinberg, F. (2004). Motivationsdiagnostik. Göttingen, Bern, Toronto, Seattle, Oxford, Prag: Hogrefe.

Rheinberg, F. (2006). Motivation. Stuttgart: W. Kohlhammer.

Rheinberg, F. \& Bromme, R. (2001). Lehrende in Schulen. In: A. Krapp \& B. Weidenmann (Hrsg.), Pädagogische Psychologie (S. 295-332). Weinheim: Beltz.

Rheinpfalz (2003). Gleichgültigkeit im Schulsport am Pranger. Zugriff am 09.12.2008 unter http://djkfussball.dj.funpic.de/sportunterricht/index03-2.html.

Rohnstock, D. (2000). Belastungsschwerpunkte im Sportlehreralltag und Anregungen für gezielte Entlastungen. Sportunterricht, 49 (4), 108-115. 
Rost, D. H. \& Sparfeldt, J. R. (2002). Facetten des schulischen Selbstkonzepts: Ein Verfahren zur Messung des differentiellen Selbstkonzepts schulischer Leistungen und Fähigkeiten (DISK-Gitter). Diagnostica, 48, 130-140.

Roth, G. (2008, 22. November). Mit Bauch und Hirn. In: DIE ZEIT, 48, S. 43.

Röthig, P. \& Prohl, R. (Hrsg.), (2004). Sportwissenschaftliches Lexikon. Schorndorf: Hofmann.

Rothland, M. (2007a). Belastung und Beanspruchung im Lehrerberuf. Zur Einführung in das Studienbuch. In: M. Rothland (Hrsg.), Belastung und Beanspruchung im Lehrerberuf. Modelle, Befunde, Interventionen (S. 7-10). Wiesbaden: Verlag für Sozialwissenschaften.

Rothland, M. (Hrsg.), (2007b). Belastung und Beanspruchung im Lehrerberuf. Modelle, Befunde, Interventionen. Wiesbaden: Verlag für Sozialwissenschaften.

Rothland, M. \& Terhart, E. (2007). Beruf: Lehrer - Arbeitsplatz: Schule. Charakteristika der Arbeitstätigkeit und Bedingungen der Berufssituation. In: M. Rothland (Hrsg.), Belastung und Beanspruchung im Lehrerberuf. Modelle, Befunde, Interventionen (S. 11-31). Wiesbaden: VS Verlag für Sozialwissenschaften.

Rotter, J. B. \& Mulry R. C. (1965). Internal Versus External Control Of Reinforcement And Decision Time. Journal of Personality and Social Psychology, 2 (4), 598-604.

Rotter, J. B. (1975). Some problems and misconceptions related to the construct of internal versus external control of reinforcement. Journal of Consulting and Clinical Psychology, 43, 56-67.

Rudow, B. (1995). Die Arbeit des Lehrers. Zur Psychologie der Lehrertätigkeit, Lehrerbelastung und Lehrergesundheit. Bern: Huber.

Rüttinger, B. (1977). Konflikt und Konfliktlösung. München: Goldmann.

Sarges, W. (2000). Fragebogen zur Messung der Arbeitsmotivation: „Avem - Arbeitsbezogene Verhaltens- und Erlebensmuster" von U. Schaarschmidt und A. Fischer (1996). Zeitschrift für Arbeits- und Organisationspsychologie, 44, 38-42.

Scarth, J. (1987). Teacher Strategies: A Review and a Critique. British Journal of Sociology of Education, 8, 249-262.

Scarth, J. (1991). Handlungsstrategien von Lehrern: Übersicht und Kritik. In: E. Terhart (Hrsg.), Unterrichten als Beruf. Neuere amerikanische und englische Arbeiten zur Berufskultur von Lehrern und Lehrerinnen (S. 23-40). Köln: Böhlau. 
Schaarschmidt, U. (Hrsg.), (2005a). Halbtagsjobber? Psychische Gesundheit im Lehrerberuf-Analyse eines veränderungsbedürftigen Zustandes. Weinheim: Beltz.

Schaarschmidt, U. (2005b). Die Belastungssituation von Lehrkräften. Was ist dagegen zu tun? Schulmagazin 5 bis 10, 12, 5-8.

Schaarschmidt, U (2005c). Psychische Belastung im Lehrerberuf. Und wie sieht es für Sportlehrkräfte aus? Sportunterricht, 54 (5), 132-140.

Schaarschmidt, U. (2008). Beanspruchungsmuster als Indikatoren psychischer Gesundheit im Lehrerberuf. Sportunterricht, 57 (9), 276-281.

Schaarschmidt, U. \& Fischer, A.W. (1996). AVEM - Arbeitsbezogenes Verhaltens- und Erlebensmuster (Manual). Frankfurt am Main: Swets.

Schaarschmidt, U. \& Fischer, A. W. (2001). Bewältigungsmuster im Beruf: Persönlichkeitsunterschiede in der Auseinandersetzung mit der Arbeitsbelastung. Göttingen: Vandenhoeck \& Ruprecht.

Schaarschmidt, U. \& Herlt, S. (2007). Fit für den Lehrerberuf? Zugriff am 3.12.2008 unter http://www.dbb.de/dbb-beamtenbund-2006/3151 3676.php.

Schaarschmidt, U. \& Kieschke, U. (2006). Arbeits-Bewertungs-Check für Lehrkräfte (ABC-L). Zugriff am 19.03.2009 unter http://www.dbb.de/events/projekte Lpotsdamer-lehrerstudie.html.

Schaarschmidt, U. \& Kieschke, U. (2007). Beanspruchungsmuster im Lehrerberuf. Ergebnisse und Schlussfolgerungen aus der Potsdamer Lehrerstudie. In: M. Rothland (Hrsg.), Belastung und Beanspruchung im Lehreberuf. Modelle, Befunde, Interventionen (S. 81-118). Wiesbaden: Verlag für Sozialwissenschaften.

Schaufeli, W. B. \& Enzmann, D. (1998). The Burnout Companion to Study and Practice: A Critical Analysis. London: Taylor \& Francis.

Scheffel, H. \& Palzkill, B. (1994). Macht und Ohnmacht von Sportlehrerinnen im koedukativen Unterricht. Sportunterricht, 43 (4), 159-166.

Scherer, H.-G. (2008). Zum Gegenstand von Sportunterricht: Bewegung, Spiel und Sport. In: H. Lange \& S. Sinning (Hrsg.), Handbuch Sportdidaktik (S. 24-39). Balingen: Spitta.

Scherler, K. (2004). Sportunterricht auswerten. Eine Unterrichtslehre. Hamburg: Feldhaus. 
Scheunpflug, A. (2005). Job oder Berufung? - Gibt es eine Berufsethik für Lehrkräfte. In: G.-B. von Carlsburg \& M. Heitger (Hrsg.), Der Lehrer - ein (un)möglicher Beruf (S. 57-66). Frankfurt am Main: Peter Lang.

Schilling, S. R., Sparfeldt, J. R., Rost, D. H. \& Nickels, G. (2004). Schulische Selbstkonzepte - zur Validität einer erweiterten Version des Differentiellen Selbstkonzept Gitters (DISK-Gitter). Diagnostica, 51, 21-28.

Schmitz, E. (2004). Burnout: Befunde, Modelle und Grenzen eines populären Konzepts. In: A. Hillert \& E. Schmitz (Hrsg.), Psychosomatische Erkrankungen bei Lehrerinnen und Lehrern (S. 51-68). Stuttgart, New York: Schattauer.

Schmitz, E., Jehle, P. \& Gayler,B. (2004). Innere Kündigung im Lehrerberuf. In: A. Hillert \& E. Schmitz (Hrsg.), Psychosomatische Erkrankungen bei Lehrerinnen und Lehrern (S. 69-81). Stuttgart, New York: Schattauer.

Schmitz, G. (2001). Kann Selbstwirksamkeitserwartung Lehrer vor Burnout schützen? Psychologie in Erziehung und Unterricht, 48, 49-67.

Schmitz, G. \& Schwarzer, R. (1999). Skala Lehrer-Selbstwirksamkeitserwartung. In: R. Schwarzer \& M. Jerusalem (Hrsg.), Skalen zur Erfassung von Lehrer- und Schülermerkmalen. Zugriff am 17.02.2008 unter http://userpage.fuberlin.de/ health/self/skalendoku selbstwirksame schulen.pdf.

Schmitz, G. \& Schwarzer, R. (2000). Selbstwirksamkeitserwartung von Lehrern: Längsschnittbefunde mit einem neuen Instrument. Zeitschrift für Pädagogische Psychologie, 14 (1), 12-25.

Schönwälder, H.-G. (1988). Die Arbeitssituation der Lehrer als Bestimmungsfaktor der Arbeitssituation der Schüler. In J. Berndt, D. Busch \& H.-G. Schönwälder (Hrsg.), Schulstre $\beta$ - Schülerstre $\beta$ - Elternstre $\beta$ (S. 97-130). Bremen: Universität Bremen.

Schönwälder, H.-G., Berndt, J., Ströver, F. \& Tiesler, G. (2003). Belastung und Beanspruchung von Lehrerinnen und Lehrern. Bremerhaven: Wirtschaftsverlag NW.

Schunk, D. (1995). Self-Efficacy, Motivation and Performance. Journal of Applied Sport Psychology, 7, 112-137.

Schwänke, U. (1988): Der Beruf des Lehrers. Professionalisierung und Autonomie im historischen Prozess. Weinheim: Juventa.

Schwarz, G. (1997). Konfliktmanagement. Sechs Grundmodelle der Konfliktlösung. Wiesbaden: Gabler. 
Schwarzer R. \& Jerusalem, M. (Hrsg.), (1999). Skalen zur Erfassung von Lehrer- und Schülermerkmalen. Dokumentation der psychometrischen Verfahren im Rahmen der Wissenschaftlichen Begleitung des Modellversuchs Selbstwirksame Schulen. Zugriff am 17.02.2008 unter http://userpage.fuberlin.de/ health/self/skalendoku selbstwirksame schulen.pdf.

Seegerer, K., Wulsten, A.-R. \& Ulich, E. (1975).Die zeitliche Verteilung von Tätigkeitselementen bei Lehrern an Grund- und Hauptschulen in Nordrhein Westfahlen. Schriftenreihe: Max Träger Stiftung. Forschungsberichte 10. München: Max Traeger Stiftung.

Seligman, M. E. P. (1979). Erlernte Hilflosigkeit. München: Urban \& Schwarzenberg.

Selten, Reinhard (1990). Bounded Rationality. Journal of Institutional and Theoretical Economics, 146, 649-658.

Semmer, N., Tschan, F. \& Schade, V. (1991). Umgang mit Stress. Deutsche Übersetzung des „Coping Inventory for Stressful Situations" (CISS) von N.S. Endler \& J. D. A. Parker (unveröffentlichter Fragebogen). Bern: Universität, Institut für Psychologie.

Siegrist, J. (1991). Contributions of sociology to the prediction of heart disease and their implications for public health. European Journal of Public Health, 1, 10-21.

Sieland, B. \& Nieskens, B.(2001). Wollen Sie Lehrerin oder Lehrer werden? Die Gelbe Mappe Orientierungshilfe für Abiturientinnen und Abiturienten. Zugriff am 4.12.2008 unter http://www.bsieland.de/download-files/Orientierungshilfen \%20fuer\%20 Lehrerinnen\%20und\%20Lehrer.pdf.

Söll, W. (2000). Das Sportartenkonzept in Vergangenheit und Gegenwart. Sportunterricht, 49 (1), 4-8.

Sosnowsky, N. (2007). Burnout - Kritische Diskussion eines vielseitigen Phänomens. In: M. Rothland (Hrsg.), Belastung und Beanspruchung im Lehreberuf. Modelle, Befunde, Interventionen (S. 119-139). Wiesbaden: Verlag für Sozialwissenschaften.

Sosnowsky-Waschek, N. (2010). Burn-Out! Burn-Out? Anmerkungen zu einem fragwürdigen Konstrukt. In: A. Feindt, T. Klaffke, E. Röbe, M. Rothland, E. Terhart \& K.-J. Tillmann (Hrsg.), Friedrich Jahresheft 2010 (S. 76-77). Seelze: FriedrichVerlag.

Spoerl, H. (1933). Die Feuerzangenbowle. Düsseldorf: Droste.

Spranger, E. (1963). Der geborene Erzieher. Heidelberg: Quelle \& Meyer. 
Statistisches Bundesamt (2005). Lehrer gehen durchschnittlich mit 62 Jahren in Pension. Pressemitteilung Nr. 519 vom 9.12.2005.

Statistisches Bundesamt (2007a). Lehrerinnen und Lehrer gehen durchschnittlich mit 62 Jahren in Pension. Pressemitteilung Nr. 104 vom 13.03.2007.

Statistisches Bundesamt (2007b). Aktuelle Fakten zum Tag des Lehrers. Pressemitteilung Nr. 399 vom 04.10.2007.

Steger, M. F. (2006). An illustration of issues in factor extraction and identification of dimensionality in psychological assessment data. Journal of personality assessment, 86 (3), 263-272.

Steiner, G. (2001). Lernen und Wissenserwerb. In: A. Krapp \& B. Weidemann (Hrsg.), Pädagogische Psychologie (S. 137-205). Weinheim: Beltz.

Stevens, J. P. (1992). Applied multivariate statistics fort he social sciences. Hillsdale: Erlbaum.

Stibbe, G. (2000). Vom Sportartenprogramm zum erziehenden Sportunterricht. Zur curricularen Neubesinnung über den Schulsport in Nordrhein-Westfalen. Sportunterricht, 49 (7), 212-219.

Stiegelbauer, S. (1992, April). “Why We Want to Be Teachers." Paper presented at the Annual Meeting of the American Educational Research Association, San Francisco.

Storf, V. (1981). Wenn Schüler aggressiv sind. Sportpädagogik, 5 (6), 41-45.

Struck, P (1994). Neue Lehrer braucht das Land. Ein Plädoyer für eine zeitgemäße Schule. Darmstadt: Wissenschaftliche Buchgesellschaft.

Struck, P. (2007). Lehrer der Zukunft. Vom Pauker zum Coach. Darmstadt: Primus.

Stündl, H. \& Zimmermann, H. (2006). Belastung und Beanspruchung von Sportlehrer/innen in der Schule. Teil 4: Der außerunterrichtliche Schulsport: Ein Arbeitsfeld ohne Grenzen. Sportunterricht, 55 (11), 334-338.

Süßlin, W. (2012). Die Situation in deutschen Schulen aus Sicht von Lehrern und Eltern. In: Institut Allensbach: Lehre(r) in Zeiten der Bildungspanik. Zugriff am: 11.05.2012 unter http://www.vodafone-stiftung.de/scripts/getdata.php?DOW NLOAD=YES\&id $=16556$.

Sust, C. A. \& Lazarus, H. (1997). Auswirkungen von Geräuschen mittlerer Intensität in Schule, Aus- und Weiterbildung. Dortmund: Bundesanstalt für Arbeitsschutz und Arbeitsmedizin. 
Sutherland, S. \& Scherl, D. J. (1970). Patterns of response among victims of rape. American journal of orthopsychiatry, 40, 503-511.

Tenorth, H.-E. (2004). Lehrerarbeit - Strukturprobleme und Wandel der Anforderungen. In: U. Beckmann, H. Brandt \& H. Wagner (Hrsg.), Ein neues Bild vom Lehrerberuf? Pädagogische Professionalität nach Pisa (S. 14-23). Weinheim: Beltz.

Tenorth, H.-E. (2006). Professionalität im Lehrerberuf. Ratlosigkeit der Theorie, gelingende Praxis. Zeitschrift für Erziehungswissenschaft, 9, 580-597.

Terhart, E. (Hrsg.), (1991). Unterrichten als Beruf. Neuere amerikanische und englische Arbeiten zur Berufskultur von Lehrern und Lehrerinnen. Köln: Böhlau.

Terhart, E. (2001). Lehrerberuf und Lehrerbildung. Forschungsbefunde, Problemanalysen, Reformkonzepte. Weinheim: Beltz.

Terhart, E. (2006). Was wissen wir über gute Lehrer? Pädagogik, 58 (5), 42-47.

Thomae, H. (1983a). Motivationsbegriffe und Motivationstheorien. In: H. Thomae (Hrsg.), Enzyklopädie der Psychologie (Themenbereich C, Serie IV Motivation und Emotion, Band Theorien und Formeln der Motivation. Göttingen: Hogrefe.

Thomae, H. (Hrsg.), (1983b). Enzyklopädie der Psychologie. Themenbereich C, Serie IV Motivation und Emotion, Band Theorien und Formeln der Motivation. Göttingen: Hogrefe.

Thomann, C. (2006). Sportlehrer - ein Traumberuf. Sportunterricht, 55 (7). 206-207.

Thurn, S. \& Tillmann, K.-J. (Hrsg.), (1997). Unsere Schule ist ein Haus des Lernens. Das Beispiel der Laborschule Bielefeld. Reinbek bei Hamburg: Rowolth.

Trachsler, E. (1990). Bewegung in der Lehrerfortbildung. Themenentwicklung einer Lehrershaft auf der Grundlage eines Modells aus der Organisationsentwicklung. Forschungsbericht. Zürich: Universität Zürich, Pädagogisches Institut, Fachbereich Pädagogische Psychologie.

Treutlein, G., Janalik, H. \& Hanke, U. (1996). Wie Sportlehrer wahrnehmen, denken, fühlen und handeln. Ein Arbeitsbuch zur Diagnose und Veränderung Ihres sportunterrichtlichen Handelns. Köln: Sport und Buch Strauß.

Tschannen-Moran, M., Woolfolk Hoy, A. \& Hoy, W. K. (1998). Teacher efficacy: its meaning and measure. Review of Educational Research, 68 (2), 202-248.

Ulich, D. (1985). Psychologie die Krisenbewältigung. Eine Längsschnittuntersuchung mit arbeitslosen Lehrern. Weinheim: Beltz. 
Ulich, K. (1996). Beruf Lehrer/in. Arbeitsbelastungen, Beziehungskonflikte, Zufriedenheit. Weinheim: Beltz.

Ulich, K. (2004). Ich will Lehrer/in werden - Eine Untersuchung zu den Berufsmotiven von Studierenden. Weinheim: Beltz.

Urban, W. (1985). Berufszufriedenheit und Berufsbelastung bei österreichen Hauptschullehrern (Forschungsbericht). Wien: Bundesministerium für Unterricht und Kunst.

van Dick, R. (2001). Der AVEM im Lehrerberuf: Eine Validierungsstudie. Zeitschrift für Differentielle und Diagnostische Psychologie, 22, 267-278.

van Dick, R. (2006). Stress und Arbeitszufriedenheit bei Lehrerinnen und Lehrern. Zwischen „Horrorjob" und Erfüllung. Marburg: Tectum.

van Dick, R. \& Stegmann, S. (2007). Belastung, Beanspruchung und Stress im Lehrerberuf - Theorien und Modell. In: M. Rothland (Hrsg.), Belastung und Beanspruchung im Lehrerberuf. Modelle, Befunde, Interventionen (S. 34-51). Wiesbaden: Verlag für Sozialwissenschaften.

Vandenberghe R. \& A. M. Huberman (Hrsg.), (1999). Understanding and preventing teacher burnout. A sourcebook of international research and practice. Cambridge: Cambridge University Press.

Verwoerdt, A. (1972). Psychopathological responses to the stress of physical illness. Adv. Psychosomatic medicine, 8, 119-141.

Volkery, C. (2006). Berliner Rede zur Bildung. Ein neuer Köhler verlangt alte Werte. Zugriff am 18.07.2007 unter http://www.spiegel.de/politik/deutschland $\angle 0,1518,438462,00 \cdot \mathrm{html}$.

Vollstädt, W. (2004). Konsequenzen aus Pisa: Auf dem Weg zu einer neuen Lernkultur. In: D. Bosse (Hrsg), Unterricht der Schülerinnen und Schüler herausfordert (S. 123-138). Bad Heilbrunn: Klinkhardt.

Voltmann-Hummes, I. (2008). Belastungserleben und Selbstwirksamkeitserwartung von Schulsportlehrkräften. Göttingen: Cuvillier.

von Engelhardt, G. (1982). Die pädagogische Arbeit des Lehrers. Paderborn: Schöningh.

von Thadden, E. (1994, 16. Juni). Professor Unrat ade. Was sagen Lehrerinnen und Lehrer über ihren Beruf? Wochenpost, 25, IV-V. 
Wagner-Link, A. (2008). Der Stress. Stressoren erkennen, Belastungen vermeiden, Stress bewältigen. Zugriff am 06.03.2009 unter: http://www.tk-online.de/ tk/broschueren-und-mehr/gesundheitsbroschueren/der-stress/ 49152.

Watt, H. M. G. \& Richardson, P. W. (2007). Motivational factors influencing teaching as a career choice: Development and validation of the FIT-Choice-Scale. The Journal of Experimental Education, 75 (3), 167-202.

Weiner (1993, Februar). Choosing teaching as a carreer. Comparing motivations of Harvard and urban college students. Paper presented to the eastern educational research association. Clearwater, Florida.

Weiss, H. (1944). Die Feuerzangenbowle [Spielfilm]. Berlin: Terra.

Weiß, H. (2004). Wo Sportlehrerinnen und Sportlehrern „der Schuh drückt“. Erfahrungen und Ratschläge aus zehn Jahren Beraterarbeit in Nordrhein-Westfalen (NRW). Sportunterricht, 53 (1), 20-24.

Weiß, S. \& Kiel, E. (2010). Berufswunsch Sportlehrer/in. Sportunterricht, 59 (10), 308311.

Wendt. W. (2001). Belastung von Lehrkräften. Fakten zu Schwerpunkten, Strukturen und Belastungstypen. Eine repräsentative Befragung von Lehrerinnen und Lehrern. Landau: Verlag Empirische Pädagogik.

Wolters, P. (2008). Schwierige Schülerinnen und Schüler. Sportpädagogik, 57 (2), 4-9.

Woolfolk Hoy, A., \& Burke-Spero, R. (2005). Changes in teacher efficacy during the early years of teaching: A Comparison of four measures. Teaching and Teacher Education, 21, 343-356.

Wopp, C. (1999). Lebenswelt, Jugendkulturen und Sport in der Schule. In: W. Günzel \& R. Laging (Hrsg.), Neues Taschenbuch des Sportunterrichts. Band 1. Grundlagen und pädagogische Orientierungen (S. 342-359). Hohengehren: Schneider.

Wulk, J. (1988). Lehrerbelastung. Qualitative und quantitative Aspekte der psychischen und physischen Belastung von Lehrern. Eine arbeitspsychologische Untersuchung an Lehrern beruflicher Schulen. Frankfurt: Peter Lang.

Yong, B. C. S. (1995). Teacher Trainees'motives for entering into teaching career in Brunei Darussalam. Teaching and Teacher Education, 11 (3), 275-280.

Zentrum für Lehrerbildung (2008). Trainingsmodul Psychosoziale Basiskompetenzen im Lehrerberuf. Zugriff am 3.12.2008 unter http://www.uni-kassel.de/zlb/ Trainingsmodul 81028.pdf. 
Zimmer, R. \& Volkamer, M. (1981). Zur Bedeutung des Modelllernens im Sportunterricht. Sportpädagogik, 5 (6), 18-23.

Zimmer, R. (Hrsg.), (2001). Erziehen als Aufgabe. Sportpädagogische Reflexionen. Festschrift anlässlich der Emeritierung von Meinhardt Volkamer. Schorndorf: Hofmann.

Zimmermann, H. (2006). Belastung und Beanspruchung von Sportlehrerinnen und Sportlehrern in der Schule. Ein Beitrag in Fortsetzungen. Sportunterricht, 55 (7), 205.

Zoglowek, H. (2008). Lehrer und Sportunterricht. In: H. Lange \& S. Sinning (Hrsg.), Handbuch Sportdidaktik (S. 117-132). Balingen: Spitta.

Zymek, Bernd (2004): Geschichte der Schule und des Lehrerberufs. In: W. Helsper \& J. Böhme (Hrsg.), Handbuch der Schulforschung (S. 205-240). Wiesbaden: VS Verlag für Sozialwissenschaften. 


\section{Anhang}

I. 1. Fragebogenentwurf (Pilotierung 1)

II. 2. Fragebogenentwurf (Pilotierung 2)

III. Informationsflyer

IV. Fragebogen Erhebungsinstrument evasiver Sportunterricht - EESU (Endversion)

V. Lebenslauf 


\section{1. Fragebogenentwurf (Pilotierung 1)}

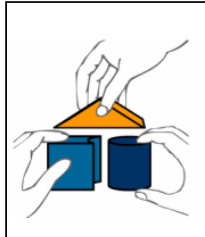

\section{DFG GRADUIERTENKOLLEG 1195 Passungsverhältnisse schulischen Lernens}

Projekt: Belastungen/Stressoren im Sportunterricht und Bewältigungsstrategien

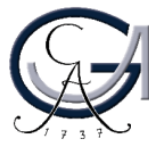

\section{Liebe Sportstudentinnen und Sportstudenten,}

im Rahmen meines Promotionsvorhabens an der Universität Göttingen beschäftige ich mich mit der Bewältigung von alltäglichen Belastungen/Stressoren/Konflikten von Sportlehrerinnen und Sportlehrern. Gegenstand des Vorhabens ist es, aufzuschlüsseln welche Verhaltensmuster Sportlehrerinnen und Sportlehrer ${ }^{1}$ im Umgang mit den stetig zunehmenden an sie gerichteten Ansprüche durch z.B. steigende Schülerzahlen, Erweiterung des Erziehungsauftrages und fehlende Unterrichtsmaterialien zeigen, wie Sie ihre Arbeit erleben und welche Einstellungen sie gegenüber dem Sportunterricht vertreten.

Lehrende im Sportunterricht stehen unter einem permanenten Handlungs- und Orientierungsdruck und sehen sich mit der Anforderung konfrontiert, innerhalb kürzester Zeit vielfältige Situationen einzuschätzen, zu bewerten und durch die Auswahl einer angemessenen Handlungsentscheidung zu bewältigen.

Noch einige wichtige Informationen zum Ausfüllen des Fragebogens:

- Grundsätzlich ist die Teilnahme an der Untersuchung freiwillig, jedoch sind die Ergebnisse umso aussagekräftiger und repräsentativer je mehr Sportlehrer bzw. Studenten sich beteiligen.

- Die Befragung erfolgt absolut anonym. Alle Daten und Angaben werden ausschließlich für wissenschaftliche Zwecke verwendet und im Bezug auf datenschutzrechtliche Bestimmungen streng vertraulich behandelt.

- Das Ausfüllen des Fragebogens sollte insgesamt nicht länger als 15min dauern. Bitte überlege bei den einzelnen Fragen nicht zu lange sondern antworte möglichst spontan.

- Es gibt keine richtigen oder falschen Antworten! Gib ehrliche Antworten und überlege nicht welche Antworten den besten Eindruck entstehen lassen. Wenn du dich nicht für eine bestimmte Antwort entscheiden kannst wähle die am ehesten auf dich zutreffende!

- Die Fragen sind auch für dich als Studenten zu beantworten, bei den Fragen zu „deinem Sportunterricht" beziehe dich auf deine Lehrerfahrungen in der Schule und/oder beantworte perspektivisch für deine spätere Lehrtätigkeit.

${ }^{1}$ Aus Gründen der besseren Lesbarkeit wird im weiteren Verlauf des Fragebogens ausschließlich die männliche Form verwendet (im Fragebogen auch bei Schülerinnen bzw. Schülern), was jedoch stets sinngemäß die weibliche mit einschließt. 


\section{Projekt: Belastungen/Stressoren im Sportunterricht und Bewältigungsstrategien}

- Bitte kreuze das Kästchen mit der für dich zutreffenden Antwort deutlich an. Wenn du etwas falsch angekreuzt hast streiche das Kreuz einfach durch und mache an der richtigen Stelle ein neues Kreuz und umkreise dieses (siehe Beispiel).

\section{Geschlecht (bitte ankreuzen) \\ weiblich $\otimes$ männlich}

- wenn du eine Rückmeldung hinsichtlich des für dich errechneten „Arbeitsbezogenen Verhaltens- und Erlebensmuster" (Gesundheit, Anstrengung, Burn-Out, Schonung) haben möchtest trage einen Code (Zahlen und/oder Buchstaben) in das dafür vorgesehen Feld auf der ersten Seite des Fragebogens ein (optional).

Es würde uns sehr freuen, wenn wir mit deiner Teilnahme rechnen könnten, da nur durch Forschungsvorhaben wie diesem die Situation der Sportlehrer besser eingeschätzt werden und Maßnahmen ergriffen bzw. entwickelt werden können, die Entlastung und Verbesserungen des Alltags ermöglichen. Je mehr Sportlehrer bzw. Sportstudenten sich an dieser Untersuchung beteiligen, desto freier von Verzerrungen und Ungenauigkeiten fällt das Ergebnis aus und desto gezielter und erfolgreicher können Verbesserungsvorschläge erarbeitet werden.

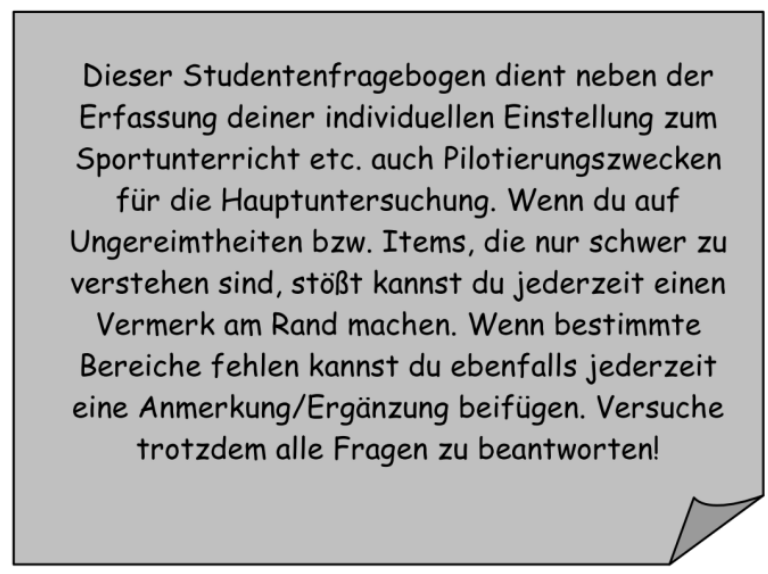

Mit herzlichem Dank für deine Mitarbeit und freundlichem Gruß

\section{Christian Belz (Projektverantwortlicher) ${ }^{2}$}

Juniorprofessorin Dr. Katja Schmitt (Institut für Sportwissenschaft Göttingen)

Prof. Dr. Marcus Hasselhorn (Georg-Elias-Müller-Institut für Psychologie Göttingen)

${ }^{2}$ Bei Rückfragen wenden Sie sich bitte an Christian Belz, Institut für Sportwissenschaft Göttingen, Sprangerweg 2, 37075 Göttingen, Tel. 0551-395656, E-Mail: ChBelz@sport.uni-goettingen.de 
1. Geschlecht (bitte ankreuzen)

\begin{tabular}{|l|l|l|l|}
\hline & weiblich & männlich
\end{tabular}

2. Alter:

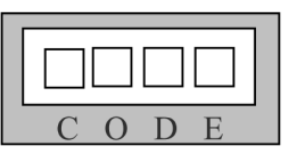

3. Fühltest du dich bei der Studienwahl für den Lehrerberuf berufen oder gab es andere Gründe (sicherer Arbeitsplatz, fehlende Alternativen, Freunde...) diesen Weg anzustreben? $\square$ fühlte mich berufen $\square$ andere Gründe

4. Welches Fach bzw. welche Fächer studierst du außer Sport?

5. Welches Lehramt studierst du bzw. strebst du an?

\begin{tabular}{|l|l|l|l|l|l|l|l|}
\hline$\square$ & Grundschule & $\square$ & Haupt-/Realschule & $\square$ & Gymnasium & $\square$ & Berufsschule \\
\hline
\end{tabular}

6. Seit wie vielen Semestern studierst du Sport? Semester

7. Hast du schon eigenverantwortlichen Sportunterricht in der Schule erteilt?

\begin{tabular}{|l|l|l|l|}
\hline$\square$ & ja & $\square$ & nein (weiter bei 11.) \\
\hline
\end{tabular}

8. Es macht mir Spaß das Fach Sport zu unterrichten!

\begin{tabular}{|l|l|l|l|l|l|l|l|l|l|}
\hline$\square$ & nie & $\square$ & selten & $\square$ & ab und zu & $\square$ & oft & $\square$ & immer \\
\hline
\end{tabular}

9. Der Stellenwert des Sportunterrichts im Vergleich zu anderen Fächern an meiner Schule (z.B. im Praktikum) ist...

\begin{tabular}{|l|l|l|l|l|l|l|l|l|l|}
\hline$\square$ & deutlich niedriger & $\square$ & eher niedriger & $\square$ & gleich & $\square$ & eher höher & $\square$ & deutlich höher \\
\hline
\end{tabular}

10. Wie würdest du die Atmosphäre in deinem Sportkollegium beschreiben?

\begin{tabular}{|l|l|l|l|l|l|l|l|l|l|}
\hline$\square$ & schlecht & $\square$ & eher schlecht & $\square$ & mittel & $\square$ & eher gut & $\square$ & gut \\
\hline
\end{tabular}

11. In welchem Umfang (pro Woche) betreibst du in deiner Freizeit Sport?

\begin{tabular}{|l|l|l|l|l|l|l|l|l|l|}
\hline$\square$ & nie & $\square$ & $<2 \mathrm{~h}$ & $\square$ & $2-4 \mathrm{~h}$ & $\square$ & $4-6 \mathrm{~h}$ & $\square$ & $>$ als $6 \mathrm{~h}$ \\
\hline
\end{tabular}

12. Hast du früher aktiv Wettkampfsport betrieben bzw. bist du noch aktiv?

\begin{tabular}{|l|l|l|l|l|l|}
\hline$\square$ & ja (früher) & $\square$ & ja (jetzt noch) & $\square$ & nein \\
\hline
\end{tabular}

13. Bist du als Vereinstrainer, Übungsleiter, Kadertrainer o. Ä. in den letzten Jahren aktiv gewesen?

\begin{tabular}{|l|l|l|l|}
\hline$\square$ & ja & $\square$ & nein \\
\hline
\end{tabular}

14. Welchen Stellenwert besitzt der Sportunterricht für dich ganz persönlich (im Vergleich mit anderen Schulfächern)?

\begin{tabular}{|l|l|l|l|l|l|l|l|l|l|}
\hline$\square$ & niedriger & $\square$ & eher niedriger & $\square$ & mittel & $\square$ & eher höher & $\square$ & höher \\
\hline
\end{tabular}


Projekt: Belastungen/Stressoren im Sportunterricht und Bewältigungsstrategien

15. Welchen Stellenwert räumst du Sport (aktiv oder passiv (z.B. TV)) in deinem Privatleben ein?

\begin{tabular}{|l|l|l|l|l|l|l|l|l|l|}
\hline$\square$ & niedrig & $\square$ & eher niedrig & $\square$ & mittel & $\square$ & eher hoch & $\square$ & hoch \\
\hline
\end{tabular}

16. Wie schätzt du dein eigenes sportliches Können in den folgenden Aktivitäten ein?

\begin{tabular}{|l|c|c|c|c|}
\cline { 2 - 5 } \multicolumn{1}{l|}{} & schlecht & $\begin{array}{c}\text { eher } \\
\text { schlecht }\end{array}$ & eher gut & gut \\
\hline Leichtathletik & $\square$ & $\square$ & $\square$ & $\square$ \\
\hline Fußball & $\square$ & $\square$ & $\square$ & $\square$ \\
\hline Handball & $\square$ & $\square$ & $\square$ & $\square$ \\
\hline Turnen & $\square$ & $\square$ & $\square$ & $\square$ \\
\hline Badminton & $\square$ & $\square$ & $\square$ & $\square$ \\
\hline Konditionstraining & $\square$ & $\square$ & $\square$ & $\square$ \\
\hline Gymnastik/Tanz & $\square$ & $\square$ & $\square$ & $\square$ \\
\hline Basketball & $\square$ & $\square$ & $\square$ & $\square$ \\
\hline Schwimmen & $\square$ & $\square$ & $\square$ & $\square$ \\
\hline Hockey & $\square$ & $\square$ & $\square$ & $\square$ \\
\hline Kleine Spiele & $\square$ & $\square$ & $\square$ & $\square$ \\
\hline Volleyball & $\square$ & $\square$ & $\square$ & $\square$ \\
\hline Trendsportarten & $\square$ & $\square$ & $\square$ & $\square$ \\
\hline
\end{tabular}

17. Wie siehst du das Verhältnis vom Sportunterricht gegenüber dem außerschulischen Sport?

Eigenwelt

Sportunterricht nimmt eine unabhängige Position gegenüber dem außerschulischen Sport ein. Schüler werden nicht mit der gesamten Komplexität des Sports konfrontiert sondern nur mit Inhalten die aus pädagogischen Überlegungen gewählt werden und auf die außerschulische Realität vorbereiten.

\section{Gegenwelt}

Sportunterricht stellt bewusst einen Kontrast zu dem außerschulischen Sport dar, der für pädagogische Zwecke ungeeignet scheint. Der Schulsport versteht sich als eine Alternative zum außerschulischen Sport.

Mitwelt

Schulsport und außerschulischer Sport beziehen sich wahlweise aufeinander. Schulsport knüpft zwar an den außerschulischen Sport an, aber nicht um ihn zu reproduzieren sondern geht über ihn hinaus und nutzt ihn als Ausgangspunkt für die didaktische Auswahl und Vermittlung im Sportunterricht.

Doppelwelt

Der außerschulische Sport wird möglichst unverfälscht in den Sportunterricht hereingeholt. Die Aufgabe des Unterrichts wird darin gesehen, zu simulieren, was außerhalb der Schule an Sport stattfindet.

18. Meine Lehrweise kann man als ...beschreiben!

\begin{tabular}{|l|l|l|l|}
\hline$\square$ & induktiv & $\square$ & deduktiv \\
\hline
\end{tabular}

19. Bitte schätze die Organisationsform deines Sportunterrichts ein!

\begin{tabular}{|l|l|l|l|l|l|}
\hline$\square$ & stark lehrerzentriert & $\square$ & eher lehrerzentriert & $\square$ & ausgewogen (mal so mal so) \\
\hline$\square$ & eher schülerorientiert & $\square$ & schülerorientiert \\
\hline
\end{tabular}




\section{Projekt: Belastungen/Stressoren im Sportunterricht und Bewältigungsstrategien}

20. In meinem Sportunterricht mache ich selber aktiv mit!

\begin{tabular}{|l|l|l|l|l|l|l|l|l|l|}
\hline$\square$ & nie & $\square$ & selten & $\square$ & ab und zu & $\square$ & oft & $\square$ & immer \\
\hline
\end{tabular}

21. Schätze die Bedeutsamkeit folgender Vermittlungsaspekte für „deinen“ Unterricht ein!

\begin{tabular}{|l|c|c|c|c|}
\hline & unwichtig & eher unwichtig & eher wichtig & wichtig \\
\hline Bewegungsfertigkeit & $\square$ & $\square$ & $\square$ & $\square$ \\
\hline Soziales Lernen & $\square$ & $\square$ & $\square$ & $\square$ \\
\hline Gesundheitserziehung & $\square$ & $\square$ & $\square$ & $\square$ \\
\hline Fachliche Kenntnisse (Theorie) & $\square$ & $\square$ & $\square$ & $\square$ \\
\hline Kondition & $\square$ & $\square$ & $\square$ & $\square$ \\
\hline Kreativität & $\square$ & $\square$ & $\square$ & $\square$ \\
\hline Freude an Bewegung & $\square$ & $\square$ & $\square$ & $\square$ \\
\hline Leistungserziehung & $\square$ & $\square$ & $\square$ & $\square$ \\
\hline Persönlichkeitsentwicklung & $\square$ & $\square$ & $\square$ & $\square$ \\
\hline Selbständigkeit & $\square$ & $\square$ & $\square$ & $\square$ \\
\hline Körperbildung & $\square$ & $\square$ & $\square$ & $\square$ \\
\hline Lebensbegleitendes Sporttreiben & $\square$ & $\square$ & $\square$ & $\square$ \\
\hline Taktik & $\square$ & $\square$ & $\square$ & $\square$ \\
\hline Teamfähigkeit, Teamgeist & $\square$ & $\square$ & $\square$ & $\square$ \\
\hline Technik & $\square$ & $\square$ & $\square$ & $\square$ \\
\hline Koordinative Fähigkeiten & $\square$ & $\square$ & $\square$ & $\square$ \\
\hline (Wett)-Kampfgeist & $\square$ & $\square$ & $\square$ & $\square$ \\
\hline Körpererfahrung & $\square$ & $\square$ & $\square$ & $\square$ \\
\hline Spielen & $\square$ & $\square$ & $\square$ & $\square$ \\
\hline Disziplin & $\square$ & $\square$ & $\square$ \\
\hline
\end{tabular}

22. Schätze deine Vermittlungsfähigkeit in den folgenden Aktivitäten ein!

\begin{tabular}{|l|c|c|c|c|}
\cline { 2 - 5 } \multicolumn{1}{c|}{} & schlecht & $\begin{array}{c}\text { eher } \\
\text { schlecht }\end{array}$ & eher gut & gut \\
\hline Leichtathletik & $\square$ & $\square$ & $\square$ & $\square$ \\
\hline Fußball & $\square$ & $\square$ & $\square$ & $\square$ \\
\hline Handball & $\square$ & $\square$ & $\square$ & $\square$ \\
\hline Turnen & $\square$ & $\square$ & $\square$ & $\square$ \\
\hline Badminton & $\square$ & $\square$ & $\square$ & $\square$ \\
\hline Konditionstraining & $\square$ & $\square$ & $\square$ & $\square$ \\
\hline Gymnastik/Tanz & $\square$ & $\square$ & $\square$ & $\square$ \\
\hline Basketball & $\square$ & $\square$ & $\square$ & $\square$ \\
\hline Schwimmen & $\square$ & $\square$ & $\square$ & $\square$ \\
\hline Hockey & $\square$ & $\square$ & $\square$ & $\square$ \\
\hline Volleyball & $\square$ & $\square$ & $\square$ & $\square$ \\
\hline Kleine Spiele & $\square$ & $\square$ & $\square$ \\
\hline Trendsportarten & $\square$ & $\square$ & $\square$ \\
\hline
\end{tabular}


Projekt: Belastungen/Stressoren im Sportunterricht und Bewältigungsstrategien

23. Inwieweit fühlst du dich durch die folgenden Faktoren des Sportunterrichts belastet?

\begin{tabular}{|c|c|c|c|c|}
\hline & wenig & eher wenig & eher stark & stark \\
\hline Hoher Lärmpegel & $\square$ & $\square$ & $\square$ & $\square$ \\
\hline Hilfestellungen & $\square$ & $\square$ & $\square$ & $\square$ \\
\hline Auf- und Abbau & $\square$ & $\square$ & $\square$ & $\square$ \\
\hline Demonstration von Bewegungen & $\square$ & $\square$ & $\square$ & $\square$ \\
\hline Stetig latente Unfallgefahr & $\square$ & $\square$ & $\square$ & $\square$ \\
\hline Mangelnde Disziplin der Schüler & $\square$ & $\square$ & $\square$ & $\square$ \\
\hline Geringe Pausen- bzw. Erholungszeiten & $\square$ & $\square$ & $\square$ & $\square$ \\
\hline Vielzahl von gleichzeitig auftretenden Aufgaben & $\square$ & $\square$ & $\square$ & $\square$ \\
\hline Häufig wechselnder Ordnungsrahmen & $\square$ & $\square$ & $\square$ & $\square$ \\
\hline Inadäquate räumlich-materielle Bedingungen & $\square$ & $\square$ & $\square$ & $\square$ \\
\hline Unangemessene curriculare Vorgaben & $\square$ & $\square$ & $\square$ & $\square$ \\
\hline Körperliche Beanspruchungen & $\square$ & $\square$ & $\square$ & $\square$ \\
\hline $\begin{array}{l}\text { Mangelnde Schülermotivation und } \\
\text { Benotungsprobleme }\end{array}$ & $\square$ & $\square$ & $\square$ & $\square$ \\
\hline Hohe Schülerzahl & $\square$ & $\square$ & $\square$ & $\square$ \\
\hline $\begin{array}{l}\text { Rechtfertigungsdruck gegenüber dem Kollegium } \\
\text { (nicht Sport) }\end{array}$ & $\square$ & $\square$ & $\square$ & $\square$ \\
\hline Außerunterrichtliche Aktivitäten (z.B. Sportfeste) & $\square$ & $\square$ & $\square$ & $\square$ \\
\hline $\begin{array}{l}\text { Erhöhter Aufwand zur Erhaltung der Fitness und } \\
\text { dem Erlernen von Trend- und/oder Schulsportarten }\end{array}$ & $\square$ & $\square$ & $\square$ & $\square$ \\
\hline $\begin{array}{l}\text { Schwierigkeiten im Umgang mit Eltern, Kollegen } \\
\text { und Schulleitung }\end{array}$ & $\square$ & $\square$ & $\square$ & $\square$ \\
\hline
\end{tabular}

\section{Fragen zu den Inhalten deines Sportunterrichts}

Anhand welcher Kriterien wählst du die Inhalte für deinen Sportunterricht aus?

\begin{tabular}{|c|c|c|c|c|}
\hline & Trifft zu & $\begin{array}{c}\text { Trifft eher } \\
\text { zu }\end{array}$ & $\begin{array}{l}\text { Trifft eher } \\
\text { nicht zu }\end{array}$ & $\begin{array}{c}\text { Trifft } \\
\text { nicht zu }\end{array}$ \\
\hline Curriculare Vorgaben & $\square$ & $\square$ & $\square$ & $\square$ \\
\hline Schülerinteresse & $\square$ & $\square$ & $\square$ & $\square$ \\
\hline Organisations- bzw. Aufbauaufwand & $\square$ & $\square$ & $\square$ & $\square$ \\
\hline Eigene Vorlieben (z.B. durch sportliche Aktivität in der Sportart) & $\square$ & $\square$ & $\square$ & $\square$ \\
\hline Geringes Konfliktpotential zwischen den Schülern & $\square$ & $\square$ & $\square$ & $\square$ \\
\hline Geringes Verletzungsrisiko & $\square$ & $\square$ & $\square$ & $\square$ \\
\hline Eigene Vermittlungskompetenz in der Sportart & $\square$ & $\square$ & $\square$ & $\square$ \\
\hline Ausstattung der Sportstätte & $\square$ & $\square$ & $\square$ & $\square$ \\
\hline Eigene sportliche Kompetenz in der Sportart. & $\square$ & $\square$ & $\square$ & $\square$ \\
\hline
\end{tabular}


Projekt: Belastungen/Stressoren im Sportunterricht und Bewältigungsstrategien

\section{Die Anforderungen an die Schüler in meinem Sportunterricht im technisch-taktischen} Bereich sind...!

\begin{tabular}{|l|l|l|l|l|l|l|l|l|l|}
\hline$\square$ & niedrig & $\square$ & eher niedrig & $\square$ & mittel & $\square$ & eher hoch & $\square$ & hoch \\
\hline
\end{tabular}

26. Die Anforderungen an die Schüler in meinem Sportunterricht im konditionellen Bereich sind....!

\begin{tabular}{|l|l|l|l|l|l|l|l|l|l|}
\hline$\square$ & niedrig & $\square$ & eher niedrig & $\square$ & mittel & $\square$ & eher hoch & $\square$ & hoch \\
\hline
\end{tabular}

\section{Fragen zu deinem Sportunterricht:}

\begin{tabular}{|c|c|c|c|c|}
\hline & Trifft zu & $\begin{array}{l}\text { Trifft eher } \\
\text { zu }\end{array}$ & $\begin{array}{l}\text { Trifft eher } \\
\text { nicht zu }\end{array}$ & $\begin{array}{l}\text { Trifft nicht } \\
\text { zu }\end{array}$ \\
\hline $\begin{array}{l}\text { Wenn ich mitbekomme, dass einzelne Schüler bzw. } \\
\text { Schülergruppen ausgegrenzt bzw. gemobbt werden, spreche ich } \\
\text { die Situation an und versuche sie zu lösen. }\end{array}$ & $\square$ & $\square$ & $\square$ & $\square$ \\
\hline $\begin{array}{l}\text { Bevor Schüler sich beschweren, biete ich lieber andere Inhalte } \\
\text { bzw. geringere Schwierigkeitsstufen an. }\end{array}$ & $\square$ & $\square$ & $\square$ & $\square$ \\
\hline $\begin{array}{l}\text { Es ist mir wichtig, dass mein Sportunterricht nach außen hin } \\
\text { (Kollegen, Rektor) geregelt erscheint. }\end{array}$ & $\square$ & $\square$ & $\square$ & $\square$ \\
\hline $\begin{array}{l}\text { Wenn ich sehe, dass ein guter Schüler sich nicht anstrengt greife } \\
\text { ich ein. }\end{array}$ & $\square$ & $\square$ & $\square$ & $\square$ \\
\hline $\begin{array}{l}\text { Wenn ich Probleme in meinem Sportunterricht habe, kann ich } \\
\text { diese mit meinen Kollegen besprechen und an einer Lösung arbei- } \\
\text { ten. }\end{array}$ & $\square$ & $\square$ & $\square$ & $\square$ \\
\hline $\begin{array}{l}\text { Wenn ich Äußerungen (z.B. Beleidigungen, Meckereien) von } \\
\text { Schülern wahrnehme, die mich bloßstellen, ignoriere ich diese. }\end{array}$ & $\square$ & $\square$ & $\square$ & $\square$ \\
\hline $\begin{array}{l}\text { Ich bin zufrieden, wenn eine Sportstunde in geregelten Bahnen } \\
\text { abläuft, auch wenn ich die von mir vorher gesetzten inhaltlichen } \\
\text { Ziele nicht erreicht habe. }\end{array}$ & $\square$ & $\square$ & $\square$ & $\square$ \\
\hline $\begin{array}{l}\text { Ich bin über Unfallverhütungsmaßnahmen, Erlasse und Vorschrif- } \\
\text { ten im Sportunterricht gut informiert. }\end{array}$ & $\square$ & $\square$ & $\square$ & $\square$ \\
\hline $\begin{array}{l}\text { Auch in schwierigen Klassen nutze ich nicht nur sichere Unter- } \\
\text { richtskonzepte. }\end{array}$ & $\square$ & $\square$ & $\square$ & $\square$ \\
\hline $\begin{array}{l}\text { Ich gehe in meinem Sportunterricht ungern Risiken ein, damit } \\
\text { meine Kollegen nicht denken "bei dem geht alles drunter und } \\
\text { drüber". }\end{array}$ & $\square$ & $\square$ & $\square$ & $\square$ \\
\hline $\begin{array}{l}\text { Ich probiere gerne neue Inhalte und Methoden in meinem Sport- } \\
\text { unterricht aus, auch auf die Gefahr hin, dass sie sich als un- } \\
\text { brauchbar erweisen. }\end{array}$ & $\square$ & $\square$ & $\square$ & $\square$ \\
\hline $\begin{array}{l}\text { Um keine zeitlichen Probleme im Sportunterricht zu bekommen, } \\
\text { greife ich meist auf bereits erprobte sichere Unterrichtskonzepte } \\
\text { zurück, auch wenn sie nicht zu 100\% auf den Lehrplan und die } \\
\text { Lerngruppe abgestimmt sind. }\end{array}$ & $\square$ & $\square$ & $\square$ & $\square$ \\
\hline $\begin{array}{l}\text { Solange ein Großteil der Schüler aktiv und motiviert am Sportun- } \\
\text { terricht teilnimmt, akzeptiere ich es, wenn einzelne Schüler nur } \\
\text { herumstehen. }\end{array}$ & $\square$ & $\square$ & $\square$ & $\square$ \\
\hline $\begin{array}{l}\text { Auch auf die Gefahr hin, dass ich verstärkt durch Regelkunde und } \\
\text { Erklärungen im Unterricht gefordert werde, führe ich mit den } \\
\text { Schülerinnen/Schülern auch unbekannte Spiele bzw. Übungsfor- } \\
\text { men in meinen Unterricht durch. }\end{array}$ & $\square$ & $\square$ & $\square$ & $\square$ \\
\hline Die Rechtsunsicherheit in vielen Bereichen des Sportunterrichts & $\square$ & $\square$ & $\square$ & $\square$ \\
\hline
\end{tabular}




\begin{tabular}{|c|c|c|c|c|}
\hline $\begin{array}{l}\text { führt dazu, dass ich in der Gestaltung des Unterrichts lieber auf } \\
\text { Nummer sicher gehe. }\end{array}$ & & & & \\
\hline $\begin{array}{l}\text { Wenn Schüler zu spät zum Unterricht kommen, stelle ich sie zur } \\
\text { Rede. }\end{array}$ & $\square$ & $\square$ & $\square$ & $\square$ \\
\hline $\begin{array}{l}\text { Um alles im Blick zu haben biete ich den Schülern meist begrenz- } \\
\text { te Bewegungsmöglichkeiten an, auch wenn dadurch Wartezeiten } \\
\text { entstehen, wie z.B. durch Anstehen in Schlangen. }\end{array}$ & $\square$ & $\square$ & $\square$ & $\square$ \\
\hline Konflikte mit Schülern im Unterricht versuche ich zu vermeiden. & $\square$ & $\square$ & $\square$ & $\square$ \\
\hline $\begin{array}{l}\text { Um eine motivierte Klasse im Sportunterricht zu haben, wähle ich } \\
\text { Inhalte, von denen ich weiß, dass die „beliebten Schüler“ diese } \\
\text { gut finden, da die anderen sich an ihnen orientieren. }\end{array}$ & $\square$ & $\square$ & $\square$ & $\square$ \\
\hline $\begin{array}{l}\text { Problemen mit Vorschriften und Erlassen versuche ich bewusst } \\
\text { aus dem Weg zu gehen. }\end{array}$ & $\square$ & $\square$ & $\square$ & $\square$ \\
\hline $\begin{array}{l}\text { Wenn einzelne Schüler in Mannschaftsspielen zwar sehr gut aber } \\
\text { auch sehr egoistisch spielen, greife ich ein. }\end{array}$ & $\square$ & $\square$ & $\square$ & $\square$ \\
\hline $\begin{array}{l}\text { Ich organisiere meinen Unterricht so, dass ich mich auch } \\
\text { zurückziehen und ausruhen kann. }\end{array}$ & $\square$ & $\square$ & $\square$ & $\square$ \\
\hline Ich weiß wie ich mich im Gefahrenfall zu verhalten habe. & $\square$ & $\square$ & $\square$ & $\square$ \\
\hline $\begin{array}{l}\text { Ich toleriere es, wenn Schüler sich in der Schlange immer wieder } \\
\text { nach hinten orientieren, um einem Scheitern bei schwierigen An- } \\
\text { forderungen aus dem Weg zu gehen }\end{array}$ & $\square$ & $\square$ & $\square$ & $\square$ \\
\hline $\begin{array}{l}\text { Um Probleme beim Aufbau zu verhindern, baue ich Unterrichts- } \\
\text { arrangements oder zumindest Teile bereits alleine in der Pause } \\
\text { auf. }\end{array}$ & $\square$ & $\square$ & $\square$ & $\square$ \\
\hline $\begin{array}{l}\text { Da die Note in Sport nicht so wichtig wie in anderen Fächern ist, } \\
\text { gebe ich überwiegend gute und sehr gute Noten um Streitereien } \\
\text { mit Schülern und Eltern aus dem Weg zu gehen. }\end{array}$ & $\square$ & $\square$ & $\square$ & $\square$ \\
\hline
\end{tabular}

\begin{tabular}{|c|c|c|c|c|c|c|c|}
\hline & $\begin{array}{l}\text { Im Folgenden findest du unterschiedliche Aussagen zu verschiedenen } \\
\text { Themen. Wahrscheinlich wirst du einigen Aussagen sehr zustimmen } \\
\text { und andere stark ablehnen. Bei wieder anderen bist du vielleicht } \\
\text { unentschieden. Nimm bitte zu jeder der folgenden Aussagen Stellung } \\
\text { und entscheide, inwieweit jede auf dich ganz persönlich zutrifft. } \\
\text { Kreuze dazu bitte jeweils ein Kästchen zwischen } 1 \text { (stimmt genau) und } \\
6 \text { (stimmt überhaupt nicht) an, und lasse bitte keine Aussage aus. }\end{array}$ & 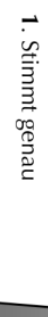 & 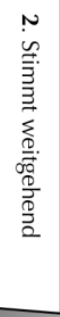 & 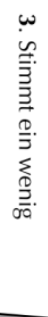 & 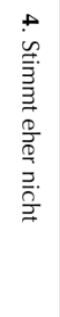 & 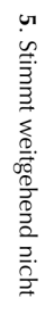 & 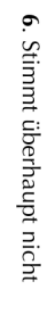 \\
\hline 1. & $\begin{array}{l}\text { Ich probiere gerne Dinge aus, auch wenn nicht immer etwas dabei } \\
\text { herauskommt. }\end{array}$ & $\square$ & $\square$ & $\square$ & $\square$ & $\square$ & $\square$ \\
\hline 2. & Ich beschäftige mich nur mit Aufgaben, die lösbar sind. & $\square$ & $\square$ & $\square$ & $\square$ & $\square$ & $\square$ \\
\hline 3. & Ich mag es, wenn unverhofft Überraschungen auftreten. & $\square$ & $\square$ & $\square$ & $\square$ & $\square$ & $\square$ \\
\hline 4. & Ich lasse die Dinge gerne auf mich zukommen. & $\square$ & $\square$ & $\square$ & $\square$ & $\square$ & $\square$ \\
\hline 5. & Ich habe es gerne, wenn die Arbeit gleichmäßig verläuft. & $\square$ & $\square$ & $\square$ & $\square$ & $\square$ & $\square$ \\
\hline 6. & Ich warte geradezu darauf, dass etwas Aufregendes passiert. & $\square$ & $\square$ & $\square$ & $\square$ & $\square$ & $\square$ \\
\hline 7. & $\begin{array}{l}\text { Wenn um mich herum alles drunter und drüber geht, fühle ich mich so } \\
\text { richtig wohl. }\end{array}$ & $\square$ & $\square$ & $\square$ & $\square$ & $\square$ & $\square$ \\
\hline 8. & Ich weiß gerne, was auf mich zukommt. & $\square$ & $\square$ & $\square$ & $\square$ & $\square$ & $\square$ \\
\hline
\end{tabular}




\begin{tabular}{|c|c|c|c|c|c|c|}
\hline & $\begin{array}{l}\text { Wir bitten dich einige deiner üblichen Verhaltensweisen, Einstellungen und } \\
\text { Gewohnheiten zu beschreiben, die dich in deiner Rolle als Sportlehrer } \\
\text { kennzeichnen. } \\
\text { Aussagen: } \\
\text { Lese jeden dieser Sätze gründlich durch und entscheide, in welchem Maße } \\
\text { er auf dich persönlich zutrifft. } \\
\text { Bitte kreuze das jeweilige Kästchen an! }\end{array}$ & 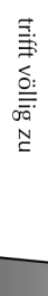 & 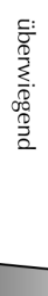 & 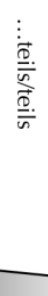 & 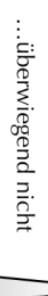 & 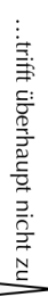 \\
\hline 1. & Die Arbeit ist für mich der wichtigste Lebensinhalt. & $\square$ & $\square$ & $\square$ & $\square$ & $\square$ \\
\hline 2. & $\begin{array}{l}\text { Ich möchte beruflich weiter kommen, als es die meisten meiner Bekannten } \\
\text { geschafft haben. }\end{array}$ & $\square$ & $\square$ & $\square$ & $\square$ & $\square$ \\
\hline 3. & Wenn es sein muss, arbeite ich bis zur Erschöpfung. & $\square$ & $\square$ & $\square$ & $\square$ & $\square$ \\
\hline 4. & Meine Arbeit soll stets ohne Fehl und Tadel sein. & $\square$ & $\square$ & $\square$ & $\square$ & $\square$ \\
\hline 5. & Zum Feierabend ist die Arbeit für mich gegessen. & $\square$ & $\square$ & $\square$ & $\square$ & $\square$ \\
\hline 6. & Wenn ich keinen Erfolg habe, resigniere ich schnell. & $\square$ & $\square$ & $\square$ & $\square$ & $\square$ \\
\hline 7. & Für mich sind Schwierigkeiten dazu da, dass ich sie überwinde. & $\square$ & $\square$ & $\square$ & $\square$ & $\square$ \\
\hline 8. & Mich bringt so leicht nichts aus der Ruhe. & $\square$ & $\square$ & $\square$ & $\square$ & $\square$ \\
\hline 9. & Mein bisheriges Berufsleben war recht erfolgreich. & $\square$ & $\square$ & $\square$ & $\square$ & $\square$ \\
\hline 10. & Mit meinem bisherigen Leben kann ich zufrieden sein. & $\square$ & $\square$ & $\square$ & $\square$ & $\square$ \\
\hline 11. & Mein Partner zeigt Verständnis für meine Arbeit. & $\square$ & $\square$ & $\square$ & $\square$ & $\square$ \\
\hline 12. & Die Arbeit ist mein ein und alles. & $\square$ & $\square$ & $\square$ & $\square$ & $\square$ \\
\hline 13. & Berufliche Karriere bedeutet mir wenig. & $\square$ & $\square$ & $\square$ & $\square$ & $\square$ \\
\hline 14. & Bei der Arbeit kenne ich keine Schonung. & $\square$ & $\square$ & $\square$ & $\square$ & $\square$ \\
\hline 15. & $\begin{array}{l}\text { Ich kontrolliere lieber noch dreimal nach, als dass ich fehlerhafte } \\
\text { Arbeitergebnisse abliefere. }\end{array}$ & $\square$ & $\square$ & $\square$ & $\square$ & $\square$ \\
\hline 16. & Auch in der Freizeit beschäftigen mich viele Arbeitsprobleme. & $\square$ & $\square$ & $\square$ & $\square$ & $\square$ \\
\hline 17. & Misserfolg kann ich nur schwer verkraften. & $\square$ & $\square$ & $\square$ & $\square$ & $\square$ \\
\hline 18. & Wenn mir etwas nicht gelingt, sage ich mir: Jetzt erst recht! & $\square$ & $\square$ & $\square$ & $\square$ & $\square$ \\
\hline 19. & Ich bin ein ruheloser Mensch. & $\square$ & $\square$ & $\square$ & $\square$ & $\square$ \\
\hline 20. & $\begin{array}{l}\text { In meiner bisherigen Berufslaufbahn habe ich mehr Erfolg als Enttäuschungen } \\
\text { erlebt. }\end{array}$ & $\square$ & $\square$ & $\square$ & $\square$ & $\square$ \\
\hline 21. & Im Großen und Ganzen bin ich glücklich und zufrieden. & $\square$ & $\square$ & $\square$ & $\square$ & $\square$ \\
\hline 22. & Meine Familie interessiert sich nur wenig für meine Arbeitsprobleme. & $\square$ & $\square$ & $\square$ & $\square$ & $\square$ \\
\hline 23. & Ich könnte auch ohne meine Arbeit ganz glücklich sein. & $\square$ & $\square$ & $\square$ & $\square$ & $\square$ \\
\hline 24. & $\begin{array}{l}\text { Was meine berufliche Entwicklung angeht, so halte ich mich für ziemlich } \\
\text { ehrgeizig. }\end{array}$ & $\square$ & $\square$ & $\square$ & $\square$ & $\square$ \\
\hline 25. & Ich arbeite wohl mehr als ich sollte. & $\square$ & $\square$ & $\square$ & $\square$ & $\square$ \\
\hline 26. & Bei meiner Arbeit habe ich den Ehrgeiz, keinerlei Fehler zu machen. & $\square$ & $\square$ & $\square$ & $\square$ & $\square$ \\
\hline 27. & Nach der Arbeit kann ich ohne Probleme abschalten. & $\square$ & $\square$ & $\square$ & $\square$ & $\square$ \\
\hline 28. & Berufliche Fehlschläge können mich leicht entmutigen. & $\square$ & $\square$ & $\square$ & $\square$ & $\square$ \\
\hline 29. & $\begin{array}{l}\text { Misserfolge werfen mich nicht um, sondern veranlassen mich zu noch stärkerer } \\
\text { Anstrengung. }\end{array}$ & $\square$ & $\square$ & $\square$ & $\square$ & $\square$ \\
\hline 30. & Ich glaube, dass ich ziemlich hektisch bin. & $\square$ & $\square$ & $\square$ & $\square$ & $\square$ \\
\hline 31. & Wirkliche berufliche Erfolge sind mir bisher versagt geblieben. & $\square$ & $\square$ & $\square$ & $\square$ & $\square$ \\
\hline 32. & Ich habe allen Grund, meine Zukunft optimistisch zu sehen. & $\square$ & $\square$ & $\square$ & $\square$ & $\square$ \\
\hline
\end{tabular}




\section{Projekt: Belastungen/Stressoren im Sportunterricht und Bewältigungsstrategien}

\begin{tabular}{|c|c|c|c|c|c|c|}
\hline 33. & $\begin{array}{l}\text { Von meinem Partner wünschte ich mir mehr Rücksichtnahme auf meine } \\
\text { beruflichen Aufgaben und Probleme. }\end{array}$ & $\square$ & $\square$ & $\square$ & $\square$ & $\square$ \\
\hline 34. & Ich brauche die Arbeit wie die Luft zum Atmen. & $\square$ & $\square$ & $\square$ & $\square$ & $\square$ \\
\hline 35. & Ich strebe nach höheren beruflichen Zielen als die meisten anderen. & $\square$ & $\square$ & $\square$ & $\square$ & $\square$ \\
\hline 36. & Ich neige dazu, über meine Kräfte hinaus zu arbeiten. & $\square$ & $\square$ & $\square$ & $\square$ & $\square$ \\
\hline 37. & Was immer ich tue, es muss perfekt sein. & $\square$ & $\square$ & $\square$ & $\square$ & $\square$ \\
\hline 38. & $\begin{array}{l}\text { Feierabend ist Feierabend, da verschwende ich keine Gedanken mehr an die } \\
\text { Arbeit. }\end{array}$ & $\square$ & $\square$ & $\square$ & $\square$ & $\square$ \\
\hline 39. & Wenn ich in der Arbeit erfolglos bin, deprimiert mich das sehr. & $\square$ & $\square$ & $\square$ & $\square$ & $\square$ \\
\hline 40. & $\begin{array}{l}\text { Ich bin mir sicher, dass ich auch die künftigen Anforderungen des Lebens gut } \\
\text { bewältigen kann. }\end{array}$ & $\square$ & $\square$ & $\square$ & $\square$ & $\square$ \\
\hline 41. & Ich glaube, ich bin ein ruhender Pol in meinem Umfeld. & $\square$ & $\square$ & $\square$ & $\square$ & $\square$ \\
\hline 42. & In meiner beruflichen Entwicklung ist mir bisher fast alles gelungen. & $\square$ & $\square$ & $\square$ & $\square$ & $\square$ \\
\hline 43. & Ich kann mich über mein Leben in keiner Weise beklagen. & $\square$ & $\square$ & $\square$ & $\square$ & $\square$ \\
\hline 44. & Bei meiner Familien finde ich jede Unterstützung. & $\square$ & $\square$ & $\square$ & $\square$ & $\square$ \\
\hline 45. & Ich wüsste nicht wie ich ohne Arbeit leben sollte. & $\square$ & $\square$ & $\square$ & $\square$ & $\square$ \\
\hline 46. & Für meine berufliche Zukunft habe ich mir viel vorgenommen. & $\square$ & $\square$ & $\square$ & $\square$ & $\square$ \\
\hline 47. & Mein Tagesablauf ist durch chronischen Zeitmangel bestimmt. & $\square$ & $\square$ & $\square$ & $\square$ & $\square$ \\
\hline 48. & $\begin{array}{l}\text { Für mich ist die Arbeit erst dann getan, wenn ich rundum mit dem Ergebnis } \\
\text { zufrieden bin. }\end{array}$ & $\square$ & $\square$ & $\square$ & $\square$ & $\square$ \\
\hline 49. & Arbeitsprobleme beschäftigen mich eigentlich den ganzen Tag. & $\square$ & $\square$ & $\square$ & $\square$ & $\square$ \\
\hline 50. & Ich verliere leicht den Mut, wenn ich trotz Anstrengung keinen Erfolg habe. & $\square$ & $\square$ & $\square$ & $\square$ & $\square$ \\
\hline 51. & Ein Misserfolg kann bei mir neue Kräfte wecken. & $\square$ & $\square$ & $\square$ & $\square$ & $\square$ \\
\hline 52. & Ich kann mich in fast allen Situationen ruhig und bedächtig verhalten. & $\square$ & $\square$ & $\square$ & $\square$ & $\square$ \\
\hline 53. & Mein bisheriges Leben ist durch beruflichen Erfolg gekennzeichnet. & $\square$ & $\square$ & $\square$ & $\square$ & $\square$ \\
\hline 54. & Von manchen Seiten des Lebens bin ich ziemlich enttäuscht. & $\square$ & $\square$ & $\square$ & $\square$ & $\square$ \\
\hline 55. & $\begin{array}{l}\text { Manchmal wünschte ich mir mehr Unterstützung durch die Menschen meiner } \\
\text { Umgebung. }\end{array}$ & $\square$ & $\square$ & $\square$ & $\square$ & $\square$ \\
\hline 56. & Es gibt wichtigeres im Leben als die Arbeit. & $\square$ & $\square$ & $\square$ & $\square$ & $\square$ \\
\hline 57. & Beruflicher Erfolg ist für mich ein wichtiges Lebensziel. & $\square$ & $\square$ & $\square$ & $\square$ & $\square$ \\
\hline 58. & In der Arbeit verausgabe ich mich stark. & $\square$ & $\square$ & $\square$ & $\square$ & $\square$ \\
\hline 59. & $\begin{array}{l}\text { Es widerstrebt mir, wenn ich eine Arbeit abschließen muss, obwohl sie noch } \\
\text { verbessert werden könnte. }\end{array}$ & $\square$ & $\square$ & $\square$ & $\square$ & $\square$ \\
\hline 60. & Meine Gedanken kreisen fast nur um die Arbeit. & $\square$ & $\square$ & $\square$ & $\square$ & $\square$ \\
\hline 61. & Wenn ich irgendwo versagt habe, kann mich das ziemlich mutlos machen. & $\square$ & $\square$ & $\square$ & $\square$ & $\square$ \\
\hline 62. & $\begin{array}{l}\text { Wenn mir etwas nicht gelingt, bleibe ich hartnäckig und strenge mich umso } \\
\text { mehr an. }\end{array}$ & $\square$ & $\square$ & $\square$ & $\square$ & $\square$ \\
\hline 63. & Hektik und Aufregung um mich herum lassen mich kalt. & $\square$ & $\square$ & $\square$ & $\square$ & $\square$ \\
\hline 64. & Meine beruflichen Leistungen können sich sehen lassen. & $\square$ & $\square$ & $\square$ & $\square$ & $\square$ \\
\hline 65. & Es dürfte nur wenige glücklichere Menschen geben als ich es bin. & $\square$ & $\square$ & $\square$ & $\square$ & $\square$ \\
\hline 66. & Wenn ich mal Rat und Hilfe brauche ist immer jemand da. & $\square$ & $\square$ & $\square$ & $\square$ & $\square$ \\
\hline
\end{tabular}

\section{Vielen Dank für deine Mitarbeit!}




\section{2. Fragebogenentwurf (Pilotierung 2)}

\section{0 \\ DFG GRADUIERTENKOLLEG 1195 \\ Passungsverhältnisse schulischen Lernens}

Projekt: Belastungen/Stressoren im Sportunterricht und deren Bewältigung

\section{Liebe(r) Sportstudent/In, Referendar/In, Sportlehrer/In,}

im Rahmen meines Promotionsvorhabens an der Universität Göttingen beschäftige ich mich mit der Bewältigung von alltäglichen Belastungen, Stressoren und Konflikten von Sportlehrerinnen und Sportlehrern. Gegenstand des Vorhabens ist es, aufzuschlüsseln welche Verhaltensmuster Sportlehrerinnen und Sportlehrer ${ }^{1}$ im Umgang mit den stetig zunehmenden an sie gerichteten Ansprüche durch z.B. steigende Schülerzahlen, Erweiterung des Erziehungsauftrages und fehlende Unterrichtsmaterialien zeigen, wie Sie ihre Arbeit erleben und welche Einstellungen sie gegenüber dem Sportunterricht vertreten.

\section{Noch einige wichtige Informationen zum Ausfüllen des Fragebogens:}

- Grundsätzlich ist die Teilnahme an der Untersuchung freiwillig, jedoch sind die Ergebnisse umso aussagekräftiger und repräsentativer je mehr Sportlehrer bzw. Studenten sich beteiligen.

- $\quad$ Die Befragung erfolgt absolut anonym. Alle Daten und Angaben werden ausschließlich für wissenschaftliche Zwecke verwendet und im Bezug auf datenschutzrechtliche Bestimmungen streng vertraulich behandelt.

Das Ausfüllen des Fragebogens sollte insgesamt nicht länger als 20min dauern. Bitte überlegen Sie bei den einzelnen Fragen nicht zu lange sondern antworten möglichst spontan.

- $\quad$ Es gibt keine richtigen oder falschen Antworten! Geben Sie ehrliche Antworten und überlegen Sie nicht welche Antworten den besten Eindruck entstehen lassen. Wenn Sie sich nicht für eine bestimmte Antwort entscheiden können wählen Sie die am ehesten auf Sie zutreffende!

Die Fragen sind auch für dich als Studenten zu beantworten, bei den Fragen zu "deinem Sportunterricht" beziehe dich auf deine Lehrerfahrungen in der Schule und/oder beantworte perspektivisch für deine spätere Lehrtätigkeit.

Bitte kreuzen Sie das Kästchen mit der für Sie zutreffenden Antwort deutlich an. Wenn Sie etwas falsch angekreuzt haben streichen Sie das Kreuz einfach durch und machen an der richtigen Stelle ein neues Kreuz und umkreisen dieses (siehe Beispiel).

$$
\begin{aligned}
& \text { Geschlecht (bitte ankreuzen) } \\
& \text { weiblich Männlich }
\end{aligned}
$$

Es würde uns sehr freuen, wenn wir mit deiner/Ihrer Teilnahme rechnen könnten, da nur durch Forschungsvorhaben wie diesem die Situation der Sportlehrer besser eingeschätzt werden und Maßnahmen ergriffen bzw. entwickelt werden können, die Entlastung und Verbesserungen des Alltags ermöglichen. Je mehr Sportlehrer bzw. Sportstudenten sich an dieser Untersuchung beteiligen, desto freier von Verzerrungen und Ungenauigkeiten fällt das Ergebnis aus und desto gezielter und erfolgreicher können Verbesserungsvorschläge erarbeitet werden.

Mit herzlichem Dank für Ihre Mitarbeit und freundlichem Gruß

Christian Belz (Projektverantwortlicher) $)^{2}$

Juniorprofessorin Dr. Katja Schmitt (Institut für Sportwissenschaft Göttingen)

Prof. Dr. Marcus Hasselhorn (Georg-Elias-Müller-Institut für Psychologie Göttingen)

${ }^{1}$ Aus Gründen der besseren Lesbarkeit wird im weiteren Verlauf des Fragebogens ausschließlich die männliche Form verwendet (im Fragebogen auch bei Schülerinnen

bzw. Schülern), was jedoch stets sinngemäß die weibliche mit einschließt.

2 Bei Rückfragen wenden Sie sich bitte an Herrn Christian Belz, Institut für Sportwissenschaft Göttingen, Sprangerweg 2, 37075 Göttingen, Tel. 0551-395656, E-Mail: ChBelz@sport.uni-goettingen.de 


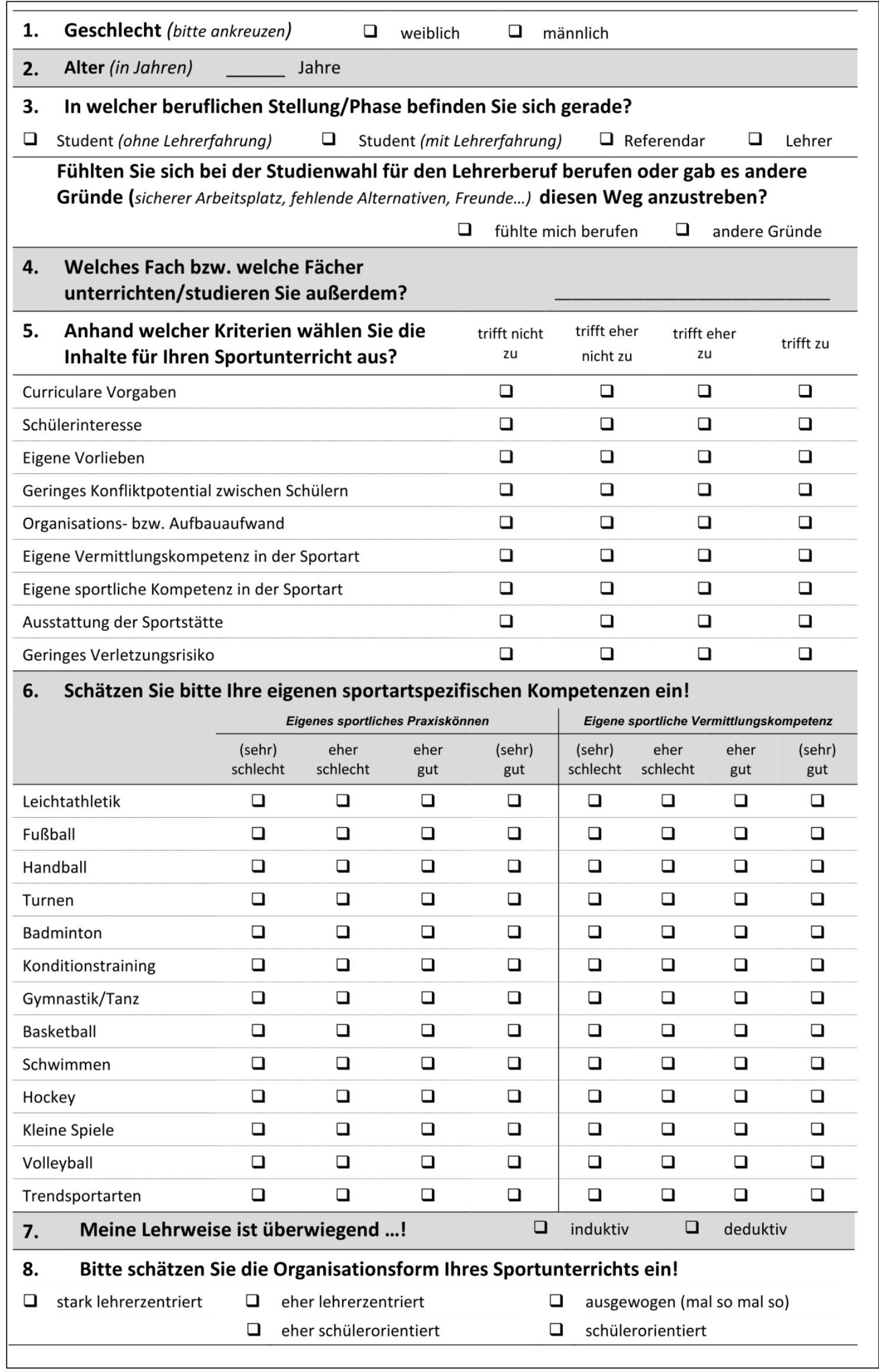




\begin{tabular}{|c|c|c|c|c|c|}
\hline 9. & $\begin{array}{l}\text { Wie oft führen Sie die folgenden } \\
\text { Aktivitäten im Unterricht durch? }\end{array}$ & selten/nie & eher selten & eher oft & oft/sehr oft \\
\hline \multicolumn{2}{|c|}{ Leichtathletik } & $\square$ & $\square$ & $\square$ & a \\
\hline \multicolumn{2}{|c|}{ Fußball } & $\square$ & $\square$ & $\square$ & $\square$ \\
\hline \multicolumn{2}{|c|}{ Handball } & $\square$ & $\square$ & $\square$ & $\square$ \\
\hline \multicolumn{2}{|c|}{ Turnen } & $\square$ & $\square$ & $\square$ & $\square$ \\
\hline \multicolumn{2}{|c|}{ Badminton } & a & $\square$ & $\square$ & $\square$ \\
\hline \multicolumn{2}{|c|}{ Konditionstraining } & $\square$ & $\square$ & $\square$ & $\square$ \\
\hline \multicolumn{2}{|c|}{ Gymnastik/Tanz } & $\square$ & $\square$ & $\square$ & $\square$ \\
\hline \multicolumn{2}{|c|}{ Basketball } & a & $\square$ & $\square$ & $\square$ \\
\hline \multicolumn{2}{|c|}{ Schwimmen } & $\square$ & $\square$ & $\square$ & $\square$ \\
\hline \multicolumn{2}{|c|}{ Hockey } & $\square$ & $\square$ & $\square$ & $\square$ \\
\hline \multicolumn{2}{|c|}{ Kleine Spiele } & a & $\square$ & $\square$ & $\square$ \\
\hline \multicolumn{2}{|c|}{ Volleyball } & 口 & 口 & 口 & 口 \\
\hline \multicolumn{2}{|c|}{ Trendsportarten } & $\square$ & $\square$ & $\square$ & $\square$ \\
\hline & $\begin{array}{l}\text { Inwieweit fühlen Sie sich durch die folgenden } \\
\text { Faktoren im Sportunterricht belastet? }\end{array}$ & $\begin{array}{l}\text { (sehr) } \\
\text { wenig }\end{array}$ & $\begin{array}{l}\text { eher } \\
\text { wenig }\end{array}$ & $\begin{array}{l}\text { eher } \\
\text { stark }\end{array}$ & $\begin{array}{l}\text { (sehr) } \\
\text { stark }\end{array}$ \\
\hline \multicolumn{2}{|c|}{ Hoher Lärmpegel } & 口 & 口 & $\square$ & a \\
\hline \multicolumn{2}{|c|}{ Hilfestellungen } & $\square$ & 口 & $\square$ & $\square$ \\
\hline \multicolumn{2}{|c|}{ Auf- und Abbau } & $\square$ & 口 & $\square$ & $\square$ \\
\hline \multicolumn{2}{|c|}{ Demonstration von Bewegungen } & 口 & $\square$ & $\square$ & $\square$ \\
\hline \multicolumn{2}{|c|}{ Stetig latente Unfallgefahr } & a & $\square$ & $\square$ & $\square$ \\
\hline \multicolumn{2}{|r|}{ Soziales Schülerfehlverhalten und Motivationsprobleme } & a & $\square$ & $\square$ & $\square$ \\
\hline \multicolumn{2}{|r|}{ Geringe Pausen bzw. Erholungszeiten } & 口 & $\square$ & $\square$ & $\square$ \\
\hline \multicolumn{2}{|c|}{ Vielzahl von gleichzeitig auftretenden Aufgaben } & a & 口 & $\square$ & $\square$ \\
\hline \multicolumn{2}{|r|}{ Rechtfertigungsdruck gegenüber dem Kollegium (nicht Sport) } & $\square$ & $\square$ & $\square$ & $\square$ \\
\hline \multicolumn{2}{|r|}{ Außerunterrichtliche Aktivitäten (z.B. Sportfeste, BuJu-Spiele) } & 口 & $\square$ & $\square$ & $\square$ \\
\hline \multicolumn{2}{|c|}{ Häufig wechselnder Ordnungsrahmen im Unterricht } & $\square$ & $\square$ & $\square$ & $\square$ \\
\hline \multicolumn{2}{|r|}{$\begin{array}{l}\text { Erhöhter Aufwand zur Erhaltung der eigenen Fitness und dem } \\
\text { Erlernen neuer Sportarten/Inhalte }\end{array}$} & $\square$ & $\square$ & $\square$ & $\square$ \\
\hline \multicolumn{2}{|r|}{ Schwierigkeiten im Umgang mit Eltern, Kollegen, Schulleitung } & a & 口 & a & a \\
\hline \multicolumn{2}{|c|}{ Schlechte räumlich-materielle Bedingungen } & 口 & $\square$ & $\square$ & $\square$ \\
\hline Klass & engröße & $\square$ & $\square$ & $\square$ & $\square$ \\
\hline Unan & gemessene curriculare Vorgaben & a & a & $\square$ & a \\
\hline Körp & rliche Beanspruchung (allgemein) & $\square$ & $\square$ & $\square$ & $\square$ \\
\hline 11. & $\begin{array}{l}\text { Im Bezug auf den Umgang mit Schülern im } \\
\text { Sportunterricht... }\end{array}$ & $\begin{array}{c}\text { trifft } \\
\text { nicht zu }\end{array}$ & $\begin{array}{l}\text { trifft } \\
\text { eher } \\
\text { nicht zu }\end{array}$ & $\begin{array}{l}\text { trifft } \\
\text { eher zu }\end{array}$ & trifft zu \\
\hline $\begin{array}{r}\text {...reiz } \\
\text { zu t }\end{array}$ & $\begin{array}{l}\text { en mich Situationen in denen ich auch mal mit Konflikten } \\
\text { un habe. }\end{array}$ & $\square$ & $\square$ & $\square$ & $\square$ \\
\hline ...ver & suche ich möglichst nicht auf Widerstände zu stoßen. & $\square$ & $\square$ & $\square$ & $\square$ \\
\hline $\begin{array}{l}\text {...war } \\
\text { von }\end{array}$ & $\begin{array}{l}\text { te ich bei Dingen die nicht so gut laufen lieber, bis sie sich } \\
\text { selbst regeln. }\end{array}$ & $\square$ & $\square$ & $\square$ & $\square$ \\
\hline
\end{tabular}




\begin{tabular}{|c|c|c|c|c|c|}
\hline ...habe ich das Ziel nicht anzuecken. & 口 & 口 & 口 & 口 & $\square$ \\
\hline $\begin{array}{l}\text {...beschäftige ich mich intensiv mit der Lösung eines Konfliktes } \\
\text { nach dem Auftreten. }\end{array}$ & 口 & $\square$ & 口 & 口 & $\square$ \\
\hline $\begin{array}{l}\text {...lasse ich die Dinge und Probleme manchmal wie sie sind und } \\
\text { kann damit auch gelassen umgehen. }\end{array}$ & 口 & $\square$ & $\square$ & $\square$ & $\square$ \\
\hline $\begin{array}{l}\text {... nehme ich Konflikte bewusst in Kauf um meine Ziele zu } \\
\text { erreichen. }\end{array}$ & $\square$ & $\square$ & $\square$ & $\square$ & $\square$ \\
\hline $\begin{array}{l}\text {...baue ich nur auf sicherem Boden und lasse die Finger von } \\
\text { Experimenten. }\end{array}$ & $\square$ & $\square$ & $\square$ & a & $\square$ \\
\hline ...setze ich mich gegen Widerstände durch. & 口 & $\square$ & 口 & 口 & $\square$ \\
\hline $\begin{array}{l}\text {...nutze ich das Entwicklungspotential, das in Konfliktsituationen } \\
\text { steckt. }\end{array}$ & 口 & $\square$ & $\square$ & a & $\square$ \\
\hline $\begin{array}{l}\text {...schlage ich lieber mir vertraute Wege ein bevor ich bei Neuem } \\
\text { das Risiko eingehe zu scheitern. }\end{array}$ & 口 & $\square$ & 口 & $\square$ & $\square$ \\
\hline $\begin{array}{l}\text {...lasse ich ein Problem erst einmal „auf kleiner Flamme } \\
\text { köcheln“, bevor ich es anpacke. }\end{array}$ & $\square$ & $\square$ & $\square$ & a & $\square$ \\
\hline $\begin{array}{l}\text {...nehme ich schwierige Situationen in denen viel von mir } \\
\text { abhängt gerne an. }\end{array}$ & $\square$ & $\square$ & $\square$ & 口 & $\square$ \\
\hline ...achte ich bewusst darauf Schwierigkeiten zu vermeiden. & 口 & 口 & 口 & 口 & $\square$ \\
\hline ...ist mir Ruhe und Gelassenheit besonders wichtig. & $\square$ & $\square$ & 口 & a & $\square$ \\
\hline ...mache ich mir keinen Stress. & $\square$ & $\square$ & 口 & a & $\square$ \\
\hline ...gehören Konflikte einfach dazu. & $\square$ & $\square$ & 口 & a & $\square$ \\
\hline ...warte ich erst mal ab (wenn ein Problem auftritt). & 口 & 口 & 口 & 口 & $\square$ \\
\hline ...gehe ich lieber auf Nummer sicher um Ärger zu vermeiden. & $\square$ & $\square$ & a & a & $\square$ \\
\hline $\begin{array}{l}\text {...antizipiere ich mögliche Konflikte und agiere bewusst so, dass } \\
\text { diese nicht entstehen. }\end{array}$ & $\square$ & 口 & 口 & $\square$ & $\square$ \\
\hline $\begin{array}{l}\text {...empfinde ich es als beunruhigend wenn sich ein Konflikt } \\
\text { anbahnt. }\end{array}$ & $\square$ & 口 & 口 & 口 & $\square$ \\
\hline $\begin{array}{l}\text {...gebe ich mich in Konfliktsituationen nicht geschlagen und } \\
\text { versuche zumindest, einen Kompromiss herauszuschlagen. }\end{array}$ & 口 & $\square$ & 口 & 口 & $\square$ \\
\hline $\begin{array}{l}\text {... versuche ich mich der Situation anzupassen wenn mal wieder } \\
\text { etwas schief läuft }\end{array}$ & 口 & 口 & 口 & 口 & $\square$ \\
\hline ...bin ich von meinen Zielvorstellungen nicht abzubringen. & 口 & $\square$ & 口 & 口 & $\square$ \\
\hline $\begin{array}{l}\text {...stecke ich meine Ziele nicht allzu hoch, weil ich oft befürchte, } \\
\text { dass ich scheitere. }\end{array}$ & 口 & 口 & 口 & a & $\square$ \\
\hline $\begin{array}{l}\text {...suche ich mir gern Herausforderungen und gehe dafür auch } \\
\text { Wagnisse ein. }\end{array}$ & $\square$ & $\square$ & $\square$ & a & 口 \\
\hline $\begin{array}{l}\text {...lasse ich ein Problem so lange ruhen, bis ich mich ihm } \\
\text { gewachsen fühle, wenn es mir zu schwierig erscheint. }\end{array}$ & 口 & 口 & 口 & 口 & 口 \\
\hline $\begin{array}{l}\text {...suche ich mir eine größere Herausforderung wenn ich ein Ziel } \\
\text { erreicht habe. }\end{array}$ & 口 & 口 & 口 & 口 & 口 \\
\hline $\begin{array}{l}\text {...kann mich nichts mehr aufhalten wenn ich mir etwas } \\
\text { vorgenommen habe. }\end{array}$ & a & 口 & 口 & 口 & 口 \\
\hline
\end{tabular}




\begin{tabular}{|c|c|c|c|c|c|}
\hline Im Bezug auf Inhalte im Sportunterricht... & $\begin{array}{c}\text { trifft } \\
\text { nicht zu }\end{array}$ & $\begin{array}{c}\text { trifft } \\
\text { eher } \\
\text { nicht zu }\end{array}$ & $\begin{array}{l}\text { teils/ } \\
\text { teils }\end{array}$ & $\begin{array}{l}\text { trifft } \\
\text { eher zu }\end{array}$ & trifft zu \\
\hline $\begin{array}{l}\text {...reizen mich Situationen in denen ich auch mal mit Konflikten zu tun } \\
\text { habe. }\end{array}$ & $\square$ & a & $\square$ & $\square$ & $\square$ \\
\hline ...versuche ich möglichst nicht auf Widerstände zu stoßen. & 口 & 口 & $\square$ & 口 & 口 \\
\hline $\begin{array}{l}\text {...warte ich bei Dingen die nicht so gut laufen lieber, bis sie sich von } \\
\text { selbst regeln. }\end{array}$ & 口 & 口 & 口 & 口 & $\square$ \\
\hline ...habe ich das Ziel nicht anzuecken. & $\square$ & 口 & $\square$ & $\square$ & $\square$ \\
\hline $\begin{array}{l}\text {...beschäftige ich mich intensiv mit der Lösung eines Konfliktes nach } \\
\text { dem Auftreten. }\end{array}$ & $\square$ & $\square$ & $\square$ & $\square$ & $\square$ \\
\hline $\begin{array}{l}\text {...lasse ich die Dinge und Probleme manchmal wie sie sind und kann } \\
\text { damit auch gelassen umgehen. }\end{array}$ & 口 & 口 & 口 & 口 & 口 \\
\hline ... nehme ich Konflikte bewusst in Kauf um meine Ziele zu erreichen. & $\square$ & $\square$ & $\square$ & $\square$ & $\square$ \\
\hline $\begin{array}{l}\text {...baue ich nur auf sicherem Boden und lasse die Finger von } \\
\text { Experimenten. }\end{array}$ & $\square$ & $\square$ & $\square$ & 口 & $\square$ \\
\hline ...setze ich mich gegen Widerstände durch. & $\square$ & $\square$ & $\square$ & $\square$ & $\square$ \\
\hline $\begin{array}{l}\text {...nutze ich das Entwicklungspotential, das in Konfliktsituationen } \\
\text { steckt. }\end{array}$ & $\square$ & $\square$ & $\square$ & $\square$ & $\square$ \\
\hline $\begin{array}{l}\text {...schlage ich lieber mir vertraute Wege ein bevor ich bei Neuem das } \\
\text { Risiko eingehe zu scheitern. }\end{array}$ & $\square$ & 口 & 口 & 口 & $\square$ \\
\hline $\begin{array}{l}\text {...lasse ich ein Problem erst einmal „auf kleiner Flamme köcheln“, } \\
\text { bevor ich es anpacke. }\end{array}$ & $\square$ & $\square$ & $\square$ & 口 & $\square$ \\
\hline $\begin{array}{l}\text {...nehme ich schwierige Situationen in denen viel von mir abhängt } \\
\text { gerne an. }\end{array}$ & $\square$ & $\square$ & $\square$ & $\square$ & 口 \\
\hline ....achte ich bewusst darauf Schwierigkeiten zu vermeiden. & a & a & $\square$ & a & a \\
\hline ...ist mir Ruhe und Gelassenheit besonders wichtig. & $\square$ & $\square$ & $\square$ & $\square$ & $\square$ \\
\hline ...mache ich mir keinen Stress. & $\square$ & 口 & $\square$ & 口 & 口 \\
\hline ...gehören Konflikte einfach dazu. & $\square$ & $\square$ & $\square$ & $\square$ & $\square$ \\
\hline ...warte ich erst mal ab (wenn ein Problem auftritt). & $\square$ & $\square$ & $\square$ & $\square$ & $\square$ \\
\hline ...gehe ich lieber auf Nummer sicher um Ärger zu vermeiden. & $\square$ & a & $\square$ & a & a \\
\hline $\begin{array}{l}\text {...antizipiere ich mögliche Konflikte und agiere bewusst so, dass diese } \\
\text { nicht entstehen. }\end{array}$ & 口 & 口 & $\square$ & 口 & $\square$ \\
\hline ...empfinde ich es als beunruhigend wenn sich ein Konflikt anbahnt. & $\square$ & $\square$ & $\square$ & $\square$ & $\square$ \\
\hline $\begin{array}{l}\text {...gebe ich mich in Konfliktsituationen nicht geschlagen und versuche } \\
\text { zumindest, einen Kompromiss herauszuschlagen. }\end{array}$ & 口 & 口 & 口 & 口 & 口 \\
\hline $\begin{array}{l}\text {... versuche ich mich der Situation anzupassen wenn mal wieder } \\
\text { etwas schief läuft. }\end{array}$ & $\square$ & $\square$ & $\square$ & 口 & $\square$ \\
\hline ...bin ich von meinen Zielvorstellungen nicht abzubringen. & $\square$ & $\square$ & $\square$ & 口 & $\square$ \\
\hline $\begin{array}{l}\text {...stecke ich meine Ziele nicht allzu hoch, weil ich oft befürchte, dass } \\
\text { ich scheitere. }\end{array}$ & $\square$ & 口 & 口 & 口 & 口 \\
\hline $\begin{array}{l}\text {...suche ich mir gern Herausforderungen und gehe dafür auch } \\
\text { Wagnisse ein. }\end{array}$ & a & 口 & $\square$ & 口 & 口 \\
\hline $\begin{array}{l}\text {...lasse ich ein Problem so lange ruhen, bis ich mich inm gewachsen } \\
\text { fühle, wenn es mir zu schwierig erscheint. }\end{array}$ & 口 & a & 口 & 口 & 口 \\
\hline $\begin{array}{l}\text {...suche ich mir eine größere Herausforderung wenn ich ein Ziel } \\
\text { erreicht habe. }\end{array}$ & $\square$ & $\square$ & $\square$ & 口 & $\square$ \\
\hline $\begin{array}{l}\text {...kann mich nichts mehr aufhalten wenn ich mir etwas } \\
\text { vorgenommen habe. }\end{array}$ & 口 & 口 & 口 & a & 口 \\
\hline
\end{tabular}




\begin{tabular}{|c|c|c|c|c|c|}
\hline $\begin{array}{l}\text { 13. Im Bezug auf äußere Bedingungen } \\
\text { (Vorschriften und Erlasse, Beziehungen zum Kollegium/zur Schulleitung, } \\
\text { Zeitordnungen, Notengebung, materielle Ausstattung...)... }\end{array}$ & $\begin{array}{c}\text { trifft } \\
\text { nicht zu }\end{array}$ & $\begin{array}{l}\text { trifft } \\
\text { eher } \\
\text { nicht zu }\end{array}$ & $\begin{array}{l}\text { teils/ } \\
\text { teils }\end{array}$ & $\begin{array}{l}\text { trifft } \\
\text { eher zu }\end{array}$ & trifft zu \\
\hline $\begin{array}{l}\text {...reizen mich Situationen in denen ich auch mal mit Konflikten zu tun } \\
\text { habe. }\end{array}$ & $\square$ & $\square$ & $\square$ & $\square$ & $\square$ \\
\hline ...versuche ich möglichst nicht auf Widerstände zu stoßen. & 口 & $\square$ & 口 & $\square$ & $\square$ \\
\hline $\begin{array}{l}\text {...warte ich bei Dingen die nicht so gut laufen lieber, bis sie sich von } \\
\text { selbst regeln. }\end{array}$ & a & a & $\square$ & $\square$ & $\square$ \\
\hline ...habe ich das Ziel nicht anzuecken. & 口 & 口 & $\square$ & $\square$ & $\square$ \\
\hline $\begin{array}{l}\text {...beschäftige ich mich intensiv mit der Lösung eines Konfliktes nach } \\
\text { dem Auftreten. }\end{array}$ & 口 & $\square$ & a & $\square$ & $\square$ \\
\hline $\begin{array}{l}\text {...lasse ich die Dinge und Probleme manchmal wie sie sind und kann } \\
\text { damit auch gelassen umgehen. }\end{array}$ & $\square$ & $\square$ & $\square$ & $\square$ & $\square$ \\
\hline ... nehme ich Konflikte bewusst in Kauf um meine Ziele zu erreichen. & a & $\square$ & a & $\square$ & $\square$ \\
\hline $\begin{array}{l}\text {...baue ich nur auf sicherem Boden und lasse die Finger von } \\
\text { Experimenten. }\end{array}$ & a & $\square$ & $\square$ & $\square$ & $\square$ \\
\hline ...setze ich mich gegen Widerstände durch. & 口 & $\square$ & $\square$ & $\square$ & $\square$ \\
\hline ...nutze ich das Entwicklungspotential, das in Konfliktsituationen steckt. & $\square$ & $\square$ & $\square$ & $\square$ & $\square$ \\
\hline $\begin{array}{l}\text {...schlage ich lieber mir vertraute Wege ein bevor ich bei Neuem das } \\
\text { Risiko eingehe zu scheitern. }\end{array}$ & a & $\square$ & a & $\square$ & $\square$ \\
\hline $\begin{array}{l}\text {...lasse ich ein Problem erst einmal „auf kleiner Flamme köcheln“, } \\
\text { bevor ich es anpacke. }\end{array}$ & $\square$ & $\square$ & 口 & $\square$ & $\square$ \\
\hline $\begin{array}{l}\text {...nehme ich schwierige Situationen in denen viel von mir abhängt } \\
\text { gerne an. }\end{array}$ & a & $\square$ & a & $\square$ & $\square$ \\
\hline ....achte ich bewusst darauf Schwierigkeiten zu vermeiden. & a & $\square$ & a & $\square$ & $\square$ \\
\hline ...ist mir Ruhe und Gelassenheit besonders wichtig. & a & 口 & a & $\square$ & a \\
\hline ...mache ich mir keinen Stress. & 口 & 口 & $\square$ & $\square$ & 口 \\
\hline ...gehören Konflikte einfach dazu. & 口 & $\square$ & 口 & $\square$ & $\square$ \\
\hline ...warte ich erst mal ab (wenn ein Problem auftritt). & 口 & 口 & 口 & $\square$ & 口 \\
\hline ...gehe ich lieber auf Nummer sicher um Ärger zu vermeiden. & 口 & $\square$ & 口 & $\square$ & 口 \\
\hline $\begin{array}{l}\text {...antizipiere ich mögliche Konflikte und agiere bewusst so, dass diese } \\
\text { nicht entstehen. }\end{array}$ & a & $\square$ & $\square$ & $\square$ & $\square$ \\
\hline ...empfinde ich es als beunruhigend wenn sich ein Konflikt anbahnt. & $\square$ & a & a & $\square$ & $\square$ \\
\hline $\begin{array}{l}\text {...gebe ich mich in Konfliktsituationen nicht geschlagen und versuche } \\
\text { zumindest, einen Kompromiss herauszuschlagen. }\end{array}$ & 口 & $\square$ & 口 & $\square$ & $\square$ \\
\hline $\begin{array}{l}\text {... versuche ich mich der Situation anzupassen wenn mal wieder } \\
\text { etwas schief läuft. }\end{array}$ & a & $\square$ & $\square$ & $\square$ & $\square$ \\
\hline ...bin ich von meinen Zielvorstellungen nicht abzubringen. & a & a & a & ם & 口 \\
\hline $\begin{array}{l}\text {...stecke ich meine Ziele nicht allzu hoch, weil ich oft befürchte, dass } \\
\text { ich scheitere. }\end{array}$ & a & $\square$ & a & $\square$ & 口 \\
\hline $\begin{array}{l}\text {...suche ich mir gern Herausforderungen und gehe dafür auch } \\
\text { Wagnisse ein. }\end{array}$ & $\square$ & $\square$ & $\square$ & $\square$ & 口 \\
\hline $\begin{array}{l}\text {...lasse ich ein Problem so lange ruhen, bis ich mich inm gewachsen } \\
\text { fühle, wenn es mir zu schwierig erscheint. }\end{array}$ & $\square$ & $\square$ & a & $\square$ & 口 \\
\hline $\begin{array}{l}\text {...suche ich mir eine größere Herausforderung wenn ich ein Ziel } \\
\text { erreicht habe. }\end{array}$ & 口 & 口 & 口 & 口 & 口 \\
\hline $\begin{array}{l}\text {...kann mich nichts mehr aufhalten wenn ich mir etwas } \\
\text { vorgenommen habe. }\end{array}$ & 口 & 口 & 口 & 口 & 口 \\
\hline
\end{tabular}




\begin{tabular}{|c|c|c|c|c|c|c|}
\hline \multicolumn{2}{|c|}{$\begin{array}{l}\text { 14. Schätzen Sie die Bedeutsamkeit } \\
\text { folgender Vermittlungsaspekte für } \\
\text { „Ihren“ Unterricht ein! }\end{array}$} & $\begin{array}{l}\text { (sehr) } \\
\text { unwichtig }\end{array}$ & \multicolumn{2}{|c|}{$\begin{array}{c}\text { eher } \\
\text { unwichtig }\end{array}$} & $\begin{array}{l}\text { eher } \\
\text { wichtig }\end{array}$ & $\begin{array}{l}\text { (sehr) } \\
\text { wichtig }\end{array}$ \\
\hline \multicolumn{2}{|c|}{ Soziales Lernen } & $\square$ & $\square$ & & $\square$ & $\square$ \\
\hline \multicolumn{2}{|c|}{ Gesundheitserziehung } & $\square$ & $\square$ & & $\square$ & $\square$ \\
\hline \multicolumn{2}{|c|}{ Fachliche (theoretische) Kenntnisse } & $\square$ & $\square$ & & $\square$ & $\square$ \\
\hline \multicolumn{2}{|c|}{ Kondition } & $\square$ & $\square$ & & $\square$ & $\square$ \\
\hline \multicolumn{2}{|c|}{ Kreativität } & $\square$ & $\square$ & & $\square$ & $\square$ \\
\hline \multicolumn{2}{|c|}{ Freude an Bewegung } & $\square$ & $\square$ & & $\square$ & $\square$ \\
\hline \multicolumn{2}{|c|}{ Leistungserziehung } & $\square$ & $\square$ & & $\square$ & $\square$ \\
\hline \multicolumn{2}{|c|}{ Persönlichkeitsentwicklung } & $\square$ & $\square$ & & $\square$ & $\square$ \\
\hline \multicolumn{2}{|c|}{ Selbständigkeit } & $\square$ & $\square$ & & $\square$ & $\square$ \\
\hline \multicolumn{2}{|c|}{ Körperbildung } & $\square$ & 口 & & $\square$ & $\square$ \\
\hline \multicolumn{2}{|c|}{ Lebensbegleitendes Sporttreiben } & $\square$ & $\square$ & & $\square$ & $\square$ \\
\hline \multicolumn{2}{|c|}{ Taktik } & $\square$ & 口 & & $\square$ & $\square$ \\
\hline \multicolumn{2}{|c|}{ Teamfähigkeit, Teamgeist } & $\square$ & $\square$ & & $\square$ & 口 \\
\hline \multicolumn{2}{|c|}{ Technik } & $\square$ & $\square$ & & $\square$ & $\square$ \\
\hline \multicolumn{2}{|c|}{ Fitness } & $\square$ & $\square$ & & $\square$ & $\square$ \\
\hline \multicolumn{2}{|c|}{ Koordinative Fähigkeiten } & $\square$ & $\square$ & & $\square$ & $\square$ \\
\hline \multicolumn{2}{|c|}{ (Wett-) Kampfgeist } & $\square$ & $\square$ & & $\square$ & $\square$ \\
\hline \multicolumn{2}{|c|}{ Körpererfahrung } & $\square$ & $\square$ & & $\square$ & $\square$ \\
\hline \multicolumn{2}{|c|}{ Spielen (Spielfähigkeit)) } & $\square$ & $\square$ & & $\square$ & $\square$ \\
\hline \multicolumn{2}{|c|}{ Disziplin } & 口 & 口 & & 口 & $\square$ \\
\hline \multicolumn{2}{|c|}{ Bewegungsfertigkeit } & $\square$ & \multicolumn{2}{|l|}{ a } & $\square$ & $\square$ \\
\hline & Berufliche Belastung & $\begin{array}{l}\text { trifft gar } \\
\text { nicht zu }\end{array}$ & $\begin{array}{l}\text { trifft } \\
\text { wenig zu }\end{array}$ & $\begin{array}{c}\text { trifft } \\
\text { mittel- } \\
\text { mäßig zu }\end{array}$ & $\begin{array}{l}\text { trifft über- } \\
\text { wiegend zu }\end{array}$ & trifft zu \\
\hline Meir & Beruf macht mir Spaß. & $\square$ & $\square$ & $\square$ & $\square$ & $\square$ \\
\hline In $\mathrm{m}$ & nem Beruf wird man ständig überfordert. & $\square$ & $\square$ & $\square$ & $\square$ & $\square$ \\
\hline $\begin{array}{l}\text { Ich h } \\
\text { ausz }\end{array}$ & $\begin{array}{l}\text { be mir schon ernsthaft überlegt aus dem Beruf } \\
\text { teigen. }\end{array}$ & $\square$ & $\square$ & $\square$ & $\square$ & $\square$ \\
\hline $\begin{array}{l}\text { Was } \\
\text { zufri }\end{array}$ & $\begin{array}{l}\text { heine Arbeit betrifft, bin ich eigentlich rundum } \\
\text { den. }\end{array}$ & 口 & a & a & a & a \\
\hline $\begin{array}{l}\text { Ich } f( \\
\text { kont }\end{array}$ & $\begin{array}{l}\text { le mich von meinem Arbeitgeber ständig } \\
\text { lliert und überwacht. }\end{array}$ & $\square$ & $\square$ & $\square$ & $\square$ & $\square$ \\
\hline $\begin{array}{l}\text { Ich h } \\
\text { zu m }\end{array}$ & $\begin{array}{l}\text { be das Gefühl auch vor Kollegen ständig betonen } \\
\text { ssen, dass ich viel arbeite. }\end{array}$ & $\square$ & $\square$ & $\square$ & $\square$ & $\square$ \\
\hline Ich $\mathrm{h}$ & Ite mich gerne an meinem Arbeitsplatz auf. & $\square$ & 口 & a & $\square$ & $\square$ \\
\hline Ich $\mathrm{h}$ & be Konflikte mit Vorgesetzten, die mich belasten. & $\square$ & 口 & $\square$ & $\square$ & 口 \\
\hline Ich fi & le mich häufig überfordert. & $\square$ & $\square$ & $\square$ & $\square$ & 口 \\
\hline $\begin{array}{l}\text { Ich h } \\
\text { könn }\end{array}$ & $\begin{array}{l}\text { be selten das Gefühl, einmal richtig abschalten zu } \\
\text { n. }\end{array}$ & $\square$ & a & 口 & 口 & a \\
\hline Ich $\mathrm{k}$ & nn in meinem Beruf meine Fähigkeiten voll & $\square$ & $\square$ & $\square$ & $\square$ & $\square$ \\
\hline
\end{tabular}




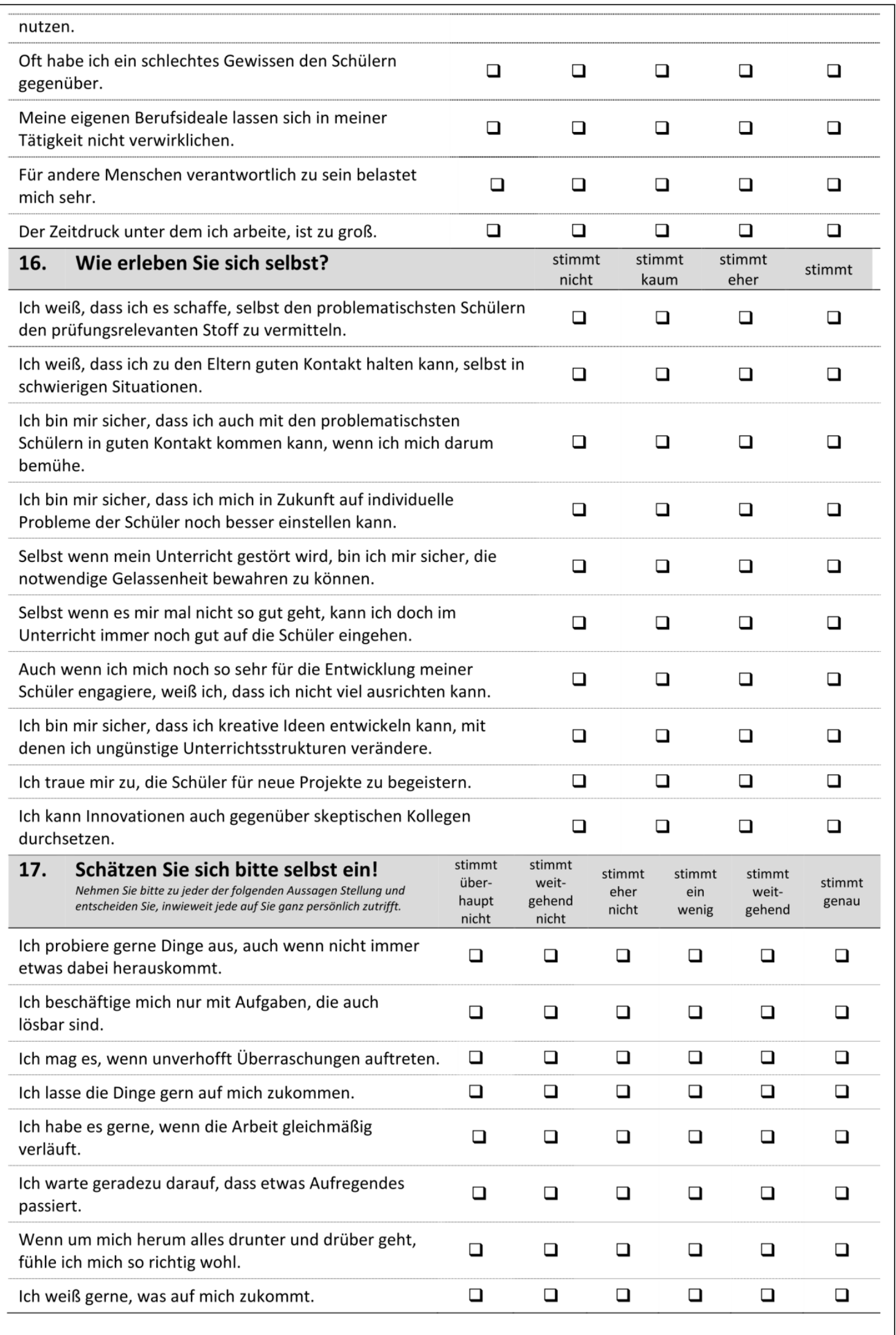




\begin{tabular}{|c|c|c|c|c|c|}
\hline $\begin{array}{l}\text { Wir bitten Sie hier einige Ihrer üblichen Verhaltensweisen, } \\
\text { Einstellungen und Gewohnheiten zu beschreiben, die Sie in Ihrem } \\
\text { Beruf als Sportlehrer vertreten bzw. kennzzeichnen. } \\
\text { Aussagen: Lesen Sie jeden dieser Sätze gründlich durch und } \\
\text { entscheiden Sie, in welchem Maße er auf Sie persönlich zutrifft. }\end{array}$ & $\begin{array}{c}\text { trifft } \\
\text { überhaupt } \\
\text { nicht zu }\end{array}$ & $\begin{array}{l}\text { über- } \\
\text { wiegend } \\
\text { nicht zu }\end{array}$ & teils/teils & $\begin{array}{c}\text { trifft } \\
\text { über- } \\
\text { wiegend } \\
\text { zu }\end{array}$ & $\begin{array}{c}\text { trifft völlig } \\
z u\end{array}$ \\
\hline Die Arbeit ist für mich der wichtigste Lebensinhalt. & $\square$ & $\square$ & $\square$ & ם & ם \\
\hline $\begin{array}{l}\text { Ich möchte beruflich weiter kommen, als es die } \\
\text { meisten meiner Bekannten geschafft haben. }\end{array}$ & $\square$ & व & $\square$ & $\square$ & ם \\
\hline Wenn es sein muss, arbeite ich bis zur Erschöpfung. & 口 & $\square$ & ם & ם & $\square$ \\
\hline Meine Arbeit soll stets ohne Fehl und Tadel sein. & $\square$ & a & 口 & $\square$ & $\square$ \\
\hline Zum Feierabend ist die Arbeit für mich gegessen. & $\square$ & $\square$ & $\square$ & $\square$ & $\square$ \\
\hline Wenn ich keinen Erfolg habe, resigniere ich schnell. & $\square$ & $\square$ & $\square$ & $\square$ & $\square$ \\
\hline $\begin{array}{l}\text { Für mich sind Schwierigkeiten dazu da, dass ich sie } \\
\text { überwinde. }\end{array}$ & $\square$ & $\square$ & व & $\square$ & $\square$ \\
\hline Mich bringt so leicht nichts aus der Ruhe. & $\square$ & $\square$ & $\square$ & $\square$ & $\square$ \\
\hline Mein bisheriges Berufsleben war recht erfolgreich. & $\square$ & $\square$ & $\square$ & ם & $\square$ \\
\hline $\begin{array}{l}\text { Mit meinem bisherigen Leben kann ich zufrieden } \\
\text { sein. }\end{array}$ & ם & $\square$ & a & $\square$ & $\square$ \\
\hline Mein Partner zeigt Verständnis für meine Arbeit. & $\square$ & $\square$ & $\square$ & $\square$ & 口 \\
\hline Die Arbeit ist mein ein und alles. & $\square$ & $\square$ & $\square$ & ם & $\square$ \\
\hline Berufliche Karriere bedeutet mir wenig. & $\square$ & $\square$ & $\square$ & $\square$ & $\square$ \\
\hline Bei der Arbeit kenne ich keine Schonung. & $\square$ & $\square$ & $\square$ & ם & 口 \\
\hline $\begin{array}{l}\text { Ich kontrolliere lieber noch dreimal nach, als dass ich } \\
\text { fehlerhafte Arbeitsergebnisse abliefere. }\end{array}$ & ם & ם & a & ם & ם \\
\hline $\begin{array}{l}\text { Auch in der Freizeit beschäftigen mich viele } \\
\text { Arbeitsprobleme. }\end{array}$ & $\square$ & $\square$ & $\square$ & $\square$ & $\square$ \\
\hline Misserfolg kann ich nur schwer verkraften. & $\square$ & $\square$ & $\square$ & $\square$ & $\square$ \\
\hline $\begin{array}{l}\text { Wenn mir etwas nicht gelingt, sage ich mir: Jetzt erst } \\
\text { recht! }\end{array}$ & $\square$ & $\square$ & व & $\square$ & $\square$ \\
\hline Ich bin ein ruheloser Mensch. & $\square$ & $\square$ & $\square$ & $\square$ & $\square$ \\
\hline $\begin{array}{l}\text { In meiner bisherigen Berufslaufbahn habe ich mehr } \\
\text { Erfolg als Enttäuschungen erlebt. }\end{array}$ & $\square$ & $\square$ & ם & $\square$ & ם \\
\hline $\begin{array}{l}\text { Im Großen und Ganzen bin ich glücklich und } \\
\text { zufrieden. }\end{array}$ & 口 & व & व & व & ם \\
\hline $\begin{array}{l}\text { Meine Familie interessiert sich nur wenig für meine } \\
\text { Arbeitsprobleme. }\end{array}$ & व & ם & $\square$ & $\square$ & $\square$ \\
\hline Ich könnte auch ohne meine Arbeit ganz glücklich sein. & a & 口 & a & a & a \\
\hline $\begin{array}{l}\text { Was meine berufliche Entwicklung angeht, so halte } \\
\text { ich mich für ziemlich ehrgeizig. }\end{array}$ & व & ם & ם & ם & ם \\
\hline Ich arbeite wohl mehr als ich sollte. & ם & $\square$ & ם & $\square$ & $\square$ \\
\hline $\begin{array}{l}\text { Bei meiner Arbeit habe ich den Ehrgeiz, keinerlei } \\
\text { Fehler zu machen. }\end{array}$ & व & $\square$ & ם & ם & ם \\
\hline Nach der Arbeit kann ich ohne Probleme abschalten. & $\square$ & $\square$ & ם & $\square$ & $\square$ \\
\hline $\begin{array}{l}\text { Berufliche Fehlschläge können mich leicht } \\
\text { entmutigen. }\end{array}$ & ם & $\square$ & ם & ם & $\square$ \\
\hline Misserfolge werfen mich nicht um, sondern & ם & ם & a & ם & ם \\
\hline
\end{tabular}




\begin{tabular}{|c|c|c|c|c|c|}
\hline Ich glaube, dass ich ziemlich hektisch bin. & $\square$ & $\square$ & $\square$ & $\square$ & $\square$ \\
\hline $\begin{array}{l}\text { Wirkliche berufliche Erfolge sind mir bisher versagt } \\
\text { geblieben. }\end{array}$ & $\square$ & $\square$ & $\square$ & $\square$ & $\square$ \\
\hline $\begin{array}{l}\text { Ich habe allen Grund, meine Zukunft optimistisch zu } \\
\text { sehen. }\end{array}$ & $\square$ & $\square$ & $\square$ & $\square$ & $\square$ \\
\hline Von meinem Partner wünschte ich mir mehr & & & & & \\
\hline $\begin{array}{l}\text { Rücksichtnahme auf meine beruflichen Aufgaben und } \\
\text { Probleme. }\end{array}$ & $\square$ & $\square$ & $\square$ & $\square$ & $\square$ \\
\hline Ich brauche die Arbeit wie die Luft zum Atmen. & $\square$ & $\square$ & $\square$ & $\square$ & $\square$ \\
\hline $\begin{array}{l}\text { Ich strebe nach höheren beruflichen Zielen als die } \\
\text { meisten anderen. }\end{array}$ & $\square$ & $\square$ & 口 & 口 & $\square$ \\
\hline Ich neige dazu, über meine Kräfte hinaus zu arbeiten. & $\square$ & $\square$ & $\square$ & $\square$ & $\square$ \\
\hline Was immer ich tue, es muss perfekt sein. & $\square$ & $\square$ & $\square$ & $\square$ & $\square$ \\
\hline $\begin{array}{l}\text { Feierabend ist Feierabend, da verschwende ich keine } \\
\text { Gedanken mehr an die Arbeit. }\end{array}$ & $\square$ & 口 & 口 & 口 & $\square$ \\
\hline $\begin{array}{l}\text { Wenn ich in der Arbeit erfolglos bin, deprimiert mich } \\
\text { das sehr. }\end{array}$ & $\square$ & a & a & a & $\square$ \\
\hline $\begin{array}{l}\text { Ich bin mir sicher, dass ich auch die künftigen } \\
\text { Anforderungen des Lebens gut bewältigen kann. }\end{array}$ & $\square$ & $\square$ & $\square$ & $\square$ & $\square$ \\
\hline Ich glaube, ich bin ein ruhender Pol in meinem Umfeld. & $\square$ & $\square$ & $\square$ & $\square$ & $\square$ \\
\hline $\begin{array}{l}\text { In meiner beruflichen Entwicklung ist mir bisher fast } \\
\text { alles gelungen. }\end{array}$ & $\square$ & $\square$ & $\square$ & a & $\square$ \\
\hline $\begin{array}{l}\text { Ich kann mich über mein Leben in keiner Weise } \\
\text { beklagen. }\end{array}$ & $\square$ & $\square$ & $\square$ & $\square$ & $\square$ \\
\hline Bei meiner Familien finde ich jede Unterstützung. & $\square$ & $\square$ & $\square$ & $\square$ & $\square$ \\
\hline Ich wüsste nicht wie ich ohne Arbeit leben sollte. & $\square$ & a & a & 口 & $\square$ \\
\hline $\begin{array}{l}\text { Für meine berufliche Zukunft habe ich mir viel } \\
\text { vorgenommen. }\end{array}$ & $\square$ & 口 & a & a & $\square$ \\
\hline $\begin{array}{l}\text { Mein Tagesablauf ist durch chronischen Zeitmangel } \\
\text { bestimmt. }\end{array}$ & $\square$ & $\square$ & 口 & 口 & $\square$ \\
\hline $\begin{array}{l}\text { Für mich ist die Arbeit erst dann getan, wenn ich } \\
\text { rundum mit dem Ergebnis zufrieden bin. }\end{array}$ & $\square$ & $\square$ & $\square$ & $\square$ & $\square$ \\
\hline $\begin{array}{l}\text { Arbeitsprobleme beschäftigen mich eigentlich den } \\
\text { ganzen Tag. }\end{array}$ & $\square$ & a & $\square$ & $\square$ & $\square$ \\
\hline $\begin{array}{l}\text { Ich verliere leicht den Mut, wenn ich trotz } \\
\text { Anstrengung keinen Erfolg habe. }\end{array}$ & $\square$ & $\square$ & $\square$ & $\square$ & $\square$ \\
\hline Ein Misserfolg kann bei mir neue Kräfte wecken. & $\square$ & $\square$ & $\square$ & 口 & $\square$ \\
\hline $\begin{array}{l}\text { Ich kann mich in fast allen Situationen ruhig und } \\
\text { bedächtig verhalten. }\end{array}$ & $\square$ & $\square$ & a & a & $\square$ \\
\hline $\begin{array}{l}\text { Mein bisheriges Leben ist durch beruflichen Erfolg } \\
\text { gekennzeichnet. }\end{array}$ & $\square$ & a & $\square$ & a & $\square$ \\
\hline $\begin{array}{l}\text { Von manchen Seiten des Lebens bin ich ziemlich } \\
\text { enttäuscht. }\end{array}$ & $\square$ & $\square$ & $\square$ & $\square$ & $\square$ \\
\hline $\begin{array}{l}\text { Manchmal wünschte ich mir mehr Unterstützung } \\
\text { durch die Menschen meiner Umgebung. }\end{array}$ & $\square$ & $\square$ & $\square$ & $\square$ & $\square$ \\
\hline Es gibt wichtigeres im Leben als die Arbeit. & $\square$ & $\square$ & $\square$ & $\square$ & $\square$ \\
\hline
\end{tabular}




\begin{tabular}{|c|c|c|c|c|c|c|}
\hline $\begin{array}{l}\text { Beruflicher Erfolg ist für mich ein wichtiges } \\
\text { Lebensziel. }\end{array}$ & $\square$ & $\square$ & $\square$ & $\square$ & & $\square$ \\
\hline In der Arbeit verausgabe ich mich stark. & $\square$ & $\square$ & $\square$ & $\square$ & & $\square$ \\
\hline $\begin{array}{l}\text { Es widerstrebt mir, wenn ich eine Arbeit abschließen } \\
\text { muss, obwohl sie noch verbessert werden könnte. }\end{array}$ & $\square$ & $\square$ & $\square$ & $\square$ & & $\square$ \\
\hline Meine Gedanken kreisen fast nur um die Arbeit. & $\square$ & $\square$ & $\square$ & $\square$ & & $\square$ \\
\hline $\begin{array}{l}\text { Wenn ich irgendwo versagt habe, kann mich das } \\
\text { ziemlich mutlos machen. }\end{array}$ & $\square$ & $\square$ & $\square$ & $\square$ & & $\square$ \\
\hline $\begin{array}{l}\text { Wenn mir etwas nicht gelingt, bleibe ich hartnäckig } \\
\text { und strenge mich umso mehr an. }\end{array}$ & $\square$ & $\square$ & $\square$ & $\square$ & & $\square$ \\
\hline Hektik und Aufregung um mich herum lassen mich kalt. & 口 & 口 & $\square$ & $\square$ & & $\square$ \\
\hline Meine beruflichen Leistungen können sich sehen lassen. & $\square$ & 口 & 口 & $\square$ & & 口 \\
\hline $\begin{array}{l}\text { Es dürfte nur wenige glücklichere Menschen geben } \\
\text { als ich es bin. }\end{array}$ & $\square$ & $\square$ & $\square$ & $\square$ & & $\square$ \\
\hline $\begin{array}{l}\text { Wenn ich mal Rat und Hilfe brauche ist immer jemand } \\
\text { da. }\end{array}$ & a & $\square$ & $\square$ & $\square$ & & $\square$ \\
\hline \multicolumn{7}{|c|}{ 19. In meinem Sportunterricht mache ich selber aktiv mit! } \\
\hline a ab und $\mathrm{zu}$ & & 口 oft & & 口 & immer & \\
\hline
\end{tabular}

20. Es macht mir Spaß das Fach Sport zu unterrichten!
口 nie
selten
a $a b$ und $z u$
$\square$ oft
a immer

21. Die Anforderungen an die Schüler in meinem Sportunterricht im technisch-taktischen 21. Bereich sind...
ㄱ niedrig
a eher niedrig
a mittel
eher hoch
a hoch

22. Die Anforderungen an die Schüler in meinem Sportunterricht im konditionellen Bereich 22. sind...

口 niedrig $\square$ eher niedrig $\square$ mittel $\square$ eherhoch $\square$ hoch

23. Welchen Stellenwert hat der Sportunterricht für Sie persönlich im Vergleich mit anderen 23. Fächern?

$\square$ deutlich geringer $\square$ eher geringer $\square$ gleichwertig $\square$ eherhöher $\square$ höher

24. Welchen Stellenwert räumen Sie Sport in Ihrem Privatleben ein?
gering
a eher geringer
a mittel
a eher hoch
a hoch

25. Wie schätzen Sie Ihren allgemeinen Fitnesszustand ein?
口 (sehr) schlecht
a eher schlecht
a mittel
$\square$ eher gut
(sehr) gut

26. In welchem Umfang (pro Woche) betreiben Sie in Ihrer Freizeit Sport?
ㅁ nie
a bis zu $2 \mathrm{~h}$
a 2 bis $4 \mathrm{~h}$
․ 4 bis $6 \mathrm{~h}$
mehr als $6 \mathrm{~h}$

27. Haben Sie früher aktiv Wettkampfsport betrieben bzw. sind Sie noch aktiv?
a ja (früher)
口 ja (jetzt noch)
a nein

28. Sind Sie als Vereinstrainer, Übungsleiter, Kadertraining o.Ä. in den letzten 5 Jahren 28. aktiv gewesen?
a ja
nein 


\begin{tabular}{|c|c|c|c|c|c|c|c|}
\hline 29. & \multicolumn{2}{|l|}{$\begin{array}{l}\text { Bitte schätzen Sie ein inwieweit die folgenden } \\
\text { Aussagen auf sie persönlich zutreffen! }\end{array}$} & $\begin{array}{c}\text { Stimmt } \\
\text { nicht }\end{array}$ & $\begin{array}{c}\text { Stimmt } \\
\text { kaum }\end{array}$ & \multicolumn{2}{|c|}{$\begin{array}{l}\text { Stimmt } \\
\text { eher }\end{array}$} & Stimmt \\
\hline \multicolumn{3}{|c|}{$\begin{array}{l}\text { Es macht mir Spaß an Problemen zu arbeiten, die für mich ein } \\
\text { bisschen schwierig sind. }\end{array}$} & a & a & \multicolumn{2}{|c|}{ a } & $\square$ \\
\hline \multicolumn{3}{|c|}{ Ich mag Situationen, in denen ich feststellen kann, wie gut ich bin. } & 口 & $\square$ & \multicolumn{2}{|c|}{$\square$} & 口 \\
\hline \multicolumn{3}{|c|}{$\begin{array}{l}\text { Wenn eine Sache etwas schwierig ist, hoffe ich, dass ich es nicht } \\
\text { machen muss, weil ich Angst habe, es nicht zu schaffen. }\end{array}$} & a & 口 & \multicolumn{2}{|c|}{ 口 } & $\square$ \\
\hline \multicolumn{3}{|c|}{ Probleme, die schwierig zu lösen sind, reizen mich. } & $\square$ & $\square$ & \multicolumn{2}{|c|}{$\square$} & $\square$ \\
\hline \multicolumn{3}{|c|}{$\begin{array}{l}\text { Es beunruhigt mich, etwas zu tun, wenn ich nicht sicher bin, dass ich es } \\
\text { kann. }\end{array}$} & $\square$ & 口 & \multicolumn{2}{|c|}{$\square$} & $\square$ \\
\hline \multicolumn{3}{|c|}{ Ich möchte gern vor eine etwas schwierige Arbeit gestellt werden. } & $\square$ & $\square$ & \multicolumn{2}{|c|}{$\square$} & $\square$ \\
\hline \multicolumn{3}{|c|}{$\begin{array}{l}\text { Auch bei Aufgaben, von denen ich glaube, dass ich sie kann, habe ich } \\
\text { Angst zu versagen. }\end{array}$} & $\square$ & $\square$ & \multicolumn{2}{|c|}{$\square$} & 口 \\
\hline \multicolumn{3}{|c|}{ Dinge, die etwas schwierig sind, beunruhigen mich. } & $\square$ & 口 & \multicolumn{2}{|c|}{$\square$} & $\square$ \\
\hline \multicolumn{3}{|c|}{$\begin{array}{l}\text { Mich reizen Situationen, in denen ich meine Fähigkeiten testen } \\
\text { kann. }\end{array}$} & $\square$ & a & \multicolumn{2}{|c|}{$\square$} & $\square$ \\
\hline \multicolumn{3}{|c|}{ Wenn ich ein Problem nicht sofort verstehe, werde ich ängstlich. } & $\square$ & $\square$ & \multicolumn{2}{|c|}{$\square$} & $\square$ \\
\hline 30. & $\begin{array}{l}\text { Bitte treffen Sie auch hier eine Auswahl } \\
\text { inwieweit die folgenden allgemein } \\
\text { formulierten Aussagen auf Sie zutreffen! }\end{array}$ & $\begin{array}{c}\text { trifft } \\
\text { über- } \\
\text { haupt } \\
\text { nicht zu }\end{array}$ & $\begin{array}{l}\text { trifft } \\
\text { weit- } \\
\text { gehend } \\
\text { nicht } \\
\text { zu }\end{array}$ & $\begin{array}{l}\text { trifft } \\
\text { eher } \\
\text { nicht } \\
\text { zu }\end{array}$ & $\begin{array}{l}\text { trifft } \\
\text { eher zu }\end{array}$ & $\begin{array}{l}\text { trifft } \\
\text { weit- } \\
\text { gehend } \\
\text { zu }\end{array}$ & $\begin{array}{l}\text { trifft } \\
\text { genau } \\
\text { zu }\end{array}$ \\
\hline \multicolumn{2}{|c|}{$\begin{array}{l}\text { Es hängt hauptsächlich von mir ab, ob sich andere Menschen } \\
\text { nach meinen Wünschen richten oder nicht. }\end{array}$} & $\square$ & 口 & 口 & 口 & $\square$ & $\square$ \\
\hline \multicolumn{2}{|c|}{$\begin{array}{l}\text { Zufällige Geschehnisse bestimmen einen großen Teil meines } \\
\text { Lebens und Alltags. }\end{array}$} & a & a & a & a & $\square$ & $\square$ \\
\hline \multicolumn{2}{|c|}{$\begin{array}{l}\text { Ich habe das Gefühl, dass vieles von dem, was in meinem } \\
\text { Leben passiert, von anderen Menschen abhängt. }\end{array}$} & 口 & a & a & a & a & a \\
\hline \multicolumn{2}{|c|}{ Ich komme mir manchmal taten- und ideenlos vor. } & a & a & ם & ם & a & a \\
\hline \multicolumn{2}{|c|}{$\begin{array}{l}\text { Ob ich einen Unfall habe oder nicht, hängt alleine von mir } \\
\text { und meinem Verhalten ab. }\end{array}$} & 口 & 口 & a & 口 & a & a \\
\hline \multicolumn{2}{|c|}{$\begin{array}{l}\text { Wenn ich Pläne schmiede, bin ich mir ganz sicher, dass das } \\
\text { Geplante auch Wirklichkeit wird. }\end{array}$} & ם & ם & a & ם & ב & a \\
\hline \multicolumn{2}{|c|}{$\begin{array}{l}\text { Ich habe oft einfach keine Möglichkeiten, mich vor Pech zu } \\
\text { schützen. }\end{array}$} & ם & ם & 口 & ב & ב & 口 \\
\hline \multicolumn{2}{|c|}{$\begin{array}{l}\text { Mehrdeutige Situationen mag ich nicht, da ich nicht weiß, } \\
\text { wie ich mich verhalten soll. }\end{array}$} & ב & ם & 口 & ב & ב & 口 \\
\hline \multicolumn{2}{|c|}{$\begin{array}{l}\text { Wenn ich bekomme, was ich will, so spielt Glück meistens } \\
\text { auch eine Rolle. }\end{array}$} & 口 & 口 & 口 & 口 & 口 & a \\
\hline \multicolumn{2}{|c|}{$\begin{array}{l}\text { Andere Menschen verhindern oft die Verwirklichung meiner } \\
\text { Pläne. }\end{array}$} & ם & ם & a & 口 & ב & a \\
\hline $\begin{array}{l}\text { Ich ka } \\
\text { Krank }\end{array}$ & $\begin{array}{l}\text { In mich am besten selbst durch mein Verhalten vor } \\
\text { eiten schützen. }\end{array}$ & ם & ם & ב & ב & ב & a \\
\hline Ich w & iß oft nicht, wie ich meine Wünsche verwirklichen soll. & a & a & $\square$ & a & a & $\square$ \\
\hline $\begin{array}{l}\text { Vieles } \\
\text { Zufall }\end{array}$ & $\begin{array}{l}\text { von dem, was in meinem Leben passiert, hängt vom } \\
\text { ab. }\end{array}$ & $\square$ & a & a & a & a & $\square$ \\
\hline $\begin{array}{l}\text { Mein } \\
\text { ander }\end{array}$ & $\begin{array}{l}\text { eben und Alltag werden in vielen Bereichen von } \\
\text { en Menschen bestimmt. }\end{array}$ & a & $\square$ & $\square$ & $\square$ & $\square$ & $\square$ \\
\hline Ob ic & einen Unfall habe oder nicht, ist vor allem & $\square$ & $\square$ & 口 & $\square$ & $\square$ & 口 \\
\hline
\end{tabular}




\begin{tabular}{|c|c|c|c|c|c|c|c|c|c|}
\hline \multicolumn{10}{|c|}{ Glücksache. } \\
\hline \multicolumn{3}{|c|}{$\begin{array}{l}\text { Ich kenne viele Möglichkeiten, mich vor Erkrankungen zu } \\
\text { schützen. }\end{array}$} & 口 & & a & 口 & a & a & 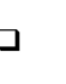 \\
\hline \multicolumn{3}{|c|}{$\begin{array}{l}\text { Ich habe nur geringe Möglichkeiten, meine Interessen gegen } \\
\text { andere Leute durchzusetzen. }\end{array}$} & 口 & & 口 & $\square$ & $\square$ & 口 & 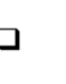 \\
\hline \multicolumn{3}{|c|}{$\begin{array}{l}\text { Es ist für mich nicht gut, weit im Voraus zu planen, da häufig } \\
\text { das Schicksal dazwischenkommt. }\end{array}$} & 口 & & a & 口 & $\square$ & $\square$ & $\square$ \\
\hline \multicolumn{3}{|c|}{$\begin{array}{l}\text { Um das zu bekommen, was ich will, muss ich zu anderen } \\
\text { Menschen freundlich und zuvorkommend sein. }\end{array}$} & $\square$ & & 口 & 口 & 口 & 口 & $\square$ \\
\hline \multicolumn{3}{|c|}{$\begin{array}{l}\text { In unklaren oder gefährlichen Situationen weiß ich immer, } \\
\text { was ich tun kann. }\end{array}$} & 口 & & 口 & 口 & $\square$ & a & 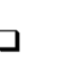 \\
\hline \multicolumn{3}{|c|}{$\begin{array}{l}\text { Es ist reiner Zufall, wenn sich andere Menschen einmal nach } \\
\text { meinen Wünschen richten. }\end{array}$} & a & & $\square$ & $\square$ & $\square$ & a & 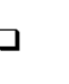 \\
\hline \multicolumn{3}{|c|}{$\begin{array}{l}\text { Mein Wohlbefinden hängt in starkem Maße vom Verhalten } \\
\text { anderer Menschen ab. }\end{array}$} & 口 & & a & $\square$ & $\square$ & a & 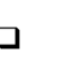 \\
\hline \multicolumn{3}{|c|}{$\begin{array}{l}\text { Ich kann sehr viel von dem, was in meinem Leben passiert, } \\
\text { selbst bestimmen. }\end{array}$} & a & & a & a & a & a & 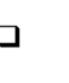 \\
\hline \multicolumn{3}{|c|}{$\begin{array}{l}\text { Manchmal weiß ich überhaupt nicht, was ich in einer } \\
\text { Situation machen soll. }\end{array}$} & 口 & & $\square$ & $\square$ & $\square$ & a & 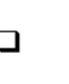 \\
\hline \multicolumn{3}{|c|}{$\begin{array}{l}\text { Gewöhnlich kann ich meine Interessen selbst vertreten und } \\
\text { erreiche dabei das, was ich will. }\end{array}$} & 口 & & a & a & a & a & $\square$ \\
\hline \multicolumn{3}{|c|}{$\begin{array}{l}\text { Ob ich einen Unfall habe oder nicht, hängt in starkem Maße } \\
\text { von dem Verhalten anderer ab. }\end{array}$} & 口 & & 口 & $\square$ & $\square$ & 口 & 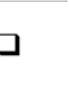 \\
\hline \multicolumn{3}{|c|}{$\begin{array}{l}\text { Wenn ich bekomme, was ich will, so ist das immer eine Folge } \\
\text { meiner Anstrengung und meines persönlichen Einsatzes. }\end{array}$} & 口 & & a & $\square$ & $\square$ & a & $\square$ \\
\hline \multicolumn{3}{|c|}{$\begin{array}{l}\text { Auch in schwierigen Situationen fallen mir immer viele } \\
\text { Handlungsalternativen ein. }\end{array}$} & $\square$ & & 口 & $\square$ & 口 & 口 & 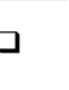 \\
\hline \multicolumn{3}{|c|}{$\begin{array}{l}\text { Damit meine Pläne eine Chance haben, richte ich mich beim } \\
\text { Planen nach den Wünschen anderer Leute. }\end{array}$} & a & & a & 口 & 口 & a & 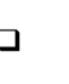 \\
\hline \multicolumn{3}{|c|}{$\begin{array}{l}\text { Mein Lebenslauf und mein Alltag werden alleine durch mein } \\
\text { Verhalten und meine Wünsche bestimmt. }\end{array}$} & 口 & & 口 & 口 & $\square$ & a & 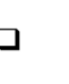 \\
\hline \multicolumn{3}{|c|}{ Es hängt vom Schicksal ab, ob ich krank werde oder nicht. } & D & & a & $\square$ & $\square$ & 口 & 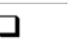 \\
\hline \multicolumn{3}{|c|}{$\begin{array}{l}\text { Für die Lösung von Problemen fallen mir immer viele } \\
\text { Möglichkeiten ein. }\end{array}$} & 口 & & a & 口 & $\square$ & a & 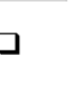 \\
\hline & $\begin{array}{l}\text { Wenn ich durch irgendetwas oder } \\
\text { irgendjemand beeinträchtigt, } \\
\text { innerlich erregt oder aus dem } \\
\text { Gleichgewicht gebracht worden bin... }\end{array}$ & gar nicht & & kaum & & $\begin{array}{l}\text { möglicher- } \\
\text { weise }\end{array}$ & $\begin{array}{c}\text { eher } \\
\text { wahr- } \\
\text { scheinlich }\end{array}$ & $\begin{array}{l}\text { wahr- } \\
\text { scheinlic }\end{array}$ & \\
\hline ...sag & ich mir, alles ist halb so schlimm & $\square$ & & $\square$ & & $\square$ & 口 & $\square$ & \\
\hline $\begin{array}{r}\text {...üb } \\
\text { aus }\end{array}$ & $\begin{array}{l}\text { lege ich, wie ich von nun an solchen Situationen } \\
\text { eichen kann }\end{array}$ & a & & $\square$ & & $\square$ & $\square$ & $\square$ & \\
\hline $\begin{array}{r}\ldots \text { we } \\
\text { ank }\end{array}$ & $\begin{array}{l}\text { ich nicht, wie ich gegen die Situation } \\
\text { mmen könnte }\end{array}$ & $\square$ & & $\square$ & & $\square$ & 口 & 口 & \\
\hline ...sag & ich mir, es geht schon alles wieder in Ordnung & $\square$ & & $\square$ & & $\square$ & $\square$ & $\square$ & \\
\hline ...der & e ich, mich trifft keine Schuld & $\square$ & & $\square$ & & $\square$ & $\square$ & $\square$ & \\
\hline ...der & e ich, morgen ist sicher alles vergessen & $\square$ & & $\square$ & & $\square$ & a & $\square$ & \\
\hline ...füh & ich mich irgendwie hilflos & 口 & & a & & 口 & a & a & \\
\hline
\end{tabular}




\begin{tabular}{|c|c|c|c|c|c|}
\hline ...sage ich mir, ich habe mir nichts vorzuwerfen & $\square$ & $\square$ & $\square$ & $\square$ & $\square$ \\
\hline $\begin{array}{l}\text {...sage ich mir, Gewissensbisse brauche ich mir nicht } \\
\text { zu machen }\end{array}$ & $\square$ & $\square$ & $\square$ & $\square$ & $\square$ \\
\hline $\begin{array}{l}\text {...versuche ich mir einzureden, dass es nicht so wichtig } \\
\text { ist }\end{array}$ & $\square$ & $\square$ & $\square$ & a & $\square$ \\
\hline ...versuche ich, mich der Situation zu entziehen & 口 & 口 & a & a & 口 \\
\hline ...neige ich dazu, alles sinnlos zu finden & 口 & a & a & a & 口 \\
\hline ...kommen mir Fluchtgedanken & $\square$ & $\square$ & $\square$ & $\square$ & $\square$ \\
\hline $\begin{array}{l}\text {...denke ich, an mir liegt es nicht, dass es dazu } \\
\text { gekommen ist }\end{array}$ & $\square$ & $\square$ & $\square$ & a & $\square$ \\
\hline ...tue ich etwas, was mich davon ablenkt & 口 & $\square$ & $\square$ & 口 & $\square$ \\
\hline $\begin{array}{l}\text {...denke ich, ich habe die Situation nicht zu } \\
\text { verantworten }\end{array}$ & $\square$ & $\square$ & $\square$ & a & $\square$ \\
\hline ...gehe ich irgendeiner anderen Beschäftigung nach & $\square$ & $\square$ & $\square$ & a & $\square$ \\
\hline $\begin{array}{l}\text {...passe ich auf, dass es in Zukunft gar nicht erst zu } \\
\text { solchen Situationen kommt }\end{array}$ & $\square$ & $\square$ & $\square$ & a & $\square$ \\
\hline ...stürze ich mich in die Arbeit & $\square$ & $\square$ & $\square$ & $\square$ & $\square$ \\
\hline $\begin{array}{l}\text {...versuche ich meine Aufmerksamkeit davon } \\
\text { abzuwenden }\end{array}$ & $\square$ & $\square$ & $\square$ & $\square$ & $\square$ \\
\hline ...lenke ich mich irgendwie $a b$ & 口 & a & 口 & a & $\square$ \\
\hline ...vermeide ich von nun an solche Situationen & $\square$ & $\square$ & $\square$ & $\square$ & $\square$ \\
\hline $\begin{array}{l}\text {...sage ich mir, das wird sich mit der Zeit schon wieder } \\
\text { einrenken }\end{array}$ & $\square$ & $\square$ & $\square$ & a & $\square$ \\
\hline $\begin{array}{l}\text {...nehme ich mir vor, solchen Situationen in Zukunft } \\
\text { aus dem Weg zu gehen }\end{array}$ & $\square$ & $\square$ & $\square$ & a & $\square$ \\
\hline ...denke ich „möglichst von hier weg“ & $\square$ & $\square$ & $\square$ & $\square$ & $\square$ \\
\hline ...möchte ich am liebsten einfach weglaufen & $\square$ & $\square$ & $\square$ & $\square$ & $\square$ \\
\hline $\begin{array}{l}\text {...versuche ich meine Gedanken auf etwas anderes zu } \\
\text { konzentrieren }\end{array}$ & $\square$ & $\square$ & $\square$ & a & $\square$ \\
\hline ...neige ich dazu, die Flucht zu ergreifen & $\square$ & $\square$ & $\square$ & $\square$ & $\square$ \\
\hline ...sage ich mir, ich kann nichts dafür & $\square$ & $\square$ & $\square$ & $\square$ & $\square$ \\
\hline $\begin{array}{l}\text {...habe ich nur den Wunsch, dieser Situation so schnell } \\
\text { wie möglich zu entkommen }\end{array}$ & $\square$ & $\square$ & $\square$ & $\square$ & $\square$ \\
\hline $\begin{array}{l}\text {...denke ich, in Zukunft will ich nicht mehr in solche } \\
\text { Situationen geraten }\end{array}$ & $\square$ & $\square$ & $\square$ & $\square$ & $\square$ \\
\hline ...neige ich dazu zu resignieren & $\square$ & $\square$ & $\square$ & $\square$ & $\square$ \\
\hline ...neige ich dazu, schnell aufzugeben & $\square$ & $\square$ & $\square$ & 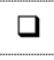 & $\square$ \\
\hline $\begin{array}{l}\text {...entziehe ich mich in Zukunft schon bei den ersten } \\
\text { Anzeichen solchen Situationen }\end{array}$ & $\square$ & $\square$ & $\square$ & a & $\square$ \\
\hline ...erscheint mir alles so hoffnungslos & $\square$ & $\square$ & $\square$ & $\square$ & $\square$ \\
\hline $\begin{array}{l}\text {...sage ich mir, die Zeit hat schon manche Träne } \\
\text { getrocknet }\end{array}$ & $\square$ & $\square$ & $\square$ & 口 & $\square$ \\
\hline
\end{tabular}




\begin{tabular}{|c|c|c|c|c|}
\hline $\begin{array}{l}\text { Bitte schätzen Sie ein inwieweit die folgenden } \\
\text { Aussagen auf sie persönlich zutreffen! }\end{array}$ & $\begin{array}{c}\text { stimmt } \\
\text { nicht }\end{array}$ & $\begin{array}{l}\text { stimmt } \\
\text { kaum }\end{array}$ & $\begin{array}{l}\text { stimmt } \\
\text { eher }\end{array}$ & stimmt \\
\hline $\begin{array}{l}\text { Bei meiner Arbeit entdecke ich immer wieder neue, interessante } \\
\text { Aspekte. }\end{array}$ & $\square$ & $\square$ & $\square$ & a \\
\hline Es gibt Tagen, dass ich mich schon vor der Arbeit müde fühle. & 口 & $\square$ & 口 & a \\
\hline $\begin{array}{l}\text { Es passiert mir immer öfter, dass ich mich abwertend über meine } \\
\text { Arbeitstätigkeit äußere. }\end{array}$ & $\square$ & 口 & 口 & a \\
\hline $\begin{array}{l}\text { Nach der Arbeit brauche ich jetzt oft längere Erholungszeiten als } \\
\text { früher, um wieder fit zu werden. }\end{array}$ & 口 & 口 & $\square$ & 口 \\
\hline Die Belastung durch meine Arbeit ist ganz gut zu ertragen. & a & a & a & a \\
\hline $\begin{array}{l}\text { Ich neige in letzter Zeit vermehrt dazu, bei meiner Arbeit wenig zu } \\
\text { denken, sondern sie fast mechanisch zu erledigen. }\end{array}$ & $\square$ & 口 & 口 & a \\
\hline Meine Arbeit stellt viele Herausforderungen an mich. & 口 & ם & $\square$ & a \\
\hline $\begin{array}{l}\text { Ich habe bei der Arbeit immer häufiger das Gefühl, emotional } \\
\text { ausgelaugt zu sein. }\end{array}$ & $\square$ & 口 & $\square$ & a \\
\hline Mit der Zeit verliert man die innere Beziehung zur eigenen Arbeit. & 口 & $\square$ & $\square$ & 口 \\
\hline $\begin{array}{l}\text { Nach der Arbeit bin ich in der Regel noch ganz fit für meine } \\
\text { Freizeitaktivitäten. }\end{array}$ & 口 & 口 & 口 & 口 \\
\hline $\begin{array}{l}\text { Manchmal bin ich von meiner Arbeitstätigkeit richtiggehend } \\
\text { angewidert. }\end{array}$ & $\square$ & $\square$ & $\square$ & a \\
\hline $\begin{array}{l}\text { Nach der Arbeit fühle ich mich in der Regel schlapp } \\
\text { und abgespannt. }\end{array}$ & $\square$ & 口 & $\square$ & a \\
\hline Ich kann mir für mich keinen anderen Beruf vorstellen. & a & 口 & a & a \\
\hline In der Regel kann ich meine Arbeitsmenge gut schaffen. & $\square$ & $\square$ & $\square$ & a \\
\hline Mit der Zeit engagiere ich mich immer mehr bei meiner Arbeit. & a & a & a & a \\
\hline Während meiner Arbeit fühle ich mich total belebt. & 口 & ם & ם & a \\
\hline
\end{tabular}

\section{Vielen Dank für Ihre/deine Mitarbeit! :-}

(Bitte kontrollieren Sie ob sie auch wirklich alle Fragen beantwortet haben!) 
III. Informationsflyer
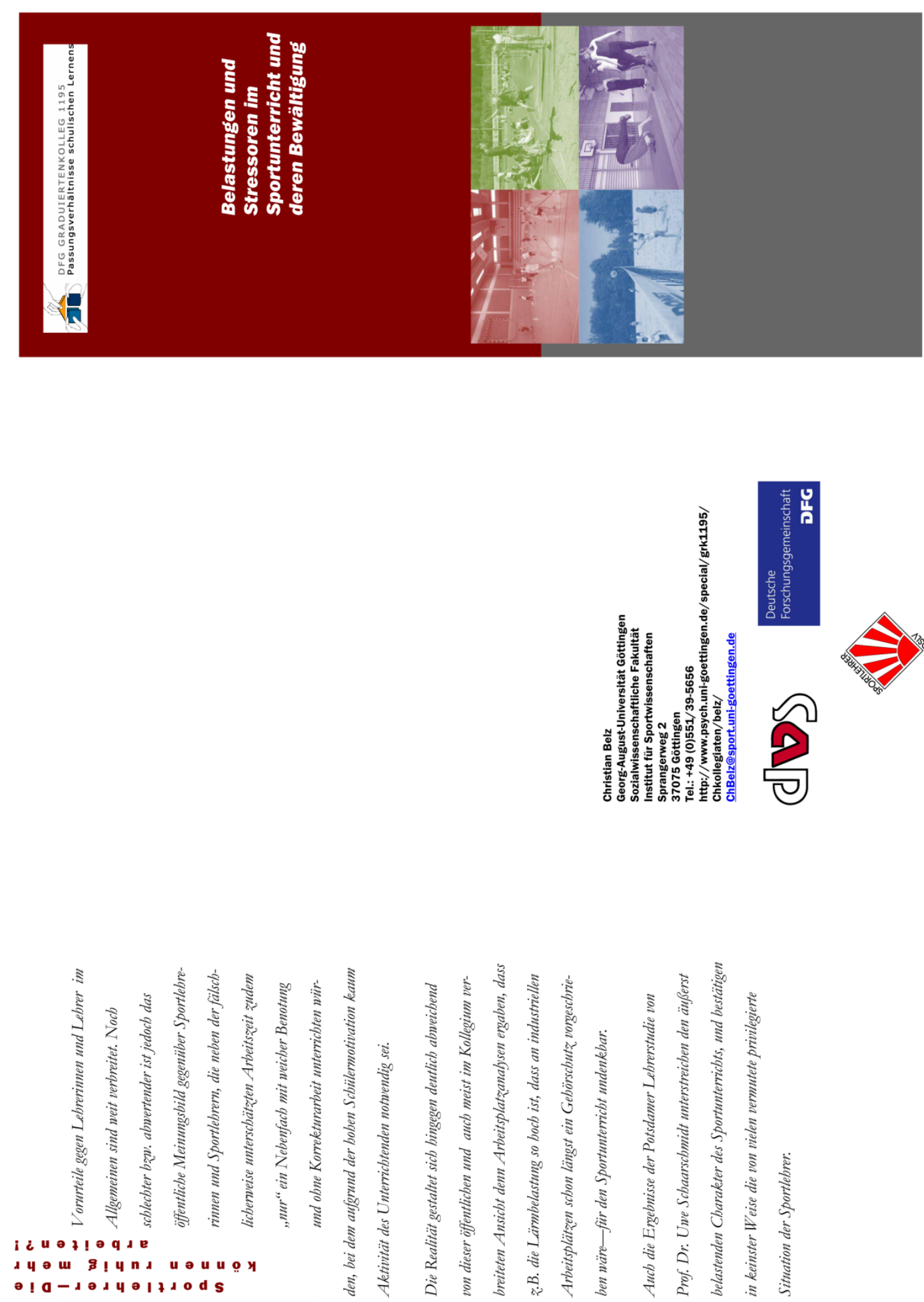

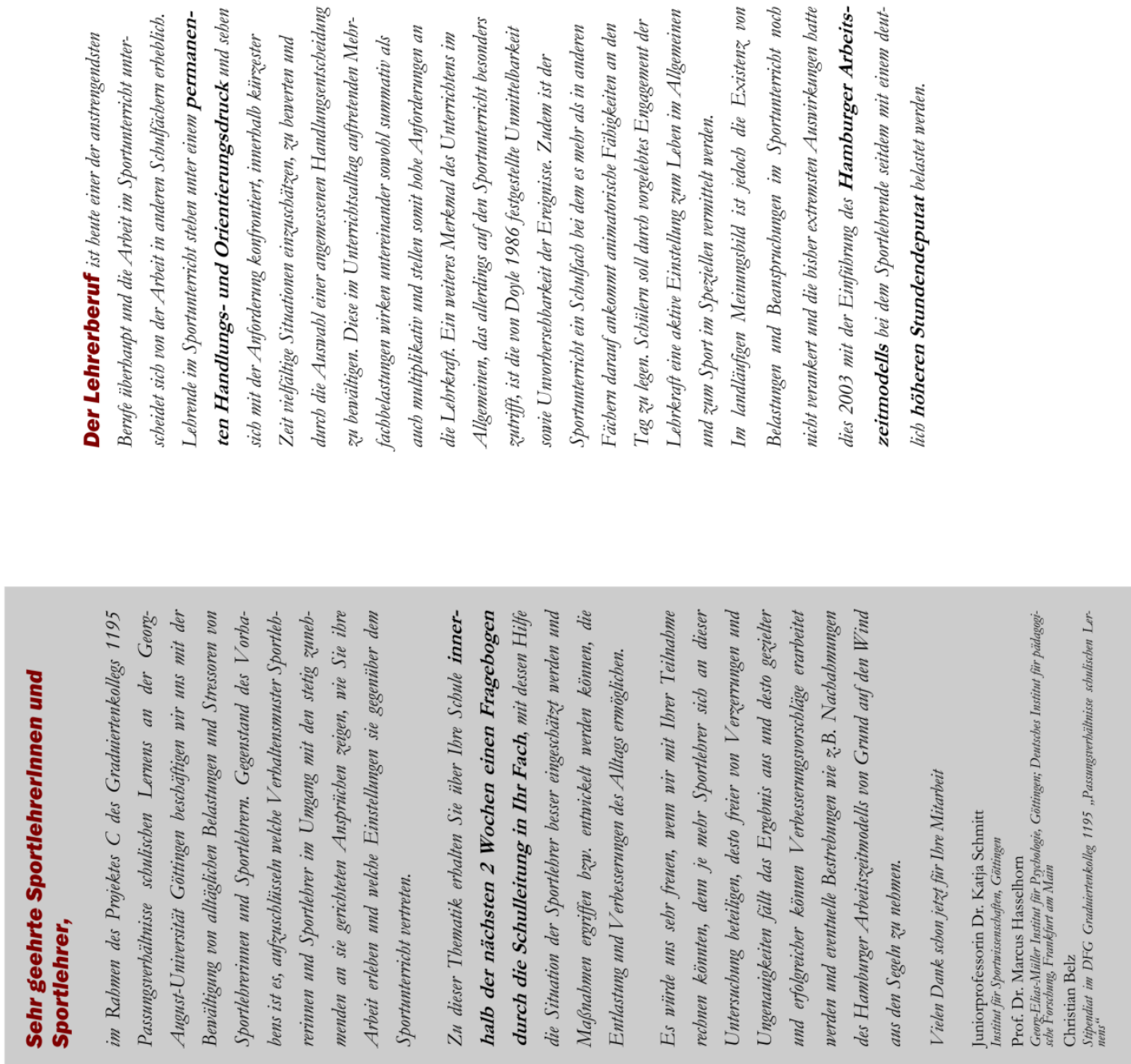


\section{Fragebogen Erhebungsinstrument evasiver Sportunterricht - EESU (Endversi- on)}

\section{DFG GRADUIERTENKOLLEG 1195 Passungsverhältnisse schulischen Lernens}

Projekt: Belastungen/Stressoren im Sportunterricht und deren Bewältigung

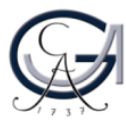

\section{Sehr geehrte(r) Sportlehrer/In,}

\section{Noch einige wichtige Informationen zum Ausfüllen des Fragebogens:}

- Grundsätzlich ist die Teilnahme an der Untersuchung freiwillig, jedoch sind die Ergebnisse umso aussagekräftiger und repräsentativer je mehr SportlehrerInnen sich beteiligen.

- Die Befragung erfolgt absolut anonym. Alle Daten und Angaben werden ausschließlich für wissenschaftliche Zwecke verwendet und in Bezug auf datenschutzrechtliche Bestimmungen ( $§ 3$ niedersächsisches Datenschutzgesetz) streng vertraulich behandelt.

- Das Ausfüllen des Fragebogens sollte insgesamt nicht länger als 25min dauern. Bitte überlegen Sie bei den einzelnen Fragen nicht zu lange sondern antworten möglichst spontan.

- Es gibt keine richtigen oder falschen Antworten! Geben Sie ehrliche Antworten und überlegen Sie nicht welche Antworten den besten Eindruck entstehen lassen. Wenn Sie sich nicht für eine bestimmte Antwort entscheiden können, wählen Sie die am ehesten auf Sie zutreffende!

- Bitte kreuzen Sie das Kästchen mit der für Sie zutreffenden Antwort deutlich an. Wenn Sie etwas falsch angekreuzt haben streichen Sie das Kreuz einfach durch und machen an der richtigen Stelle ein neues Kreuz und umkreisen dieses (siehe Beispiel).

\begin{tabular}{|l|} 
Geschlecht (bitte ankreuzen) \\
weiblich $\bigotimes$ Männlich
\end{tabular}

- Natürlich sind wir auch gerne bereit Ihnen eine individuelle Rückmeldung zu liefern inwieweit Sie beispielsweise berufsbedingt belastet sind und wie erfolgreich Sie damit umgehen (z.B. Burn-Out-Risiko etc.). Geben Sie hierzu optional einen für Sie individuellen 6stelligen Code an (die Rückmeldung wird in einem verschlossenen mit Ihrem anonymen Code beschrifteten Umschlag an Ihre Schule nach Abschluss der Auswertung gesendet).

\begin{tabular}{|l|l|}
\hline 1. Stelle: Der 2. Buchstabe des Vornamens Ihres Vaters & 2./3. Stelle: Der 2. Und 3. Buchstabe des Geburtsnamens Ihrer Mutter \\
\hline 4. Stelle: Der 2. Buchstabe Ihres Geburtsortes & 5./6. Stelle: Der 1. Und 2. Buchstabe des Vornamens Ihrer Mutter \\
\hline
\end{tabular}

Es würde uns sehr freuen, wenn wir mit Ihrer Teilnahme rechnen könnten, da nur durch Forschungsvorhaben wie diesem die Situation der Sportlehrer besser eingeschätzt werden und Maßnahmen ergriffen bzw. entwickelt werden können, die Entlastung und Verbesserungen des Alltags ermöglichen.

Bitte geben Sie den ausgefüllten Fragebogen in dem verschlossenen Umschlag spätestens bis zum $\underline{\mathbf{1 5 . 0 4 . 0 8}}$ in Ihrem Sekretariat bzw. Ihrer Schulsportleitung ab, von wo aus die Fragebögen zentral in einem vorfrankierten Sammelumschlag zurückgesendet werden. Selbstverständlich können Sie auch den Fragebogen per Post an folgende Adresse senden: Christian Belz, Institut für Sportwissenschaft, Sprangerweg 2, 37075 Göttingen.

Mit herzlichem Dank für Ihre Mitarbeit und freundlichem Gruß

Christian Belz (Stipendiat im Graduiertenkolleg 1195) ${ }^{1}$

Juniorprofessorin Dr. Katja Schmitt (Institut für Sportwissenschaften Göttingen)

Prof. Dr. Marcus Hasselhorn (Zentrum für empirische Unterrichts- und Schulforschung (ZEUS) Göttingen \&

Deutsches Institut für Internationale Pädagogische Forschung AE: Bildung und Entwicklung, Frankfurt am Main

${ }^{1}$ Bei Rückfragen wenden Sie sich bitte an Herrn Christian Belz, Institut für Sportwissenschaften, Sprangerweg 2, 37075 Göttingen, Tel. 0551-39-5656, E-Mail: ChBelz@sport.uni-goettingen.de 


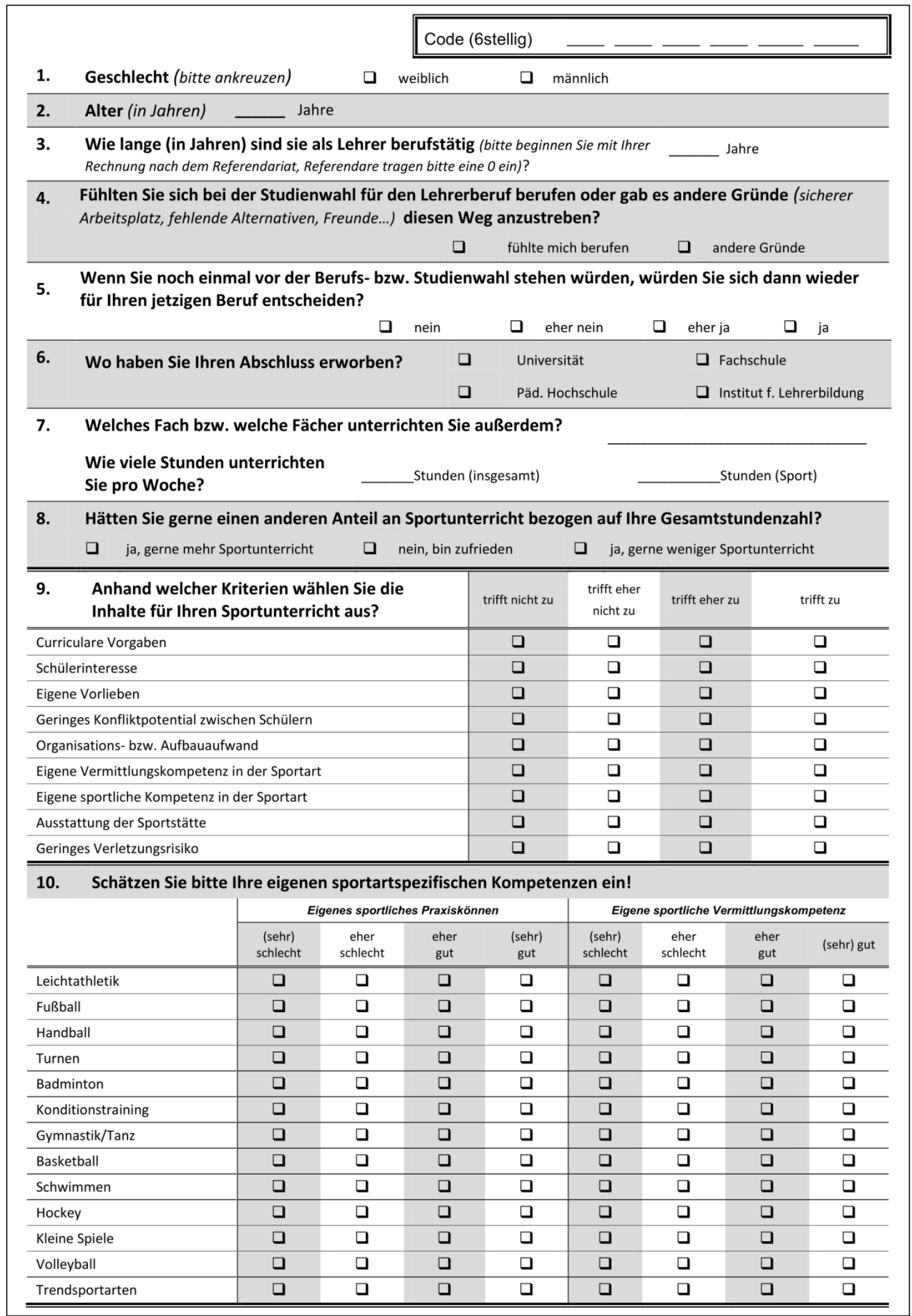




\begin{tabular}{|c|c|c|c|c|c|}
\hline $\begin{array}{l}\text { 11. Wie oft führen Sie die folgenden Aktivitäten im } \\
\text { Unterricht durch? }\end{array}$ & selten/nie & eher selten & \multicolumn{2}{|c|}{ eher oft } & oft/sehr oft \\
\hline Leichtathletik & 口 & 口 & \multicolumn{2}{|c|}{$\square$} & 口 \\
\hline Fußball & $\square$ & $\square$ & \multicolumn{2}{|c|}{ 口 } & $\square$ \\
\hline Handball & 口 & $\square$ & \multicolumn{2}{|c|}{ 口 } & 口 \\
\hline Turnen & $\square$ & $\square$ & \multicolumn{2}{|c|}{ 口 } & $\square$ \\
\hline Badminton & 口 & 口 & \multicolumn{2}{|c|}{ a } & 口 \\
\hline Konditionstraining & a & 口 & \multicolumn{2}{|c|}{ 口 } & 口 \\
\hline Gymnastik/Tanz & $\square$ & $\square$ & \multicolumn{2}{|c|}{$\square$} & $\square$ \\
\hline Basketball & a & ב & \multicolumn{2}{|c|}{ 口 } & 口 \\
\hline Schwimmen & $\square$ & $\square$ & \multicolumn{2}{|c|}{ 口 } & $\square$ \\
\hline Hockey & a & ב & \multicolumn{2}{|c|}{$\square$} & a \\
\hline Kleine Spiele & 口 & 口 & \multicolumn{2}{|c|}{ 口 } & $\square$ \\
\hline Volleyball & $\square$ & $\square$ & \multicolumn{2}{|c|}{ 口 } & $\square$ \\
\hline Trendsportarten & $\square$ & $\square$ & & & $\square$ \\
\hline $\begin{array}{l}\text { 12. Inwieweit fühlen Sie sich durch die folgenden Faktoren } \\
\text { im Sportunterricht belastet? }\end{array}$ & $\begin{array}{l}\text { (sehr) } \\
\text { wenig }\end{array}$ & eher wenig & & & $\begin{array}{l}\text { (sehr) } \\
\text { stark }\end{array}$ \\
\hline Hoher Lärmpegel & प & ב & & & 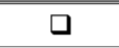 \\
\hline Hilfestellungen & $\square$ & $\square$ & & & $\square$ \\
\hline Auf- und Abbau & 口 & 口 & & & 口 \\
\hline Demonstration von Bewegungen & $\square$ & $\square$ & & & $\square$ \\
\hline Stetig latente Unfallgefahr & $\square$ & $\square$ & & & $\square$ \\
\hline Soziales Schülerfehlverhalten und Motivationsprobleme & $\square$ & $\square$ & & & $\square$ \\
\hline Geringe Pausen bzw. Erholungszeiten & $\square$ & $\square$ & & & $\square$ \\
\hline Vielzahl von gleichzeitig auftretenden Aufgaben & $\square$ & 口 & & & 口 \\
\hline Rechtfertigungsdruck gegenüber dem Kollegium (nicht Sport) & $\square$ & $\square$ & & & $\square$ \\
\hline Außerunterrichtliche Aktivitäten (z.B. Sportfeste, BuJu-Spiele) & $\square$ & $\square$ & & & $\square$ \\
\hline Häufig wechselnder Ordnungsrahmen im Unterricht & $\square$ & $\square$ & & & $\square$ \\
\hline $\begin{array}{l}\text { Erhöhter Aufwand zur Erhaltung der eigenen Fitness/Erlernen neuer } \\
\text { Sportarten }\end{array}$ & 口 & 口 & & & $\square$ \\
\hline Schwierigkeiten im Umgang mit Eltern, Kollegen, Schulleitung & $\square$ & $\square$ & & & $\square$ \\
\hline Schlechte räumlich-materielle Bedingungen & $\square$ & $\square$ & & & $\square$ \\
\hline Klassengröße & $\square$ & $\square$ & & & $\square$ \\
\hline Unangemessene curriculare Vorgaben & 口 & 口 & & & 口 \\
\hline Körperliche Beanspruchung (allgemein) & $\square$ & $\square$ & & & $\square$ \\
\hline $\begin{array}{l}\text { 13. Im Bezug auf den Umgang mit Schülerinnen und } \\
\text { Schülern im Sportunterricht... }\end{array}$ & $\begin{array}{l}\text { trifft nicht } \\
\text { zu }\end{array}$ & $\begin{array}{l}\text { trifft eher } \\
\text { nicht zu }\end{array}$ & $\begin{array}{l}\text { teils/ } \\
\text { teils }\end{array}$ & $\begin{array}{l}\text { trifft eher } \\
\mathrm{zu}\end{array}$ & trifft zu \\
\hline $\begin{array}{l}\text {...schlage ich lieber mir vertraute Wege ein bevor ich bei Neuem das Risiko } \\
\text { eingehe zu scheitern. }\end{array}$ & 口 & 口 & ם & $\square$ & 口 \\
\hline $\begin{array}{l}\text {...suche ich mir umgehend eine größere Herausforderung wenn ich ein Ziel } \\
\text { erreicht habe. }\end{array}$ & $\square$ & $\square$ & ם & $\square$ & $\square$ \\
\hline $\begin{array}{l}\text {...lasse ich ein Problem erst einmal „vor sich hinköcheln“, bevor ich es } \\
\text { anpacke. }\end{array}$ & ם & ם & ם & ם & ם \\
\hline ...nehme ich schwierige Situationen in denen viel von mir abhängt gerne an. & 口 & 口 & ם & $\square$ & $\square$ \\
\hline ...achte ich bewusst darauf Schwierigkeiten zu vermeiden. & 口 & ב & a & (ם & 口 \\
\hline ...suche ich mir gern Herausforderungen und gehe dafür auch Risiken ein. & 口 & 口 & ם & $\square$ & 口 \\
\hline ...gehe ich lieber auf Nummer sicher um Ärger zu vermeiden. & a & a & a & a & a \\
\hline ...habe ich das Ziel nicht anzuecken. & 口 & 口 & ם & a & 口 \\
\hline
\end{tabular}




\begin{tabular}{|c|c|c|c|c|c|}
\hline 14. Im Bezug auf Inhalte im Sportunterricht... & $\begin{array}{l}\text { trifft nicht } \\
\text { zu }\end{array}$ & $\begin{array}{l}\text { trifft eher } \\
\text { nicht zu }\end{array}$ & $\begin{array}{l}\text { teils/ } \\
\text { teils }\end{array}$ & $\begin{array}{l}\text { trifft eher } \\
\text { zu }\end{array}$ & trifft zu \\
\hline $\begin{array}{l}\text {...schlage ich lieber mir vertraute Wege ein bevor ich bei Neuem das Risiko } \\
\text { eingehe zu scheitern. }\end{array}$ & $\square$ & a & $\square$ & $\square$ & $\square$ \\
\hline $\begin{array}{l}\text {...suche ich mir umgehend eine größere Herausforderung wenn ich ein Ziel } \\
\text { erreicht habe. }\end{array}$ & a & a & $\square$ & 口 & $\square$ \\
\hline $\begin{array}{l}\text {...lasse ich ein Problem erst einmal „vor sich hinköcheln“, bevor ich es } \\
\text { anpacke. }\end{array}$ & $\square$ & a & $\square$ & 口 & $\square$ \\
\hline ...nehme ich schwierige Situationen in denen viel von mir abhängt gerne an. & $\square$ & $\square$ & $\square$ & $\square$ & $\square$ \\
\hline ...achte ich bewusst darauf Schwierigkeiten zu vermeiden. & $\square$ & $\square$ & $\square$ & 口 & $\square$ \\
\hline ...suche ich mir gern Herausforderungen und gehe dafür auch Risiken ein. & 口 & a & 口 & 口 & ם \\
\hline ...gehe ich lieber auf Nummer sicher um Ärger zu vermeiden. & $\square$ & $\square$ & $\square$ & $\square$ & $\square$ \\
\hline ...habe ich das Ziel nicht anzuecken. & $\square$ & 口 & 口 & 口 & ם \\
\hline $\begin{array}{l}\text { 15. Im Bezug auf äußere Bedingungen... } \\
\text { (Vorschriften und Erlasse, Beziehungen zum Kollegium/zur Schulleitung, } \\
\text { Zeitordnungen, Notengebung, materielle Ausstattung) }\end{array}$ & $\begin{array}{c}\text { trifft nicht } \\
\mathrm{zu}\end{array}$ & $\begin{array}{l}\text { trifft eher } \\
\text { nicht zu }\end{array}$ & $\begin{array}{l}\text { teils/ } \\
\text { teils }\end{array}$ & $\begin{array}{l}\text { trifft eher } \\
z \mathrm{zu}\end{array}$ & trifft zu \\
\hline $\begin{array}{l}\text {...schlage ich lieber mir vertraute Wege ein bevor ich bei Neuem das Risiko } \\
\text { eingehe zu scheitern. }\end{array}$ & $\square$ & a & $\square$ & $\square$ & $\square$ \\
\hline $\begin{array}{l}\text {...suche ich mir umgehend eine größere Herausforderung wenn ich ein Ziel } \\
\text { erreicht habe. }\end{array}$ & 口 & a & $\square$ & $\square$ & a \\
\hline $\begin{array}{l}\text {...lasse ich ein Problem erst einmal „vor sich hinköcheln“, bevor ich es } \\
\text { anpacke. }\end{array}$ & $\square$ & a & $\square$ & 口 & ם \\
\hline ...nehme ich schwierige Situationen in denen viel von mir abhängt gerne an. & 口 & 口 & 口 & 口 & 口 \\
\hline ...achte ich bewusst darauf Schwierigkeiten zu vermeiden. & a & a & 口 & a & ם \\
\hline ...suche ich mir gern Herausforderungen und gehe dafür auch Risiken ein. & $\square$ & a & $\square$ & $\square$ & $\square$ \\
\hline ...gehe ich lieber auf Nummer sicher um Ärger zu vermeiden. & $\square$ & $\square$ & $\square$ & $\square$ & $\square$ \\
\hline ...habe ich das Ziel nicht anzuecken. & ב & ב & $\square$ & ב & ם \\
\hline $\begin{array}{l}\text { 16. Schätzen Sie die Bedeutsamkeit folgender } \\
\text { Vermittlungsaspekte für „Ihren“ Unterricht ein! }\end{array}$ & $\begin{array}{c}\text { (sehr) } \\
\text { unwichtig }\end{array}$ & $\begin{array}{c}\text { eher } \\
\text { unwichtig }\end{array}$ & & $\begin{array}{l}\text { her } \\
\text { chtig }\end{array}$ & $\begin{array}{l}\text { (sehr) } \\
\text { wichtig }\end{array}$ \\
\hline Soziales Lernen/Persönlichkeitsentwicklung & 口 & $\square$ & & 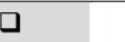 & 口 \\
\hline Gesundheitserziehung/Fitness & $\square$ & $\square$ & & 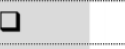 & $\square$ \\
\hline Fachliche (theoretische) Kenntnisse & a & $\square$ & & 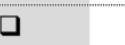 & a \\
\hline Kondition & 口 & $\square$ & & 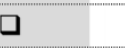 & $\square$ \\
\hline Kreativität & 口 & $\square$ & & 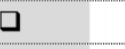 & $\square$ \\
\hline Freude an Bewegung/Lebensbegleitendes Sporttreiben & $\square$ & $\square$ & & 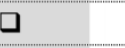 & $\square$ \\
\hline Leistungserziehung/(Wett-) Kampfgeist & $\square$ & $\square$ & & 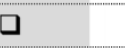 & $\square$ \\
\hline Selbständigkeit & 口 & $\square$ & & 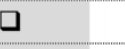 & $\square$ \\
\hline Körperbildung & $\square$ & $\square$ & & 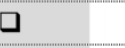 & $\square$ \\
\hline Taktik & 口 & $\square$ & & 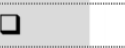 & $\square$ \\
\hline Technik & 口 & $\square$ & & $\square$ & $\square$ \\
\hline Koordinative Fähigkeiten & $\square$ & $\square$ & & 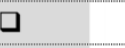 & $\square$ \\
\hline Körpererfahrung & 口 & $\square$ & & 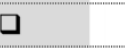 & $\square$ \\
\hline Spielen (Spielfähigkeit) & 口 & $\square$ & & 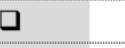 & $\square$ \\
\hline Disziplin & $\square$ & $\square$ & & 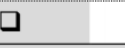 & 口 \\
\hline \multicolumn{6}{|l|}{ 17. In meinem Sportunterricht mache ich selber aktiv mit! } \\
\hline nie/sehr selten & $\square$ oft & & $\square$ & \multicolumn{2}{|c|}{ sehr oft/immer } \\
\hline \multicolumn{6}{|l|}{ 18. Es macht mir Spaß das Fach Sport zu unterrichten! } \\
\hline nie/sehr selten & $\square$ oft & & 口 & \multicolumn{2}{|c|}{ sehr oft/immer } \\
\hline \multicolumn{6}{|c|}{$\begin{array}{l}\text { 19. Wie hoch schätzen Sie Ihren Arbeitsaufwand für das Fach Sport in Stunden }(60 \mathrm{~min}) \text { durchschnittlich pro Woche } \\
\text { ein, den sie zusätzlich zu Ihrer regulären Stundenverpflichtung aufbringen? }\end{array}$} \\
\hline
\end{tabular}




\begin{tabular}{|c|c|c|c|c|c|c|c|c|}
\hline 20. & \multicolumn{2}{|l|}{ Berufliche Belastung } & $\begin{array}{l}\text { trifft gar } \\
\text { nicht zu }\end{array}$ & $\begin{array}{r}\text { trifft } \\
\text { wenig }\end{array}$ & $\begin{array}{r}\text { tri } \\
\text { mitte } \\
z\end{array}$ & \begin{tabular}{l|l} 
rifft & t \\
elmäßig \\
zu
\end{tabular} & $\begin{array}{l}\text { trifft } \\
\text { über- } \\
\text { wiegend } \\
\text { zu }\end{array}$ & trifft zu \\
\hline \multicolumn{3}{|c|}{ Mein Beruf macht mir Spaß. } & $\square$ & $\square$ & & 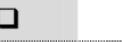 & $\square$ & $\square$ \\
\hline \multicolumn{3}{|c|}{ In meinem Beruf wird man ständig überfordert. } & $\square$ & $\square$ & & $\square$ & $\square$ & $\square$ \\
\hline \multicolumn{3}{|c|}{ Ich habe mir schon ernsthaft überlegt aus dem Beruf auszusteigen. } & $\square$ & $\square$ & & $\square$ & $\square$ & $\square$ \\
\hline \multicolumn{3}{|c|}{ Was meine Arbeit betrifft, bin ich eigentlich rundum zufrieden. } & $\square$ & 口 & & a & ב & a \\
\hline \multicolumn{3}{|c|}{ Ich fühle mich von meinem Arbeitgeber ständig kontrolliert und überwacht. } & $\square$ & a & & 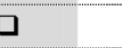 & $\square$ & 口 \\
\hline \multicolumn{3}{|c|}{$\begin{array}{l}\text { Ich habe das Gefühl auch vor Kollegen ständig betonen zu müssen, dass ich } \\
\text { viel arbeite. }\end{array}$} & $\square$ & a & & 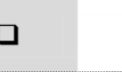 & $\square$ & a \\
\hline \multicolumn{3}{|c|}{ Ich halte mich gerne an meinem Arbeitsplatz auf. } & $\square$ & $\square$ & & 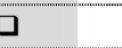 & $\square$ & 口 \\
\hline \multicolumn{3}{|c|}{ Ich habe Konflikte mit Vorgesetzten, die mich belasten. } & $\square$ & a & & 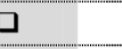 & $\square$ & $\square$ \\
\hline \multicolumn{3}{|c|}{ Ich fühle mich häufig überfordert. } & $\square$ & $\square$ & & $\square$ & $\square$ & 口 \\
\hline \multicolumn{3}{|c|}{ Ich habe selten das Gefühl, einmal richtig abschalten zu können. } & $\square$ & $\square$ & & $\square$ & $\square$ & $\square$ \\
\hline \multicolumn{3}{|c|}{ Ich kann in meinem Beruf meine Fähigkeiten voll nutzen. } & $\square$ & $\square$ & & $\square$ & $\square$ & $\square$ \\
\hline \multicolumn{3}{|c|}{ Oft habe ich ein schlechtes Gewissen den Schülern gegenüber. } & $\square$ & $\square$ & & 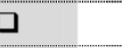 & $\square$ & $\square$ \\
\hline \multicolumn{3}{|c|}{$\begin{array}{l}\text { Meine eigenen Berufsideale lassen sich in meiner Tätigkeit nicht } \\
\text { verwirklichen. }\end{array}$} & $\square$ & ב & & 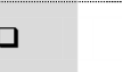 & $\square$ & 口 \\
\hline \multicolumn{3}{|c|}{ Für andere Menschen verantwortlich zu sein belastet mich sehr. } & a & a & & $\square$ & a & 口 \\
\hline \multicolumn{3}{|c|}{ Der Zeitdruck unter dem ich arbeite, ist zu groß. } & $\square$ & ב & & 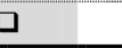 & $\square$ & $\square$ \\
\hline & \multicolumn{2}{|l|}{ Wie erleben Sie sich selbst? } & \multicolumn{2}{|c|}{ stimmt nicht } & $\begin{array}{c}\text { stimmt } \\
\text { kaum }\end{array}$ & \multicolumn{2}{|c|}{ stimmt eher } & stimmt \\
\hline \multicolumn{3}{|c|}{$\begin{array}{l}\text { Ich weiß, dass ich es schaffe, selbst den problematischsten Schülern den Stoff } \\
\text { zu vermitteln. }\end{array}$} & \multicolumn{2}{|l|}{ a } & 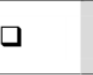 & \multicolumn{2}{|l|}{$\square$} & $\square$ \\
\hline \multicolumn{3}{|c|}{$\begin{array}{l}\text { Ich weiß, dass ich zu den Eltern guten Kontakt halten kann, selbst in } \\
\text { schwierigen Situationen. }\end{array}$} & \multicolumn{2}{|l|}{ ב } & $\square$ & $\square$ & & a \\
\hline $\begin{array}{l}\text { Ich bir } \\
\text { Konta }\end{array}$ & $\begin{array}{l}\text { mir sicher, dass ich auch mit den problematischen Schü } \\
\text { kommen kann, wenn ich mich darum bemühe. }\end{array}$ & rn in guten & $\square$ & & $\square$ & 口 & & $\square$ \\
\hline $\begin{array}{l}\text { Ich bir } \\
\text { Schüle }\end{array}$ & $\begin{array}{l}\text { mir sicher, dass ich mich in Zukunft auf individuelle Pro } \\
\text { noch besser einstellen kann. }\end{array}$ & eme der & a & & $\square$ & $\square$ & & $\square$ \\
\hline $\begin{array}{l}\text { Selbst } \\
\text { Gelass }\end{array}$ & $\begin{array}{l}\text { Jenn mein Unterricht gestört wird, bin ich mir sicher, } d \\
\text { nheit bewahren zu können. }\end{array}$ & notwendige & a & & $\square$ & $\square$ & & $\square$ \\
\hline $\begin{array}{l}\text { Selbst } \\
\text { noch \& }\end{array}$ & $\begin{array}{l}\text { venn es mir mal nicht so gut geht, kann ich doch im Un } \\
\text { t auf die Schüler eingehen. }\end{array}$ & rricht immer & $\square$ & & $\square$ & $\square$ & & a \\
\hline $\begin{array}{l}\text { Auch } \\
\text { engag }\end{array}$ & $\begin{array}{l}\text { enn ich mich noch so sehr für die Entwicklung meiner S } \\
\text { re, weiß ich, dass ich nicht viel ausrichten kann. }\end{array}$ & & $\square$ & & $\square$ & $\square$ & & 口 \\
\hline $\begin{array}{l}\text { Ich bir } \\
\text { ungün }\end{array}$ & $\begin{array}{l}\text { mir sicher, dass ich kreative Ideen entwickeln kann, mit } \\
\text { ige Unterrichtsstrukturen verändere. }\end{array}$ & nen ich & a & & 口 & $\square$ & & $\square$ \\
\hline Ich tra & e mir zu, die Schüler für neue Projekte zu begeistern. & & 口 & & $\square$ & $\square$ & & $\square$ \\
\hline Ich ka & Innovationen auch gegenüber skeptischen Kollegen d & rchsetzen. & 口 & & 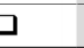 & 口 & & $\square$ \\
\hline 22. & $\begin{array}{l}\text { Schätzen Sie sich bitte selbst ein! } \\
\text { Nehmen Sie bitte zu jeder der folgenden Aussagen Stellung und } \\
\text { entscheiden Sie, inwieweit jede auf Sie ganz persönlich zutrifft. }\end{array}$ & $\begin{array}{c}\text { stimmt } \\
\text { überhaupt } \\
\text { nicht }\end{array}$ & $\begin{array}{c}\text { stimmt } \\
\text { weit- } \\
\text { gehend } \\
\text { nicht } \\
\end{array}$ & $\begin{array}{l}\text { stimmt } \\
\text { eher } \\
\text { nicht }\end{array}$ & $\begin{array}{l}\text { stimmt } \\
\text { ein } \\
\text { wenig }\end{array}$ & $\begin{array}{l}\text { stimmt } \\
\text { weit- } \\
\text { gehend }\end{array}$ & stim & mt genau \\
\hline $\begin{array}{l}\text { Ich pr } \\
\text { dabei }\end{array}$ & $\begin{array}{l}\text { jiere gerne Dinge aus, auch wenn nicht immer etwas } \\
\text { erauskommt. }\end{array}$ & $\square$ & $\square$ & 口 & $\square$ & $\square$ & & 口 \\
\hline Ich be & häftige mich nur mit Aufgaben, die lösbar sind. & $\square$ & $\square$ & $\square$ & $\square$ & $\square$ & & a \\
\hline Ich $\mathrm{me}$ & es, wenn unverhofft Überraschungen auftreten. & $\square$ & $\square$ & $\square$ & $\square$ & $\square$ & & $\square$ \\
\hline Ich las & die Dinge gern auf mich zukommen. & 口 & $\square$ & $\square$ & $\square$ & $\square$ & & 口 \\
\hline Ich ha & es gerne, wenn die Arbeit gleichmäßig verläuft. & $\square$ & $\square$ & $\square$ & $\square$ & $\square$ & & $\square$ \\
\hline Ich wa & te geradezu darauf, dass etwas Aufregendes passiert. & $\square$ & $\square$ & $\square$ & $\square$ & $\square$ & & $\square$ \\
\hline $\begin{array}{l}\text { Wenn } \\
\text { ich mi }\end{array}$ & $\begin{array}{l}\mathrm{m} \text { mich herum alles drunter und drüber geht, fühle } \\
\text { so richtig wohl. }\end{array}$ & 口 & $\square$ & 口 & $\square$ & $\square$ & & 口 \\
\hline Ich we & gerne, was auf mich zukommt. & 口 & a & a & a & 口 & & 口 \\
\hline
\end{tabular}


23. Arbeitsbezogene Verhaltens- und Erlebensmuster

Wir bitten Sie hier einige Ihrer üblichen Verhaltensweisen, Einstellungen und Gewohnheiten zu beschreiben, die Sie in Ihrem Beruf als Sportlehrer vertreten bzw. kennzeichnen.

Aussagen: Lesen Sie jeden dieser Sätze gründlich durch und entscheiden Sie, in welchem Maße er auf Sie persönlich zutrifft.

Die Arbeit ist für mich der wichtigste Lebensinhalt.

Ich möchte beruflich weiter kommen, als es die meisten meiner

Bekannten geschafft haben.

Wenn es sein muss, arbeite ich bis zur Erschöpfung.

Meine Arbeit soll stets ohne Fehl und Tadel sein.

Zum Feierabend ist die Arbeit für mich gegessen.

Wenn ich keinen Erfolg habe, resigniere ich schnell.

Für mich sind Schwierigkeiten dazu da, dass ich sie überwinde.

Mich bringt so leicht nichts aus der Ruhe.

Mein bisheriges Berufsleben war recht erfolgreich.

Mit meinem bisherigen Leben kann ich zufrieden sein.

Mein(e) Partner(in) ${ }^{2}$ zeigt Verständnis für meine Arbeit.

Die Arbeit ist mein ein und alles.

Berufliche Karriere bedeutet mir wenig.

Bei der Arbeit kenne ich keine Schonung.

Ich kontrolliere lieber noch dreimal nach, als dass ich fehlerhafte

Arbeitsergebnisse abliefere.

Auch in der Freizeit beschäftigen mich viele Arbeitsprobleme

Misserfolg kann ich nur schwer verkraften.

Wenn mir etwas nicht gelingt, sage ich mir: Jetzt erst recht!

Ich bin ein ruheloser Mensch.

In meiner bisherigen Berufslaufbahn habe ich mehr Erfolg als

Enttäuschungen erlebt.

Im Großen und Ganzen bin ich glücklich und zufrieden.

Meine Familie interessiert sich nur wenig für meine Arbeitsprobleme.

Ich könnte auch ohne meine Arbeit ganz glücklich sein.

Was meine berufliche Entwicklung angeht, so halte ich mich für ziemlich ehrgeizig.

Ich arbeite wohl mehr als ich sollte.

Bei meiner Arbeit habe ich den Ehrgeiz, keinerlei Fehler zu machen.

Nach der Arbeit kann ich ohne Probleme abschalten.

Berufliche Fehlschläge können mich leicht entmutigen.

Misserfolge werfen mich nicht um, sondern veranlassen mich zu noch stärkerer Anstrengung.

Ich glaube, dass ich ziemlich hektisch bin.

Wirkliche berufliche Erfolge sind mir bisher versagt geblieben.

Ich habe allen Grund, meine Zukunft optimistisch zu sehen.

Von meinem Partner/meiner Partnerin ${ }^{3}$ wünschte ich mir mehr

Rücksichtnahme auf meine beruflichen Aufgaben und Probleme.

Ich brauche die Arbeit wie die Luft zum Atmen.

Ich strebe nach höheren beruflichen Zielen als die meisten anderen.

Ich neige dazu, über meine Kräfte hinaus zu arbeiten.

Was immer ich tue, es muss perfekt sein.

Feierabend ist Feierabend, da verschwende ich keine Gedanken mehr an die Arbeit.

Wenn ich in der Arbeit erfolglos bin, deprimiert mich das sehr.

\begin{tabular}{|c|c|c|c|c|}
\hline $\begin{array}{c}\text { trifft } \\
\text { überhaupt } \\
\text { nicht zu }\end{array}$ & $\begin{array}{c}\text { über- } \\
\text { wiegend } \\
\text { nicht zu }\end{array}$ & teils/teils & $\begin{array}{l}\text { trifft über- } \\
\text { wiegend zu }\end{array}$ & $\begin{array}{c}\text { trifft völlig } \\
z u\end{array}$ \\
\hline 口 & 口 & 口 & 口 & 口 \\
\hline 口 & 口 & $\square$ & 口 & 口 \\
\hline 口 & 口 & $\square$ & $\square$ & D \\
\hline 口 & 口 & 口 & 口 & 口 \\
\hline 口 & 口 & 口 & 口 & 口 \\
\hline 口 & 口 & 口 & 口 & 口 \\
\hline 口 & a & D & a & a \\
\hline 口 & 口 & 口 & D & 口 \\
\hline$\square$ & 口 & 口 & 口 & 口 \\
\hline D & D & D & D & D \\
\hline a & D & 口 & a & a \\
\hline 口 & a & a & D & a \\
\hline 口 & $\square$ & 口 & a & a \\
\hline a & a & 口 & 口 & 口 \\
\hline 口 & a & 口 & 口 & 口 \\
\hline 口 & a & a & 口 & a \\
\hline a & a & 口 & 口 & 口 \\
\hline 口 & 口 & 口 & 口 & 口 \\
\hline a & a & 口 & 口 & a \\
\hline 口 & ص & ص & 口 & a \\
\hline 口 & a & 口 & a & 口 \\
\hline 口 & 口 & $\square$ & $\square$ & a \\
\hline a & 口 & $\square$ & $\square$ & a \\
\hline ם & ص & 口 & 口 & 口 \\
\hline a & a & 口 & 口 & a \\
\hline a & ט & a & a & a \\
\hline 口 & $\square$ & $\square$ & $\square$ & 口 \\
\hline a & a & a & 口 & a \\
\hline 口 & ص & ص & 口 & ט \\
\hline 口 & a & 口 & 口 & a \\
\hline 口 & $\square$ & $\square$ & $\square$ & $\square$ \\
\hline 口 & 口 & $\square$ & $\square$ & 口 \\
\hline a & ص & 口 & 口 & 口 \\
\hline 口 & $\square$ & 口 & 口 & 口 \\
\hline a & a & a & 口 & 口 \\
\hline 口 & 口 & $\square$ & $\square$ & $\square$ \\
\hline a & a & a & a & 口 \\
\hline 口 & ص & ص & 口 & 口 \\
\hline 口 & D & 口 & D & 口 \\
\hline
\end{tabular}

2/3 Bzw. die Person, zu der die engste persönliche Bindung besteht. 


\begin{tabular}{|c|c|c|c|c|c|}
\hline & $\begin{array}{c}\text { trifft } \\
\text { überhaupt } \\
\text { nicht zu }\end{array}$ & $\begin{array}{l}\text { trifft über- } \\
\text { wiegend } \\
\text { nicht zu }\end{array}$ & teils/teils & $\begin{array}{l}\text { trifft über- } \\
\text { wiegend zu }\end{array}$ & $\begin{array}{l}\text { trifft völlig } \\
\mathrm{zu}\end{array}$ \\
\hline $\begin{array}{l}\text { Ich bin mir sicher, dass ich auch die künftigen Anforderungen des } \\
\text { Lebens gut bewältigen kann. }\end{array}$ & a & a & $\square$ & 口 & 口 \\
\hline Ich glaube, ich bin ein ruhender Pol in meinem Umfeld. & a & $\square$ & $\square$ & $\square$ & $\square$ \\
\hline In meiner beruflichen Entwicklung ist mir bisher fast alles gelungen. & $\square$ & $\square$ & $\square$ & $\square$ & $\square$ \\
\hline Ich kann mich über mein Leben in keiner Weise beklagen. & ב & a & a & ב & a \\
\hline Bei meiner Familie finde ich jede Unterstützung. & $\square$ & $\square$ & $\square$ & $\square$ & $\square$ \\
\hline Ich wüsste nicht wie ich ohne Arbeit leben sollte. & $\square$ & $\square$ & $\square$ & $\square$ & $\square$ \\
\hline Für meine berufliche Zukunft habe ich mir viel vorgenommen. & 口 & 口 & ב & 口 & 口 \\
\hline Mein Tagesablauf ist durch chronischen Zeitmangel bestimmt. & $\square$ & $\square$ & $\square$ & 口 & $\square$ \\
\hline $\begin{array}{l}\text { Für mich ist die Arbeit erst dann getan, wenn ich rundum mit dem } \\
\text { Ergebnis zufrieden bin. }\end{array}$ & $\square$ & $\square$ & $\square$ & $\square$ & $\square$ \\
\hline Arbeitsprobleme beschäftigen mich eigentlich den ganzen Tag. & $\square$ & $\square$ & $\square$ & $\square$ & $\square$ \\
\hline $\begin{array}{l}\text { Ich verliere leicht den Mut, wenn ich trotz Anstrengung keinen Erfolg } \\
\text { habe. }\end{array}$ & $\square$ & $\square$ & ם & व & $\square$ \\
\hline Ein Misserfolg kann bei mir neue Kräfte wecken. & $\square$ & $\square$ & $\square$ & $\square$ & $\square$ \\
\hline Ich kann mich in fast allen Situationen ruhig und bedächtig verhalten. & $\square$ & $\square$ & $\square$ & $\square$ & $\square$ \\
\hline Mein bisheriges Leben ist durch beruflichen Erfolg gekennzeichnet. & ב & ב & ב & ב & ב \\
\hline Von manchen Seiten des Lebens bin ich ziemlich enttäuscht. & $\square$ & $\square$ & 口 & $\square$ & $\square$ \\
\hline $\begin{array}{l}\text { Manchmal wünschte ich mir mehr Unterstützung durch die Menschen } \\
\text { meiner Umgebung. }\end{array}$ & $\square$ & 口 & ב & 口 & 口 \\
\hline Es gibt wichtigeres im Leben als die Arbeit. & $\square$ & $\square$ & $\square$ & $\square$ & $\square$ \\
\hline Beruflicher Erfolg ist für mich ein wichtiges Lebensziel. & $\square$ & $\square$ & $\square$ & 口 & $\square$ \\
\hline In der Arbeit verausgabe ich mich stark. & $\square$ & $\square$ & $\square$ & $\square$ & $\square$ \\
\hline $\begin{array}{l}\text { Es widerstrebt mir, wenn ich eine Arbeit abschließen muss, obwohl } \\
\text { sie noch verbessert werden könnte. }\end{array}$ & a & a & ם & a & a \\
\hline Meine Gedanken kreisen fast nur um die Arbeit. & $\square$ & $\square$ & $\square$ & 口 & $\square$ \\
\hline $\begin{array}{l}\text { Wenn ich irgendwo versagt habe, kann mich das ziemlich mutlos } \\
\text { machen. }\end{array}$ & $\square$ & $\square$ & $\square$ & $\square$ & $\square$ \\
\hline $\begin{array}{l}\text { Wenn mir etwas nicht gelingt, bleibe ich hartnäckig und strenge mich } \\
\text { umso mehr an. }\end{array}$ & $\square$ & a & $\square$ & $\square$ & $\square$ \\
\hline Hektik und Aufregung um mich herum lassen mich kalt. & 口 & 口 & 口 & 口 & $\square$ \\
\hline Meine beruflichen Leistungen können sich sehen lassen. & $\square$ & $\square$ & $\square$ & $\square$ & $\square$ \\
\hline Es dürfte nur wenige glücklichere Menschen geben als ich es bin. & $\square$ & $\square$ & $\square$ & a & $\square$ \\
\hline Wenn ich mal Rat und Hilfe brauche ist immer jemand da. & $\square$ & $\square$ & $\square$ & 口 & $\square$ \\
\hline 24. Meine Lehrweise ist überwiegend ...! & $\square$ & induktiv & $\square$ & deduktiv & \\
\hline $\begin{array}{l}\text { 25. Bitte schätzen Sie die Organisationsform Ihres Spor } \\
\square \quad \text { stark lehrerzentriert } \\
\square\end{array}$ & unterricht & \multicolumn{4}{|c|}{ ausgewogen ( $\mathrm{mal}$ so mal so) } \\
\hline \multicolumn{6}{|c|}{ 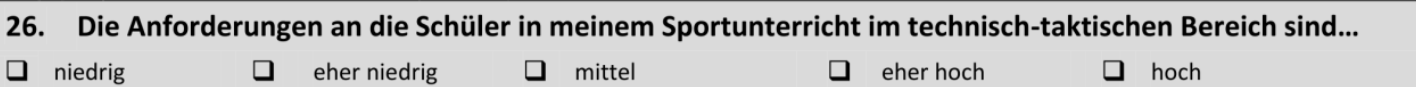 } \\
\hline \multicolumn{6}{|c|}{ 27. Die Anforderungen an die Schüler in meinem Sportunterricht im konditionellen Bereich sind... } \\
\hline \multicolumn{6}{|c|}{ 28. Wie würden Sie die Atmosphäre im gesamten Kolleqium Ihrer Schule beschreiben? } \\
\hline$\square$ (sehr) schlecht $\quad \square \quad$ eher schlecht $\quad \square$ & mittel & 口 & eher gut & 口 (sehr) & \\
\hline \multicolumn{6}{|c|}{ 29. Wie würden Sie die Atmosphäre im Sportkollegium Ihrer Schule beschreiben? } \\
\hline \multicolumn{6}{|c|}{ 30. Welchen Stellenwert hat der Sportunterricht für Sie persönlich im Vergleich mit anderen Fächern? } \\
\hline
\end{tabular}




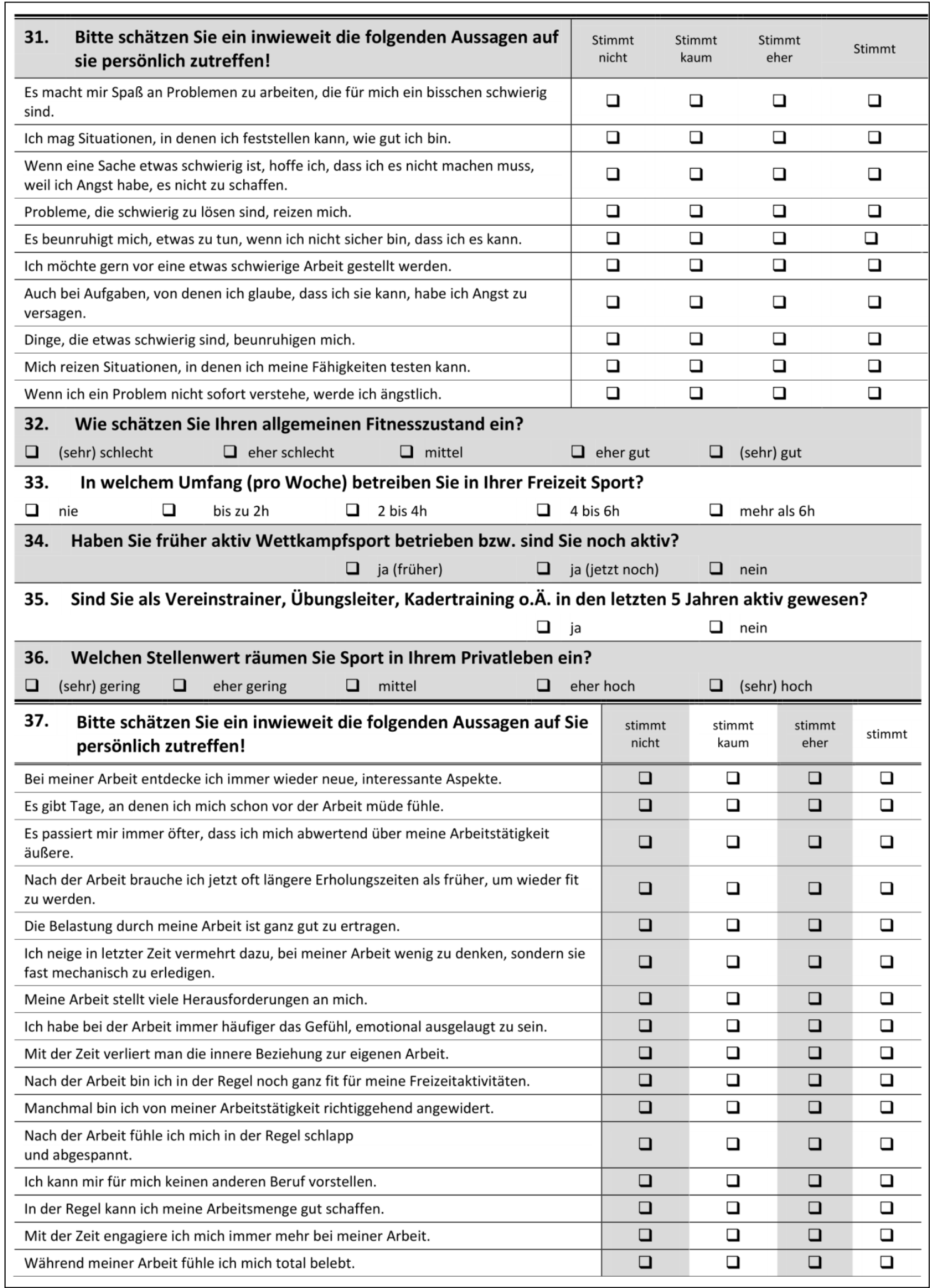




\begin{tabular}{|c|c|c|c|c|c|c|}
\hline $\begin{array}{l}\text { 38. Bitte treffen Sie auch hier eine Auswahl inwieweit die } \\
\text { folgenden allgemein formulierten Aussagen auf Sie } \\
\text { zutreffen! }\end{array}$ & $\begin{array}{l}\text { trifft } \\
\text { über- } \\
\text { haupt } \\
\text { nicht zu }\end{array}$ & $\begin{array}{l}\text { trifft } \\
\text { weit- } \\
\text { gehend } \\
\text { nicht } \\
\text { zu }\end{array}$ & $\begin{array}{l}\text { trifft } \\
\text { eher } \\
\text { nicht } \\
\text { zu }\end{array}$ & $\begin{array}{l}\text { trifft } \\
\text { eher zu }\end{array}$ & $\begin{array}{l}\text { trifft } \\
\text { weit- } \\
\text { gehend } \\
\text { zu }\end{array}$ & $\begin{array}{c}\text { trifft } \\
\text { genau } \\
\text { zu }\end{array}$ \\
\hline $\begin{array}{l}\text { Es hängt hauptsächlich von mir ab, ob sich andere Menschen nach meinen } \\
\text { Wünschen richten oder nicht. }\end{array}$ & $\square$ & $\square$ & a & $\square$ & $\square$ & $\square$ \\
\hline $\begin{array}{l}\text { Zufällige Geschehnisse bestimmen einen großen Teil meines Lebens und } \\
\text { Alltags. }\end{array}$ & $\square$ & $\square$ & a & $\square$ & $\square$ & 口 \\
\hline $\begin{array}{l}\text { Ich habe das Gefühl, dass vieles von dem, was in meinem Leben passiert, von } \\
\text { anderen Menschen abhängt. }\end{array}$ & ם & $\square$ & a & $\square$ & 口 & $\square$ \\
\hline Ich komme mir manchmal taten- und ideenlos vor. & 口 & $\square$ & ב & ב & 口 & 口 \\
\hline $\begin{array}{l}\text { Ob ich einen Unfall habe oder nicht, hängt alleine von mir und meinem } \\
\text { Verhalten ab. }\end{array}$ & 口 & $\square$ & a & $\square$ & $\square$ & $\square$ \\
\hline $\begin{array}{l}\text { Wenn ich Pläne schmiede, bin ich mir ganz sicher, dass das Geplante auch } \\
\text { Wirklichkeit wird. }\end{array}$ & 口 & $\square$ & a & $\square$ & 口 & $\square$ \\
\hline Ich habe oft einfach keine Möglichkeiten, mich vor Pech zu schützen. & $\square$ & a & a & a & $\square$ & 口 \\
\hline $\begin{array}{l}\text { Mehrdeutige Situationen mag ich nicht, da ich nicht weiß, wie ich mich } \\
\text { verhalten soll. }\end{array}$ & 口 & $\square$ & ם & $\square$ & $\square$ & $\square$ \\
\hline Wenn ich bekomme, was ich will, so spielt Glück meistens auch eine Rolle. & $\square$ & $\square$ & 口 & $\square$ & $\square$ & $\square$ \\
\hline Andere Menschen verhindern oft die Verwirklichung meiner Pläne. & $\square$ & $\square$ & $\square$ & $\square$ & $\square$ & $\square$ \\
\hline $\begin{array}{l}\text { Ich kann mich am besten selbst durch mein Verhalten vor Krankheiten } \\
\text { schützen. }\end{array}$ & 口 & $\square$ & a & 口 & $\square$ & $\square$ \\
\hline Ich weiß oft nicht, wie ich meine Wünsche verwirklichen soll. & 口 & a & a & $\square$ & 口 & a \\
\hline Vieles von dem, was in meinem Leben passiert, hängt vom Zufall ab. & $\square$ & $\square$ & ם & $\square$ & $\square$ & $\square$ \\
\hline $\begin{array}{l}\text { Mein Leben und Alltag werden in vielen Bereichen von anderen Menschen } \\
\text { bestimmt. }\end{array}$ & $\square$ & $\square$ & 口 & $\square$ & $\square$ & $\square$ \\
\hline Ob ich einen Unfall habe oder nicht, ist vor allem Glücksache. & 口 & $\square$ & 口 & $\square$ & 口 & $\square$ \\
\hline Ich kenne viele Möglichkeiten, mich vor Erkrankungen zu schützen. & $\square$ & $\square$ & $\square$ & $\square$ & $\square$ & $\square$ \\
\hline $\begin{array}{l}\text { Ich habe nur geringe Möglichkeiten, meine Interessen gegen andere Leute } \\
\text { durchzusetzen. }\end{array}$ & $\square$ & $\square$ & 口 & $\square$ & $\square$ & $\square$ \\
\hline $\begin{array}{l}\text { Es ist für mich nicht gut, weit im Voraus zu planen, da häufig das Schicksal } \\
\text { dazwischenkommt. }\end{array}$ & 口 & $\square$ & a & 口 & $\square$ & $\square$ \\
\hline $\begin{array}{l}\text { Um das zu bekommen, was ich will, muss ich zu anderen Menschen } \\
\text { freundlich und zuvorkommend sein. }\end{array}$ & 口 & $\square$ & 口 & $\square$ & $\square$ & $\square$ \\
\hline In unklaren oder gefährlichen Situationen weiß ich immer, was ich tun kann. & $\square$ & $\square$ & $\square$ & $\square$ & $\square$ & $\square$ \\
\hline $\begin{array}{l}\text { Es ist reiner Zufall, wenn sich andere Menschen einmal nach meinen } \\
\text { Wünschen richten. }\end{array}$ & 口 & $\square$ & a & 口 & $\square$ & $\square$ \\
\hline $\begin{array}{l}\text { Mein Wohlbefinden hängt in starkem Maße vom Verhalten anderer } \\
\text { Menschen ab. }\end{array}$ & $\square$ & $\square$ & 口 & $\square$ & 口 & $\square$ \\
\hline Ich kann sehr viel von dem, was in meinem Leben passiert, selbst bestimmen. & $\square$ & $\square$ & 口 & $\square$ & $\square$ & $\square$ \\
\hline Manchmal weiß ich überhaupt nicht, was ich in einer Situation machen soll. & $\square$ & $\square$ & $\square$ & $\square$ & $\square$ & $\square$ \\
\hline $\begin{array}{l}\text { Gewöhnlich kann ich meine Interessen selbst vertreten und erreiche dabei } \\
\text { das, was ich will. }\end{array}$ & 口 & 口 & 口 & $\square$ & 口 & $\square$ \\
\hline $\begin{array}{l}\text { Ob ich einen Unfall habe oder nicht, hängt in starkem Maße von dem } \\
\text { Verhalten anderer ab. }\end{array}$ & $\square$ & $\square$ & 口 & $\square$ & $\square$ & $\square$ \\
\hline $\begin{array}{l}\text { Wenn ich bekomme, was ich will, so ist das immer eine Folge meiner } \\
\text { Anstrengung und meines persönlichen Einsatzes. }\end{array}$ & 口 & $\square$ & a & $\square$ & $\square$ & $\square$ \\
\hline $\begin{array}{l}\text { Auch in schwierigen Situationen fallen mir immer viele } \\
\text { Handlungsalternativen ein. }\end{array}$ & $\square$ & $\square$ & a & $\square$ & $\square$ & $\square$ \\
\hline $\begin{array}{l}\text { Damit meine Pläne eine Chance haben, richte ich mich beim Planen nach den } \\
\text { Wünschen anderer Leute. }\end{array}$ & 口 & $\square$ & a & 口 & $\square$ & 口 \\
\hline $\begin{array}{l}\text { Mein Lebenslauf und mein Alltag werden alleine durch mein Verhalten und } \\
\text { meine Wünsche bestimmt. }\end{array}$ & 口 & $\square$ & 口 & $\square$ & 口 & $\square$ \\
\hline Es hängt vom Schicksal ab, ob ich krank werde oder nicht. & $\square$ & $\square$ & $\square$ & $\square$ & $\square$ & $\square$ \\
\hline Für die Lösung von Problemen fallen mir immer viele Möglichkeiten ein. & $\square$ & $\square$ & $\square$ & $\square$ & $\square$ & $\square$ \\
\hline
\end{tabular}




\begin{tabular}{|c|c|c|c|c|c|c|}
\hline 39. & $\begin{array}{l}\text { Wenn ich durch irgendetwas oder irgendjemand } \\
\text { beeinträchtigt, innerlich erregt oder aus dem } \\
\text { Gleichgewicht gebracht worden bin... }\end{array}$ & gar nicht & kaum & $\begin{array}{l}\text { möglicher- } \\
\text { weise }\end{array}$ & $\begin{array}{l}\text { eher wahr- } \\
\text { scheinlich }\end{array}$ & $\begin{array}{l}\text { wahr- } \\
\text { scheinlich }\end{array}$ \\
\hline \multicolumn{2}{|r|}{...überlege ich, wie ich von nun an solchen Situationen ausweichen kann } & $\square$ & $\square$ & $\square$ & $\square$ & a \\
\hline \multicolumn{2}{|r|}{...weiß ich nicht, wie ich gegen die Situation ankommen könnte } & ב & $\square$ & ב & a & a \\
\hline \multicolumn{2}{|c|}{...fühle ich mich irgendwie hilflos } & ב & ב & a & ב & a \\
\hline \multicolumn{2}{|c|}{...versuche ich, mich der Situation zu entziehen } & $\square$ & 口 & a & $\square$ & $\square$ \\
\hline \multicolumn{2}{|c|}{...neige ich dazu, alles sinnlos zu finden } & $\square$ & 口 & $\square$ & 口 & $\square$ \\
\hline \multicolumn{2}{|c|}{...kommen mir Fluchtgedanken } & $\square$ & $\square$ & $\square$ & $\square$ & $\square$ \\
\hline \multicolumn{2}{|c|}{...tue ich etwas, was mich davon ablenkt } & $\square$ & $\square$ & $\square$ & $\square$ & $\square$ \\
\hline \multicolumn{2}{|c|}{...gehe ich irgendeiner anderen Beschäftigung nach } & ב & a & ב & ב & ב \\
\hline \multicolumn{2}{|r|}{$\begin{array}{l}\text {...passe ich auf, dass es in Zukunft gar nicht erst zu solchen Situationen } \\
\text { kommt }\end{array}$} & $\square$ & $\square$ & 口 & a & a \\
\hline \multicolumn{2}{|c|}{...stürze ich mich in die Arbeit } & $\square$ & $\square$ & $\square$ & $\square$ & $\square$ \\
\hline \multicolumn{2}{|r|}{...versuche ich meine Aufmerksamkeit davon abzuwenden } & $\square$ & 口 & $\square$ & a & $\square$ \\
\hline \multicolumn{2}{|c|}{...lenke ich mich irgendwie ab } & $\square$ & $\square$ & $\square$ & $\square$ & $\square$ \\
\hline \multicolumn{2}{|c|}{...vermeide ich von nun an solche Situationen } & $\square$ & 口 & $\square$ & ב & a \\
\hline \multicolumn{2}{|r|}{$\begin{array}{l}\text {...nehme ich mir vor, solchen Situationen in Zukunft aus dem Weg zu } \\
\text { gehen }\end{array}$} & $\square$ & $\square$ & 口 & 口 & 口 \\
\hline \multicolumn{2}{|c|}{...denke ich „möglichst von hier weg“ } & ב & ב & ב & ב & ב \\
\hline \multicolumn{2}{|c|}{...möchte ich am liebsten einfach weglaufen } & $\square$ & 口 & $\square$ & 口 & a \\
\hline \multicolumn{2}{|r|}{...versuche ich meine Gedanken auf etwas anderes zu konzentrieren } & $\square$ & $\square$ & $\square$ & $\square$ & $\square$ \\
\hline \multicolumn{2}{|c|}{...neige ich dazu, die Flucht zu ergreifen } & $\square$ & $\square$ & $\square$ & 口 & $\square$ \\
\hline \multicolumn{2}{|r|}{$\begin{array}{l}\text {...habe ich nur den Wunsch, dieser Situation so schnell wie möglich zu } \\
\text { entkommen }\end{array}$} & $\square$ & 口 & 口 & $\square$ & 口 \\
\hline \multicolumn{2}{|r|}{...denke ich, in Zukunft will ich nicht mehr in solche Situationen geraten } & $\square$ & 口 & $\square$ & $\square$ & 口 \\
\hline \multicolumn{2}{|c|}{...neige ich dazu zu resignieren } & $\square$ & $\square$ & $\square$ & $\square$ & $\square$ \\
\hline \multicolumn{2}{|c|}{...neige ich dazu, schnell aufzugeben } & $\square$ & $\square$ & $\square$ & $\square$ & $\square$ \\
\hline \multicolumn{2}{|r|}{$\begin{array}{l}\text {...entziehe ich mich in Zukunft schon bei den ersten Anzeichen solchen } \\
\text { Situationen }\end{array}$} & $\square$ & $\square$ & $\square$ & 口 & $\square$ \\
\hline \multicolumn{2}{|c|}{...erscheint mir alles so hoffnungslos } & $\square$ & $\square$ & $\square$ & a & a \\
\hline \multirow[t]{2}{*}{40.} & \multicolumn{4}{|c|}{$\begin{array}{l}\text { Wie viele fächerübergreifende Fortbildungen haben Sie im letzten Jahr (in } \\
\text { den letzten } 365 \text { Tagen ab dem heutigen Tag gerechnet) besucht (in Tagen)? }\end{array}$} & \multicolumn{2}{|c|}{- Tage } \\
\hline & \multicolumn{4}{|c|}{$\begin{array}{l}\text { Wie viele sportunterrichtsspezifische Fortbildungen haben Sie im letzten } \\
\text { Jahr (in den letzten } 365 \text { Tagen ab dem heutigen Tag gerechnet) besucht (in Tagen)? }\end{array}$} & \multicolumn{2}{|c|}{ — Tage } \\
\hline
\end{tabular}

41. Wie oft bilden Sie sich privat sportunterrichtsspezifisch fort (z.B. durch Zeitschriften, Fachbücher etc.)?
a sehr selten/nie
a selten
a $a b$ und zu
a oft
sehr oft

42. Welche Gefühle und Erfahrungen verbinden Sie mit Ihrer bisherigen Karriere als Sportlehrer?
ㅁ negative
a eher negative
口 neutrale
$\square$ eher positive
positive

43. Mit welchen Gefühle und Erfahrungen gehen Sie in Ihre Zukunft als Sportlehrer?
a negative
a eher negative
口 neutrale
a eher positive
positive

44. An wie vielen Tagen fehlten Sie im letzten Jahr (in den letzen 365 Tagen ab dem heutigen Tag gerechnet) krankheitsbedingt?

45. Planen Sie in Zukunft einen Berufswechsel bzw. Ausstieg?

口 ja (schon bald)

$\square$ ja (auf längere Sicht) $\square$ nein

Vielen Dank für Ihre Mitarbeit! :-

(Bitte kontrollieren Sie ob Sie auch wirklich alle Fragen beantwortet haben!) 


\title{
V. Lebenslauf
}

\section{Persönliche Daten}

Name:

\author{
Christian Belz \\ 26.10.1980 in Fulda \\ deutsch \\ katholisch \\ verheiratet
}

Geburtstag und -ort:

Staatsangehörigkeit:

Konfession:

Familienstand:

\section{Schulbildung und Studium}

$1987-1991$

Grundschule Flieden

$1991-2000$

09.06 .2000

$2001-2006$

02.05.2006

01.08.2006-31-07.2009

13.12.2006

23.05.2008

01.08.2009-31.07.2012

05.04.2011

seit 05.08.2011

Freiherr-vom-Stein-Gymnasium Fulda

Abschluss mit der Allgemeinen Hochschulreife am Freiherr-vomStein-Gymnasium Fulda

Studium an der Universität Kassel: Lehramt an Gymnasien Fächer: Sport und Biologie

1. Staatsexamen (mit Auszeichnung bestanden) für Lehramt an Gymnasien an der Universität Kassel

Promotionsstipendium der Deutschen Forschungsgesellschaft im interdisziplinären Graduiertenkolleg 1195 „Passungsverhältnisse schulischen Lernens - Verstehen und Optimieren" an der GeorgAugust-Universität-Göttingen

„Dietrich-Martin-Preis“ für die besten Studien- und Prüfungsleistungen des Institut für Sportwissenschaft der Universität Kassel

1. Posterpreis der Deutschen Vereinigung für Sportwissenschaft (DVS) auf der Jahrestagung der Sektion Sportpädagogik in Köln

Referendariat (Diltheyschule Wiesbaden)

2. Staatsexamen (Abschlussnote "sehr gut") in den Fächern Biologie und Sport

Studienrat an der Diltheyschule -alt- und neusprachliches Gymnasium in Wiesbaden

\section{Praktische Erfahrungen}

$05 / 2003-01 / 2006$

2007-2009

Studentischer Mitarbeiter im Projektteam „Talentsuche Talentförderung" des IFSS an der Universität Kassel unter der Leitung von Prof. Dr. Volker Scheid und Mitarbeit am Abschlussbericht.

Lehrtätigkeit am Institut für Sportwissenschaft, Georg-AugustUniversität Göttingen

\section{Sonstige Qualifikationen /Sprachen}

- EDV-Kenntnisse (Office, SPSS)

- Englisch, Französisch 